Institut für Konstruktion und Entwurf

Stahl- Holz- und Verbundbau

Universität Stuttgart

\title{
Zur Bestimmung der Tragfähigkeit von Kehlnahtverbindungen höherfester Baustähle
}

Christina Rasche

Mitteilungen 



\title{
Zur Bestimmung der Tragfähigkeit von Kehlnahtverbindungen höherfester Baustähle
}

\author{
Von der Fakultät Bau- und Umweltingenieurwissenschaften \\ der Universität Stuttgart zur Erlangung der Würde eines Doktors \\ der Ingenieurwissenschaften (Dr.-Ing.) genehmigte Abhandlung
}

Vorgelegt von

Christina Rasche

aus Marsberg

Hauptberichterin: Prof. Dr.-Ing. Ulrike Kuhlmann

Mitberichter: Prof. Dr.-Ing. habil. Frank Werner

Tag der mündlichen Prüfung: 15.12.2011

Institut für Konstruktion und Entwurf der Universität Stuttgart 
Mitteilungen des Instituts für Konstruktion und Entwurf; Nr. 2012-1

Christina Rasche Zur Bestimmung der Tragfähigkeit von Kehlnahtverbindungen höherfester Baustähle

Herausgeber

Prof. Dr.-Ing. Ulrike Kuhlmann

Pfaffenwaldring 7

70569 Stuttgart

Telefon: (0711) 68566245

Telefax: (0711) 68566236

Redaktion Bernadette Froschmeier

D 93

(c) Institut für Konstruktion und Entwurf; Stuttgart 2012

Alle Rechte vorbehalten

ISSN 1439-3751 


\section{Kurzdarstellung}

Im Stahlbau gibt es einen Trend zu zunehmend schlankeren und leichten Konstruktionen aus Baustählen mit höherer Festigkeit. Für die Verwendung dieser Stähle spricht neben rein ästhetischen Gründen das Einsparpotential beim Material und damit einhergehend die Reduktion des Gewichts. In den letzten Jahren wurden hierzu moderne Stähle entwickelt, die neben einer hohen Festigkeit gute Schweißeigenschaften und eine hohe Zähigkeit besitzen.

Trotz der guten Schweißeigenschaften ist die normative Tragfähigkeit von Kehlnahtverbindungen dieser Stähle gering. In den meisten bisher geltenden Normen wird für die Tragfähigkeit der Schweißnaht nur die Festigkeit des Grundwerkstoffs und nicht die des Schweißzusatzwerkstoffs berücksichtigt. Für normalfeste Baustähle S235 und S355 ist diese Vereinfachung sinnvoll, da die zur Verfügung stehenden Schweißzusatzwerkstoffe immer höhere Festigkeiten als diese normalfesten Stähle besitzen. Für höherfeste Stähle S460 und S690 kann für die Fertigung oder in Abhängigkeit von der Beanspruchung sowohl der Einsatz von Schweißzusatzwerkstoffen mit geringerer als auch mit höherer Festigkeit sinnvoll sein. Um diese Werkstoffkombinationen verwenden zu können, müssen die Bemessungsregeln neben der Festigkeit des Grundwerkstoffs auch die Festigkeit des Schweißzusatzwerkstoffes berücksichtigen. Voraussetzung dafür ist die genaue Kenntnis des Einflusses, den die Festigkeit des Schweißzusatzwerkstoffs auf die Tragfähigkeit von Kehlnahtverbindungen höherfester Baustähle hat.

Das Ziel dieser Arbeit ist daher die Entwicklung eines erweiterten Bemessungsmodells zur Bestimmung der Tragfähigkeit von Kehlnahtverbindungen für höherfester Baustähle S460 und S690 unter Berücksichtigung der Festigkeit des Schweißzusatzwerkstoffs.

In einem ersten Schritt werden im Rahmen dieser Arbeit die vorhandenen Bemessungskonzepte und experimentellen Untersuchungen aus der Literatur analysiert. Für höherfeste Stähle existiert nur eine sehr begrenzte Anzahl an Versuchsergebnissen. Aus diesem Grund bilden die experimentellen Untersuchungen zur Tragfähigkeit von Schweißverbindungen mit den Grundwerkstoffen S460 und S690 unter Variation der Festigkeit des Schweißzusatzwerkstoffs den Schwerpunkt dieser Arbeit. Je nach Belastungsrichtung werden die untersuchten Schweißverbindungen in Flankenkehlnahtverbindungen und Kreuzstöße mit Stirnkehlnähten unterteilt. Eine detaillierte Analyse des Tragverhaltens der Schweißverbindungen in den Versuchen erfolgt über numerische Berechnungen.

Die Ergebnisse der Untersuchungen zeigen einen deutlichen Einfluss des Schweißzusatzwerkstoffs sowie einen geringeren Einfluss des Grundwerkstoffs auf die Tragfähigkeit von Kehlnahtverbindungen. Eine Abhängigkeit der Belastungsrichtung lässt sich durch eine höhere Tragfähigkeit von Kreuzstößen mit Stirnkehlnähten gegenüber Flankenkehlnahtverbindungen nachweisen.

Aufbauend auf diesen Erkenntnissen der experimentellen und numerischen Untersuchungen und auf Basis hieraus gewonnener statistischer Kennwerte erfolgt die Entwicklung des erweiterten Bemessungsmodells, das die Festigkeit von Grund- und Schweißzusatzwerkstoff berücksichtigt. Mit diesem Bemessungsmodell ist eine sichere und wirtschaftliche Auslegung von Kehlnahtverbindungen insbesondere höherfester Baustähle mit unterschiedlichen Schweißzusatzwerkstoffen möglich. 


\begin{abstract}
In recent years a trend towards an increasing number of slim and lightweight steel constructions using high-strength steels can be observed. Beside aesthetic reasons the material and thus the weight saving potential increasingly leads to an application of these steels. Therefore, modern high-strength steels have been developed, which comprise aside a high strength, good welding characteristics and a high ductility.

Despite the good welding characteristics there is a low normative load-bearing capacity of welded joints of high-strength steels. In most current standards, in which the load-bearing capacity of the weld is regulated, only the base metal strength is taken into consideration. The filler metal strength is not taken into account. For the steel grades S235 and S355 this simplification is reasonable, since the applicable filler metals always exhibit a higher strength than the base metals. For high-strength steels S460 and S690 it can be useful in some cases to choose filler metals with higher or with lower strength, dependent on the applied loading or the manufacturing process. To be able to consider different combinations of materials the design rules have to take the base metal strength as well as the filler metal strength into account. For design reasons it is important to have the exact knowledge about the effect of the filler metal strength on the load-bearing capacity of fillet welded joints of high-strength steels.
\end{abstract}

Thus, the main goal of this thesis is the development of an expanded design model to determine the load-bearing capacity of fillet welded joints of high-strength steels S460 and S690 that explicitly considers the filler metal strength.

In a first step the existing design concepts and experimental investigations from literature are analyzed. For high-strength steels only a limited number of test results exists. Therefore the experimental investigations on the load-bearing capacity of fillet welds with the base metals S460 and S690 and the variation of the filler metal strength are in the main focus. Dependent on the load-direction the welded joints can be divided into longitudinal fillet welded connections or cruciform joints with transverse welds. A detailed analysis of the load-bearing behaviour of the welded joints in the tests is done by numeric investigations.

The test results show a dominant influence of the filler metal and in addition a minor influence of the base metal on the load-bearing capacity of fillet welded connections. The dependence on the load direction is evident in a higher load-bearing capacity of cruciform joints with transverse fillet welds in comparison with longitudinal fillet welded connections.

Basing on experimental and numerical investigations and on statistic criteria applied on these results an expanded design model is developed, which considers both, the strength of the base and the filler metal. With this model a safe and economic design of fillet welded joints particularly of high-strength steels and different filler metals is possible. 


\section{Vorwort}

Die vorliegende Arbeit entstand während meiner Tätigkeit als wissenschaftliche Mitarbeiterin am Institut für Konstruktion und Entwurf der Universität Stuttgart.

Mein ganz besonderer Dank gilt Frau Prof. Dr.-Ing. U. Kuhlmann, Leiterin des Instituts, für die Ermöglichung und Förderung meiner Arbeit, das entgegengebrachte Vertrauen während meiner Zeit am Institut für Konstruktion und Entwurf sowie für die Übernahme des Hauptberichts.

Bei Herrn Prof. Dr.-Ing. habil. F. Werner, Leiter des Instituts für Konstruktiven Ingenieurbau, Professur Stahlbau, Bauhaus-Universität Weimar, darf ich mich für das Interesse an der Arbeit, die kritische Durchsicht und die Übernahme des Mitberichts bedanken.

Ein weiterer Dank gilt der Arbeitsgemeinschaft industrieller Forschungsvereinigungen (AiF) „Otto von Guericke“ e.V. für die finanzielle Unterstützung der experimentellen und theoretischen Untersuchungen.

Allen an den Projekten beteiligten Partnern danke ich für die gute Zusammenarbeit.

Auch allen Mitarbeitern der beteiligten Prüfeinrichtungen sei hiermit für die konstruktive und freundliche Unterstützung bei der Herstellung und Durchführung der Versuche gedankt.

Bei meinen ehemaligen Kolleginnen und Kollegen möchte ich mich herzlich für die angenehme Arbeitsatmosphäre und eine schöne Zeit bedanken.

Weiterhin bedanke ich mich bei allen Diplomandinnen und wissenschaftlichen Hilfskräften, die diese Arbeit in verschiedenster Form unterstützt haben.

Jürgen, meiner Familie, meinen Freundinnen und Freunden danke ich aufrichtig für die vielseitige und uneingeschränkte Unterstützung und Geduld. 


\section{Inhaltsverzeichnis}

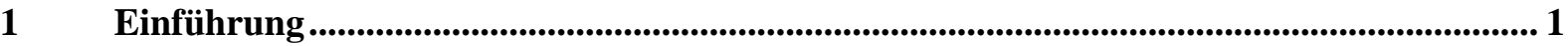

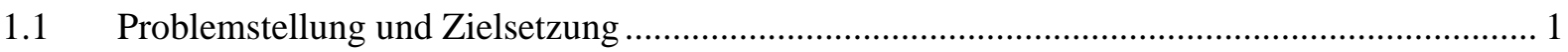

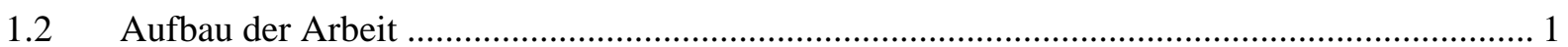

2 Höherfeste Baustähle und ihr Verhalten beim Schweißen ................................................... 3

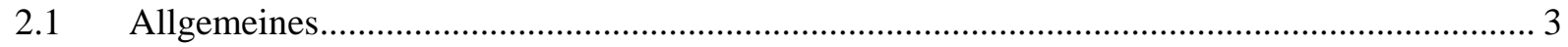

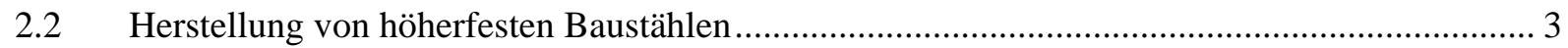

2.3 Werkstoffeigenschaften und Verwendung höherfester Baustähle .............................................. 5

2.4 Verhalten von höherfesten Baustählen beim Schweißen ......................................................... 6

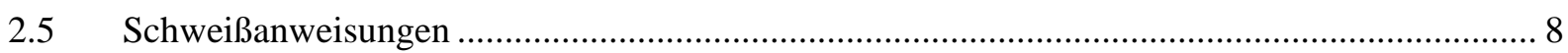

2.6 Bestimmung der Festigkeit im Schweißgut.......................................................................... 11

2.7 Bestimmung der Eigenschaften in der Wärmeeinflusszone................................................... 11

2.8 Bruchmechanische Eigenschaften und Zähigkeit................................................................. 12

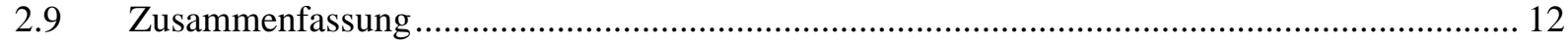

3 Vergleich bestehender Bemessungsverfahren von Schweißverbindungen .......................... 13

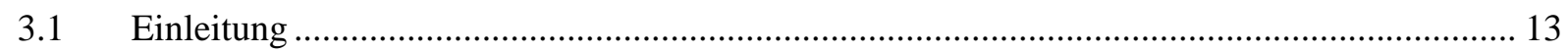

3.2 Beschreibung der verglichenen Normenregeln ................................................................ 13

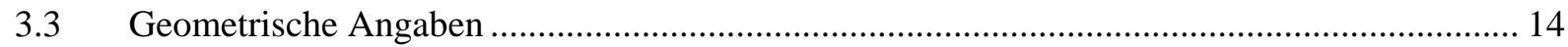

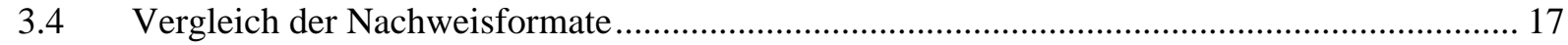

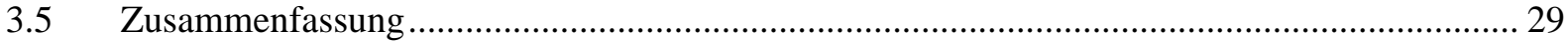

$4 \quad$ Tragverhalten von Kehlnahtverbindungen .................................................................................. 31

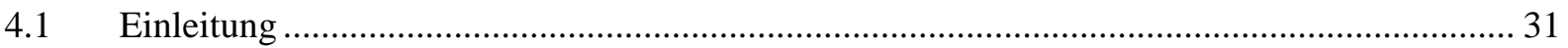

4.2 Bestimmung und Erläuterung zur Festigkeit des Schweißgutes .............................................. 31

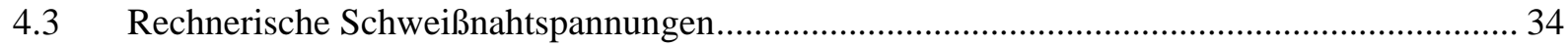

4.4 Ermittlung der Spannungen aus Versuchsergebnissen............................................................... 38

4.5 Beschreibung der statistischen Auswertung............................................................................... 39

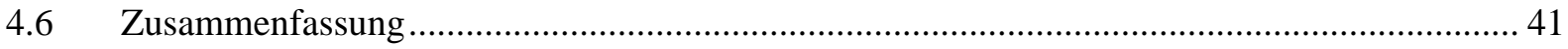

$5 \quad$ Auswertung bekannter Versuchsreihen der Literatur ....................................................... 43

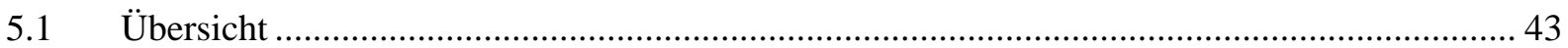

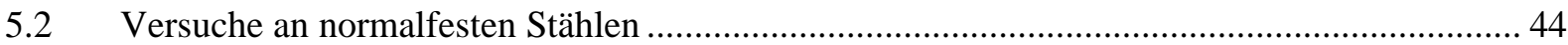

5.3 Kehlnahtverbindungen höherfester Stähle S690 mit niederfestem Schweißzusatzwerkstoff ... 45

5.4 Kehlnahtverbindungen höherfester Stähle HSF 640 mit höherfestem Schweißzusatzwerkstoff

5.5 Kehlnahtverbindungen S460 mit zugehörigem Schweißzusatzwerkstoff ................................ 50

5.6 Kehlnahtverbindungen höherfester Stähle S460 mit höherfestem Schweißzusatzwerkstoff .... 51

5.7 Kehlnahtverbindungen höherfester Stähle S690 und niederfestem Schweißzusatzwerkstoff... 53

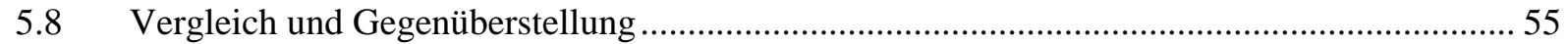

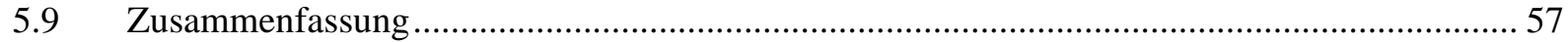


$6 \quad$ Experimentelle Untersuchungen an manuell geschweißten Versuchskörpern.................... 59

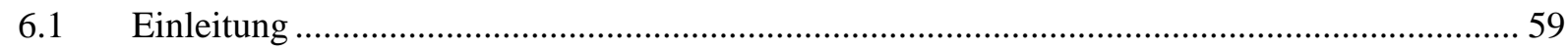

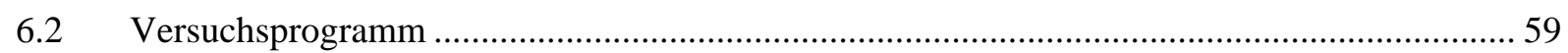

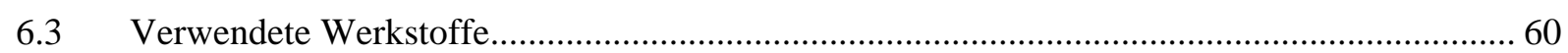

6.4 Herstellung der Versuchskörper und Versuchsdurchführung ................................................. 61

6.5 Überblick über die Versuchsergebnisse an Flankenkehlnahtverbindungen ............................... 62

6.6 Flankenkehlnahtverbindungen mit gleichen Grund- und zugehörigen

Schweißzusatzwerkstoffen

6.7 Flankenkehlnahtverbindungen mit gleichen Grund- und Variation der

Schweißzusatzwerkstoffe

6.8 Flankenkehlnahtverbindungen mit unterschiedlichen Grundwerkstoffen................................. 70

6.9 Überblick über die Versuchsergebnisse an Kreuzstößen ........................................................ 71

6.10 Kreuzstöße mit gleichen Grund- und zugehörigen Schweißzusatzwerkstoffen (BUW) ........... 72

6.11 Kreuzstöße mit gleichen Grund- und Variation der Schweißzusatzwerkstoffe (BUW) ........... 75

6.12 Kreuzstöße mit unterschiedlichen Grundwerkstoffen ................................................................ 76

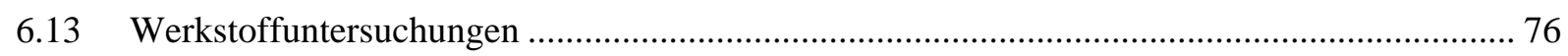

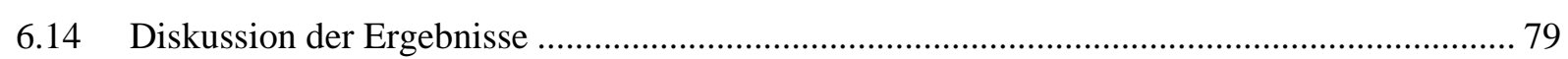

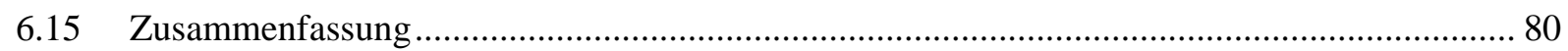

$7 \quad$ Voruntersuchungen an vollmechanisiert geschweißten Versuchskörpern ........................... 81

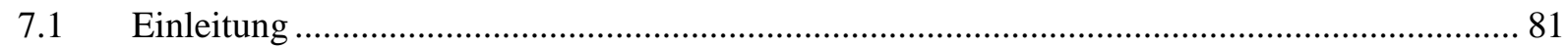

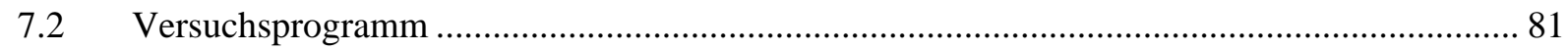

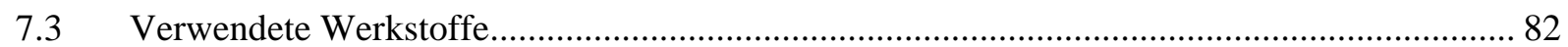

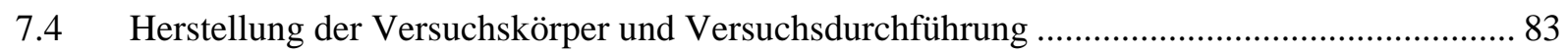

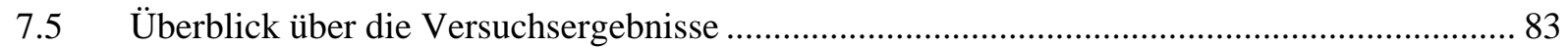

7.6 Tragverhalten bei gleichen Grundwerkstoffen und zugehörigen Schweißzusatzwerkstoffen .. 84

7.7 Tragverhalten bei gleichen Grundwerkstoffen und Variation der Schweißzusatzwerkstoffe ... 84

7.8 Einfluss von Stahlsorte und Schweißzusatzwerkstoff (Serie 5) ................................................ 86

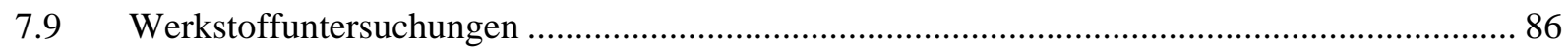

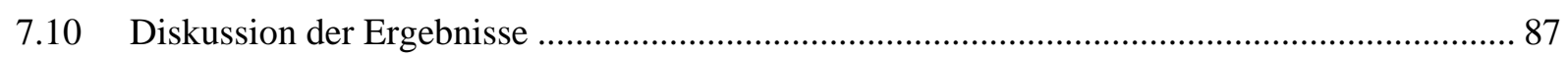

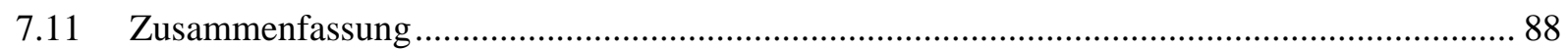

8 Untersuchungen an vollmechanisiert geschweißten Versuchskörpern ................................. 89

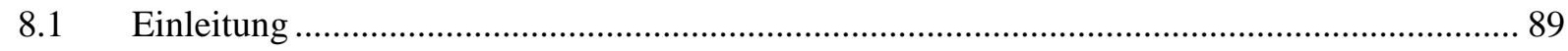

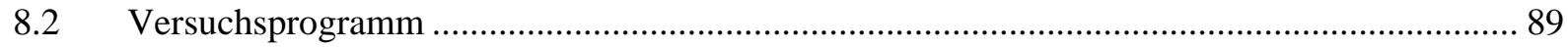

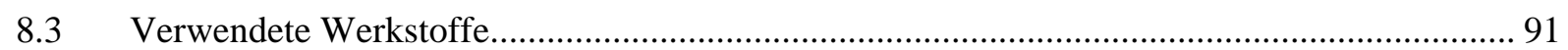

8.4 Herstellung der Versuchskörper und Versuchsdurchführung …............................................... 92

8.5 Überblick über die Versuchsergebnisse an Flankenkehlnahtverbindungen ................................ 93

8.6 Flankenkehlnahtverbindungen mit gleichen Grund- und zugehörigen

Schweißzusatzwerkstoffen 
8.7 Flankenkehlnahtverbindungen mit gleichen Grundwerkstoffen und Variation der

Schweißzusatzwerkstoffe (Serie 5) 109

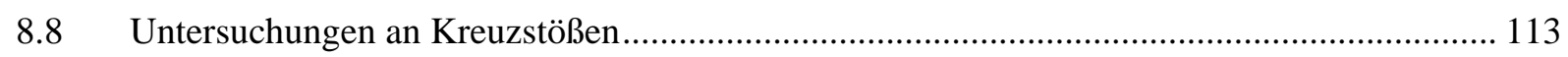

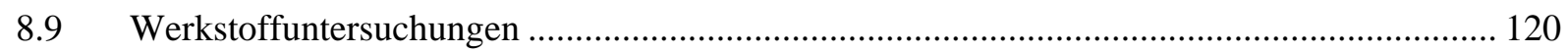

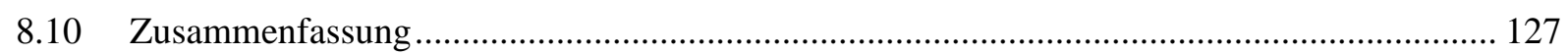

$9 \quad$ Analyse des Tragverhaltens anhand numerischer Untersuchungen .................................. 129

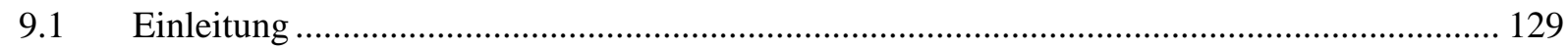

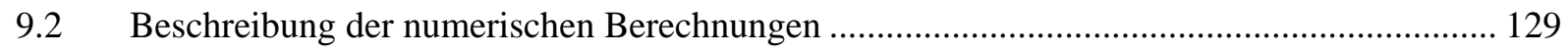

$9.3 \quad$ Voruntersuchungen an Flankenkehlnahtverbindungen ......................................................... 131

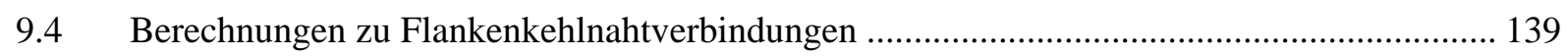

9.5 Berechnungen zu Kreuzstößen...................................................................................... 146

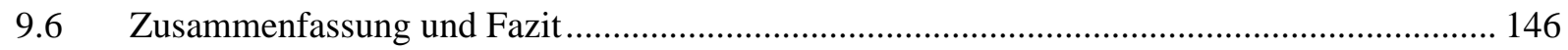

10 Entwicklung eines Bemessungsvorschlags ................................................................................. 149

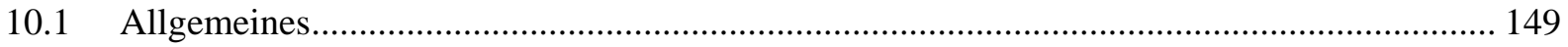

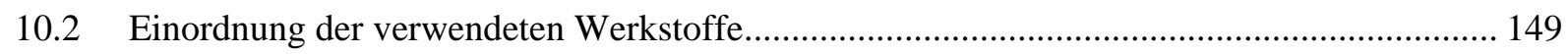

10.3 Beurteilung der Versuchsergebnisse der Flankenkehlnähte................................................. 151

10.4 Beurteilung der Versuchsergebnisse der Kreuzstöße ................................................................. 153

10.5 Vorschlag für das bestehende Bemessungsmodell................................................................. 155

10.6 Bestimmung der Tragfähigkeit in Abhängigkeit von Grund- und Schweißzusatzwerkstoff .. 160

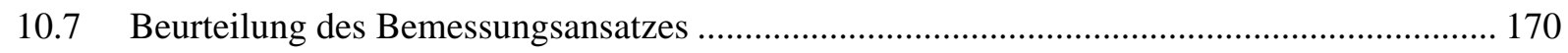

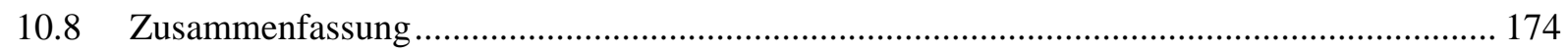

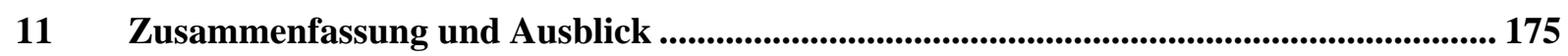

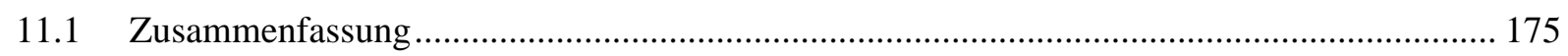

11.2 Beschreibung der Tragfähigkeit von Kehlnähten........................................................... 179

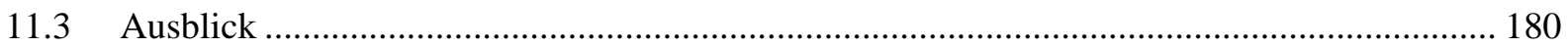

12 Schrifttum .............................................................................................................................................. 181

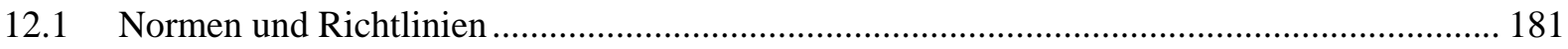

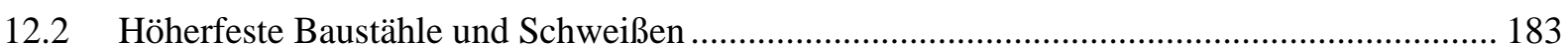

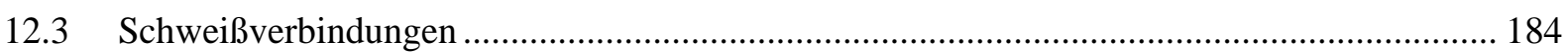

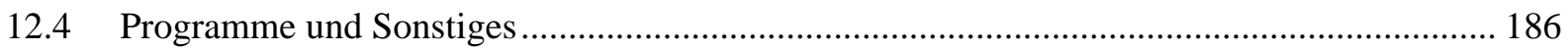

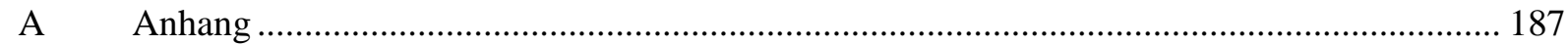

A.1 Auswertung bekannter Versuchsreihen der Literatur.............................................................. 187

A.2 Überblick Schweißverbindungen nach [FOSTA P652, 2008] ............................................. 210

A.3 Überblick Schweißverbindungen nach [Zusatzversuche, 2008] ........................................... 216

A.4 Überblick Schweißverbindungen nach [FOSTA P812, 2011] ................................................ 218

A.5 Statistische Auswertung zur Entwicklung eines Bemessungsvorschlags .............................. 222 


\section{Einführung}

\subsection{Problemstellung und Zielsetzung}

Im Bauwesen werden zunehmend schlanke und leichte Konstruktionen angestrebt. In den letzten Jahren wurden hierzu moderne höherfeste Baustähle entwickelt, die neben einer hohen Festigkeit gute Schweißeigenschaften und eine hohe Zähigkeit besitzen. Für die Verwendung dieser Stähle ist neben ästhetischen Gründen das Einsparpotential von Material und damit Gewicht relevant. Als höherfeste Baustähle bezeichnet man Stähle mit einer Streckgrenze $\mathrm{R}_{\mathrm{eH}}>355 \mathrm{~N} / \mathrm{mm}^{2}$.

Trotz der guten Schweißeigenschaften ist die normative Tragfähigkeit dieser Schweißverbindungen jedoch gering. Dies betrifft insbesondere Kehlnähte, die im Bauwesen häufig verwendet werden, da sie kostengünstig ohne Nahtvorbereitung gefertigt werden können. Es werden demnach sehr großen Kehlnahtdicken benötigt.

In den meisten bisher geltenden Normen wird für die Tragfähigkeit der Schweißnaht nur die Festigkeit des Grundwerkstoffs berücksichtigt, während für den Schweißzusatzwerkstoff mindestens die gleiche Festigkeit wie die des Grundwerkstoffs gefordert wird. Für normalfeste Baustähle S235 und S355 ist diese Vereinfachung sinnvoll, da die zugehörigen Schweißzusatzwerkstoffe immer eine höhere Festigkeit als die Grundwerkstoffe haben. Für höherfeste Stähle S460 und S690 kann es auch sinnvoll sein, Schweißzusatzwerkstoffe mit geringerer oder höherer Festigkeit einzusetzen. Dazu müssen die Bemessungsregeln neben der Festigkeit des Grundwerkstoffs auch die Festigkeit des Schweißzusatzwerkstoffes berücksichtigen. Voraussetzung dafür ist die genaue Kenntnis des Einflusses der Festigkeit des Schweißzusatzwerkstoffs auf die Tragfähigkeit von Kehlnahtverbindungen höherfester Baustähle.

Ziel dieser Arbeit ist die Entwicklung eines realitätsnahen, zeitgemäßen Bemessungsmodells zur Bestimmung der Tragfähigkeit von Kehlnahtverbindungen höherfester Baustähle S460 und S690 für die Anwendung im Stahlbau. Angestrebt wird die Verbesserung der normativen Tragfähigkeit dieser Schweißverbindungen.

Als Grundlage dienen experimentelle und numerische Untersuchungen zur Tragfähigkeit. Insbesondere der Einfluss der Festigkeit des Schweißzusatzwerkstoffs und der Einfluss der Kraftrichtung werden betrachtet. Der Schwerpunkt liegt auf der Untersuchung von Flankenkehlnähten, weil diese aufgrund ihrer Beanspruchungsrichtung die geringste Tragfähigkeit haben. Darüber hinaus wird der Einfluss des Schweißprozesses und der Schweißnahtgeometrie auf die mechanischen Eigenschaften von Schweißverbindungen höherfester Baustähle untersucht.

Angestrebt wird ein vermehrter Einsatz von höherfesten Stählen S690, indem durch eine Verbesserung der normativen Tragfähigkeit mit Hilfe von angepassten, neuen Bemessungskonzepten eine bessere Wirtschaftlichkeit erreicht wird.

\subsection{Aufbau der Arbeit}

Im Rahmen dieser Arbeit wird die Tragfähigkeit von Kehlnahtverbindungen höherfester Baustähle bestimmt. Dazu werden die vorhandenen Bemessungskonzepte und experimentelle Untersuchungen aus der Literatur analysiert. Der Schwerpunkt liegt aber auf eigenen experimentellen und numerischen Untersuchungen. Als Ergebnis daraus wird ein eigener Bemessungsvorschlag entwickelt.

Die Arbeit gliedert sich in die folgenden Kapitel:

Die Tragfähigkeit von Schweißverbindungen wird zum einen durch die verwendeten Werkstoffe und zum anderen durch das Schweißen selbst bestimmt. Nach der Einleitung werden deshalb im zweiten Kapitel dieser Arbeit die Eigenschaften von höherfesten Stählen beschrieben. Neben der Herstellung und den Werkstoffeigenschaften wird insbesondere das Verhalten von höherfesten Baustählen beim Schweißen erläutert. Diese Eigenschaften sind wichtig für die Erklärung des Tragverhaltens der in den folgenden Kapiteln betrachteten Versuche. 
Im dritten Kapitel werden die bestehenden europäischen und internationalen Bemessungsvorschriften für Kehlnähte verglichen und die Besonderheiten herausgearbeitet. Insbesondere für die höherfesten Baustähle zeigen sich große Unterschiede. Vor allem der Einfluss des Schweißzusatzwerkstoffs wird nicht ausreichend berücksichtigt. Die Ergebnisse dieses Kapitels dienen als Grundlage für einen eigenen Bemessungsvorschlag.

Als Einleitung und Hintergrund zur Beurteilung der experimentellen Untersuchungen wird im vierten Kapitel das Tragverhalten von Kehlnähten hinsichtlich der Werkstoffeigenschaften von Kehlnähten und der theoretischen Spannungsverteilung beschrieben. Für die Beurteilung der Versuchsergebnisse wird das Vorgehen bei der statistischen Auswertung vorgestellt.

Die durchgeführte Literaturrecherche wird in Kapitel 5 vorgestellt. Die bestehenden Versuchsreihen mit Flanken- und Stirnkehlnähten werden statistisch neu ausgewertet. Für Schweißverbindungen höherfester Baustähle sind nur sehr wenige Daten bekannt. Mit den wenigen Versuchen kann der Einfluss sowohl des Grundwerkstoffs als auch des Schweißzusatzwerkstoffs auf die Tragfähigkeit von Kehlnahtverbindungen gezeigt werden.

Im sechsten Kapitel werden eigene Untersuchungen [FOSTA P652, 2008] an praxisgerechten Handschweißungen höherfester Stähle S460 und S690 beschrieben und ausgewertet. Ziel der Untersuchungen ist die Ermittlung der Tragfähigkeit von Kehlnahtverbindungen mit den Grundwerkstoffen S460 und S690 und verschiedenen Schweißzusatzwerkstoffen. Die Untersuchungen ergeben eine Verbesserungsmöglichkeit der normativen Tragfähigkeit für Kehlnahtverbindungen mit dem Grundwerkstoff S460, während für Verbindungen mit dem Grundwerkstoff S690 Tragfähigkeiten unterhalb der damals gültigen Normen festgestellt werden.

Zur weiteren Klärung der Tragfähigkeit von Schweißverbindungen mit dem Grundwerkstoff S690 sind im Anschluss an dieses Vorhaben erste Testversuche [Zusatzversuche, 2008] an Schweißverbindungen durchgeführt worden, die gezielt die Tragfähigkeit bei vollmechanisierter Schweißung, aufwändig hergestellter Prüfkörperform und Verwendung höherfester Schweißzusatzwerkstoffe untersucht haben. Die Ergebnisse werden in Kapitel 7 präsentiert.

Aufbauend auf den in Kapitel 6 und 7 vorgestellten Ergebnissen werden in Kapitel 8 weitere umfassende Untersuchungen [FOSTA P812, 2011] an vollmechanisiert geschweißten Versuchen mit Kehlnähten zum Tragverhalten von Schweißverbindungen höherfester Baustähle S690Q und S700M vorgestellt. Durch die experimentelle Untersuchung der Parameter Nahtdicke, Nahtlänge und Schweißparameter kann die Tragfähigkeit von Flankenkehlnahtverbindungen mit dem Grundwerkstoff S690 und passendem Schweißzusatzwerkstoff bestimmt werden. Als weiterer Parameter wird der Einfluss von Unter- und Überfestigkeiten des Schweißzusatzwerkstoffs auf die Tragfähigkeit der Verbindung S690 ermittelt. Vergleichend werden Versuche mit dem Grundwerkstoff S460 betrachtet. Außerdem wird die Tragfähigkeit von Kreuzstößen an Verbindungen gleicher Grundwerkstoffe und unterschiedlicher Schweißzusatzwerkstoffe bestimmt. Als Werkstoffuntersuchungen werden neben Zugversuchen am Grund- und Schweißzusatzwerkstoff auch Härtemessungen an Makroschliffen durchgeführt, deren Ergebnisse für die numerischen Untersuchungen verwendet werden. Zudem ermöglichen diese Untersuchungen Rückschlüsse auf die Festigkeit von Kehlnähten im Vergleich zur Festigkeit des Schweißzusatzwerkstoffs.

In Kapitel 9 werden numerisch erzeugte Ergebnisse vorgestellt. Mit Hilfe der genauen Geometrie und der ermittelten Werkstoffkennwerte werden die Versuche an Flankenkehlnähten nachgerechnet und damit das Tragverhalten beschrieben.

Aufbauend auf den experimentellen und numerischen Untersuchungen und im Zusammenhang mit der statistischen Auswertung erfolgt in Kapitel 10 die Entwicklung eines Bemessungsmodells, das die Festigkeit von Grund- und Schweißzusatzwerkstoff berücksichtigt. Dazu werden sowohl die Versuche aus der Literatur als auch die eigenen experimentellen Ergebnisse verwendet.

Die Arbeit schließt mit einer Zusammenfassung und einem Ausblick in Kapitel 11. 


\section{Höherfeste Baustähle und ihr Verhalten beim Schweißen}

\subsection{Allgemeines}

Ziel dieser Arbeit ist die Bestimmung der Tragfähigkeit von Schweißverbindungen höherfester Baustähle. Beeinflusst wird die Tragfähigkeit zum einen durch die verwendeten Werkstoffe und zum anderen durch das Schweißen selbst. Beim Schweißen kommt es zu großen Wärmeentwicklungen und extremen Abkühlgeschwindigkeiten, die zu Gefügeänderungen und damit zu Eigenschaftsänderungen im Schweißgut und führen.

Im Rahmen dieses Kapitels werden daher einleitend die Herstellung von höherfesten Baustählen sowie deren wesentliche Werkstoffeigenschaften dargestellt. Der Schwerpunkt liegt auf dem Verhalten der höherfesten Baustähle beim Schweißen und auf der Charakteristik der Schweißverbindung. Außerdem werden insbesondere die Ermittlung und der Einfluss der Schweißparameter erläutert. Diese Vorstellung der wichtigsten Eigenschaften und ihrer Hintergründe in diesem Kapitel ist wichtig für die Interpretation und Erklärung der Versuchsergebnisse in den folgenden Kapiteln.

Neben den Versuchen werden numerische Untersuchungen durchgeführt, für die die Werkstoffeigenschaften in der Schweißnaht und der Wärmeeinflusszone besonders wichtig sind. Die Werkstoffkennwerte können bei Kehlnähten oft nicht direkt über Zugversuche bestimmt werden. Aus diesem Grund wird eine Möglichkeit zur Abschätzung der Zugfestigkeit der Schweißverbindung mithilfe von Härtemessungen gezeigt. Zudem werden die Möglichkeiten zur Bestimmung der Eigenschaften in der Wärmeeinflusszone beschrieben.

Abschließend wird ein kurzer Ausblick in die bruchmechanischen Eigenschaften und die Zähigkeit gegeben, die in dieser Arbeit jedoch nicht weiter betrachtet werden.

\subsection{Herstellung von höherfesten Baustählen}

\subsubsection{Allgemeines}

Als höherfeste Baustähle werden im Bauwesen Stähle mit einer Streckgrenze $R_{e H}>355 \mathrm{~N} / \mathrm{mm}^{2}$ bezeichnet. Betrachtet werden höherfeste Feinkornbaustähle, die eine höhere Streckgrenze bei gleichzeitig hoher Zähigkeit haben. Der Kohlenstoffgehalt dieser Stähle liegt fast immer unter 0,2 \%.

Die Feinkörnigkeit des Gefüges wird durch Wärmebehandlung und durch Mikrolegierungselemente, wie z. B. Niob, Titan oder Vanadin, erreicht. Durch die Legierungselemente entstehen außerdem sehr gute Schweißeigenschaften [Schröter, 2007]. Das feine Korn führt durch die Versetzungsbehinderung an den Korngrenzen zu höheren Streckgrenzen. Durch die Wärmebehandlungen im festen Zustand sind die Eigenschaften von Stählen wesentlich veränderbar, da die Wärmebehandlungen die $\alpha / \gamma$ Gitterumwandlung (kubisch-flächenzentriert $\rightarrow$ kubisch-raumzentriert) beeinflussen.

Man unterscheidet bei diesen höherfesten Feinkornbaustählen in Abhängigkeit der Wärmebehandlung zwischen normalgeglühten, thermomechanisch gewalzten und vergüteten Stählen.

\subsubsection{Normalgeglühte Stähle}

Als Normalglühen bezeichnet man die Behandlung von Stählen bei Temperaturen oberhalb von $900^{\circ} \mathrm{C}$. Dabei gibt es zwei klassische Möglichkeiten: Entweder wird bei hohen Temperaturen gewalzt und anschließend normalgeglüht oder normalisierend gewalzt. Der Stahl wird dabei in beiden Fällen auf Temperaturen oberhalb der Rekristallisationstemperatur des Austenits $\left(\mathrm{A}_{\mathrm{c} 3}\right)$ erwärmt und an ruhender Luft abgekühlt. Er durchläuft damit zweimal die $\alpha / \gamma$-Umwandlung und es bildet sich so ein sehr feinkörniges ferritisch-perlitisches Gefüge. Diese Kornfeinung wird auch als Korngrenzenhärtung bezeichnet. Die Rekristallisationstemperatur des Austenits $\left(\mathrm{A}_{\mathrm{c} 3}\right)$ ist in Abbildung 2.1 als Linie zwischen Perlit oder $\gamma$-Mischkristallen mit $\alpha$-Mischkristallen und $\gamma$-Mischkristallen dargestellt. Durch diese Feinkörnigkeit entsteht eine hohe Versetzungsdichte und damit Stähle mit hohen Streckgrenzen und hervorragender Zähigkeit. 


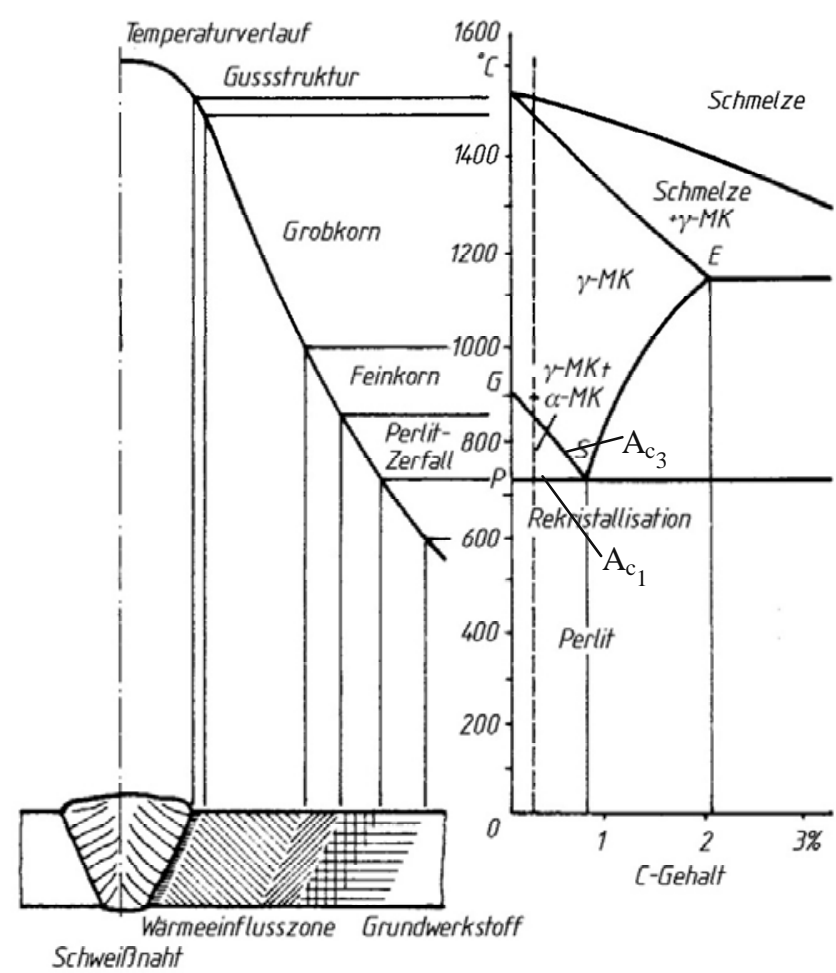

Abbildung 2.1: Exemplarisches Eisen-Kohlenstoff-Schaubild mit Temperaturverlauf und Ausbildung der Wärmeeinflusszone bei einer Stumpfnaht nach [Fahrenwaldt, Schuler, 2009]

\subsubsection{Thermomechanisch gewalzte Stähle}

Im Gegensatz zu normalgeglühten Stählen haben thermomechanisch gewalzte Stähle einen noch geringeren Kohlenstoffgehalt. Um den Festigkeitsabfall durch den geringen Anteil an Kohlenstoff auszugleichen, werden diese Stähle extrem feinkörnig hergestellt, und es wird eine Ausscheidungshärtung durch Mikrolegierungselemente erzeugt.

Thermomechanisch gewalzte Stähle (TM-Stähle) werden durch eine exakt festgelegte Folge von mehreren Walz- und Kühlsequenzen thermisch und mechanisch bei Temperaturen zwischen $700^{\circ} \mathrm{C}$ und $900^{\circ} \mathrm{C}$ behandelt. Mit Hilfe der Wärmebehandlung wird eine Kornfeinung (Korngrenzenhärtung) erreicht, die bereits für die normalgeglühten Stähle erläutert wurde.

Mithilfe von Mikrolegierungselementen wie z. B. Niob, Titan oder Vanadin wird zudem eine Ausscheidungshärtung erreicht. Die Ausscheidungen behindern die Versetzungen und führen zu höheren Festigkeiten. Es entsteht ein stark verspanntes feinkörniges Gefüge mit einer hohen Streckgrenze und großer Zähigkeit, [Schulze, 2010].

\subsubsection{Vergütete Stähle}

Als Vergüten bezeichnet man die kombinierte Wärmebehandlung aus Härten und anschließendem Anlassen. Vergütete Stähle werden von Temperaturen oberhalb $900^{\circ} \mathrm{C}$ mit Wasser abgeschreckt (gehärtet), dabei entsteht ein Ungleichgewichtsgefüge aus Perlit, Bainit und Martensit (tetragonal verspannt). Durch das Abschrecken kann der Kohlenstoff sich nicht ausscheiden, so dass eine tetragonale Verzerrung des Gitters entsteht. Das anschließende Anlassen bei Temperaturen oberhalb $600^{\circ} \mathrm{C}\left(\mathrm{A}_{\mathrm{c} 1}\right.$, Temperaturlinie der Perlitumwandlung, vgl. Abbildung 2.1) führt zu einer Entspannung des Martensit in ein gleichgewichtsnäheres Gefüge. Dabei nehmen Festigkeit und Härte ab, die Zähigkeit nimmt zu, [Schröter, 2007], [Schulze, 2010]. 


\subsubsection{Vergleich von thermomechanisch gewalzten und vergüteten Stählen}

In dieser Arbeit werden vorwiegend thermomechanisch gewalzte und vergütete höherfeste Stähle behandelt. Die Festigkeit von vergüteten Stählen beruht auf einer Martensitbildung, die das Vorhandensein von Kohlenstoff voraussetzt. Die Festigkeit von thermomechanisch gewalzten Stählen wird durch die beschriebene Feinkörnigkeit infolge der Wärmebehandlung erreicht. Es wird nur ein sehr geringer Anteil von Kohlenstoff benötigt. Vergütete Stähle haben in der Regel einen höheren Anteil an Kohlenstoff als thermomechanisch gewalzte Stähle.

\subsection{Werkstoffeigenschaften und Verwendung höherfester Baustähle}

Zur Beschreibung der Werkstoffeigenschaften höherfester Baustähle S460 und S690 werden deren Eigenschaften mit denen normalfester Baustähle S355 verglichen. Für Blechdicken $\mathrm{t} \leq 16 \mathrm{~mm}$ ergeben sich nach DIN EN 10025-2 (2005), DIN EN 10025-4 (2005) und DIN EN 10025-6 (2005) die in Tabelle 2.1 aufgeführten Grenzwerte.

Tabelle 2.1: Mechanische Eigenschaften der verwendeten Grundwerkstoffe nach DIN EN 10025

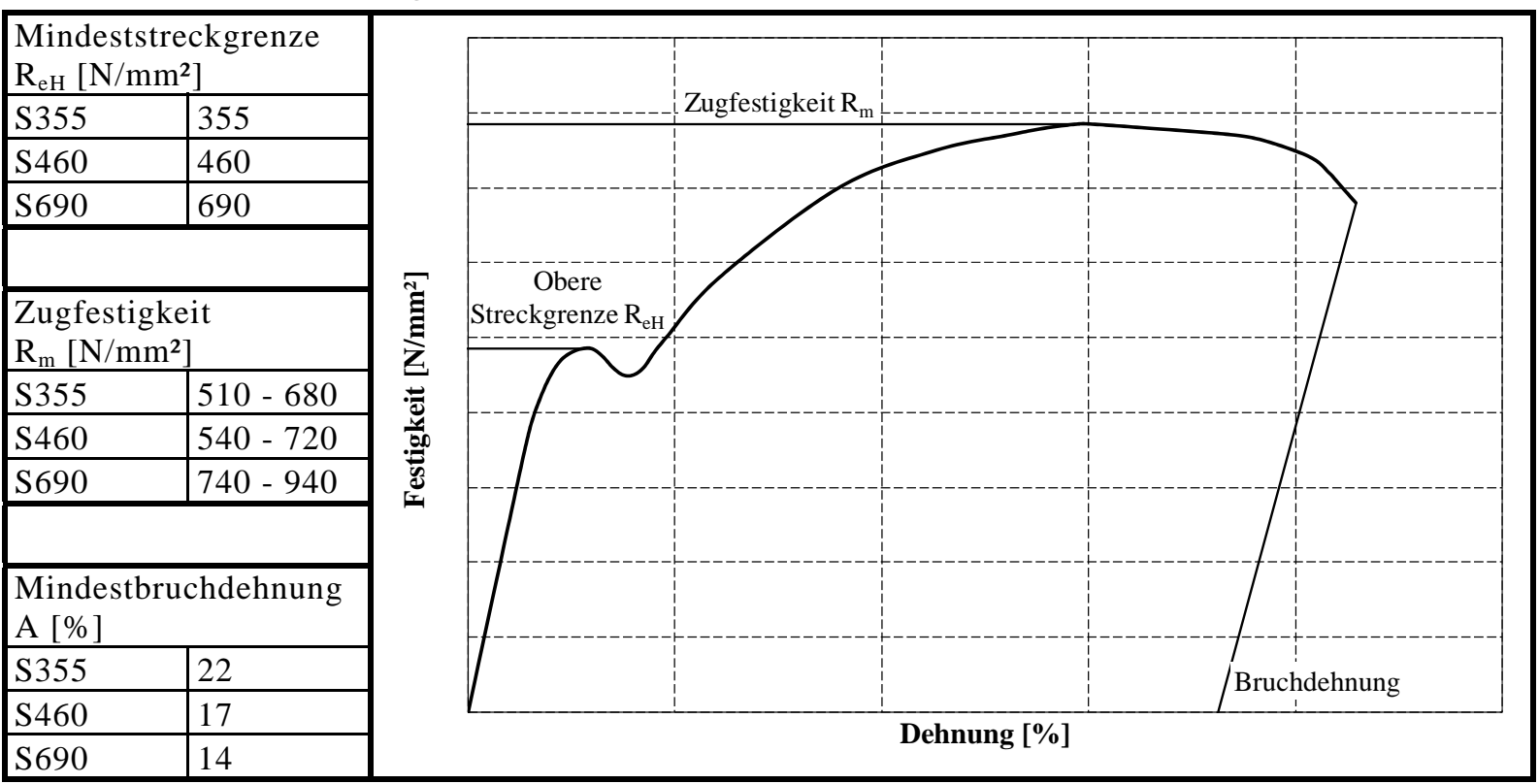

In Abbildung 2.2 werden exemplarisch drei typische Spannungs-Dehnungs-Linien für diese Stähle dargestellt. Die höherfesten Baustähle zeichnen sich durch eine höhere Streckgrenze und ein geringes Verhältnis von Zugfestigkeit zu Streckgrenze sowie durch geringere plastische Dehnungen aus. Bei den höherfesten Stählen sind nicht immer ausgeprägte Streckgrenzen vorhanden.

Der Vorteil höherfester Baustähle besteht in der höheren Streckgrenze und der daraus folgenden höheren maximalen Beanspruchbarkeit des Werkstoffs. Infolge der damit möglichen geringeren Werkstoffdicken können Eigenspannungen reduziert werden. Durch diese hohe Festigkeit können Material und damit Gewicht und Herstellungskosten eingespart werden. Insgesamt kann sich ein wirtschaftlicher Vorteil beim Einsatz höherfester Baustähle ergeben.

Technisch sinnvoll lassen sich diese Stähle insbesondere bei zugbeanspruchten Bauteilen einsetzen. Ist Stabilitätsversagen maßgebend, können die Vorteile bei Verwendung höherfester Baustähle nicht genutzt werden. Das Stabilitätsverhalten wird vor allem durch den E-Modul bestimmt, der bei normalund höherfesten Baustählen gleich ist. Die Ermüdungsfestigkeit steigt nicht proportional zur Streckgrenze, so dass sich beim Nachweis der Ermüdung keine Vorteile durch die Verwendung höherfester Stähle ergeben, [Schulze, 2010]. Steigerungen der Ermüdungsfestigkeiten von Schweißverbindungen höherfester Baustähle können jedoch durch Schweißnahtnachbehandlungsmethoden erreicht werden [Kuhlmann et al., 2006]. Außerdem ist zu bedenken, dass infolge der geringeren QuerschnittsAbmessungen größere Verformungen auftreten. 


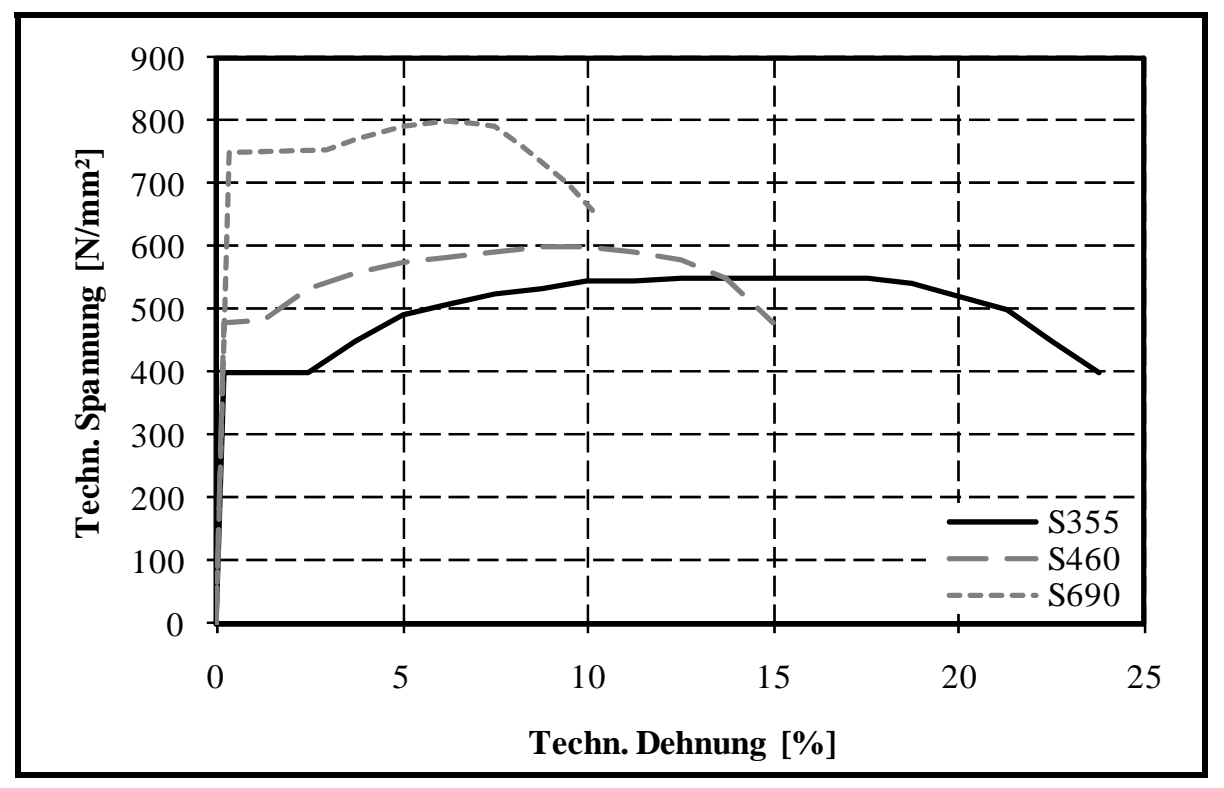

Abbildung 2.2: Typische Spannungs-Dehnungs-Linien unterschiedlicher Stähle S355, S460 und S690

\subsection{Verhalten von höherfesten Baustählen beim Schweißen}

\subsubsection{Schweißeignung von Stählen}

Die Schweißbarkeit des Bauteils ergibt sich aus Schweißsicherheit, Schweißmöglichkeit und Schweißeignung. Die Schweißsicherheit ist abhängig von der Konstruktion, die die konstruktive Gestaltung, den Beanspruchungszustand und die Betriebstemperatur umfasst. Mit Schweißmöglichkeit werden die Fertigungsbedingungen beschrieben, [Schulze, 2010]. Die Schweißeignung definiert sich über den Werkstoff und dessen chemischer Zusammensetzung, d. h. die metallurgischen und physikalischen Eigenschaften. Die Schweißeignung wird im Weiteren beschrieben.

Die wesentlichen Werkstoffeigenschaften, die die Schweißeignung bestimmen, sind zum einen die Aufhärtungsneigung in der Wärmeeinflusszone, die zu Kaltrissen führen kann. Kaltrisse können durch das Vorhandensein von Wasserstoff in der Wärmeeinflusszone entstehen. Liegt Wasserstoff im erstarrten Schweißgut vor, kommt es zu einer Versprödung des Gitters. Da Wasserstoff auch bei Raumtemperatur diffundiert, treten die Risse zeitverzögert im „kalten“ Zustand auf. Durch eine gezielte Wärmebehandlung (Vgl. Kapitel 2.4.6 Abkühlbedingungen) können Kaltrisse vermieden werden, indem der Wasserstoff schnell aus der Schweißverbindung diffundiert.

Neben den Kaltrissen in der Wärmeeinflusszone können zum anderen Heißrisse im Schweißgut entstehen. Heißrisse können während des Schweißens entstehen, wenn Entmischungsvorgänge von Legierungselementen zu unterschiedlichen Schmelzpunkten führen. Dann können isolierte flüssige Phasen entstehen, die Zugspannungen aus Schrumpfungen nicht aufnehmen können, so dass Mikrorisse entstehen.

Diese Werkstoffeigenschaften sind von der Art der Herstellung und der chemische Zusammensetzung abhängig und im Folgenden beschrieben.

\subsubsection{Aufbau der Schweißverbindung}

Schweißverbindungen bestehen aus verschiedenen Gefügebereichen, die exemplarisch in Abbildung 2.1 dargestellt sind. Die aus Grund- und Schweißzusatzwerkstoff bestehende Schweißschmelze erstarrt in einem Gussgefüge zur Schweißnaht. Bei einlagigen Nähten erstarrt das Primärgefüge unter Bildung von Dendriten (stängliges Gefüge, vgl. Abbildung 2.1). Neben der Schmelzlinie entsteht die Wärmeeinflusszone (WEZ). 


\subsubsection{Charakteristik der Wärmeeinflusszone}

Die Wärmeeinflusszone (WEZ) besteht aus verschiedenen Bereichen, wie in Abbildung 2.1 dargestellt. Direkt neben der Schweißraupe findet sich eine unvollständig aufgeschmolzene Gussstruktur, an die sich die Überhitzungszone oder Grobkornzone anschließt. Das grobkörnige Gefüge entsteht durch sehr hohe Spitzentemperaturen und wird durch die Abkühlgeschwindigkeit beeinflusst. Dort entsteht ein Bereich großer Härte. Neben der Grobkornzone findet sich die Normalisierungs- oder Feinkornzone. Die Feinkornzone entsteht durch die in diesem Bereich vorhandenen Temperaturen oberhalb von $\mathrm{A}_{\mathrm{C} 3}$ wie bei einer Normalisierung, vgl. Kapitel 2.2.2. Die vierte Zone ist ein Bereich des teilweisen Umkristallisierens (Perlit-Zerfall). An diesen Bereich schließt sich die Anlasszone an, die in den unbeeinflussten Grundwerkstoff übergeht, [Dilthey, 2005], [Fahrenwaldt, Schuler, 2009].

Bereiche der Wärmeeinflusszone haben eine Neigung zur Aufhärtung, die mit der Kaltrissbildung einher geht. Beide werden durch die chemische Zusammensetzung (vgl. Kapitel 2.4.5) und die Abkühlgeschwindigkeit (vgl. Kapitel 2.4.6) beeinflusst. Insgesamt entsteht ein sprödes, rissanfälliges Gefüge in der Wärmeeinflusszone.

\subsubsection{Charakteristik des Schweißgutes}

Für die Herstellung von höherfesten Baustählen ist die Temperaturführung und die Einhaltung von Haltezeiten, wie in Kapitel 2.2 erläutert, besonders wichtig. Beim Schweißen herrschen andere Abkühlbedingungen, und die erforderlichen Zeiten zur Ausbildung gewisser Eigenschaften stehen nicht zur Verfügung. Aus diesem Grund haben Schweißzusatzwerkstoffe andere chemische Zusammensetzungen als die zugehörigen Grundwerkstoffe. In den meisten Fällen liegt im Schweißzusatzwerkstoff eine erhöhte Konzentration von Legierungselementen vor.

\subsubsection{Chemische Zusammensetzung}

Für die chemische Zusammensetzung spielt insbesondere der Anteil von Kohlenstoff eine große Rolle. Die chemische Zusammensetzung von Schweißverbindungen wird mithilfe des KohlenstoffÄquivalents CET (Gleichung (2.2)) beschrieben. Neben Kohlenstoff werden die Elemente Mangan, Molybdän, Chrom, Kupfer und Nickel berücksichtigt.

Bei Betrachtung der höherfesten Baustähle lassen sich insbesondere bei thermomechanisch gewalzten Stählen niedrige Kohlenstoffgehalte und geringe Werte für die Kohlstoff-Äquivalente CET feststellen. Dies deutet auf eine geringe Aufhärtungsneigung und damit eine geringe Gefahr von Kaltrissen des Stahls hin.

Die thermomechanisch gewalzten Baustähle haben geringere Kohlenstoffgehalte als die vergüteten Stähle. Für das Schweißen der vergüteten Stähle ist eine sehr große Sorgfalt erforderlich, da infolge der höheren Streckgrenzen nur eine geringe Duktilität vorhanden ist. Dadurch können mehrachsige Eigenspannungszustände, die durch das Schweißen entstehen, nur schlecht abgebaut werden. Das erhöht die Gefahr von Kaltrissen deutlich. Wie in Kapitel 2.2.4 beschrieben, wird mit Hilfe der Mikrolegierungselemente die Festigkeit von Vergütungsstählen erhöht. Beim Schweißen wird durch diese Mikrolegierungselemente die Anlassbeständigkeit verbessert. Wenn beim Schweißen die Anlasstemperatur des Stahls überschritten wird, kann es zu einem Festigkeitsabfall in der Wärmeeinflusszone kommen, der durch einen Abfall der Härte gekennzeichnet ist, [Schulze, 2010].

Mithilfe des Kohlenstoff-Äquivalents (Gleichung (2.2)) wird dieser Einflussfaktor bei der Ermittlung von möglichen Schweißparametern begrenzt, wie in Kapitel 2.5 beschrieben.

Die Neigung zu Heißrissen im Schweißgut wird durch Verunreinigungen insbesondere durch Schwefel begünstigt. Bei der Erstarrung kann es zu Entmischungsvorgängen von Legierungselementen kommen, wodurch noch flüssige Schmelzanteile eingeschlossen und isoliert werden können. Durch Schrumpfung verursachte Zugspannungen können von diesen flüssigen Phasenanteilen noch nicht aufgenommen werden, so dass Mikrotrennungen entstehen können, [Dorn et al., 1986]. 


\subsubsection{Abkühlbedingungen}

Die Abkühlgeschwindigkeit beeinflusst die Eigenschaften der Schweißnaht. Bei zu geringen Abkühlzeiten besteht die Gefahr von Kaltrissen. Durch eine zu langsame Abkühlung können sich weitere Ferrit- und Perlitanteile bilden, die zu einer Erweichung und damit zu Festigkeitsverlusten führen können. Diese Erweichung hinterlässt in anderen Bereichen eine Anreicherung von Kohlenstoff, so dass sich vermehrt Martensit bilden kann. Dieses Mischgefüge führt zu einem großen Zähigkeitsverlust, [Schulze, 2010]. Die Abkühlzeiten können über die Schweißparameter (vgl. 2.5) beeinflusst werden.

Beim Schweißen von thermomechanischen Stählen kommt es zu Erweichungen der Bereiche in der Wärmeeinflusszone, die bis zur $\mathrm{A}_{1}$-Temperatur oder darunter erwärmt wurden. Durch diese Erwärmung werden die mechanischen Eigenschaften dieser Stähle zerstört. Damit geht ein Verlust an Härte und Festigkeit einher, [Dilthey, 2005].

\subsection{Schweißanweisungen}

Die bisher beschriebenen Eigenschaften und Gefahren, insbesondere die Kaltrissgefahr, sind bei der Wahl der Schweißparameter zu berücksichtigen. Die Schweißparameter beeinflussen wesentlich die Eigenschaften der Schweißverbindungen. Die festzulegenden Parameter Strom, Spannung, Drahtvorschub, Schweißgeschwindigkeit und Vorwärmtemperatur werden in Schweißanweisungen festgelegt. Beim Erstellen von Schweißanweisungen sind DIN EN 1011-2 (2001), [SEW 088, 1993], [SEW 088 Beiblatt 1, 1993] und [SEW 088 Beiblatt 2, 1993] zu beachten. Zur Erläuterung des Vorgehens bei der Erstellung von Schweißanweisungen werden zunächst die Auswahl und Berechnung einzelner Parameter vorgestellt, die dann in einem zweiten Schritt überprüft werden müssen.

Die Werte für Strom I und Spannung U werden so gewählt, dass der gewünschte spritzerarme Sprühlichtbogen entsteht. Die erforderliche Schweißgeschwindigkeit v kann für die angestrebte Kehlnahtdicke in Abhängigkeit des Drahtdurchmessers und der Drahtvorschubgeschwindigkeit ermittelt werden. Damit wird der Wärmeeintrag Q mit dem thermischen Wirkungsgrad $\varepsilon$ nach Gleichung (2.1) berechnet:

$$
\mathrm{Q}=\varepsilon \cdot \mathrm{E}=\varepsilon \cdot \frac{\mathrm{U} \cdot \mathrm{I}}{\mathrm{V}} \cdot 1000 \mathrm{in}[\mathrm{kJ} / \mathrm{mm}]
$$

mit Wärmeeintrag Q [kJ/mm], thermischer Wirkungsgrad $\varepsilon[-]$,

Streckenenergie E $[\mathrm{kJ} / \mathrm{mm}]$,

Spannung U [V],

Strom I [A],

Schweißgeschwindigkeit $\mathrm{v}[\mathrm{mm} / \mathrm{s}]$

Die Schweißparameter müssen so gewählt werden, dass keine Kaltrisse entstehen. Das Phänomen des Kaltrisses ist in Kapitel 2.4.3 beschrieben. Mit Hilfe des Kohlenstoff-Äquivalents CET wird die Kaltrissneigung in Abhängigkeit der Legierungselemente berechnet, vgl. Gleichung (2.2). Die Kaltrissneigung wird mit zunehmendem CET-Wert größer.

$$
\mathrm{CET}=\mathrm{C}+\left(\frac{\mathrm{Mn}+\mathrm{Mo}}{10}\right)+\left(\frac{\mathrm{Cr}+\mathrm{Cu}}{20}\right)+\left(\frac{\mathrm{Ni}}{40}\right) \text { in [\%] }
$$

mit Kohlenstoff-Äquivalent CET

Kohlenstoff C, Mangan Mn, Molybdän Mo, Chrom Cr, Kupfer Cu und Nickel Ni in [\%]

In Abhängigkeit des Kohlenstoff-Äquivalents CET nach Gleichung (2.2), des Wärmeeintrags Q nach Gleichung (2.1), der Blechdicke d und des Gehalt an diffusiblem Wasserstoff HD wird eine Mindestvorwärmtemperatur $\mathrm{T}_{\mathrm{P}}$ nach (2.3) errechnet:

$$
\mathrm{T}_{\mathrm{P}}=697 \cdot \mathrm{CET}+160 \cdot \tanh \left(\frac{\mathrm{d}}{35}\right)+62 \cdot \mathrm{HD}^{0,35}+(53 \cdot \mathrm{CET}-32) \cdot \mathrm{Q}-328 \text { in }\left[{ }^{\circ} \mathrm{C}\right]
$$


mit Mindestvorwärmtemperatur $\mathrm{T}_{\mathrm{P}}\left[{ }^{\circ} \mathrm{C}\right]$,

Kohlenstoff-Äquivalent CET nach Gleichung (2.2),

Blechdicke d [mm],

Gehalt an diffusiblem Wasserstoff HD [ml/100g abgeschmolzenes Schweißgut]

Wärmeeintrag Q nach Gleichung (2.1)

In Abhängigkeit der gewählten Vorwärmtemperatur $\mathrm{T}_{0}$, des Wärmeeintrags $\mathrm{Q}$ und der Blechdicke $\mathrm{d}$ kann die Abkühlzeit $t_{8 / 5}$ bestimmt werden. Die Abkühlzeit $t_{8 / 5}$ definiert den Zeitraum der Abkühlung der Schweißnaht zwischen $800^{\circ} \mathrm{C}$ und $500^{\circ} \mathrm{C}$. Für höherfeste Baustähle wird eine Abkühlzeit $t_{8 / 5} \mathrm{zwi}$ schen $t_{8 / 5}=5 \mathrm{~s}$ und $t_{8 / 5}=15 \mathrm{~s}$ von den Herstellern empfohlen. Die Bestimmung der Abkühlzeit $\mathrm{t}_{8 / 5}$ unterscheidet zwischen zwei- und dreidimensionaler Wärmeableitung. Für dünne Bleche gilt die zweidimensionale Wärmeableitung, während für dicke Bleche eine dreidimensionale Wärmeableitung herrscht. Die Berechnung der Abkühlzeit $\mathrm{t}_{8 / 5}$ kann mit folgenden Formeln in Abhängigkeit der Vorwärm- oder Zwischenlagentemperatur $T_{0}$, der Blechdicke d und geometrieabhängiger Formfaktoren $F_{2}$ und $\mathrm{F}_{3}$ nach Gleichung (2.4) oder (2.5) durchgeführt werden. Die Formfaktoren berücksichtigen den

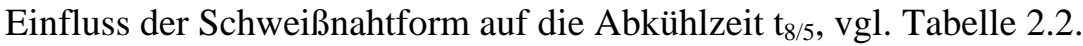

Für zweidimensionale Wärmeableitung gilt:

$$
\mathrm{t}_{8 / 5}=\left(4300-4,3 \cdot \mathrm{T}_{0}\right) \cdot 10^{5} \cdot \frac{\mathrm{Q}^{2}}{\mathrm{~d}^{2}} \cdot\left[\left(\frac{1}{500-\mathrm{T}_{0}}\right)^{2}-\left(\frac{1}{800-\mathrm{T}_{0}}\right)^{2}\right] \cdot \mathrm{F}_{2}
$$

Für dreidimensionale Wärmeableitung gilt:

$$
\mathrm{t}_{8 / 5}=\left(6700-5 \cdot \mathrm{T}_{0}\right) \cdot \mathrm{Q} \cdot\left(\frac{1}{500-\mathrm{T}_{0}}-\frac{1}{800-\mathrm{T}_{0}}\right) \cdot \mathrm{F}_{3}
$$

mit Vorwärm- oder Zwischenlagentemperatur $\mathrm{T}_{0}\left[{ }^{\circ} \mathrm{C}\right]$

Blechdicke d [mm]

Wärmeeintrag Q nach Gleichung (2.1)

$\mathrm{F}_{2}, \mathrm{~F}_{3}$ Formfaktoren nach Tabelle 2.2

Tabelle 2.2: Formfaktoren $F_{2}$ und $F_{3}$ zur Berücksichtigung des Einflusses der Schweißnahtform auf die

\begin{tabular}{|c|c|c|c|c|}
\hline & \multicolumn{4}{|l|}{ Schweißnahtform } \\
\hline & $\begin{array}{l}\text { Schweißraupe auf } \\
\text { Blech }\end{array}$ & \begin{tabular}{|l} 
Zwischen- \\
Schweißraupen in \\
Stumpfnähten
\end{tabular} & $\begin{array}{l}\text { Einlagige Kehlnaht } \\
\text { in einem Eckstoß }\end{array}$ & $\begin{array}{l}\text { Einlagige Kehlnaht } \\
\text { in einem T-Stoß }\end{array}$ \\
\hline \begin{tabular}{|l} 
Formfaktor $\mathrm{F}_{2}$ \\
zweidimensionale \\
Wärmeableitung
\end{tabular} & 1 & 0,9 & 0,9 bis 0,67 & 0,45 bis 0,67 \\
\hline \begin{tabular}{|l} 
Formfaktor $\mathrm{F}_{3}$ \\
dreidimensionale \\
Wärmeableitung
\end{tabular} & 1 & 0,9 & 0,67 & 0,67 \\
\hline
\end{tabular}
Abkühlzeit t 8 $/ 5_{5}$, DIN EN 1011-2 (2001)

Zusammenfassend lässt sich das Vorgehen beim Erstellen von Schweißanweisungen wie folgt beschreiben.

Zunächst werden sinnvolle Schweißparameter ausgewählt und das Kohlenstoff-Äquivalent CET bestimmt. Damit kann die Mindestvorwärmtemperatur errechnet werden. In Abhängigkeit der gewählten Vorwärmtemperatur, der Schweißparameter und des Kohlenstoff-Äquivalents CET wird die Abkühlzeit $t_{8 / 5}$ berechnet. Zur Überprüfung der so festgelegten Schweißparameter wird die berechnete Abkühlzeit $\mathrm{t}_{8 / 5}$ mit den Angaben des Herstellers (z. B. 5 - 15 s) verglichen.

Um dieses rechnerische Vorgehen grafisch zu verdeutlichen, kann ein Arbeitsfenster mit vier Rändern erstellt werden. Innerhalb dieses Arbeitsfensters sollten der Wärmeeintrag Q und die Vorwärmtemperatur $\mathrm{T}_{0}$ liegen, vgl. Abbildung 2.3. 
Für eine gegebene Geometrie (bekannte Blechdicke, bekannter Formfaktor $\mathrm{F}_{2}$ oder $\mathrm{F}_{3}$ aus Tabelle 2.2) mit festgelegten Werkstoffen können für jede gewünschte Abkühlzeit $t_{8 / 5}$ (hier mit $t_{8 / 5}=5 \mathrm{~s}$ und $t_{8 / 5}=$ 15 s) die erforderliche Wärmeeinbringung in Abhängigkeit der Vorwärmtemperatur ermittelt werden. Dazu werden die oben genannten Formeln (2.4) und (2.5) zur Berechnung der Abkühlzeit $t_{8 / 5}$ umgestellt. Es ergibt sich der obere und untere Rand des Arbeitsfensters in Abbildung 2.3.

Für zweidimensionale Wärmeableitung ergibt sich aus Gleichung (2.4):

$$
\mathrm{Q}\left(\mathrm{T}_{0}\right)=\sqrt{\frac{\mathrm{t}_{8 / 5} \cdot \mathrm{d}^{2}}{\left(4300-4,3 \cdot \mathrm{T}_{0}\right) \cdot 10^{5} \cdot \mathrm{F}_{2} \cdot\left(\left(\frac{1}{500-\mathrm{T}_{0}}\right)^{2}-\left(\frac{1}{800-\mathrm{T}_{0}}\right)^{2}\right)}}
$$

Für dreidimensionale Wärmeableitung ergibt sich aus Gleichung (2.5):

$$
\mathrm{Q}\left(\mathrm{T}_{0}\right)=\frac{\mathrm{t}_{8 / 5}}{\left(6700-5 \cdot \mathrm{T}_{0}\right) \cdot \mathrm{F}_{3}\left(\frac{1}{500-\mathrm{T}_{0}}-\frac{1}{800-\mathrm{T}_{0}}\right)}
$$

Um die Wärmeeinbringung Q in Abhängigkeit der Vorwärmtemperatur zu berechnen, wird Gleichung (2.3) zur Bestimmung der Mindestvorwärmtemperatur zur Vermeidung von Kaltrissen umgestellt. Daraus wird der linke Rand des Arbeitsfensters in Abbildung 2.3 ermittelt:

$$
\mathrm{Q}\left(\mathrm{T}_{\mathrm{P}}\right)=\frac{\mathrm{T}_{\mathrm{P}}+328-697 \cdot \mathrm{CET}-160 \cdot \tanh \left(\frac{\mathrm{d}}{35}\right)-62 \mathrm{HD}^{0,35}}{(53 \cdot \mathrm{CET}-32)}
$$

Der rechte Rand ergibt sich aus einer maximalen Vorwärmtemperatur, die vom Stahlhersteller vorgegeben wird (z. B. $250^{\circ} \mathrm{C}$ ).

Der Punkt beschreibt den vorhandenen Wärmeeintrag Q und die vorhandene Vorwärmtemperatur $\mathrm{T}_{0}$.

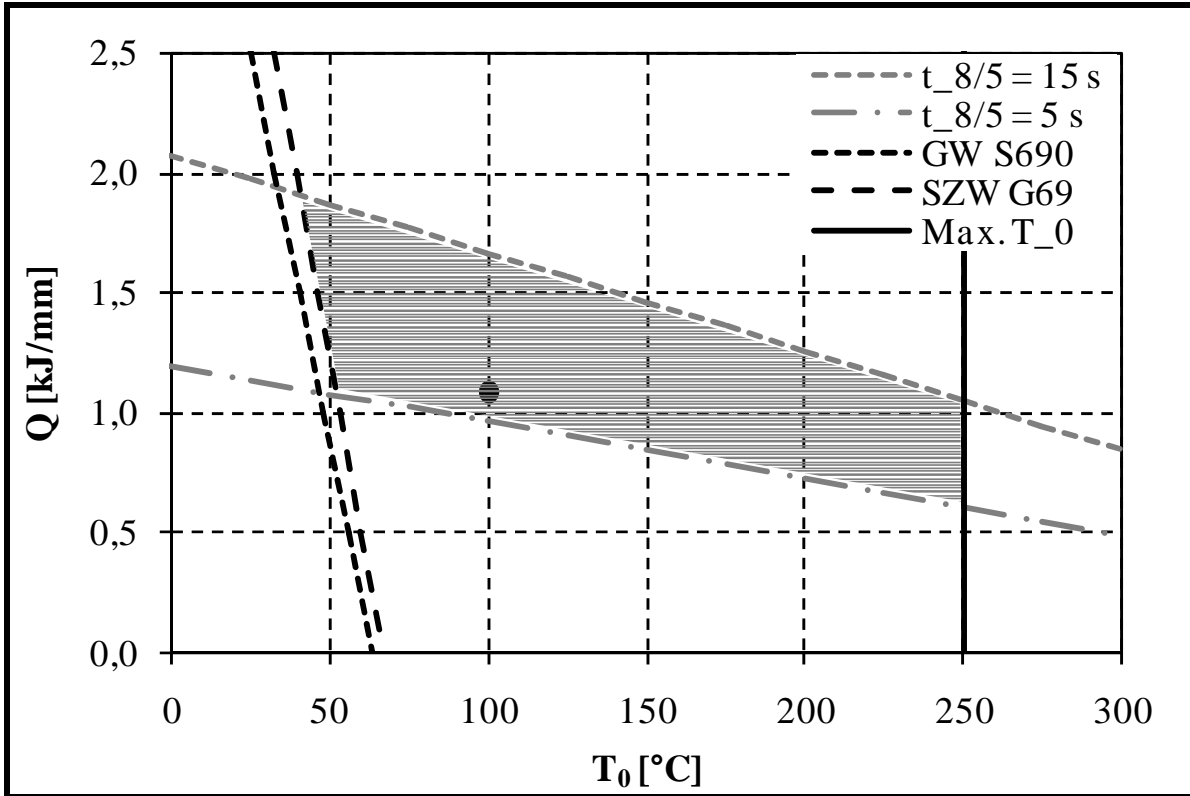

Abbildung 2.3: Exemplarische Darstellung des Arbeitsfensters bei bekannter Geometrie

Um den Punkt im Arbeitsfenster zu ändern, müssen die Vorwärmtemperatur (Abszisse) und/oder der Wärmeeintrag (Ordinate) geändert werden. Durch beide Parameter werden die Abkühlbedingungen beeinflusst.

Der Wärmeeintrag Q wird durch die Schweißparameter Strom, Spannung und Schweißgeschwindigkeit ermittelt. Mit der Änderung der Streckenenergie, vor allem der Schweißgeschwindigkeit, ändert sich die Nahtdicke. Bei höherer Schweißgeschwindigkeit wird die Nahtdicke geringer, bei geringerer Schweißgeschwindigkeit ergibt sich eine größere Nahtdicke. 
Wird die Vorwärmtemperatur geändert, ändern sich ebenfalls die Abkühlbedingungen. Werden die anderen Schweißparameter beibehalten, bleibt die äußere Schweißnahtgeometrie gleich. Infolge der Vorwärmtemperatur und einer Veränderung der Abkühlung ergibt sich jedoch ein anderer Nahteinbrand.

\subsection{Bestimmung der Festigkeit im Schweißgut}

Zur Beurteilung der Tragfähigkeit der Schweißverbindung ist die Kenntnis der Zugfestigkeit der eingesetzten Werkstoffe, insbesondere des Schweißgutes besonders wichtig. Da es nicht immer möglich ist, die Zugfestigkeit einer Schweißnaht oder bestimmter Bereiche der Schweißnaht direkt zu bestimmen, wird in diesem Abschnitt ein Verfahren vorgestellt, wie mit Hilfe von Härtemessungen die Zugfestigkeit abgeschätzt werden kann.

Nach DIN EN ISO 18265 (2004) ist geregelt, wie ermittelte Härtewerte in eine Zugfestigkeit umgerechnet werden können, da Härtewerte und Zugfestigkeiten korrelieren. Die Umrechnung unterliegt jedoch gewissen Unsicherheiten. Die folgende Abbildung 2.4 setzt die Angaben nach Tabelle A.1 aus DIN EN ISO 18265 (2004) zur Umrechnung der Vickers-Härte HV 10 in die Zugfestigkeit grafisch um. Es ergibt sich eine Steigung von $\frac{\text { Zugfestigkeit }}{\text { Härte }}=3,2$.

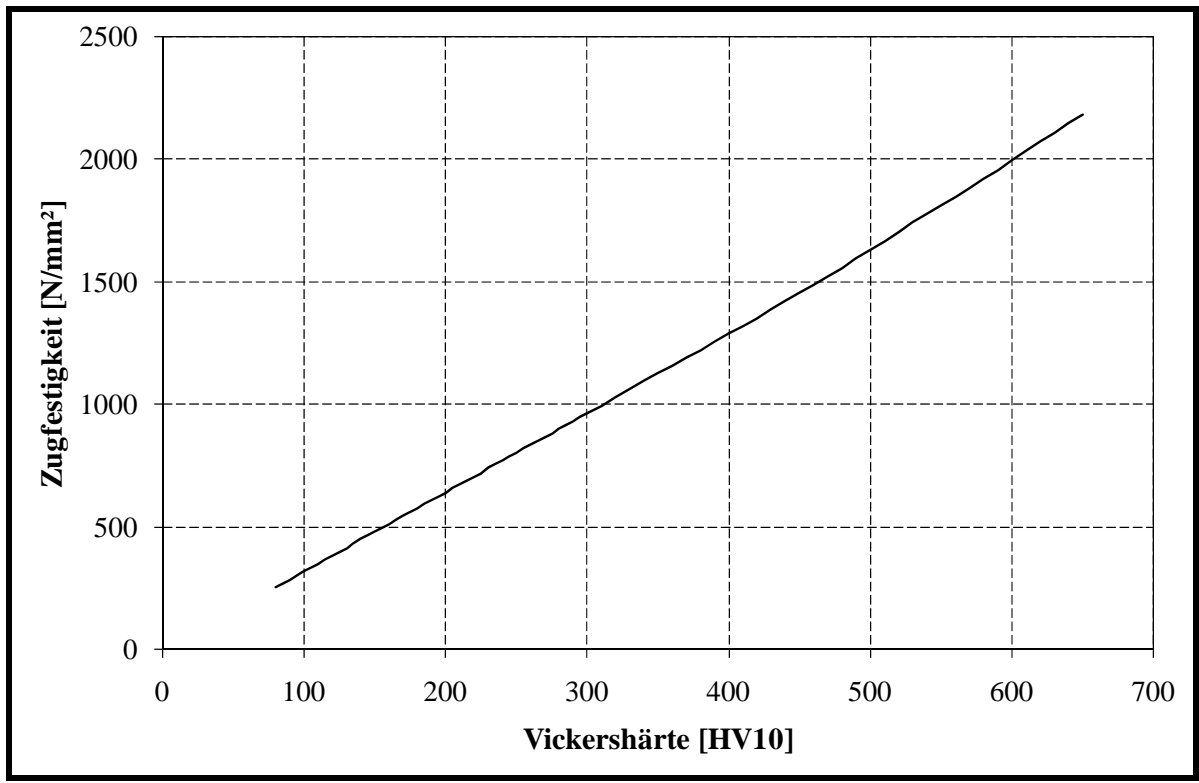

Abbildung 2.4: Korrelation zwischen Härte und Zugfestigkeit nach DIN EN ISO 18265 (2004)

Die vorgestellte Art, die Zugfestigkeiten mithilfe von Härtemessungen abzuschätzen, wird in dieser Arbeit für die numerischen Berechnungen verwendet. Die numerischen Berechnungen werden durchgeführt, um die Tragfähigkeit von Kehlnähten zu bestimmen. Für die numerischen Berechnungen ist die Angabe der Zugfestigkeit für die Kehlnaht erforderlich. Ist diese Zugfestigkeit nicht bekannt, liegt hiermit eine Möglichkeit vor, um sie über die Ergebnisse von Härtemessungen zu ermitteln.

Ein ähnliches Vorgehen für numerische Berechnungen von ein- und mehrlagigen Flankenkehlnähten wird in [Hölbling et al., 2005] angewendet.

\subsection{Bestimmung der Eigenschaften in der Wärmeeinflusszone}

Die Werkstoffeigenschaften in der Wärmeeinflusszone sind in Kapitel 2.4.3 beschrieben. Der Bereich der Wärmeeinflusszone ist im Allgemeinen nur wenige Millimeter groß, besteht aber aus verschiedenen Bereichen. Insbesondere wird sie in die sehr unterschiedlichen Bereiche Fein- und Grobkornzone unterteilt. Da der Bereich der Wärmeeinflusszone nur sehr gering ist, ist es nicht möglich, Härtemessungen in den verschiedenen Bereichen durchzuführen und damit die Festigkeit zu bestimmen. 
Eine neuere Möglichkeit, die mechanischen Eigenschaften in der Wärmeeinflusszone zu bestimmen, bietet die numerische Schweißsimulation. In der Dissertation von [Hildebrand, 2008] werden u. a. numerische Schweißsimulationen von Kehlnähten von höherfesten Baustählen durchgeführt. In der Arbeit von [Hildebrand, 2008] werden die metallurgischen Vorgänge in der Wärmeeinflusszone erläutert und numerisch simuliert. Durch die numerische Simulation war die Ermittlung der Gefügestruktur in der Schweißnaht und der Wärmeeinflusszone möglich.

\subsection{Bruchmechanische Eigenschaften und Zähigkeit}

Neben den mechanischen und chemischen Eigenschaften der Schweißverbindungen spielen bruchmechanische Eigenschaften und Zähigkeit eine Rolle. Bruchmechanische Eigenschaften und die Zähigkeitseigenschaften von höherfesten Feinkornbaustählen wurden z. B. in [Müsch, 1999] oder [Krebs, 1990] untersucht. In [Müsch, 1999] werden bruchmechanische Untersuchungen von Stumpfnähten an Feinkornbaustählen mit einer Streckgrenze von 460 - $550 \mathrm{~N} / \mathrm{mm}^{2}$ beschrieben, die auch den Einfluss von Über- und Unterfestigkeiten der verwendeten Schweißzusatzwerkstoffe betrachten. Es wurden hohe Zähigkeiten sowohl im Schweißgut als auch in der Wärmeeinflusszone festgestellt. In [Krebs, 1990] wurden Stumpfnähte an Feinkornbaustählen S355 und S460 hinsichtlich ihres Bruchverhaltens untersucht. Es konnten hohe Zähigkeiten festgestellt werden.

In der Dissertation von [Hölbling, 2008] wird u. a. bruchmechanisch die statische Tragfähigkeit von teilweise durchgeschweißten Nähten untersucht.

In der Dissertation von [Volz, 2009] wird die Rissentstehung infolge statischer Beanspruchung unter dem Aspekt der Sprödbruchsicherheit untersucht.

In dieser Arbeit werden die bruchmechanischen Eigenschaften und die Zähigkeit nicht weiter betrachtet, es wird aber auf oben stehende Arbeiten verwiesen.

\subsection{Zusammenfassung}

In diesem Kapitel wurde neben der Herstellung und den Werkstoffeigenschaften von höherfesten Baustählen insbesondere das Verhalten von höherfesten Baustählen beim Schweißen erläutert, da diese Eigenschaften in den folgenden Kapiteln zur Erklärung der Versuchsergebnisse benötigt werden.

Im Einzelnen wurden die auftretenden Gefahren von Rissen und deren Begrenzung beschrieben. Das Vorgehen zur Ermittlung der Schweißparameter wurde vorgestellt, da diese Gefahren durch die Wahl der Schweißparameter begrenzt werden können.

Für die numerischen Berechnungen, die in Kapitel 9 vorgestellt werden, sind die mechanischen Kennwerte der Schweißverbindung erforderlich. Da diese teilweise nicht direkt (über Zugversuche) ermittelt werden können, wurde eine Möglichkeit zur Abschätzung der Zugfestigkeit der Schweißverbindung mithilfe von Härtemessungen veranschaulicht. Zudem wurden die Möglichkeiten zur Bestimmung der Eigenschaften in der Wärmeeinflusszone beschrieben.

Abschließend wurde ein kurzer Ausblick in die bruchmechanischen Eigenschaften und die Zähigkeit gegeben, die in dieser Arbeit jedoch nicht weiter betrachtet werden. 


\section{Vergleich bestehender Bemessungsverfahren von Schweiß- verbindungen}

\subsection{Einleitung}

In den meisten bisher geltenden Normen wird für die Tragfähigkeit der Schweißnaht nur die Festigkeit des Grundwerkstoffs berücksichtigt, während für den Schweißzusatzwerkstoff mindestens die gleiche Festigkeit wie die des Grundwerkstoffs gefordert wird. Für normalfeste Baustähle S235 und S355 ist diese Vereinfachung sinnvoll, da die zugehörigen Schweißzusatzwerkstoffe immer eine höhere Festigkeit als die Grundwerkstoffe haben. Für höherfeste Stähle S460 und S690 kann es für die Fertigung oder in Abhängigkeit der Beanspruchung auch sinnvoll sein, Schweißzusatzwerkstoffe mit geringerer oder höherer Festigkeit einzusetzen. Dazu müssen die Bemessungsregeln neben der Festigkeit des Grundwerkstoffs auch die Festigkeit des Schweißzusatzwerkstoffes aufnehmen. Die bestehenden Normen berücksichtigen die Besonderheiten von höherfesten Baustählen jedoch nicht ausreichend. Daraus ergibt sich das Ziel dieser Arbeit, ein Bemessungsmodell zur Bestimmung der Tragfähigkeit von Kehlnahtverbindungen höherfester Baustähle zu entwickeln.

Um einen Überblick über die bestehenden Regelungen zu geben, werden in diesem Kapitel die bestehenden europäischen und internationalen Bemessungsregeln zur Ermittlung der Tragfähigkeit von Kehlnahtverbindungen verglichen und die Besonderheiten erläutert. Die betrachteten Normen sind in Tabelle 3.1 mit Titel und Herkunft zusammengestellt. Im Weiteren werden verkürzt anstelle des gesamten Titels nur die genannten Bezeichnungen verwendet.

Tabelle 3.1: Zusammenstellung der betrachteten Normen

\begin{tabular}{|l|l|l|}
\hline Bezeichnung & Herkunft & Titel \\
\hline $\begin{array}{l}\text { DIN 18800-1 (1990) } \\
\text { DIN 18800-1 (2008) }\end{array}$ & Deutschland & $\begin{array}{l}\text { Stahlbauten, Teil 1: Bemessung und Konstruktion, Deut- } \\
\text { shes Institut für Normung e.V. (DIN) }\end{array}$ \\
\hline EN 1993-1-8 (2005) & Europa & $\begin{array}{l}\text { Eurocode 3: Bemessung und Konstruktion von Stahlbauten } \\
- \text { Teil 1-8: Berechnung und Bemessung von Anschlüssen, } \\
\text { CEN }\end{array}$ \\
\hline AISC 360-05 (2005) & Europa & $\begin{array}{l}\text { Eurocode 3: Bemessung und Konstruktion von Stahlbauten } \\
- \text { Teil 1-12: Zusätzliche Regeln zur Erweiterung von EN } \\
\text { 1993 auf Stahlsorten bis S700, CEN }\end{array}$ \\
\hline CAN/NSA S16.1-94 (2006) & Kanada & $\begin{array}{l}\text { Specification for structural steel buildings: American In- } \\
\text { stitute of steel construction, Inc, Chicago 2005 }\end{array}$ \\
\hline BSK 99 (2003) & Schweden & $\begin{array}{l}\text { Limit states design of steel structures, Canadian Standard } \\
\text { Association }\end{array}$ \\
\hline
\end{tabular}

Unterschiede werden hinsichtlich der geometrischen Angaben und der Nachweisformate festgestellt. Auch unterscheiden sich die geometrischen Angaben zu Nahtdicke und Nahtlänge. Der Schwerpunkt der Untersuchungen liegt auf dem Vergleich der Nachweisformate. Es werden die Unterschiede hinsichtlich der Verwendung von Fließgrenze oder Zugfestigkeit von Grund- oder Schweißzusatzwerkstoff herausgearbeitet. Außerdem werden die Unterschiede zwischen der normativen Tragfähigkeit von Kreuzstößen mit Stirnkehlnähten und Flankenkehlnahtverbindungen besprochen. Anhand der Beispiele der Flankenkehlnaht und des Kreuzstoßes werden die Tragfähigkeiten der einzelnen Bemessungsregeln verglichen.

\subsection{Beschreibung der verglichenen Normenregeln}

In Deutschland werden Schweißverbindungen nach DIN 18800 bemessen. Seit 1990 wurden nach DIN 18800-1 (1990) nur Stähle bis zur Stahlsorte S355 betrachtet. Die 2001 erschienene Anpassungsrichtlinie [ARiLi Stahl, 2001] ergänzte die Regelungen für den Stahl S460. Die Norm DIN 18800 wurde überarbeitet und erschien im Jahr 2008 als DIN 18800-1 (2008) unter Berücksichtigung der 
Anpassungsrichtlinie [ARiLi Stahl, 2001] neu. Zusätzlich gibt es Zulassungen für die Stähle S460 [Zulassung Z-30.2-5] und S690 [Zulassung Z-30-1.1].

Auf europäischer Ebene ist die Bemessung von Schweißverbindungen für Stähle bis zu einer Streckgrenze von $\mathrm{f}_{\mathrm{y}}=460 \mathrm{~N} / \mathrm{mm}^{2}$ in EN 1993-1-8 (2005) geregelt. Regelungen für Stahlsorten im Streckgrenzenbereich $500 \mathrm{~N} / \mathrm{mm}^{2} \leq \mathrm{f}_{\mathrm{y}} \leq 700 \mathrm{~N} / \mathrm{mm}^{2}$ (Stahlsorten S500 bis S700) finden sich in EN 1993-1-12 (2007).

In den USA werden Schweißverbindungen im Hochbau nach AISC 360-05 (2005) bemessen. Danach sind jedoch nur Stähle bis zur einer Streckgrenze von etwa $\mathrm{f}_{\mathrm{y}}=480 \mathrm{~N} / \mathrm{mm}^{2}$ geregelt. Die Bemessung von Schweißverbindungen in Kanada nach CAN/NSA S16.1-94 (2006) regelt Stähle bis zu einer Streckgrenze von $\mathrm{f}_{\mathrm{y}}=700 \mathrm{~N} / \mathrm{mm}^{2}$. Die Bemessung der Schweißnähte gemäß dem Schwedischen Regelwerk BSK 99 (2003) regelt Stahlsorten bis S690.

\subsection{Geometrische Angaben}

\subsubsection{Einleitung}

Als geometrische Parameter wird die rechnerische Tragfähigkeit von Kehlnahtverbindungen durch die Nahtdicke und die Nahtlänge bestimmt. Aus dem Produkt aus Nahtdicke und Nahtlänge ergibt sich die Nahtfläche, die rechnerisch zur Bestimmung der Tragfähigkeit herangezogen wird.

\subsubsection{Vergleich der Angaben zur Nahtdicke}

Über die Nahtdicke wird die Nahtfläche berechnet, mit der die rechnerische Tragfähigkeit ermittelt wird. Unterschiede in der Ermittlung der Nahtdicke bewirken somit Änderungen in der rechnerischen Tragfähigkeit. Zudem gibt es unterschiedliche Begrenzungen für minimale und maximale Nahtdicken.

Die rechnerische Nahtdicke kann aus der tatsächlichen Nahtdicke über einen Makroschliff bestimmt werden. Dies geschieht über ein Dreieck, das in die Naht eingeschrieben wird. Es muss dabei vollständig innerhalb der Naht liegen. Für die rechnerische Nahtdicke wird in allen Normen die Höhe des einschreibbaren Dreiecks festgelegt.

Nach Deutscher Norm DIN 18800 ist für die Nahtdicke a die Höhe eines gleichschenkligen rechtwinkligen Dreiecks zu verwenden. Im Gegensatz dazu darf nach Europäischer, nach US-amerikanischer, Kanadischer und Schwedischer Norm auch ein ungleichschenkliges Dreieck verwendet werden. Nach allen betrachteten Normen ist die Berücksichtigung eines Einbrands bei einer durchgeführten Verfahrensprüfung möglich. Die rechnerische Nahtdicke mit oder ohne Einbrand nach Deutscher und Europäischer Norm ist in Abbildung 3.1 dargestellt. Mit dem Einbrand bezeichnet man die Tiefe des aufgeschmolzenen Bereichs des Grundwerkstoffs. Damit vergrößert sich die Nahtfläche, die durch den aufgeschmolzenen Schweißzusatzwerkstoff entsteht. Der theoretische Wurzelpunkt ergibt sich als Verlängerung der Außenkanten.

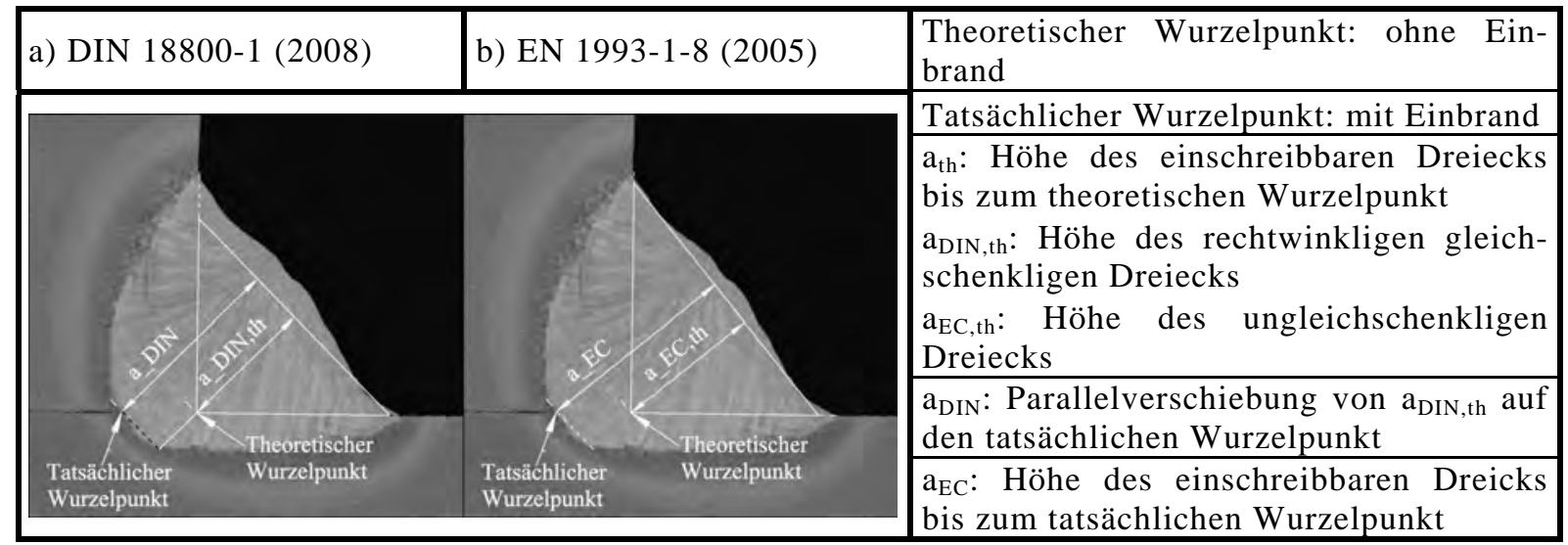

Abbildung 3.1: Rechnerische Nahtdicke a) nach DIN 18800-1 (2008) und b) nach EN 1993-1-8 (2005) 
Die theoretische Nahtdicke $\mathrm{a}_{\mathrm{th}}$ bezeichnet die Höhe des einschreibbaren Dreiecks bis zur theoretischen Nahtwurzel. Dabei ist nach DIN 18800 als Nahtdicke $a_{\text {DIN,th }}$ die Höhe eines rechtwinkligen gleichschenkligen Dreiecks und nach Europäischer Norm als Nahtdicke $\mathrm{a}_{\mathrm{EC} \text {,th }}$ die Höhe eines ungleichschenkligen Dreiecks zu verwenden. Die tatsächliche Nahtdicke $\mathrm{a}_{\mathrm{EC}}$ wird als Höhe des einschreibbaren Dreiecks bezogen auf die tatsächliche Nahtwurzel (ungleichschenkliges Dreieck, im Makroschliff gemessen) bestimmt. Für die tatsächliche Nahtdicke $a_{\text {DIN }}$ wird die Höhe des gleichschenkligen Dreiecks $a_{\text {DIN,th }}$ parallel auf die tatsächliche Nahtwurzel verschoben.

Die Angaben für minimale und maximale Kehlnahtdicken unterscheiden sich in den jeweiligen Normen. Die Mindestdicken und die maximalen Kehlnahtdicken sind wichtig, um schweißtechnisch einwandfreie Nähte zu ermöglichen. Die Angaben für die minimale und maximale Kehlnahtdicke der verschiedenen Normen sind im Folgenden aufgeführt. Einen Überblick über die Mindestabmessungen gibt Abbildung 3.2. Da die amerikanischen Normen die Nahtdicke nicht als Höhe sondern als Schenkellänge des einschreibbaren Dreiecks angeben, werden die Angaben mit dem Faktor $\sqrt{2}$ für ein gleichschenkliges Dreieck umgerechnet, um einen Vergleich zu ermöglichen. In den meisten Fällen variieren die geforderten Mindestnahtdicken jedoch nur um etwa $1 \mathrm{~mm}$.

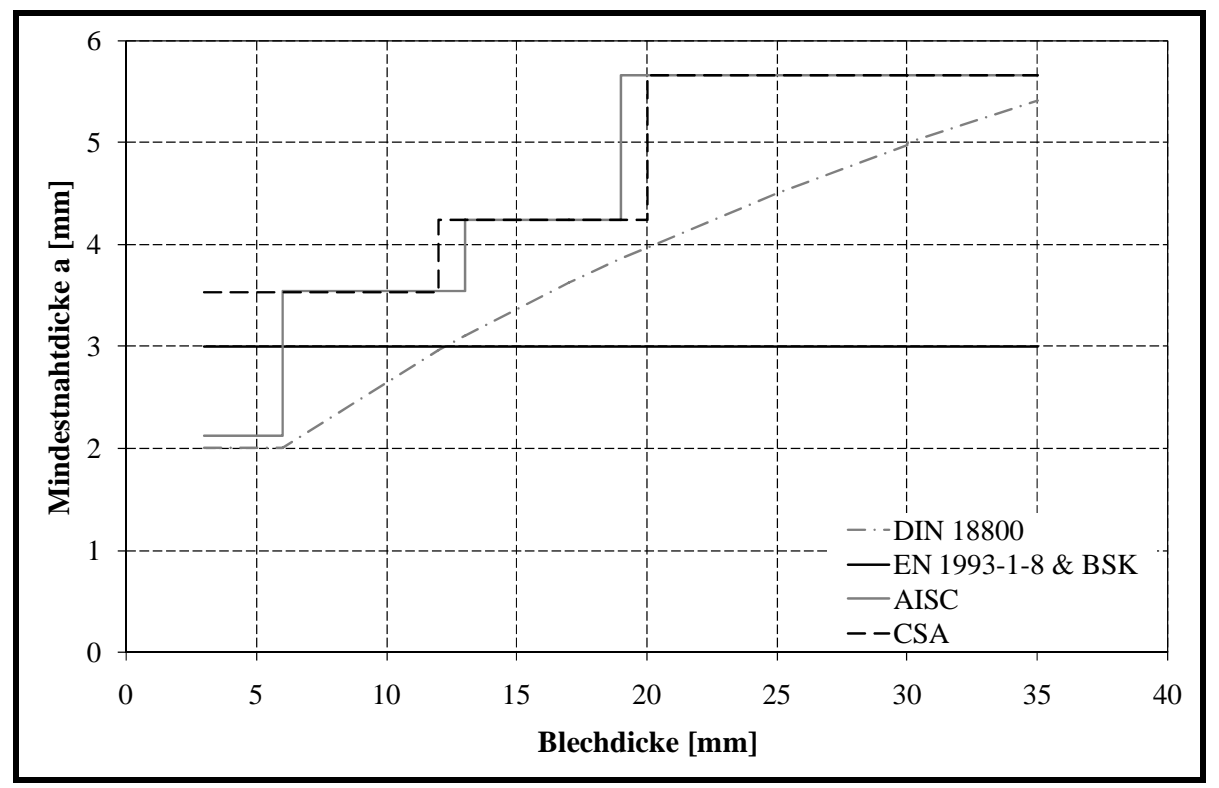

Abbildung 3.2: Vergleich der Mindestnahtdicke nach verschiedenen Normen

Nach deutscher Norm DIN 18800-1 (2008) gibt es für die Schweißnahtdicke a von Kehlnähten folgende Grenzwerte:

$$
\begin{aligned}
& \mathrm{a} \geq 2 \mathrm{~mm} \\
& \mathrm{a} \geq(\sqrt{\max \mathrm{t}})-0,5
\end{aligned}
$$

Für Blechdicken $\mathrm{t} \geq 30 \mathrm{~mm}$ a $\geq 5 \mathrm{~mm}$

$\mathrm{a} \leq 0,7 \cdot t_{\min }$, mit $\mathrm{t}_{\min }$ als Blechdicke des dünneren Bleches.

Nach Europäischer Norm EN 1993-1-8 (2005) beträgt die Mindestschweißnahtdicke von Kehlnähten $3 \mathrm{~mm}$. Auf nationaler Ebene wurden die oben genannten Regeln für die Mindestdicken nach DIN 18800 in den Nationalen Anhang DIN EN 1993-1-8/NA (2010) übernommen.

In der US-amerikanischen Norm wird anstelle der Höhe des einschreibbaren Dreiecks die Schenkellänge des Dreiecks verwendet. Dort gibt es für die minimale Schweißnahtdicke w (Schenkellänge) von Kehlnähten in Abhängigkeit des dünneren Bleches folgende Grenzwerte:

$\mathrm{w} \geq 3 \mathrm{~mm}$ für $\mathrm{t} \leq 6 \mathrm{~mm}$

$\mathrm{w} \geq 5 \mathrm{~mm}$ für $6 \mathrm{~mm}<\mathrm{t} \leq 13 \mathrm{~mm}$

$\mathrm{w} \geq 6 \mathrm{~mm}$ für $13 \mathrm{~mm}<\mathrm{t} \leq 19 \mathrm{~mm}$

$\mathrm{w} \geq 8 \mathrm{~mm}$ für $\mathrm{t}>19 \mathrm{~mm}$.

Außerdem darf nach US-amerikanischer Norm AISC 360-05 (2005) die maximale Dicke 0,25 · die Nahtlänge nicht überschreiten. 
Die minimale Schweißnahtdicke w (Schenkellänge) von Kehlnähten nach Kanadischer Norm CAN/NSA S16.1-94 (2006) wird in Abhängigkeit des dünneren Bleches $t_{\min }$ begrenzt:

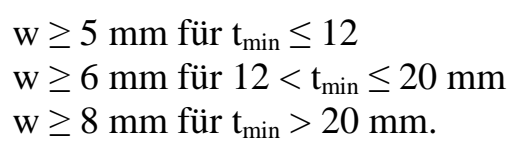

Nach Schwedischer Norm BSK 99 (2003) wird eine Mindestnahtdicke von a = 3 mm vorgegeben. Für eine Nahtdicke a $>15 \mathrm{~mm}$ muss die Festigkeit der Naht abgemindert werden, nähere Angaben zu dieser Abminderung werden jedoch nicht gemacht.

Insgesamt lässt sich zusammenfassen, dass die vorhandenen Unterschiede in der Ermittlung der Nahtdicke nur dann einen Einfluss haben, wenn die tatsächliche Nahtgeometrie z. B. anhand eines Makroschliffes vorliegt. Für theoretische Berechnungen ohne Kenntnis der tatsächlichen Naht, ergeben sich für alle Nachweisformate die gleichen Nahtdicken. Dann wird für die Bestimmung der Nahtdicke vereinfachend immer von einem gleichschenkligen Dreieck ausgegangen.

In allen Normen ergeben sich ähnliche Werte für die Mindestnahtdicken. Diese sind technisch sinnvoll, um schweißtechnisch einwandfreie Nähte zu erhalten.

\subsubsection{Vergleich der Angaben zur Nahtlänge}

Im Folgenden werden die Angaben für die Nahtlänge nach verschiedenen Normen verglichen. Für höherfeste Baustähle gibt es Begrenzungen der Nahtlänge. Im Rahmen dieser Arbeit werden experimentelle Untersuchungen zum Einfluss die Nahtlänge auf die Tragfähigkeit von Flankenkehlnähten durchgeführt, um diese Begrenzungen zu überprüfen. Als Grundlage dazu werden die aktuellen Angaben nach verschiedenen Normen verglichen. Die Nahtlänge l wird immer auf die Nahtdicke a bezogen als Größe 1/a angegeben.

Abbildung 3.3 zeigt den Vergleich der maximal zu berücksichtigenden Nahtlängen $\mathrm{L}_{\text {eff }}$ nach Deutscher, Europäischer und US-amerikanischer Norm. Für den Vergleich wird die Schenkellänge w mit dem Faktor $\sqrt{2}$ in die Nahtdicke a umgerechnet. Bei der europäischen Norm fehlt eine Obergrenze, so dass ein Abfallen des Verhältnisses Nahtlänge/Nahtdicke möglich ist.

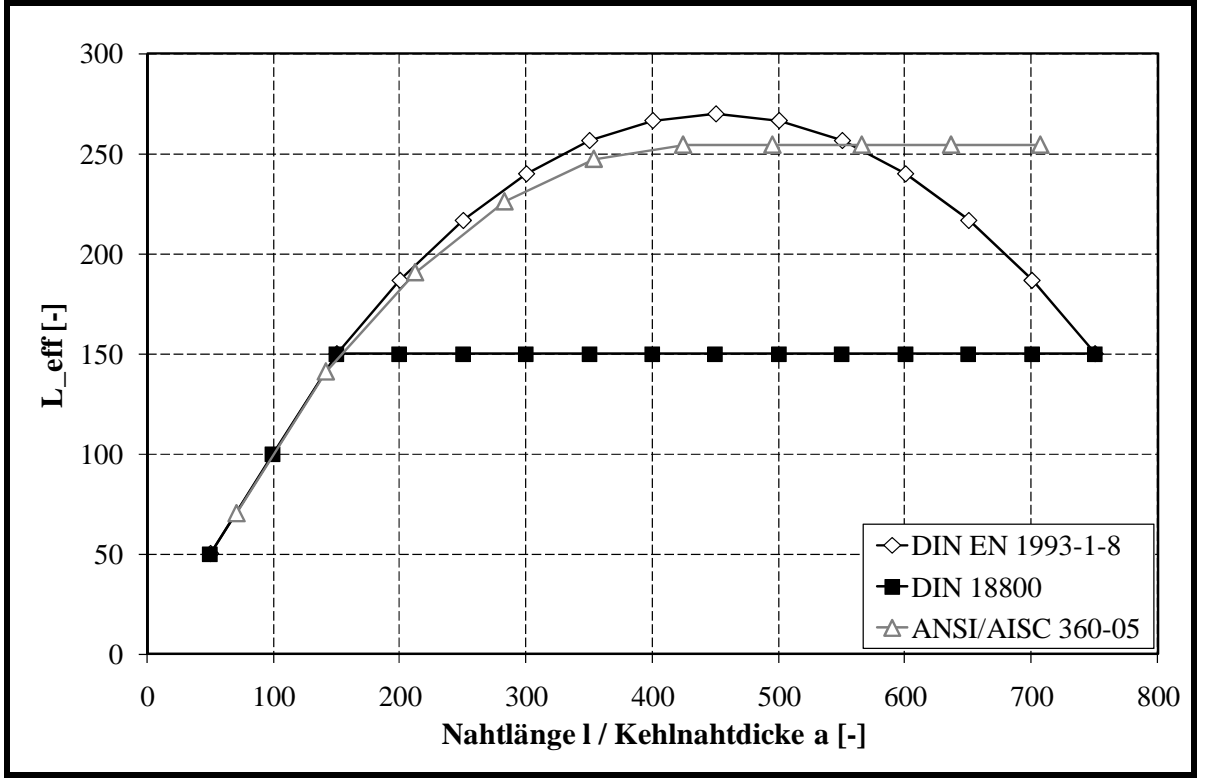

Abbildung 3.3: Vergleich der maximalen Nahtlänge bei Kehlnähten nach verschiedenen Normen

Nach DIN 18800-1 (2008) und EN 1993-1-8 (2005) darf als rechnerische Länge die geometrische Länge der Kehlnaht in der Wurzellinie verwendet werden, allerdings nur wenn die Länge l die Bedingung $\mathrm{l} \geq 6$ a und mindestens $\mathrm{l} \geq 30 \mathrm{~mm}$ erfüllt. 
Die Regelungen für die rechnerische Schweißnahtlänge unmittelbarer Stabanschlüsse unterscheiden sich in den verschiedenen deutschen Regelwerken. Nach DIN 18800-1 (1990) und [ARiLi Stahl, 2001] gilt für unmittelbare Stabanschlüsse mit normalfesten Stählen eine maximale Nahtlänge von l/a = 150. Für höherfeste Baustähle S460 gilt eine maximale Nahtlänge von l/a = 50. Die Empfehlungen der eigenen Untersuchungen [FOSTA P652, 2008], die in Kapitel 6 vorgestellt werden, konnten bei der Überarbeitung von DIN 18800-1 (2008) mit einer maximalen Nahtlänge von l/a = 100 berücksichtigt werden. Für den Stahl S460 wird in der Zulassung [Zulassung Z-30.2-5] eine maximale Nahtlänge von 1/a=150 festgelegt. Für den Stahl S690 wird in der Zulassung [Zulassung Z-30-1.1] die maximale Nahtlänge auf $1 / \mathrm{a}=50$ begrenzt.

Nach EN 1993-1-8 (2005) wird die rechnerische Nahtlänge bei unmittelbarer Lasteinleitung für Stähle bis Stahlgüte S460 bis zu einer Länge von l/a $=150$ nicht abgemindert. Für größere Längen l/a $>150$ gibt es einen Abminderungsfaktor $\beta_{\mathrm{Lw}, 1}=1,2-0,2 \cdot \mathrm{L}_{\mathrm{j}} /(150 \cdot \mathrm{a}) \leq 1,0$. Nach EN 1993-1-12 (2007) darf für lange Anschlüsse mit den Stahlsorten S500 bis S700 nur eine rechnerische Nahtlänge von $1 / a=50$ angesetzt werden.

Nach US-amerikanischer Norm AISC 360-05 (2005) muss für Verbindungen mit zwei Flankenkehlnähten die Länge jeder Naht mindestens so lang sein wie der Abstand zwischen den Nähten. Als rechnerische Länge darf die geometrische Länge $l$ der Kehlnaht bezogen auf die Schenkellänge $\mathrm{w}$ bis zu einer Länge von $\mathrm{l} / \mathrm{w}=100$ verwendet werden. Gleichzeitig muss die Länge $\mathrm{l}$ die Bedingung $\mathrm{l} \geq 4 \mathrm{w}$ erfüllen. Oberhalb von $\mathrm{l} / \mathrm{w}=100$ muss die maximale Nahtlänge mit einen Faktor $\beta=1,2-0,2 \cdot \mathrm{l} /$ $(100 \cdot w) \leq 1,0$ abgemindert werden. Oberhalb von $\mathrm{l} / \mathrm{w}=300$ muss als effektive Nahtlänge $\mathrm{l} / \mathrm{w}=180$ verwendet werden. Die rechnerische Länge wird in Abhängigkeit der Schenkellänge w angegeben.

Die Kanadische Norm CAN/NSA S16.1-94 (2006) macht keine Angaben zur Nahtlänge.

Nach Schwedischer Norm BSK 99 (2003) ist für Flankenkehlnähte eine maximale Nahtlänge von 1/a = 60 gegeben.

In den experimentellen Untersuchungen, die in Kapitel 6 - 8 vorgestellt werden, wird untersucht, ob sich für höherfeste Baustähle S460 und S690 mit zunehmender Nahtlänge eine Verringerung der Tragfähigkeit ergibt.

\subsection{Vergleich der Nachweisformate}

\subsubsection{Allgemeines}

Im Folgenden werden die verschiedenen Nachweisformate verglichen. Dabei wird der Schwerpunkt auf die Berücksichtigung des Schweißzusatzwerkstoffs und den Einfluss der Kraftrichtung gelegt. Für höherfeste Baustähle zeigen sich große Unterschiede, die eine systematische Untersuchung notwendig machen. Es zeigt sich, dass die bestehenden Nachweisformate die Besonderheiten von höherfesten Baustählen, wie die Berücksichtigung des Schweißzusatzwerkstoffs nicht ausreichend berücksichtigen. Daraus ergibt sich das Ziel dieser Arbeit, ein Bemessungsmodell in Abhängigkeit des Schweißzusatzwerkstoffs zu entwickeln.

Der Nachweis der Tragfähigkeit von Schweißverbindungen erfolgt immer durch den Nachweis, dass die Beanspruchung kleiner sein muss als die Beanspruchbarkeit. Die Beanspruchung ergibt sich aus der einwirkenden Kraft, die in Spannungskomponenten berücksichtigt wird, und der Nahtfläche. Für die Beanspruchbarkeit gibt es neben verschiedenen Formaten Unterschiede hinsichtlich der Verwendung von Fließgrenze oder Zugfestigkeit von Grund- oder Schweißzusatzwerkstoff. Dazu werden auf verschiedene Arten Beiwerte verwendet. Die Beanspruchbarkeit wird auch als Widerstand bezeichnet. Mit der Beanspruchbarkeit wird die Festigkeit der Kehlnaht im Verhältnis zur Festigkeit der eingesetzten Werkstoffe beschrieben.

Die Unterschiede in den normativen Tragfähigkeiten werden zum einen anhand der Tragfähigkeit von Flankenkehlnahtverbindungen und zum anderen an Kreuzstößen mit Stirnkehlnähten verglichen. 


\subsubsection{Nachweisformat nach Deutscher Norm DIN 18800}

Die Bestimmung der Schweißnahtspannung nach DIN 18800-1 (2008) erfolgt über einen Vergleichswert $\sigma_{\mathrm{w}, \mathrm{v}}$, in dem als Spannungskomponenten die Normalspannung $\sigma_{\perp}$ und die Schubspannungen $\tau_{\perp}$ und $\tau_{\text {II }}$ enthalten sind, vgl. Abbildung 3.4. Die Normalspannung $\sigma_{\text {II }}$ parallel zur Nahtachse wird vernachlässigt. Die Spannungskomponenten ergeben sich aus dem Quotienten aus Kraft und Nahtfläche.

Die Nahtfläche berechnet sich aus dem Produkt von Nahtdicke $a_{w}$ und Nahtlänge $a_{1}$. Die Nahtdicke ergibt sich als Höhe des einschreibbaren Dreiecks, wie in Kapitel 3.3.2 beschrieben. Die Kraft wird in die Komponenten bezogen auf eine Nachweiseisebene aufgeteilt, die nicht die Nahtfläche, sondern eine horizontale oder vertikale Ebene ist.

Für die Grenzschweißnahtspannung $\sigma_{\mathrm{w}, \mathrm{R}, \mathrm{d}}$ werden als Komponenten die Streckgrenze des Grundwerkstoffs $f_{\mathrm{y}, \mathrm{k}}$, der Beiwert $\alpha_{\mathrm{w}}$ und der Teilsicherheitsbeiwert $\gamma_{\mathrm{M}}$ verwendet. Der Vergleichswert $\sigma_{\mathrm{w}, \mathrm{v}}$ muss kleiner als die Grenzschweißnahtspannung $\sigma_{\mathrm{w}, \mathrm{R}, \mathrm{d}}$ sein:

$$
\sigma_{\mathrm{w}, \mathrm{v}}=\sqrt{\sigma_{\perp}^{2}+\tau_{\perp}^{2}+\tau_{\mathrm{II}}^{2}} \leq \alpha_{\mathrm{w}} \cdot \frac{\mathrm{f}_{\mathrm{y}, \mathrm{k}}}{\gamma_{\mathrm{M}}}=\sigma_{\mathrm{w}, \mathrm{R}, \mathrm{d}}
$$

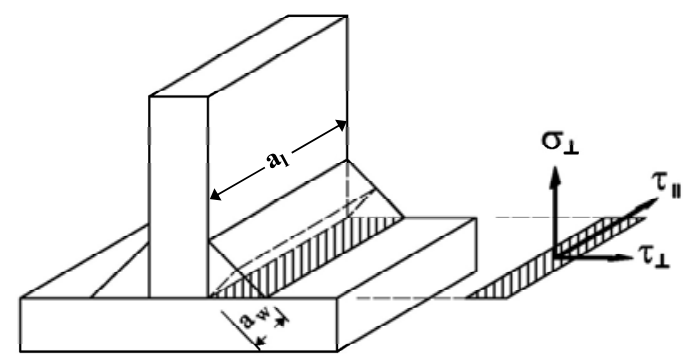

Abbildung 3.4: Spannungskomponenten parallel und senkrecht zur Nahtachse

Die charakteristischen Werte $\mathrm{f}_{\mathrm{y}, \mathrm{k}}$ sind nach DIN 18800-1 (2008) blechdickenabhängig (Blechdicke $\mathrm{t} \leq$ $40 \mathrm{~mm}$ oder $40<\mathrm{t} \leq 100 \mathrm{~mm}$ ) gestaffelt. Mit zunehmender Blechdicke dürfen nur geringere Streckgrenzen berücksichtigt werden. Für Bauteile mit einer Blechdicke $\mathrm{t}>40 \mathrm{~mm}$ darf für die Bemessung von Schweißverbindungen der charakteristische Wert der Streckgrenze für Blechdicken bis $\mathrm{t}=40 \mathrm{~mm}$ verwendet werden. Nach DIN 18800-1 (2008) und [Beuth-Kommentare, 1998] ist bei Schweißverbindungen mit Grundwerkstoffen unterschiedlicher Festigkeit die Streckgrenze des Grundwerkstoffs mit geringerer Festigkeit zu verwenden. Die Festigkeit des Schweißzusatzwerkstoffs wird nicht berücksichtigt. Als Teilsicherheitsbeiwert ist $\gamma_{M}=1,1$ zu verwenden.

Der Beiwert $\alpha_{\mathrm{w}}$ liegt zwischen 0,95 für normalfeste Stähle und 0,6 für höherfeste Stähle. Bei der Überarbeitung von DIN 18800-1 (2008) wurde für den Stahl S460 der Beiwert $\alpha_{w}$ von 0,6 auf 0,7 angehoben. Diese Änderung ist durch die Ergebnisse der in Kapitel 6 vorgestellten eigenen Untersuchungen [FOSTA P652, 2008] ermöglicht worden.

Die Anwendung der bisherigen Angaben auf die verschiedenen Stähle ist in Tabelle 3.2 dargestellt. Es sind die Bemessungswiderstände der Regelungen nach DIN 18800-1 (1990) und der Anpassungsrichtlinie [ARiLi Stahl, 2001] sowie der neuen Regelungen nach DIN 18800-1 (2008) zusammengestellt. Zusätzlich sind die Regelungen der Zulassungen ([Zulassung Z-30.2-5], [Zulassung Z-30-1.1]) aufgeführt.

Tabelle 3.2: Bemessungswiderstand für Kehlnähte nach Deutscher Norm

\begin{tabular}{|l|l|l|l|l|l|l|}
\hline \multirow{2}{*}{ Stahlsorte } & $\begin{array}{l}\text { DIN 18800-1 (1990) } \\
\text { DIN 18800-1 (2008) }\end{array}$ & $\begin{array}{l}\text { DIN 18800-1 (1990) } \\
\text { [ARiLi Stahl, 2001] }\end{array}$ & $\begin{array}{l}\text { DIN 18800- } \\
\text { 1 (2008) }\end{array}$ & $\begin{array}{l}\text { Zulassung } \\
\text { Z-30.2-5] }\end{array}$ & $\begin{array}{l}\text { [Zulassung } \\
\text { Z-30-1.1] }\end{array}$ \\
\cline { 2 - 7 } & S235 & S355 & S460 & S460 & S460 & S690 \\
\hline $\mathrm{f}_{\mathrm{y}, \mathrm{k}}\left[\mathrm{N} / \mathrm{mm}^{2}\right]$ & 240 & 360 & 460 & 460 & 460 & 690 \\
\hline$\alpha_{\mathrm{w}}[-]$ & 0,95 & 0,80 & 0,60 & 0,70 & 0,75 & 0,60 \\
\hline$\gamma_{\mathrm{M}}[-]$ & 1,1 & \multicolumn{5}{|l}{} \\
\hline$\alpha_{\mathrm{w}} \cdot \frac{\mathrm{f}_{\mathrm{y}, \mathrm{k}}}{\gamma_{\mathrm{M}}}\left[\frac{\mathrm{N}}{\mathrm{mm}^{2}}\right]$ & 207 & 262 & 251 & 293 & 314 & 376 \\
\hline
\end{tabular}


Nach DIN 18800-1 (1990) und [ARiLi Stahl, 2001] ergeben sich damit für Schweißverbindungen höherfester Stähle S460 etwas geringere Bemessungswiderstände als für die der Stahlsorte S355. Für die Stahlsorte S690 hingegen ergeben sich deutlich höhere Bemessungswiderstände.

Eine Beispielrechnung in Tabelle 3.3 ermittelt die maximale Beanspruchbarkeit von Kreuzstößen mit Stirnkehlnähten und Flankenkehlnähten. Nach Gleichung (3.1) ergibt sich kein Unterschied in der Tragfähigkeit zwischen Stirn- und Flankenkehlnähten. Somit wird kein Einfluss der Kraftrichtung berücksichtigt.

Tabelle 3.3: Beispielrechnung für Stirn- und Flankenkehlnähte nach DIN 18800-1 (2008)

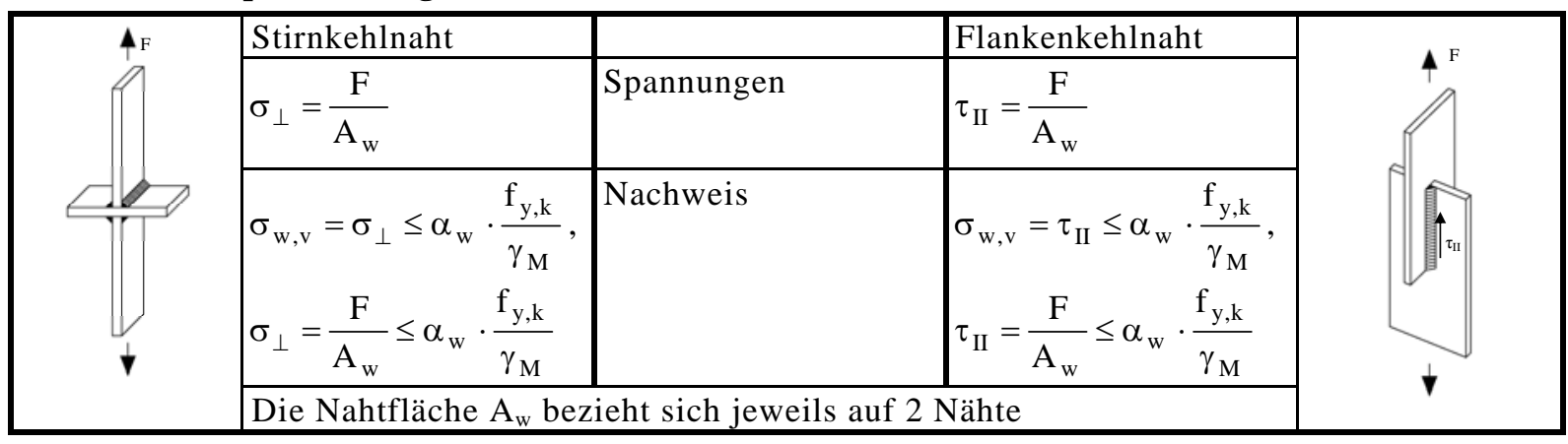

\subsubsection{Nachweisformat nach Europäischer Norm DIN EN 1993}

Im Eurocode gibt es zwei mögliche Bemessungsverfahren: das Richtungsbezogene Verfahren und das Vereinfachte Verfahren.

Beim Richtungsbezogenen Verfahren werden die in der Naht wirkenden Kräfte in Spannungskomponenten $\sigma_{\perp}, \tau_{\perp}$ und $\tau_{\text {II }}$ aufgeteilt, vgl. Abbildung 3.5. Die Spannungskomponenten werden auf die Nahtachse bezogen. Es wird eine Gewichtung der Schubspannungskomponenten mit dem Faktor 3 vorgenommen, vgl. (3.2). Die Normalspannung $\sigma_{\text {II }}$ parallel zur Nahtachse wird vernachlässigt.

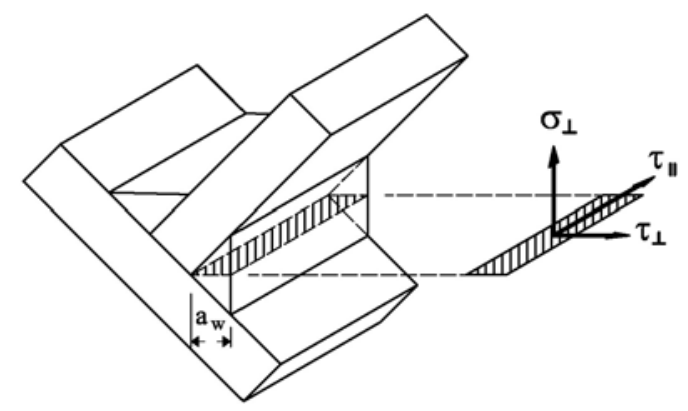

Abbildung 3.5: Spannungskomponenten parallel und senkrecht zur Nahtachse

Die Spannungskomponenten werden aus den Bemessungslasten unter der Annahme einer gleichmäßigen Spannungsverteilung bestimmt und in einer Vergleichsspannung zusammengefasst. Die Vergleichsspannung $\sigma_{\mathrm{v}, \mathrm{Ed}}$ gemäß Gleichung (3.2) muss kleiner als die Beanspruchbarkeit der Naht sein, die im Folgenden als Bemessungsgrenzspannung $\sigma_{\mathrm{w}, \mathrm{Rd}}$ bezeichnet wird. Gleichzeitig ist die Zusatzbedingung nach Gleichung (3.3) einzuhalten:

$$
\begin{aligned}
& \sigma_{\mathrm{v}, \mathrm{Ed}} \leq \sigma_{\mathrm{w}, \mathrm{Rd}} \rightarrow \sqrt{\sigma_{\perp}^{2}+3 \cdot \tau_{\perp}^{2}+3 \cdot \tau_{\mathrm{II}}^{2}} \leq \frac{\mathrm{f}_{\mathrm{u}, \mathrm{k}}}{\beta_{\mathrm{w}} \cdot \gamma_{\mathrm{M} 2}} \\
& \sigma_{\perp} \leq \frac{0,9 \cdot \mathrm{f}_{\mathrm{u}, \mathrm{k}}}{\gamma_{\mathrm{M} 2}}
\end{aligned}
$$

Der Nachweis gemäß Gleichung (3.3) kann nur für nicht durchgeschweißte Stumpfnähte maßgebend werden, während für Kehlnahtverbindungen stets die Grenzbeanspruchbarkeit gemäß Gleichung (3.2) maßgebend wird. Aus diesem Grund wird der Nachweis nach Gleichung (3.3) nicht weiterbetrachtet. 
Das „Vereinfachte Verfahren“ vergleicht die Resultierende aller auf die wirksame Kehlnahtfläche einwirkenden Kräfte $F_{w, E d}$ mit dem Bemessungswert der Scherfestigkeit $F_{w, R d}$ der Schweißnaht:

$$
\mathrm{F}_{\mathrm{w}, \mathrm{Ed}} \leq \mathrm{F}_{\mathrm{w}, \mathrm{Rd}} \text { und damit auf Spannungsebene } \frac{\mathrm{F}_{\mathrm{w}, \mathrm{Ed}}}{\mathrm{A}_{\mathrm{w}}} \leq \frac{\mathrm{f}_{\mathrm{u}, \mathrm{k}} / \sqrt{3}}{\beta_{\mathrm{w}} \cdot \gamma_{\mathrm{M} 2}}
$$

In beiden Verfahren werden als Komponenten für den Bemessungswiderstand die Zugfestigkeit $\mathrm{f}_{\mathrm{u}, \mathrm{k}}$, der Korrelationsbeiwert $\beta_{\mathrm{w}}$ und der Teilsicherheitsbeiwert $\gamma_{\mathrm{M} 2}$ verwendet.

Für die Zugfestigkeit $\mathrm{f}_{\mathrm{u}, \mathrm{k}}$ wird die Zugfestigkeit des schwächeren Grundwerkstoffs der beiden Bauteile verwendet. Nach EN 1993-1-8 (2005) wird die Zugfestigkeit des Schweißzusatzwerkstoffs nicht berücksichtigt. Stattdessen werden für den Schweißzusatzwerkstoff mindestens die spezifizierten Werte des Grundwerkstoffs, wie Streckgrenze, Bruchfestigkeit, Bruchdehnung und Mindestkerbschlagarbeit gefordert. Für Bauteile mit einer Blechdicke $\mathrm{t}>40 \mathrm{~mm}$ ist die Zugfestigkeit abzumindern. Diese Regelung wird als konfliktfreie Ergänzung nach DIN EN 1993-1-8/NA (2010) ersetzt, so dass eine ähnliche Regel wie nach DIN 18800-1 (2008) entsteht, wonach bei größeren Blechdicken der charakteristische Wert der Zugfestigkeit für Blechdicken bis $\mathrm{t}=40 \mathrm{~mm}$ verwendet werden darf.

Der Korrelationsbeiwert $\beta_{\mathrm{w}}$ liegt zwischen 0,8 für normalfeste Stähle und 1,0 für höherfeste Stähle.

Als Teilsicherheitsbeiwert für die Beanspruchbarkeit von Schweißnähten bei Bruchversagen infolge Zugbeanspruchung ist $\gamma_{\mathrm{M} 2}=1,25$ vorgeschrieben.

Für $\mathrm{f}_{\mathrm{u}, \mathrm{k}}$ wird nach EN 1993-1-12 (2007) bei Stählen der Stahlsorte höher als S460 bis S700 die Zugfestigkeit des Schweißzusatzwerkstoffs eingesetzt, wenn niederfestere Schweißzusatzwerkstoffe verwendet werden. Dann ist ein Beiwert $\beta_{w}=1,0$ zu verwenden. Somit gibt es eine Regelung für die Verwendung von niederfesten Schweißzusatzwerkstoffen. Ein Vergleich zum Einfluss der unterschiedlichen Festigkeiten des Schweißzusatzwerkstoffs (SZW) und des Grundwerkstoffs (GW) ist in Tabelle 3.4 zusammengestellt. Nach DIN EN 1993-1-12/NA (2011) wird jedoch gefordert, dass die Festigkeit des Schweißzusatzwerkstoffs mindestens so groß ist wie die des Grundwerkstoffs.

Tabelle 3.4: Bemessungsgrenzspannungen bei unterschiedlichen Werkstoffen nach EN 1993-1-12 (2007)

\begin{tabular}{|l|l|l|l|}
\hline Kombination & S690Q-G42 & S690Q-G46 & S690Q-G69 \\
\hline $\mathrm{SZW} \mathrm{f}_{\mathrm{u}, \mathrm{k}}\left[\mathrm{N} / \mathrm{mm}^{2}\right]$ & 770 & 770 & 770 \\
\hline $\mathrm{GW} \mathrm{f}_{\mathrm{u}, \mathrm{k}}\left[\mathrm{N} / \mathrm{mm}^{2}\right]$ & 500 & 530 & 770 \\
\hline \begin{tabular}{l}
$\mathrm{f}_{\mathrm{u}, \mathrm{k}, \mathrm{SZW}} / \sqrt{3}$ \\
\hline$\beta_{\mathrm{w}} \cdot \gamma_{\mathrm{M} 2}$
\end{tabular} & $\frac{500 / \sqrt{3}}{1,0 \cdot 1,25}=231$ & $\frac{530 / \sqrt{3}}{1,0 \cdot 1,25}=245$ & $\left.\frac{770 / \sqrt{3}}{1,0 \cdot 1,25}=356 / \frac{770 / \sqrt{3}}{1,2 \cdot 1,25}=296^{*}\right)$ \\
\hline mit $\left.\beta_{\mathrm{w}}=1,0 ; *\right)$ unter Berücksichtigung des Nationalen Anhangs, vgl. Tabelle 3.5 \\
\hline
\end{tabular}

Daraus ergeben sich die Bemessungsgrenzspannungen, die in Tabelle 3.5 für einzelne Stähle zusammengestellt sind. Je nach Lieferzustand der Stähle ändern sich die Zugfestigkeiten nach EN 1993-1-1 (2005). Die in Kapitel 6 vorgestellten Ergebnisse des Projekts [FOSTA P652, 2008] führten zu Empfehlungen für den Korrelationsbeiwert: für $\mathrm{S} 460 \beta_{\mathrm{w}}=0,85$ und für $\mathrm{S} 690 \beta_{\mathrm{w}}=1$,2. Für den Stahl S460 wurden diese Empfehlungen in den Nationalen Anhang DIN EN 1993-1-8/NA (2010) und für den Stahl S690 in den Nationalen Anhang DIN EN 1993-1-12/NA (2011) übernommen. In Tabelle 3.5 sind jeweils beide Werte aufgeführt.

Im Eurocode ergeben sich damit für Schweißverbindungen höherfester Stähle S460 etwas geringere Beanspruchbarkeiten als für die der Stahlsorte S355. Der Grund dafür liegt nach [Background D.03, 1990] und [Gresnigt, 2002] in der begrenzten Anzahl von Versuchen und deren großer Streuung. Für die Stahlsorte S690 hingegen ergeben sich deutlich höhere Tragfähigkeiten im Eurocode, die jedoch bei Anwendung des Nationalen Anhangs nicht angesetzt werden dürfen. 
Tabelle 3.5: Bemessungsgrenzspannung nach EN 1993-1-8 (2005), DIN EN 1993-1-8/NA (2010), EN 19931-12 (2007) und DIN EN 1993-1-12/NA (2011) für Kehlnähte in N/mm²

\begin{tabular}{|c|c|c|c|c|c|c|c|c|c|}
\hline \multirow[b]{2}{*}{ Stahlsorte } & \multirow{2}{*}{\begin{tabular}{|l|} 
S235 \\
$1)$ \\
\end{tabular}} & \multicolumn{3}{|l|}{ S355 } & \multicolumn{3}{|l|}{5460} & \multirow{2}{*}{\begin{tabular}{|l|}
$\mathrm{S} 690$ \\
$\mathrm{Q} / \mathrm{QL} /$ \\
$\mathrm{QL1}^{4)}$ \\
\end{tabular}} & \multirow{2}{*}{$\begin{array}{l}\text { S700 } \\
\text { MC }\end{array}$} \\
\hline & & \begin{tabular}{|l|}
$\mathbf{J R} / \mathbf{J O}$ \\
$/ \mathbf{J} 2^{1)}$ \\
\end{tabular} & \begin{tabular}{|l}
$\mathbf{N} /$ \\
$\mathbf{N L}^{2)}$ \\
\end{tabular} & $\begin{array}{l}\mathbf{M} / \\
\mathbf{M L} \mathbf{L}^{3)} \\
\end{array}$ & \begin{tabular}{|l|} 
\\
$2)$
\end{tabular} & $\begin{array}{l}\mathbf{M} / \mathbf{M L} \\
\text { 3) }\end{array}$ & \begin{tabular}{|l|}
$\mathbf{Q} / \mathbf{Q L} /$ \\
$\mathbf{Q L 1}^{\mathbf{4}}$ \\
\end{tabular} & & \\
\hline $\mathrm{f}_{\mathrm{v}, \mathrm{k}}\left[\mathrm{N} / \mathrm{mm}^{2}\right]$ & 235 & 355 & 355 & 355 & 460 & 460 & 460 & 690 & 700 \\
\hline $\mathrm{f}_{\mathrm{u}, \mathrm{k}}\left[\mathrm{N} / \mathrm{mm}^{2}\right]$ & 360 & 510 & 490 & 470 & 540 & 540 & 570 & 770 & 750 \\
\hline$\beta_{w}[-]$ & 0,8 & 0,9 & & & $1,0 / 0$, & & & $1,0 / 1,2$ & \\
\hline$\gamma_{\mathrm{M} 2}[-]$ & & & & & & 25 & & & \\
\hline$\frac{\mathrm{f}_{\mathrm{u}, \mathrm{k}}}{\beta_{\mathrm{w}} \cdot \gamma_{\mathrm{M} 2}}(3.2)$ & 360 & 453 & 436 & 418 & |432/50 & & |456/536 & \begin{tabular}{|l}
$616 / 3$ \\
513 \\
\end{tabular} & $\begin{array}{l}600 / \\
500\end{array}$ \\
\hline$\frac{\mathrm{f}_{\mathrm{u}, \mathrm{k}} / \sqrt{3}}{\beta_{\mathrm{w}} \cdot \gamma_{\mathrm{M} 2}}$ & 208 & 262 & 251 & 241 & |249/29 & & 263/310 & \begin{tabular}{|l}
$356 / /$ \\
296
\end{tabular} & $\begin{array}{l}346 / \\
289\end{array}$ \\
\hline
\end{tabular}

Eine Beispielrechnung zur Anwendung der Bemessungsgrenzbeanspruchbarkeit in Tabelle 3.6 zeigt den Unterschied des Richtungsbezogenen und des Vereinfachten Verfahren sowohl für Kreuzstöße mit Stirnkehlnähten als auch für Flankenkehlnahtverbindungen. Für Flankenkehlnahtverbindungen, also im Grenzfall der reinen Scherbeanspruchung, ist das Vereinfachte Verfahren mit dem Richtungsbezogenen Verfahren identisch. Für Kreuzstöße ergibt sich mit dem Richtungsbezogenen Verfahren eine um ca. $22 \%$ höhere Bemessungsgrenzspannung als mit dem Vereinfachten Verfahren. Zwischen Flanken- und Stirnkehlnahtverbindungen ergibt sich die gleiche Beanspruchbarkeit beim Vereinfachten Verfahren, während sich beim Richtungsbezogenen Verfahren ein Unterschied von 22 \% ergibt.

Tabelle 3.6: Beispielrechnung für Stirn- und Flankenkehlnähte nach EN 1993-1-8 (2005)

\begin{tabular}{|c|c|c|}
\hline \multirow{3}{*}{$\overbrace{1}^{\sigma_{1}^{I_{1}}}$} & \multicolumn{2}{|c|}{ Kreuzstoß mit Stirnkehlnaht } \\
\hline & Spannungen & $\sigma_{\perp}=\tau_{\perp}=\frac{\mathrm{F}}{\sqrt{2} \mathrm{~A}_{\mathrm{w}}}$ \\
\hline & $\begin{array}{l}\text { Nachweis Richtungs- } \\
\text { bezogen }\end{array}$ & $\sqrt{\sigma_{\perp}^{2}+3 \tau_{\perp}^{2}} \leq \frac{\mathrm{f}_{\mathrm{u}, \mathrm{k}}}{\beta_{\mathrm{w}} \cdot \gamma_{\mathrm{M} 2}} \rightarrow \frac{\mathrm{F}}{\mathrm{A}_{\mathrm{w}}} \leq \frac{\mathrm{f}_{\mathrm{u}, \mathrm{k}}}{\sqrt{2} \cdot \beta_{\mathrm{w}} \cdot \gamma_{\mathrm{M} 2}}$ \\
\hline \multirow{3}{*}{$\boldsymbol{\nabla}$} & Nachweis Verein- & $\mathrm{F} \leq \mathrm{f}_{\mathrm{u}, \mathrm{k}}$ \\
\hline & & $A_{w} \sqrt{3} \cdot \beta_{w} \cdot \gamma_{M 2}$ \\
\hline & Vergleich & Um $22 \%$ höhere Beanspruchbarkeit \\
\hline \multirow{6}{*}{$\underbrace{\mathrm{F}}_{V}$} & \multicolumn{2}{|c|}{ Flankenkehlnahtverbindung } \\
\hline & Spannungen & $\tau_{\text {II }}=\frac{F}{A_{w}}$ \\
\hline & $\begin{array}{l}\text { Nachweis Richtungs- } \\
\text { bezogen }\end{array}$ & $\sqrt{3 \cdot \tau_{\mathrm{II}}^{2}} \leq \frac{\mathrm{f}_{\mathrm{u}, \mathrm{k}}}{\beta_{\mathrm{w}} \cdot \gamma_{\mathrm{M} 2}} \rightarrow \frac{\mathrm{F}}{\mathrm{A}_{\mathrm{w}}} \leq \frac{\mathrm{f}_{\mathrm{u}, \mathrm{k}}}{\sqrt{3} \cdot \beta_{\mathrm{w}} \cdot \gamma_{\mathrm{M} 2}}$ \\
\hline & \multirow{2}{*}{$\begin{array}{l}\text { Nachweis Verein- } \\
\text { facht }\end{array}$} & $\mathrm{F}<\mathrm{f}_{\mathrm{u}, \mathrm{k}}$ \\
\hline & & $\overline{A_{w}}=\frac{\sqrt{3} \cdot \beta_{w} \cdot \gamma_{M 2}}{\sqrt{2}}$ \\
\hline & Vergleich & Gleiche Beanspruchbarkeit \\
\hline \multicolumn{3}{|c|}{ Vergleich zwischen Flankenkehlnahtverbindungen und Stirnkehlnahtverbindungen } \\
\hline \multicolumn{2}{|c|}{ Nachweis Richtungsbezogen } & Um 22 \% höhere Beanspruchbarkeit \\
\hline \multicolumn{2}{|c|}{ Nachweis Vereinfacht } & Gleiche Beanspruchbarkeit \\
\hline
\end{tabular}

Der Hintergrund der Eurocode-Regeln wird im Folgenden vorgestellt. Eine internationale Veröffentlichung des Institute of Welding (IIW) zu Bemessungsregeln von Schweißverbindungen [IIW, 1976] liefert einen Bemessungsansatz für normalfeste Stähle. Dieser wird im Detail in [Gresnigt, 1989] vorgestellt. In diesem Bemessungsansatz werden die vorhandenen Vergleichsspannungen mit einem Abminderungsfaktor $\beta$ abgemindert und der Zugfestigkeit des Grundwerkstoffs gegenüberstellt: 


$$
\beta \cdot \sqrt{\sigma_{\perp}^{2}+3 \cdot\left(\tau_{\perp}^{2}+\tau_{\text {II }}^{2}\right)} \leq \mathrm{f}_{\mathrm{u}} \text { mit } \beta=0,7 \text { für S235 und } \beta=0,85 \text { für S355 }
$$

Daraus wurde der Ansatz für den Eurocode [Snijder et al., 1988] entwickelt. Diese Werte sind günstiger als die Werte im Eurocode EN 1993-1-8 (2005) (mit $\beta=0,8$ für S235 und $\beta=0,9$ für S355). Zitiert und angewendet wird diese Formel für die statistische Auswertung für Stähle S460 nach [Background D.03, 1990]. Eine Weiterentwicklung wird in [Gresnigt, 2002] für höherfeste Stähle S460 diskutiert.

\subsubsection{Nachweisformat nach US-amerikanischer Norm}

Im Bemessungsmodell nach AISC 360-05 (2005) sind zwei mögliche Vorgehensweisen für die Berechnung der Tragfähigkeit vorhanden: LRFD und ASD. Für die Nachweise nach LRFD werden im Gegensatz zum Nachweis nach ASD Teilsicherheitsbeiwerte $(\Phi)$ verwendet. Im Folgenden wird nur das Verfahren LRFD nach Gleichung (3.6) betrachtet, da dieses Konzept der Teilsicherheitsbeiwerte der aktuellen deutschen und europäischen Norm ähnlich ist.

LRFD: Load and Resistance Factor Design $\mathrm{R}_{\mathrm{u}} \leq \Phi \cdot \mathrm{R}_{\mathrm{n}}$ und $\mathrm{R}_{\mathrm{n}}=\mathrm{F}_{\mathrm{w}} \cdot \mathrm{A}_{\mathrm{w}}$

Mit: $\mathrm{R}_{\mathrm{u}}$ : Beanspruchung

$\mathrm{R}_{\mathrm{n}}$ : Beanspruchbarkeit

$\mathrm{F}_{\mathrm{w}}$ : nach Gleichung (3.7) oder (3.8)

$\mathrm{A}_{\mathrm{w}}$ : Schweißnahtfläche

Für den Nachweis (3.6) (LRFD) gibt es zwei Verfahren zur Bestimmung der Tragfähigkeit von Schweißverbindungen. Das erste Verfahren berücksichtigt nur die Festigkeit des Schweißzusatzwerkstoffs $F_{\text {EXX }}$, Gleichung (3.7). Im zweiten Verfahren, Gleichung (3.8), wird die Tragfähigkeit von Schweißverbindungen in Abhängigkeit der Festigkeit des Schweißzusatzwerkstoffs $\mathrm{F}_{\text {EXx }}$ und des Winkels $\theta$ zwischen Naht- und Kraftrichtung bestimmt. Die Tragfähigkeit wird mit folgender Gleichung beschrieben:

1. Verfahren: $\mathrm{F}_{\mathrm{w}}=0,60 \cdot \mathrm{F}_{\mathrm{EXX}}$

2. Verfahren: $F_{w}=0,60 \cdot F_{\mathrm{EXX}} \cdot\left(1,0+0,50 \cdot \sin ^{1,5}(\theta)\right)$

Mit: $\Phi:$ Teilsicherheitsbeiwert auf der Widerstandsseite, $\Phi=0,75$

$\mathrm{F}_{\mathrm{EXX}}$ : Klassifikationsnummer, Mindestzugfestigkeit des Schweißzusatzwerkstoffs

$\theta$ : Winkel zwischen Kraft- und Nahtlängsrichtung

Beim zweiten Verfahren wird die geringste Tragfähigkeit bei scherbeanspruchten Nähten erreicht, die höchste bei einer Belastung senkrecht zur Naht. Beim ersten Verfahren ergibt sich immer die Tragfähigkeit der scherbeanspruchten Nähte. Die Herleitung dieser Formel erfolgte an normalfesten Stählen, [Callele et al., 2005].

Die Mindestzugfestigkeiten des Schweißzusatzwerkstoffs $\mathrm{F}_{\mathrm{Exx}}$ sind in Tabelle 3.7 angegeben. Es wird für den Schweißzusatzwerkstoff immer mindestens die Festigkeit des Grundwerkstoffs gefordert. Da die Tragfähigkeit in Abhängigkeit des Schweißzusatzwerkstoffs berechnet wird, ist für die Vergleichbarkeit mit den anderen Normen die Angabe der zu den Schweißzusatzwerkstoffen gehörigen Stähle erforderlich. Dazu wird in Tabelle 3.7 eine Auswahl der möglichen Kombinationen von Stählen und Schweißzusatzwerkstoffen gegeben.

Tabelle 3.7: Festigkeiten der Stähle mit zugehörigen Schweißzusatzwerkstoffen nach AISC 360-05 (2005), [AWS D1.1, 2004]

\begin{tabular}{|l|l|l|l|}
\hline Stahl & $\mathrm{f}_{\mathrm{y}}\left[\mathrm{N} / \mathrm{mm}^{2}\right]$ & $\mathrm{f}_{\mathrm{u}}\left[\mathrm{N} / \mathrm{mm}^{2}\right]$ & $\begin{array}{l}\text { Mindestzugfestigkeit des } \\
\text { Schweißzusatzwerkstoff } \mathrm{F}_{\text {EXx }}\end{array}$ \\
\hline A36 ( $20 \mathrm{~mm})$ & 250 & $400-550$ & $60 \mathrm{ksi} / 70 \mathrm{ksi}\left(414 / 483 \mathrm{~N} / \mathrm{mm}^{2}\right)$ \\
\hline A913 (Gr. 50) & 345 & min. 455 & $70 \mathrm{ksi}\left(483 \mathrm{~N} / \mathrm{mm}^{2}\right)$ \\
\hline A913 (Gr. 60) & 415 & min. 520 & $80 \mathrm{ksi}\left(552 \mathrm{~N} / \mathrm{mm}^{2}\right)$ \\
\hline A913 (Gr. 65) & 450 & min. 550 & $80 \mathrm{ksi}\left(552 \mathrm{~N} / \mathrm{mm}^{2}\right)$ \\
\hline A852 & 485 & $620-760$ & $90 \mathrm{ksi}\left(621 \mathrm{~N} / \mathrm{mm}^{2}\right)$ \\
\hline
\end{tabular}


Es hat sich gezeigt, dass die US-amerikanischen Normen die Bemessungswiderstände in Abhängigkeit der Kraftrichtung bestimmen. Um die Unterschiede zu ermitteln und zu beurteilen, sind die Bemessungswiderstände in Tabelle 3.8 für einzelne Schweißzusatzwerkstoffe zusammengestellt.

In Tabelle 3.9 wird eine Beispielrechnung für beide Verfahren für Kreuzstöße mit Stirnkehlnähten und Flankenkehlnahtverbindungen gezeigt. Für Flankenkehlnähte, also für den Grenzfall der reinen Scherbeanspruchung, sind die Ergebnisse des ersten und zweiten Verfahrens identisch. Für Kreuzstöße ergibt sich ein Unterschied zwischen den Verfahren von 50 \%. Zwischen Flanken- und Stirnkehlnähten ergeben sich beim ersten Verfahren keine Unterschiede, und beim 2. Verfahren unterscheiden sich die Ergebnisse um $50 \%$.

Tabelle 3.8: Bemessungswiderstand für Kehlnähte nach AISC 360-05 (2005), [AWS D1.1, 2004]

\begin{tabular}{|c|c|c|c|c|}
\hline & \multicolumn{4}{|c|}{ Schweißzusatzwerkstoff } \\
\hline & 60 ksi & 70 ksi & 80 ksi & 90 ksi \\
\hline $\mathrm{f}_{\mathrm{y}}\left[\mathrm{ksi} / \mathrm{N} / \mathrm{mm}^{2}\right]$ & $48 / 331$ & $57 / 393$ & $67 / 462$ & $77 / 531$ \\
\hline $\mathrm{f}_{\mathrm{u}}\left[\mathrm{ksi} / \mathrm{N} / \mathrm{mm}^{2}\right]$ & $60 / 414$ & $70 / 483$ & $80 / 552$ & $90 / 621$ \\
\hline $\mathrm{F}_{\mathrm{EXX}}\left[\mathrm{N} / \mathrm{mm}^{2}\right]$ & 414 & 483 & 552 & 621 \\
\hline$\Phi[-]$ & \multicolumn{4}{|l|}{0,75} \\
\hline$\Phi \cdot 0,6 \cdot \mathrm{F}_{\mathrm{EXX}}$ & 186 & 217 & 248 & 279 \\
\hline \multicolumn{5}{|c|}{$\Phi \cdot 0,6 \cdot \mathrm{F}_{\mathrm{EXX}} \cdot\left(1,0+0,50 \cdot \sin ^{1,5}(\theta)\right)$} \\
\hline$\theta=0^{\circ}$ & 186 & 217 & 248 & 279 \\
\hline$\theta=90^{\circ}$ & 279 & 326 & 373 & 419 \\
\hline
\end{tabular}

Tabelle 3.9: Beispielrechnung für Stirn- und Flankenkehlnähte nach AISC 360-05 (2005)

\begin{tabular}{|c|c|c|c|c|}
\hline \multirow{5}{*}{ 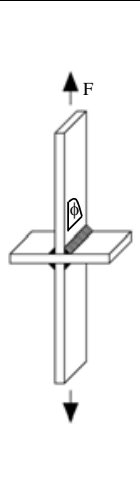 } & Stirnkehlnahtverbindungen & $\begin{array}{l}\text { Nach- } \\
\text { weis }\end{array}$ & Flankenkehlnahtverbindungen & \multirow{5}{*}{$\underbrace{q_{\tau_{\mathrm{II}}}^{\mathrm{F}}}_{V}$} \\
\hline & $\begin{array}{l}\mathrm{F} \leq \Phi \cdot \mathrm{R}_{\mathrm{n}} \\
\mathrm{F} \leq \Phi \cdot 0,6 \cdot \mathrm{F}_{\mathrm{EXX}} \cdot\left(1,0+0,50 \cdot \sin ^{1,5}(90)\right) \\
\mathrm{F} \leq \Phi \cdot 0,6 \cdot \mathrm{F}_{\mathrm{EXX}} \cdot 1,50\end{array}$ & $\begin{array}{l}\text { 2. Ver- } \\
\text { fahren }\end{array}$ & $\begin{array}{l}\mathrm{F} \leq \Phi \cdot \mathrm{R}_{\mathrm{n}} \\
\mathrm{F} \leq \Phi \cdot 0,6 \cdot \mathrm{F}_{\mathrm{EXX}} \cdot\left(1,0+0,50 \cdot \sin ^{1,5}(0)\right) \\
\mathrm{F} \leq \Phi \cdot 0,6 \cdot \mathrm{F}_{\mathrm{EXX}}\end{array}$ & \\
\hline & $\begin{array}{l}\mathrm{F} \leq \Phi \cdot \mathrm{R}_{\mathrm{n}} \\
\mathrm{F} \leq \Phi \cdot 0,6 \cdot \mathrm{F}_{\mathrm{EXX}}\end{array}$ & $\begin{array}{l}1 . \\
\text { Ver- } \\
\text { fahren }\end{array}$ & $\begin{array}{l}\mathrm{F} \leq \Phi \cdot \mathrm{R}_{\mathrm{n}} \\
\mathrm{F} \leq \Phi \cdot 0,6 \cdot \mathrm{F}_{\mathrm{EXX}}\end{array}$ & \\
\hline & Um 50 \% höhere Beanspruchbarkeit & $\begin{array}{l}\text { Ver- } \\
\text { gleich }\end{array}$ & Gleiche Beanspruchbarkeit & \\
\hline & \multicolumn{3}{|c|}{ Nahtfläche $A_{w}$ bezieht sich jeweils auf 2 Nähte } & \\
\hline
\end{tabular}

\subsubsection{Nachweisformat nach Kanadischer Norm}

Das Nachweisverfahren nach Kanadischer Norm ist dem Verfahren nach US-amerikanischer Norm sehr ähnlich. Es unterscheidet sich die Bemessungsgleichung (3.9) lediglich in den Vorfaktoren. Nach Kanadischer Norm ergibt sich ein Vorfaktor von $0,67 \cdot \Phi_{\mathrm{w}}=0,45$, während die US-amerikanische Norm 0,60 $\cdot \Phi=0,45$ fordert. Die Bemessungsgleichungen führen damit zu vergleichbaren Ergebnissen. Die Bestimmung der Tragfähigkeit von Schweißverbindungen berücksichtigt die Festigkeit des Schweißzusatzwerkstoffs und den Winkel zwischen Nahtlängs- und Kraftrichtung. In der kanadischen Bemessungsvorschrift CAN/NSA S16.1-94 (2006) wird die Tragfähigkeit mit folgender Gleichung beschrieben:

$$
\mathrm{F}_{\mathrm{w}}=0,67 \cdot \Phi_{\mathrm{w}} \cdot \mathrm{A}_{\mathrm{w}} \cdot \mathrm{X}_{\mathrm{u}} \cdot\left(1,0+0,50 \cdot \sin ^{1,5}(\theta)\right)
$$

mit $\Phi_{\mathrm{w}}$ : Widerstandsbeiwert für Schweißnähte $\Phi_{\mathrm{w}}=0,67$

$\mathrm{X}_{\mathrm{u}}$ : Klassifikationsnummer, Mindestzugfestigkeit des Schweißzusatzwerkstoffs

$\theta$ : Winkel zwischen Kraft- und Nahtlängsrichtung

$\mathrm{A}_{\mathrm{w}}$ : Schweißnahtfläche

Die geringste Tragfähigkeit ergibt sich bei scherbeanspruchten Nähten $\left(\theta=0^{\circ}\right)$, während die höchste Tragfähigkeit bei einer Belastung senkrecht zur Naht $\left(\theta=90^{\circ}\right)$ erreicht werden kann. Die Festigkeit des Schweißzusatzwerkstoffs muss mindestens der des Grundwerkstoffs entsprechen. Da die Berech- 
nung in Abhängigkeit des Schweißzusatzwerkstoffs erfolgt, wird in Tabelle 3.10 für die Vergleichbarkeit auf Basis der Grundwerkstoffe mit anderen Normen eine Auswahl der möglichen Kombinationen von Stählen und Schweißzusatzwerkstoffen gegeben. Die Bemessungswiderstände sind in Tabelle 3.11 für einzelne Schweißzusatzwerkstoffe zusammengestellt. Das Kanadische Verfahren unterscheidet sich vom US-amerikanischen durch die Vorfaktoren, wobei der Nachweis zu denselben Werten führt. Somit ergibt sich analog zu der Berechnung in Tabelle 3.9 zwischen Flanken- und Stirnkehlnahtverbindungen ein Unterschied von 50 \% für die Beanspruchbarkeit nach Gleichung (3.9).

Tabelle 3.10: Zugehörige Stähle und Schweißzusatzwerkstoffe nach CAN/NSA S16.1-94 (2006), HSC (2000)

\begin{tabular}{|l|l|l|l|}
\hline Stahl CSA G40.21 grade & $\mathrm{f}_{\mathrm{v}}\left[\mathrm{N} / \mathrm{mm}^{2}\right]$ & $\mathrm{f}_{\mathrm{u}}\left[\mathrm{N} / \mathrm{mm}^{2}\right]$ & Schweißzusatzwerkstoff \\
\hline $260 \mathrm{~W}$ & 260 & min. 410 & E410/ E60 \\
\hline $350 \mathrm{~W}$ & 350 & min. 450 & E480/ E70 \\
\hline $400 \mathrm{~W}$ & 400 & min. 520 & E550/ E80 \\
\hline $550 \mathrm{~W}$ & 550 & min. 620 & E620 / E90 \\
\hline $700 \mathrm{Q}$ & 700 & min. 800 & E830 / E120 \\
\hline
\end{tabular}

Tabelle 3.11: Bemessungswiderstand nach CAN/NSA S16.1-94 (2006), HSC (2000) für Kehlnähte

\begin{tabular}{|c|c|c|c|c|c|c|}
\hline & & \multicolumn{5}{|c|}{ Schweißzusatzwerkstoff } \\
\hline & & E410 / E60 & E480 / E70 & E550 / E80 & E620 / E90 & E830/E120 \\
\hline \multicolumn{2}{|l|}{$X_{u}=f_{u}\left[N / m^{2}\right]$} & 410 & 480 & 550 & 620 & 820 \\
\hline \multicolumn{2}{|l|}{$\Phi_{\mathrm{w}}[-]$} & \multicolumn{5}{|l|}{0,67} \\
\hline \multirow{2}{*}{$\begin{array}{l}F_{w}=0,67 \cdot \Phi_{w} \cdot A_{w} \cdot X_{u} \\
\cdot\left(1,0+0,50 \cdot \sin ^{1,5}(\theta)\right)\end{array}$} & $\theta=0^{\circ}$ & 184 & 215 & 247 & 278 & 368 \\
\hline & $\theta=90^{\circ}$ & 276 & 323 & 370 & 417 & 552 \\
\hline
\end{tabular}

\subsubsection{Nachweisformat der Schwedischen Norm}

Nach Schwedischer Norm BSK 99 (2003) ist der Nachweis der Schweißverbindung im kleinsten Querschnitt der Naht und an der Nahtflanke zu führen. Im Nachweis wird zwischen Kräften in Nahtrichtung $\mathrm{F}_{\mathrm{SII}}$ und Kräften $\mathrm{F}_{\mathrm{S} \alpha}$ senkrecht dazu unterschieden. Bei den Kräften senkrecht zur Naht wird zusätzlich der Winkel $\alpha$ zwischen Kraft und Nahtquerschnitt berücksichtigt. Die Aufteilung der Kräfte ist in Abbildung 3.6 dargestellt.

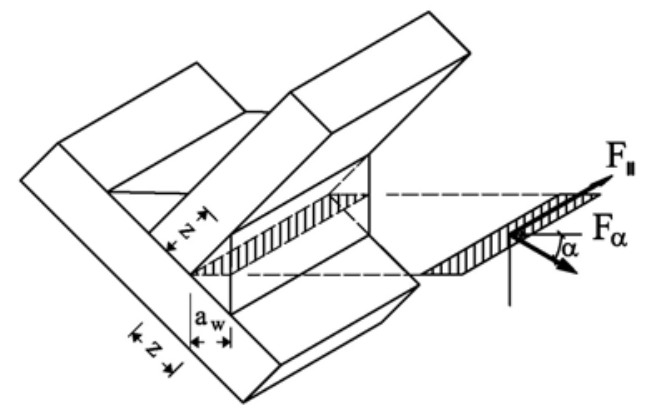

Abbildung 3.6: Spannungskomponenten parallel und senkrecht zur Nahtachse

Für den Widerstand werden zum einen die Zugfestigkeit des Grundwerkstoffs $f_{\text {uk }}$ und/oder des Schweißzusatzwerkstoffs $\mathrm{f}_{\text {euk }}$ und zum anderen die Kraftrichtung als Winkel $\alpha$ berücksichtigt. Die Widerstandkräfte $F_{R I I}$ und $F_{R \alpha}$ werden mit Hilfe der Spannung $f_{w d}$ berechnet. Dabei wird $f_{w d}$ in Abhängigkeit der Festigkeit des Grund- und Schweißzusatzwerkstoffs bestimmt, vgl. Gleichung (3.12) und (3.13). Der Nachweis ist nach Gleichung (3.10) und (3.11) zu führen.

$$
\begin{aligned}
& \left(\frac{\mathrm{F}_{\mathrm{SII}}}{\mathrm{F}_{\mathrm{RII}}}\right)^{2}+\left(\frac{\mathrm{F}_{\mathrm{S} \alpha}}{\mathrm{F}_{\mathrm{R} \alpha}}\right)^{2} \leq 1 \\
& \mathrm{~F}_{\mathrm{RII}}=0,6 \cdot \mathrm{a}_{\mathrm{w}} \cdot l \cdot \mathrm{f}_{\mathrm{wd}} \text { und } \mathrm{F}_{\mathrm{R} \alpha}=\frac{\mathrm{a}_{\mathrm{w}} \cdot 1 \cdot \mathrm{f}_{\mathrm{wd}}}{\sqrt{2+\cos 2 \alpha}}
\end{aligned}
$$


Wenn $\mathrm{f}_{\text {euk }}>\mathrm{f}_{\mathrm{uk}}$ :

$\mathrm{f}_{\mathrm{wd}}=\frac{\varphi \cdot \sqrt{\mathrm{f}_{\mathrm{uk}} \cdot \mathrm{f}_{\text {euk }}}}{\gamma_{\mathrm{n}} \cdot 1,2}$, zusätzlich Nachweis des Schenkels z mit $\mathrm{f}_{\mathrm{ud}}$, ür $\mathrm{f}_{\mathrm{ud}}<\mathrm{f}_{\mathrm{wd}}$

oder wenn $\mathrm{f}_{\text {euk }}<\mathrm{f}_{\mathrm{uk}}: \mathrm{f}_{\mathrm{wd}}=\frac{\varphi \cdot \mathrm{f}_{\text {euk }}}{\gamma_{\mathrm{n}} \cdot 1,2}$

mit $\mathrm{f}_{\mathrm{uk}}$ : charakteristische Zugfestigkeit des Grundwerkstoffs

$\mathrm{f}_{\text {euk }}$ : Zugfestigkeit des Schweißzusatzwerkstoffes

$\varphi$ : Abminderungsfaktor mit $\varphi=0,9$

$\gamma_{\mathrm{n}}$ : Sicherheitsbeiwert mit $\gamma_{\mathrm{n}}=1,0$ bis 1,2 in Abhängigkeit der Ausführungsklasse

$\mathrm{a}_{\mathrm{w}}$ : Nahtdicke

z: Schenkelquerschnitt

Die Zusatzbedingung gemäß Gleichung (3.12), wonach ein Nachweis am Nahtschenkel zu führen ist, wird nur maßgebend, wenn die Zugfestigkeit des Schweißzusatzwerkstoffs $\mathrm{f}_{\text {euk }}$ mindestens um den Faktor 1,44 größer ist als die Zugfestigkeit des Grundwerkstoffs. Im Nachweisformat (3.10) gibt es keinen weiteren Faktor, der sich in Abhängigkeit der Werkstofffestigkeiten ändert. Es ist daher mit diesen Regeln möglich, unterschiedliche Festigkeiten von Schweißzusatzwerkstoff und Grundwerkstoff zu berücksichtigen. Eine Auflistung möglicher Werkstoffe ist in Tabelle 3.12 gegeben.

Tabelle 3.12: Stähle und Schweißzusatzwerkstoffe nach BSK 99 (2003)

\begin{tabular}{|c|c|c|c|c|c|}
\hline Stahl & $\mathrm{f}_{\mathrm{y}}\left[\mathrm{N} / \mathrm{mm}^{2}\right]$ & $\mathrm{f}_{\mathrm{u}}\left[\mathrm{N} / \mathrm{mm}^{2}\right]$ & Schweißzusatzwerkstoff & $\mathrm{f}_{\mathrm{y}}\left[\mathrm{N} / \mathrm{mm}^{2}\right]$ & $\mathrm{f}_{\mathrm{u}}\left[\mathrm{N} / \mathrm{mm}^{2}\right]$ \\
\hline S235 & 235 & 340 & E35 & 355 & 440 \\
\hline S355 JR/JO/J2 & 355 & 490 & E42 & 420 & 500 \\
\hline S355 N/NL & 355 & 470 & $\overline{E 62}$ & 620 & 690 \\
\hline S355 M/ML & 355 & 450 & & & \\
\hline S460M/ML & 460 & 530 & & & \\
\hline S460Q/QL/QL1 & 460 & 550 & & & \\
\hline S690 Q/QL/QL1 & 690 & 770 & & & \\
\hline
\end{tabular}

In Tabelle 3.13 werden als Berechnungsbeispiel die Ergebnisse von Gleichung (3.10) für Kreuzstöße mit Stirnkehlnähten und Flankenkehlnahtverbindungen ermittelt. Die geringste Tragfähigkeit wird bei scherbeanspruchten Nähten erreicht, die höchste Tragfähigkeit bei einer Belastung senkrecht zur Naht. Zwischen Flanken- und Stirnkehlnahtverbindungen ergibt sich ein Unterschied von $1 / \sqrt{2} / 0,6=1,18$ und somit $18 \%$.

Tabelle 3.13: Beispielrechnung für Stirn- und Flankenkehlnähte nach BSK 99 (2003)

\begin{tabular}{|l|l|l|}
\hline$\Lambda_{\mathrm{F}}$ & Stirnkehlnaht - Nachweis & Flankenkehlnaht - Nachweis \\
\cline { 2 - 3 } & $\left(\frac{\mathrm{F}_{\mathrm{S} \alpha}}{\mathrm{F}_{\mathrm{R} \alpha}}\right)^{2} \leq 1 \Leftrightarrow \mathrm{F}_{\mathrm{S} \alpha} \leq \mathrm{F}_{\mathrm{R} \alpha}$ & $\left(\frac{\mathrm{F}_{\mathrm{SII}}}{\mathrm{F}_{\mathrm{RII}}}\right)^{2} \leq 1 \Leftrightarrow \mathrm{F}_{\mathrm{SII}} \leq \mathrm{F}_{\mathrm{RII}}$ \\
\hline & $\mathrm{F}_{\mathrm{S} \alpha} \leq \frac{\mathrm{a} \cdot 1 \cdot \mathrm{f}_{\mathrm{wd}}}{\sqrt{2+\cos (2 \cdot 45)}}=\frac{\mathrm{a} \cdot \mathrm{l} \cdot \mathrm{f}_{\mathrm{wd}}}{\sqrt{2}}$ & $\mathrm{~F}_{\mathrm{SII}} \leq 0,6 \cdot \mathrm{a} \cdot \mathrm{l} \cdot \mathrm{f}_{\mathrm{wd}}$
\end{tabular}

Anhand des Beispiels in Tabelle 3.14 wird der Einfluss der unterschiedlichen Festigkeiten des Schweißzusatzwerkstoffs und des Grundwerkstoffs aufgezeigt. Für die Werkstoffkennwerte wurden Annahmen getroffen. Es zeigt sich, dass bei den gewählten Werkstoffkombinationen der Zusatznachweis der Schenkelfläche maßggebend werden kann. Wenn der Nachweis mit der Schenkelfläche geführt wird, erhöht sich im idealen Fall die Nahtfläche um den Faktor $\sqrt{2}$. Der Nachweis wird somit erst maßgebend, wenn der Bemessungswert der Zugfestigkeit $f_{\text {ud }}$ um diesen Faktor höher liegt als der Wert $\mathrm{f}_{\mathrm{wd}}$. Bei den gewählten Werkstoffkombinationen wird das nicht maßgebend. 
Tabelle 3.14: Beispiel für Bemessungsgrenzspannungen bei unterschiedlichen Werkstoffen nach BSK 99 (2003)

\begin{tabular}{|c|c|c|c|c|c|}
\hline Kombination & S460-G46 & S460-G69 & S690-G46 & S690-G69 & S690-G89 \\
\hline $\mathrm{SZW} \mathrm{f}_{\text {euk }}\left[\mathrm{N} / \mathrm{mm}^{2}\right]$ & 530 & 770 & 530 & 770 & 940 \\
\hline $\mathrm{GW} \mathrm{f}_{\mathrm{uk}}\left[\mathrm{N} / \mathrm{mm}^{2}\right]$ & 540 & 540 & 770 & 770 & 770 \\
\hline $\mathrm{GW} \mathrm{f}_{\mathrm{ud}}\left[\mathrm{N} / \mathrm{mm}^{2}\right]$ & 442 & 442 & 642 & 642 & 642 \\
\hline $\begin{array}{l}\mathrm{f}_{\mathrm{wd}}=\frac{\varphi \cdot \mathrm{f}_{\text {euk }}}{\gamma_{\mathrm{n}} \cdot 1,2} \\
\text { wenn } \mathrm{f}_{\text {euk }}<\mathrm{f}_{\mathrm{uk}}\end{array}$ & $\frac{0,9 \cdot 530}{1,0 \cdot 1,2}=398$ & -- & $\frac{0,9 \cdot 530}{1,0 \cdot 1,2}=398$ & $\frac{0,9 \cdot 770}{1,0 \cdot 1,2}=578$ & -- \\
\hline $\begin{array}{l}\mathrm{f}_{\mathrm{wd}}=\frac{\varphi \cdot \sqrt{\mathrm{f}_{\mathrm{uk}} \cdot \mathrm{f}_{\text {euk }}}}{\gamma_{\mathrm{n}} \cdot 1,2} \\
\text { wenn } \mathrm{f}_{\text {euk }}>\mathrm{f}_{\mathrm{uk}}\end{array}$ & -- & $\begin{array}{l}\frac{0,9 \cdot \sqrt{540 \cdot 770}}{1,0 \cdot 1,2} \\
=484\end{array}$ & -- & -- & $\begin{array}{l}\frac{0,9 \cdot \sqrt{770 \cdot 940}}{1,0 \cdot 1,2} \\
=638\end{array}$ \\
\hline Abfrage $\mathrm{f}_{\mathrm{u}, \mathrm{d}}<\mathrm{f}_{\mathrm{wd}}$ & -- & ja & -- & -- & ja \\
\hline $\begin{array}{l}\text { Nachweis mit } \\
\text { Nahtdicke a } \\
\text { oder Schenkel z }\end{array}$ & Nahtdicke a & $\begin{array}{l}\text { Nahtdicke a } \\
\text { und Schenkel z }\end{array}$ & Nahtdicke a & Nahtdicke a & $\begin{array}{l}\text { Nahtdicke a } \\
\text { und Schenkel z }\end{array}$ \\
\hline
\end{tabular}

\subsubsection{Vergleich der Bemessungsgrenzspannungen}

Für die Materialkombinationen gleicher Grund- und passender Schweißzusatzwerkstoffe werden die vorgestellten Bemessungsgrenzspannungen der beschriebenen Normen für Flankenkehlnahtverbindungen und für Kreuzstöße im Folgenden verglichen. Da die Materialkennwerte nicht exakt übereinstimmen, werden teilweise näherungsweise gleiche und jeweils zugelassene Werkstoffe verwendet, wie in Tabelle 3.15 beschrieben.

Tabelle 3.15: Vergleich der Bemessungsgrenzspannung der unterschiedlichen Normen in [N/mm²]

\begin{tabular}{|c|c|c|c|c|}
\hline \multirow{2}{*}{$\begin{array}{l}\text { Norm/ } \\
\text { Bemessungsgrenzspannung }\end{array}$} & \multicolumn{4}{|c|}{ Werkstoffkombination } \\
\hline & S235-42 & S355-42 & $\mathrm{S} 460-46$ & S690-69 \\
\hline $\begin{array}{l}\text { DIN 18800-1 (1990) } \\
\text { [Zulassung Z-30.2-5] } \\
\text { Flankenkehlnaht }=\text { Kreuzstoß }\end{array}$ & $\begin{array}{l}\mathrm{f}_{\mathrm{y}, \mathrm{GW}}=240 \\
\alpha_{\mathrm{w}}=0,95 \\
\mathbf{2 0 7} \mathbf{N} / \mathbf{m m}^{\mathbf{2}}\end{array}$ & $\begin{array}{l}\mathrm{f}_{\mathrm{y}, \mathrm{GW}}=360 \\
\alpha_{\mathrm{w}}=0,8 \\
\mathbf{2 6 2} \mathbf{N} / \mathbf{m m}^{2}\end{array}$ & $\begin{array}{l}\mathrm{f}_{\mathrm{y}, \mathrm{GW}}=460 \\
\alpha_{\mathrm{w}}=0,6 \\
\mathbf{2 5 1} \mathbf{N} / \mathbf{m m}^{2}\end{array}$ & $\begin{array}{l}\mathrm{f}_{\mathrm{y}, \mathrm{GW}}=690 \\
\alpha_{\mathrm{w}}=0,6 \\
\mathbf{3 7 6} \mathbf{N} / \mathbf{m m}^{2}\end{array}$ \\
\hline $\begin{array}{l}\text { DIN 18800-1 (2008) } \\
\text { [Zulassung Z-30.2-5] } \\
\text { Flankenkehlnaht }=\text { Kreuzstoß }\end{array}$ & $\begin{array}{l}\mathrm{f}_{\mathrm{y}, \mathrm{GW}}=240 \\
\alpha_{\mathrm{w}}=0,95 \\
\mathbf{2 0 7} \mathbf{N} / \mathbf{m m}^{\mathbf{2}}\end{array}$ & $\begin{array}{l}\mathrm{f}_{\mathrm{y}, \mathrm{GW}}=360 \\
\alpha_{\mathrm{w}}=0,8 \\
\mathbf{2 6 2} \mathbf{N} / \mathbf{m m}^{2}\end{array}$ & $\begin{array}{l}\mathrm{f}_{\mathrm{y}, \mathrm{GW}}=460 \\
\alpha_{\mathrm{w}}=0,7 \\
\mathbf{2 9 3} \mathbf{N} / \mathbf{m m}^{2}\end{array}$ & $\begin{array}{l}\mathrm{f}_{\mathrm{y}, \mathrm{GW}}=690 \\
\alpha_{\mathrm{w}}=0,6 \\
\mathbf{3 7 6} \mathbf{N} / \mathbf{m m}^{2}\end{array}$ \\
\hline $\begin{array}{l}\text { EN 1993-1-8 (2005) } \\
\text { EN 1993-1-12 (2007) } \\
\text { Flankenkehlnaht } \\
\text { Kreuzstoß }\end{array}$ & $\begin{array}{l}\mathrm{f}_{\mathrm{u}, \mathrm{GW}}=360 \\
\beta_{\mathrm{w}}=0,8 \\
\mathbf{2 0 8} \mathbf{N} / \mathbf{m m}^{2} \\
\mathbf{2 5 5} \mathbf{N} / \mathbf{m m}^{2}\end{array}$ & $\begin{array}{l}\mathrm{f}_{\mathrm{u}, \mathrm{GW}}=510 \\
\beta_{\mathrm{w}}=0,9 \\
\mathbf{2 6 2} \mathbf{N} / \mathbf{m m}^{2} \\
\mathbf{3 2 1} \mathbf{N} / \mathbf{m m}^{2}\end{array}$ & $\begin{array}{l}\mathrm{f}_{\mathrm{u}, \mathrm{GW}}=540 \\
\beta_{\mathrm{w}}=1,0 \\
\mathbf{2 4 9} \mathbf{N} / \mathbf{m m}^{2} \\
\mathbf{3 0 5} \mathbf{N} / \mathbf{m m}^{2} \\
\end{array}$ & $\begin{array}{l}\mathrm{f}_{\mathrm{u}, \mathrm{GW}}=770 \\
\beta_{\mathrm{w}}=1,0 \\
356 \mathbf{N} / \mathbf{m m}^{2} \\
\mathbf{4 3 6} \mathbf{N} / \mathbf{m m}^{2} \\
\end{array}$ \\
\hline $\begin{array}{l}\text { DIN EN 1993-1-8/NA (2010) } \\
\text { DIN EN 1993-1-12/NA (2011) } \\
\text { Flankenkehlnaht } \\
\text { Kreuzstoß }\end{array}$ & $\begin{array}{l}\mathrm{f}_{\mathrm{u}, \mathrm{GW}}=360 \\
\beta_{\mathrm{w}}=0,8 \\
\mathbf{2 0 8} \mathbf{N} / \mathbf{m m}^{2} \\
\mathbf{2 5 5} \mathbf{N} / \mathbf{m m}^{2}\end{array}$ & $\begin{array}{l}\mathrm{f}_{\mathrm{u}, \mathrm{GW}}=510 \\
\beta_{\mathrm{w}}=0,9 \\
\mathbf{2 6 2} \mathbf{N} / \mathbf{m m}^{2} \\
\mathbf{3 2 1} \mathbf{N} / \mathbf{m m}^{2}\end{array}$ & $\begin{array}{l}\mathrm{f}_{\mathrm{u}, \mathrm{GW}}=540 \\
\beta_{\mathrm{w}}=0,85 \\
\mathbf{2 9 3} \mathbf{N} / \mathbf{m m}^{2} \\
\mathbf{3 5 9} \mathbf{N} / \mathbf{m m}^{2} \\
\end{array}$ & $\begin{array}{l}\mathrm{f}_{\mathrm{u}, \mathrm{GW}}=770 \\
\beta_{\mathrm{w}}=1,2 \\
\mathbf{2 9 6} \mathbf{N} / \mathbf{m m}^{2} \\
\mathbf{3 6 3} \mathbf{N} / \mathbf{m m}^{2}\end{array}$ \\
\hline $\begin{array}{l}\text { AISC 360-05 (2005) } \\
\text { Flankenkehlnaht } \\
\text { Kreuzstoß }\end{array}$ & $\begin{array}{l}\mathrm{f}_{\mathrm{u}, \mathrm{SZW}}=414 \\
\mathbf{1 8 6} \mathbf{N} / \mathbf{m m}^{2} \\
\mathbf{2 7 9} \mathbf{N} / \mathbf{m m}^{2}\end{array}$ & $\begin{array}{l}\mathrm{f}_{\mathrm{u}, \mathrm{SZW}}=483 \\
217 \mathrm{~N} / \mathbf{m m}^{2} \\
326 \mathrm{~N} / \mathbf{m m}^{2}\end{array}$ & $\begin{array}{l}\mathrm{f}_{\mathrm{u}, \mathrm{SZW}}=552 \\
\mathbf{2 4 8} \mathbf{N} / \mathbf{m m}^{2} \\
\mathbf{3 7 3} \mathbf{N} / \mathbf{m m}^{\mathbf{2}} \\
\end{array}$ & $*)$ \\
\hline $\begin{array}{l}\text { CAN/NSA S16.1-94 (2006) } \\
\text { Flankenkehlnaht } \\
\text { Kreuzstoß }\end{array}$ & $\begin{array}{l}\mathrm{f}_{\mathrm{u}, \mathrm{SZW}}=410 \\
\mathbf{1 8 4} \mathbf{N} / \mathbf{m m}^{2} \\
\mathbf{2 7 6} \mathbf{N} / \mathbf{m m}^{2}\end{array}$ & $\begin{array}{l}\mathrm{f}_{\mathrm{u}, \mathrm{SZW}}=480 \\
215 \mathrm{~N} / \mathbf{m m}^{2} \\
323 \mathrm{~N} / \mathbf{m m}^{2} \\
\end{array}$ & $*)$ & $\begin{array}{l}\mathrm{f}_{\mathrm{u}, \mathrm{SZW}}=820 \\
368 \mathrm{~N} / \mathbf{m m}^{2} \\
552 \mathrm{~N} / \mathbf{m m}^{2} \\
\end{array}$ \\
\hline $\begin{array}{l}\text { BSK } 99 \text { (2003) } \\
\text { Flankenkehlnaht } \\
\text { Kreuzstoß }\end{array}$ & $\begin{array}{l}\mathrm{f}_{\mathrm{u}, \mathrm{GW}}=340 \\
\mathrm{f}_{\mathrm{u}, \mathrm{SZW}}=500 \\
\mathbf{1 8 6} \mathbf{N} / \mathbf{m m}^{2} \\
\mathbf{2 1 9} \mathbf{N} / \mathbf{m m}^{2}\end{array}$ & $\begin{array}{l}\mathrm{f}_{\mathrm{u}, \mathrm{GW}}=490 \\
\mathrm{f}_{\mathrm{u}, \mathrm{SZW}}=500 \\
\mathbf{2 2 3} \mathbf{N} / \mathbf{m m}^{2} \\
\mathbf{2 6 3} \mathbf{N} / \mathbf{m m}^{2}\end{array}$ & $\begin{array}{l}\mathrm{f}_{\mathrm{u}, \mathrm{GW}}=530 \\
\mathrm{f}_{\mathrm{u}, \mathrm{SZW}}=530 \\
\mathbf{2 3 9} \mathbf{N} / \mathbf{m m}^{2} \\
\mathbf{2 8 1} \mathbf{N} / \mathbf{m m}^{2}\end{array}$ & $\begin{array}{l}\mathrm{f}_{\mathrm{u}, \mathrm{GW}}=770 \\
\mathrm{f}_{\mathrm{u}, \mathrm{SZW}}=760 \\
\mathbf{3 4 2} \mathbf{N} / \mathbf{m m}^{2} \\
\mathbf{4 0 3} \mathbf{N} / \mathbf{m m}^{2}\end{array}$ \\
\hline
\end{tabular}


Abbildung 3.7 vergleicht die Bemessungsgrenzspannungen von Flankenkehlnahtverbindungen, während in Abbildung 3.8 die Beanspruchbarkeiten von Kreuzstößen mit Stirnkehlnähten dargestellt sind. Verglichen werden jeweils die in Tabelle 3.15 zitierten Normen. Es zeigt sich, dass die Werte sehr unterschiedlich sind. Es zeigt sich auch, dass sich die normativen Tragfähigkeiten nicht proportional zur Änderung der Festigkeit des Werkstoffs ändern.

Die in Abbildung 3.7 dargestellten Tragfähigkeiten von Flankenkehlnahtverbindungen zeigen sehr ähnliche Werte für die beiden Nordamerikanischen Normen CAN/NSA S16.1-94 (2006) und AISC 360-05 (2005). Für die normalfesten Verbindungen mit den Grundwerkstoffen S235 und S355 liegen die Deutschen (DIN 18800-1 (1990) und DIN 18800-1 (2008)) und Europäischen Werte (EN 1993-1-8 (2005) und EN 1993-1-12 (2007) jeweils mit Nationalem Anhang) um ca. 10 \% und $20 \%$ höher als die Werte nach beiden Nordamerikanischen Normen und der Schwedischen Norm BSK 99 (2003). Für die Verbindungen mit dem Grundwerkstoff S460 ergeben sich sehr ähnliche Beanspruchbarkeiten für die beiden Nordamerikanischen Normen und die Schwedische Norm. Eine Ausnahme bildet die neue Deutsche Norm (DIN 18800-1 (2008), DIN EN 1993-1-8/NA (2010)), nach der die Werte deutlich höher liegen. Diese Verbesserung der normativen Tragfähigkeit konnte infolge der in Kapitel 6 vorgestellten eigenen Ergebnisse nach [FOSTA P652, 2008] bereits berücksichtigt werden. Für Verbindungen mit dem Grundwerkstoff S690 zeigen sich Unterschiede zwischen den beiden Nordamerikanischen Normen und der Schwedischen Norm von bis zu 10 \%, während die normative Tragfähigkeit nach DIN EN 1993-1-12/NA (2011) deutlich darunter liegen. Dies ist ebenfalls in den neueren Ergebnissen nach [FOSTA P652, 2008] begründet.

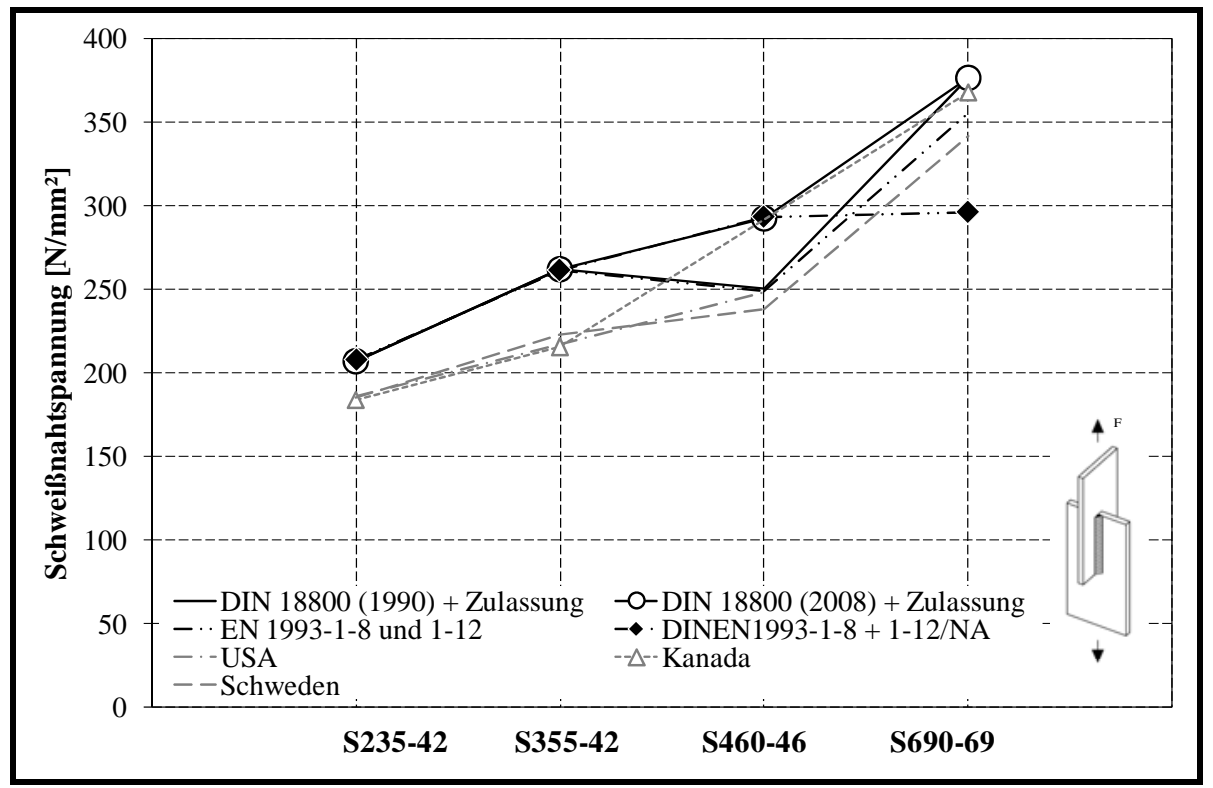

Abbildung 3.7: Bemessungsgrenzspannungen von Flankenkehlnähten 
In Abbildung 3.8 werden die Beanspruchbarkeiten von Kreuzstößen mit Stirnkehlnähten verglichen. Es zeigen sehr ähnliche Werte für die vergleichbaren Werte der beiden Nordamerikanischen Normen. Für die normalfesten Verbindungen mit den Grundwerkstoffen S235 und S355 liegen die Schwedischen und Deutschen Werte um ca. $35 \%$ und $23 \%$ niedriger als die Amerikanischen und Europäischen Werte. Für die Verbindungen mit dem Grundwerkstoff S460 liegen die Amerikanischen und neuen Deutschen Werte deutlich höher als die Europäischen, Schwedischen und Deutschen Werte. Die Schwedischen und die Deutschen Werte sind jeweils ähnlich und liegen um den Faktor $\sqrt{2}$ unterhalb der Werte nach EN 1993-1-8 und 1-12. Für die Verbindungen mit dem Grundwerkstoff S690 liegen die nationalen Deutschen Werte unterhalb der Europäischen Werte, da in den neueren Werten bereits die in Kapitel 6 beschriebenen Forschungsergebnisse nach [FOSTA P652, 2008] berücksichtigt sind. Der Wert der Kanadischen Norm ist deutlich höher als die anderen Werte, da in diesem Konzept keine verschiedenen Beiwerte in Abhängigkeit der verschiedenen Werkstoffgüten berücksichtigt werden, und somit ein linearer Anstieg in Abhängigkeit der Festigkeit erfolgt.

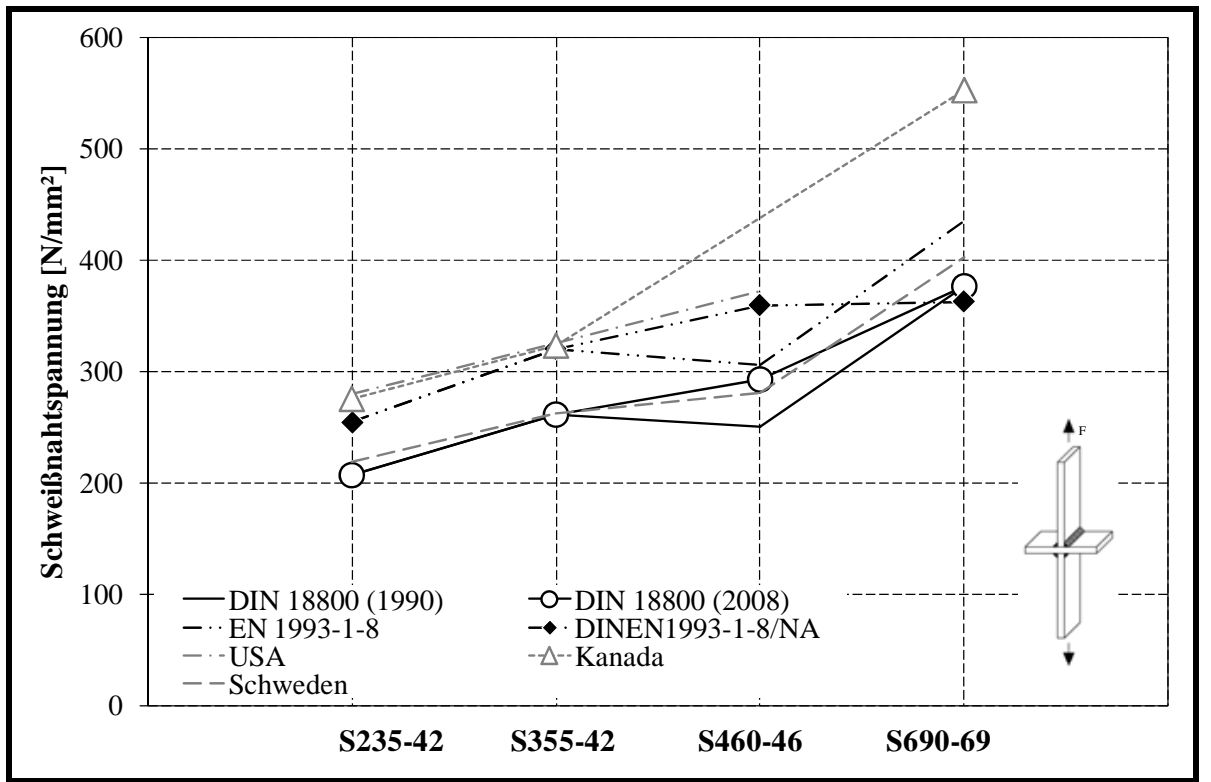

Abbildung 3.8: Bemessungsgrenzspannungen von Kreuzstößen 
Unterschiede in der Festigkeit zwischen Schweißzusatzwerkstoff und Grundwerkstoff können nur nach Schwedischer Norm BSK 99 (2003) und für höherfeste Stähle mit einer nominellen Streckgrenze $\mathrm{f}_{\mathrm{y}}>460 \mathrm{~N} / \mathrm{mm}^{2}$ nach EN 1993-1-12 (2007) berücksichtigt werden. Aus diesem Grund werden in Abbildung 3.9 nur Werkstoffkombinationen mit dem Grundwerkstoff S690Q verglichen. In beiden Normen wird für die höherfesten Stähle nur die Festigkeit des Schweißzusatzwerkstoffs verwendet. Sie unterscheiden sich nur geringfügig in den Vorfaktoren für die Schwedische Norm BSK 99 (2003) mit $\frac{0,6 \cdot 0,9}{1,2}=0,45$ im Vergleich zu EN 1993-1-12 (2007) mit $\frac{1}{1,25 \cdot 1,0 \cdot \sqrt{3}}=0,46$.

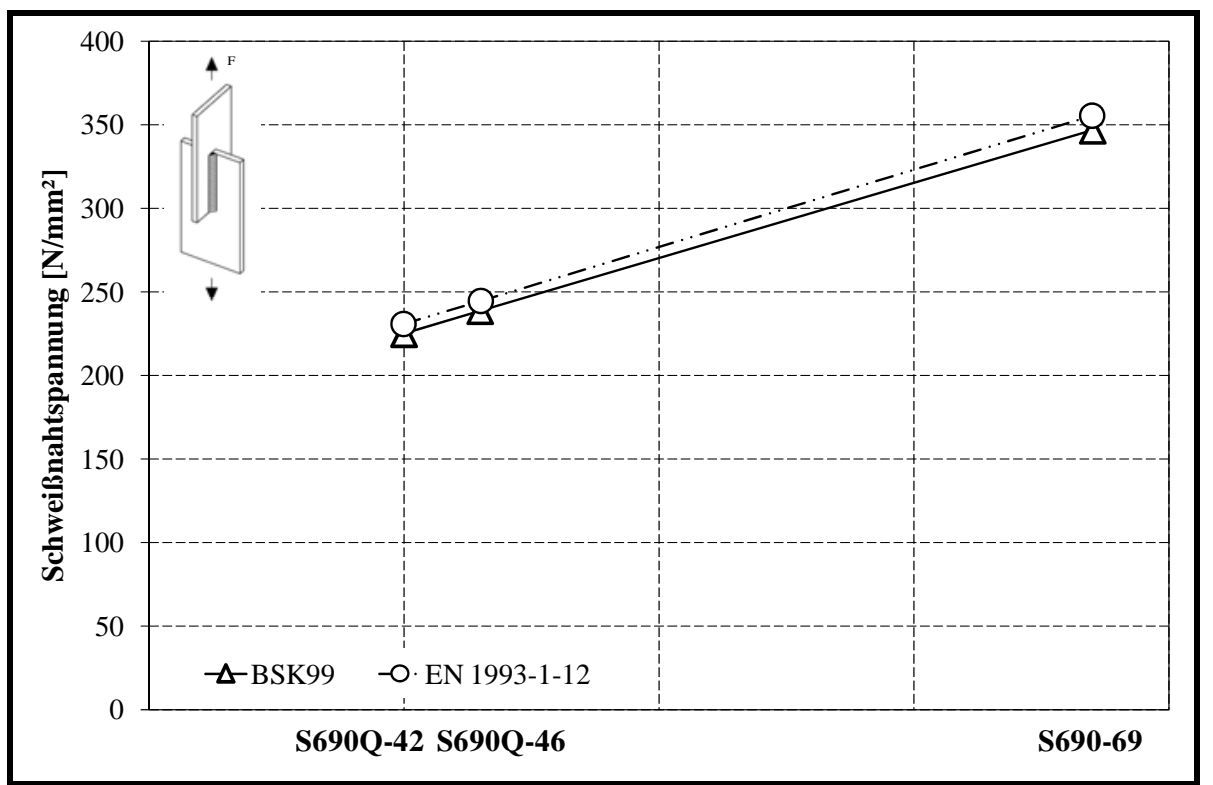

Abbildung 3.9: Vergleich der Bemessungsgrenzspannungen von Flankenkehlnähten nach BSK 99 (2003) und EN 1993-1-12 (2007)

\subsection{Zusammenfassung}

Als geometrische Angaben der verschiedenen Normen wurden die Angaben zu Nahtdicke und Nahtlänge verglichen. Die Ermittlung der Nahtdicke ist nach den verschiedenen Normen unterschiedlich. Diese Unterschiede haben allerdings im Bemessungsfall keinen Einfluss, sondern werden nur maßgebend, wenn die tatsächliche Nahtgeometrie z. B. anhand eines Makroschliffes vorliegt. In allen Normen ergeben sich ähnliche Werte für die Mindestnahtdicken. Diese sind technisch sinnvoll, um schweißtechnisch einwandfreie Nähte zu erhalten. Die Vergleiche zur Nahtlänge haben ergeben, dass für höherfeste Stähle S460 und S690 Begrenzungen der Nahtlänge vorliegen. In den experimentellen Untersuchungen, die in Kapitel 6 - 8 vorgestellt werden, wird untersucht, ob sich auch experimentell mit zunehmender Nahtlänge eine Verringerung der Tragfähigkeit ergibt.

Der Schwerpunkt des Kapitels lag auf dem Vergleich der Nachweisformate zur Bestimmung der normativen Tragfähigkeit. Es wurde gezeigt, dass die Tragfähigkeiten in Abhängigkeit der Streckgrenze des Grundwerkstoffs oder der Zugfestigkeit von Grund- oder Schweißzusatzwerkstoff bestimmt werden. Nur in der Schwedischen Norm und nach EN 1993-1-12 (2007) für höherfeste Stähle mit $\mathrm{f}_{\mathrm{y}}>460$ $\mathrm{N} / \mathrm{mm}^{2}$ ist eine Berücksichtigung von niederfesten Schweißzusatzwerkstoffen möglich. In allen anderen Normen wird immer für den Schweißzusatzwerkstoff mindestens die gleiche Festigkeit wie für den Grundwerkstoff gefordert.

Der Vergleich der Nachweisformate zeigt, dass in den meisten Normen die Tragfähigkeiten von Kreuzstößen höher sind als die von Flankenkehlnahtverbindungen. Die Tragfähigkeiten sind unterschiedlich in den verschiedenen Normen, so dass keine eindeutigen Aussagen möglich sind.

Die großen Unterschiede insbesondere für höherfeste Baustähle zeigen, dass eine systematische Untersuchung notwendig ist. 


\section{$4 \quad$ Tragverhalten von Kehlnahtverbindungen}

\subsection{Einleitung}

In diesem Kapitel werden die Bestimmung der Tragfähigkeit von Kehlnähten und das Tragverhalten erklärt. Dazu wird als Werkstoffeigenschaft die Zugfestigkeit von Kehlnähten hergeleitet. Außerdem werden theoretische Untersuchungen zum Tragverhalten und zur Spannungsverteilung vorgestellt. Zur Bestimmung der Tragfähigkeit wird das Vorgehen bei der statistischen Auswertung der Versuchsergebnisse erläutert. Dieses Kapitel dient als Einleitung und als Hintergrund für die in den folgenden Kapiteln vorgestellten experimentellen Untersuchungen.

\subsection{Bestimmung und Erläuterung zur Festigkeit des Schweißgutes}

\subsubsection{Allgemeines}

Um die Tragfähigkeit von Schweißverbindungen bestimmen zu können, ist die Kenntnis der Zugfestigkeit der betrachteten Naht besonders wichtig. Eine Bestimmung der Zugfestigkeit ist allerdings nicht einfach zu erreichen, da sie von der Festigkeit des Schweißzusatzwerkstoffs abweichen kann. Die Festigkeit von Kehlnähten kann aufgrund von anderer Geometrie, anderen Abkühlbedingungen, anderen Schweißparametern und metallurgischen Effekten von den Werten des Schweißzusatzwerkstoffs abweichen. Im Folgenden werden die Eigenschaften und die Bestimmung der Festigkeit des Schweißzusatzwerkstoffs und von Kehlnähten erläutert und verglichen.

\subsubsection{Festigkeit des reinen Schweißgutes}

Wie in Kapitel 2.4.4 beschrieben, haben Schweißzusatzwerkstoffe andere chemische Zusammensetzungen als die zugehörigen Grundwerkstoffe. Die Legierungselemente von Schweißzusatzwerkstoffen werden so gewählt, dass im reinen Schweißgut die jeweils normativ geforderten Kennwerte für die Festigkeit und Zähigkeit erreicht werden.

Diese im Materialprüfzeugnis angegebene Zugfestigkeit des Schweißzusatzwerkstoffs wird normgerecht an Zugproben bestimmt, die aus einer Stumpfnaht entnommen werden. Diese Stumpfnaht hat eine festgelegte Geometrie (siehe Abbildung 4.1) und vorgegebene Schweißparameter. Die Geometrie einer solchen Schweißnaht ist in DIN EN ISO 15792-1 (2008) geregelt, während die zugehörigen Schweißparameter in der jeweiligen Produktnorm der Schweißzusatzwerkstoffe gemeinsam mit den normativen Festigkeiten festgelegt sind. Das Volumen der Schweißnaht ist so groß gewählt, damit reines Schweißgut entsteht. Eine Beeinflussung des Schweißgutes durch eine Aufmischung mit den eingesetzten Grundwerkstoffen findet kaum statt.

In dieser Arbeit wurden neben Zugversuchen an Stumpfstößen auch Härtemessungen durchgeführt. Die Härtemessungen wurden waagerecht und senkrecht durch den Querschnitt gelegt, siehe Abbildung 4.1 und Abbildung 8.35.
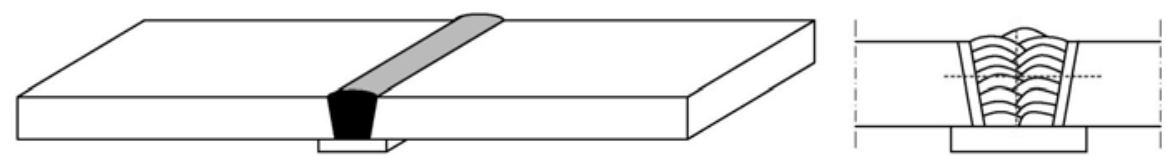

Abbildung 4.1: Versuchskörper mit Stumpfnaht (links) DIN EN ISO 15792-1 (2008) und Härteverläufe (rechts)

Neben dem Einfluss der Legierungselemente wird die Festigkeit des Schweißgutes durch die Mehrlagigkeit bestimmt. Durch das Überschweißen bei mehrlagigen Schweißnähten stellen sich Vergütungseffekte ein. Wie in Kapitel 2.2.4 für vergütete Stähle beschrieben, entspricht der Vorgang des Überschweißens der Wärmebehandlung der überschweißten Lage. Der Effekt ist mit dem Anlassen von Vergütungsstählen zu vergleichen. 


\subsubsection{Beschreibung der Festigkeit von Kehlnähten}

Die Festigkeit von Kehlnähten kann sich infolge mehrerer Gründe von der Festigkeit von Stumpfnähten unterscheiden. Zum einen beeinflusst der Grundwerkstoff die Schweißverbindung. Zum anderen herrschen andere Abkühlbedingungen als bei Stumpfnähten, die zu unterschiedlicher Ausbildung des Gefüges führen.

Das Schweißgut setzt sich aus Grund- und Schweißzusatzwerkstoff in unterschiedlicher Aufteilung der Massenanteile zusammen. Diese sind abhängig von Art und Größe der Naht. Bei Kehlnähten ist der Anteil des Grundwerkstoffs besonders groß, vgl. Abbildung 4.2.

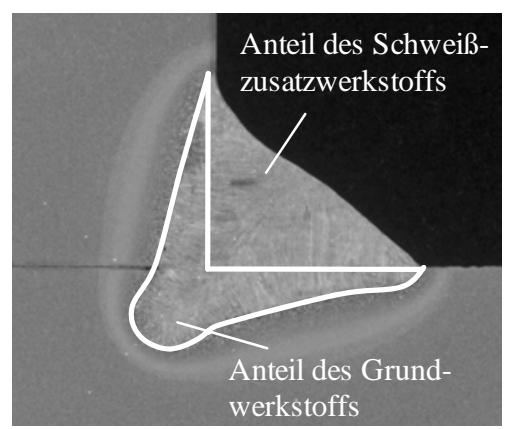

\section{Abbildung 4.2: Exemplarische Darstellung einer Kehlnaht}

In der Wurzellage, bei einlagigen Kehlnähten die einzige Lage, stellt sich eine deutliche Vermischung zwischen Grundwerkstoff und Schweißzusatzwerkstoff ein. Der aufgeschmolzene Grundwerkstoff wird damit Teil der Schweißverbindung und beeinflusst die chemische Zusammensetzung und damit auch die Festigkeit in der Naht, vgl. [Hildebrand, 2008]. Die chemische Zusammensetzung wird über den Kohlenstoffgehalt und den Gehalt an weiteren Legierungselementen gekennzeichnet. Ein Maß für den Legierungsgehalt liefert das Kohlenstoff-Äquivalent CET, vgl. (2.2). Wenn Grund- und Schweißzusatzwerkstoff ähnliche CET-Werte besitzen, ergibt sich eine Naht mit Eigenschaften, die denen der eingesetzten Werkstoffe ähneln. Wenn die Legierungselemente jedoch stark unterschiedlich sind, führt die Aufmischung zu veränderten Bedingungen. Während bei den Grundwerkstoffen eine Steigerung der Festigkeit neben einem erhöhten Anteil von Legierungselementen durch Wärmebehandlungen erreicht werden kann, steht bei den Schweißzusatzwerkstoffen nur ein höherer Anteil von Legierungselementen zur Verfügung. Bei den Grundwerkstoffen, die in dieser Arbeit betrachtetet werden, wird eine Erhöhung der Festigkeit vorwiegend durch Wärmebehandlungen erreicht. Die höhere Festigkeit der Schweißzusatzwerkstoffe entsteht durch einen höheren Anteil an Legierungselementen. Damit ergibt sich bei höherfesten Schweißverbindungen ein ausgeprägter Unterschied des KohlenstoffÄquivalents zwischen Grund- und Schweißzusatzwerkstoff.

Bei Kehlnähten stellen sich extreme Abkühlbedingungen ein, da die Nahtfläche gegenüber der Fläche des Grundwerkstoffs sehr klein ist. Die Geschwindigkeit der Abkühlung beeinflusst, wie in Kapitel 2.4.6 beschrieben, die Bildung von Martensit, Ferrit und Perlit. Durch die schnelle Abkühlung kommt es zu einer Aufhärtung, die zu größeren Festigkeiten führt. Bei mehrlagigen Kehlnähten können beim Schweißen der zweiten Lage Vergütungseffekte in der Wurzellage entstehen. Sie können dazu führen, dass die Aufhärtungen und Festigkeitssteigerungen der zuvor einlagigen Kehlnähte durch das erneute Aufschmelzen oder Anlassen zurückgeführt werden.

Kehlnähte der Werkstoffkombination S460-G46 zeigen in Versuchen eine höhere Tragfähigkeit als sich aus der Festigkeit der eingesetzten Werkstoffe ergibt. Dies lässt sich durch die extremen Abkühlbedingungen erklären. Durch diese ergeben sich Aufhärtungen, die zu höheren Festigkeiten führen. Die chemischen Zusammensetzungen von Grund- und Schweißzusatzwerkstoffen sind ähnlich, Einflüsse der Aufmischung machen sich somit nicht bemerkbar.

Bei Kehlnähten der Werkstoffkombination S690-G69 zeigen sich bei den Kehlnahttragfähigkeiten ähnliche oder sogar geringere Tragfähigkeiten als aus den Festigkeiten der eingesetzten Werkstoffe zu erwarten wäre. Die höherfesten Schweißzusatzwerkstoffe G69 erzielen die hohen Festigkeiten durch die chemischen Zusammensetzungen, während die zugehörigen Grundwerkstoffe ihre Festigkeit auch über die Wärmeführung bei der Herstellung generieren. Durch die oben beschriebene Aufmischung in der Kehlnaht ändert sich die chemische Zusammensetzung, der Legierungsgehalt wird geringer. Im 
gleichen Zug verringert sich damit auch die Tragfähigkeit der Naht. Der Tragfähigkeitserhöhung infolge der extremen Abkühlbedingungen bei Kehlnähten steht dieser Verlust an Tragfähigkeit durch die chemische Zusammensetzung entgegen.

\subsubsection{Bestimmung der Festigkeit von Kehlnähten}

Eine direkte Bestimmung der Zugfestigkeit von einlagigen Kehlnähten an einachsigen Zugproben ist nicht möglich, da aus den einlagigen Kehlnähten keine Zugproben gefertigt werden können, vgl. Abbildung 4.3. Es ist aber möglich, die Zugfestigkeit von einlagigen Kehlnähten über Härtemessungen abzuschätzen, vgl. Kapitel 2.6.

Um die Festigkeit von einlagigen Kehlnähten zu bestimmen, werden in dieser Arbeit Versuchskörper als T-Stoß verwendet, die mit ein- und mehrlagigen Kehlnähten geschweißt wurden, vgl. Abbildung 4.3. Sowohl an den einlagigen Kehlnähten als auch an den mehrlagigen Kehlnähten wurden Härtemessungen in drei Reihen durchgeführt: eine Reihe nahe der Oberfläche, eine Reihe in der Nahtmitte, eine Reihe nahe der Nahtwurzel und eine Reihe senkrecht dazu, vgl. Abbildung 4.3. Zusätzlich wurden an den dreiraupigen Kehlnähten Rundzugproben entnommen, an denen Zugversuche durchgeführt wurden. Die Ergebnisse dieser Härtemessungen und Zugversuche wurden mit den Härtemessungen an einlagigen Kehlnähten verglichen, um von der Zugfestigkeit der mehrlagigen Naht auf die Zugfestigkeit der einlagigen Naht Rückschlüsse ziehen zu können.

Bei den in Kapitel 8 vorgestellten Versuchen wurden die Härtemessungen der einlagigen Naht nicht an diesem T-Stoß sondern an der Naht des Versuchskörpers (in der ungebrochenen Naht) durchgeführt.
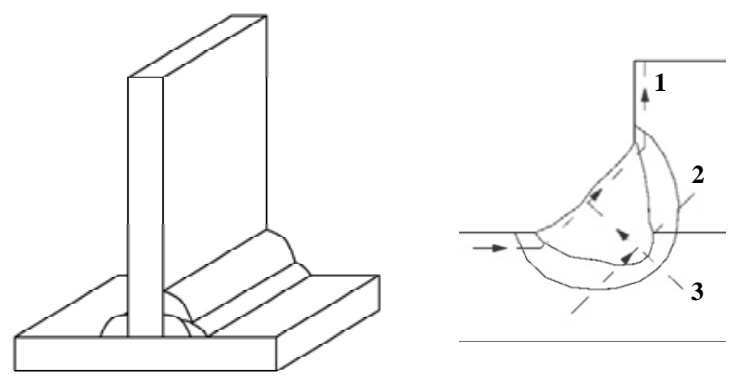

Abbildung 4.3: Versuchskörper mit ein- und dreiraupiger Kehlnaht (links) und Härteverläufe (rechts)

Ein typisches Ergebnis der Härtemessungen wird in Abbildung 4.4 gezeigt.

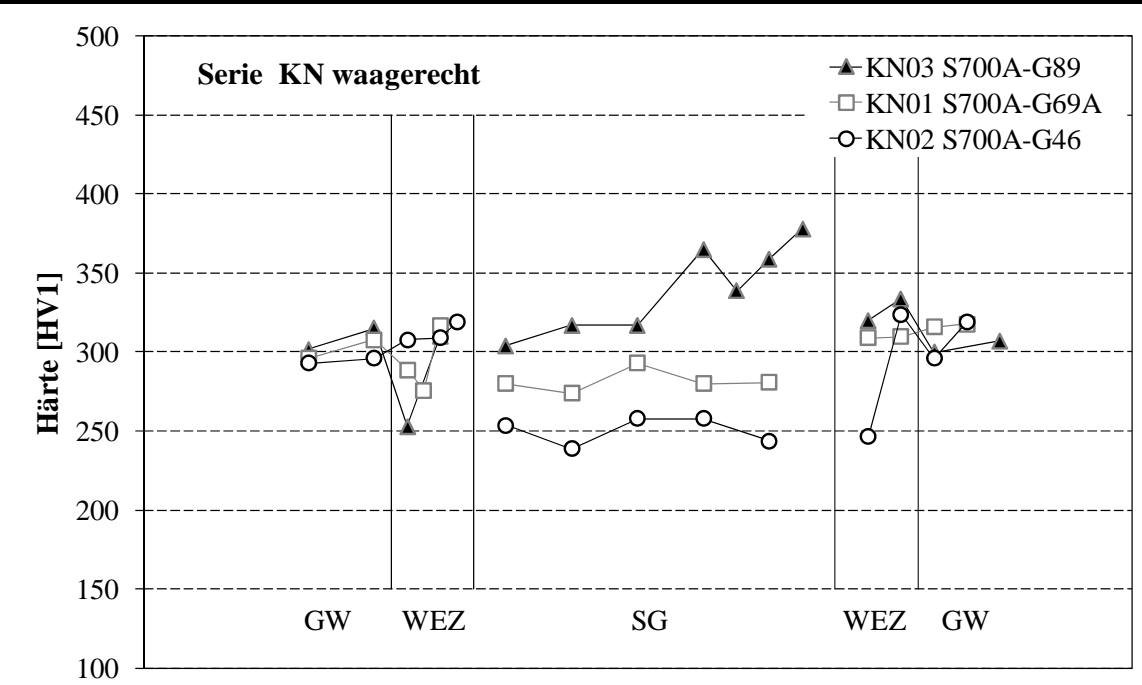

KN-01 S700A-G69A

SG MW: 282 HV1

$\mathrm{f}_{\mathrm{u}}=837 \mathrm{~N} / \mathrm{mm}^{2}$

KN-02 S700A-G46

SG MW: 251 HV1

$\mathrm{f}_{\mathrm{u}}=785 \mathrm{~N} / \mathrm{mm}^{2}$

KN-03 S700A-G89

SG MW: $340 \mathrm{HV} 1$

$\mathrm{f}_{\mathrm{u}}=932-952 \mathrm{~N} / \mathrm{mm}^{2}$

Abbildung 4.4: Typischer Vergleich der Ergebnisse der Härtemessungen für verschiedene Schweißzusatzwerkstoffe 
Dargestellt sind die ermittelten Härtewerte (HV1) in Abhängigkeit der Lage. Für die Lage sind an der Abszisse keine Werte angegeben sondern die unterschiedlichen Werkstoffbereiche. Als Bereiche werden der Grundwerkstoff (GW), die Wärmeeinflusszone (WEZ) und das Schweißgut (SG) unterschieden. Zusätzlich werden die Mittelwerte der Härtepunkte im Schweißgut und die zugehörigen ermittelten Zugfestigkeiten neben dem Diagramm angegeben. Diese werden für Vergleiche herangezogen. In der Wärmeeinflusszone ergeben sich stark variierende Werte, da der Bereich der Wärmeeinflusszone aus Grobkorn- und Feinkornzone und damit wiederum aus mehreren Bereichen besteht. In der Wärmeeinflusszone findet sich oft sowohl ein Abfall in der Härte als auch ein Bereich mit Aufhärtung. Da die Werte in der Wärmeeinflusszone keine Aussage über die Festigkeit in der Naht zulassen, werden diese Werte nicht weiter betrachtet.

Durch den Vergleich der beschriebenen Härtemessungen von zweilagigen (dreiraupigen) und einlagigen Kehlnähten und den zugehörigen Zugversuchen aus dreiraupigen Kehlnähten kann die Zugfestigkeit von einlagigen Kehlnähten abgeschätzt werden.

\subsection{Rechnerische Schweißnahtspannungen}

\subsubsection{Allgemeines}

Die Tragfähigkeit der Kehlnaht wird zum einen von der bisher beschriebenen Festigkeit des Schweißgutes bestimmt. Zum anderen ist die Tragfähigkeit von der Spannungsverteilung in der Naht abhängig, die in diesem Abschnitt beschrieben wird.

Im Allgemeinen geht man davon aus, dass für den Werkstoff Stahl als zähem Werkstoff die Gestaltänderungsenergiehypothese gilt, wie Gleichung (4.1) zeigt. Diese wird auch nach Huber, von Mises und Hencky benannt (z. B. [Schnell et al., 1998]).

$$
\sigma_{v}=\sqrt{\sigma_{x}^{2}+\sigma_{y}^{2}+\sigma_{z}^{2}-\sigma_{x} \cdot \sigma_{y}-\sigma_{x} \cdot \sigma_{z}-\sigma_{y} \cdot \sigma_{z}+3 \cdot\left(\tau_{x y}^{2}+\tau_{x z}^{2}+\tau_{y z}^{2}\right)}
$$

Betrachtet man Kehlnähte, lassen sich die Beanspruchungen in Spannungskomponenten $\sigma_{\perp}, \tau_{\perp}$ und $\tau_{\text {II }}$ aufteilen, vgl. Abbildung 4.5. Die Spannungen ergeben sich aus dem Quotienten von Kraft und Nahtfläche. In Gleichung (4.2) ist der Nachweis der Tragfähigkeit von Kehlnähten gegeben. Aus Gleichung (4.1) lässt sich die Beanspruchung auf der linken Seite von Gleichung (4.2) ableiten, während die rechte Seite die Beanspruchbarkeit als Bemessungsgrenzspannung definiert. Nach der Gestaltänderungsenergiehypothese ist der Grenzzustand erreicht, wenn die Vergleichsspannung diese Bemessungsgrenzspannung $\sigma_{\mathrm{w}, \mathrm{Rd}}$ erreicht, wie Gleichung (4.2) zeigt.

Dieses Format bildet die Grundlage für den dargestellten Nachweis nach EN 1993-1-8 (2005), dessen Definitionen im Folgenden gefolgt wird. Danach wird die Spannungskomponente $\sigma_{\text {II }}$ vernachlässigt.

$$
\sigma_{\mathrm{v}}=\sqrt{\sigma_{\perp}^{2}+3 \cdot \tau_{\perp}^{2}+3 \cdot \tau_{\mathrm{II}}^{2}} \leq \sigma_{\mathrm{w}, \mathrm{Rd}}=\frac{\mathrm{f}_{\mathrm{u}, \mathrm{k}}}{\beta_{\mathrm{w}} \cdot \gamma_{\mathrm{M} 2}}
$$
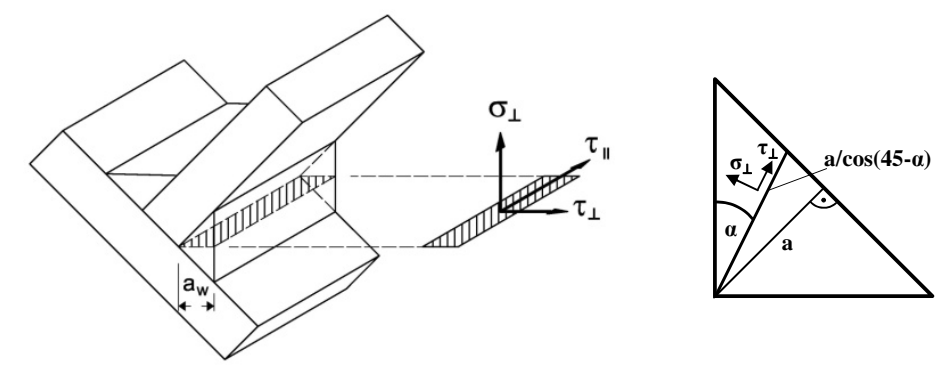

\section{Abbildung 4.5: Spannungskomponenten parallel und senkrecht zur Nahtachse}

In den folgenden Abschnitten werden die Beanspruchungen und Beanspruchbarkeit betrachtet. In Abschnitt 4.3.2 wird die Spannungsverteilung in Flankenkehlnähten beschrieben, während in Abschnitt 4.3.3 die Spannungskomponenten von Kreuzstößen erläutert werden. In Abschnitt 4.3.4 wird die Bemessungsgrenzspannung betrachtet. 


\subsubsection{Flankenkehlnähte}

Für die Beanspruchung von Flankenkehlnähten ergeben sich für Gleichung (4.2) als Beanspruchung nur Längsschubspannungen $\tau_{\mathrm{II}}$, vgl. Gleichung (4.3). Die größte Spannung $\sigma_{\mathrm{v}}$ entsteht infolge der größten Kraft dividiert durch die kleinste Nahtfläche $A_{w}$. Der Grenzzustand ist erreicht, wenn diese größte Spannung $\sigma_{\mathrm{v}}$ die Größe der Bemessungsgrenzspannung $\sigma_{\mathrm{w}, \mathrm{Rd}}$ erreicht. Bei den schubbeanspruchten Flankenkehlnähten bestimmt sich die Bemessungsgrenzspannung $\sigma_{\mathrm{w}, \mathrm{Rd}}$ immer mit dem Faktor $\sqrt{3}$. Im Ideal-Fall eines gleichschenkligen Dreiecks als Nahtgeometrie entspricht die maßgebende kleinste Fläche der Höhe des Dreiecks multipliziert mit der Nahtlänge.

$$
\sigma_{\mathrm{v}}=\sqrt{3 \cdot \tau_{\mathrm{II}}^{2}} \leq \sigma_{\mathrm{w}, \mathrm{Rd}}=\frac{\mathrm{f}_{\mathrm{u}, \mathrm{k}}}{\beta_{\mathrm{w}} \cdot \gamma_{\mathrm{M} 2}} \rightarrow \sigma_{\mathrm{w}, \mathrm{Rd}}=\sqrt{3} \cdot \frac{\mathrm{F}}{\mathrm{A}_{\mathrm{w}}}
$$

\subsubsection{Stirnkehlnähte}

Werden die Nähte als Stirnkehlnähte belastet, herrschen Spannungen $\sigma_{\perp}$ und $\tau_{\perp}$ vor, und es gilt Gleichung (4.4). Die maximale Vergleichsspannung $\sigma_{\mathrm{v}}$ erreicht mit der Bemessungsgrenzspannung $\sigma_{\mathrm{w}, \mathrm{Rd}}$ den Grenzzustand.

$$
\sigma_{\mathrm{v}}=\sqrt{\sigma_{\perp}^{2}+3 \cdot \tau_{\perp}^{2}} \leq \sigma_{\mathrm{w}, \mathrm{Rd}}=\frac{\mathrm{f}_{\mathrm{u}, \mathrm{k}}}{\beta_{\mathrm{w}} \cdot \gamma_{\mathrm{M} 2}}
$$

Um die Bemessungsgrenzspannung für Stirnkehlnähte zu bestimmen, muss ermittelt werden, wann die Vergleichsspannung $\sigma_{\mathrm{v}}$ maximal wird. In diesem Fall lässt sich die größte Spannung $\sigma_{\mathrm{v}}$ nicht allein in Abhängigkeit der kleinsten Nahtfläche ermitteln wie bei den Flankenkehlnähten. Sondern es muss eine maßgebende Nahtdicke bestimmt werden, die durch den Winkel $\alpha$ definiert ist, wie Abbildung 4.5 zeigt. Die Spannungen $\sigma_{\perp}$ und $\tau_{\perp}$ sind von der Nahtdicke und zusätzlich von der Kraftaufteilung parallel und senkrecht zur Naht abhängig. Die Kraftaufteilung ändert sich mit dem Winkel $\alpha$.

Für ein gleichschenkliges Dreieck kann die maßgebende Nahtdicke in Abhängigkeit des Winkels $\alpha$ mit $\cos \left(45^{\circ}-\alpha\right)$ definiert werden. Mit den Spannungskomponenten $\sigma_{\perp}=\frac{F \cdot \sin \alpha}{A_{\alpha}}$ und $\tau_{\perp}=\frac{F \cdot \cos \alpha}{A_{\alpha}}$ und der Nahtfläche $A_{\alpha}=\frac{a \cdot 1}{\cos \left(45^{\circ}-\alpha\right)}$ ergibt sich die Vergleichsspannung $\sigma_{v}$ nach Gleichung (4.5).

$$
\sigma_{\mathrm{v}}=\sqrt{\sigma_{\perp}^{2}+3 \cdot \tau_{\perp}^{2}}=\sqrt{\left(\frac{\mathrm{F} \cdot \sin \alpha}{\mathrm{A}_{\alpha}}\right)^{2}+3 \cdot\left(\frac{\mathrm{F} \cdot \cos \alpha}{\mathrm{A}_{\alpha}}\right)^{2}}=\frac{\mathrm{F}}{\mathrm{a} \cdot \mathrm{l}} \cdot \cos (45-\alpha) \cdot \sqrt{(\sin \alpha)^{2}+3 \cdot(\cos \alpha)^{2}}
$$

Zur Bestimmung der Bemessungsgrenzspannung muss ermittelt werden, für welchen Winkel $\alpha$ die Vergleichsspannung $\sigma_{\mathrm{v}}$ nach Gleichung (4.5) maximal wird. Zur Veranschaulichung ist diese Vergleichsspannung in Abbildung 4.6 in Abhängigkeit des Winkels $\alpha$ dargestellt.

Es zeigt sich, dass die Vergleichsspannung $\sigma_{\mathrm{v}}$ bei einem Winkel von etwa $\alpha=27,4^{\circ}$ mit einem Faktor von 1,53 maximal wird, wie in Gleichung (4.6) ausgeführt.

$$
\sigma_{\mathrm{v}, \max }\left(\alpha=27,4^{\circ}\right)=1,53 \cdot \frac{\mathrm{F}}{\mathrm{a} \cdot \mathrm{l}} \text { mit } \mathrm{A}_{\alpha}=1,05 \cdot \mathrm{a} \cdot \mathrm{l}, \sigma_{\perp}=\frac{\mathrm{F} \cdot 0,46}{1,05 \cdot \mathrm{a} \cdot \mathrm{l}} \text { und } \tau_{\perp}=\frac{\mathrm{F} \cdot 0,89}{1,05 \cdot \mathrm{a} \cdot \mathrm{l}}
$$

Diese Vergleichsspannung $\sigma_{\mathrm{v}, \max }$ erreicht mit der Bemessungsgrenzspannung $\sigma_{\mathrm{w}, \mathrm{Rd}}$ den Grenzzustand, vgl. Gleichung (4.7):

$$
\sigma_{\mathrm{v}, \max }\left(\alpha=27,4^{\circ}\right)=1,53 \cdot \frac{\mathrm{F}}{\mathrm{a} \cdot \mathrm{l}}=\sigma_{\mathrm{w}, \mathrm{Rd}}
$$

Im Gegensatz dazu ergibt sich nach EN 1993-1-8 (2005), wie in Kapitel 3, Tabelle 3.5 beschrieben, mit der dort geforderten kleinsten Fläche $\left(\alpha=45^{\circ}\right)$ der Faktor $\sqrt{2}$, wie Gleichung (4.8) zeigt: 


$$
\sigma_{\mathrm{v}, \mathrm{EC}}=\sqrt{2} \cdot \frac{\mathrm{F}}{\mathrm{a} \cdot \mathrm{l}} \text { mit } \mathrm{A}_{\mathrm{w}}=\mathrm{a} \cdot \mathrm{l}, \sigma_{\perp}=\frac{\mathrm{F}}{\sqrt{2} \cdot \mathrm{a} \cdot \mathrm{l}} \text { und } \tau_{\perp}=\frac{\mathrm{F}}{\sqrt{2} \cdot \mathrm{a} \cdot \mathrm{l}}
$$

Nach EN 1993-1-8 (2005) ergibt sich der Grenzzustand, wenn diese Vergleichsspannung $\sigma_{\mathrm{v}, \mathrm{EC}}$ den Wert der Bemessungsgrenzspannung $\sigma_{\mathrm{w}, \mathrm{Rd}}$ erreicht, vgl. Gleichung (4.9):

$$
\sigma_{\mathrm{v}, \mathrm{EC}}=\sqrt{2} \cdot \frac{\mathrm{F}}{\mathrm{a} \cdot \mathrm{l}}=\sigma_{\mathrm{w}, \mathrm{Rd}}
$$

Theoretisch tritt der Grenzzustand erst ein, wenn die Vergleichsspannung $\sigma_{\mathrm{v}}$ den Wert $1,53 \cdot \frac{\mathrm{F}}{\mathrm{a} \cdot \mathrm{l}}$ erreicht. Der normative Grenzzustand wird bereits mit dem Wert $\sqrt{2} \cdot \frac{\mathrm{F}}{\mathrm{a} \cdot \mathrm{l}}$ erreicht.

Der Vergleichsspannungsnachweis gemäß EN 1993-1-8 (2005) für ein gleichschenkliges Dreieck liegt somit um $1,53 / \sqrt{2}=1,08$, also um $8 \%$ auf der sicheren Seite. Damit konnte gezeigt werden, dass für die Ermittlung der größten Vergleichsspannung $\sigma_{v}$ nicht die kleinste Fläche maßgebend wird, wie nach EN 1993-1-8 (2005).

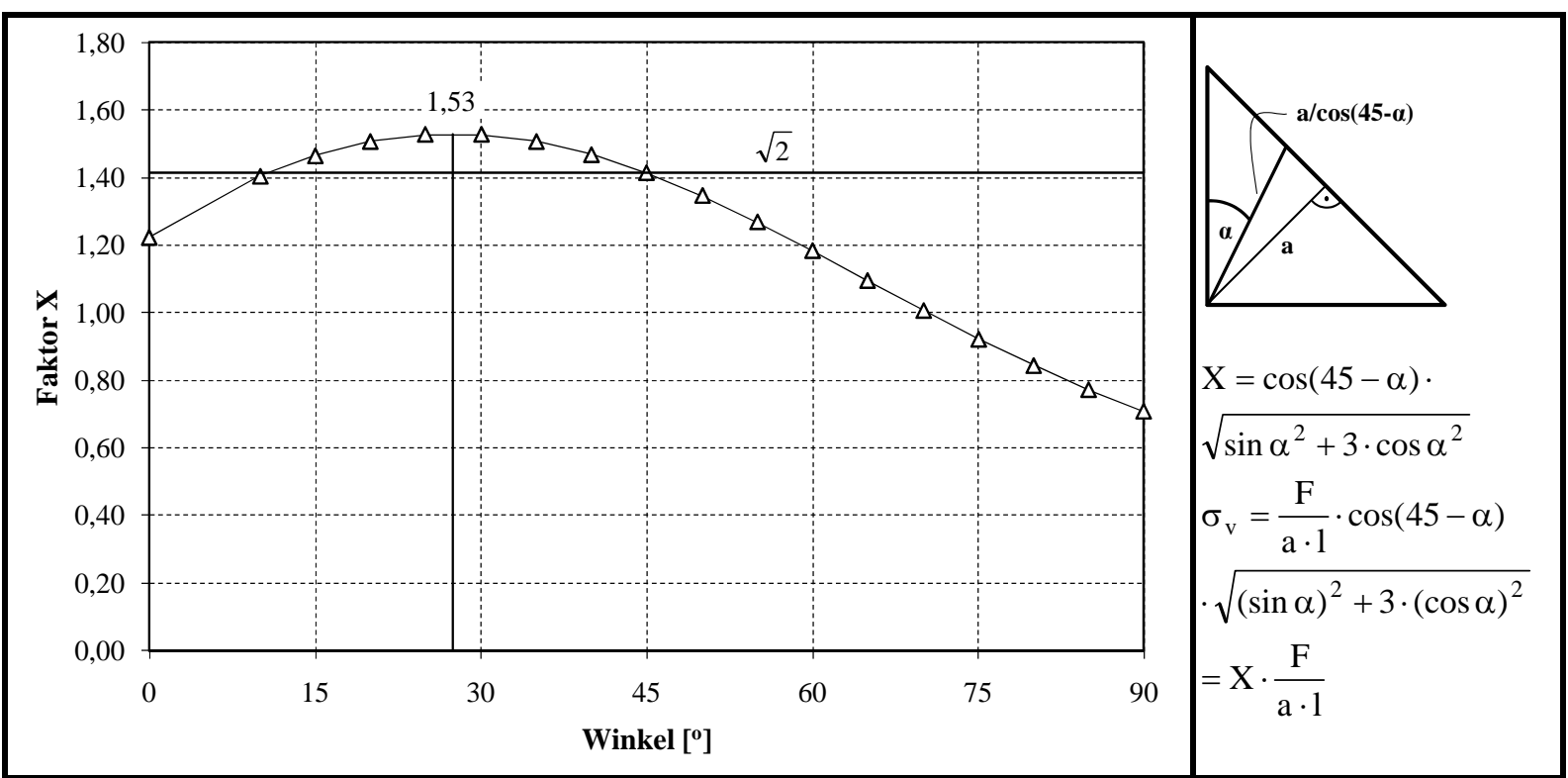

Abbildung 4.6: Vergleichsspannung bei gleichschenkligen Dreiecken in Abhängigkeit des Winkels $\alpha$

Nach dem einfachen Fall des gleichschenkligen Dreiecks wird nun das ungleichschenklige Dreieck betrachtet. Bei ungleichschenkligen Dreiecken muss zusätzlich zum Winkel $\alpha$ ein Winkel $\beta$ berücksichtigt werden. Der Winkel $\beta$ definiert gemeinsam mit der Höhe h das ungleichschenklige Dreieck, während mit dem Winkel $\alpha$ die maßgebende Nahtdicke definiert wird.

Mit den Spannungskomponenten $\sigma_{\perp}=\frac{F \cdot \sin \alpha}{A_{\alpha}}$ und $\tau_{\perp}=\frac{F \cdot \cos \alpha}{A_{\alpha}}$ und der Nahtfläche $A_{\alpha}=\frac{a \cdot l}{\cos (\beta-\alpha)}$ ergibt sich die Vergleichsspannung $\sigma_{\mathrm{v}}$ nach Gleichung (4.10).

$$
\sigma_{\mathrm{v}}=\sqrt{\sigma_{\perp}^{2}+3 \cdot \tau_{\perp}^{2}}=\sqrt{\left(\frac{\mathrm{F} \cdot \sin \alpha}{\mathrm{A}_{\alpha}}\right)^{2}+3 \cdot\left(\frac{\mathrm{F} \cdot \cos \alpha}{\mathrm{A}_{\alpha}}\right)^{2}}=\frac{\mathrm{F}}{\mathrm{a} \cdot \mathrm{l}} \cdot \cos (\beta-\alpha) \cdot \sqrt{(\sin \alpha)^{2}+3 \cdot(\cos \alpha)^{2}}
$$

Der Winkel $\alpha$, der die für die maximalen Spannungen maßgebende Nahtdicke definiert, ergibt sich gemäß dem Diagramm in Abbildung 4.7.

Für einen Winkel $\beta=30^{\circ}$ ergibt sich die maximale Vergleichsspannung mit dem Faktor 1,64 beim Winkel $\alpha=20^{\circ}$. Ein Dreieck mit dem Winkel $\beta=45^{\circ}$ errechnet sich die maximale Vergleichsspannung $\sigma_{\mathrm{v}}$ mit dem Faktor 1,53 beim Winkel $\alpha=30^{\circ}$. Für $\beta=60^{\circ}$ kann man die maximale Vergleichsspannung $\sigma_{\mathrm{v}}$ mit dem Faktor 1,4 beim Winkel $\alpha=40^{\circ}$ ablesen. 


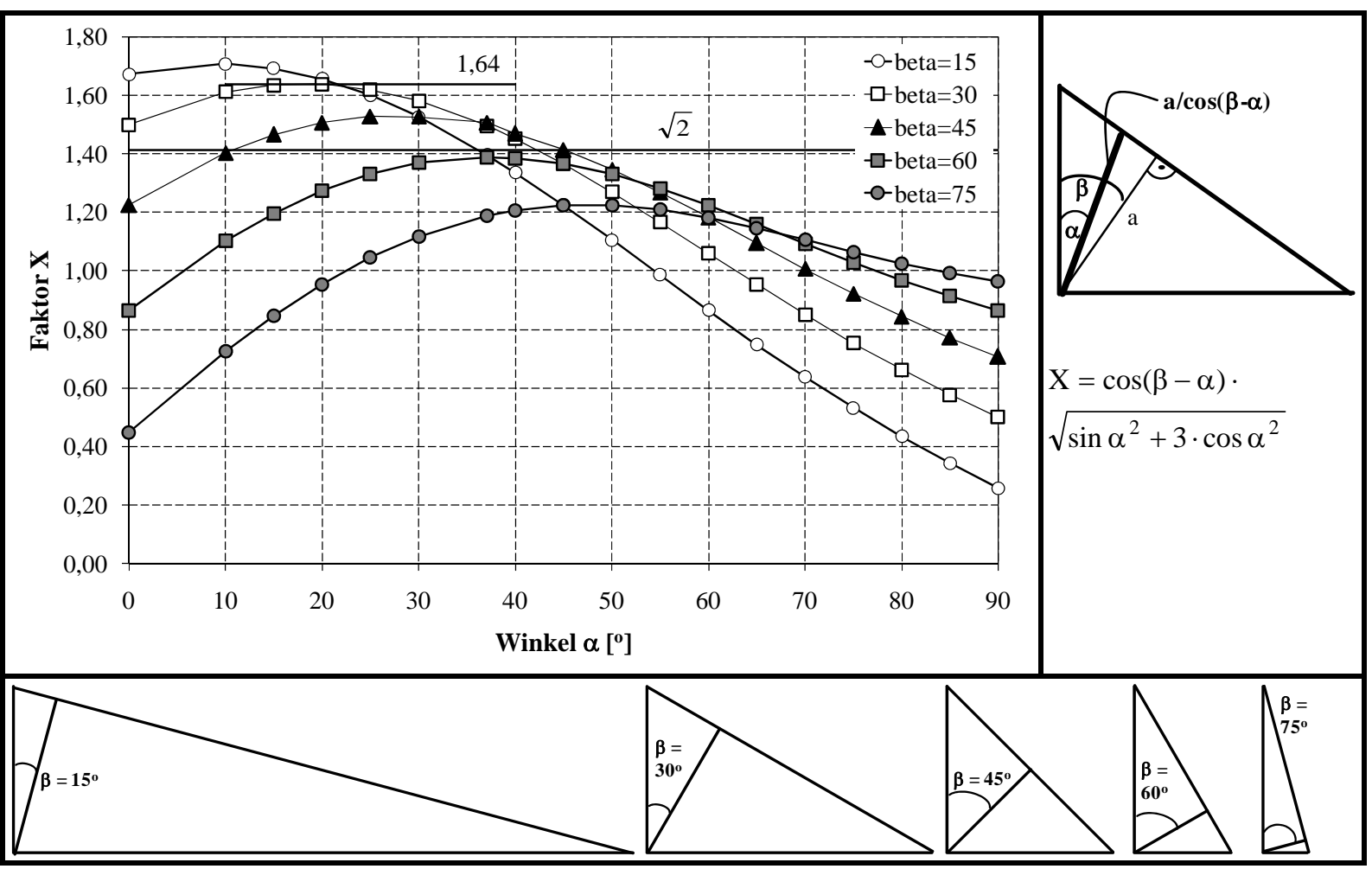

Abbildung 4.7: Vergleichsspannung bei ungleichschenkligen Dreiecken in Abhängigkeit des Winkels $\alpha$

Zur Veranschaulichung sind Dreiecke mit unterschiedlichen Winkeln $\beta$ in Abbildung 4.7 in der unteren Zeile dargestellt. Eine unsymmetrische Naht mit Abmessungen $\beta>60^{\circ}$ oder $\beta<30^{\circ}$ (vgl. Abbildung 4.7, untere Zeile) ist nach DIN EN ISO 5817 (2006) nicht zulässig.

Für ungleichschenklige Dreiecke, deren Winkel $\beta$ kleiner als $60^{\circ}$ sein müssen, ergeben sich maximalen Vergleichsspannungen $\sigma_{v}$, die mit einem Faktor zwischen 1,4 und 1,64 gleich oder größer als der Faktor $\sqrt{2}$ sind, der vom Nachweisformat nach EN 1993-1-8 (2005) gefordert wird. Die maximale Vergleichsspannung $\sigma_{\mathrm{v}}$ erreicht mit der Bemessungsgrenzspannung $\sigma_{\mathrm{w}, \mathrm{Rd}}$ den Grenzzustand. Da die Faktoren zwischen 1,4 und 1,64 stets gleich oder größer als $\sqrt{2}$ sind, lässt sich auch für ungleichschenklige Dreiecke sagen, dass der Nachweis nach EN 1993-1-8 (2005) immer auf der sicheren Seite liegt.

Somit liegt der Nachweis gemäß EN 1993-1-8 (2005) (mit der Annahme, dass die Höhe des einschreibbaren Dreiecks zu verwenden ist) sowohl für gleichschenklige als auch für ungleichschenklige Dreiecke, immer auf der sicheren Seite. In Abhängigkeit der Nahtform liegt die Größe der Überschätzung sehr unterschiedlich zwischen 1,0 und $1,64 / \sqrt{2}=1,16$.

\subsubsection{Beschreibung der Bemessungsgrenzspannung im Nachweisformat}

Nach den in Kapitel 4.3.2 und 4.3.3 beschriebenen Beanspruchungen in der Kehlnaht werden nun die Beanspruchbarkeiten als Bemessungsgrenzspannung betrachtet.

Im Kapitel 3 wurden die unterschiedlichen Nachweisformate zur Bestimmung der Tragfähigkeiten von Kehlnähten vorgestellt. Die Tragfähigkeit von Kehlnähten unterscheidet sich von der Festigkeit von Grund- und Schweißzusatzwerkstoff, was durch verschiedene Faktoren in den diversen Normen berücksichtigt wird. Im Folgenden wird die Beschreibung der Bemessungsgrenzspannung von Kehlnähten im Nachweisformat in der europäischen Norm EN 1993-1-8 (2005) erläutert, vgl. Gleichung (3.2) oder (4.2).

Das Nachweisformat gemäß Gleichung (4.2) bestimmt die Bemessungsgrenzspannung in Abhängigkeit der Zugfestigkeit des Grundwerkstoffs $\mathrm{f}_{\mathrm{u}, \mathrm{k}}$, eines Teilsicherheitsbeiwerts $\gamma_{\mathrm{M} 2}$ und des Beiwerts $\beta_{\mathrm{w}}$. Bei Verbindungen gleicher Grund- und zugehöriger Schweißzusatzwerkstoffe kann vereinfachend 
davon ausgegangen werden, dass die Festigkeit des Grundwerkstoffs der Festigkeit des reinen Schweißgutes, somit der normativen Festigkeit des Schweißzusatzwerkstoffs entspricht, wie in Kapitel 4.2 beschrieben.

Die Unterschiede in der Festigkeit von Kehlnähten im Vergleich zur Festigkeit des reinen Schweißgutes werden durch den Beiwert $\beta_{w}$ definiert. Überfestigkeit der Kehlnaht gegenüber der Festigkeit der eingesetzten Werkstoffe wird durch einen Beiwert $\beta_{w}<1,0$ gekennzeichnet. Liegt die Festigkeit der Kehlnaht unterhalb der Festigkeit des reinen Schweißgutes, ergibt sich ein Beiwert $\beta_{\mathrm{w}}>1,0$.

Zusätzlich berücksichtigt der Beiwert $\beta_{w}$, dass die Tragfähigkeiten der Kehlnähte gewissen Streuungen unterliegen, die statistisch erfasst werden.

\subsubsection{Schlussfolgerung}

Zur Bestimmung der Kehlnahttragfähigkeit werden im Nachweisformat der Europäischen Norm EN 1993-1-8 (2005) als Beanspruchung die unterschiedlichen Spannungskomponenten $\sigma_{\perp}, \tau_{\perp}$ und $\tau_{\text {II }}$ berücksichtigt. Zur Bestimmung der Bemessungsgrenzspannung werden neben der normativen Festigkeit der eingesetzten Werkstoffe Unterschiede zur Festigkeit der Kehlnaht mit dem Beiwert $\beta_{\mathrm{w}}$ berücksichtigt.

Die Annahme nach EN 1993-1-8 (2005), die Höhe des einschreibbaren Dreiecks als maßgebende Nahtdicke zu verwenden, wurde diskutiert. Für die Flankenkehlnaht wurde gezeigt, dass diese Fläche maßgebend wird, da infolge der kleinsten Fläche die größte Spannung entsteht. Die Berechnungen zur Stirnkehlnaht zeigen, dass die Forderung, die Höhe des einschreibbaren Dreiecks als maßgebende Nahtdicke zu verwenden, sowohl für gleichschenklige als auch für ungleichschenklige Dreiecke auf der sicheren Seite liegt. In Abhängigkeit der Nahtform liegt die Größe der Überschätzung sehr unterschiedlich zwischen 1,0 und 1,16.

\subsection{Ermittlung der Spannungen aus Versuchsergebnissen}

\subsubsection{Flankenkehlnahtverbindungen}

Nach der theoretischen Spannungsverteilung wird in diesem Abschnitt die Ermittlung der Spannungen aus Versuchen vorgestellt. Wie in Kapitel 4.3.2 beschrieben, ergeben sich rechnerisch bei Versuchen an Flankenkehlnähten nur Längsschubspannungen $\tau_{\text {II. }}$. Die größte Spannung entsteht als Quotient der größten Kraft im Versuch und der kleinsten Fläche. Das Nahtversagen tritt immer in der kleinsten Fläche, also der Bruchfläche auf. Die Bruchfläche enthält ggf. plastische Verformungen, die nach Erreichen der Maximalkraft entstehen können. Da die Verformungen insgesamt sehr klein sind, werden diese plastischen Verformungen vernachlässigt.

In dieser Arbeit werden die Längsschubspannungen bestimmt als maximale Kraft aus dem Versuch dividiert durch die Bruchfläche. Die Versuchsergebnisse an Flankenkehlnahtverbindungen, die in Kapitel 6 - 8 vorgestellt werden, zeigen vorwiegend Bruchflächen, die eine Neigung von etwa $45^{\circ}$ haben. Dies entspricht der kleinsten Fläche und damit der Höhe des Dreiecks.

\subsubsection{Kreuzstöße mit Stirnkehlnähten}

Wie in Abschnitt 4.3.3 beschrieben, ergeben sich die größten Spannungen bei Kreuzstößen nicht in der kleinsten Fläche. Aus dem Nachweis in Gleichung (4.4) kann ein Winkel $\alpha$ abgeleitet werden, der die Bruchfläche definiert. Dieser Winkel $\alpha$ definiert nicht die Höhe des einschreibbaren Dreiecks, sondern die Lage der Bruchfläche über die maßgebende Nahtdicke.

Um die maximalen Spannungen in der Schweißnaht von Versuchen an Kreuzstößen mit Stirnkehlnähten zu bestimmen, müssen somit der Bruchwinkel und die Bruchfläche bestimmt werden. Dann muss die maximale Kraft entsprechend in die Komponenten aufgeteilt werden. 
Praktisch ist dieses Vorgehen nur schwierig umsetzbar, da die Versuchskörper im Allgemeinen zwei Nähte haben, die eine unterschiedliche Geometrie haben können. Neben der theoretisch möglichen Aufteilung in die Komponenten muss die Kraft auf diese beiden unterschiedlichen Nähte verteilt werden. Es kann im Versuch nicht festgestellt werden, wie sich die Kraft auf die beiden Nähte verteilt. Hierzu muss daher eine Annahme getroffen werden. Nach dem Versagen der ersten Naht erfährt die zweite Naht Biegung und versagt durch eine nicht bekannte Spannungsbeanspruchung. Eine theoretisch korrekte Aufteilung der Kraft auf die beiden Nähte ist nicht möglich, wenn nicht bekannt ist, welche Naht zuerst versagt. Das ist meistens der Fall.

In der Literatur wird typischerweise bei den bisherigen Versuchsauswertungen so vorgegangen, dass die Spannung als Quotient von Kraft F und Gesamtbruchfläche $A_{\alpha}$ bestimmt wird. Damit wird die maximale Kraft aus dem Versuch gleich auf die beiden Nähte aufgeteilt.

Bezogen auf die Bruchfläche $\mathrm{A}_{\alpha}$ lassen sich die Spannungskomponenten $\sigma_{\perp}$ und $\tau_{\perp}$ der Versuchsergebnisse aus der Vergleichsspannung aus Gleichung (4.5) als Vergleichsspannung nach Gleichung (4.11) darstellen. Diese Vergleichsspannung der Versuche wird für die Versuchsauswertung der Vergleichsspannung nach Gleichung (4.8) gegenübergestellt, wie in Gleichung (4.11) dargestellt. Dabei wird von einem gleichschenkligen Dreieck ausgegangen, so dass ein Faktor von $\sqrt{2}$ zu beachten ist.

$$
\begin{aligned}
& \sigma_{\mathrm{v}}=\sqrt{\sigma_{\perp}^{2}+3 \cdot \tau_{\perp}^{2}}=\sqrt{\left(\frac{\mathrm{F} \cdot \sin \alpha}{\mathrm{A}_{\alpha}}\right)^{2}+3 \cdot\left(\frac{\mathrm{F} \cdot \cos \alpha}{\mathrm{A}_{\alpha}}\right)^{2}}=\frac{\mathrm{F}}{\mathrm{A}_{\alpha}} \cdot \sqrt{\sin \alpha^{2}+3 \cdot \cos \alpha^{2}} \leq \sigma_{\mathrm{w}, \mathrm{Rd}} \text { und } \\
& \sigma_{\mathrm{w}, \mathrm{Rd}, \mathrm{EC}}=\sqrt{2} \cdot \frac{\mathrm{F}}{\mathrm{a} \cdot \mathrm{l}}
\end{aligned}
$$

Der Fehler zwischen Versuchsspannungen und dem Bemessungsansatz ergibt sich aus dem Vergleich der beiden Faktoren $\sqrt{\sin \alpha^{2}+3 \cdot \cos \alpha^{2}}$ und $\sqrt{2}$. Je nach Bruchwinkel liegen die Versuchsergebnisse mehr oder weniger oberhalb der rechnerischen Werte, die sich im Nachweis nach EN 1993-1-8 (2005) ergeben. Die Versuchsergebnisse, die in den Kapiteln 5 bis 8 vorgestellt werden, zeigen in allen Fällen einen steilen Bruchwinkel $\alpha$ von $15-30^{\circ}$. Damit ist der Beiwert $\sqrt{\sin \alpha^{2}+3 \cdot \cos \alpha^{2}}$ immer größer als $\sqrt{2}$. Das Vorgehen liegt somit auf der sicheren Seite.

Versuchstechnisch ist es kaum möglich, die Spannungskomponenten korrekt zu ermitteln. Deshalb wird auch in dieser Arbeit auf die oben beschriebene Vereinfachung zurückgegriffen. Nach Möglichkeit wird die Spannung als Quotient aus Kraft und Gesamtbruchfläche bestimmt.

\subsection{Beschreibung der statistischen Auswertung}

\subsubsection{Allgemeines}

Um die eigenen Versuchsergebnisse sowie die Versuche aus der Literatur beurteilen zu können, wurden diese statistisch ausgewertet. Die statistische Auswertung der Versuche wurde in Anlehnung an DIN EN 1990 (2002), Anhang D durchgeführt. Danach können Bemessungswerte aus Versuchen entweder durch die statistische Bestimmung einer einzelnen Eigenschaft oder durch die statistische Bestimmung eines Widerstandsmodells abgeleitet werden.

Mit Hilfe der statistischen Bestimmung einer einzelnen Eigenschaft werden einzelne Eigenschaften auf Basis einer gewissen Anzahl von Versuchen als charakteristischer Wert oder als Bemessungswert festgelegt.

Bei dem verwendeten Verfahren der statistischen Bestimmung eines Widerstandsmodells handelt es sich um ein Verfahren zur Kalibrierung von Widerstandsmodellen und die Bestimmung von Bemessungswerten. Es wird ein Bemessungsmodell vorgeschlagen, dessen Gültigkeit auf Basis von Versuchen statistisch überprüft wird. Die Bemessungswerte können entweder über die charakteristischen Werte (Methode A) oder direkt als Bemessungswerte (Methode B) abgeleitet werden. Einzelheiten der statistischen Auswertung können dem Anhang A.1 entnommen werden. 
Die verwendeten Bemessungsmodelle und das Vorgehen bei der statistischen Auswertung werden im Folgenden beschrieben.

\subsubsection{Bemessungsmodell für Flankenkehlnahtverbindungen}

Das Bemessungsmodell für Schweißverbindungen mit Flankenkehlnähten nach EN 1993-1-8 (2005) ist in Kapitel 3 beschrieben. Da Flankenkehlnähte planmäßig nur in Nahtlängsrichtung mit der Spannung $\tau_{\text {II }}$ beansprucht werden, unterscheiden sich das Vereinfachte und das Richtungsbezogene Verfahren nicht. Daraus ergibt sich als folgende Vereinfachung aus Gleichung (3.2) das Bemessungsmodell in Gleichung (4.12).

$$
\begin{aligned}
& \sigma_{\mathrm{v}, \mathrm{EC}}=\sqrt{\sigma_{\perp}^{2}+3 \cdot \tau_{\perp}^{2}+3 \cdot \tau_{\mathrm{II}}^{2}} \leq \frac{\mathrm{f}_{\mathrm{u}}}{\beta_{\mathrm{w}} \cdot \gamma_{\mathrm{M} 2}} \Rightarrow \tau_{\mathrm{II}}=\frac{\mathrm{F}_{\mathrm{II}}}{\mathrm{A}} \leq \frac{\mathrm{f}_{\mathrm{u}}}{\sqrt{3} \cdot \beta_{\mathrm{w}} \cdot \gamma_{\mathrm{M} 2}} \\
& \text { Bemessungsmodell: } \mathrm{r}_{\mathrm{t}}=\mathrm{A}_{\mathrm{w}} \cdot \frac{\mathrm{f}_{\mathrm{u}}}{\sqrt{3} \cdot \beta_{\mathrm{w}} \cdot \gamma_{\mathrm{M} 2}}
\end{aligned}
$$

Das Bemessungsmodell berücksichtigt als Variablen die Bruchfläche der Schweißnaht und die Zugfestigkeit des Grundwerkstoffs. Für die statistische Auswertung bei den Flankenkehlnahtverbindungen wird als Nahtfläche $A_{w}$ die ausgemessene Bruchfläche $A_{B r u c h}$ verwendet.

\subsubsection{Bemessungsmodell für Stirnkehlnahtverbindungen}

Das Bemessungsmodell für Schweißverbindungen mit Stirnkehlnähten nach EN 1993-1-8 (2005) ist in Kapitel 3 beschrieben. Im richtungsbezogenen Verfahren, vgl. Gleichung (3.2), werden Spannungen $\sigma_{\perp}$ und $\tau_{\perp}$ berücksichtigt:

$$
\begin{aligned}
& \sigma_{\mathrm{v}, \mathrm{EC}}=\sqrt{\sigma_{\perp}^{2}+3 \tau_{\perp}^{2}} \leq \frac{\mathrm{f}_{\mathrm{u}}}{\beta_{\mathrm{w}} \cdot \gamma_{\mathrm{M} 2}} \text { mit } \sigma_{\perp}=\tau_{\perp}=\frac{\mathrm{F}}{\sqrt{2} \mathrm{~A}} \text { und damit } \frac{\mathrm{F}}{\mathrm{A}} \leq \frac{\mathrm{f}_{\mathrm{u}}}{\sqrt{2} \cdot \beta_{\mathrm{w}} \cdot \gamma_{\mathrm{M} 2}} \\
& \text { Bemessungsmodell: } \mathrm{r}_{\mathrm{t}}=\mathrm{A}_{\mathrm{w}} \cdot \frac{\mathrm{f}_{\mathrm{u}}}{\sqrt{2} \cdot \beta_{\mathrm{w}} \cdot \gamma_{\mathrm{M} 2}}
\end{aligned}
$$

Das Bemessungsmodell berücksichtigt als Variablen die Bruchfläche der Schweißnaht und die Zugfestigkeit des Grundwerkstoffs. Für die Kreuzstöße wurden für die Nahtfläche die Werte aus dem Makroschliff oder die ermittelten Bruchflächen verwendet, soweit sie bekannt sind. Eine Aufteilung des Kraftvektors wird nicht vorgenommen, wie in Kapitel 4.4.2 erläutert.

\subsubsection{Bemessungsmodell für Flanken- und Stirnkehlnahtverbindungen}

Wie in Kapitel 3 beschrieben, erlaubt EN 1993-1-8 (2005) mit dem richtungsbezogenen Verfahren eine gemeinsame Betrachtung der Spannungskomponenten $\sigma_{\perp}, \tau_{\perp}$ und $\tau_{\mathrm{II}}$.

$$
\sigma_{\mathrm{EC}}=\sqrt{\sigma_{\perp}^{2}+3 \cdot \tau_{\perp}^{2}+3 \cdot \tau_{\mathrm{II}}^{2}} \leq \frac{\mathrm{f}_{\mathrm{u}}}{\beta_{\mathrm{w}} \cdot \gamma_{\mathrm{M} 2}}
$$

Damit könnten Versuchsergebnisse für Flankenkehlnähte und Stirnkehlnähte gemeinsam ausgewertet werden, um einen gemeinsamen Beiwert $\beta_{\mathrm{w}}$ zu bestimmen. Darauf wird in den folgenden Kapiteln verzichtet, um die Unterschiede zwischen der Tragfähigkeit von Flanken- und Stirnkehlnähten herauszuarbeiten.

Im Gegensatz zu den Flankenkehlnähten ist der Bemessungswiderstand von Stirnkehlnähten um $\sqrt{2} / \sqrt{3}$, somit um 22 \% höher. Nach EN 1993-1-8 (2005) ergeben sich theoretisch dieselben Beiwerte für Flanken- und Stirnkehlnähte, da der Richtungseinfluss in der Spannungsaufteilung enthalten ist. 


\subsubsection{Vorgehen bei der statistischen Auswertung}

\section{Einleitung}

Für die statistische Auswertung wird auf das bestehende Bemessungsmodell nach Eurocode 3 (EN 1993-1-8 (2005)) zurückgegriffen, das in Kapitel 3, Gleichung (3.2) beschrieben ist. Die Versuche mit Flankenkehlnähten und Stirnkehlnähten werden getrennt ausgewertet. Einzelheiten der jeweils verwendeten Bemessungsmodelle sind in Kapitel 4.5.2 für Flankenkehlnahtverbindungen und in Kapitel 4.5.3 für Kreuzstöße beschrieben. Als Eingangswerte für die statistische Auswertung sind neben den Werkstoffeigenschaften als Versuchsergebnisse die maximale Kraft im Versuch und die Nahtfläche erforderlich. Das Bemessungsmodell berücksichtigt als Basisvariable die Nahtfläche und die Zugfestigkeit.

\section{Basisvariable Nahtfläche}

Die Nahtfläche ergibt sich entweder aus dem Produkt aus Nahtdicke und Nahtlänge oder entspricht der Bruchfläche. Die Bruchfläche $A_{\text {Bruch }}$ ist die nach dem Versuch ausgemessene Bruchfläche (enthält ggf. plastische Verformungen). Für die Nahtdicke a gibt es verschiedene Definitionen, die in Kapitel 3.3.2, Abbildung 3.1 erläutert sind. Für die statistische Auswertung werden die Bruchflächen oder die im Makroschliff bestimmten Nahtdicken $\mathrm{a}_{\mathrm{EC}}$, also die Höhe des einschreibbaren Dreiecks bezogen auf den tatsächlichen Wurzelpunkt, verwendet. Für die Nahtfläche der Schweißnaht wird ein Variationskoeffizient von $\mathrm{V}_{\mathrm{Aw}}=0,10$ angenommen, wie auch in [Background D.03, 1990].

\section{Basisvariable Zugfestigkeit}

Die Festigkeitswerte des Grundwerkstoffs und des Schweißzusatzwerkstoffs basieren auf Standardzugversuchen gemäß DIN EN 10002-1 (2001), DIN EN 876 (1995) und DIN EN 14532-1 (2005).

Das gewählte Bemessungsmodell berücksichtigt die Zugfestigkeit des Grundwerkstoffs, obwohl das Versagen in der Schweißnaht auftritt und durch die Festigkeit des Schweißgutes geprägt wird. Die Auswertung der einzelnen Versuchsreihen wurde deshalb sowohl mit der Festigkeit des Grundwerkstoffs als auch der Festigkeit des Schweißzusatzwerkstoffs durchgeführt. Die berechneten Werte sind jeweils angegeben. Der Variationskoeffizient für die Zugfestigkeit des Grundwerkstoffs und des Schweißgutes wird mit $\mathrm{V}_{\mathrm{fu}, \mathrm{GW}}=\mathrm{V}_{\mathrm{fu}, \mathrm{SzW}}=0,07$ angenommen, wie auch in [Background D.03, 1990].

\section{Vorgehen und Darstellung der statistischen Auswertung}

Für Flankenkehlnähte wird das Bemessungsmodell nach Gleichung (4.12) verwendet, für Stirnkehlnähte gilt Gleichung (4.13).

Mit dem jeweiligen Bemessungsmodell wird die theoretische Widerstandskraft $r_{t}$ berechnet, in der die Versuchsgrößen der Nahtfläche A und der Zugfestigkeit $f_{u}$ und der Beiwert $\beta_{w}$ enthalten sind. Diese theoretische Widerstandskraft $r_{t}$ wird der maximalen Kraft $r_{e}$ im Versuch gegenüber gestellt. Der statistischen Auswertung liegt ein Sicherheitsindex von $\beta=3,8$ für den Grenzzustand der Tragfähigkeit mit einem Bezugszeitraum von 50 Jahren zu Grunde.

Ergebnis der statistischen Auswertung ist jeweils die Festlegung des Beiwerts $\beta_{w}$, der so gewählt wird, dass sich der Sicherheitsbeiwert $\gamma_{\mathrm{M} 2 *}$ gegen Bruch entsprechend Eurocode 3 und DIN EN 1990 (2002) zu $\gamma_{\mathrm{M}^{*}}=1,25$ ergibt. Auch zur Festlegung der Beiwerte $\beta_{\mathrm{w}}$ für die ursprünglichen Eurocodes wurde dieses Verfahren verwendet, [Snijder et al., 1988], [Background D.03, 1990].

\subsection{Zusammenfassung}

In diesem Kapitel wurde das Tragverhalten von Kehlnähten beschrieben. Dazu wurden die Möglichkeiten zur Bestimmung der Festigkeiten von Kehlnähten vorgestellt. Für Flanken- und Stirnkehlnähte wurden die rechnerische Schweißnahtspannung und die Ermittlung der Spannungen in den Versuchen hergeleitet. Für die Bestimmung der Spannungen an Kreuzstoßversuchen wurde das angewandte vereinfachte Verfahren erläutert. Abschließend wurde das Vorgehen bei der statistischen Versuchsauswertung vorgestellt. 


\section{Auswertung bekannter Versuchsreihen der Literatur}

\section{1 Übersicht}

Um das Ziel dieser Arbeit, die Tragfähigkeit von Kehlnahtverbindungen der höherfesten Baustähle zu bestimmen, wird neben eigenen Versuchen auf bereits bestehende Versuchsergebnisse zurückgegriffen. Im Rahmen dieses Kapitels werden bisher unabhängige Versuchsreihen aus der Literatur zusammengestellt, systematisch ausgewertet und so die Tragfähigkeit bestimmt. Die Versuche werden getrennt statistisch ausgewertet und anschließend verglichen und beurteilt.

Die in diesem Kapitel vorgestellten Versuche behandeln vorwiegend Werkstoffkombinationen mit Schweißzusatzwerkstoffen, deren Festigkeiten von der des Grundwerkstoffs abweichen, und somit Über- oder Unterfestigkeiten verursachen. Sie unterscheiden sich von den Werkstoffkombinationen der eigenen Untersuchungen und ergänzen diese damit. Insgesamt sind nur sehr wenige Versuchsdaten von Schweißverbindungen höherfester Baustähle bekannt. Die Ergebnisse der statistischen Auswertung werden neben den Ergebnissen der eigenen Untersuchungen für die Bestimmung der Tragfähigkeit verwendet.

Zur Bestimmung der Tragfähigkeit wurden in den verschiedenen Versuchsreihen aus der Literatur unterschiedliche Versuchskörperformen verwendet. An den längsschubbeanspruchten Kehlnähten ebenso wie an den senkrecht zur Nahtlängsrichtung beanspruchten Kehlnähten wurden Traglastversuche an jeweils zwei Typen von Versuchskörpern durchgeführt, wie in Abbildung 5.1 dargestellt. Bei den längsbeanspruchten Kehlnähten wurden zum einen Überlappverbindungen (d) und (e) und zum anderen Stecklaschenverbindungen (c) untersucht. Als senkrecht zur Nahtlängsrichtung beanspruchte Kehlnähte wurden Kreuzstöße (a) betrachtet. Eine weitere Form von Versuchskörpern für senkrecht beanspruchte Nähte waren Überlappverbindungen mit Stirnkehlnähten (b).

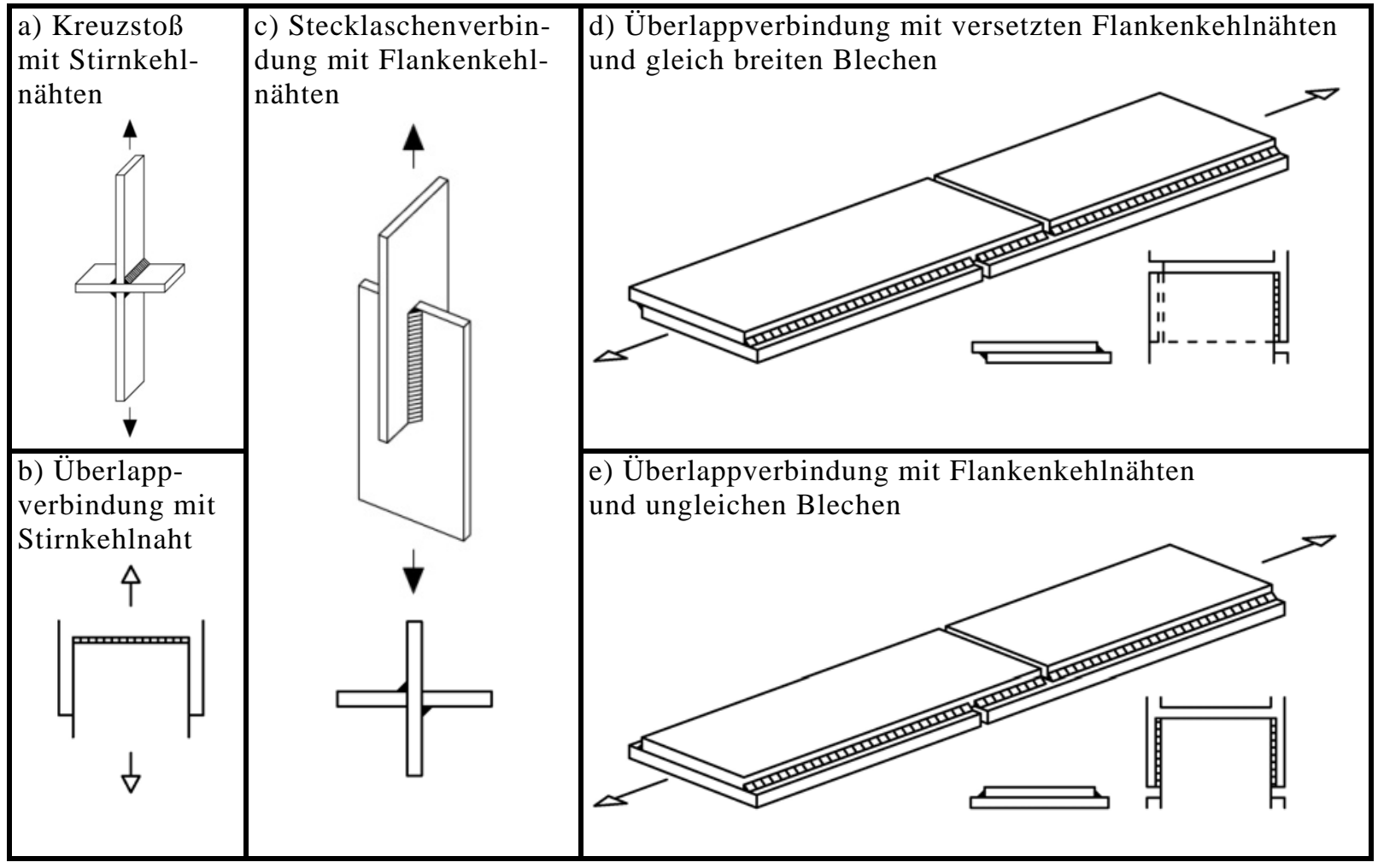

Abbildung 5.1: Darstellung der untersuchten Kehlnahtverbindungen

Um die Versuche aus der Literatur beurteilen zu können, wurden die Versuchsergebnisse statistisch neu ausgewertet, wie in Kapitel 4.5 vorgestellt. Für die statistische Auswertung in diesem Kapitel wird auf das bestehende Bemessungsmodell nach Eurocode 3 (EN 1993-1-8 (2005)) zurückgegriffen. Durch das einheitliche Vorgehen bei der Neuauswertung der Versuche aus der Literatur kann eine 
Vergleichbarkeit zwischen den einzelnen Versuchsreihen erzielt werden. Es können sich auch Abweichungen zu den bereits durchgeführten Auswertungen aus der Literatur ergeben.

Die Ergebnisse der statistischen Auswertung sind für jede Versuchsserie in einem Diagramm dargestellt, in dem die theoretischen Tragfähigkeiten $r_{t}$ als Wertepaare mit den Versuchskräften $r_{e}$ dargestellt sind. Angegeben werden jeweils die Mittelwertabweichung $b$, der Schätzwert $\mathrm{s}_{\Delta}$, der Fraktilenfaktor für den charakteristischen Wert $k_{n}$, der Fraktilenfaktor für den Bemessungswert $k_{n, d}$, die Widerstandsfunktionen $r_{k}$ und $r_{d}$, der Teilsicherheitsbeiwert $\gamma_{\mathrm{M} 2}$ und der korrigierte Sicherheitsbeiwert $\gamma_{\mathrm{M} 2 *}$.

Um eine übersichtliche Darstellung zu ermöglichen, werden die Versuchsdaten und die statistische Auswertung der einzelnen Versuchsreihen im Anhang aufgeführt. Innerhalb jedes Kapitels erfolgt die Beurteilung.

\subsection{Versuche an normalfesten Stählen}

In diesem Abschnitt werden die Versuche zur Bestimmung der Tragfähigkeit normalfester Baustähle S235 und S355 kurz erläutert, aber nicht statistisch ausgewertet werden. Diese Untersuchungen werden vorgestellt, um einen Überblick zu geben.

Im Zuge der europäischen Harmonisierung und der Erstellung der Eurocodes wurden Ende der 80er Jahre des 20. Jahrhunderts in [Snijder et al., 1988] die bekannten Versuche von Kehlnahtverbindungen normalfester Stähle S235 und S355 für das Vereinfachte Verfahren und das Richtungsbezogene Verfahren ausgewertet. Als Ergebnis dieser Auswertung wurden die Beiwerte $\beta_{w}$ für die Bestimmung der Tragfähigkeit von Kehlnahtverbindungen nach Eurocode festgelegt, die in Tabelle 5.1 zusammengestellt sind. In der dritten Spalte sind die statistisch ermittelten Beiwerte angegeben, während in der vierten Spalte der auf der sicheren Seite liegende Normvorschlag gezeigt wird. Der Bemessungsvorschlag ist in ENV 1993-1-1 und als Nachfolger heute in EN 1993-1-8 (2005) enthalten.

Tabelle 5.1: Statistische Auswertung und Empfehlung für den Beiwert $\beta_{w}$, [Snijder et al., 1988]

\begin{tabular}{|l|l|l|l|}
\hline Stahlgüte & Zugfestigkeit $\mathrm{f}_{\mathrm{u}}\left[\mathrm{N} / \mathrm{mm}^{2}\right]$ & $\begin{array}{l}\text { Statistisch ermittelte } \\
\text { Beiwerte } \beta_{\mathrm{w}}\end{array}$ & Normvorschlag $\beta_{\mathrm{w}}$ \\
\hline FeE235 (heute S235) & 360 & 0,74 & 0,8 \\
\hline FeE355 (heute S355) & 510 & 0,77 & 0,9 \\
\hline
\end{tabular}

In [Bornscheuer, Feder, 1966] und [Klöppel, Petri, 1966] werden Versuche an Schweißverbindungen mit dem Grundwerkstoff St37 (S235) vorgestellt. Die Ergebnisse von [Bornscheuer, Feder, 1966] zur Nahtlänge und Nahtdicke zeigen eine abnehmende Tragfähigkeit mit zunehmender Nahtdicke, aber keine Abnahme der Tragfähigkeit infolge der Nahtlänge. Im Gegensatz hierzu wurde in [Klöppel, Petri, 1966] der Einfluss der Nahtlänge auf die Tragfähigkeit an Flankenkehlnähten untersucht. Es wurde eine abnehmende Tragfähigkeit mit zunehmender Nahtlänge festgestellt.

In [Klöppel, Petri, 1969] werden zusätzliche Untersuchungen zu Schweißverbindungen mit dem Stahl S235 und S355 vorgestellt. Diese Versuche wurden anlässlich der Überarbeitung der Stahlbaunorm DIN 4100 (1968) durchgeführt. Die überarbeitete Norm erlaubte höhere Beanspruchbarkeiten, die mit den Versuchen bestätigt werden konnten.

In den 60er und 70er Jahren des 20. Jahrhunderts wurden einige Untersuchungen zum Tragverhalten von Kehlnahtverbindungen von normalfesten Baustählen veröffentlicht. In [Werner, Bornscheuer, 1978] werden jeweils 9 Versuchsergebnisse an Stumpfnahtproben, Kreuzstößen und Flankenkehlnahtverbindungen diskutiert, bei denen die Festigkeit des Stahls (StE36, 51, 70) und des Schweißzusatzwerkstoffs $\left(R_{m}=520\right.$ bis $\left.880 \mathrm{~N} / \mathrm{mm}^{2}\right)$ variiert wurde. An diesen vereinzelten Versuchsergebnissen wurde vorgeschlagen, die Bemessungsansätze zu modifizieren, um die Festigkeit des Schweißzusatzwerkstoffes und die Beanspruchungsrichtung zu berücksichtigen.

Neuere Untersuchungen an normalfesten Stählen zum Einfluss der Lastrichtung wurden an der Universität Alberta durchgeführt: [Callele et al., 2005], [Deng et al., 2003], [Gilbert et al., 2002], [Ng et al., 2005]. 


\subsection{Kehlnahtverbindungen höherfester Stähle S690 mit niederfestem Schweißzusatzwerkstoff}

\subsubsection{Allgemeines}

Am Otto-Graf-Institut, MPA, Universität Stuttgart wurde mit Hilfe der Deutschen Forschungsgemeinschaft ein Forschungsvorhaben durchgeführt, das die Tragfähigkeit von Stirn- und Flankenkehlnahtverbindungen mit den Grundwerkstoffen S355 und S690 untersucht [Kob et al., 1987].

Insgesamt wurden ca. 180 Versuche mit Flanken- und Stirnkehlnähten geprüft. Es wurden zwei Stähle St52-3 (normalisiert) und StE690 (luftvergütet) verwendet, die heute als S355J0+N und S690Q bezeichnet werden. Die Schweißverbindungen beider Stahlgüten wurden mit einem Schweißzusatzwerkstoff SG2, heute G42, hergestellt. Der Schweißzusatzwerkstoff hatte im Vergleich zum Stahl S355J0 zwar eine höhere Festigkeit, dieser Schweißzusatzwerkstoff wird jedoch im Allgemeinen für das Schweißen des Stahls S355J0 verwendet. Damit ergaben sich mit heutigen Bezeichnungen zum einen Werkstoffkombinationen mit zugehörigen Grund- und Schweißzusatzwerkstoffen S355J0-G42 und zum anderen Verbindungen mit einer geringeren Festigkeit des Schweißzusatzwerkstoffs S690Q-G42.

Als Ergebnis dieses Kapitels kann der Einfluss der Richtung zwischen Kraft und Nahtlängsrichtung, also Flankenkehlnaht oder Stirnkehlnaht, für die beiden Werkstoffkombinationen S355-G42 und S690-G42 ermittelt werden. Durch die Verwendung des gleichen Schweißzusatzwerkstoffs G42 kann der Einfluss des Grundwerkstoffs auf die Tragfähigkeit von Stirn- und Flankenkehlnahtverbindungen bestimmt werden. Der Einfluss von Lastrichtung und Grundwerkstoff wird in den folgenden Abschnitten durch eine eigene statistische Auswertung beurteilt.

Die mechanischen Eigenschaften der verwendeten Grund- und Schweißzusatzwerkstoffe und die Versuchsergebnisse sind mit den Ergebnissen der statistischen Auswertung in Anhang A.1.2 aufgeführt.

\subsubsection{Versuche an Flanken- und an Stirnkehlnahtverbindungen}

Am Otto-Graf-Institut wurden 50 Versuche mit Flankenkehlnähten mit dem Grundwerkstoff S355J0+N und zugehörigem Schweißzusatzwerkstoff durchgeführt. Als Parameter wurden zwei Blechdicken mit $\mathrm{t}=10 \mathrm{~mm}$ und $\mathrm{t}=20 \mathrm{~mm}$ und die Nahtdicken zwischen 3 und $10 \mathrm{~mm}$ variiert. Mit dem Grundwerkstoff S690Q standen 39 Versuchsergebnisse mit Flankenkehlnähten zur Verfügung. Die Versuche variierten zwei Blechdicken $(10 \mathrm{~mm}$ und $20 \mathrm{~mm}$ ) und die Nahtdicken zwischen $3 \mathrm{~mm}$ und $12 \mathrm{~mm}$.

In [Kob et al., 1987] werden 38 Versuchsergebnisse von Kreuzstößen mit dem Grundwerkstoff S355J0+N und zugehörigem Schweißzusatzwerkstoff vorgestellt. Die Versuche umfassten die Parameter Blechdicke mit $\mathrm{t}=10,20$ und $30 \mathrm{~mm}$ und Nahtdicke zwischen 3 und $11 \mathrm{~mm}$. Mit dem Grundwerkstoff S690Q und einem Schweißzusatzwerkstoff G42 werden dort 42 Versuchsergebnisse präsentiert, die die Parameter Blechdicke mit $\mathrm{t}=10 \mathrm{~mm}$ und $\mathrm{t}=20 \mathrm{~mm}$ die Nahtdicken zwischen 3 und $11 \mathrm{~mm}$ variierten. Bei den Kreuzstößen wurden zum einen die Bruchflächen $A_{\text {Bruch }}$ bestimmt, zum anderen wurden am Makroschliff die Nahtdicken $\mathrm{A}_{\mathrm{EC}}$ bestimmt, vgl. Kapitel 3.3.2.

Die Nahtlänge war mit l = $60 \mathrm{~mm}$ bei allen Versuchen gleich .

\subsubsection{Diskussion der Ergebnisse}

\section{Allgemeines}

Die durchgeführten Untersuchungen an Flanken- und Stirnkehlnähten ermöglichen eine Beurteilung des Einflusses des Grundwerkstoffs bei gleichem Schweißzusatzwerkstoff auf die Tragfähigkeit der Schweißverbindung. Außerdem ist ein Vergleich der Tragfähigkeiten von Stirn- und Flankenkehlnahtverbindungen möglich. 
Vergleich der Tragfähigkeiten von Flankenkehlnähten bei unterschiedlichen Grundwerkstoffen Im Folgenden werden die Tragfähigkeiten von Flankenkehlnähten bei unterschiedlichen Grundwerkstoffen verglichen. In Tabelle 5.2 sind die Ergebnisse der statistischen Auswertung für die beiden Werkstoffkombinationen S355-G42 und S690-G42 dargestellt. Für beide Werkstoffkombinationen wurde die Auswertung in Abhängigkeit des Schweißzusatzwerkstoffs (SZW) und in Abhängigkeit des Grundwerkstoffs (GW) durchgeführt. Aufgeführt sind neben den Werkstofffestigkeiten der ermittelte Beiwert $\beta_{\mathrm{w}}$ und die daraus zu berechnende Bemessungsgrenzspannung.

Tabelle 5.2: Bemessungsgrenzspannung für Flankenkehlnähte

\begin{tabular}{|c|c|c|c|c|c|}
\hline & & \multicolumn{2}{|c|}{ S355J0-G42 } & \multicolumn{2}{|c|}{ S690Q-G42 } \\
\hline & & GW & SZW & GW & SZW \\
\hline & $\mathrm{f}_{\mathrm{u}}\left[\mathrm{N} / \mathrm{mm}^{2}\right]$ & $\begin{array}{l}550-552 \\
\text { MW } 551\end{array}$ & 590 & $\begin{array}{l}826-854 \\
\text { MW } 846\end{array}$ & 577 \\
\hline & $\beta_{w}[-]$ & 0,796 & 0,853 & 1,136 & 0,762 \\
\hline & $\gamma_{\mathrm{M} 2}[-]$ & \multicolumn{4}{|c|}{1,25} \\
\hline & $\mathrm{f}_{\mathrm{u}}$ & |319-320 & 310 & $336-347$ & 350 \\
\hline SZW: Schweißzusatzwerkstoff & $\sqrt{3} \cdot \gamma_{\mathrm{M} 2} \cdot \beta_{\mathrm{w}}\left[\mathrm{mm}^{2}\right.$ & MW 319 & & MW 344 & \\
\hline
\end{tabular}

Beim gleichem Schweißzusatzwerkstoff G42 wurde bezogen auf den Schweißzusatzwerkstoff für die Flankenkehlnähte und dem Grundwerkstoff S355J0 eine Bemessungsgrenzspannung von $319 \mathrm{~N} / \mathrm{mm}^{2}$ ermittelt, während die Versuche mit dem Grundwerkstoff S690Q eine Bemessungsgrenzspannung von $350 \mathrm{~N} / \mathrm{mm}^{2}$ und somit eine Steigerung der Bemessungsgrenzspannung um $10 \%$ erreichten. Der Mittelwert änderte sich von $437 \mathrm{~N} / \mathrm{mm}^{2}$ auf $478 \mathrm{~N} / \mathrm{mm}^{2}$ um $9 \%$, wie Abbildung 5.2 zeigt. Damit kann ein deutlicher Einfluss des Grundwerkstoffs bei gleichem Schweißzusatzwerkstoff auf die Tragfähigkeit der Schweißverbindung gezeigt werden.

Die Ergebnisse der Flankenkehlnahtverbindungen S355J0-G42 zeigen mit $\beta_{\mathrm{w}}=0,853$ in Abhängigkeit des Schweißzusatzwerkstoffs bzw. $\beta_{w}=0,796$ in Abhängigkeit des Grundwerkstoffs einen besseren Wert als $\beta_{\mathrm{w}}=0,9$ nach den aktuellen Regelungen nach EN 1993-1-8 (2005) und bestätigen diese somit. Bezogen auf den Schweißzusatzwerkstoff bedeutet ein Beiwert $\beta_{w}=0,853$, dass die Tragfähigkeit der Kehlnaht um $1 / \beta_{\mathrm{w}}=1,17$ höher liegt als die Festigkeit des Schweißzusatzwerkstoffs. Die Ergebnisse der Flankenkehlnahtverbindungen S690Q ergaben bezogen auf die Festigkeit des Schweißzusatzwerkstoffs einen Beiwert $\beta_{\mathrm{w}}=0,762$. Die Überfestigkeit ist somit auch bei Verwendung des höherfesten Grundwerkstoffs S690Q vorhanden. Das heißt die Überfestigkeit von $1 / \beta_{\mathrm{w}}=1 / 0,762=1,31$ setzt sich zusammen aus dem Produkt einer Steigerung von 1,17 aufgrund der Kehlnaht und weitere 1,12 infolge des höherfesten Grundwerkstoffs S690Q.

\section{Vergleich der Tragfähigkeiten von Kreuzstößen mit Stirnkehlnähten bei unterschiedlichen Grundwerkstoffen}

Für Kreuzstöße werden die Tragfähigkeiten bei unterschiedlichen Grundwerkstoffen verglichen. In Tabelle 5.3 sind die Ergebnisse der statistischen Auswertung für die beiden Werkstoffkombinationen S355-G42 und S690Q-G42 in Abhängigkeit des Schweißzusatzwerkstoffs und in Abhängigkeit des Grundwerkstoffs zusammengefasst. Es wird nach der Art der Nahtfläche $A_{E C}$ und $A_{B r u c h}$ unterschieden.

Tabelle 5.3: Bemessungsgrenzspannung für Stirnkehlnähte

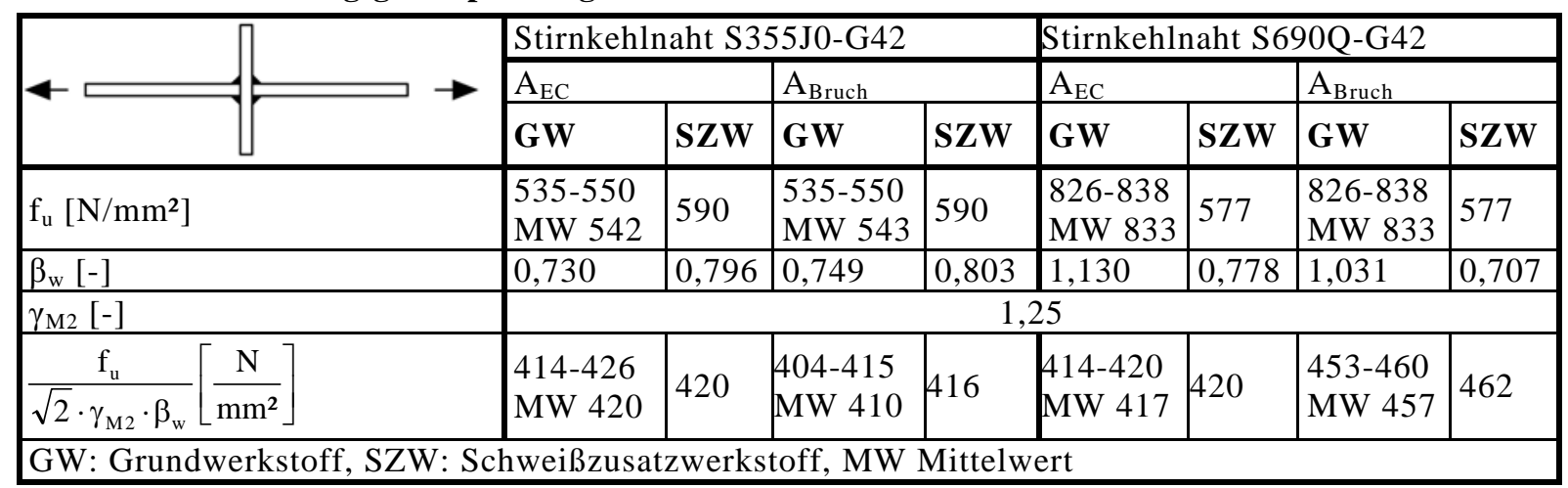


Bei Betrachtung der Ergebnisse der statistischen Auswertung infolge der Nahtfläche $A_{\text {Bruch }}$ weist die Kehlnaht eine Überfestigkeit gegenüber dem Schweißzusatzwerkstoff von $1 / \beta_{\mathrm{w}}=1,26$ auf. Diese Überfestigkeit ist auch bei Verwendung des höherfesten Grundwerkstoffs S690Q vorhanden. Das heißt die Überfestigkeit von $1 / \beta_{\mathrm{w}}=1 / 0,707=1,41$ setzt sich zusammen aus dem Produkt einer Steigerung von 1,26 aufgrund der Kehlnaht und weitere 1,13 infolge des höherfesten Grundwerkstoffs S690Q.

Bei gleichem Schweißzusatzwerkstoff G42 wurde bei Verwendung der Fläche $\left(\mathrm{A}_{\mathrm{EC}}\right)$ sowohl für die Stirnkehlnähte und den Grundwerkstoff S355J0 als auch für die Stirnkehlnähte mit dem Grundwerkstoff S690Q eine Bemessungsgrenzspannung von $420 \mathrm{~N} / \mathrm{mm}^{2}$ ermittelt. Die Mittelwerte steigen um $7 \%$. Damit zeigt sich kein eindeutiger Einfluss des Grundwerkstoffs auf die Tragfähigkeit.

Abbildung 5.2 zeigt die Bemessungsgrenzspannungen der Kreuzstöße unter Berücksichtigung der Nahtfläche $A_{\text {Bruch. }}$ Beim gleichen Schweißzusatzwerkstoff G42 wurde für die Stirnkehlnähte und den Grundwerkstoff S355J0 eine Bemessungsgrenzspannung von $416 \mathrm{~N} / \mathrm{mm}^{2}$ ermittelt, für die Nähte mit dem Grundwerkstoff S690Q ergibt sich eine Bemessungsgrenzspannung von $462 \mathrm{~N} / \mathrm{mm}^{2}$. Bei Betrachtung der Bemessungsgrenzspannungen bezogen auf die Bruchfläche zeigt sich eine Steigerung der Tragfähigkeit um $11 \%$.

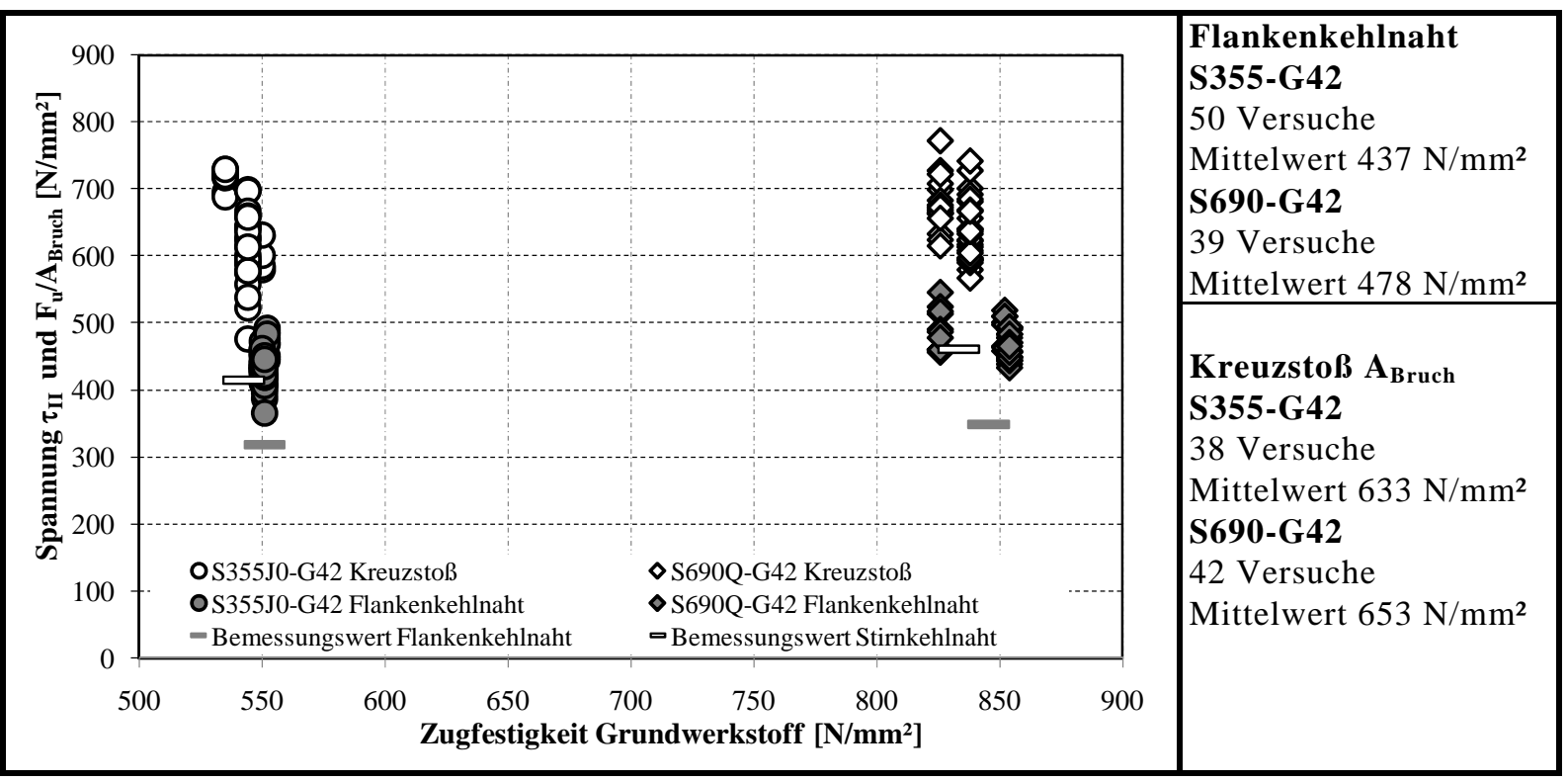

Abbildung 5.2: Vergleich der Ergebnisse von Kreuzstößen $\left(A_{\text {Bruch }}\right)$ und Flankenkehlnahtverbindungen S355J0 und S690Q

Der Vergleich der Bemessungsgrenzspannungen mit der Bruchfläche $A_{B r u c h}$ und $A_{E C}$ in Tabelle 5.3 zeigt für den Stahl S355J0 kaum Unterschiede. Für den Stahl S355J0 liegen die mit $A_{B r u c h}$ ermittelten Spannungen im Mittel höher als mit $A_{E C}$, die Streuung ist aber größer. Bei den Verbindungen mit dem Stahl S690Q zeigt sich ein Unterschied von $10 \%$ in den Bemessungsgrenzspannungen zwischen $A_{B r u c h}$ und $A_{E C}$. Die mit $A_{B r u c h}$ ermittelten Spannungen liegen auch im Mittel höher, die Streuung ist in beiden Fällen ähnlich. Für die Mischverbindung S690Q-G42 deutet der Unterschied in der Bemessungsgrenzspannung zwischen den verschiedenen Nahtdicken darauf hin, dass die Bestimmung der Nahtdicke $A_{E C}$ nicht die Bruchfläche widerspiegelt.

\section{Vergleich der Tragfähigkeiten von Flankenkehlnahtverbindungen und Kreuzstößen}

Abbildung 5.2 zeigt den Vergleich von Flanken- und Stirnkehlnähten $\left(\mathrm{A}_{\text {Bruch }}\right)$. Die Bemessungsgrenzspannungen der Kreuzstöße sind um ca. 30 \% höher als die der Flankenkehlnähte. Die Tragfähigkeit von Kreuzstößen liegt höher als die der Flankenkehlnahtverbindungen, obwohl die Richtungsabhängigkeit bereits berücksichtigt ist.

Im Vergleich dazu zeigt Abbildung 5.3 den Vergleich von Flanken- und Stirnkehlnähten $\left(A_{E C}\right)$. Für die Verbindungen mit dem Grundwerkstoff S355J0 zeigt sich ein Unterschied in den Bemessungs- 
grenzspannungen zwischen Flankenkehlnähten und Kreuzstößen von 32 \%. Für die Verbindungen mit dem Grundwerkstoff S690Q ergibt sich ein Unterschied von $20 \%$.

Für die Mischverbindung S690Q-G42 zeigen sich deutliche Unterschiede in den Bemessungsgrenzspannungen zwischen den verschiedenen Nahtdicken. Da die Nahtdicke $A_{E C}$ am Makroschliff der ungebrochenen Naht bestimmt wird, ist sie unabhängig von der Bruchfläche. Somit können unterschiedliche Bruchwinkel nicht abgebildet werden, obwohl sie die Größe der Bruchfläche beeinflussen.

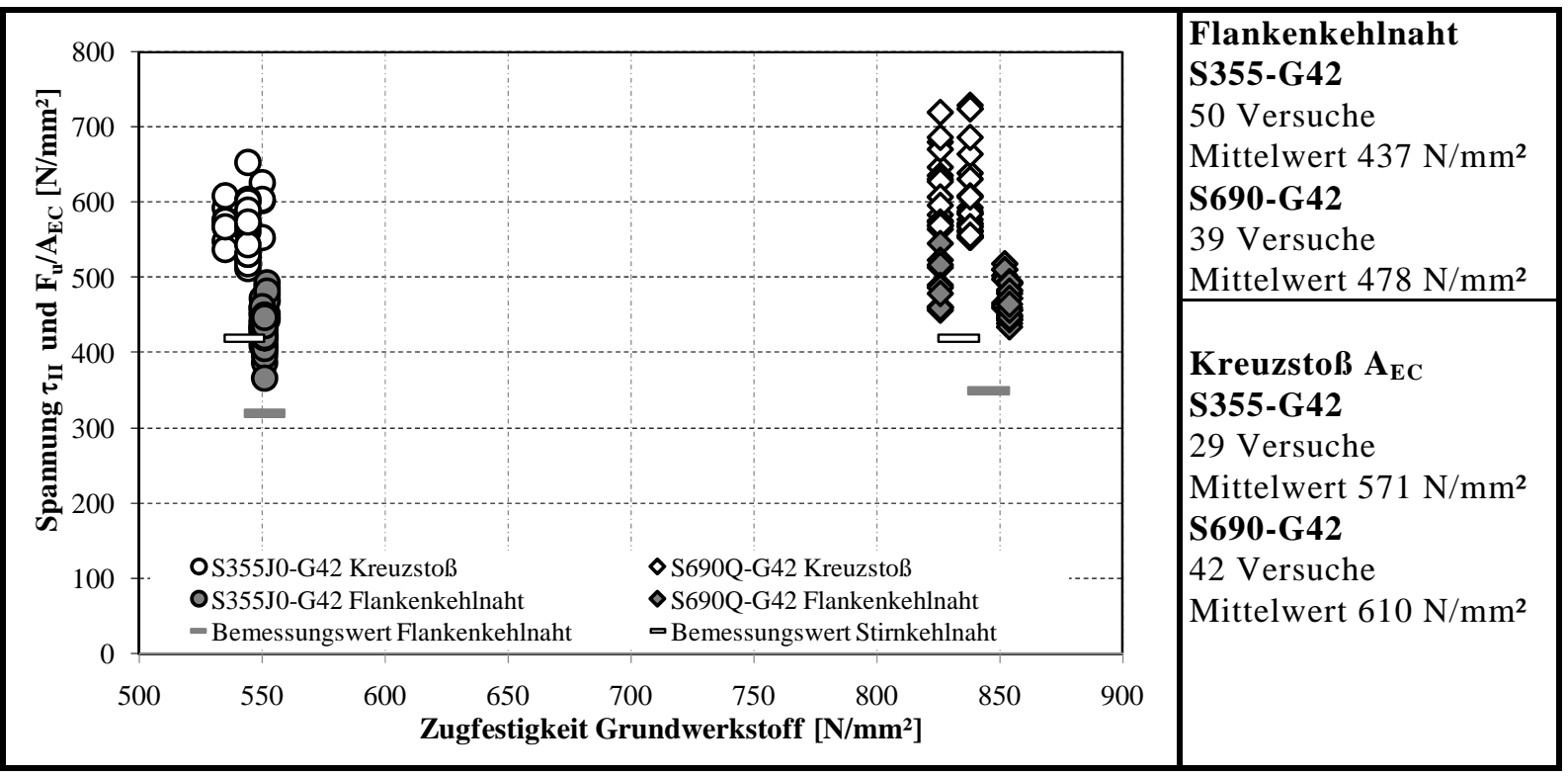

Abbildung 5.3: Vergleich der Ergebnisse von Kreuzstößen $\left(A_{\mathrm{EC}}\right)$ und Flankenkehlnahtverbindungen S355J0 und S690Q

\section{Zusammenfassung}

Insgesamt bestätigen die Ergebnisse der Flankenkehlnahtverbindungen normalfester Baustähle die aktuellen Regelungen. Durch die Variation der Grundwerkstoffe konnte gezeigt werden, dass der Grundwerkstoff einen Einfluss auf die Tragfähigkeit der Verbindung hat. Der Vergleich von Kreuzstößen und Flankenkehlnähten zeigt eine um ca. 30 \% höhere Tragfähigkeit für die Kreuzstöße, wenn die Bruchfläche verwendet wird. Der Vergleich der Bemessungsgrenzspannungen mit der Bruchfläche $\mathrm{A}_{\mathrm{Bruch}}$ und $\mathrm{A}_{\mathrm{EC}}$ darauf hin, dass die Bestimmung der Nahtdicke $\mathrm{A}_{\mathrm{EC}}$ nicht die Bruchfläche widerspiegelt. Da die Nahtdicke $A_{E C}$ am Makroschliff der ungebrochenen Naht bestimmt wird, ist sie unabhängig von der Bruchfläche. Damit wird ein wesentlicher Faktor für die Bestimmung der Tragfähigkeit nicht berücksichtigt.

\subsection{Kehlnahtverbindungen höherfester Stähle HSF 640 mit höherfestem Schweißzusatzwerkstoff}

\subsubsection{Allgemeines}

In [Niemi, 1988] werden Versuche zur Bestimmung der Tragfähigkeit von Schweißverbindungen höherfester Stähle vorgestellt. Untersucht wurden insgesamt 38 Versuche an Versuchskörpern mit Flankenkehlnähten, an Versuchskörpern mit Stirnkehlnähten und an Versuchskörpern mit einer Kombination aus Flanken- und Stirnkehlnähten. Die kombinierten Verbindungen werden in dieser Arbeit nicht weiter betrachtet. Für die Versuchskörper wurde ein Stahl HSF 640 nach ISO 5951 (1980) mit einer Blechdicke von 8 mm verwendet. Dieser lässt sich den europäischen Stählen nicht eindeutig zuordnen. Die Festigkeit ist etwas höher als beim Stahl S620, erreicht aber die Eigenschaften von S690 nicht.

Mit diesem Grundwerkstoff wurden Versuchskörper mit Schweißzusatzwerkstoffen unterschiedlicher Festigkeit gefertigt. Beim Schweißzusatzwerkstoff OK74.78 handelt es sich um eine Elektrode mit der normativen Festigkeitsklasse E55, deren Zugfestigkeit der Zugfestigkeit des Grundwerkstoffs ent- 
spricht, während die Streckgrenze des Schweißzusatzwerkstoffs deutlich höher liegt als die des Grundwerkstoffs. Der Schweißzusatzwerkstoff OK Autrod 13.12 (G CrMo1Si) wird zum Schweißen von warmfesten Stählen verwendet. Die Streckgrenze und die Zugfestigkeit sind mit einem Schweißzusatzwerkstoff der Güte G69 vergleichbar, die Einsatzgebiete sind jedoch unterschiedlich. Mit den beiden verschiedenen Schweißzusatzwerkstoffen ergeben sich zum einen Versuchskörper, deren Schweißzusatzwerkstoff eine Überfestigkeit gegenüber dem Grundwerkstoff hat und zum anderen Versuchskörper mit ähnlicher Festigkeit von Grund- und Schweißzusatzwerkstoff.

Diese Versuchsergebnisse werden vorgestellt, um für die beiden Werkstoffkombinationen HSF640E55 und HSF640-(G CRMo1Si) den Einfluss der Kraftrichtung, also Flankenkehlnaht oder Stirnkehlnaht, zu ermitteln. Die Verwendung des gleichen Grundwerkstoffs bei Variation des Schweißzusatzwerkstoffs ermöglicht die Beurteilung des Einflusses des Schweißzusatzwerkstoffs auf die Tragfähigkeit von Stirn- und Flankenkehlnahtverbindungen. Die Versuchsergebnisse sind mit den Ergebnissen der statistischen Auswertung ausführlich in Anhang A.1.3 aufgeführt.

\subsubsection{Versuche an Flanken- und an Stirnkehlnahtverbindungen}

In [Niemi, 1988] werden 14 Versuchsergebnisse von Flankenkehlnahtverbindungen mit dem Grundwerkstoff HSF640 präsentiert. Davon wurde bei 7 Versuchen ein zugehöriger Schweißzusatzwerkstoff (OK 74.78) verwendet, und 7 Versuche wurden mit einem Schweißzusatzwerkstoff höherer Festigkeit (OK Autrod 13.12) verschweißt. Die Blechdicke betrug $8 \mathrm{~mm}$ und die Nahtdicke $3 \mathrm{~mm}$. Die Nahtlänge variierte zwischen $50 \mathrm{~mm}$ und $400 \mathrm{~mm}$.

Zusätzlich werden 12 Versuche mit Stirnkehlnähten und dem Grundwerkstoff HSF640 vorgestellt. Bei 6 Versuchen wurde ein zugehöriger Schweißzusatzwerkstoff (OK 74.78) eingesetzt, während 6 weitere Versuche mit einem Schweißzusatzwerkstoff höherer Festigkeit (OK Autorid 13.12) gefertigt wurden. Die Blechdicke betrug $8 \mathrm{~mm}$ und die Nahtdicke $3 \mathrm{~mm}$. Die Nahtlängen variierten zwischen $50 \mathrm{~mm}$ und $200 \mathrm{~mm}$.

\subsubsection{Diskussion der Ergebnisse}

\section{Vergleich der Tragfähigkeiten von Flankenkehlnähten bei unterschiedlichen Schweißzusatz- werkstoffen}

Bei den Flankenkehlnahtverbindungen zeigt der Vergleich der Tragfähigkeiten bei unterschiedlichen Schweißzusatzwerkstoffen infolge des Schweißzusatzwerkstoffs mit höherer Festigkeit (+15\%) eine Erhöhung der Mittelwert der Tragfähigkeit um $11 \%$ von 494 N/mm² auf 550 N/mm², vgl. Abbildung 5.4. Die statistische Auswertung in Tabelle 5.4 zeigt jedoch, dass infolge der größeren Streuung ein größerer Sicherheitsabstand erforderlich ist, so dass sich die Bemessungsgrenzspannung von 384 auf $322 \mathrm{~N} / \mathrm{mm}^{2}$ verringert, siehe auch Abbildung 5.4.

Tabelle 5.4: Bemessungsgrenzspannung für Flankenkehlnähte

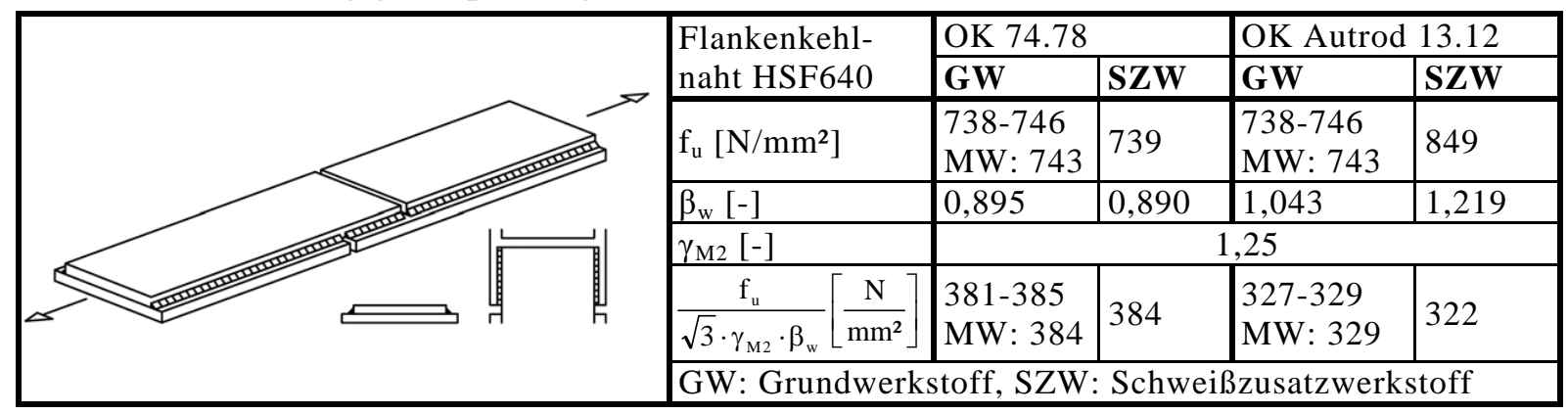

Vergleich der Tragfähigkeiten von Stirnkehlnahtverbindungen bei unterschiedlichen Schweißzusatzwerkstoffen

Die Ergebnisse der Stirnkehlnahtverbindungen mit unterschiedlichen Schweißzusatzwerkstoffen sind als Ergebnisse der statistischen Auswertung in Tabelle 5.5 aufgeführt. Infolge der höheren Festigkeit 
des Schweißzusatzwerkstoffs wurde für die Stirnkehlnähte die Bemessungsgrenzspannung von $399 \mathrm{~N} / \mathrm{mm}^{2}$ um $11 \%$ auf $445 \mathrm{~N} / \mathrm{mm}^{2}$ gesteigert. Die Zugfestigkeit des Schweißguts liegt um $15 \%$ höher. Für die Stirnkehlnähte ist ein Vergleich der Tragfähigkeiten in Abbildung 5.4 dargestellt.

Tabelle 5.5: Bemessungsgrenzspannung für Stirnkehlnähte

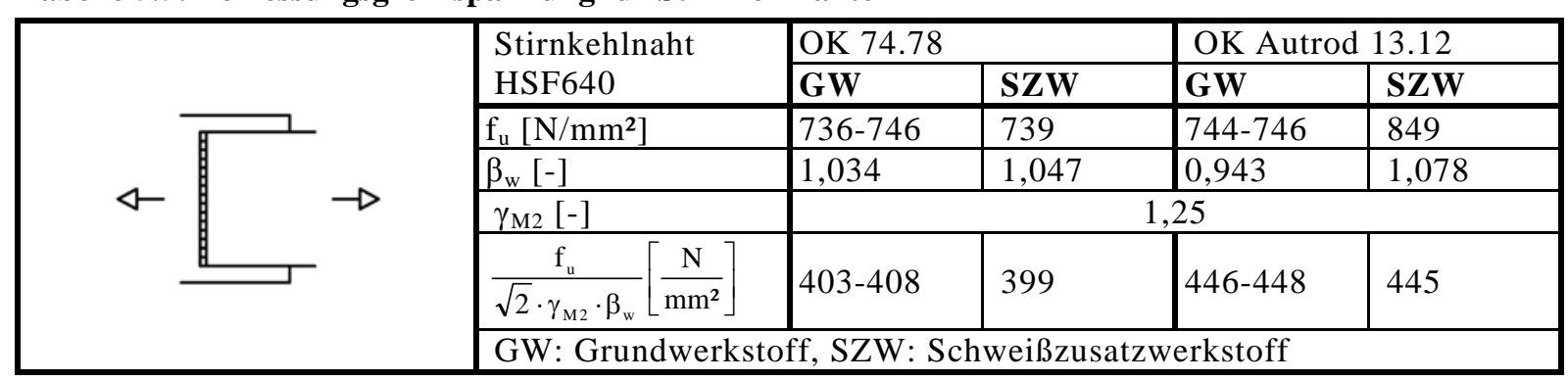

\section{Vergleich der Tragfähigkeiten von Flankenkehlnaht- und Stirnkehlnahtverbindungen}

Die zuvor besprochenen Untersuchungen an Flanken- und Stirnkehlnähten werden in Abbildung 5.4 verglichen. Die Bemessungsgrenzspannungen der Stirnkehlnähte sind um ca. $4 \%$ und 38 \% höher als die der Flankenkehlnahtverbindungen.

Die Variation des Schweißzusatzwerkstoffs zeigt den Einfluss, den der Schweißzusatzwerkstoff auf die Tragfähigkeit der Verbindung hat. Der Vergleich von Stirn- und Flankenkehlnähten zeigt eine höhere Tragfähigkeit für die Kreuzstöße.

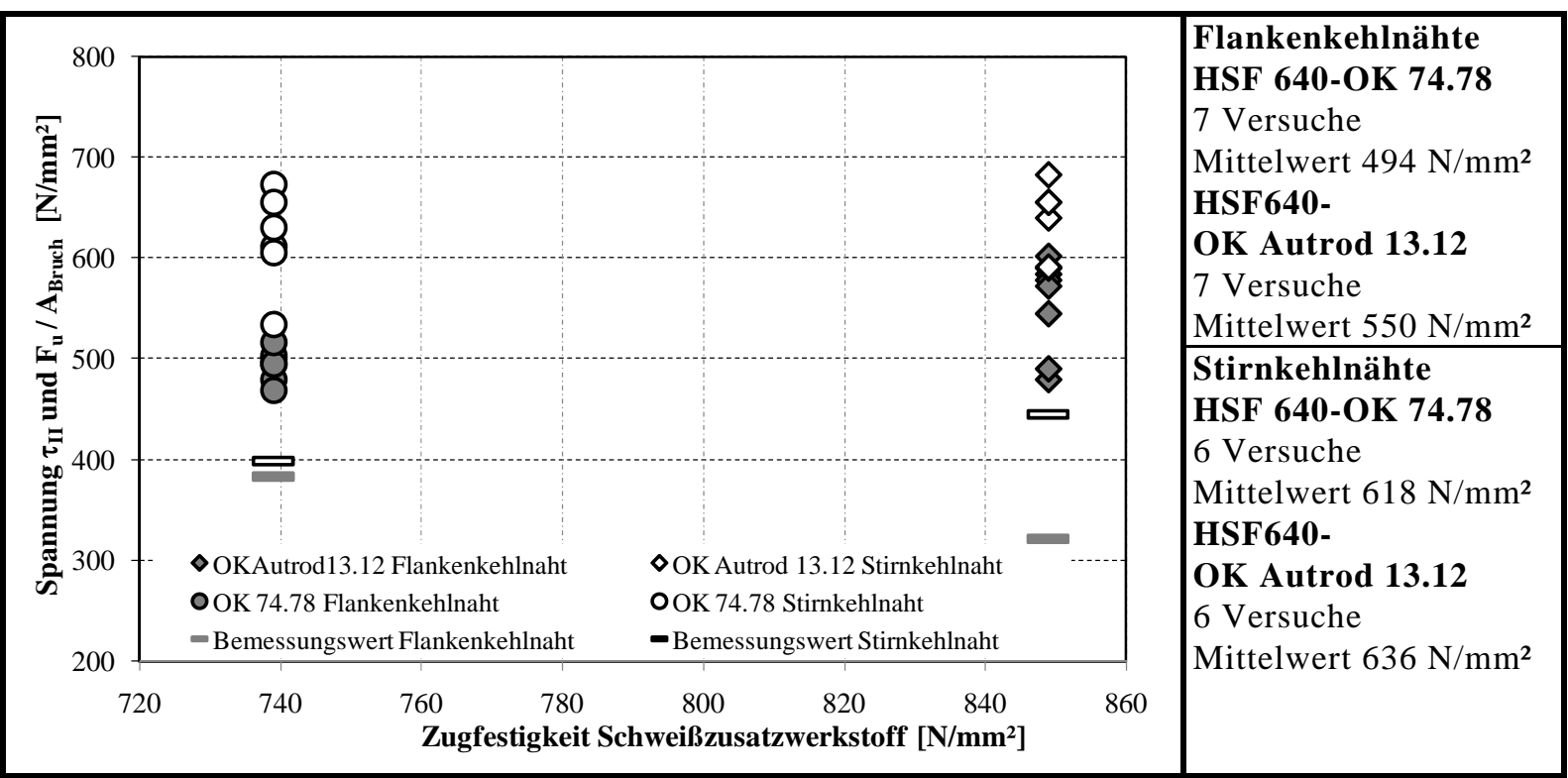

Abbildung 5.4: Vergleich der Ergebnisse von Kreuzstößen und Flankenkehlnahtverbindungen

\subsection{Kehlnahtverbindungen S460 mit zugehörigem Schweißzusatzwerk- stoff}

\subsubsection{Allgemeines}

In [Saal, 2005] werden Tragfähigkeitsversuche mit dem Stahl S460 vorgestellt. Untersucht wurden Versuche an Flankenkehlnähten mit überlappenden Blechen und ineinander gesteckten Blechen, Versuche mit Stirnkehlnähten an überlappenden Blechen und Versuche mit Kreuzstößen. Diese Untersuchungen waren die Grundlage für die Zulassung [Zulassung Z-30.2-5]. Es werden 34 Versuche an Flankenkehlnahtverbindungen betrachtet. Alle betrachteten Versuche wurden mit demselben zugehörigen Schweißzusatzwerkstoff T46 geschweißt. Diese Versuche werden vorgestellt und statistisch ausgewertet, da sie vergleichbar mit den eigenen Untersuchungen an Schweißverbindungen mit dem Grundwerkstoff S460 und zugehörigem Schweißzusatzwerkstoff sind. 
Die Versuchsergebnisse sind mit den Ergebnissen der statistischen Auswertung ausführlich in Anhang A.1.4 aufgeführt.

Weitere Versuche an Stirnkehlnähten mit überlappenden Blechen sowie an Kreuzstößen mit Stirnkehlnähten wurden in [Saal, 2005] vorgestellt. Diese Prüfkörper werden nicht weiter betrachtet.

\subsubsection{Versuche an Flankenkehlnahtverbindungen}

Zur Untersuchung von Flankenkehlnähten wurden in [Saal, 2005] zwei Arten von Versuchskörpern verwendet: Flankenkehlnahtverbindungen mit überlappenden Blechen und mit ineinander gesteckten Blechen, die in Tabelle 5.6 dargestellt sind. Die Blechdicken variierten von 20 bis 77 mm, die Nahtdicken von 5 bis $10 \mathrm{~mm}$ und die Nahtlängen von 50 bis $700 \mathrm{~mm}$.

\subsubsection{Diskussion der Ergebnisse}

In Tabelle 5.6 sind die Bemessungsgrenzspannungen als Ergebnis der in dieser Arbeit durchgeführten standardisierten statistischen Auswertung an Flankenkehlnahtverbindungen dargestellt. Das Ergebnis der statistischen Auswertung zeigt, dass der Beiwert $\beta_{\mathrm{w}}$ mit $\beta_{\mathrm{w}}=0,788$ in Abhängigkeit des Grundwerkstoffs und $\beta_{\mathrm{w}}=0,775$ in Abhängigkeit des Schweißzusatzwerkstoffs deutlich günstiger ist als der nach EN 1993-1-8 (2005) gültige Wert von $\beta_{\mathrm{w}}=1,0$.

Für die Flankenkehlnähte ergeben sich in Abhängigkeit des Grundwerkstoffs und des Schweißzusatzwerkstoffs die gleichen Tragfähigkeiten von $370 \mathrm{~N} / \mathrm{mm}^{2}$.

Tabelle 5.6: Bemessungsgrenzspannung für Flankenkehlnahtverbindungen

\begin{tabular}{|c|c|c|c|}
\hline \multicolumn{2}{|l|}{ Überlappende Bleche } & \multicolumn{2}{|l|}{ Flankenkehlnaht } \\
\hline & & GW & SZW \\
\hline & $\mathrm{f}_{\mathrm{u}}\left[\mathrm{N} / \mathrm{mm}^{2}\right]$ & 614-671 (MW: 631) & 618 \\
\hline & $\beta_{\mathrm{w}}[-]$ & & 0,775 \\
\hline & $\gamma_{\mathrm{M} 2}[-]$ & \multicolumn{2}{|c|}{1,25} \\
\hline \multicolumn{2}{|r|}{$\frac{\mathrm{f}_{\mathrm{u}}}{\sqrt{3} \cdot \gamma_{\mathrm{M} 2} \cdot \beta_{\mathrm{w}}}\left[\frac{\mathrm{N}}{\mathrm{mm}^{2}}\right]$} & 360-394 (MW 370) & 369 \\
\hline & \multicolumn{3}{|c|}{ GW: Grundwerkstoff, SZW: Schweißzusatzwerkstof } \\
\hline
\end{tabular}

\subsection{Kehlnahtverbindungen höherfester Stähle S460 mit höherfestem Schweißzusatzwerkstoff}

\subsubsection{Allgemeines}

Zur Erstellung der Eurocodes wurde 1990 ein Hintergrundbericht [Background D.03, 1990] veröffentlicht, in dem die in der Literatur vorhandenen Versuche zur Bestimmung der Tragfähigkeit von Schweißverbindungen höherfester Stähle S460 zusammengefasst und ausgewertet wurden. Untersucht wurden insgesamt ca. 65 Versuche an Versuchskörpern mit Flankenkehlnähten und/oder Stirnkehlnähten mit überlappenden Blechen sowie 16 Versuche an Kreuzstößen mit Stirnkehlnähten. Von diesen Flankenkehlnahtversuchen sind 16 Versuchsergebnisse von Versuchskörpern, die nur mit Flankenkehlnähten verbunden waren.

Der Schweißzusatzwerkstoff Conarc G60 entspricht normativ dem Schweißzusatzwerkstoff E55. Obwohl nominell die Festigkeit des verwendeten Schweißzusatzwerkstoff höher liegt als die des Grundwerkstoffs, liegt die tatsächliche Festigkeit des Schweißzusatzwerkstoff mit $\mathrm{f}_{\mathrm{u}}=620 \mathrm{~N} / \mathrm{mm}^{2}$ in einigen Fällen unterhalb der der Grundwerkstoffe mit $\mathrm{f}_{\mathrm{u}}=613-725 \mathrm{~N} / \mathrm{mm}^{2}$. 
Außerdem erfüllt diese Zugfestigkeit knapp (-3\%) nicht die normativen Anforderungen für die Güte E55 mit $\mathrm{f}_{\mathrm{u}}=640 \mathrm{~N} / \mathrm{mm}^{2}$. Die Versuchsergebnisse sind mit der statistischen Auswertung in Anhang A.1.5 aufgeführt.

\subsubsection{Versuche an Flanken- und an Stirnkehlnahtverbindungen}

In [Background D.03, 1990] werden 13 Versuchsergebnisse an Flankenkehlnähten mit dem Grundwerkstoff S460 und dem Schweißzusatzwerkstoff E55 beschrieben. Weitere Angaben über die geometrischen Parameter fehlen. Es handelt sich dabei um zwei verschiedene Überlappverbindungen, die in Tabelle 5.7 gemeinsam mit den Ergebnissen der statistischen Auswertung dargestellt sind.

An Kreuzstößen mit Stirnkehlnähten werden in [Background D.03, 1990] 16 Versuchsergebnisse an Kreuzstößen mit Stirnkehlnähten der Werkstoffkombination S460-E55 beschrieben. Weitere Angaben über die geometrischen Parameter fehlen.

\subsubsection{Diskussion der Ergebnisse}

Die statistische Auswertung zeigt für die Auswertung in Abhängigkeit vom Schweißzusatzwerkstoff eine deutlich höhere Bemessungsgrenzspannung als für die Auswertung in Abhängigkeit vom Grundwerkstoff, weil die Mittelwertabweichung b (in Abhängigkeit des Grundwerkstoffs $b=1,326$ und in Abhängigkeit des Schweißzusatzwerkstoffs $b=1,161$ ) deutlich geringer ist. Die Zugfestigkeiten der eingesetzten Grundwerkstoffe variieren stark, während beim Schweißzusatzwerkstoff nur eine Festigkeit berücksichtigt wird. Das deutet darauf hin, dass die Tragfähigkeit eher durch die Festigkeit des Schweißzusatzwerkstoffs als durch die Festigkeit des Grundwerkstoffs geprägt wird.

Für die Kreuzstöße mit Stirnkehlnähten ergibt die statistische Auswertung in Abhängigkeit des Grundwerkstoffs eine um 4 \% höhere Tragfähigkeit als die Auswertung in Abhängigkeit des Schweißzusatzwerkstoffs, vgl. Tabelle 5.8. Die Ergebnisse sind somit vergleichbar.

Tabelle 5.7: Bemessungsgrenzspannung für Flankenkehlnahtverbindungen

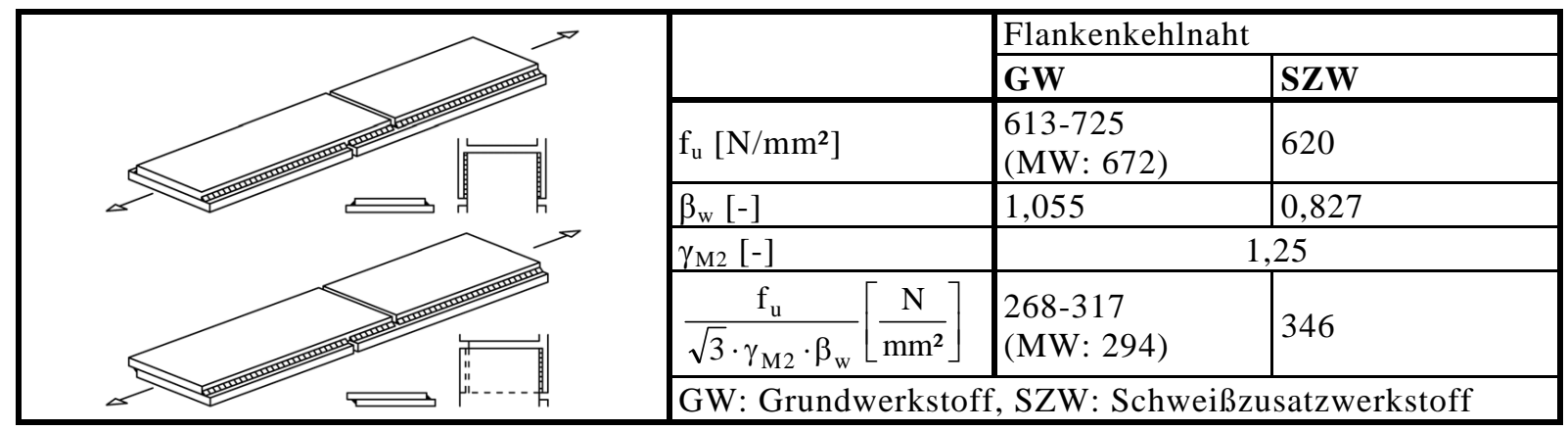

Tabelle 5.8: Bemessungsgrenzspannung für Stirnkehlnahtverbindungen

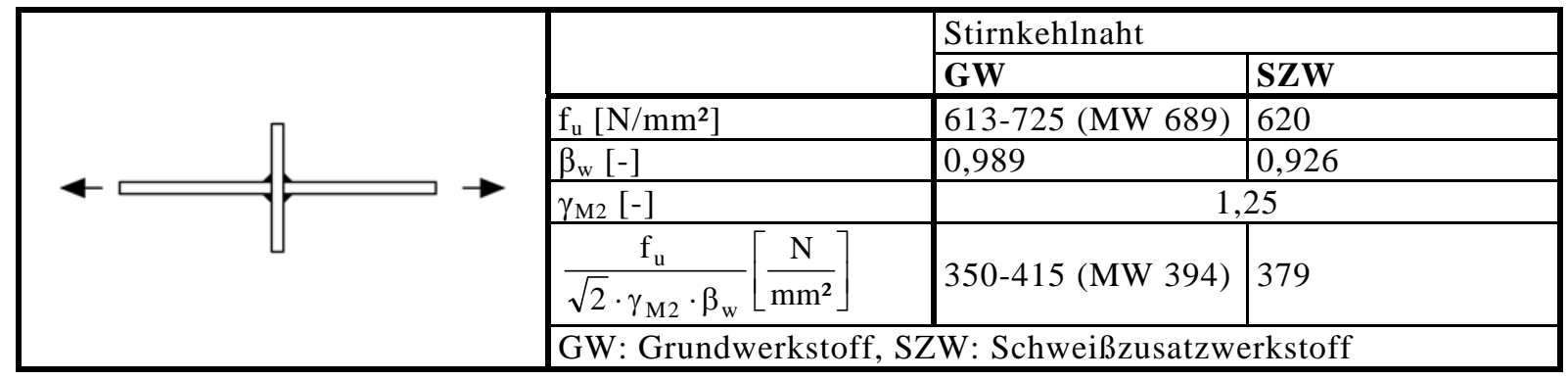


Abbildung 5.5 zeigt den Vergleich der Versuchsergebnisse zwischen Flanken- und Stirnkehlnähten. Die Bemessungsgrenzspannungen der Kreuzstöße sind um ca. 12 \% höher als die der Flankenkehlnahtverbindungen.

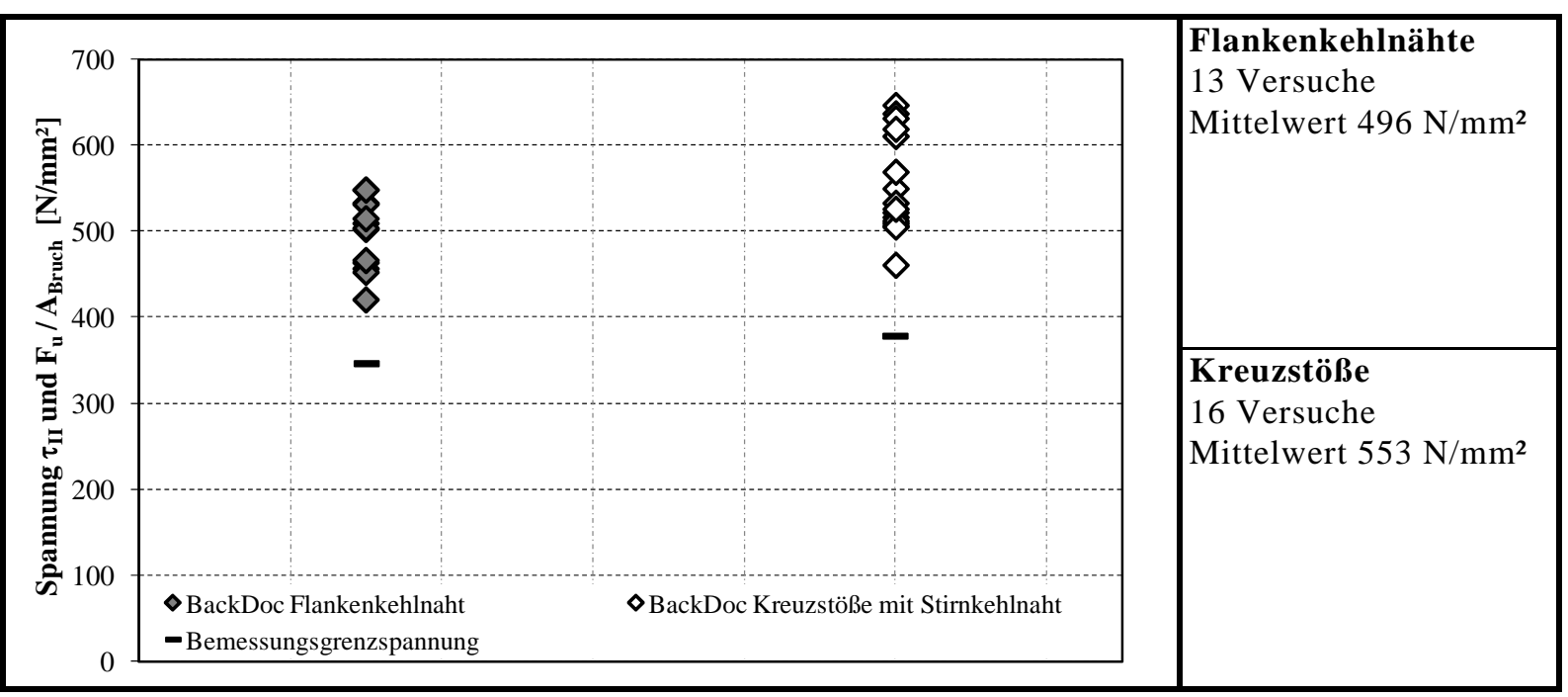

Abbildung 5.5: Vergleich der Ergebnisse von Kreuzstößen und Flankenkehlnahtverbindungen

\subsection{Kehlnahtverbindungen höherfester Stähle S690 und niederfestem Schweißzusatzwerkstoff}

\subsubsection{Allgemeines}

In [Collin, Johansson, 2005] wurden Versuche zur Bestimmung der Tragfähigkeit von Kehlnahtverbindungen höherfester Stähle veröffentlicht. Untersucht wurden insgesamt 27 Versuche an Flankenkehlnähten und Stirnkehlnähten mit überlappenden Blechen unter Variation des Schweißzusatzwerkstoffs. Zur Herstellung der Versuchskörper wurden zwei verschiedene Werkstoffkombinationen verwendet: Weldox 700 - OK75.75 und Domex 650MC - OK48.00.

Der Stahl Weldox 700 lässt sich normativ als Stahl der Güte S690Q einordnen. Bei dem Stahl Domex $650 \mathrm{MC}$ handelt es sich um einen Kaltumformstahl mit einer Mindeststreckgrenze von $\mathrm{f}_{\mathrm{y}}=650 \mathrm{~N} / \mathrm{mm}^{2}$. Die tatsächlichen Festigkeitswerte für die Streckgrenze und die Zugfestigkeit lassen auch eine normative Einordnung als Stahl S690 zu, allerdings handelt es sich um einen thermomechanisch gewalzten Stahl und nicht um einen vergüteten Werkstoff. Ein Vergleich der tatsächlichen Festigkeiten der verwendeten Stähle für Streckgrenze und Zugfestigkeit zeigt sehr ähnliche Werte, so dass diese Stähle vergleichbar sind. Der verwendete Schweißzusatzwerkstoff OK 75.75 erfüllt die Anforderungen nach DIN EN 757 (1997) für die Streckgrenze als E69 nicht, der Wert für die Zugfestigkeit (> $760 \mathrm{~N} / \mathrm{mm}^{2}$ ) ist sehr knapp mit 758 N/mm² nicht erfüllt. Der Schweißzusatzwerkstoff OK 48.00 entspricht E42.

Somit wurden Versuchskörper mit einer geringen Unterfestigkeit des Schweißzusatzwerkstoffs und mit einer deutlichen Unterfestigkeit des Schweißzusatzwerkstoffs im Vergleich mit dem Grundwerkstoff geprüft. Die Versuchsergebnisse und die statistischen Ergebnisse sind in Anhang A.1.7 aufgeführt. 


\subsubsection{Versuche an Flanken- und an Stirnkehlnahtverbindungen}

Die in [Collin, Johansson, 2005] beschriebenen Flankenkehlnahtversuche sind in Tabelle 5.9 dargestellt. 5 Versuche (WL) wurden mit einem Grundwerkstoff Weldox 700 E und einem Schweißzusatzwerkstoff OK75.75 durchgeführt, dessen Festigkeit etwas geringer als die des Grundwerkstoffs waren. Weitere 10 Versuche (DL) mit einem Grundwerkstoff Domex 650 MC und einem Schweißzusatzwerkstoff mit deutlich geringer Festigkeit werden vorgestellt.

Zusätzlich werden 12 Versuche an Stirnkehlnähten mit überlappenden Blechen vorgestellt. Wie bei den Flankenkehlnähten wurden auch für die Stirnkehlnähte zwei verschiedene Grund- und Schweißzusatzwerkstoff verwendet.

\subsubsection{Vergleich der Tragfähigkeiten}

Die Ergebnisse der statistischen Auswertung der Flankenkehlnähte sind in Tabelle 5.9 zusammengefasst. Die Bemessungswerte in Abhängigkeit des Grund- oder Schweißzusatzwerkstoffs sind identisch, da in jeder Versuchsserie nur ein Grund- und Schweißzusatzwerkstoff verwendet wurde, und somit keine Streuung zu berücksichtigen ist. Mit der Festigkeit des Schweißzusatzwerkstoffs erhöht sich die Bemessungsgrenzspannung von $360 \mathrm{~N} / \mathrm{mm}^{2}$ auf $383 \mathrm{~N} / \mathrm{mm}^{2}$ um 6 \%, vgl. Abbildung 5.6.

Tabelle 5.9: Bemessungsgrenzspannung für Flankenkehlnahtverbindungen

\begin{tabular}{|c|c|c|c|c|c|}
\hline & \multirow{2}{*}{ Flankenkehlnaht } & \multicolumn{2}{|l|}{ DL } & \multicolumn{2}{|l|}{ WL } \\
\hline & & GW & SZW & GW & SZW \\
\hline & $\mathrm{f}_{\mathrm{u}}\left[\mathrm{N} / \mathrm{mm}^{2}\right]$ & 827 & 548 & 833 & 758 \\
\hline & $\beta_{\mathrm{w}}[-]$ & 1,090 & 0,7220 & 1,004 & 0,913 \\
\hline 罗 & $\gamma_{\mathrm{M} 2}[-]$ & \multicolumn{4}{|c|}{1,25} \\
\hline $\begin{array}{l}\text { GW: Grundwerkstoff } \\
\text { SZW: Schweißzusatzwerkstoff }\end{array}$ & $\frac{\mathrm{f}_{\mathrm{u}}}{\sqrt{3} \cdot \gamma_{\mathrm{M} 2} \cdot \beta_{\mathrm{w}}}\left[\frac{\mathrm{N}}{\mathrm{mm}^{2}}\right]$ & \multicolumn{2}{|l|}{350} & \multicolumn{2}{|l|}{383} \\
\hline
\end{tabular}

Die Bemessungsgrenzspannung ändert sich infolge der höheren Festigkeit des Schweißzusatzwerkstoffs bei den Stirnkehlnahtverbindungen kaum, vgl. Tabelle 5.10. Während sich die Mittelwerte von 809 nach $947 \mathrm{~N} / \mathrm{mm}^{2}$ um 17 \% ändern, wie Abbildung 5.6 zeigt. Das liegt vor allem an der unterschiedlichen Streuung, die sich mit der Mittelwertabweichung b beschreiben lässt, der von 1,05 auf 1,22 steigt.

Tabelle 5.10: Bemessungsgrenzspannung für Stirnkehlnähte

\begin{tabular}{|c|c|c|c|c|c|c|c|}
\hline \multirow{5}{*}{$\triangleleft$} & \multirow{5}{*}{$\rightarrow$} & \multirow{2}{*}{\multicolumn{2}{|c|}{ Stirnkehlnaht }} & \multicolumn{2}{|l|}{ DT } & \multicolumn{2}{|l|}{ WT } \\
\hline & & & & GW & SZW & GW & SZW \\
\hline & & \multicolumn{2}{|l|}{$\mathrm{f}_{\mathrm{u}}\left[\mathrm{N} / \mathrm{mm}^{2}\right]$} & 827 & 548 & 833 & 758 \\
\hline & & \multicolumn{2}{|l|}{$\beta_{\mathrm{w}}[-]$} & 0,759 & 0,503 & 0,764 & 0,695 \\
\hline & & \multicolumn{2}{|l|}{$\gamma_{\mathrm{M} 2}[-]$} & \multicolumn{4}{|c|}{1,25} \\
\hline \multicolumn{2}{|c|}{$\begin{array}{l}\text { GW: Grundwerkstoff } \\
\text { SZW: Schweißzusatzwerkstoff }\end{array}$} & $\frac{\mathrm{f}_{\mathrm{u}}}{\sqrt{2} \cdot \gamma_{\mathrm{M} 2} \cdot \beta_{\mathrm{w}}}$ & $\frac{\mathrm{N}}{\mathrm{mm}^{2}}$ & 616 & 616 & 617 & 617 \\
\hline
\end{tabular}


Abbildung 5.6 zeigt den Vergleich von Flanken- und Stirnkehlnähten, die Bemessungsgrenzspannungen sind um ca. 61 \% und 71 \% höher. Die Tragfähigkeit von Stirnkehlnähten liegt hier höher als bei Flankenkehlnahtverbindungen.

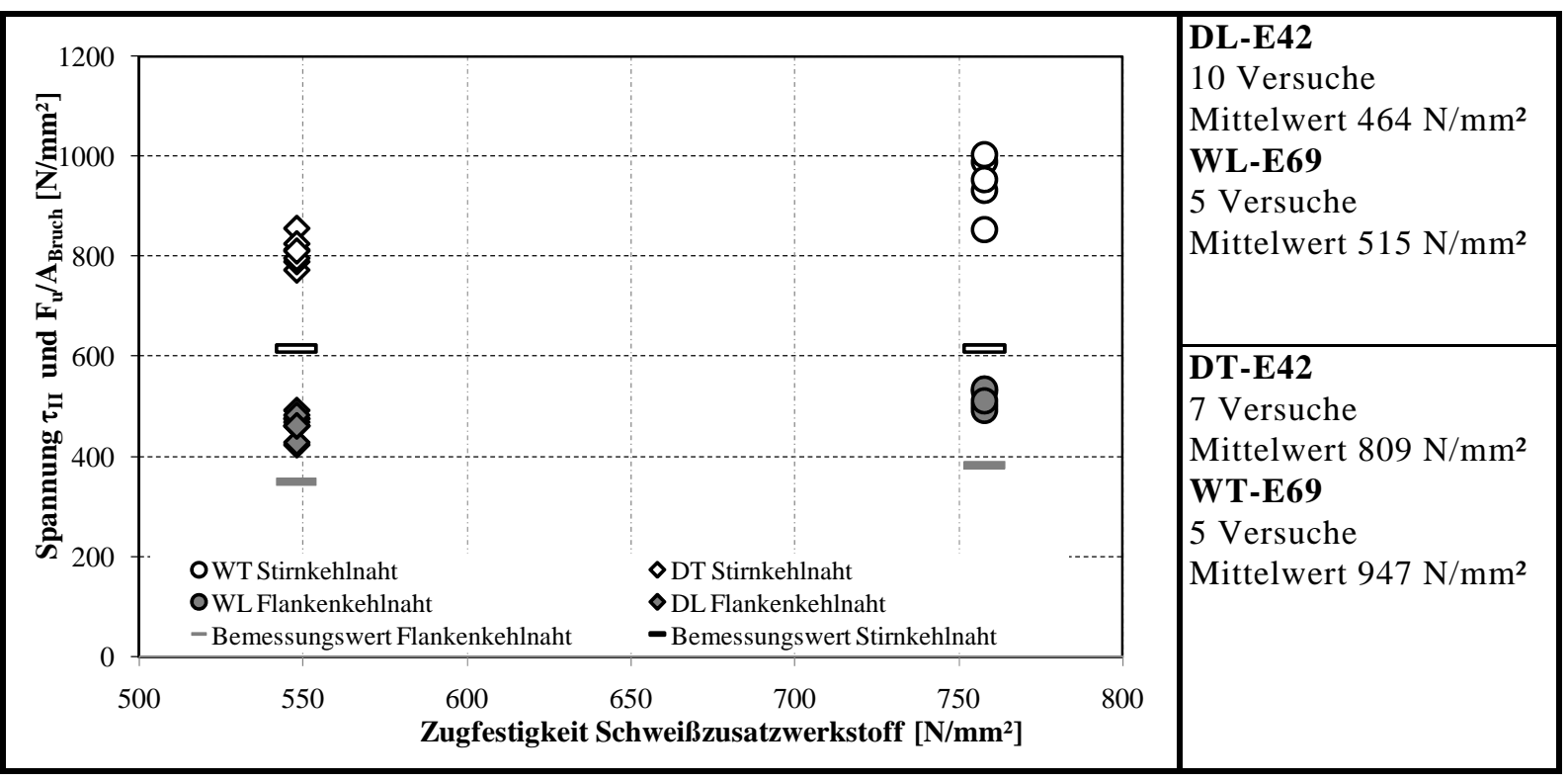

Abbildung 5.6: Vergleich der Ergebnisse von Stirn- und Flankenkehlnahtverbindungen

\subsection{Vergleich und Gegenüberstellung}

Die bisher vorgestellten Ergebnisse der statistischen Auswertung werden in diesem Kapitel verglichen und beurteilt.

In Abbildung 5.7 werden die Versuchsergebnisse von Versuchen an Flankenkehlnähten getrennt nach Werkstoffkombinationen unterschieden. Bei Betrachtung von Verbindungen gleicher Grund- und zugehöriger Schweißzusatzwerkstoffe ist eine Steigerung der Tragfähigkeit von S355-G42 zu S460-T46 und S690-E69 erkennbar.

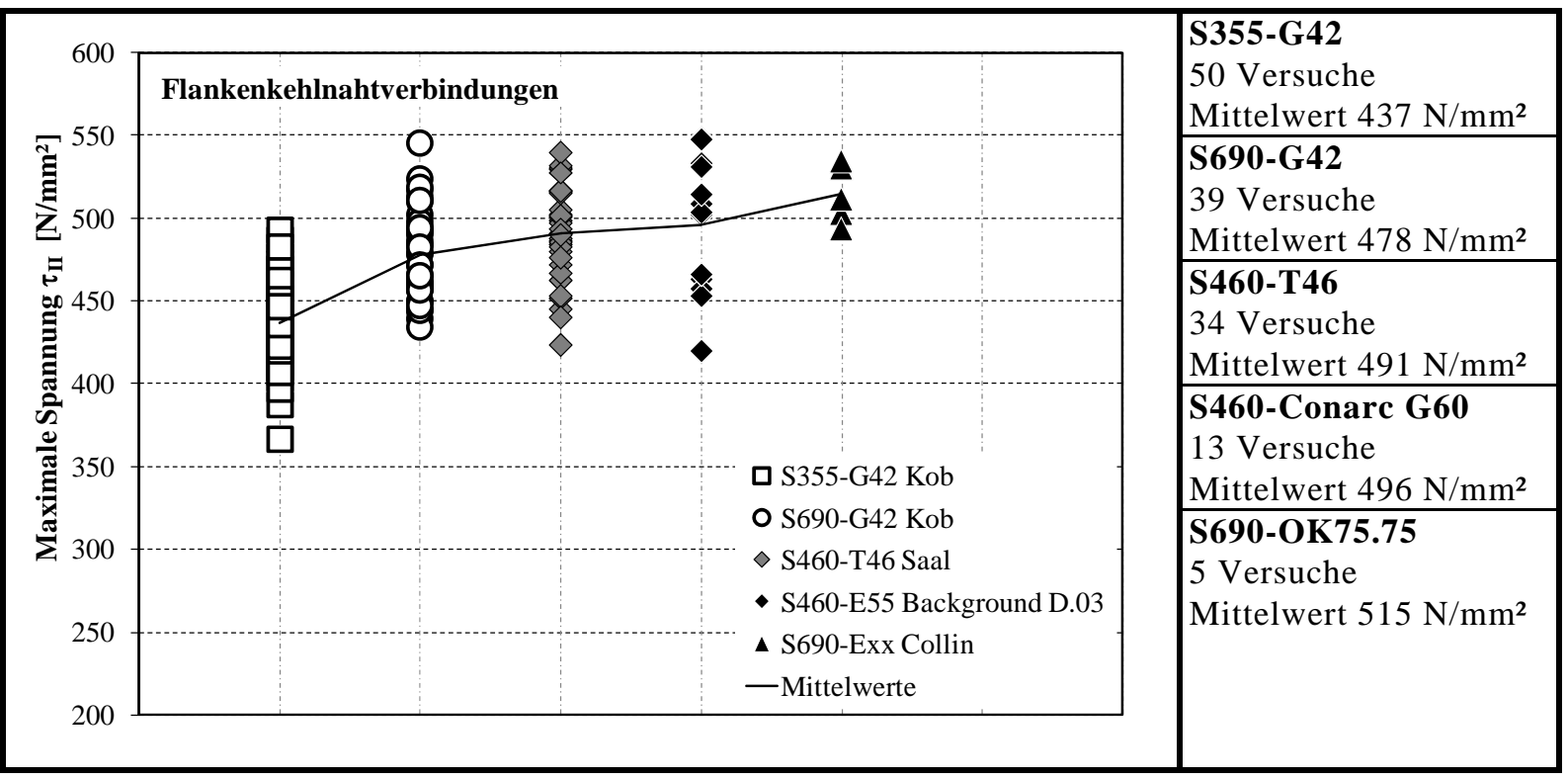

Abbildung 5.7: Darstellung der Versuchsergebnisse aller Flankenkehlnahtverbindungen 
Für einen Vergleich mit dem bestehenden Bemessungsmodell nach Eurocode 3 werden nur die Versuche herangezogen, bei denen der Grundwerkstoff und der Schweißzusatzwerkstoff zugehörig sind.

Für die Verbindung mit dem Grundwerkstoff S355 und dem zugehörigen Schweißzusatzwerkstoff G42 wurden die Versuchsergebnisse nach [Kob et al., 1987] in Kapitel 5.3 vorgestellt und beurteilt. Danach konnten die gültigen Werte nach EN 1993-1-8 (2005) von $\beta_{\mathrm{w}}=0,9$ bestätigt werden.

Die Verbindungen mit dem Grundwerkstoff S460 und dem zugehörigen Schweißzusatzwerkstoff mit der nominellen Festigkeit $\mathrm{f}_{\mathrm{y}}=460 \mathrm{~N} / \mathrm{mm}^{2}$ nach [Saal, 2005] zeigen, dass der Beiwert mit $\beta_{\mathrm{w}}=0,788$ bezogen auf den Grundwerkstoff und $\beta_{\mathrm{w}}=0,775$ bezogen auf den Schweißzusatzwerkstoff deutlich günstiger ist als nach EN 1993-1-8 (2005) mit $\beta_{\mathrm{w}}=1,0$.

Für die Verbindungen mit dem Grundwerkstoff S690 und zugehörigem Schweißzusatzwerkstoff können lediglich 5 Versuche nach [Collin, Johansson, 2005] herangezogen werden, das Schweißgut dieser Versuche erfüllt jedoch nicht die Mindestwerte für die Streckgrenze. Andere Versuche sind nicht bekannt.

Wie in Kapitel 4.5.4 beschrieben, gilt für die Auswertung mit Hilfe des Richtungsbezogenen Verfahrens nach EN 1993-1-8 (2005), dass sich für den Beiwert $\beta_{\mathrm{w}}$ ähnliche Werte für Flanken- und Stirnkehlnähte ergeben. Die Ergebnisse zeigen, dass die Beiwerte $\beta_{\mathrm{w}}$ für die Stirnkehlnähte in fast allen Fällen geringer als für die Flankenkehlnähte sind und damit auf der sicheren Seite liegen. Aufgrund der jeweils sehr geringen Anzahl von Versuchen und der großen Streuung der Versuchsergebnisse insbesondere der Stirnkehlnahtverbindungen wird der geringere Beiwert $\beta_{w}$ nicht in allen Fällen erreicht. Verdeutlicht wird diese Aussage in Abbildung 5.8.

Wenn eine gemeinsame Auswertung von Flanken- und Stirnkehlnähten erfolgt, liegen die Ergebnisse auf der unsicheren Seite, da dann die Tragfähigkeit der Flankenkehlnähte überschätzt werden kann.

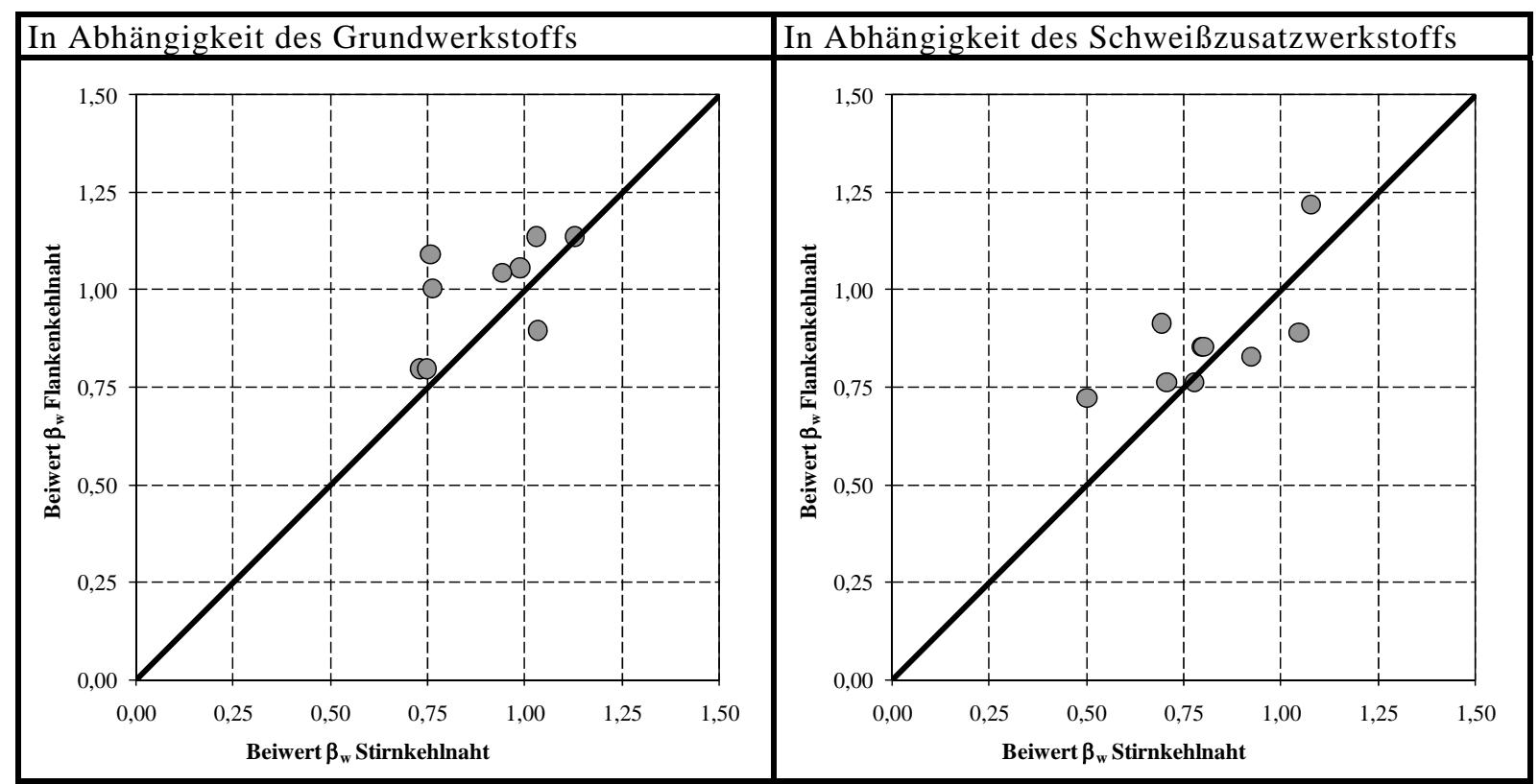

Abbildung 5.8: Vergleich der Bemessungsgrenzspannungen von Stirn- und Flankenkehlnahtverbindungen 


\subsection{Zusammenfassung}

In diesem Kapitel wurden bekannte Versuchsergebnisse aus der Literatur statistisch neu ausgewertet. Dabei wurden sowohl Flankenkehlnähte als auch Stirnkehlnähte betrachtet. Die Ergebnisse der Flankenkehlnahtverbindungen normalfester Baustähle bestätigen die aktuellen Regelungen. Es konnte ein deutlicher Einfluss des Grundwerkstoffs auf die Tragfähigkeit von Kehlnahtverbindungen nachgewiesen werden. Der Vergleich von Kreuzstößen und Flankenkehlnahtverbindungen zeigt eine höhere Tragfähigkeit für die Kreuzstöße, wobei die Steigerung sehr unterschiedlich ist. Der Vergleich der Bemessungsgrenzspannungen mit der Bruchfläche $\mathrm{A}_{\mathrm{Bruch}}$ und $\mathrm{A}_{\mathrm{EC}}$ deutet darauf hin, dass die Verwendung der Nahtdicke $A_{E C}$ für die Bestimmung der Tragfähigkeit ungenauere Ergebnisse liefert.

Der Vergleich der Tragfähigkeiten zeigt, dass das bestehende Bemessungsmodell die unterschiedlichen Festigkeiten der Schweißzusatzwerkstoffe nicht ausreichend berücksichtigt. Insgesamt kann festgestellt werden, dass für Schweißverbindungen höherfester Baustähle nur sehr wenige Daten bekannt sind. Aus diesem Grund werden diesbezüglich eigene Untersuchungen durchgeführt. 


\section{Experimentelle Untersuchungen an manuell geschweißten Versuchskörpern}

\subsection{Einleitung}

Dieses Kapitel stellt experimentelle Untersuchungen vor, die im Rahmen des FOSTA/AiFForschungsprojekts „Wirtschaftliche Schweißverbindungen höherfester Baustähle“ [FOSTA P652, 2008] durchgeführt wurden. Innerhalb des Projekts wurde die Tragfähigkeit an Flankenkehlnahtverbindungen und Kreuzstößen höherfester Baustähle S460M und S690Q bei Raumtemperatur analysiert.

Projektpartner waren das Institut für konstruktiven Ingenieurbau, Professur Stahlbau, BauhausUniversität Weimar (BUW), das Institut für Stahlbau und Werkstoffmechanik, Fachgebiet Werkstoffmechanik, Technische Universität Darmstadt (TUD) und das Günter-Köhler-Institut für Fügetechnik und Werkstoffprüfung GmbH, IFW Jena (IFW). Verwendete Ergebnisse der Projektpartner werden entsprechend der Klammerausdrücke gekennzeichnet. Insbesondere für die Ergebnisse an Kreuzstößen, die in Weimar durchgeführt und in dieser Arbeit verwendet werden dürfen, sei herzlich gedankt.

Im diesem Kapitel werden die durchgeführten Versuchsergebnisse ausführlich beschrieben. Die Versuche wurden durch manuelle Schweißung hergestellt, so dass praxisnahe, aber keine gleichmäßigen Bedingungen vorherrschten. Die tatsächlichen Schweißparameter wurden schweißtechnisch neu bewertet. Es wurden nur die Versuchsergebnisse verwendet, deren Schweißparameter die Anforderungen nach DIN EN 1011-2 (2001) erfüllen. Die Versuchsergebnisse wurden statistisch neu ausgewertet.

\subsection{Versuchsprogramm}

Der Schwerpunkt der Untersuchungen der Universität Stuttgart im Rahmen von [FOSTA P652, 2008] lag auf Untersuchungen von längsbeanspruchten Kehlnahtverbindungen. Die Referenzversuchskörper für Flankenkehlnahtverbindungen und für Kreuzstöße mit Stirnkehlnähten sind in der folgenden Tabelle 6.1 schematisch dargestellt. Die Versuchskörper für Flankenkehlnahtverbindungen bestanden aus zwei ineinander gesteckten Blechen, die manuell mit Kehlnähten verschweißt wurden. Diese Kehlnähte waren im Bereich von Nahtanfang und Nahtende herstellungsbedingt ungleichmäßig.

Für die Traglastversuche an Flankenkehlnahtverbindungen wurden jeweils zwei nominell gleiche Versuchskörper bei verschiedenen Fertigern hergestellt. Bei den Kreuzstößen wurden jeweils 3 Versuchsproben aus einem Versuchskörper entnommen.

Tabelle 6.1: Untersuchte Parameter [FOSTA P652, 2008]

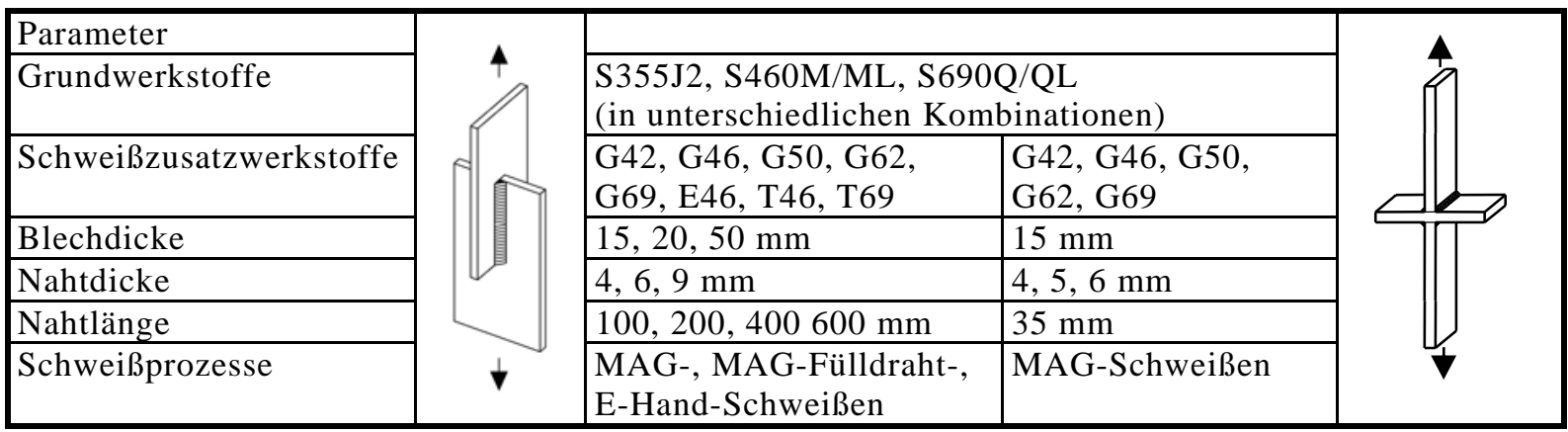

Als Parameter wurden die Grund- und Schweißzusatzwerkstoffe variiert, um den Einfluss von deren Festigkeit auf die Tragfähigkeit der Schweißverbindung zu untersuchen. Dazu wurden Verbindungen mit zwei gleichen Grundwerkstoffen und einem Schweißzusatzwerkstoff mit einer Festigkeit, die nominell gleich war wie die der Grundwerkstoffe, untersucht. Im Weiteren werden diese Verbindungen als Verbindungen mit gleichen Grund- und zugehörigen Schweißzusatzwerkstoffen bezeichnet. Es wurden Versuche mit gleichen Grund- und Schweißzusatzwerkstoffen mit höherer oder geringerer Festigkeit als die der Grundwerkstoffe getestet. Außerdem wurde die Tragfähigkeit von Verbindungen mit unterschiedlichen Grundwerkstoffen ermittelt. Die untersuchten Parameter sind in Tabelle 6.1 
zusammengefasst. Um die Tragfähigkeit der Naht zu bestimmen, wurden die Versuchskörper so dimensioniert, dass ein Versagen im Schweißgut und nicht im Grundwerkstoff auftritt. Die Parameter der einzelnen Versuche und die Versuchskörperformen sind im Detail im Anhang A.2 aufgeführt.

\subsection{Verwendete Werkstoffe}

Als Grundwerkstoffe wurden Stähle S355J2 nach DIN EN 10025-2 (2005), S460M/ML nach DIN EN 10025-4 (2005) und S690Q/QL nach DIN EN 10025-6 (2005) verwendet. Die Zeugniskennwerte der mechanischen Eigenschaften der Grundwerkstoffe sind in Tabelle 6.2 zusammengestellt. Für die Schweißzusatzwerkstoffe sind die Normen und Bezeichnungen der verwendeten Schweißzusatzwerkstoffe in Tabelle 6.3 aufgeführt. In Tabelle 6.4 sind die Zeugniskennwerte der mechanischen Kennwerte der Schweißzusatzwerkstoffe zusammengestellt. Die chemischen Kennwerte der Grund- und Schweißzusatzwerkstoffe sind in Anhang A.2.1 aufgeführt.

Tabelle 6.2: Mechanische Eigenschaften der verwendeten Grundwerkstoffe

\begin{tabular}{|c|c|c|c|c|c|c|c|c|c|c|c|c|c|c|}
\hline \multirow{2}{*}{$\begin{array}{l}\text { Werk- } \\
\text { stoff }\end{array}$} & \multirow{2}{*}{$\begin{array}{l}\mathrm{t} \\
{[\mathrm{mm}]}\end{array}$} & \multicolumn{2}{|c|}{$\mathrm{R}_{\mathrm{eH}}\left[\mathrm{N} / \mathrm{mm}^{2}\right]$} & \multicolumn{2}{|c|}{$\mathrm{R}_{\mathrm{p} 0,2}\left[\mathrm{~N} / \mathrm{mm}^{2}\right]$} & \multicolumn{2}{|c|}{$\mathrm{R}_{\mathrm{m}}\left[\mathrm{N} / \mathrm{mm}^{2}\right]$} & \multirow{2}{*}{$\begin{array}{l}\mathrm{A}_{5}[\%] \\
\mathrm{L} / \mathrm{Q}\end{array}$} & \multirow{2}{*}{$\begin{array}{l}A_{V} \\
{[J]}\end{array}$} & \multicolumn{2}{|c|}{$-20^{\circ} \mathrm{C}$} & \multicolumn{2}{|c|}{$-40^{\circ} \mathrm{C}$} & \multirow{2}{*}{$\begin{array}{l}-60^{\circ} \mathrm{C} \\
\mathrm{L}\end{array}$} \\
\hline & & $\mathrm{L}$ & $\mathrm{Q}$ & $L$ & & $\mathrm{~L}$ & Q & & & $\mathrm{L}$ & $\mathrm{Q}$ & $\mathrm{L}$ & $\mathrm{Q}$ & \\
\hline S355J2 & 15 & & 422 & & & & 557 & $-/ 34,5$ & & 286 & & & & \\
\hline S460M & 15 & & & & 466 & & 621 & $-/ 26,6$ & & & & & 393 & \\
\hline S690Q & 15 & & & & 830 & & 871 & $-/ 17$ & & & & 207 & & \\
\hline S355J2 & 20 & 389 & 418 & & & 586 & 608 & $26,9 / 29,3$ & & 171 & 103 & & & \\
\hline S460M & 20 & & & 467 & & 587 & & $30,9 /-$ & & & & 346 & & 347 \\
\hline S690Q & 20 & & & & 832 & & 876 & $-/ 14,0$ & & & & 238 & & \\
\hline S355J2 & 50 & & 356 & & 338 & & 542 & $-/ 27,5$ & & 172 & & 132 & & \\
\hline S460ML & 50 & 505 & 535 & 473 & 505 & 568 & 585 & $27,0 / 24,5$ & & 316 & & 304 & 298 & \\
\hline S690QL & 50 & & & & 797 & & 871 & $-/ 19,7$ & & & & & 121 & \\
\hline
\end{tabular}

Tabelle 6.3: Verwendete Schweißzusatzwerkstoffe

\begin{tabular}{|l|l|l|l|}
\hline Schweißprozess & Norm & \multicolumn{2}{l|}{ Bezeichnung } \\
\hline \multirow{4}{*}{$111($ LBH) } & DIN EN ISO 2560 (2006) & E46 & E 46 6 1Ni B42 H5 \\
\hline \multirow{3}{*}{ (MAG) } & DIN EN 440 (1994) & G42 & G 42 4M G3Si1 \\
& Nachfolger: DIN EN ISO & G46 & G 46 4M G4Si1 \\
& 14341 (2008) & G50 & G 50 5M G3Ni1 \\
\cline { 2 - 4 } & DIN EN ISO 16834 (2007) & G62 & G 62 5M Mn3Ni1Mo \\
& & G69 & G 69 4Mn4Ni1,5CrMo \\
\hline \multirow{2}{*}{ 136 (MAG-Fülldraht) } & DIN EN ISO 17632 (2008) & T46 & T 46 4 P M1 H10 \\
\cline { 2 - 4 } & DIN EN ISO 18276 (2006) & T69 & T 69 4 Mn2 NiCrMo M M1 H5 \\
\hline
\end{tabular}

Tabelle 6.4: Mechanische Eigenschaften der verwendeten Schweißzusatzwerkstoffe gemäß Lieferzeugnis

\begin{tabular}{|c|c|c|c|c|c|c|c|c|c|}
\hline \multicolumn{2}{|l|}{ Schweißzusatzwerkstoff } & \multirow{2}{*}{$\begin{array}{l}\mathrm{R}_{\mathrm{p} 0,2} \\
{\left[\mathrm{~N} / \mathrm{mm}^{2}\right]} \\
487 \\
\end{array}$} & \multirow{2}{*}{$\begin{array}{l}\mathrm{R}_{\mathrm{m}} \\
{\left[\mathrm{N} / \mathrm{mm}^{2}\right]} \\
583\end{array}$} & \multirow{2}{*}{$\begin{array}{l}A_{5} \\
{[\%]} \\
27,5 \\
\end{array}$} & \multirow{2}{*}{\begin{tabular}{|l|}
$\begin{array}{l}\mathrm{Z} \\
{[\%]}\end{array}$ \\
73,0 \\
\end{tabular}} & \multirow{2}{*}{$\begin{array}{l}\mathrm{A}_{\mathrm{v}}[\mathrm{J}] \\
-20^{\circ} \mathrm{C} \\
125\end{array}$} & \multirow{2}{*}{\begin{tabular}{|l}
$-40^{\circ} \mathrm{C}$ \\
112
\end{tabular}} & \multirow{2}{*}{\begin{tabular}{|l}
$-50^{\circ} \mathrm{C}$ \\
98
\end{tabular}} & \multirow[t]{2}{*}{$-60^{\circ} \mathrm{C}$} \\
\hline Union $\mathrm{K}^{2} 2^{1)}$ & G42 & & & & & & & & \\
\hline Union K56 ${ }^{1)}$ & G46 & 547 & 639 & 23,2 & 57,9 & 120 & 90 & 82 & 62 \\
\hline OK AristoRod $12.63^{1)}$ & G46B & 525 & 595 & 26 & & & 60 & & \\
\hline Union $\mathrm{K}_{5} \mathrm{Ni}^{1)}$ & G50 & 528 & 623 & 24,0 & 72,0 & 123 & 101 & 68 & 65 \\
\hline UnionMoNi $^{1)}$ & G62 & 704 & 796 & 16,6 & 39,3 & 111 & 78 & 73 & 53 \\
\hline Union NiMoCr $^{1)}$ & G69 & 724 & 836 & 15,4 & 34,6 & 105 & 90 & 74 & 66 \\
\hline Union RV 71 2) & T46 & 539 & 602 & 23,4 & 68,8 & 93 & 32 & & \\
\hline Union MV NiMoCr & T69 & 738 & 794 & 21,0 & 68,7 & 149 & 140 & 110 & 104 \\
\hline Phoenix SH Ni $2{\mathrm{~K} 70^{3)}}^{3}$ & E46 & 480 & 590 & 26 & & & & & \\
\hline
\end{tabular}

Die Ergebnisse eigener Zugversuche am Grundwerkstoff und an Zugproben aus reinem Schweißgut (Schweißzusatzwerkstoff im verschweißten Zustand) sind in Tabelle 6.5 zusammengefasst. Die Werte wurden jedoch nicht für die statistische Auswertung verwendet, da es sich nicht um Normzugproben handelte. 
Tabelle 6.5: Ermittelte mechanische Eigenschaften der Grund- und Schweißzusatzwerkstoffe

\begin{tabular}{|c|c|c|c|c|c|c|c|c|c|}
\hline $\begin{array}{l}\begin{array}{l}\text { Schweißzu- } \\
\text { satzwerkstoff }\end{array} \\
\end{array}$ & \begin{tabular}{|l}
$\mathrm{R}_{\mathrm{eH}} / \mathrm{R}_{\mathrm{p} 0,2}$ \\
{$\left[\mathrm{~N} / \mathrm{mm}^{2}\right]$} \\
\end{tabular} & $\begin{array}{l}\mathrm{R}_{\mathrm{m}} \\
{\left[\mathrm{N} / \mathrm{mm}^{2}\right]}\end{array}$ & \begin{tabular}{|l}
$\mathrm{A}_{5}$ \\
{$[\%]$} \\
$\%$
\end{tabular} & $\begin{array}{l}\text { Grund- } \\
\text { werkstoff }\end{array}$ & $\begin{array}{l}\begin{array}{l}\text { Blech- } \\
\text { dicke }\end{array} \\
\end{array}$ & $\begin{array}{l}\mathrm{R}_{\mathrm{p} 0,2} \\
{\left[\mathrm{~N} / \mathrm{mm}^{2}\right]}\end{array}$ & $\begin{array}{l}\mathrm{R}_{\mathrm{m}} \\
{\left[\mathrm{N} / \mathrm{mm}^{2}\right]}\end{array}$ & \begin{tabular}{|l}
$\mathrm{A}_{5}$ \\
{$[\%]$} \\
$\%$
\end{tabular} & \\
\hline G46 & 475,6 & 607,7 & 24,5 & \multirow{6}{*}{$\begin{array}{l}\text { S460M } \\
\text { (BUW) }\end{array}$} & \multirow[t]{6}{*}{15} & 509,1 & 616,1 & 20,6 & $\mathrm{~L}$ \\
\hline \multirow[t]{2}{*}{ (TUD) } & 476,6 & 610,3 & 25,3 & & & 522,1 & 634,8 & 14,7 & $\mathrm{~L}$ \\
\hline & 486,3 & 615,5 & 26,0 & & & 512,1 & 602,3 & 21,4 & Q \\
\hline \multirow{5}{*}{$\begin{array}{l}\text { G69 } \\
\text { G69 (TUD) }\end{array}$} & \multirow{5}{*}{$\begin{array}{l}7779,6 \\
772,4\end{array}$} & \multirow{5}{*}{\begin{tabular}{|l|}
833,0 \\
816,7 \\
810,5 \\
863,7 \\
828,6 \\
\end{tabular}} & \multirow{5}{*}{$\begin{array}{l}14,92 \\
14,52\end{array}$} & & & 533,1 & 634,5 & 20,6 & L \\
\hline & & & & & & 497,1 & 615,4 & 21,6 & $\mathrm{~L}$ \\
\hline & & & & & & 535,0 & 593,1 & 18,6 & $\mathrm{~L}$ \\
\hline & & & & \multirow{3}{*}{$\begin{array}{l}\text { S690Q } \\
\text { (BUW) }\end{array}$} & \multirow[t]{3}{*}{15} & 833,0 & 922,8 & 11,2 & $\mathrm{Q}$ \\
\hline & & & & & & 840,9 & 933,5 & 12,4 & Q \\
\hline \multirow{4}{*}{\multicolumn{4}{|c|}{$\begin{array}{l}\text { Die Werte wurden nicht für die statistische } \\
\text { Auswertung verwendet, da es sich nicht um } \\
\text { Normzugproben handelte. }\end{array}$}} & & & 786,1 & 863,6 & 13,7 & L \\
\hline & & & & S355J2 & 15 & 416,9 & 554,7 & 31,46 & $\bar{Q}$ \\
\hline & & & & S355J2 & 20 & 389,7 & 565,8 & 32,50 & $\mathrm{~L}$ \\
\hline & & & & S355J2 & 50 & 378,5 & 554,9 & 29,46 & $\mathrm{Q}$ \\
\hline
\end{tabular}

\subsection{Herstellung der Versuchskörper und Versuchsdurchführung}

\subsubsection{Allgemeines}

Für die Herstellung der Versuchskörper wurden Schweißanweisungen nach DIN EN 1011-2 (2001), [SEW 088, 1993], [SEW 088 Beiblatt 1, 1993] und [SEW 088 Beiblatt 2, 1993] erstellt. Die Versuchskörper wurden bei verschiedenen Fertigern unter Praxisbedingungen hergestellt. Der Zuschnitt der Bleche erfolgte mittels Plasmaschnitt und Brennschnitt. Die Schweißung wurde teilmechanisiert durchgeführt.

\subsubsection{Flankenkehlnahtverbindungen}

Alle Versuchskörper mit Flankenkehlnähten wurden vor dem Versuch vermessen. Die theoretische Nahtdicke wurde mit einer Schweißnahtlehre entlang der Naht gemessen. Es wurde bei allen Versuchskörpern eine zerstörungsfreie Werkstoffprüfung mittels Sichtprüfung durchgeführt. Bei den Versuchskörpern, die mit Blechen aus S690 oder mit höherfestem Schweißzusatzwerkstoff $\left(\mathrm{mit}_{\mathrm{y}}>460\right.$ $\mathrm{N} / \mathrm{mm}^{2}$ ) verschweißt waren, wurde zusätzlich eine Magnetpulverprüfung durchgeführt. Dabei wurden keine Fehler festgestellt.

Die Versuche wurden als weggeregelte Zugversuche bis zum Bruchversagen der Schweißnaht durchgeführt. Bei etwa der Hälfte der erwarteten Bruchlast wurde der Versuch 5 Minuten lang angehalten. Anschließend wurde entlastet und dann bis zum Bruch belastet. Während des Versuches wurden die Kraft und der Traversenweg gemessen. Wegmessungen erfolgten mit Hilfe von optischen Messungen und Wegaufnehmern. Die Versuche zur Untersuchung des Einflusses der Nahtlänge wurden als Druckversuche durchgeführt, da die höheren Traglasten eine andere Prüfmaschine erforderten. Nach dem Bruch der Schweißnaht und dem Ausbau der Versuchskörper wurden die Bruchflächen vermessen. Der Bruch trat stets in der Naht unter einem Winkel nahe $45^{\circ}$ ein. Die Bruchfläche wurde auf einer aufgelegten Folie nachgezeichnet. Diese Abbildung der Bruchfläche wurde digitalisiert, und die Größe der Fläche wurde grafisch bestimmt.

\subsubsection{Kreuzstöße mit Stirnkehlnähten}

Die Kreuzstöße wurden vor dem Versuch vermessen. Die theoretische Nahtdicke wurde mit einer Schweißnahtlehre entlang der Naht gemessen. Die genaue Nahtdicke wurde anhand eines Makroschliffes bestimmt. Es wurde bei allen Versuchskörpern eine zerstörungsfreie Werkstoffprüfung mittels Sichtprüfung durchgeführt. Die Versuche wurden als weggeregelte Zugversuche mit einer Geschwindigkeit von 2 - 3,6 mm/min bis zum Versagen durchgeführt. Während des Versuches wurden 
die Kraft und der Traversenweg gemessen. Nach dem Bruch und dem Ausbau der Versuchskörper wurden die Bruchwinkel vermessen.

\section{5 Überblick über die Versuchsergebnisse an Flankenkehlnahtverbin- dungen}

In Tabelle 6.6 und Tabelle 6.7 sind die Versuchsergebnisse aller durchgeführten Traglastversuche an Flankenkehlnahtverbindungen dargestellt. Es sind die maximal gemessenen Prüfkräfte, die ermittelten Bruchflächen und die sich daraus ergebenden Maximalspannungen aufgeführt. Die dargestellten Spannungen $\tau_{\| 1}$ ergeben sich aus der im Versuch gemessenen Kraft, dividiert durch die nach dem Versuch ausgemessene Bruchfläche. Da die Nähte planmäßig scherbeansprucht sind, wird die ermittelte Spannung mit $\tau_{11}$ bezeichnet. Das Versagen trat planmäßig stets in der Naht auf, nie im Grundwerkstoff. Die Werkstoffkombinationen der einzelnen Versuche sind im Anhang zusammen mit den gemessenen Schweißparametern Streckenenergie, Vorwärmtemperatur und Abkühlzeit $t_{8 / 5}$ aufgeführt. Für die statistische Auswertung wurden nur die Versuche verwendet, deren Abkühlzeit im Bereich von 4 - 15 Sekunden lag. Aufgrund der manuellen Schweißung wurden nicht immer die gleichen Bedingungen erreicht, so dass einige Versuchsergebnisse nicht verwendet werden können. Diese sind in Tabelle 6.6 und Tabelle 6.7 gekennzeichnet.

Tabelle 6.6: Flankenkehlnahtverbindungen gleicher Grund- und zugehöriger Schweißzusatzwerkstoffe

\begin{tabular}{|c|c|c|c|c|c|c|c|c|c|c|c|}
\hline$\dot{\mathrm{z}}$ & 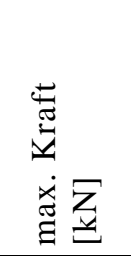 & 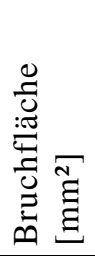 & 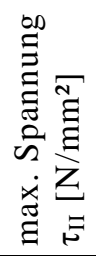 & $\dot{\mathrm{z}}$ & 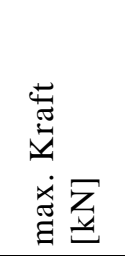 & 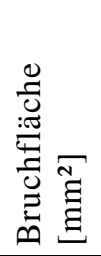 & 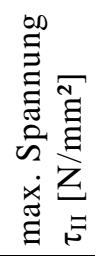 & $\dot{\mathrm{z}}$ & 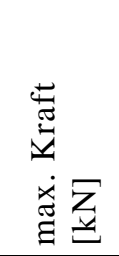 & 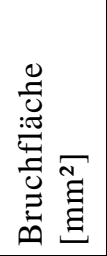 & 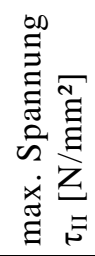 \\
\hline \multicolumn{4}{|c|}{ S690-S690-G69/T69 } & \multicolumn{4}{|c|}{ S460-S460-G46 } & \multicolumn{4}{|c|}{ S460-S460-E46/T46 } \\
\hline $1-045$ & 586,0 & 1210 & 484 & $1-001$ & 575,4 & 1129 & 510 & $1-017$ & 398,6 & 869 & 458 \\
\hline $1-047^{*}$ & 585,4 & 1383 & 423 & $1-002$ & 572,1 & 1122 & 510 & $1-019$ & 453,5 & 985 & 460 \\
\hline $1-049 *$ & 551,0 & 987 & 558 & $1-004$ & 543,0 & 1091 & 498 & $1-020 *$ & 399,0 & 800 & 499 \\
\hline $1-050 *$ & 524,9 & 844 & 622 & $1-005$ & 561,4 & 1206 & 465 & $1-021^{*}$ & 354,2 & 753 & 470 \\
\hline $1-052$ & 642,0 & 1274 & 504 & $1-006$ & 547,7 & 1247 & 439 & $1-022$ & 533,8 & 1062 & 502 \\
\hline $1-054$ & 651,7 & 1361 & 479 & $1-009$ & 597,5 & 1409 & 424 & $1-024$ & 503,3 & 1106 & 455 \\
\hline $1-055 * *$ & 1529,4 & 2112 & 724 & $1-011$ & 581,3 & 1333 & 436 & $1-025 *$ & 472,0 & 1020 & 463 \\
\hline $1-057 * *$ & 1384,2 & 2209 & 627 & $1-012$ & 672,7 & 1352 & 497 & $1-026 *$ & 486,5 & 1041 & 467 \\
\hline $1-058 * *$ & 3097,2 & 4539 & 682 & $1-013$ & 658,1 & 1391 & 473 & \multicolumn{4}{|c|}{ S355-S3555-G42 } \\
\hline $1-059 * *$ & 2824,2 & 4863 & 581 & $1-015$ & 563,8 & 1175 & 480 & $1-041$ & 459,3 & 1161 & 396 \\
\hline $1-060 * *$ & 4193,4 & 6844 & 613 & $1-016$ & 579,0 & 1163 & 498 & $1-042$ & 486,9 & 1153 & 422 \\
\hline $1-062 * *$ & 4359,6 & 8399 & 519 & $1-027 * *$ & 1412,1 & 2214 & 638 & $1-043$ & 534,8 & 1485 & 360 \\
\hline $1-063$ & 960,8 & 2150 & 447 & $1-029 * *$ & 1470,6 & 2754 & 534 & $1-044$ & 509,8 & 1286 & 396 \\
\hline $1-064$ & 1097,4 & 2445 & 449 & $1-030 * *$ & 2842,8 & 4525 & 628 & \multirow{9}{*}{\multicolumn{4}{|c|}{$\begin{array}{l}\text { * Nicht für die statistische } \\
\text { Auswertung verwendet } \\
\text { ** Druckversuch }\end{array}$}} \\
\hline $1-065$ & 2722,5 & 5361 & 508 & $1-031^{* *}$ & 2899,2 & 4987 & 581 & & & & \\
\hline 1-066* & 2473,6 & 6377 & 388 & $1-032 * *$ & 4587,0 & 6303 & 728 & & & & \\
\hline $1-067$ & 633,7 & 1143 & 554 & $1-035$ & 2319,7 & 5250 & 442 & & & & \\
\hline $1-068$ & 721,2 & 1250 & 577 & $1-036$ & 2328,9 & 5654 & 412 & & & & \\
\hline $1-146$ & 2410,8 & 5009 & 481 & $1-037$ & 1076,2 & 2537 & 424 & & & & \\
\hline $1-147$ & \multirow{3}{*}{\multicolumn{3}{|c|}{ Versuch fehlerhaft }} & $1-038$ & 1023,3 & 2428 & 421 & & & & \\
\hline & & & & 1-039* & 575,1 & 1035 & 556 & & & & \\
\hline & & & & $1-040$ & 643,9 & 1284 & 501 & & & & \\
\hline
\end{tabular}


Tabelle 6.7: Flankenkehlnahtverbindungen unterschiedlicher Grund- und/oder Schweißzusatzwerkstoffe

\begin{tabular}{|c|c|c|c|c|c|c|c|c|c|c|c|}
\hline$\dot{\mathrm{z}}$ & 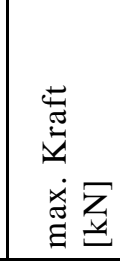 & 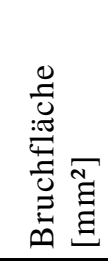 & 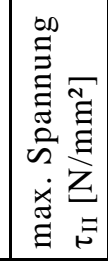 & $\dot{\mathrm{Z}}$ & 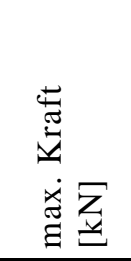 & 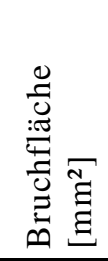 & 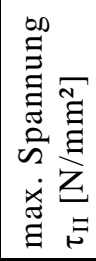 & $\dot{\mathrm{z}}$ & 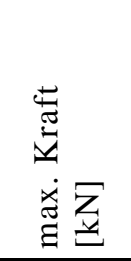 & 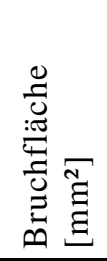 & 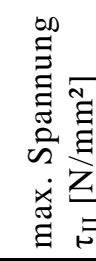 \\
\hline \multicolumn{4}{|c|}{ S690-S690-G62 } & \multicolumn{4}{|c|}{ S460-S690-G69 } & \multicolumn{4}{|c|}{ S355-S690-G69 } \\
\hline $1-080$ & 555,1 & 1133 & 490 & $1-107$ & 613,7 & 1246 & 492 & $1-098$ & 564,2 & 1232 & 458 \\
\hline $1-081$ & 649,8 & 1347 & 482 & $1-109$ & 656,3 & 1431 & 459 & $1-100$ & 618,3 & 1410 & 438 \\
\hline $1-082 * *$ & 3024,6 & 4731 & 639 & \multicolumn{4}{|c|}{ S460-S690-G62 } & $1-118 * *$ & 1336,2 & 2545 & 525 \\
\hline $1-083 * *$ & 3057,0 & 4920 & 621 & $1-104$ & 592,8 & 1264 & 469 & $1-119 * *$ & 1424,4 & 2836 & 502 \\
\hline $1-084$ & 2616,4 & 5269 & 497 & $1-106$ & 640,8 & 1588 & 404 & $1-120 * *$ & 2518,8 & 4973 & 506 \\
\hline $1-085$ & 2402,9 & 5604 & 429 & \multicolumn{4}{|c|}{ S460-S690-G46 } & $1-121 * *$ & 2797,8 & 5349 & 523 \\
\hline \multicolumn{4}{|c|}{ S690-S690-G46 } & $1-101$ & 545,9 & 1133 & 482 & $1-123 * *$ & 3784,2 & 7848 & 482 \\
\hline $1-077$ & 493,2 & 1089 & 453 & $1-103$ & 593,3 & 1404 & 422 & \multicolumn{4}{|c|}{ S355-S460-G42 } \\
\hline 1-079* & 574,7 & 1424 & 404 & \multicolumn{4}{|c|}{ S355-S690-G42 } & $1-086$ & 528,1 & 1180 & 448 \\
\hline S355-S3 & $5-G 46$ & & & $1-092$ & 511,3 & 1200 & 426 & $1-088$ & 514,7 & 1322 & 389 \\
\hline $1-075$ & 474,5 & 1159 & 409 & $1-094$ & 531,1 & 1355 & 392 & \multicolumn{4}{|c|}{ S355-S460-G46 } \\
\hline $1-076$ & 559,8 & 1487 & 376 & $1-110$ & 1944,1 & 4715 & 412 & $1-089$ & 549,8 & 1138 & 483 \\
\hline \multicolumn{4}{|c|}{ S460-S460-G42 } & $1-111$ & 2106,9 & 5986 & 352 & 1-091 & 560,5 & 1432 & 391 \\
\hline $1-069$ & 553,6 & 1178 & 470 & $1-112 *$ & 440,7 & 968 & 456 & \multirow{5}{*}{\multicolumn{4}{|c|}{$\begin{array}{l}\text { * Nicht für die statistische } \\
\text { Auswertung verwendet } \\
\text { ** Druckversuch }\end{array}$}} \\
\hline $1-071$ & 586,4 & 1432 & 410 & $1-113$ & 486,4 & 1271 & 383 & & & & \\
\hline \multicolumn{4}{|c|}{ S460-S460-G69 } & \multicolumn{4}{|c|}{ S355-S690-G50 } & & & & \\
\hline $1-072$ & 590,0 & 1173 & 503 & $1-095$ & 488,2 & 1241 & 393 & & & & \\
\hline $1-074$ & 600,8 & 1429 & 420 & $1-097$ & 579,9 & 1495 & 388 & & & & \\
\hline
\end{tabular}

Wie in Kapitel 6.4 erläutert, wurde, wenn möglich, die Tragfähigkeit mit Zugversuchen bestimmt. Bei den Versuchen mit langen Nähten waren Zugversuche nicht möglich, die Traglasten wurden mit Druckversuchen bestimmt. Abbildung 6.1 stellt die Versuche an Flankenkehlnahtverbindungen gleicher Grund- und Schweißzusatzwerkstoffe S460-G46 und S690-G69 dar. Es wird zwischen Zug- und Druckversuchen unterschieden. Es zeigt sich ein anderes Verhalten der Versuchskörper unter Druckbelastung als unter Zugbelastung, da eventuell Einflüsse aus Biegung vorhanden waren. Infolge der Handschweißung kann außerdem nicht ausgeschlossen werden, dass sich andere Schweißparameter ergeben haben. Darum werden die Druckversuche in der statistischen Auswertung nicht verwendet.

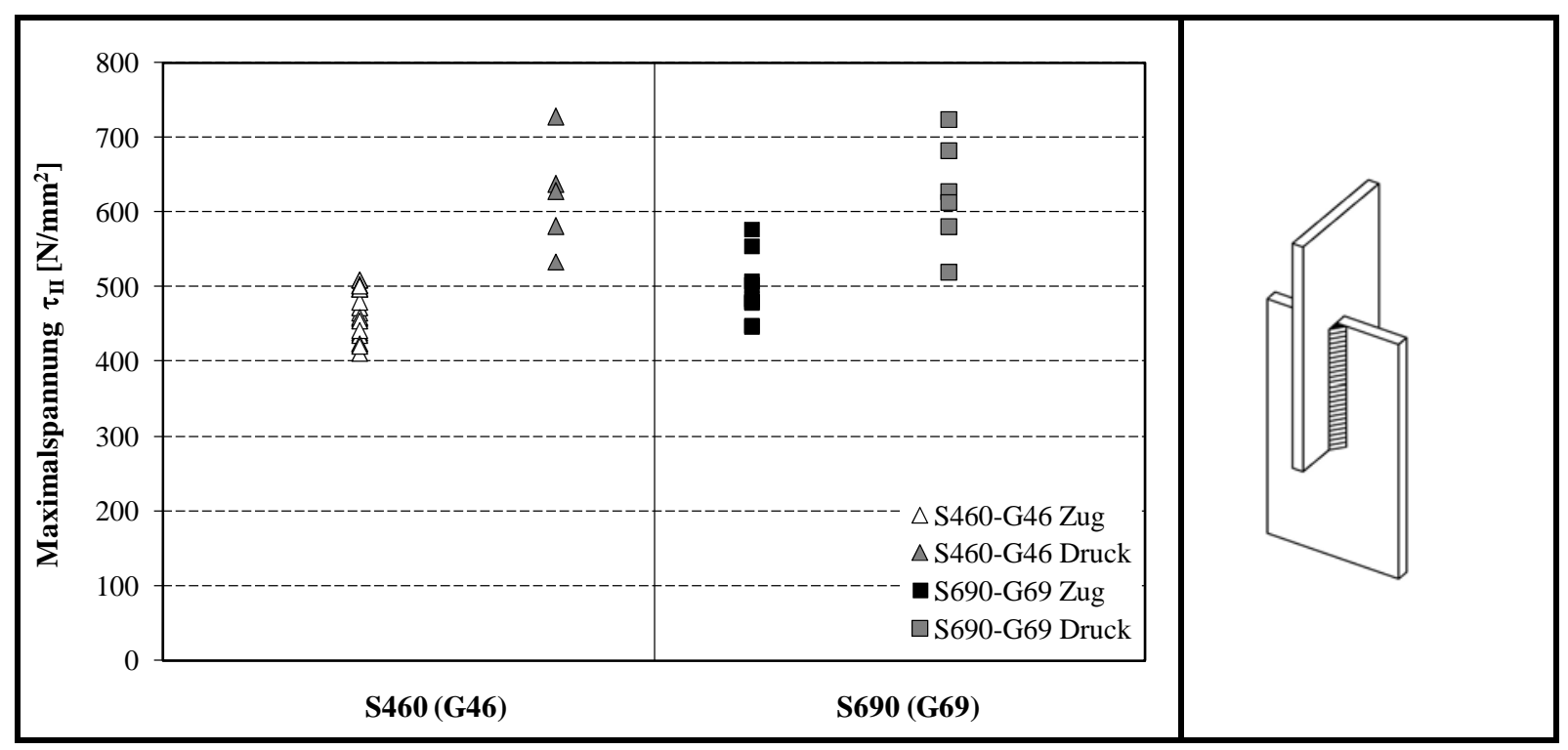

Abbildung 6.1: Einfluss von Zug- und Druckbelastung auf die Tragfähigkeit der Verbindungen S460-G46 und S690-G69 


\subsection{Flankenkehlnahtverbindungen mit gleichen Grund- und zugehörigen Schweißzusatzwerkstoffen}

\subsubsection{Einfluss der Stahlsorte}

Um den Einfluss der Stahlsorte zu bestimmen, wurden Versuche an Versuchskörpern mit zwei gleichen Grundwerkstoffen durchgeführt. Die verwendeten Schweißzusatzwerkstoffe wurden gemäß Festigkeitsklasse nach Lieferzeugnis so gewählt wie die nominelle Festigkeit des Grundwerkstoffs. Bei sonst gleichen Bedingungen wurde die Stahlsorte variiert: S355-G42, S460-G46 und S690-G69. Somit entstanden Versuchskörper mit gleichen Grund- und zugehörigen Schweißzusatzwerkstoffen. Abbildung 6.2 zeigt als Versuchsergebnis den Vergleich der Tragfähigkeiten der Schweißverbindungen unterschiedlicher Stahlsorten. Die Versuche mit dem Grundwerkstoff S460 zeigen eine höhere Tragfähigkeit als die Versuche mit dem Grundwerkstoff S355. Zwischen den Versuchen mit dem Grundwerkstoff S690 und den Versuchen mit dem Grundwerkstoff S460 zeigt sich nur eine geringe Steigerung der Tragfähigkeit.

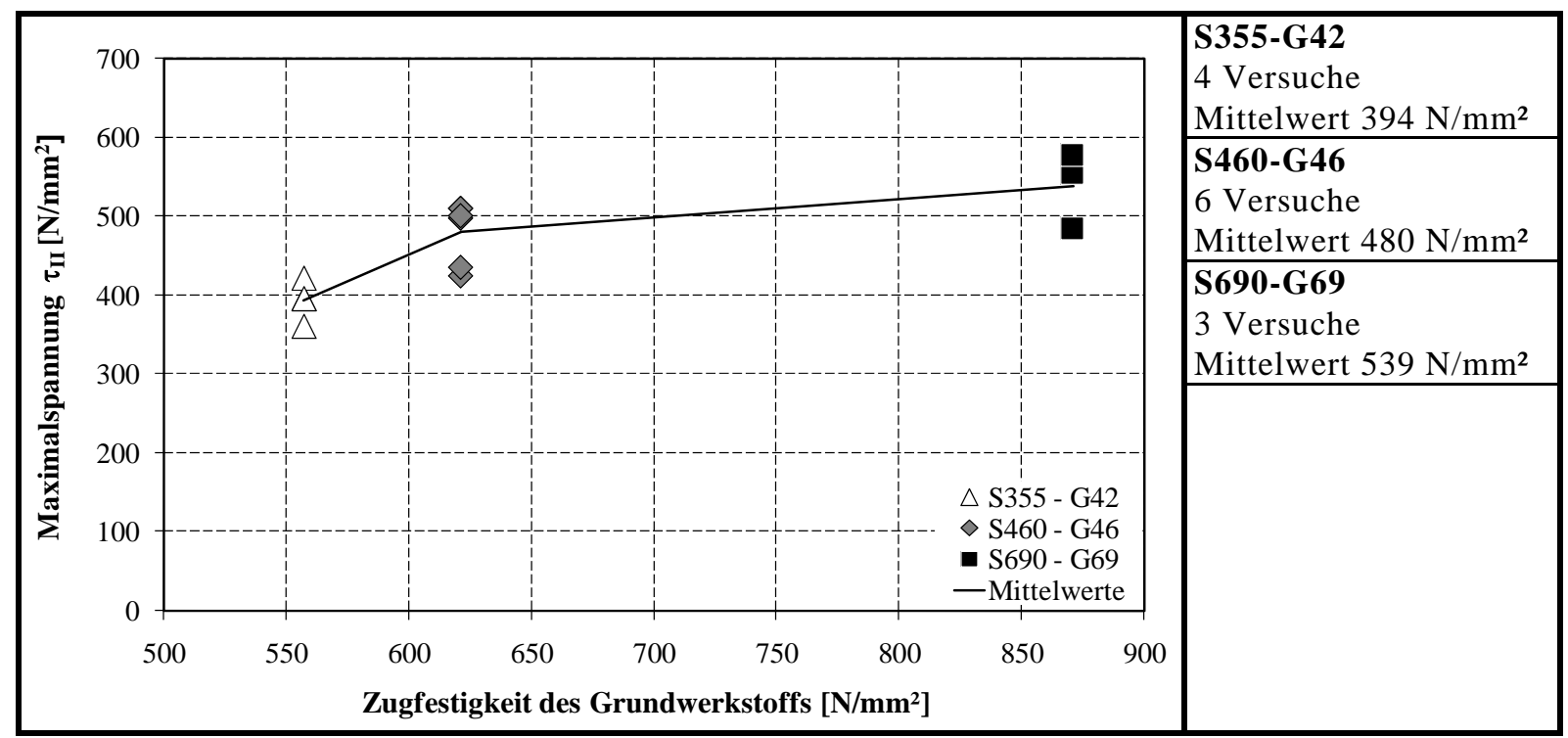

Abbildung 6.2: Einfluss der Stahlsorte auf die Tragfähigkeit beim S355-G42, S460-G46 und S690-G69

\subsubsection{Naht- und Blechdickeneinfluss}

An Versuchskörpern gleicher Grundwerkstoffe wurde unter Verwendung eines zugehörigen Schweißzusatzwerkstoffs die Blech- und Nahtdicke an den Stahlsorten S460 und S690 variiert. Abbildung 6.3 zeigt den Einfluss dieser Parameter auf die Tragfähigkeit der Schweißverbindungen. Dargestellt sind die Versuche mit einem Verhältnis von Blechdicke t zu Nahtdicke a von t/a $=15 \mathrm{~mm} / 4 \mathrm{~mm}$ (einlagige Referenzversuche) und t/a $=50 \mathrm{~mm} / 9 \mathrm{~mm}$ (1 bzw. 2 mehrlagige Versuche). Es zeigen sich bei allen Versuchen sehr ähnliche Tragfähigkeiten. Ein signifikanter Einfluss der Naht- und Blechdicke auf die Tragfähigkeit kann auch aufgrund der großen Streuung nicht festgestellt werden. 


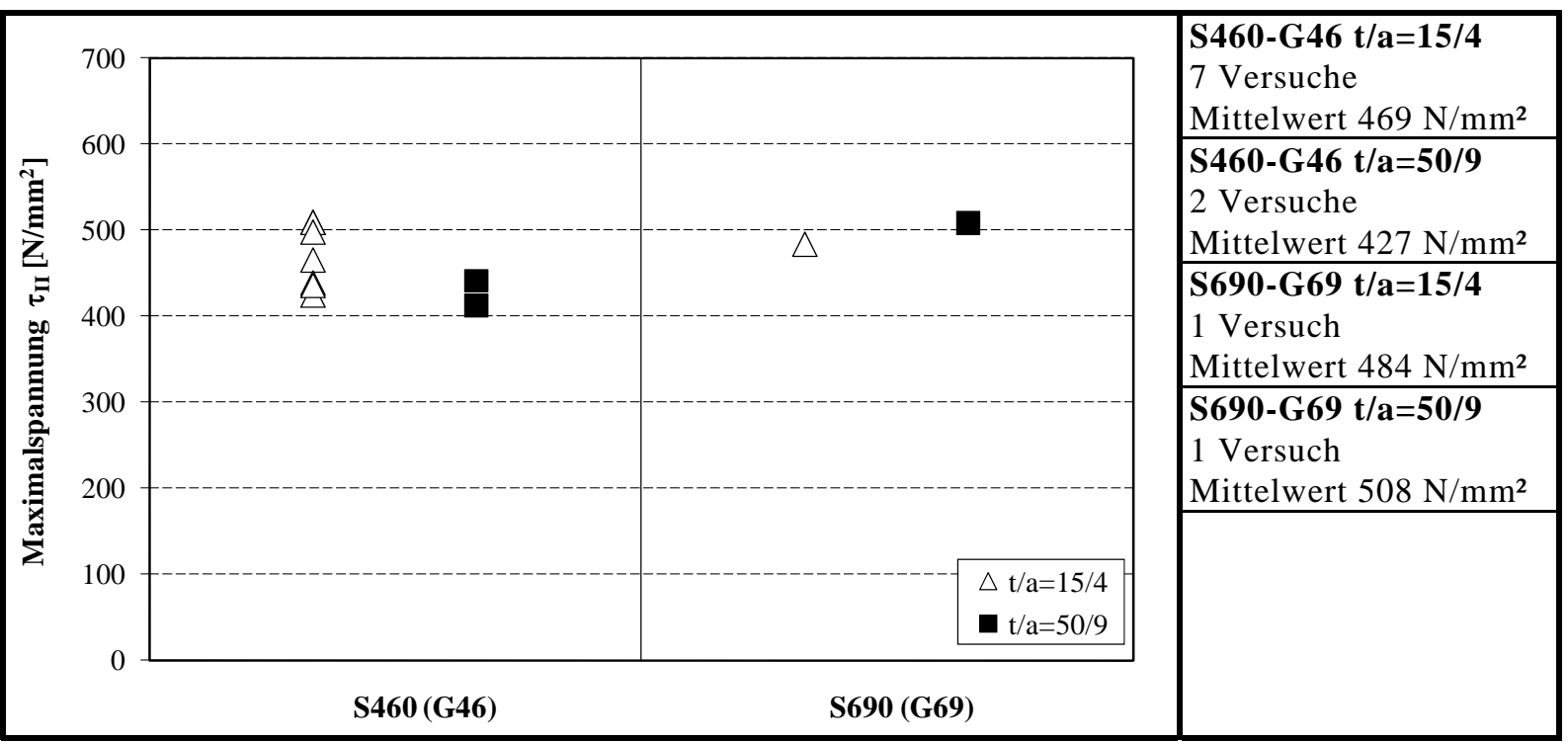

Abbildung 6.3: Einfluss der Blechdicke $t$ und der Nahtdicke a auf die Tragfähigkeit am S460-G46 und S690-G69

\subsubsection{Nahtlängeneinfluss}

An Versuchskörpern gleicher Grundwerkstoffe wurde unter Verwendung eines zugehörigen Schweißzusatzwerkstoffs die Nahtlänge als Verhältnis Nahtlänge L zur Nahtdicke a bei den Stahlsorten S460 und S690 variiert. In Abbildung 6.4 sind Versuche mit einem Nahtlängen-Nahtdickenverhältnis L/a von 50, 100 und 150 dargestellt. Bei Schweißverbindungen mit der Stahlsorte S460 lassen sich keine eindeutigen Tendenzen in der Tragfähigkeit feststellen. Bei Schweißverbindungen mit der Stahlsorte S690 zeigt sich eine abnehmende mittlere Maximalspannung $\tau_{\text {II }}$ bei zunehmender Nahtlänge. In Abbildung 6.4 zeigt sich eine deutlich geringere Duktilität bei den Versuchen mit der Stahlsorte S690 im Vergleich zu den Versuchen an Versuchskörpern mit der Stahlsorte S460. Für die Versuche am Grundwerkstoff S460 zeigt sich nur eine geringe Abnahme der Verformungsfähigkeit bei langen Nähten. Die Versuchsergebnisse weisen eine sehr große Streuung auf, da jeweils die beiden Versuche gleicher Werkstoffe und Nahtlänge bei verschiedenen Fertigern geschweißt wurden. Eindeutige Tendenzen können nicht getroffen werden, weil die Druckversuche zusätzlich durch Biegung beansprucht waren.

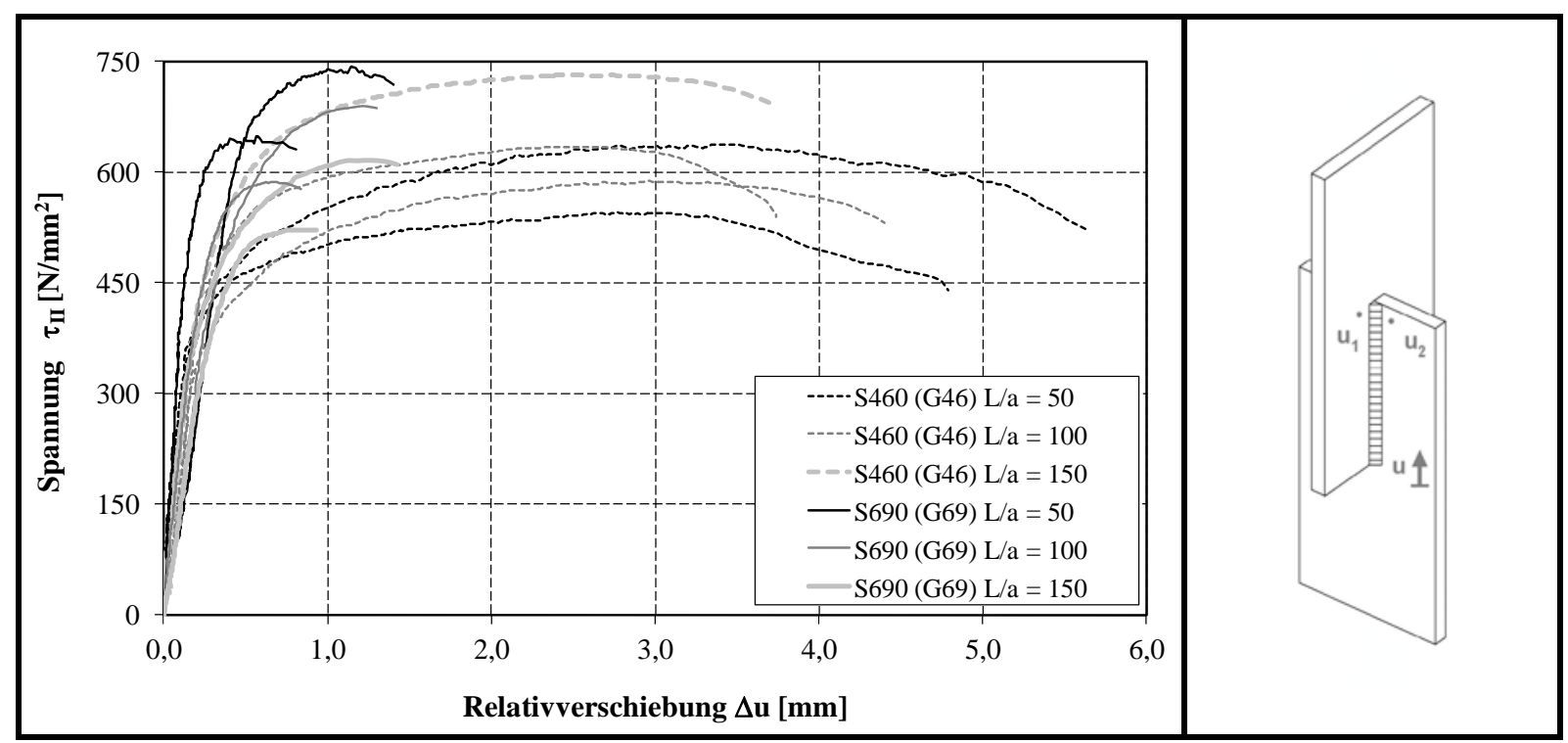

Abbildung 6.4: Einfluss der Nahtlänge auf das Verformungsverhalten beim S460-G46 und S690-G69 


\subsubsection{Einfluss der Schweißparameter}

Der Einfluss der Schweißparameter wurde durch Variation der Schweißgeschwindigkeit an Versuchen mit dem Grundwerkstoff S460 und S690 untersucht. Mit der Variation der Schweißgeschwindigkeit ändert sich neben der der Streckenenergie E auch die Nahtdicke. Abbildung 6.5 zeigt den Vergleich der Tragfähigkeiten zwischen den nach Vorgabe geschweißten Versuchskörpern $(1,5 \mathrm{~kJ} / \mathrm{mm})$ und den Versuchskörpern mit Variation der Streckenenergie.

An den Versuchen mit dem Grundwerkstoff S460 wurde innerhalb des Arbeitsfensters sowohl eine erhöhte $(1,8 \mathrm{~kJ} / \mathrm{mm})$ als auch eine verringerte $(1,4 \mathrm{~kJ} / \mathrm{mm})$ Streckenenergie untersucht. Die Maximalspannungen sind sehr ähnlich. Innerhalb des Arbeitsfensters ist der Einfluss der Streckenenergie beim Stahl S460 begrenzt.

Bei den Versuchen mit dem Grundwerkstoff S690 wurde außerhalb des Arbeitsfensters eine verringerte Streckenenergie $(0,8 \mathrm{~kJ} / \mathrm{mm})$ untersucht. Man erkennt eine deutliche Steigerung der Tragfähigkeit bei Abnahme der Streckenenergie. Mit Abnahme der Streckenenergie ändern sich die Abkühlbedingungen, dadurch kann es, wie in Kapitel 2.4.6 beschrieben, zu Aufhärtungen und damit zu einer höheren Tragfähigkeit kommen. Damit einher geht ein Verlust an Verformungsfähigkeit. Eine ausführliche Erläuterung zu den Schweißparametern und dem Arbeitsfenster findet sich in Kapitel 2.5.

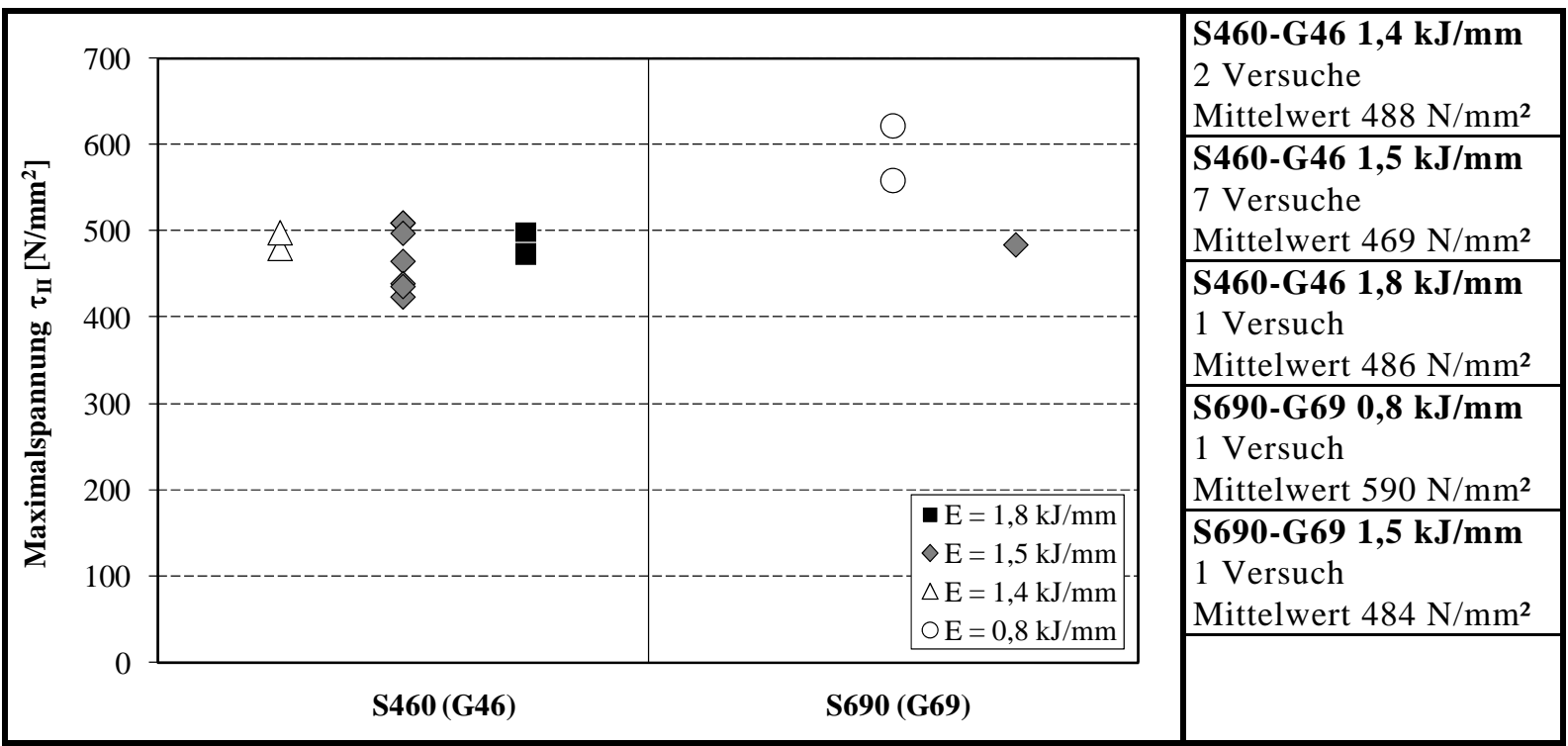

Abbildung 6.5: Einfluss der Streckenenergie auf die Tragfähigkeit beim S460-G46 und S690-G69

\subsubsection{Statistische Auswertung}

Wie in Kapitel 4.5 beschrieben, wurden die beschriebenen Versuche gleicher Grundwerkstoffe und zugehöriger Schweißzusatzwerkstoffe mit dem Bemessungsmodell nach EN 1993-1-8 (2005) statistisch ausgewertet. Die statistische Auswertung wurde sowohl in Abhängigkeit der Zugfestigkeit des Grundwerkstoffs als auch in Abhängigkeit der Festigkeit des Schweißzusatzwerkstoffs durchgeführt.

Nach Beurteilung der Versuchsergebnisse in Abhängigkeit der Schweißparameter standen für die statistische Auswertung 20 Versuche mit Flankenkehlnähten mit der Werkstoffkombination S460 und einem Schweißzusatzwerkstoff mit der nominellen Streckgrenze $\mathrm{f}_{\mathrm{y}}=460 \mathrm{~N} / \mathrm{mm}^{2}$ zur Verfügung, vgl. Tabelle 6.8.

Mit der Werkstoffkombination S690 und einem Schweißzusatzwerkstoff mit der nominellen Streckgrenze $\mathrm{f}_{\mathrm{y}}=690 \mathrm{~N} / \mathrm{mm}^{2}$ wurden 9 Versuchsergebnisse ermittelt, die statistisch ausgewertet wurden, wie in Tabelle 6.9 zusammengefasst ist. 
Tabelle 6.8: Statistische Auswertung der Flankenkehlnahtverbindungen S460 bezogen auf die Festigkeit des Grundwerkstoffs (links) oder des Schweißzusatzwerkstoffs (rechts)

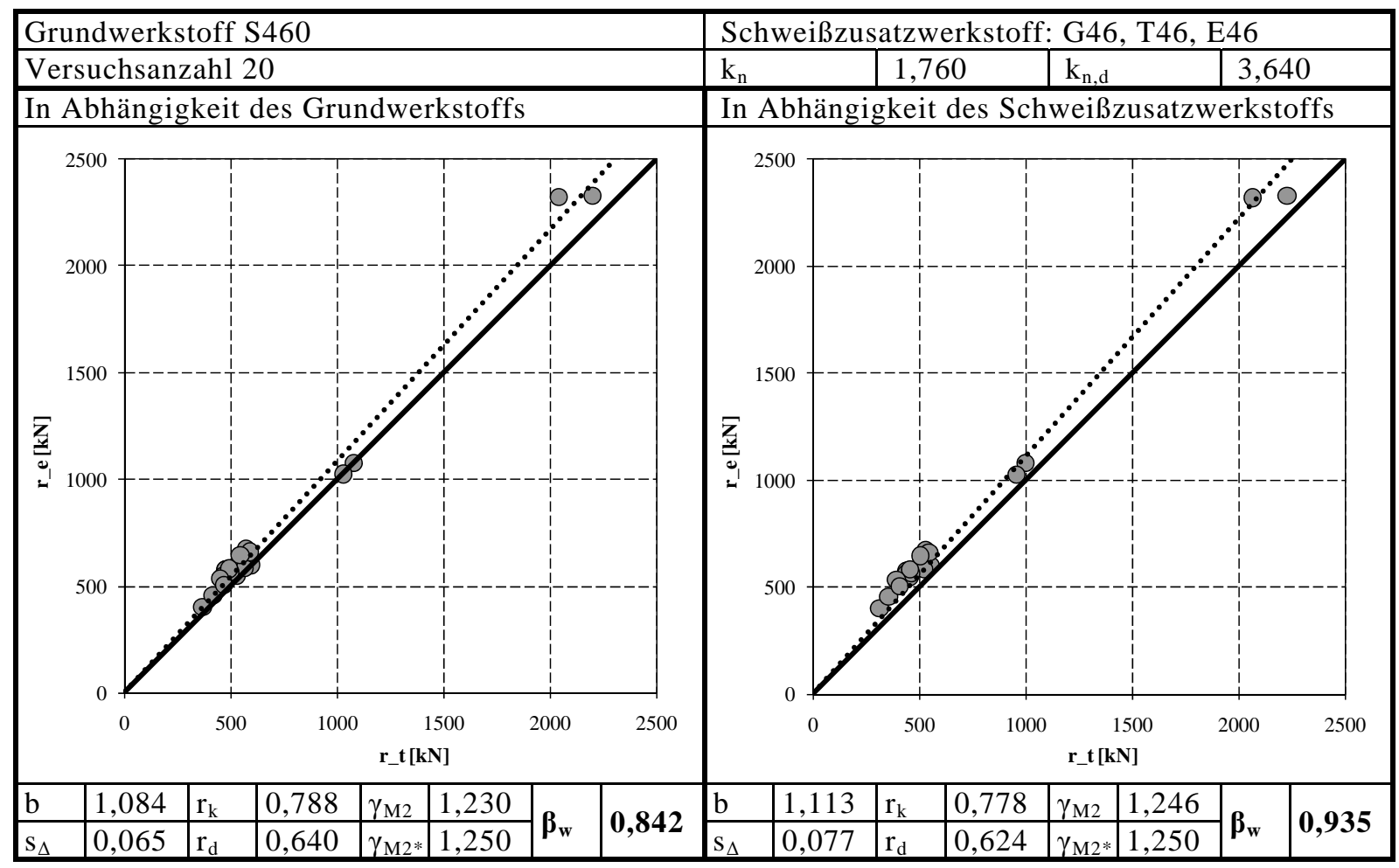

Tabelle 6.9: Statistische Auswertung der Flankenkehlnahtverbindungen S690 bezogen auf die Festigkeit des Grundwerkstoffs (links) oder des Schweißzusatzwerkstoffs (rechts)

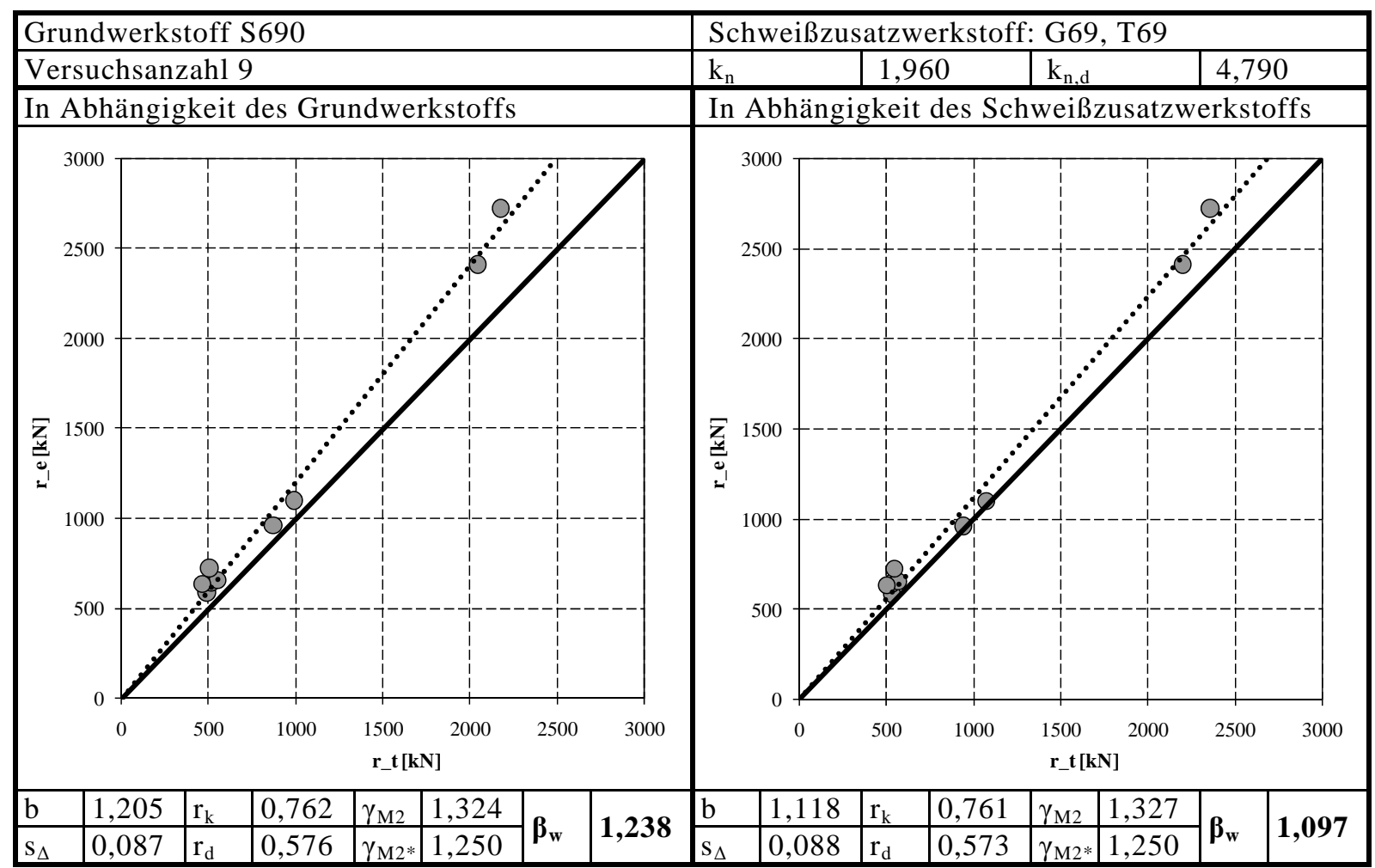

Einen Überblick über die Ergebnisse der statistischen Auswertung gibt Tabelle 6.10. Dargestellt sind die Ergebnisse der statistischen Auswertung für die beiden Werkstoffkombinationen S460-G/T/E46 und S690-G/T69. Für die Verbindungen S460-G/T/E46 wurden viele verschiedene Werkstoffkombinationen verwendet, die unterschiedliche Streuungen der Eingangsparameter provozieren. Damit ergeben sich unterschiedliche Bemessungsgrenzspannungen je nach dem, ob die Tragfähigkeit auf die Festigkeit des Schweißzusatzwerkstoffs oder auf die Festigkeit des Grundwerkstoffs bezogen wird. 
Tabelle 6.10: Bemessungsgrenzspannung für Flankenkehlnähte

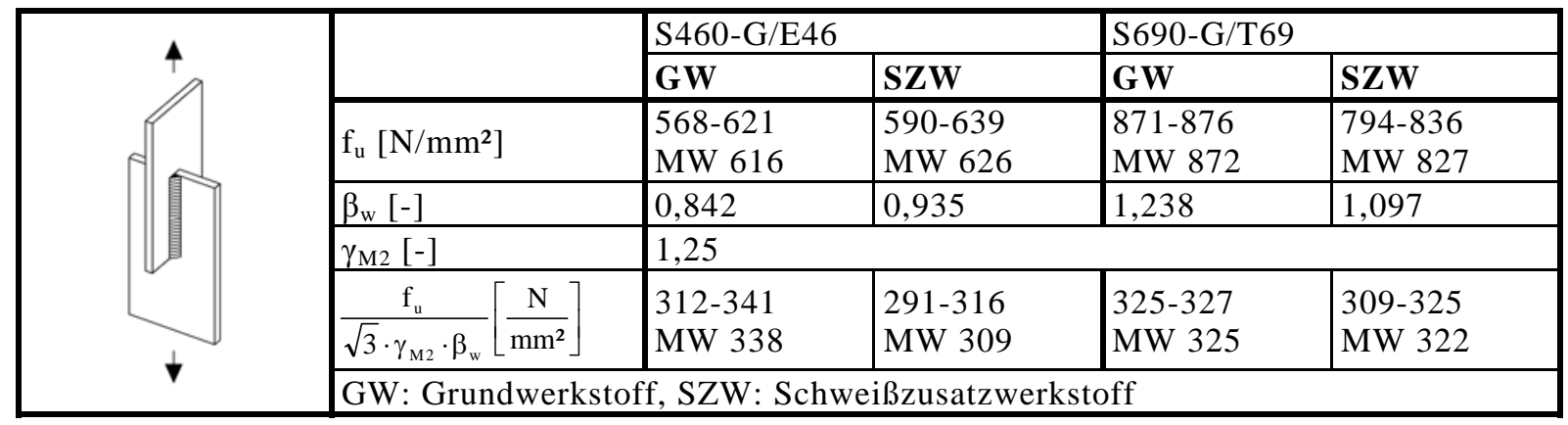

Für die Verbindungen S460-G/T/E46 zeigt der Beiwert $\beta_{\mathrm{w}}<1,0$, dass die Festikeit der Kehlnaht höher als die Festigkeit des Schweißzusatzwerkstoffs liegt. Für die Verbindungen S690-G/T69 liegt die Festigkeit der Kehlnaht unterhalb der Festigkeit des Schweißzusatzwerkstoffs, wie der Beiwert $\beta_{\mathrm{w}}>1,0$ angibt.

Infolge der unterschiedlichen Überfestigkeiten verringern sich die Unterschiede in der Tragfähigkeit zwischen S460-G/T/E46 und S690-G/T69, wie Abbildung 6.6 zeigt. Dargestellt sind die Versuchsergebnisse bezogen auf die Bemessungsgrenzspannungen $\tau_{\mathrm{Rd}}=309 \mathrm{~N} / \mathrm{mm}^{2}$ und $\tau_{\mathrm{Rd}}=322$ $\mathrm{N} / \mathrm{mm}^{2}$ in Abhängigkeit vom Schweißzusatzwerkstoff. Die Mittelwerte steigen von $465 \mathrm{~N} / \mathrm{mm}^{2}$ für S460-G/T/E46 auf $498 \mathrm{~N} / \mathrm{mm}^{2}$ für S690-G/T69 um 7 \%, die Bemessungswerte steigen um $4 \%$.

Der Vergleich der Ergebnisse wird durch die vielen Einflussparameter erschwert. Bei diesen Versuchen wurden nicht nur verschiedene Grund- und Schweißzusatzwerkstoffe gleicher Nenngüte verwendet, sondern es wurde auch bei unterschiedlichen Fertigern hergestellt. Insgesamt folgt daraus eine sehr große Streuung, wie Abbildung 6.6 zeigt.

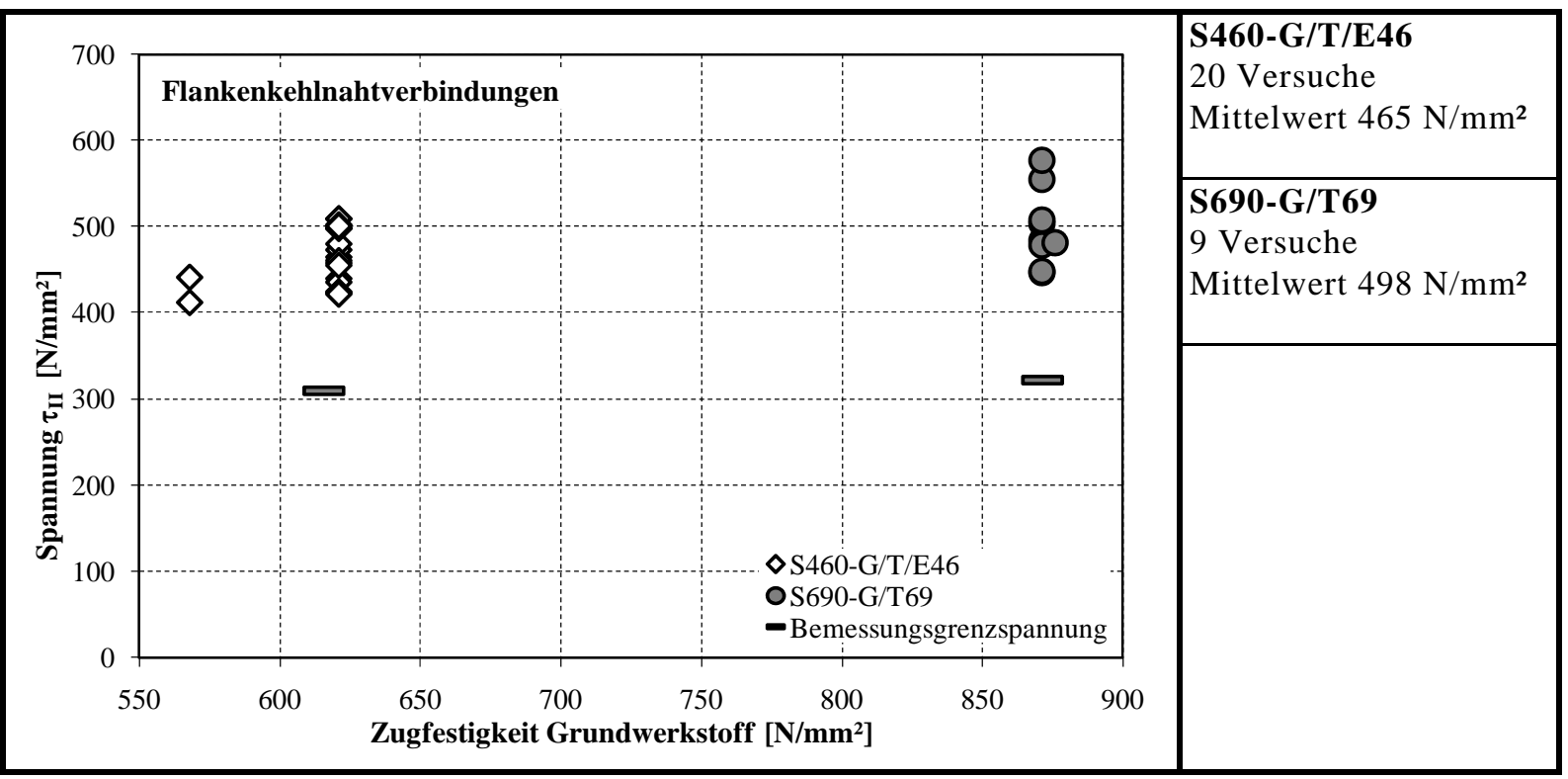

Abbildung 6.6: Vergleich der Ergebnisse der Flankenkehlnahtverbindungen S460-G/T/E46 und S690G/T69 


\subsection{Flankenkehlnahtverbindungen mit gleichen Grund- und Variation der Schweißzusatzwerkstoffe}

An Versuchskörpern gleicher Grundwerkstoffe wurden bei den Stahlsorten S460 und S690 Untersuchungen zum Einfluss des Schweißzusatzwerkstoffs auf die Tragfähigkeit der Schweißverbindungen durchgeführt. Für den Grundwerkstoff S355 wurden Tragfähigkeitsuntersuchungen mit einem Schweißzusatzwerkstoff der Festigkeitsklasse G42 und einem Schweißzusatzwerkstoff höherer Festigkeitsklasse G46 verglichen. Für den Grundwerkstoff S460 wurden die Ergebnisse von Versuchen mit einem Schweißzusatzwerkstoff geringerer Festigkeitsklasse G42, gleicher Festigkeitsklasse G46 und höherer Festigkeitsklasse G69 analysiert. Für den Grundwerkstoff S690 standen Versuche mit einem Schweißzusatzwerkstoff mit deutlich geringerer Festigkeit G46 und leicht geringerer Festigkeit G62 im Vergleich mit Versuchen mit passendem Schweißzusatzwerkstoff G69 zur Verfügung. In Abbildung 6.7 sind diese Versuchsergebnisse dargestellt. Mit zunehmender Festigkeit des Schweißzusatzwerkstoffs nimmt die Tragfähigkeit leicht zu. Eindeutige Aussagen lassen sich auf Grund der großen Streuung nicht treffen.

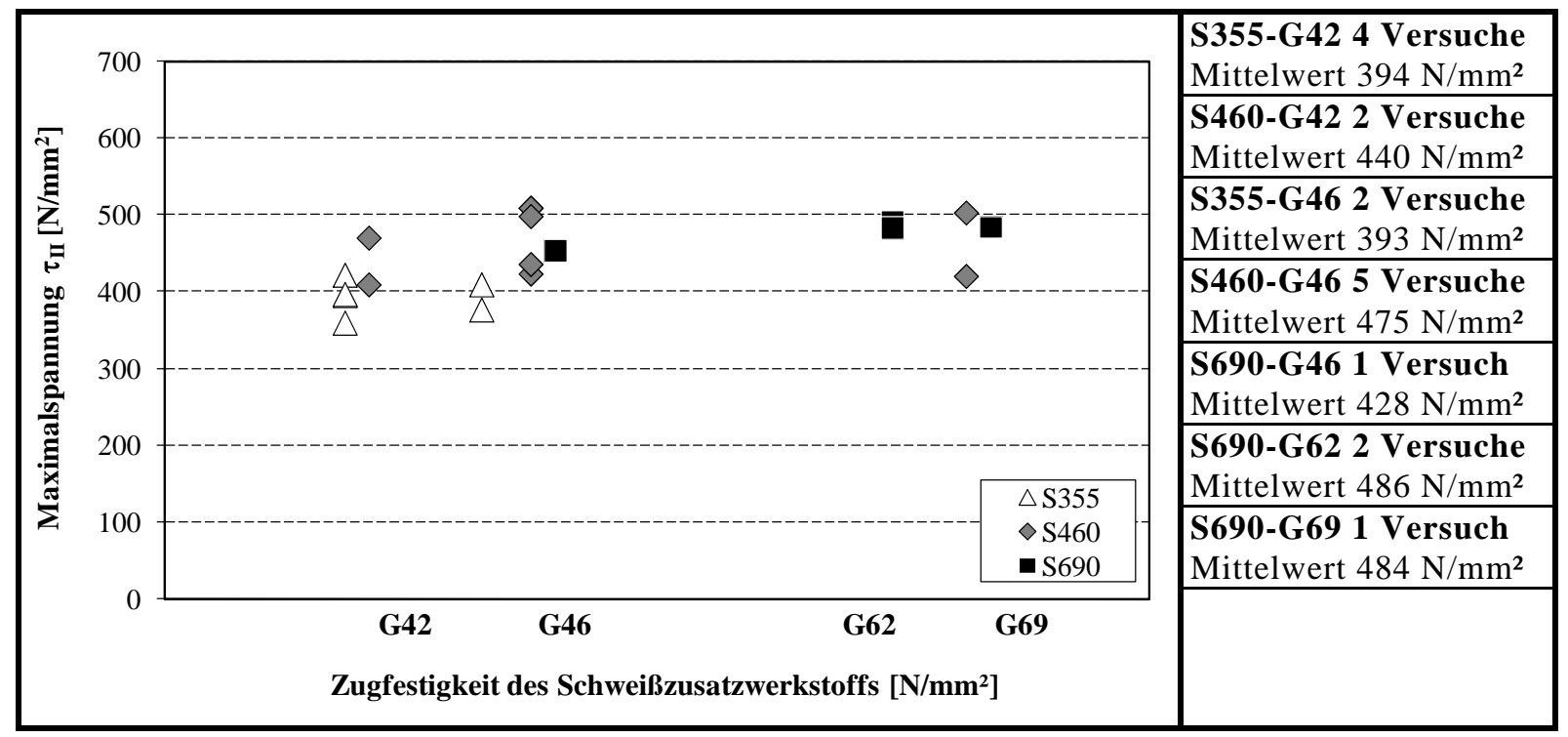

Abbildung 6.7: Einfluss des Schweißzusatzwerkstoffs auf die Tragfähigkeit 


\subsection{Flankenkehlnahtverbindungen mit unterschiedlichen Grundwerk- stoffen}

Es wurden Versuchskörper mit zwei unterschiedlichen Grundwerkstoffen (Mischverbindungen) hergestellt, um den Einfluss dieser Mischverbindungen auf die Tragfähigkeit der Schweißverbindungen zu ermitteln. In jeder Werkstoffkombination wurde der Schweißzusatzwerkstoff (SZW) variiert. Die Schweißzusatzwerkstoffe wurden gemäß Festigkeitsklasse nach Lieferzeugnis so gewählt, dass sie zum einen zur Festigkeit des niederfesteren Grundwerkstoffs GW1 (SZW $\cong$ GW1) passten, und zum anderen zur Festigkeit des höherfesten Grundwerkstoffs GW2 (SZW GW2). Zusätzlich wurde ein Schweißzusatzwerkstoff gewählt, dessen Festigkeitsklasse zwischen der beider Grundwerkstoffe liegt (GW1 $<$ SZW $<$ GW2). Abbildung 6.8 zeigt die Tragfähigkeit der verschiedenen Kombinationen. Mit zunehmender Festigkeit des Schweißzusatzwerkstoffs steigt die Tragfähigkeit der Verbindung tendenziell leicht an. Da die Tragfähigkeitsunterschiede bei den Verbindungen gleicher Grundwerkstoffe schon gering sind, ist es auf Grund der großen Streuung schwierig, eindeutige Tendenzen abzulesen.

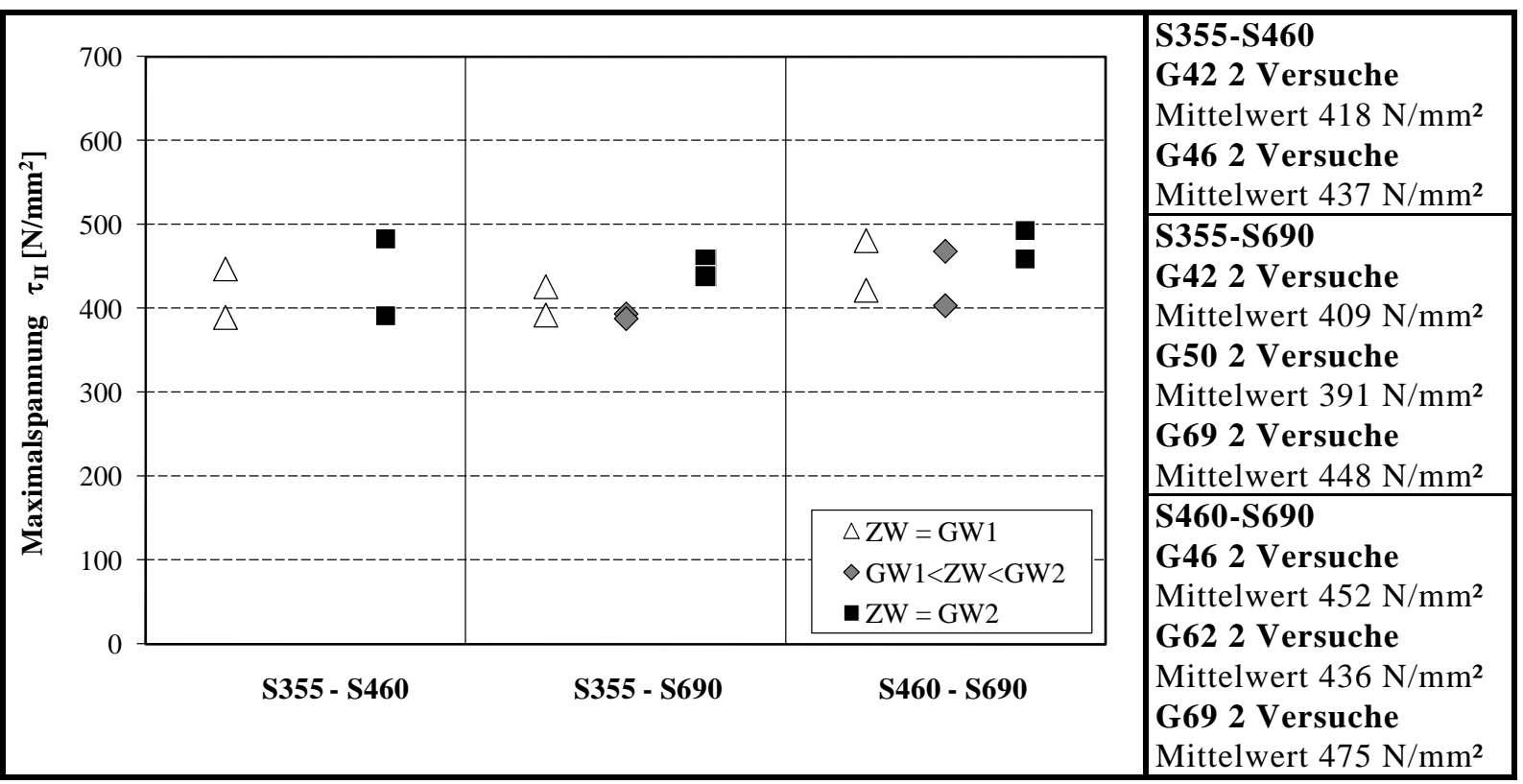

Abbildung 6.8: Einfluss des Schweißzusatzwerkstoffs auf die Tragfähigkeit bei Mischverbindungen 


\section{9 Überblick über die Versuchsergebnisse an Kreuzstößen}

In Tabelle 6.11 sind die Versuchsergebnisse aller durchgeführten Traglastversuche an Kreuzstößen dargestellt. Für alle Versuchskörper gilt, dass der Bruch stets in der Naht verlief, der Bruchwinkel war sehr steil. Die Bruchfläche wurde nicht nach dem Versuch ausgemessen, sondern es wurde die Nahtfläche $A_{E C}$ bestimmt. Die Werkstoffkombinationen der einzelnen Versuche sind im Anhang zusammen mit den gemessenen Schweißparametern Streckenenergie, Vorwärmtemperatur und Abkühlzeit $t_{8 / 5}$ aufgeführt. Für die statistische Auswertung wurden nur die Versuche verwendet, deren Abkühlzeit im Bereich von 4 - 15 Sekunden lag. Aufgrund der manuellen Schweißung wurden keine gleichmäßigen Bedingungen erreicht, so dass einige Versuchsergebnisse nicht verwendet werden können, diese sind gekennzeichnet.

Tabelle 6.11: Versuchsergebnisse Kreuzstöße

\begin{tabular}{|c|c|c|c|c|c|c|c|c|c|c|c|}
\hline 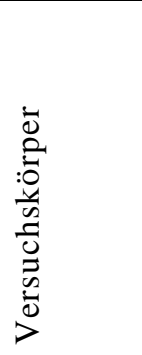 & 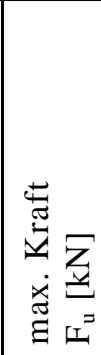 & 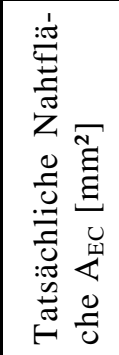 & 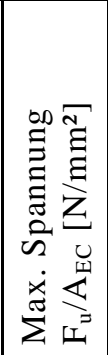 & 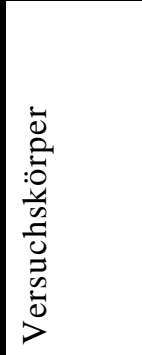 & 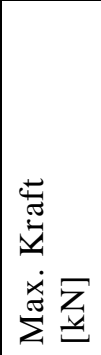 & 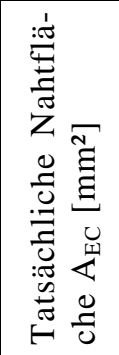 & 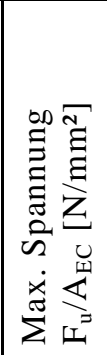 & 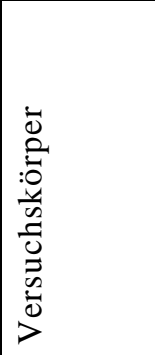 & 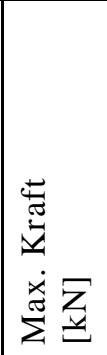 & 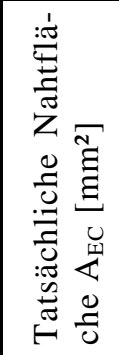 & 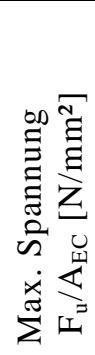 \\
\hline \multicolumn{4}{|c|}{ S355-S355-G42 } & \multicolumn{4}{|c|}{ S460-S460-G46 } & \multicolumn{4}{|c|}{ S690-S690-G69 } \\
\hline $2-001 / 1$ & 241,5 & 370,2 & 652 & $2-019 / 2$ & 294,6 & 436,5 & 675 & $2-031 / 2$ & 382,0 & 520,2 & 734 \\
\hline $2-001 / 2$ & 252,3 & 363,0 & 695 & $2-019 / 3$ & 312,0 & 456,3 & 684 & $1 / 3$ & 369,5 & 532,3 & 694 \\
\hline $2-001 / 3$ & 241,7 & 362,0 & 668 & $2-019 / 5$ & 300,2 & 447,8 & 670 & $2-031 / 5$ & 363,3 & 550,3 & 660 \\
\hline \multicolumn{4}{|c|}{ S355-S460-G46 } & $2-021 / 3$ & 280,4 & 422,5 & 664 & $2-031 / 7$ & 340,8 & 499,8 & 682 \\
\hline $2-002 / 1$ & 264,7 & 380,5 & 696 & 2-021/5 & 275,2 & 407,9 & 675 & $2-031 / 8$ & 340,4 & 493,5 & 690 \\
\hline $2-002 / 2$ & 264,2 & 391,9 & 674 & & 259,9 & 397,6 & 654 & 2-W31/3 & 377,4 & 537,5 & 702 \\
\hline $2-002 / 3$ & 268,3 & 385,2 & 696 & $2-021 / 8$ & 271,1 & 400,6 & 677 & $1 / 5$ & 381,9 & 549,7 & 695 \\
\hline \multicolumn{4}{|c|}{ S355-S690-G42 } & 2-022/2 & 313,4 & 479,0 & 654 & 2-W31/7 & 367,8 & 540,8 & 680 \\
\hline $2-003 / 1$ & 247,3 & 395,0 & 626 & $2-0$ & 318,0 & 513,1 & 620 & $2-W$ & 378,1 & 531,0 & 712 \\
\hline & 248,5 & 399,0 & 623 & & 326,1 & 513,1 & 636 & & 324,9 & 376,9 & 862 \\
\hline $2-003 / 3$ & 244,5 & 371,2 & 659 & $2-022 / 8$ & 317,5 & 476,3 & 667 & $2-032 / 3^{*}$ & 336,2 & 382,2 & 880 \\
\hline \multicolumn{4}{|c|}{ S355-S690-G69 } & \multicolumn{4}{|c|}{ S460-S460-G42 } & $2-032 / 5^{*}$ & 342,9 & 361,9 & 947 \\
\hline $2-005 / 1$ & 311,8 & 400,6 & 779 & $2-023 / 3$ & 266,6 & 449,8 & 593 & $2-032 / 7^{*}$ & 353,1 & 393,9 & 896 \\
\hline $2-005 / 2$ & 285,7 & 396,0 & 721 & & 270,4 & 425,2 & 636 & $2-032 / 8^{*}$ & 345,2 & 380,7 & 907 \\
\hline $2-005 / 3$ & 292,9 & 393,9 & 744 & 2-023/7 & 274,3 & 425,6 & 644 & \multicolumn{4}{|c|}{ S690-S690-G46 } \\
\hline \multicolumn{4}{|c|}{ S460-S690-G46 } & 2-023/8 & 270,6 & 403,6 & 670 & $2-034 / 2$ & 280,9 & 388,0 & 724 \\
\hline $2-006 / 1$ & 292,2 & 389,5 & 750 & $2-024 / 2$ & 274,2 & 417,5 & 657 & $2-034 / 3$ & 284,5 & 378,2 & 752 \\
\hline $2-006 / 2$ & 293,5 & 400,2 & 733 & $2-02$ & 286,9 & 415,0 & 691 & $1 / 5$ & 318,2 & 418,8 & 760 \\
\hline $2-006 / 3$ & 290,4 & 395,8 & 734 & $2-024 / 5$ & 293,9 & 400,0 & 735 & $4 / 7$ & 295,8 & 394,0 & 751 \\
\hline \multicolumn{4}{|c|}{ S460-S690-G69 } & $2-024 / 7$ & 292,1 & 395,8 & 738 & $2-034 / 8$ & 290,7 & 393,8 & 738 \\
\hline $2-008 / 1$ & 302,0 & 395,8 & 763 & $2-025 / 2$ & 300,7 & 430,6 & 698 & $2-035 / 2$ & 321,9 & 503,4 & 639 \\
\hline $2-008 / 2$ & 284,8 & 383,9 & 742 & $2-025 / 3$ & 289,6 & 405,4 & 714 & & 320,2 & 516,5 & 620 \\
\hline $2-008 / 3$ & 306,4 & 394,0 & 778 & $2-025 / 5$ & 282,0 & 426,3 & 662 & $2-035 / 5$ & 324,7 & 488,6 & 665 \\
\hline \multicolumn{4}{|c|}{ S460-S460-G69 } & $2-025 / 7$ & 288,1 & 430,1 & 670 & $2-035 / 7$ & 323,1 & 509,1 & 635 \\
\hline $2-020 / 3$ & 289,5 & 406,0 & 713 & 2-025/8 & 297,7 & 427,4 & 696 & $2-035 / 8$ & 306,0 & 492,0 & 622 \\
\hline $2-020 / 5$ & 282,0 & 385,3 & 732 & \multicolumn{4}{|c|}{ S690-S690-G69 } & $2-036 / 2$ & 322,4 & 482,0 & 669 \\
\hline $2-020 / 7$ & 281,1 & 404,7 & 694 & $2-030 / 3$ & 338,3 & 461,9 & 732 & $2-036 / 3$ & 316,0 & 486,8 & 649 \\
\hline $2-020 / 8$ & 278,9 & 389,8 & 715 & 2-030/5 & 336,9 & 462,0 & 729 & $2-036 / 5$ & 320,1 & 495,6 & 646 \\
\hline $2-026 / 2$ & 306,0 & 441,3 & 693 & $2-030 / 7$ & 314,5 & 470,7 & 668 & $2-036 / 7$ & 326,8 & 488,2 & 669 \\
\hline $2-026 / 3$ & 301,2 & 454,0 & 663 & 2-030/8 & 319,9 & 465,2 & 688 & $2-036 / 8$ & 334,1 & 476,9 & 701 \\
\hline $2-026 / 5$ & 285,6 & 477,4 & 598 & \multirow{2}{*}{\multicolumn{8}{|c|}{ * nicht für die statistische Auswertung verwendet }} \\
\hline $2-026 / 7$ & 286,8 & 451,4 & 636 & & & & & & & & \\
\hline
\end{tabular}




\subsection{Kreuzstöße mit gleichen Grund- und zugehörigen Schweißzusatz- werkstoffen (BUW)}

\subsubsection{Einfluss der Stahlsorte}

Zur Bestimmung des Einflusses der Stahlsorte auf die Tragfähigkeit von Kreuzstößen mit Stirnkehlnähten wurden Versuchskörper aus Blechen gleicher Grundwerkstoffe hergestellt. Die verwendeten Schweißzusatzwerkstoffe wurden so gewählt, dass die nominellen Festigkeiten der nominellen Festigkeit des Grundwerkstoffs entsprachen. In Abbildung 6.9 ist der Vergleich der Tragfähigkeiten der Schweißverbindungen unterschiedlicher Stahlsorten S355-G42, S460-G46 und S690-G69 bei sonst gleichen Bedingungen dargestellt. Zwischen den Versuchen mit dem Grundwerkstoff S355 und den Versuchen mit dem Grundwerkstoff S460 zeigt sich ein sehr geringer Anstieg der Tragfähigkeit. Die Versuche mit dem Grundwerkstoff S690 zeigen eine etwas höhere Tragfähigkeit aber auch eine größere Streuung als die Versuche mit dem Grundwerkstoff S460.

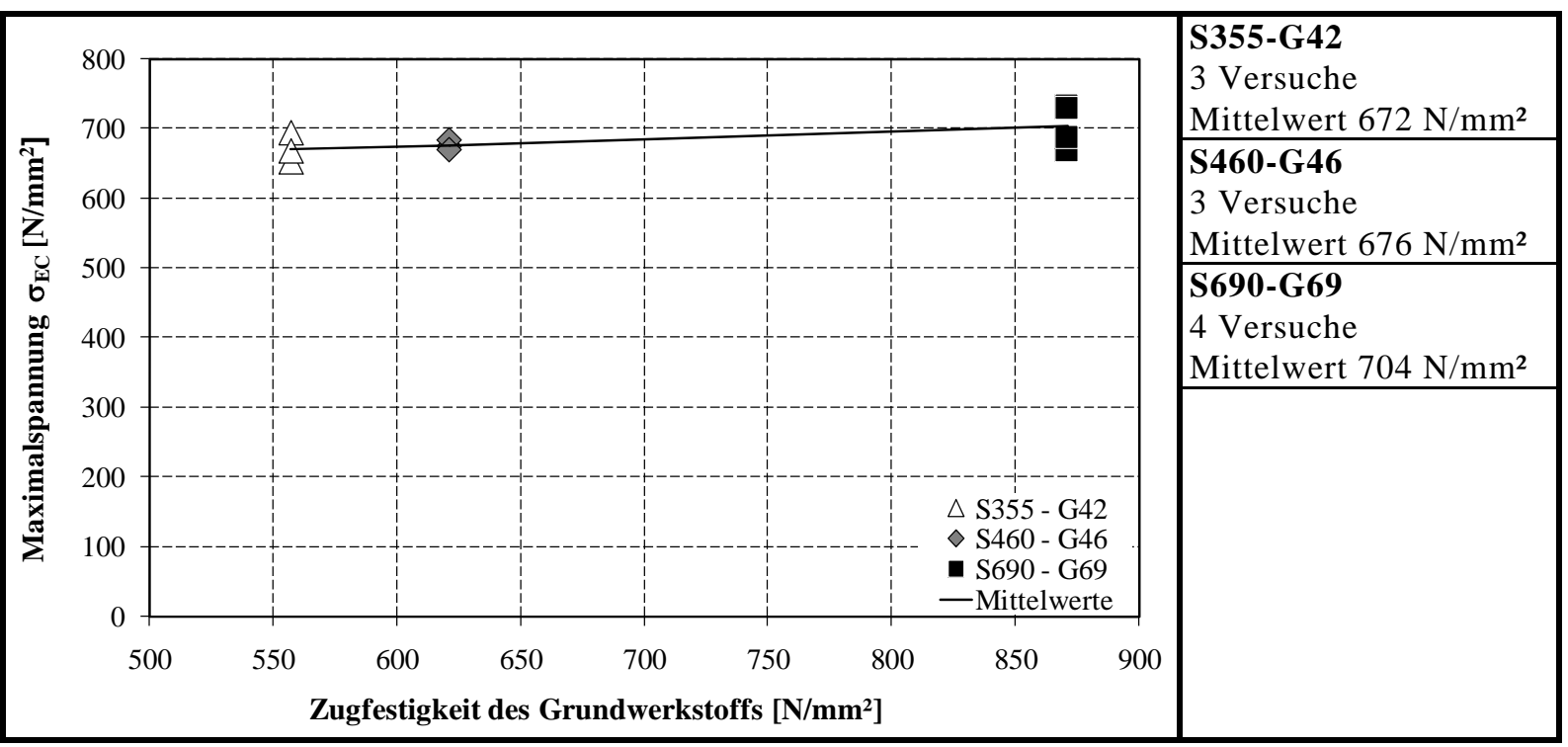

Abbildung 6.9: Einfluss der Stahlsorte auf die Tragfähigkeit beim S355-G42, S460-G46 und S690-G69

\subsubsection{Einfluss der Schweißparameter / Einfluss der Nahtdicke}

Die Schweißparameter wurden bei der Herstellung der Kreuzstöße variiert, um deren Einfluss auf die Tragfähigkeit der Verbindung zu untersuchen. Der entscheidende Parameter ist die Abkühlzeit $t_{8 / 5}$. Diese lässt sich über die Streckenenergie durch die Schweißparameter Strom, Spannung und Schweißgeschwindigkeit aber auch über die Vorwärmtemperatur ändern. Hier wurde als Parameter die Schweißgeschwindigkeit an Versuchen mit dem Grundwerkstoff S460 und S690 variiert, wodurch sich neben der Streckenenergie auch die Nahtdicke ändert.

Abbildung 6.10 zeigt den Vergleich der Tragfähigkeiten zwischen den nach Vorgabe geschweißten Versuchskörpern und den Versuchskörpern mit höherer oder geringerer Streckenenergie. Mit dem Grundwerkstoff S460 wurden neben Versuchskörpern mit der vorgegebenen Streckenenergie von $1,4 \mathrm{~kJ} / \mathrm{mm}$ sowohl Versuchskörper mit einer erhöhten $(1,8 \mathrm{~kJ} / \mathrm{mm})$ als auch einer verringerten $(1,2 \mathrm{~kJ} / \mathrm{mm})$ Streckenenergie innerhalb des Arbeitsfensters (s. Kapitel 2.5) gefertigt. Bei den Versuchen mit dem Grundwerkstoff S690 wurde neben der gewünschten Streckenenergie von 1,6 kJ/mm eine verringerte Streckenenergie $(1,1 \mathrm{~kJ} / \mathrm{mm})$ als auch eine erhöhte Streckenenergie $(1,9 \mathrm{~kJ} / \mathrm{mm})$ untersucht. Bei den Verbindungen mit dem Stahl S460 ergab sich eine gleichbleibende Tragfähigkeit trotz unterschiedlicher Abkühlbedingungen ein. Bei den Verbindungen mit dem Stahl S690 zeigt sich ein deutlicher Einfluss der Streckenenergie auf die Tragfähigkeit. Wie in Kapitel 2.4.6 beschrieben, führen die schnellen Abkühlbedingungen zu höheren Härten und damit höheren Tragfähigkeiten. Diese Versuche mit der höchsten Traglast $\left(\mathrm{S} 690 \mathrm{v}_{\max }\right.$ ) wurden mit einer Abkühlzeit von $3 \mathrm{~s}$ geschweißt 
und damit deutlich außerhalb des Arbeitsfensters von 5 - 15 s. Aus diesem Grund werden diese Versuche nicht für die statistische Auswertung verwendet.

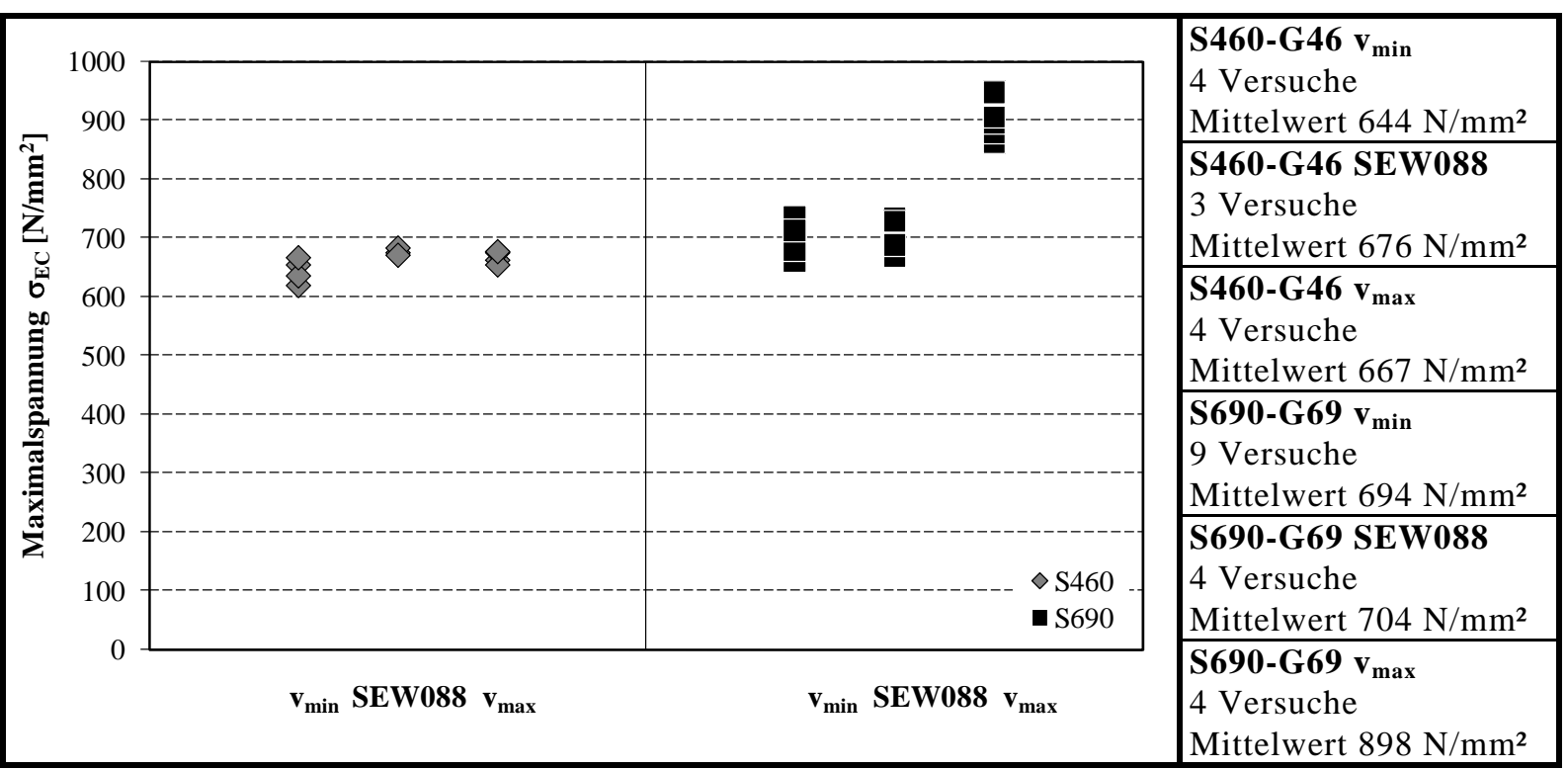

Abbildung 6.10: Einfluss der Streckenenergie auf die Tragfähigkeit beim S460-G46 und S690-G69

\subsubsection{Statistische Auswertung}

Die in Kapitel 6.10.1 und 6.10.2 vorgestellten Versuche gleicher Grund- und zugehöriger Schweißzusatzwerkstoffe wurden gemeinsam statistisch ausgewertet, wie in Kapitel 4.5 beschrieben. Dabei wurden nur die Versuchsergebnisse mit Schweißparameter innerhalb des Arbeitsfensters berücksichtigt.

Tabelle 6.12 zeigt die statistische Auswertung von 11 Versuchen mit der Werkstoffkombination S460 und einem Schweißzusatzwerkstoff mit der nominellen Streckgrenze $f_{y}=460$ N/mm².

Tabelle 6.12: Statistische Auswertung der Stirnkehlnahtverbindungen S460 bezogen auf die Festigkeit des Grundwerkstoffs (links) oder des Schweißzusatzwerkstoffs (rechts)

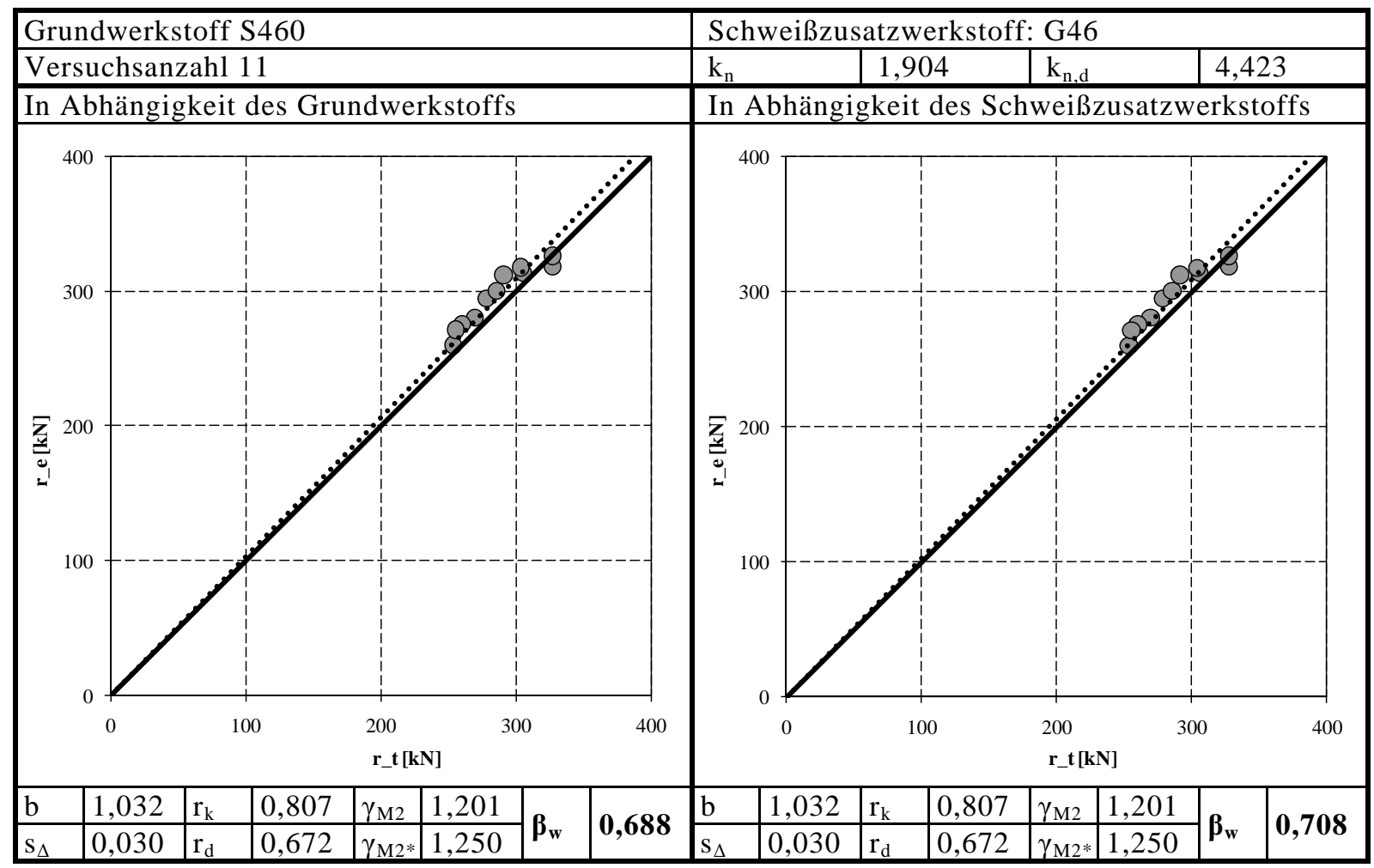


Mit der Werkstoffkombination S690 und einem Schweißzusatzwerkstoff mit der nominellen Streckgrenze $\mathrm{f}_{\mathrm{y}}=690 \mathrm{~N} / \mathrm{mm}^{2}$ wurden 13 Versuchsergebnisse ermittelt. Diese Versuchsergebnisse wurden statistisch ausgewertet, wie in Tabelle 6.13 zusammengefasst.

Tabelle 6.13: Statistische Auswertung der Stirnkehlnahtverbindungen $\mathbf{S 6 9 0}$ bezogen auf die Festigkeit des Grundwerkstoffs (links) oder des Schweißzusatzwerkstoffs (rechts)

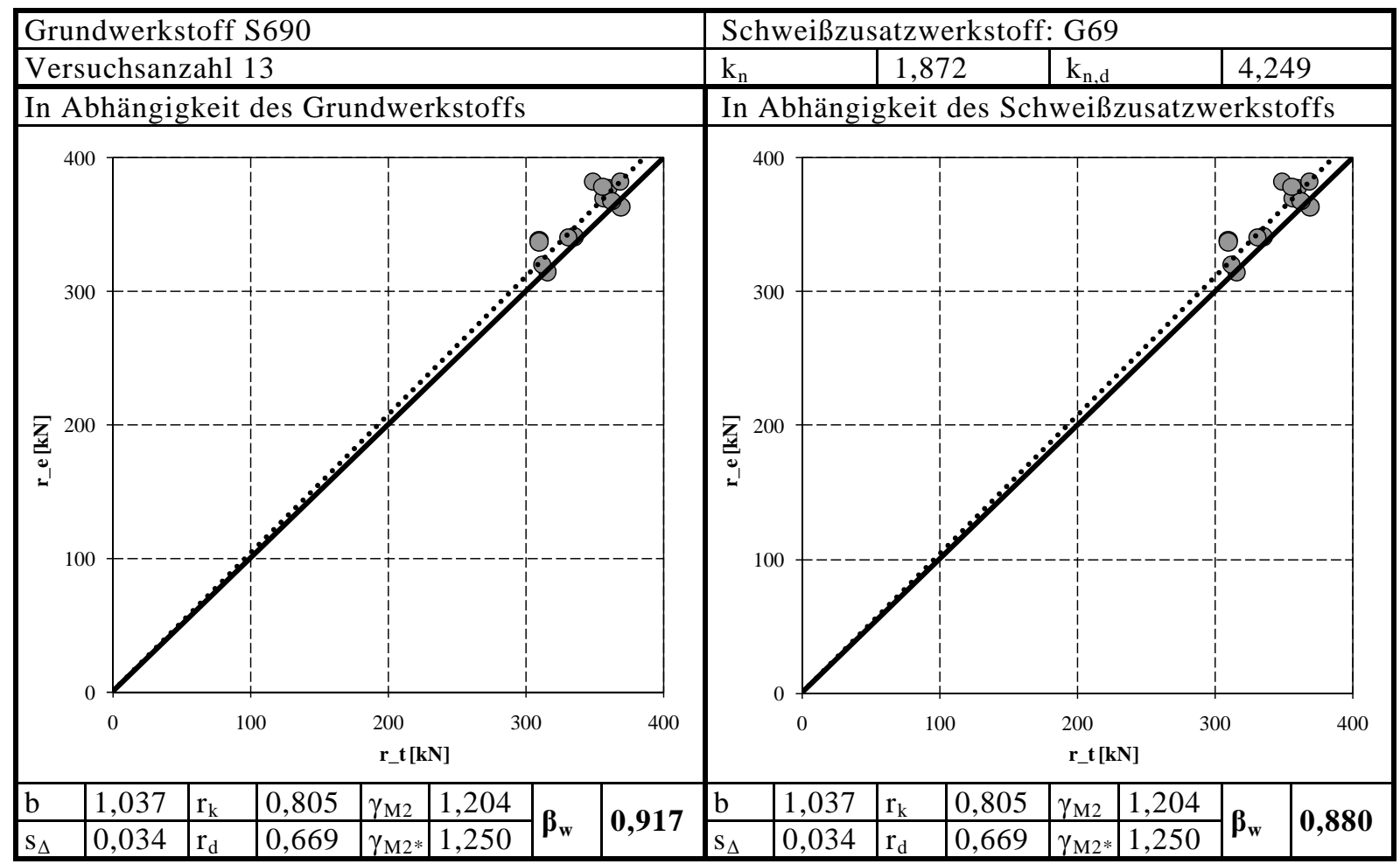

Einen Überblick über die Ergebnisse der statistischen Auswertung gibt Tabelle 6.14. Dargestellt sind die Ergebnisse der statistischen Auswertung für die beiden Werkstoffkombinationen S460-G46 und S690-G69. Es ergeben sich jeweils in Abhängigkeit des Grund- und des Schweißzusatzwerkstoffs die gleichen Bemessungsgrenzspannungen, weil weder die Grund- noch die Schweißzusatzwerkstoffe in den einzelnen Serien variierten.

Tabelle 6.14: Bemessungsgrenzspannung für Kreuzstöße mit Stirnkehlnähten

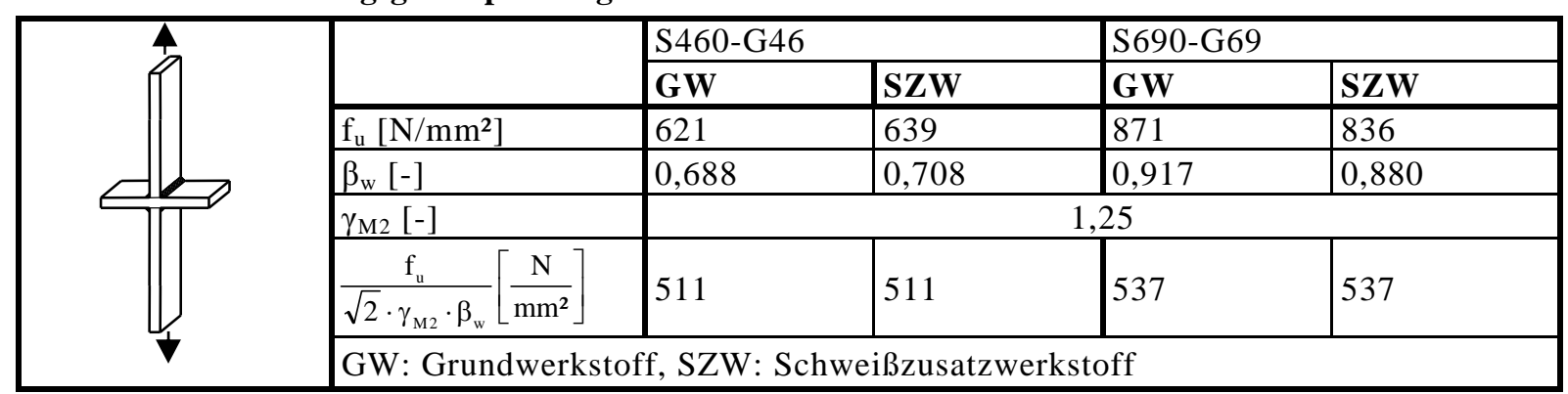

Der Vergleich der Beiwerte $\beta_{\mathrm{w}}$ zeigt bei beiden Werkstoffkombinationen eine Überfestigkeit gegenüber der Festigkeit des Schweißzusatzwerkstoffs. Für die Verbindungen S460-G46 ist diese Überfestigkeit deutlich größer als für die Verbindungen S690-G69. In Abbildung 6.11 sind die Versuchsergebnisse unter Berücksichtigung der Nahtfläche $A_{E C}$ gemeinsam mit den Bemessungsgrenzspannungen $\sigma_{\mathrm{EC}}=511 \mathrm{~N} / \mathrm{mm}^{2}$ und $\sigma_{\mathrm{EC}}=537 \mathrm{~N} / \mathrm{mm}^{2}$ in Abhängigkeit vom Schweißzusatzwerkstoff dargestellt. Sowohl die Mittelwerte als auch die Bemessungswerte zeigen eine Steigerung der Tragfähigkeit von ca. 5 \%. Es wurden jeweils nur ein Schweißzusatzwerkstoff und ein Grundwerkstoff verwendet, dennoch ist die Streuung groß. 


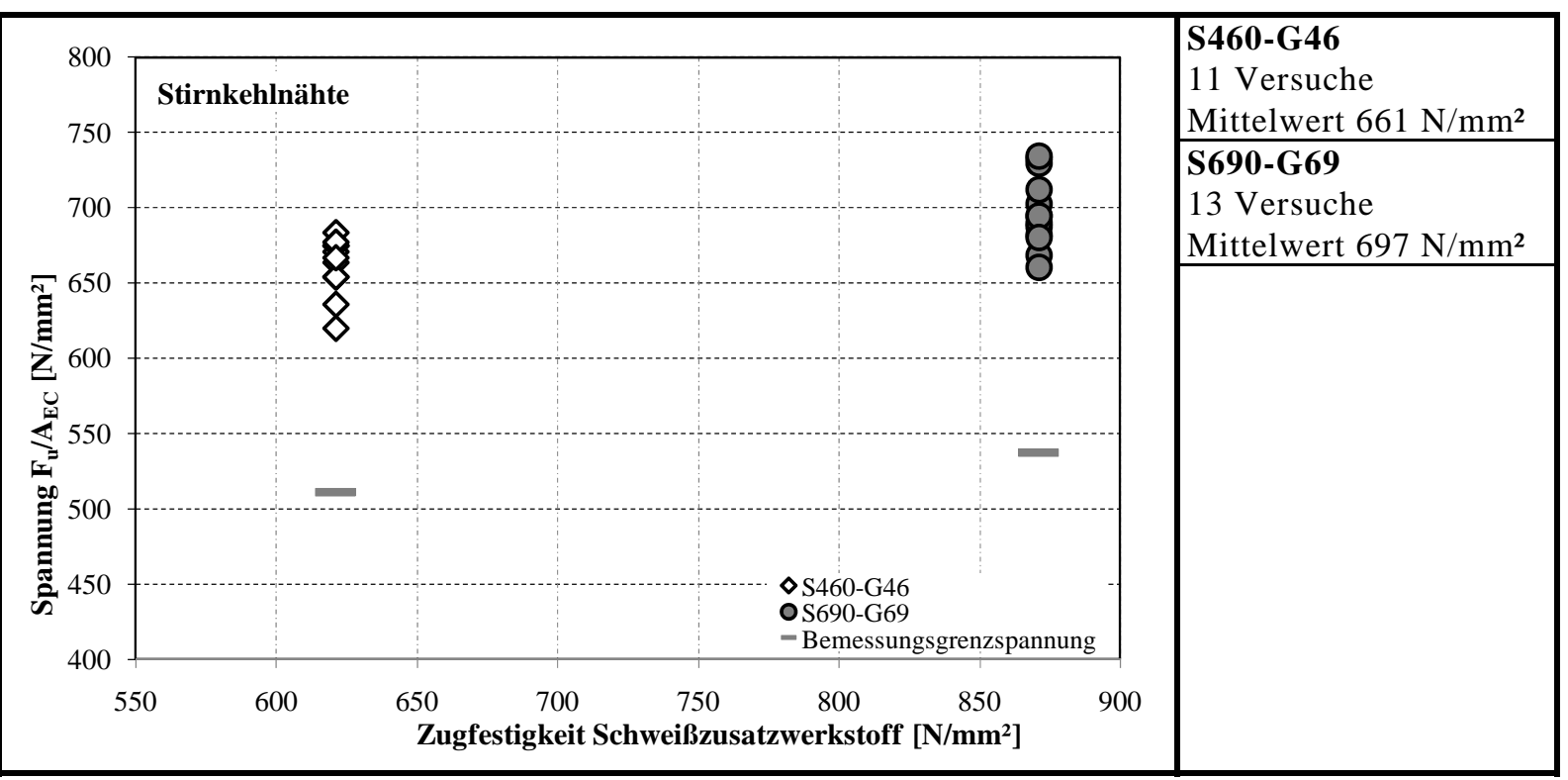

Abbildung 6.11: Vergleich der Ergebnisse der Kreuzstöße mit Stirnkehlnähten mit der Nahtfläche $A_{E C}$ S460-G46 und S690-G69

\subsection{Kreuzstöße mit gleichen Grund- und Variation der Schweißzusatz- werkstoffe (BUW)}

Um den Einfluss des Schweißzusatzwerkstoffs auf die Tragfähigkeit der Kreuzstöße zu untersuchen, wurden Versuchskörper mit gleichen Grundwerkstoffen der Stahlsorten S460 und S690 hergestellt. Für den Grundwerkstoff S460 wurden Versuche mit einem Schweißzusatzwerkstoff geringerer Festigkeitsklasse G42, gleicher Festigkeitsklasse G46 und höherer Festigkeitsklasse G69 analysiert. Versuche mit dem Grundwerkstoff S690 variierten den Schweißzusatzwerkstoff, indem eine deutlich geringere Festigkeitsklasse G46 und die zugehörige Festigkeitsklasse G69 verwendet wurde. In der folgenden Abbildung 6.12 ist die Tragfähigkeit als Versuchsergebnis bei gleichen Schweißbedingungen dargestellt. Eindeutige Aussagen lassen sich auf Grund der Streuung nicht treffen.

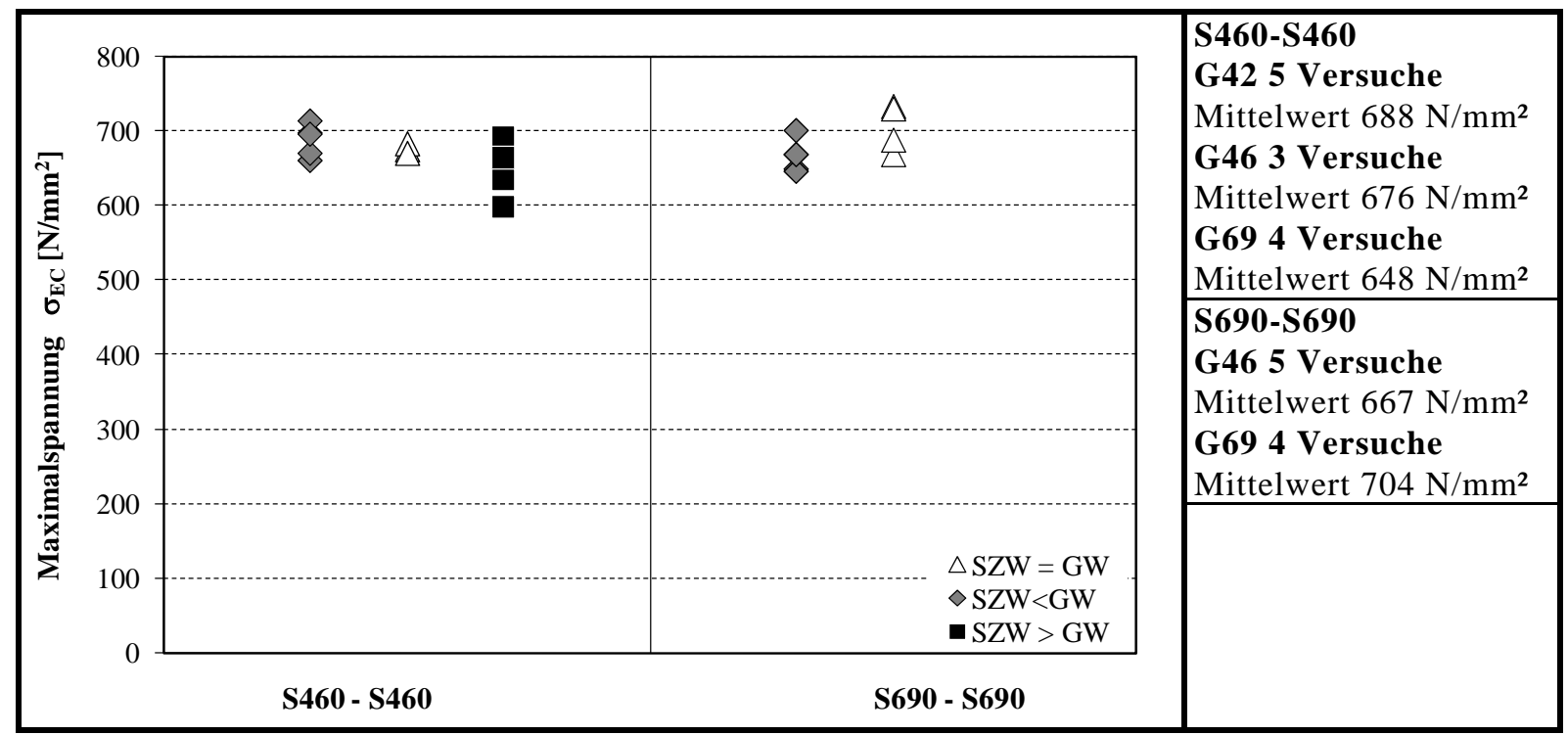

Abbildung 6.12: Einfluss des Schweißzusatzwerkstoffs auf die Tragfähigkeit 


\subsection{Kreuzstöße mit unterschiedlichen Grundwerkstoffen}

Um den Einfluss unterschiedlicher Grundwerkstoffe zu untersuchen, wurden Versuchskörper mit unterschiedlichen Grundwerkstoffkombinationen (Mischverbindungen) hergestellt. In jeder Werkstoffkombination wurde der Schweißzusatzwerkstoff variiert. Die Schweißzusatzwerkstoffe wurden so gewählt, dass sie entweder der Festigkeit des niederfesteren Grundwerkstoffs (SZW $\cong G W 1$ ) oder der Festigkeit des höherfesten Grundwerkstoffs (SZW GW2) entsprachen. Abbildung 6.13 zeigt die Tragfähigkeit der verschiedenen Kombinationen. Mit zunehmender Festigkeit des Schweißzusatzwerkstoffs steigt die Tragfähigkeit der Verbindung tendenziell leicht an. Da die Tragfähigkeitsunterschiede bei den Verbindungen gleicher Grundwerkstoffe schon sehr gering sind, ist es schwierig auf Grund der großen Streuung eindeutige Tendenzen abzulesen.

Diese Versuche wurden am Institut für Konstruktion und Entwurf, Universität Stuttgart von Herrn Dr.Ing. H.-P. Günther durchgeführt.

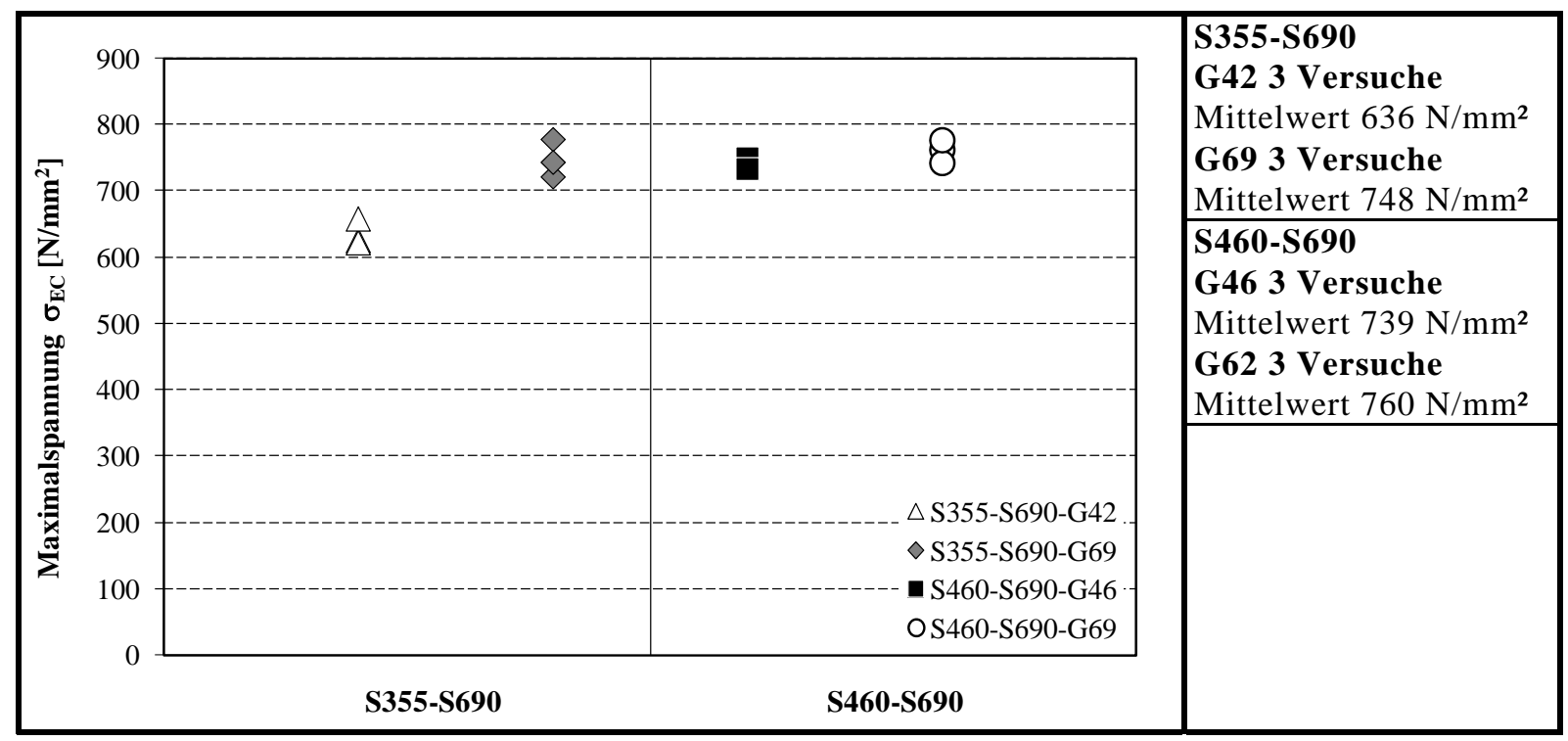

Abbildung 6.13: Einfluss des Schweißzusatzwerkstoffs auf die Tragfähigkeit bei Mischverbindungen

\subsection{Werkstoffuntersuchungen}

\subsubsection{Allgemeines}

Um die Tragfähigkeit von Schweißverbindungen zu bestimmen, ist die Kenntnis der Zugfestigkeit der betrachteten Naht besonders wichtig. Zur Bestimmung der Festigkeit in der Kehlnaht wurden die in Kapitel 4.2 beschriebenen Untersuchungen durchgeführt.

Um die Festigkeit von einlagigen Kehlnähten zu bestimmen, wurden ein- und mehrlagige Kehlnähte sowie Stumpfnähte geschweißt. An den einlagigen Kehlnähten wurden Härtemessungen durchgeführt. Während an den zweilagigen Kehlnähten und an Stumpfnähten sowohl Härtemessungen als auch Zugversuche durchgeführt wurden. Die Ergebnisse dieser Härtemessungen und Zugversuche wurden mit den Härtemessungen an einlagigen Kehlnähten verglichen.

Die verwendeten Versuchskörper bestehen aus den Werkstoffkombinationen S460-G46 und S690G69. Die Versuchskörper sind als T-Stoß mit zwei Kehlnähten aufgebaut, wobei die eine Kehlnaht einlagig und die andere zweilagig (mit drei Raupen) ausgeführt sind, vgl. Abbildung 4.3. Die Kehlnähte wurden anhand von Makroschliffen der Querschnitte als auch anhand von Härtemessungen charakterisiert. Die Härtemessungen wurden in drei Reihen durchgeführt: eine Reihe nahe der Oberfläche, eine Reihe in der Nahtmitte, eine Reihe nahe der Nahtwurzel und eine Reihe senkrecht dazu. Aus der mehrlagigen Naht wurden Rundzugproben ausgearbeitet. Bei diesen Proben handelte es sich um Handschweißungen, bei denen die Schweißparameter nicht nachvollziehbar sind. Die Ergebnisse können dennoch qualitativ verwendet werden. 
Um die Werkstoffeigenschaften des reinen Schweißgutes nach Norm zu bestimmen, wurden Zugproben aus Stumpfnähten mit den Schweißzusatzwerkstoffen G46 (TUD) und G69 gefertigt, siehe Abbildung 4.1. Zusätzlich wurden an dem Stumpfstoß mit dem Schweißzusatzwerkstoff G69 Härtemessungen durchgeführt. Die Härtemessungen wurden in vier Reihen durchgeführt: eine Reihe waagerecht nahe der Oberfläche, eine Reihe waagerecht in der Mitte, eine Reihe waagerecht nahe der Nahtwurzel und eine Reihe senkrecht dazu.

\subsubsection{Ergebnisse der Zugversuche}

Ein Vergleich der Ergebnisse der Zugversuche aus mehrlagigen Kehlnähten und Stumpfnähten sind im Vergleich mit den Zeugniswerten der Schweißzusatzwerkstoffe nach Tabelle 6.4 und Tabelle 6.5 in Tabelle 6.15 gegeben. Die Zeugniswerte und die Ergebnisse der Zugversuche an Stumpfstößen stimmen gut überein.

Ein Vergleich der Zugfestigkeit der Stumpfnaht (= reines Schweißgut) mit der Zugfestigkeit der zweilagigen Kehlnaht zeigt für den Schweißzusatzwerkstoff G46 eine deutliche Steigerung von $16 \%$. Beim Schweißgut G69 liegt die Zugfestigkeit der Kehlnaht nur geringfügig höher als die Zugfestigkeit des reinen Schweißgutes. Das heißt, dass die Kehlnaht mit dem Schweißzusatzwerkstoff G46 eine deutliche Überfestigkeit gegenüber dem reinen Schweißgut hat, während beim Schweißzusatzwerkstoff G69 die Überfestigkeit deutlich geringer ist.

Vergleicht man die Zugfestigkeit des Schweißzusatzwerkstoffs G46 mit der des Schweißzusatzwerkstoffs G69, zeigt sich beim Stumpfstoß ein Unterschied von 31 \%, bei der zweilagigen Kehlnaht nur von $19 \%$, wie in Tabelle 6.15 angegeben. Die Verringerung des Unterschiedes ergibt sich aus den beschriebenen unterschiedlichen Überfestigkeiten der Kehlnähte bezogen auf die Stumpfnähte.

Tabelle 6.15: Vergleich der Zugfestigkeiten von Proben aus Stumpf- und Kehlnähten

\begin{tabular}{|l|l|l|l|}
\hline \multirow{2}{*}{} & Zugfestigkeit $\mathrm{R}_{\mathrm{m}}\left[\mathrm{N} / \mathrm{mm}^{2}\right]$ & Unterschied \\
\cline { 2 - 4 } & $\mathrm{G} 46-\mathrm{S} 460$ & $\mathrm{G} 69-\mathrm{S690}$ & \\
\hline Zeugnis & 639 & 836 & $31 \%$ \\
\hline Zugversuch, Stumpfstoß & $608 / 610 / 616$ (TUD) & $833 / 817$ (MW 825) & $35 \%$ \\
\hline Zugversuch, zweilagige Kehlnähte & 711 & 846 & $19 \%$ \\
\hline Unterschied zw. den Zugversuchen & $16 \%$ & $3 \%$ & \\
\hline
\end{tabular}

\subsubsection{Ergebnisse der Härtemessungen}

Für die Schweißverbindung S690-G69 ist ein Vergleich der Härtewerte zwischen Stumpfnaht, einlagiger Kehlnaht und mehrlagiger Kehlnaht in Abbildung 6.14 dargestellt. Angegeben sind die Härtewerte im Bereich der Grundwerkstoffe (GW), der Wärmeeinflusszone (WEZ) und des Schweißgutes (SG). Es wurden jeweils die Härtemessungen an der Nahtoberfläche verglichen. Die Härtemessungen liefern in allen Fällen sehr ähnliche Werte.

Abbildung 6.15 zeigt den Vergleich der Härtemessungen zwischen ein- und mehrlagigen Kehlnähten S690-G69 und S460-G46. Bei den Schweißverbindungen G69 zeigen sich nur geringe Unterschiede zwischen ein- und mehrlagigen Nähten. Bei den Schweißverbindungen G46 wurden für die einlagigen Verbindungen um $8 \%$ höhere Härtewerte ermittelt als für die mehrlagigen Nähte. Bei den mehrlagigen Nähten liegt die Härte von G69 um 12 \% höher als die von G46. Bei den einlagigen Nähten ergeben sich ähnliche Werte für G69 und G46. 


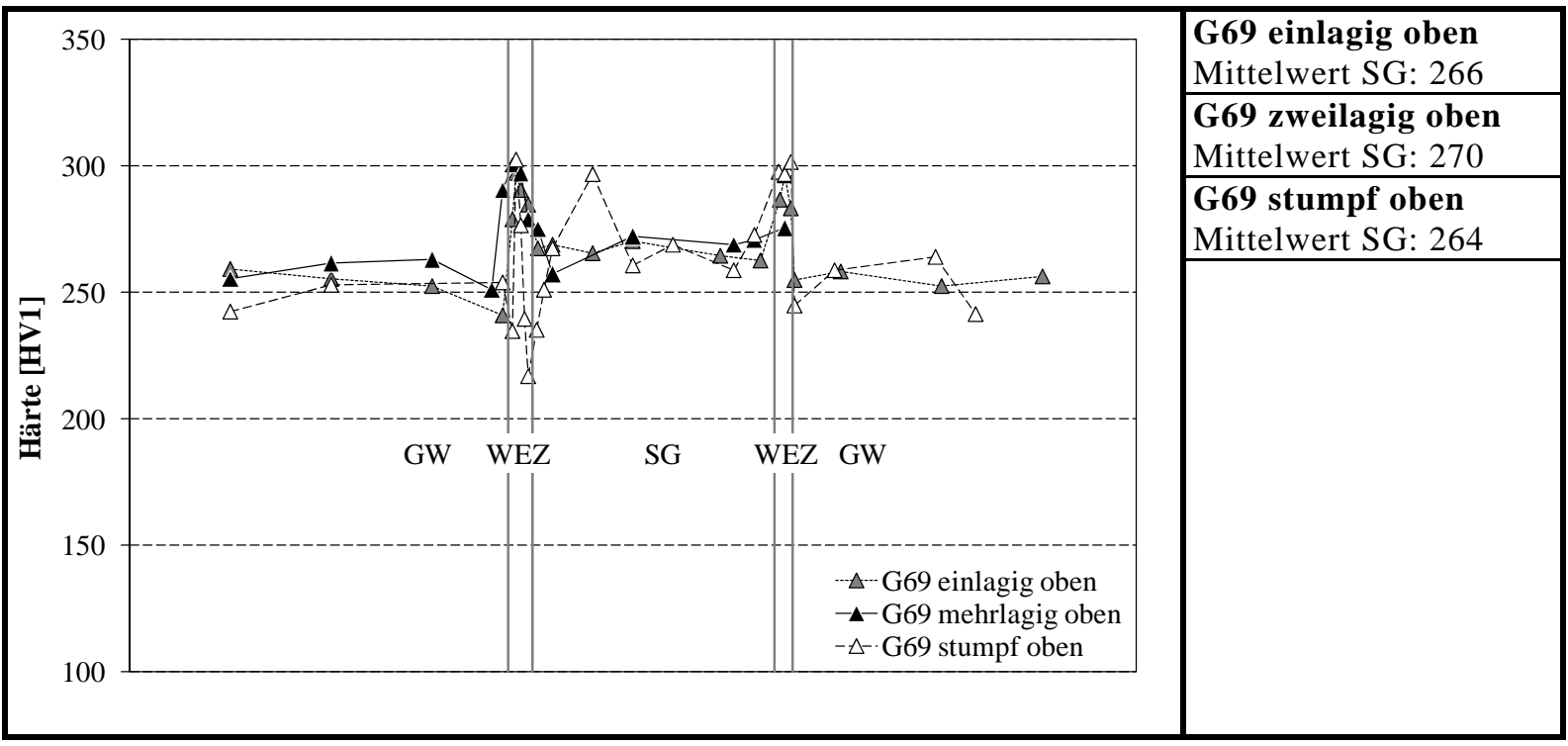

Abbildung 6.14: Härtemessungen Vergleich ein- und mehrlagige Kehlnaht mit Stumpfnaht S690 - G69

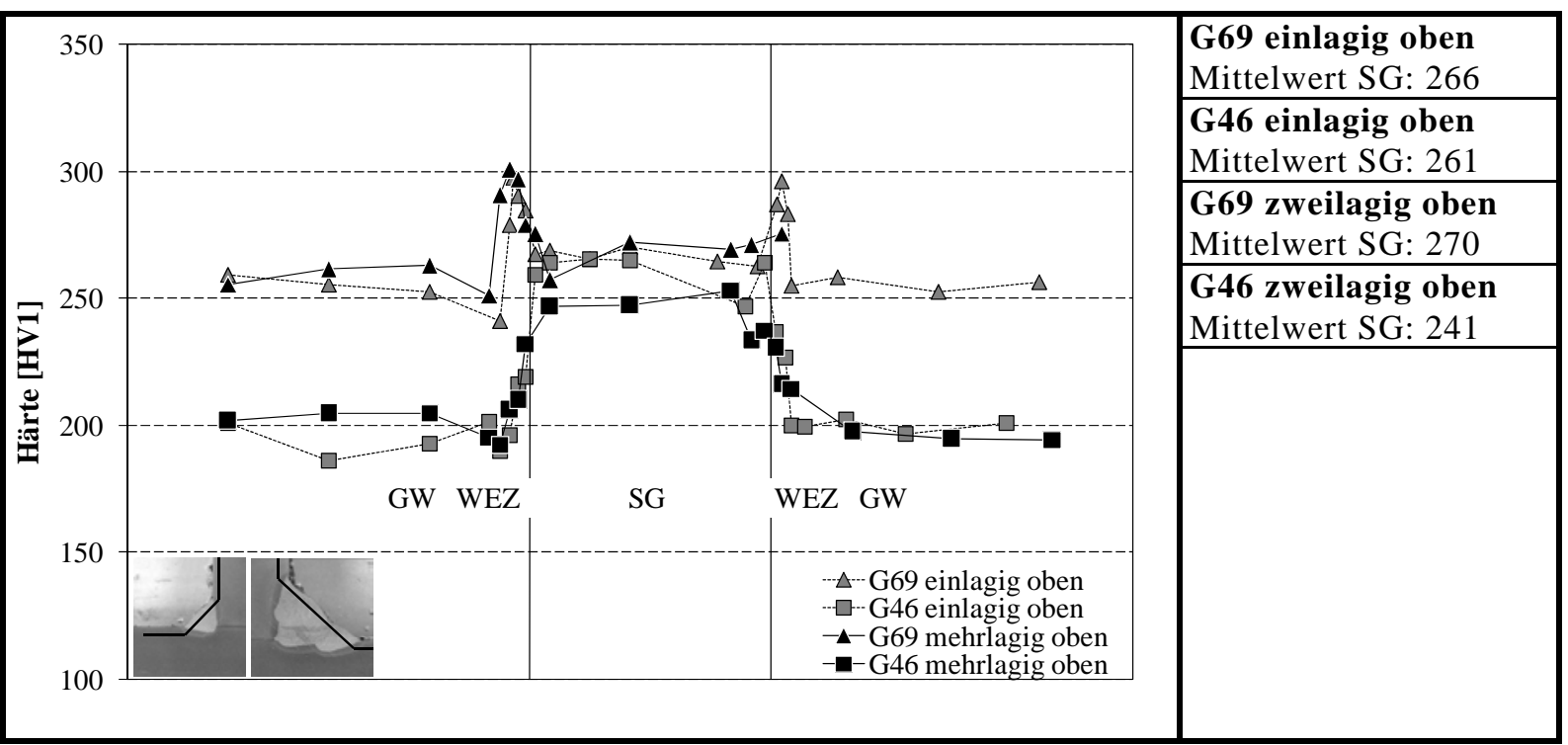

Abbildung 6.15: Härtemessungen Kehlnaht ein- und mehrlagig oben

\subsubsection{Vergleich der Ergebnisse von Härtemessungen und Zugversuchen}

Ein Vergleich von Härtemessungen und Zugfestigkeiten ist in Tabelle 6.16 gegeben.

Tabelle 6.16: Vergleich der Härtemessungen und Zugfestigkeiten von Stumpf- und Kehlnähten

\begin{tabular}{|l|l|l|l|l|l|l|}
\hline & G69 stumpf & $\begin{array}{l}\text { G69 Kehl- } \\
\text { naht einlagig }\end{array}$ & $\begin{array}{l}\text { G69 Kehlnaht } \\
\text { mehrlagig }\end{array}$ & G46 stumpf & $\begin{array}{l}\text { G46 Kehl- } \\
\text { naht einlagig }\end{array}$ & $\begin{array}{l}\text { G46 Kehlnaht } \\
\text { mehrlagig }\end{array}$ \\
\hline Härte HV1 & 266 & 267 & 269 & & 259 & 239 \\
\hline $\begin{array}{l}\text { Zugfestig- } \\
\text { keit }\end{array}$ & $\begin{array}{l}833 / 817 \\
(825)\end{array}$ & & 846 & $\begin{array}{l}608 / 610 / 616 \\
(611)\end{array}$ & & 711 \\
\hline
\end{tabular}

Vergleicht man die Zugfestigkeit der Stumpfnaht mit der Zugfestigkeit der zweilagigen Kehlnaht, so stellt man für das Schweißgut G46 eine deutliche Steigerung im Bereich von 16 \% fest. Der Vergleich der Härtemessungen von einlagigen und mehrlagigen Nähten zeigt für Schweißverbindungen G46 bei den einlagigen Nähten um 8 \% höhere Härtewerte als bei den mehrlagigen Nähten. Aus der Härte lässt sich auf die Festigkeit schließen. Somit kann daraus geschlossen werden, dass die Festigkeit der einlagigen Nähte mit dem Schweißzusatzwerkstoff deutlich höher liegt als bei den mehrlagigen Nähten. Die größere Härte und Festigkeit der einlagigen Nähte im Vergleich mit Stumpfnähten lässt sich durch 
die fehlenden Vergütungseffekte und die extremen Abkühlbedingungen erklären, wie in Kapitel 4.2.3 erläutert. Als Grund für die geringere Härte der mehrlagigen Nähte sind die Vergütungseffekte zu nennen, die durch das Überschweißen der Wurzellage entstehen. Durch das erneute Aufschmelzen stellen sich weitere Gefügeänderungen ein. Der Gefügezustand nähert sich immer mehr dem Gefüge von Stumpfnähten an, die, wie Tabelle 6.15 zeigt, eine geringere Festigkeit und, wie Tabelle 6.16 zeigt, eine geringere Härte aufweisen. Beim Schweißgut G69 liegt die Zugfestigkeit der Kehlnaht nur geringfügig höher als die Zugfestigkeit des reinen Schweißgutes. Die Härteverläufe liegen in dem Bereich, in dem die Zugproben entnommen wurden, bei der Stumpfnaht um 6 \% höher als die Härtewerte bei der dreiraupigen Kehlnaht. An der Oberfläche der Naht liefern die Ergebnisse der Härtemessungen an der Stumpfnaht, der einlagigen Kehlnaht und der mehrlagigen Kehlnaht in allen Fällen sehr ähnliche Werte. Daraus folgt, dass die Kehlnaht keine Überfestigkeit gegenüber der Festigkeit des reinen Schweißzusatzwerkstoffs hat.

Vergleicht man die Zugfestigkeit des Schweißzusatzwerkstoffs G46 mit der des Schweißzusatzwerkstoffs G69, zeigt sich bei der zweilagigen Kehlnaht ein Unterschied von $19 \%$, wie in Tabelle 6.16 angegeben. Bei diesen mehrlagigen Nähten liegen die Härtewerte von G69 um 12 \% höher als die von G46. Bei den einlagigen Nähten ergeben sich ähnliche Werte für G69 und G46.

Der Vergleich von Härtewerten und Zugfestigkeiten korrelieren, es wurden jedoch Unterschiede infolge der Streuung festgestellt. Die festgestellten Unterschiede in den Zugfestigkeiten zwischen Kehlnähten und Stumpfnähten spiegeln sich auch in der Tragfähigkeit der Schweißverbindungen wider.

\subsection{Diskussion der Ergebnisse}

Die durchgeführten Untersuchungen an Flanken- und Stirnkehlnähten werden hinsichtlich des Einflusses der Grundwerkstoffe mit Schweißzusatzwerkstoff gleicher Festigkeitsklasse auf die Tragfähigkeit der Schweißverbindung beurteilt. Dazu werden die Versuchsergebnisse den Bemessungsgrenzspannungen infolge der ermittelten Werte $\beta_{\mathrm{w}}$ gegenübergestellt. Die Bemessungsgrenzspannungen sind für Flankenkehlnähte in Tabelle 6.10 und für Stirnkehlnähte in Tabelle 6.14 zusammengestellt. Für die folgenden Abbildungen wird der Bemessungswert infolge des Schweißzusatzwerkstoffs (gemittelter Wert) verwendet. Abbildung 6.16 zeigt den Vergleich mit Flanken- und Stirnkehlnähten $\left(\mathrm{A}_{\mathrm{EC}}\right)$, die Bemessungsgrenzspannungen der Kreuzstöße mit Stirnkehlnähten sind für S460-G/T/E46 um ca. 65 \% und für S690-G/T69 um ca. 67 \% höher als die Bemessungsgrenzspannungen der Flankenkehlnähte. Die Mittelwerte liegen in beiden Fällen um 40 - 42 \% höher. Die Tragfähigkeit von Kreuzstößen liegt um mehr als 22 \% höher als bei Flankenkehlnahtverbindungen, obwohl die Richtungsabhängigkeit im Bemessungskonzept bereits berücksichtigt ist.

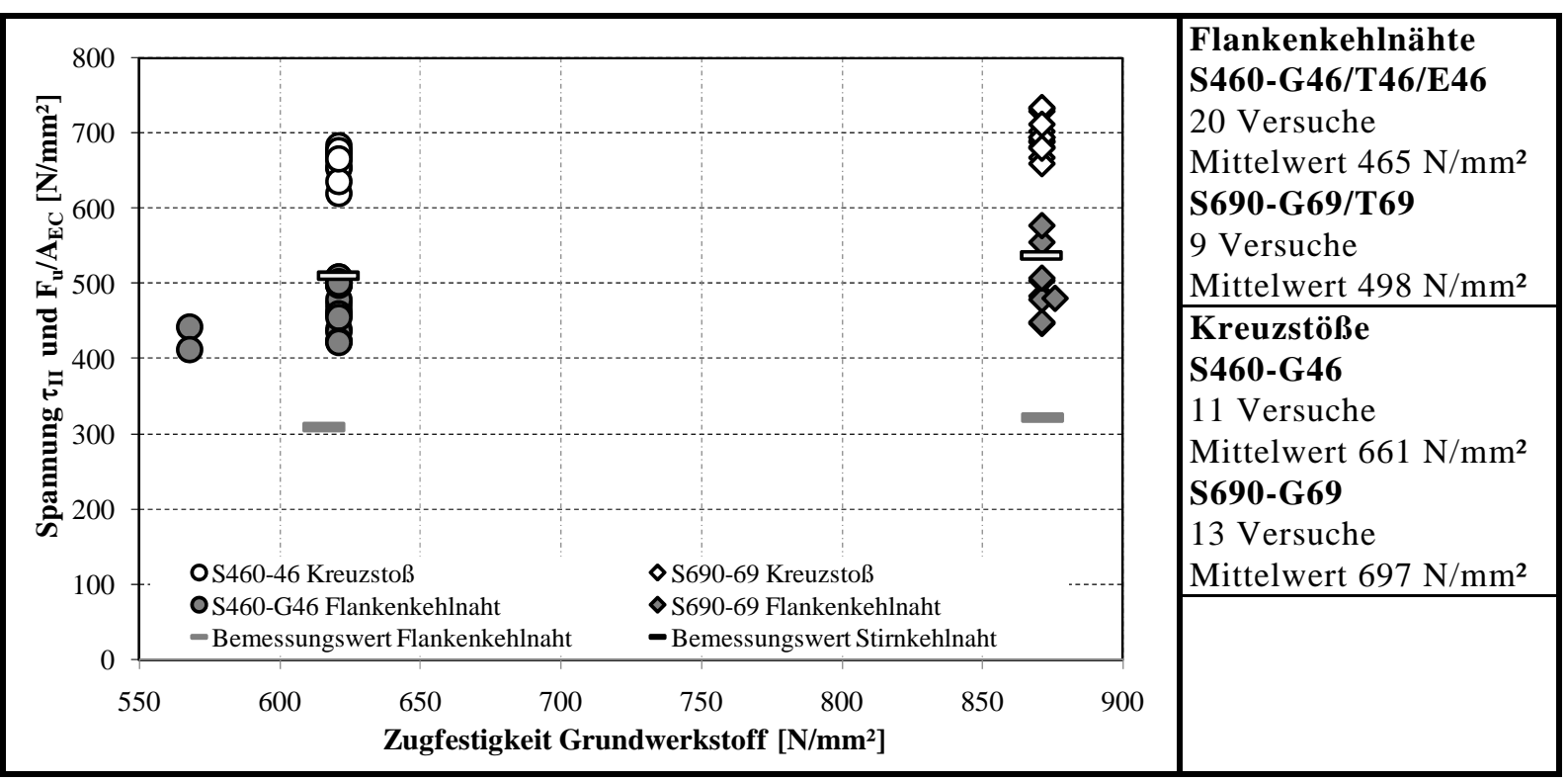

Abbildung 6.16: Vergleich der Ergebnisse von Kreuzstößen $\left(A_{\mathrm{EC}}\right)$ und Flankenkehlnahtverbindungen S460 und 5690 


\subsection{Zusammenfassung}

In diesem Kapitel wurden die im Rahmen des FOSTA/AiF-Forschungsprojekts „Wirtschaftliche Schweißverbindungen höherfester Baustähle“ [FOSTA P652, 2008] durchgeführten experimentellen Untersuchungen beschrieben und ausgewertet. Es wurden Tragfähigkeitsuntersuchungen an Flankenund Stirnkehlnähten mit den Grundwerkstoffen S355, S460 und S690 und unterschiedlichen Schweißzusatzwerkstoffen sowie Werkstoffuntersuchungen vorgestellt.

Für Flankenkehlnahtverbindungen konnte festgestellt werden, dass die Parameter Nahtdicke, Nahtlänge, Blechdicke, Schweißprozess und Schweißprozessparameter (im Anwendungsbereich von DIN EN 1011-2 (2001) und [SEW 088, 1993]) keinen bedeutenden Einfluss auf die Tragfähigkeit ausüben. Für die Parameter Festigkeit des Grundwerkstoffs (Stahlsorte) und des Schweißzusatzwerkstoffs ergaben sich jedoch folgende Tendenzen: Mit zunehmender Festigkeit sowohl des Grund- als auch des Schweißzusatzwerkstoffs ist im Allgemeinen eine Tragfähigkeitssteigerung der Schweißverbindung zu erwarten. Die starke Streuung der Ergebnisse infolge der Handschweißung bei verschiedenen Fertigern macht eine eindeutige Feststellung von Einflüssen schwierig. Tabelle 6.17 fasst die Ergebnisse zusammen.

Tabelle 6.17: Zusammenfassung der Versuchsergebnisse von Flankenkehlnahtverbindungen

\begin{tabular}{|l|l|}
\hline Parameter & Einfluss \\
\hline $\begin{array}{l}\text { Stahlsorte - } \\
\text { Schweißzusatzwerkstoff }\end{array}$ & $\begin{array}{l}\text { S460-G46: ca. 15 - 20 \% höhere Tragfähigkeit als S355-G42 } \\
\text { S690-G69: ca. 7 \% höhere Tragfähigkeit als S460-G46 } \\
\text { Steigerung der Tragfähigkeit nicht proportional zur Streckgrenze }\end{array}$ \\
\hline Nahtlänge & $\begin{array}{l}\text { S460-G46: bis L/a = 150 geringer Einfluss } \\
\text { S690-G69: bis L/a = 100 geringer Einfluss }\end{array}$ \\
\hline Naht-/ und Blechdicke & Einfluss gering \\
\hline $\begin{array}{l}\text { Festigkeit des Schweiß- } \\
\text { zusatzwerkstoffs (SZW) }\end{array}$ & $\begin{array}{l}\text { Leichte Steigerung der Tragfähigkeit bei zunehmender Festigkeit des } \\
\text { SZW, } \\
\text { Steigerung der Tragfähigkeit nicht proportional zur Festigkeit des SZW }\end{array}$ \\
\hline Schweißprozess & Kaum Einfluss auf die Tragfähigkeit \\
\hline
\end{tabular}

Für Kreuzstöße mit Stirnkehlnähten ergeben sich für die Parameter Festigkeit des Grundwerkstoffs (Stahlsorte) und des Schweißzusatzwerkstoffs Tendenzen, dass mit zunehmender Festigkeit sowohl des Grund- als auch des Schweißzusatzwerkstoffs im Allgemeinen eine Tragfähigkeitssteigerung der Schweißverbindung vorhanden ist. Die starke Streuung der Ergebnisse infolge der Handschweißung bei verschiedenen Fertigern macht eine eindeutige Feststellung von Einflüssen schwierig. Tabelle 6.18 fasst die Ergebnisse zusammen.

Tabelle 6.18: Zusammenfassung der Versuchsergebnisse von Kreuzstößen

\begin{tabular}{|l|l|}
\hline Parameter & Einfluss \\
\hline $\begin{array}{l}\text { Stahlsorte - } \\
\text { Schweißzusatzwerkstoff }\end{array}$ & $\begin{array}{l}\text { S460-G46: ähnliche Tragfähigkeit wie S355-G42 } \\
\text { S690-G69: ca. 5 \% höhere Tragfähigkeit als S460-G46 } \\
\text { Steigerung der Tragfähigkeit nicht proportional zur Streckgrenze }\end{array}$ \\
\hline $\begin{array}{l}\text { Festigkeit des Schweißzu- } \\
\text { satzwerkstoffs (SZW) }\end{array}$ & $\begin{array}{l}\text { Leichte Steigerung der Tragfähigkeit bei zunehmender Festigkeit des } \\
\text { SZW, } \\
\text { Steigerung der Tragfähigkeit nicht proportional zur Festigkeit des SZW }\end{array}$ \\
\hline
\end{tabular}

Die Versuchsergebnisse zeigen eine nur geringfügig höhere Tragfähigkeit bei Schweißverbindungen mit dem Stahl S690 im Vergleich mit Verbindungen mit dem Stahl S460, während sich die Zugfestigkeiten des reinen Schweißguts G46 zu G69 deutlich steigern. Die Festigkeit bei mehrlagigen Nähten steigt im Vergleich zur Festigkeit bei Stumpfnähten jeweils an, beim G46 deutlich um $16 \%$ und beim G69 nur wenig, um 3 \%. Für die Schweißverbindungen S690-G69 kann festgestellt werden, dass sich die Tragfähigkeit über den Faktor 1/ $\sqrt{3}$ (Von-Mises, vgl. Kapitel 4) mit Hilfe der Zugfestigkeit bestimmen lässt. Für die Verbindungen S460-G46 wurde eine Überfestigkeit der Kehlnähte im Vergleich zum reinen Schweißgut festgestellt. Der geringe Unterschied in der Härte bei einlagigen Nähten bestätigt den geringen Unterschied in der Tragfähigkeit. Der Grund dafür liegt in den in Kapitel 2.4.7 beschriebenen metallurgischen Unterschieden und Unterschieden in den Abkühlbedingungen zwischen Kehl- und Stumpfnähten. 


\section{Voruntersuchungen an vollmechanisiert geschweißten Ver- suchskörpern}

\subsection{Einleitung}

Die in Kapitel 6 vorgestellten Versuchsergebnisse stammten von manuell geschweißten Versuchskörpern mit ineinander gesteckten Blechen. Die so hergestellten Flankenkehlnahtverbindungen waren im Bereich von Nahtanfang und Nahtende herstellungsbedingt ungleichmäßig. Die in Kapitel 6 ermittelten Tragfähigkeiten der Versuchskörper mit dem Grundwerkstoff S690 lagen (mit $\beta_{\mathrm{w}}=1,1$ bezogen auf den Schweißzusatzwerkstoff) unterhalb der Tragfähigkeiten nach den damals aktuellen Normen EN 1993-1-12 (2007) $\left(\beta_{\mathrm{w}}=1,0\right)$.

Die Versuche, die in diesem Kapitel vorgestellt werden, wurden durchgeführt, um die Tragfähigkeiten der in Kapitel 6 vorgestellten Versuche mit dem Grundwerkstoff S690 zu bestätigen oder eine erhöhte Tragfähigkeit zu erreichen. Dazu wurde überprüft, ob durch eine andere Versuchskörperform im Vergleich zu den Stecklaschenverbindungen, eine vollmechanisiert geschweißte Naht oder durch die Verwendung eines höherfesten Schweißzusatzwerkstoffs eine erhöhte Tragfähigkeit von Flankenkehlnahtverbindungen S690 festgestellt werden kann. Es wird auf den detaillierten Versuchsbericht in [Zusatzversuche, 2008] verwiesen.

\subsection{Versuchsprogramm}

Als Versuchskörperform wurde eine sog. Überlappverbindung ausgewählt, wie in Abbildung 7.1 dargestellt. Es wurden zwei unterschiedlich breite Bleche gleicher Werkstoffgüte aufeinander gelegt und über die ganze Länge der Bleche mit einer vollmechanisierten MAG-Schweißung hergestellt. Nach dem Schweißen wurden die Versuchskörper mechanisch bearbeitet, indem durch Fräsen an einer Stelle das Oberblech und die Nähte und an einer anderen Stelle das Unterblech und die Schweißnähte getrennt wurden.

Die Besonderheit des vollmechanisch geschweißten Versuchskörpers liegt darin, dass durch die mechanische Trennung Schweißnähte ermöglicht wurden, die geometrisch keine großen Toleranzen und keinen Nahtanfangs- und Nahtendbereich haben. Auch schweißtechnisch befindet sich die tragende Schweißnaht nicht im Anfangs- und Endbereich, sondern in einem Bereich, in dem sehr gleichmäßige Bedingungen vorliegen.

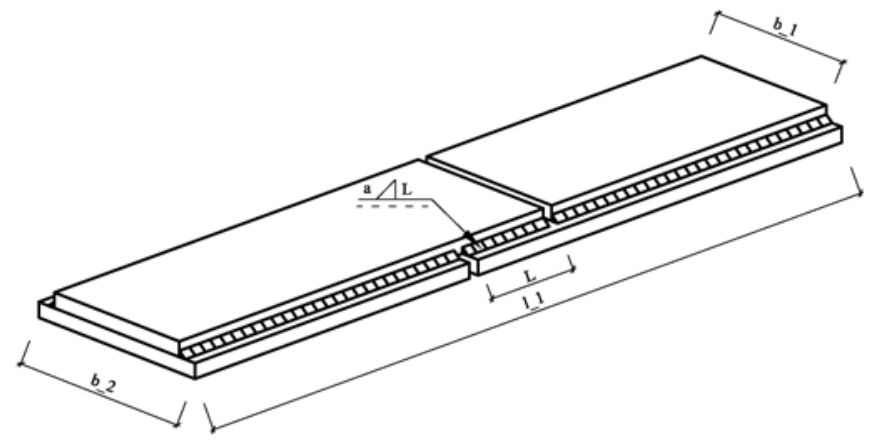

\section{Abbildung 7.1: Versuchskörper nach der mechanischen Bearbeitung}

Fünf Versuchsserien wurden durchgeführt, siehe Tabelle 7.1. In den Versuchsserien 1 - 4 wurden jeweils zwei nominell gleiche Versuchskörper verwendet. In der fünften Serie stand nur ein Versuchskörper zur Verfügung.

Als Serie 1 wurden mit der in Abbildung 7.1 dargestellten Versuchskörperform zwei Referenzversuche mit dem Grundwerkstoff S690 und zugehörigem Schweißzusatzwerkstoff G69 hergestellt, um durch den Vergleich mit den Versuchsergebnissen der manuell gefertigten Stecklaschenverbindungen (Kapitel 6) den Einfluss des Mechanisierungsgrades und der Versuchskörperform zu ermitteln. 
Als Serie 2 wurden mit dieser Werkstoffkombination S690-G69 weitere Versuchskörper gefertigt, bei denen die Schweißparameter durch die Abkühlzeit t 8/5 $_{5}$ variiert wurden, um den Einfluss der Abkühlzeit $\mathrm{t}_{8 / 5}$ auf die Tragfähigkeit zu bestimmen.

In Serie 3 wurden Bleche der Grundwerkstoffe S690 mit einem höherfesten Schweißzusatzwerkstoff G89 verschweißt. Durch den Vergleich mit den ermittelten Traglasten der Serie 1 kann der Einfluss der Zugfestigkeit des Schweißzusatzwerkstoffs auf die Tragfähigkeit bestimmt werden.

In Serie 4 wurden Versuchskörper mit mehrlagigen Kehlnähten und einem höherfestem Schweißzusatzwerkstoff G89 gefertigt, um durch den Vergleich mit den Ergebnissen der Serie 3 den Einfluss der Mehrlagigkeit bei höherfesten Schweißzusatzwerkstoffen zu bestimmen.

In Serie 5 wurde ein normalfester Grundwerkstoff S355J2 verwendet. Es wurde eine einlagige Schweißverbindung mit dem Schweißzusatzwerkstoff G89 hergestellt. Der Vergleich der Ergebnisse aus Serie 3 und Serie 5 zeigt den Einfluss des Grundwerkstoffs auf die Tragfähigkeit der Schweißverbindung.

Tabelle 7.1 fasst das Versuchsprogramm zusammen. Die Werkstoffkombinationen der einzelnen Versuche sind im Anhang A.3 zusammen mit den gemessenen Schweißparametern Streckenenergie, Vorwärmtemperatur und Abkühlzeit t 8/5 $_{5}$ aufgeführt. Die Versuchskörper wurden so dimensioniert, dass ein Versagen im Schweißgut und nicht im Grundwerkstoff auftrat.

Tabelle 7.1: Untersuchte Parameter an Überlappverbindungen mit Kehlnähten

\begin{tabular}{|c|c|c|c|c|c|c|c|c|c|c|c|}
\hline Serie & Name & GW & SZW & $\begin{array}{l}\text { Anzahl } \\
\text { Lagen }\end{array}$ & $\begin{array}{l}\text { Abkühlzeit } \\
\mathrm{t}_{8 / 5}[\mathrm{~s}]\end{array}$ & Serie & Name & GW & SZW & $\begin{array}{l}\text { Anzahl } \\
\text { Lagen }\end{array}$ & $\begin{array}{l}\text { Abkühlzeit } \\
\mathrm{t}_{8 / 5}[\mathrm{~s}]\end{array}$ \\
\hline \multirow[t]{2}{*}{1} & Z-001 & S690Q & G69 & einlagig & $5-8$ & \multirow[t]{2}{*}{3} & Z-005 & S690Q & G89 & einlagig & $5-8$ \\
\hline & Z-002 & S690Q & G69 & einlagig & $5-8$ & & Z-006 & S690Q & G89 & einlagig & $5-8$ \\
\hline \multirow[t]{2}{*}{2} & Z-003 & S690Q & G69 & einlagig & 10 & \multirow[t]{2}{*}{4} & Z-007 & S690Q & G89 & zweilagig & $5-8$ \\
\hline & Z-004 & S690Q & G69 & einlagig & 15 & & Z-008 & S690Q & G89 & zweilagig & $5-8$ \\
\hline & & & & & & 5 & Z-009 & S355J2 & G89 & einlagig & $5-8$ \\
\hline
\end{tabular}

Zusätzlich wurden Werkstoffuntersuchungen durchgeführt. An den einlagigen Verbindungen der Serien 1, 3 und 5 wurden Härtemessungen durchgeführt, um mit Hilfe der Härte die Festigkeit des Schweißgutes und damit die Tragfähigkeit der Verbindung abzuschätzen. Ein Vergleich der Härtewerte soll den Vergleich der Tragfähigkeiten ergänzen.

\subsection{Verwendete Werkstoffe}

Für diese Voruntersuchungen an vollmechanisiert geschweißten Versuchen wurden als Grundwerkstoffe Stähle S355J2 nach DIN EN 10025-2 (2005) und S690Q/QL nach DIN EN 10025-6 (2005) verwendet. Die Zeugniskennwerte der mechanischen Eigenschaften der Grundwerkstoffe sind in Tabelle 7.2 zusammengestellt.

Für das MAG-Schweißen wurden die Schweißzusatzwerkstoffe mit der Kurzbezeichnung G69 und G89 nach DIN EN ISO 16834 (2007) verwendet. In Tabelle 7.3 sind die Zeugniskennwerte der mechanischen Eigenschaften dieser Schweißzusatzwerkstoffe enthalten. Die chemischen Kennwerte der Grund- und Schweißzusatzwerkstoffe sind in Anhang A.3.1 aufgeführt.

Tabelle 7.2: Mechanische Eigenschaften der verwendeten Grundwerkstoffe

\begin{tabular}{|c|c|c|c|c|c|c|c|c|c|c|c|c|c|c|c|}
\hline \multirow[t]{3}{*}{ Werkstoff } & \multirow{3}{*}{$\begin{array}{l}\text { Blech- } \\
\text { dicke } \\
{[\mathrm{mm}]}\end{array}$} & \multicolumn{14}{|c|}{ Mechanische Eigenschaften } \\
\hline & & \multicolumn{2}{|c|}{$\begin{array}{l}\mathrm{R}_{\mathrm{eH}} \\
{\left[\mathrm{N} / \mathrm{mm}^{2}\right]}\end{array}$} & \multicolumn{2}{|c|}{$\begin{array}{l}\mathrm{R}_{\mathrm{p} 0,2} \\
{\left[\mathrm{~N} / \mathrm{mm}^{2}\right]}\end{array}$} & \multicolumn{2}{|c|}{$\begin{array}{l}\mathrm{R}_{\mathrm{m}} \\
{\left[\mathrm{N} / \mathrm{mm}^{2}\right]}\end{array}$} & \multicolumn{2}{|l|}{$\begin{array}{l}A_{5} \\
{[\%]}\end{array}$} & \multicolumn{2}{|c|}{$\begin{array}{l}A_{v}[\mathrm{~J}] \\
-20^{\circ} \mathrm{C} \\
\end{array}$} & \multicolumn{2}{|c|}{$-40^{\circ} \mathrm{C}$} & \multicolumn{2}{|c|}{$-60^{\circ} \mathrm{C}$} \\
\hline & & $\mathrm{L}$ & $\mathrm{Q}$ & $\mathrm{L}$ & $\mathrm{Q}$ & $\mathrm{L}$ & $\mathrm{Q}$ & $\mathrm{L}$ & $\mathrm{Q}$ & $\mathrm{L}$ & $\mathrm{Q}$ & $\mathrm{L}$ & $\mathrm{Q}$ & $\mathrm{L}$ & $\mathrm{Q}$ \\
\hline S355J2 & 15 & & 422 & & 557 & & & & 34,5 & 286 & & & & & \\
\hline S690QL & 20 & & & 713 & 767 & 760 & 797 & 22,5 & 19,5 & & & 264 & & 247 & \\
\hline
\end{tabular}


Tabelle 7.3: Mechanische Eigenschaften der verwendeten Schweißzusatzwerkstoffe gemäß Lieferzeugnis

\begin{tabular}{|c|c|c|c|c|c|c|c|c|c|}
\hline \multicolumn{2}{|c|}{ Schweißzusatzwerkstoff } & \multirow{2}{*}{$\begin{array}{l}\mathrm{R}_{\mathrm{p} 0,2} \\
{\left[\mathrm{~N} / \mathrm{mm}^{2}\right]} \\
724 \\
\end{array}$} & \multirow{2}{*}{$\begin{array}{l}\mathrm{R}_{\mathrm{m}} \\
{\left[\mathrm{N} / \mathrm{mm}^{2}\right]} \\
836\end{array}$} & \multirow{2}{*}{$\begin{array}{l}A_{5} \\
{[\%]} \\
15,4\end{array}$} & \multirow{2}{*}{\begin{tabular}{|l}
$\mathrm{Z}$ \\
{$[\%]$} \\
34,6 \\
\end{tabular}} & \multirow{2}{*}{$\begin{array}{l}\text { Av }[\mathrm{J}] \\
-20^{\circ} \mathrm{C} \\
105 \\
\end{array}$} & \multirow{2}{*}{$\begin{array}{l}-40^{\circ} \mathrm{C} \\
90\end{array}$} & \multirow{2}{*}{$\frac{-50^{\circ} \mathrm{C}}{74}$} & \multirow{2}{*}{$\frac{-60^{\circ} \mathrm{C}}{66}$} \\
\hline Union NiMoCr ${ }^{1)}$ & G69 & & & & & & & & \\
\hline Union X90 & G89 & 931 & 981 & 18,2 & 61,8 & 65 & 66 & 60 & 59 \\
\hline
\end{tabular}

\subsection{Herstellung der Versuchskörper und Versuchsdurchführung}

Die Schweißparameter der in Kapitel 7.2 vorgestellten Versuche wurden in Übereinstimmung mit DIN EN 1011-2 (2001) und [SEW 088 Beiblatt 2, 1993] und in Anlehnung an die Schweißparameter nach Kapitel 6 [FOSTA P652, 2008] gewählt. Der Zuschnitt der Bleche erfolgte mit Sägeschnitt. Die Versuchskörper wurden vollmechanisiert geschweißt. Alle Versuchskörper wurden bei einem Fertiger geschweißt.

Alle Versuchskörper wurden vor dem Versuch vermessen. Das Vermessen umfasste die Abmessungen der Bleche und der Nahtlänge. Die theoretische Nahtdicke wurde mit einer Schweißnahtlehre entlang der Naht gemessen. Es wurde bei allen Versuchskörpern eine zerstörungsfreie Werkstoffprüfung mittels Sichtprüfung und Magnetpulverprüfung durchgeführt. Dabei wurden keine Fehler festgestellt. Die Versuche wurden als weggeregelte Zugversuche bis zum Bruchversagen der Schweißnaht durchgeführt. Bei etwa der Hälfte der erwarteten Bruchlast wurde der Versuch 5 Minuten lang angehalten. Anschließend wurde entlastet und dann bis zum Bruch belastet. Während des Versuchs wurden die Kraft und der Traversenweg gemessen. Wegmessungen zur Ermittlung der Nahtverschiebung erfolgten mit Hilfe von Wegaufnehmern. Nach dem Bruch der Schweißnaht und dem Ausbau der Versuchskörper wurden die Bruchflächen vermessen. Der Bruch trat stets in der Naht unter einem Winkel nahe $45^{\circ}$ ein. Die Bruchfläche wurde auf einer aufgelegten Folie nachgezeichnet. Diese Abbildung der Bruchfläche wurde digitalisiert, und die Größe der Fläche wurde grafisch bestimmt.

\section{5 Überblick über die Versuchsergebnisse}

Die Versuchsergebnisse aller Voruntersuchungen sind in Tabelle 7.4 zusammengestellt. Aufgeführt sind die gemessenen maximalen Prüfkräfte, die ermittelten Bruchflächen und die aus diesen Größen ermittelten Maximalspannungen $\tau_{\| 1}$. Das Versagen trat wie geplant in der Naht auf und nie im Grundwerkstoff.

Tabelle 7.4: Versuchsergebnisse Überlappverbindungen mit Flankenkehlnähten

\begin{tabular}{|l|l|l|l|l|l|l|}
\hline Serie & Name & GW & SZW & $\begin{array}{l}\text { Max. Prüfkraft } \\
{[\mathrm{kN}]}\end{array}$ & $\begin{array}{l}\text { Bruchfläche } \\
{\left[\mathrm{mm}^{2}\right]}\end{array}$ & $\begin{array}{l}\text { Max. Spannung } \tau_{\mathrm{ll}} \\
{\left[\mathrm{N} / \mathrm{mm}^{2}\right]}\end{array}$ \\
\hline \multirow{2}{*}{1} & Z-001 & S690Q & G69 & 722,5 & 1376 & 525 \\
\cline { 2 - 7 } & Z-002 & S690Q & G69 & 721,4 & 1382 & 522 \\
\hline \multirow{2}{*}{3} & Z-003 & S690Q & G69 & 868,1 & 1640 & 529 \\
\cline { 2 - 7 } & Z-004 & S690Q & G69 & 974,5 & 1990 & 490 \\
\hline \multirow{2}{*}{3} & Z-005 & S690Q & G89 & 761,2 & 1326 & 574 \\
\cline { 2 - 7 } & Z-006 & S690Q & G89 & 743,6 & 1307 & 569 \\
\hline \multirow{2}{*}{4} & Z-007 & S690Q & G89 & 1510,2 & 2783 & 543 \\
\cline { 2 - 7 } & Z-008 & S690Q & G89 & 1560,6 & 2830 & 551 \\
\hline 5 & Z-009 & S355J2 & G89 & 720,5 & 1343 & 536 \\
\hline
\end{tabular}




\subsection{Tragverhalten bei gleichen Grundwerkstoffen und zugehörigen Schweißzusatzwerkstoffen}

Versuchskörper mit gleichen Grundwerkstoffen und zugehörigen Schweißzusatzwerkstoffen wurden als Serie 1 gefertigt, um die Versuchsergebnisse der manuell gefertigten Stecklaschenverbindungen (Kapitel 6) zu bestätigen. Zusätzlich wurden mit dieser Werkstoffkombination zwei weitere Versuchskörper gefertigt (Serie 2), bei denen die Schweißparameter durch die Abkühlzeit $t_{8 / 5}$ variiert wurden, um den Einfluss der Abkühlzeit $\mathrm{t}_{8 / 5}$ auf die Tragfähigkeit zu bestimmen.

Die Versuche der Serie 1 ergeben einen Mittelwert der Tragfähigkeit von 524 N/mm². Im Vergleich dazu wurden bei den in Kapitel 6 vorgestellten einlagigen Versuchen im Mittel Tragfähigkeiten von $539 \mathrm{~N} / \mathrm{mm}^{2}$ festgestellt, vgl. Abbildung 6.2. Die Tragfähigkeiten sind somit vergleichbar.

Bei der Herstellung der Versuchskörper der Serie 2 wurde die Abkühlzeit $t_{8 / 5}$ variiert. Durch die Variation von Schweißgeschwindigkeit, Stromstärke und Spannung wurden unterschiedliche Abkühlzeiten $t_{8 / 5}$ erreicht. Die Abkühlzeiten $t_{8 / 5}$ wurden von 7,5 s (Serie 1) auf 10 und 15 Sekunden variiert. Abbildung 7.2 zeigt den Vergleich der Tragfähigkeiten bei unterschiedlichen Abkühlzeiten. Die Tragfähigkeit der Versuche mit einer Abkühlzeit $\mathrm{t}_{8 / 5}$ von 7,5 s und $10 \mathrm{~s}$ sind nahezu identisch, während bei einer Abkühlzeit $\mathrm{t}_{8 / 5}=15 \mathrm{~s}$ eine Abnahme der Tragfähigkeit von $7 \% \mathrm{zu}$ beobachten ist.

Es zeigt sich sehr deutlich, dass die Abkühlbedingungen einen großen Einfluss auf die Tragfähigkeit haben. Langsame Abkühlzeiten können zu einer Erweichung führen, wie in Kapitel 2.4.6 beschrieben.

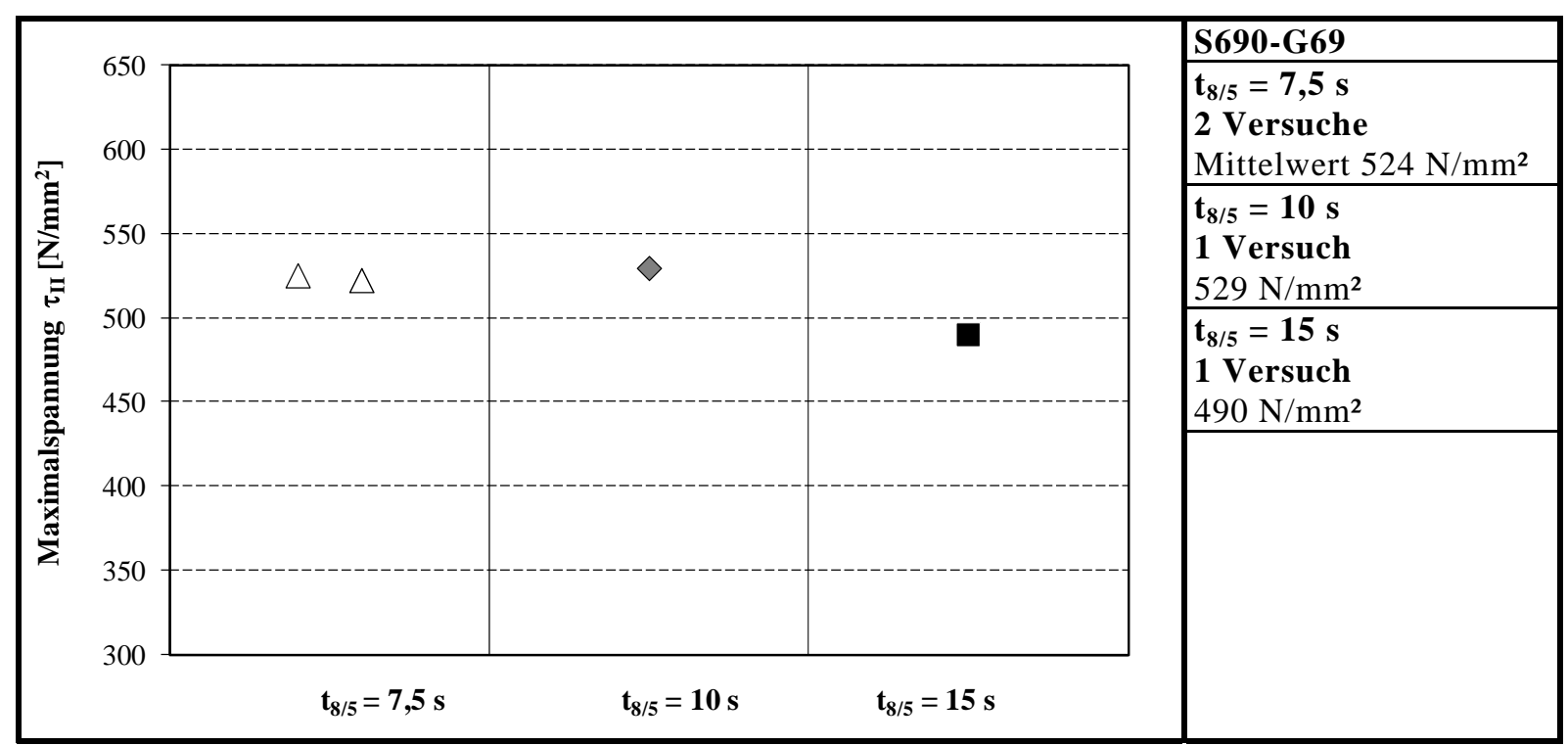

Abbildung 7.2: Einfluss der Abkühlzeit $t_{8 / 5}$ auf die Tragfähigkeit

\subsection{Tragverhalten bei gleichen Grundwerkstoffen und Variation der Schweißzusatzwerkstoffe}

\subsubsection{Einfluss der Festigkeit des Schweißzusatzwerkstoffs}

Die Versuche der Serie 3 wurden durchgeführt, um den Einfluss der Festigkeit des Schweißzusatzwerkstoffs auf die Tragfähigkeit zu ermitteln. Dazu wurden Bleche der Grundwerkstoffe S690 mit einem Schweißzusatzwerkstoff der Festigkeitsklasse G89 verschweißt. In Abbildung 7.3 werden die Tragfähigkeiten der Versuche mit zugehörigem Schweißzusatzwerkstoff G69 (Serie 1) mit den Tragfähigkeiten der Versuche mit dem höherfesten Schweißzusatzwerkstoff G89 (Serie 3) verglichen. Im Diagramm werden als Tragfähigkeiten die Maximalspannungen $\tau_{\text {II }}$ in Abhängigkeit der Zugfestigkeit des Schweißzusatzwerkstoffs dargestellt. Deutlich erkennbar ist die geringe Streuung der Versuchsergebnisse. Die jeweils nominell identischen Versuchskörper liefern nahezu gleiche Ergebnisse. Vergleicht man die Mittelwerte der Versuchsergebnisse, stellt man eine Steigerung der Tragfähigkeit infolge des höherfesten Schweißzusatzwerkstoffs um ca. 9 \% fest. Damit zeigt sich der deutliche Ein- 
fluss des Schweißzusatzwerkstoffs auf die Tragfähigkeit der Schweißverbindung. Die Steigerung der Tragfähigkeit ist mit $9 \%$ aber deutlich geringer als die Steigerung der Zugfestigkeiten der eingesetzten Schweißzusatzwerkstoffe mit 17 \%. Der Grund dafür ist darin zu sehen, dass der Grundwerkstoff die Festigkeit des Schweißgutes ebenfalls beeinflusst, da alle anderen Parameter identisch sind.

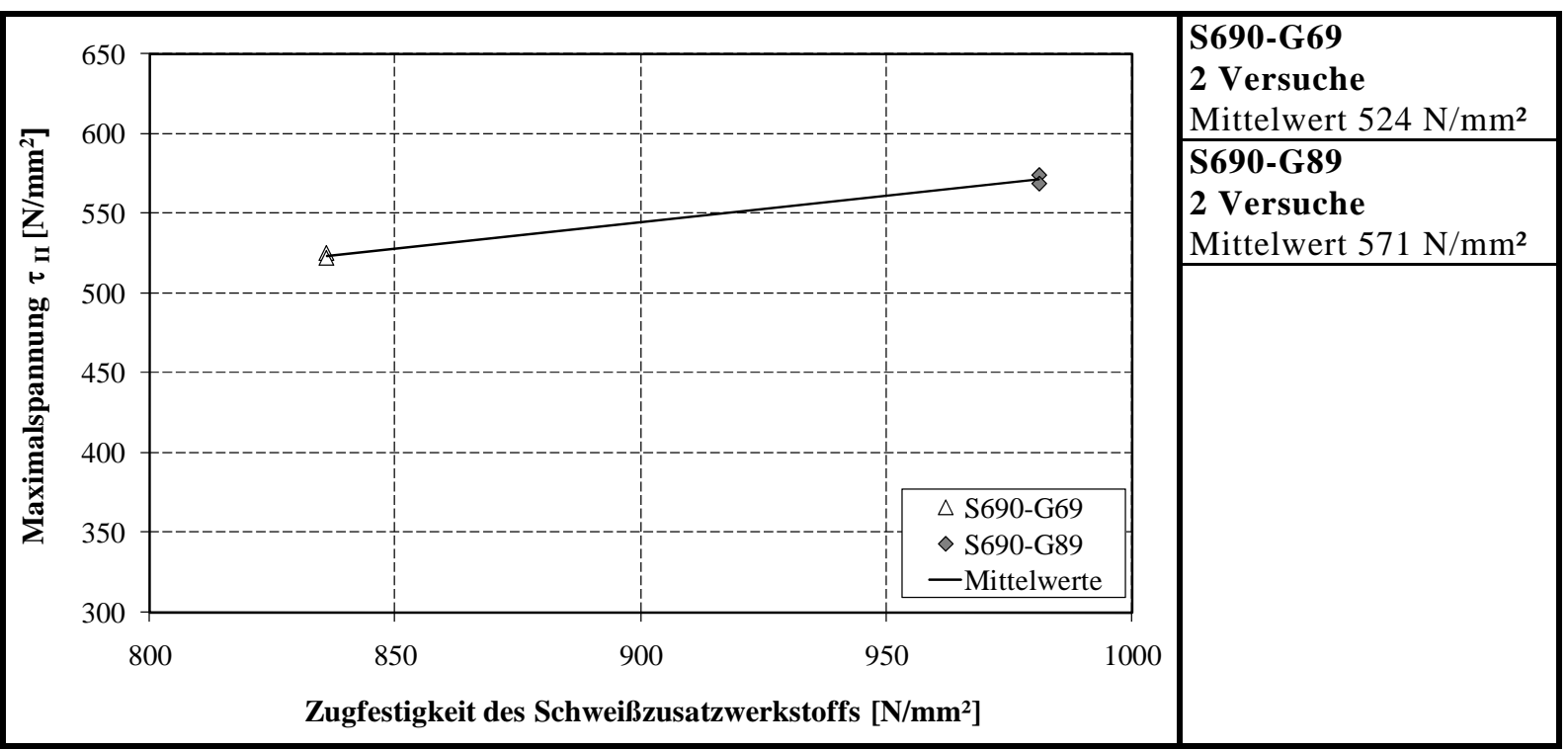

Abbildung 7.3: Einfluss des Schweißzusatzwerkstoffs auf die Tragfähigkeit

\subsubsection{Nahtdickeneinfluss}

Die Analyse des Nahtdickeneinflusses wurde als Serie 4 an Versuchskörpern der Stahlgüte S690QG89 mit zweilagigen Nähten durchgeführt. Die Tragfähigkeiten dieser zweilagigen Nähte der Serie 4 werden mit denen der einlagigen Schweißnähte der Serie 3 in Abbildung 7.4 verglichen. Wiederum ist die Streuung der Versuchsergebnisse der nominell gleichen Versuchskörper sehr gering. Die Abbildung zeigt eine leicht erhöhte Tragfähigkeit (4\%) der einlagigen Nähte im Vergleich zu den mehrlagigen Nähten.

Durch die zweite Lage wird die Wurzellage der Schweißnaht vergütet. Das heißt, dass durch das erneute Aufschmelzen Festigkeit und Härte abnehmen, vgl. Kapitel 4.2.3.

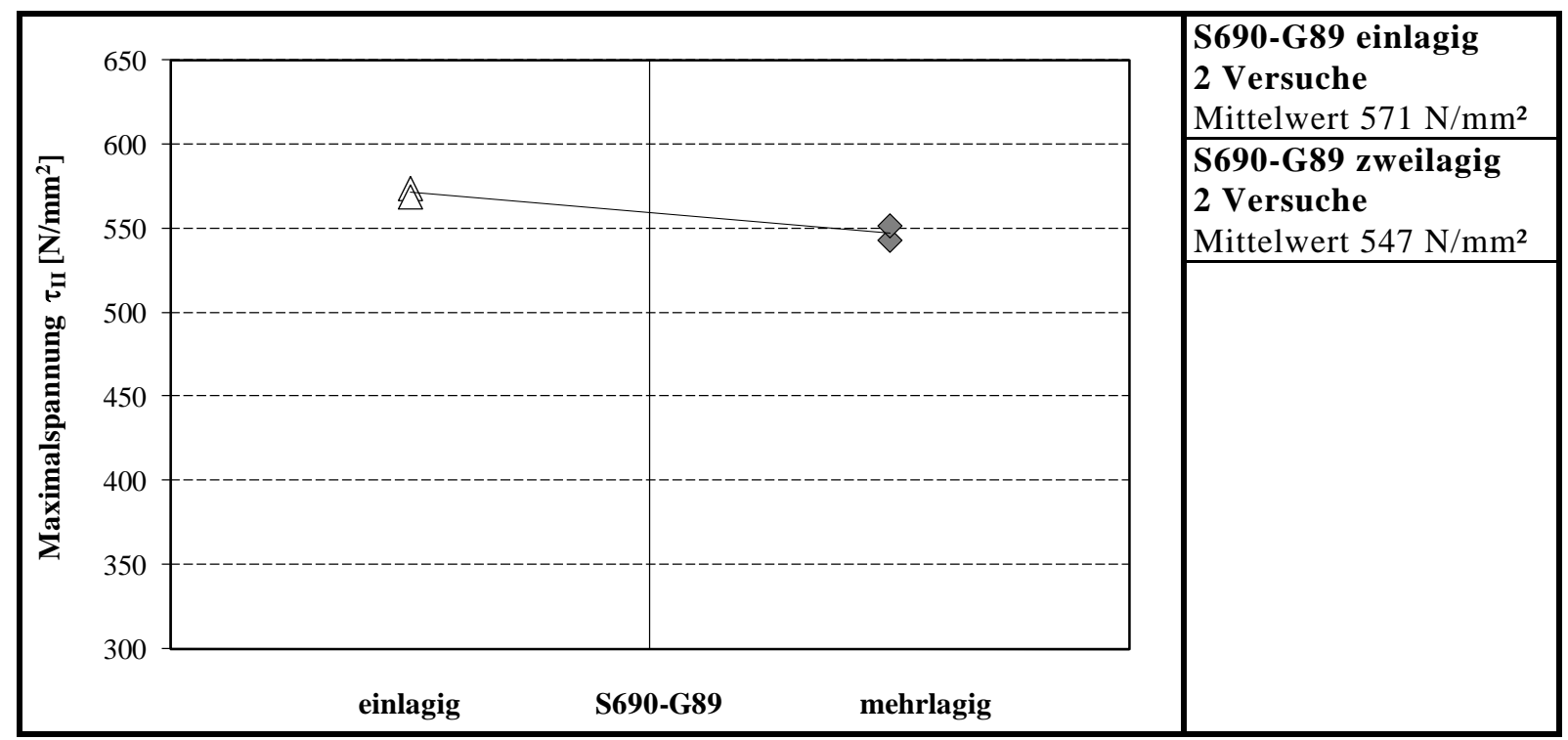

Abbildung 7.4: Einfluss der Anzahl der Lagen auf die Tragfähigkeit 


\subsection{Einfluss von Stahlsorte und Schweißzusatzwerkstoff (Serie 5)}

Um den Einfluss des Grundwerkstoffs auf die Tragfähigkeit der Schweißverbindung zu ermitteln, wurde in Serie 5 mit einem normalfesten Grundwerkstoff S355J2 eine einlagige Schweißverbindung mit dem Schweißzusatzwerkstoff G89 hergestellt. Die Tragfähigkeit wird mit den Ergebnissen der Serie 3 mit dem Grundwerkstoff S690 und dem Schweißzusatzwerkstoff G89 verglichen, vgl. Abbildung 7.5. Durch Verwendung des Schweißzusatzwerkstoffs G89 kann eine Steigerung der Tragfähigkeit bei Versuchen mit dem Grundwerkstoff S690Q im Vergleich mit Versuchen, die mit dem Schweißzusatzwerkstoff G69 verschweißt wurden, erreicht werden. Der Versuch mit dem Grundwerkstoff S355J2 und dem Schweißzusatzwerkstoff G89 zeigt eine ähnliche, nur leicht geringere Tragfähigkeit als die Versuche S690-G89. Man sieht, dass der Schweißzusatzwerkstoff den größten Anteil an der Tragfähigkeit der Schweißverbindungen hat. Es zeigt sich, dass bei identischem Zusatzwerkstoff (G89) ein Einfluss der Festigkeit des Grundwerkstoffs, hier S690Q und alternativ S355J2, vorhanden ist. Der Unterschied in der Tragfähigkeit beträgt rund $7 \%$.

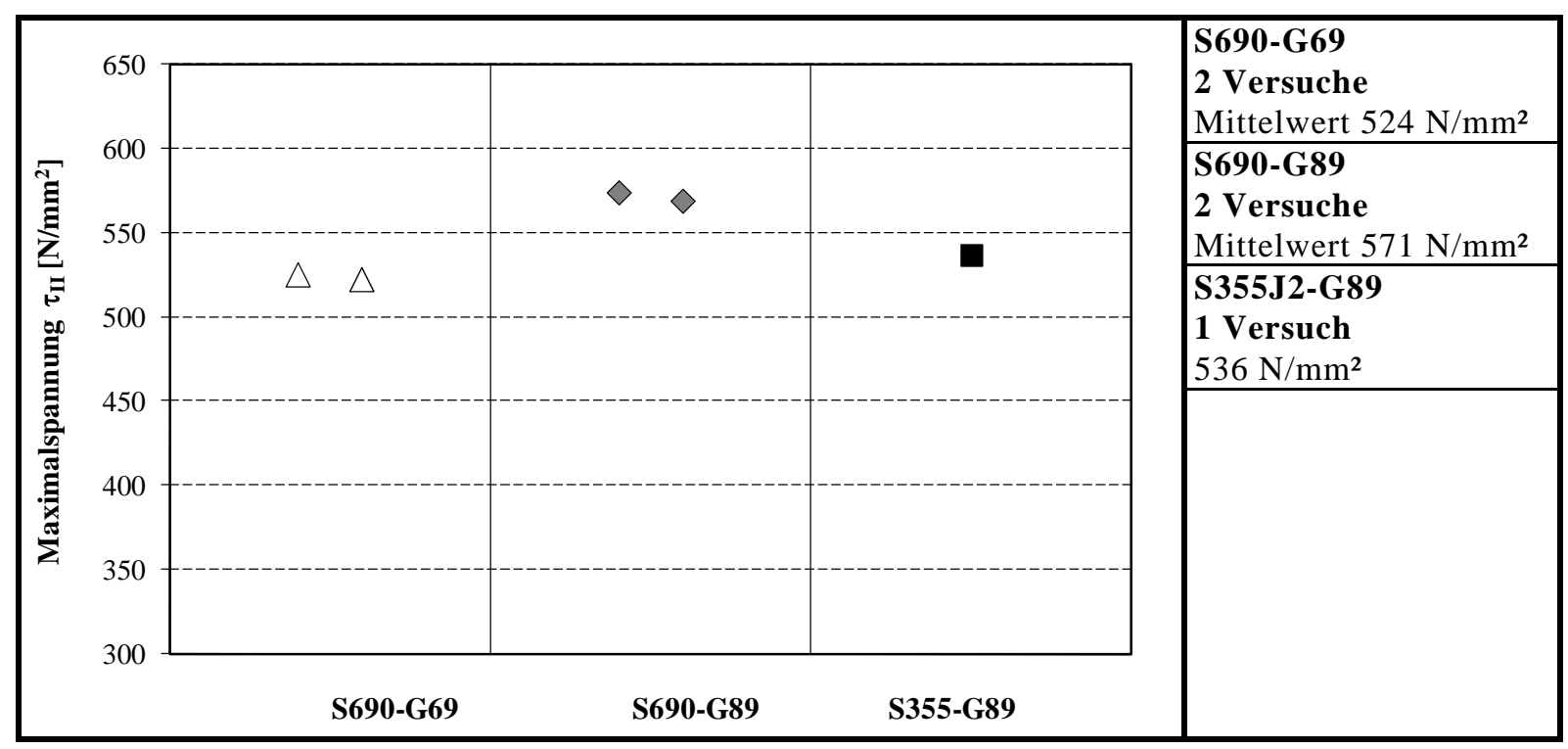

Abbildung 7.5: Einfluss von Stahlsorte und Schweißzusatzwerkstoff auf die Tragfähigkeit

\subsection{Werkstoffuntersuchungen}

An den einlagigen Verbindungen der Serien 1, 3 und 5 wurden Härtemessungen durchgeführt, um mit Hilfe der Härte die Festigkeit des Schweißgutes und damit die Tragfähigkeit der Verbindung abzuschätzen. Dazu werden nur die Härtewerte im Schweißgut betrachtet.

In Abbildung 7.6 werden die Makroschliffe der Proben Z-001 (S690-G69), Z-006 (S690-G89) und Z009 (S355-G89) gezeigt. Zur Beurteilung der Zugfestigkeiten der einlagigen Kehlnähte wurden Härtemessungen (HV1) in drei Reihen durchgeführt. Die Lage der Reihen ist in Abbildung 7.6 d) dargestellt. Reihe 1 wurde nahe der Oberfläche gelegt, Reihe 2 nahe der Wurzel und Reihe 3 verläuft senkrecht dazu entlang der Bruchfläche. Die Bereiche des Grundwerkstoffs (GW), der Wärmeeinflusszone (WEZ) und des Schweißgutes (SG) sind gekennzeichnet.

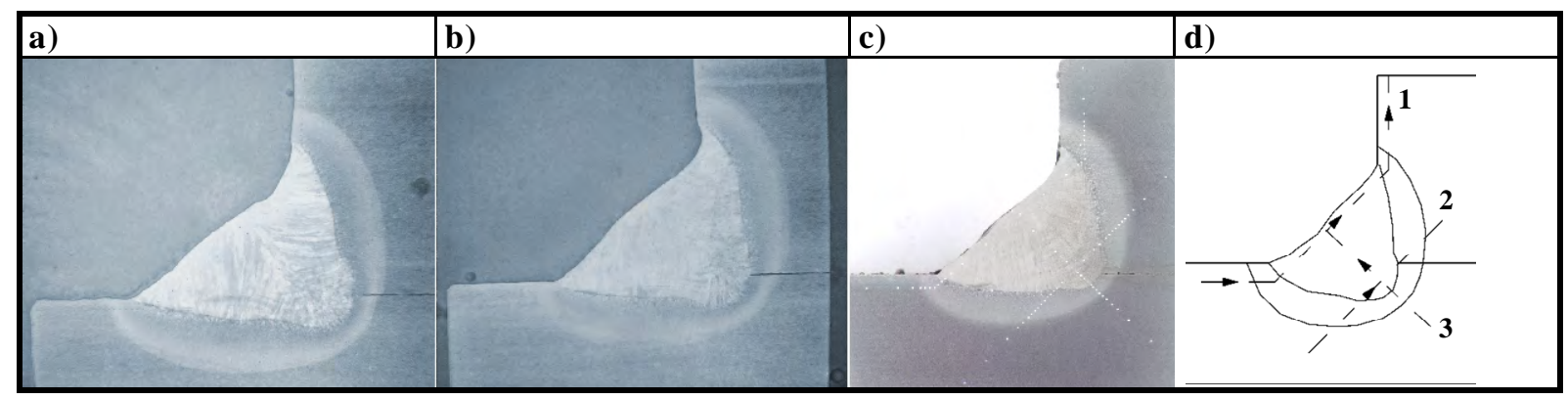

Abbildung 7.6: Makroschliffe S690 G69 (a), S690 G89 (b) und S355-G89 (c), Härteverlauf (d) 
In Abbildung 7.7 sind die Ergebnisse der Härtemessung (HV1) in Reihe 1 dargestellt. Für die Reihe 1 (nahe der Oberfläche der Schweißnaht) zeigt sich eine Steigerung der Härte im Schweißgut von ca. 10 \% zwischen S690-G69 und S690-G89. Die Härte des Schweißgutes der Verbindung S355J2-G89 liegt um 3 \% unter den Werten für S690-G89. Die Härtewerte bestätigen somit die Unterschiede in der Tragfähigkeit. Infolge des Schweißzusatzwerkstoffs G89 stellen sich höhere Härtewerte ein als bei Verwendung des Schweißzusatzwerkstoffs G69. Der Einfluss des Grundwerkstoffs kann bei gleichbleibendem Schweißzusatzwerkstoff durch eine geringere Härte bei Verwendung von S355J2 gezeigt werden.

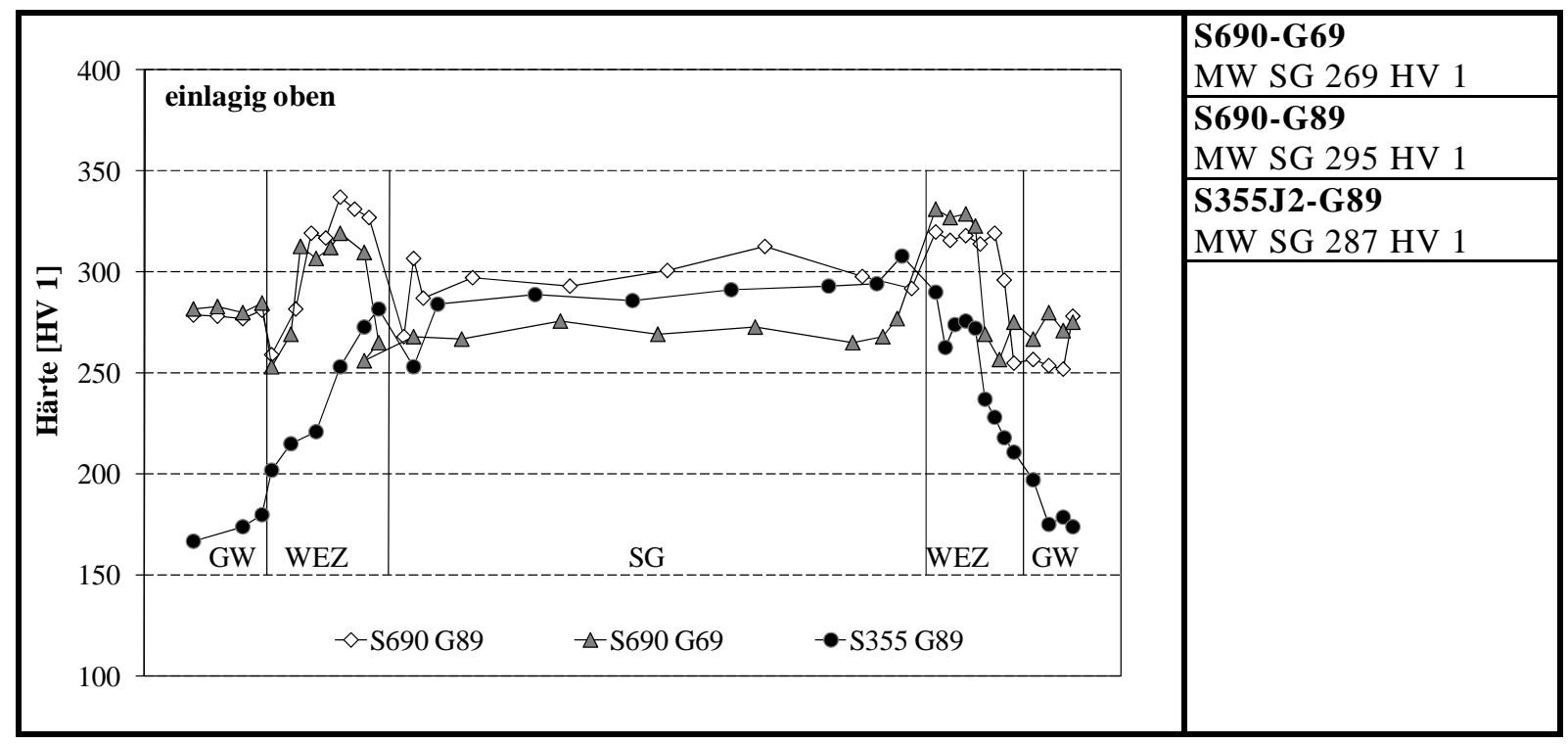

Abbildung 7.7: Vergleich der Härtewerte S690-G69, S690-G89 und S355-G89 in Reihe 1

\subsection{Diskussion der Ergebnisse}

In Abbildung 7.8 ist einen Vergleich der maximal erzielten Spannungen $\tau_{\| l}$ aller Versuche bezogen auf die Zugfestigkeit des jeweiligen Schweißzusatzwerkstoffs (G69 $\mathrm{f}_{\mathrm{u}}=836 \mathrm{~N} / \mathrm{mm}^{2}$ und G89 $f_{u}=981 \mathrm{~N} / \mathrm{mm}^{2}$ ) gemäß Lieferzeugnis (vgl. Tabelle 7.3) dargestellt. Man erkennt, dass die Versuche der einzelnen Serien nur eine geringe Streuung der Tragfähigkeit aufweisen. Außerdem zeigt sich ein deutlicher Einfluss des Schweißzusatzwerkstoffs auf die Tragfähigkeit der Schweißverbindung, während der Einfluss des Grundwerkstoffs geringer ist. Aufgrund der geringen Anzahl von Versuchen wird in diesem Fall auf eine statistische Auswertung verzichtet.

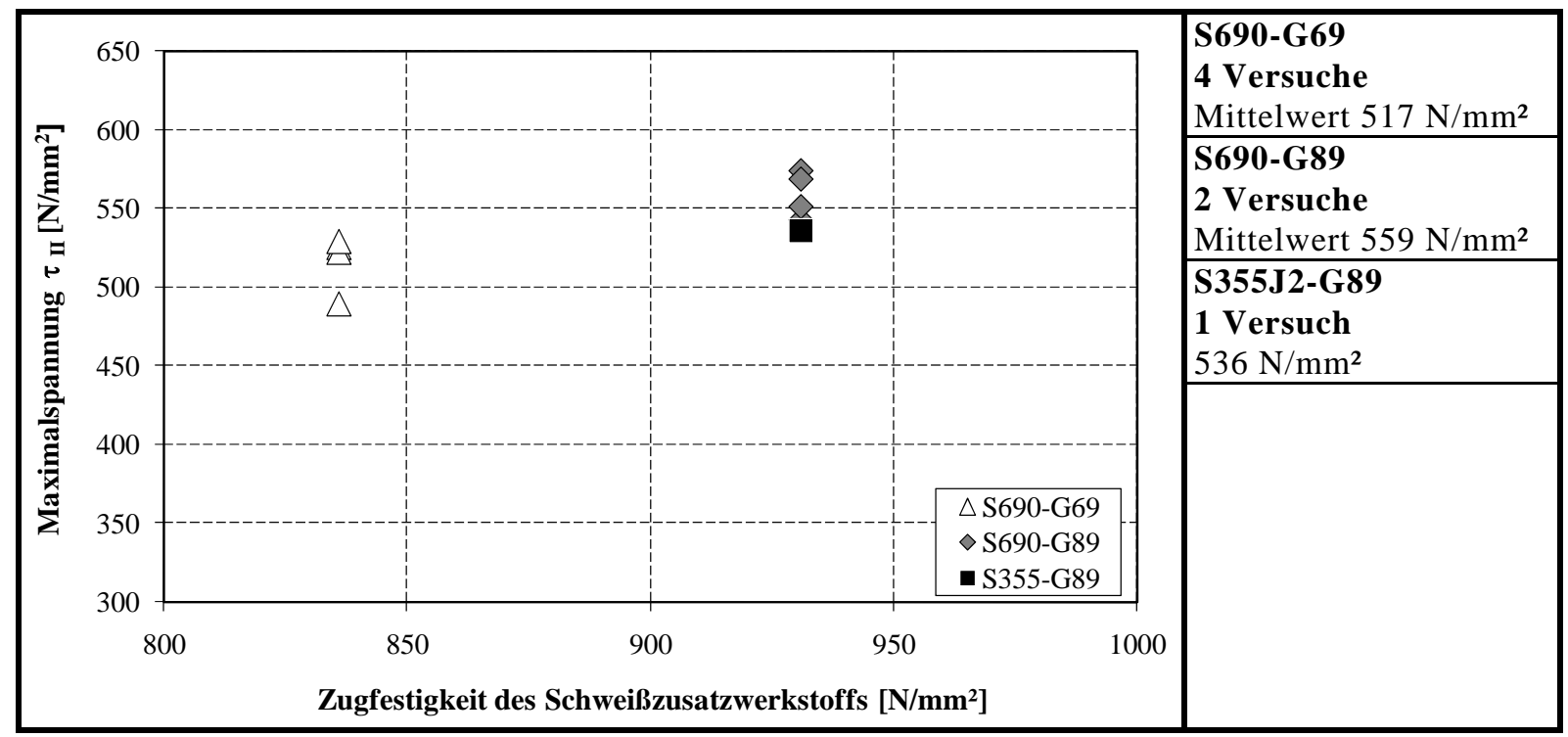

Abbildung 7.8: Darstellung der Tragfähigkeit aller durchgeführten Versuche 
In Tabelle 7.5 sind die mittleren Härtewerte der Versuche Z-001, Z-006 und Z-009 mit den Tragfähigkeiten zusammengefasst. Die Härtewerte im Schweißgut sind als Mittelwerte der einzelnen Härtereihen sowie als Gesamtmittelwert im Schweißgut angegeben. In dem Diagramm in Tabelle 7.5 sind die Tragfähigkeiten in Abhängigkeit der Härtewerte aufgetragen. Das dargestellte Diagramm zeigt eine Ausgleichsgerade mit der Steigung 3,2/ $\sqrt{3}$ (vgl. Kapitel 2.6), die eine gute Übereinstimmung liefert. Der Unterschied in der Härte zwischen S690-G69 und S690-G89 von etwa 10 \% entspricht etwa dem Unterschied in der Tragfähigkeit. Der Vergleich der Härte zwischen S355-G89 und S690-G89 liefert sehr ähnliche Werte, die Tragfähigkeit unterscheidet sich um $7 \%$.

Tabelle 7.5: Vergleich der Härtemessungen und Tragfähigkeiten von einlagigen Kehlnähten

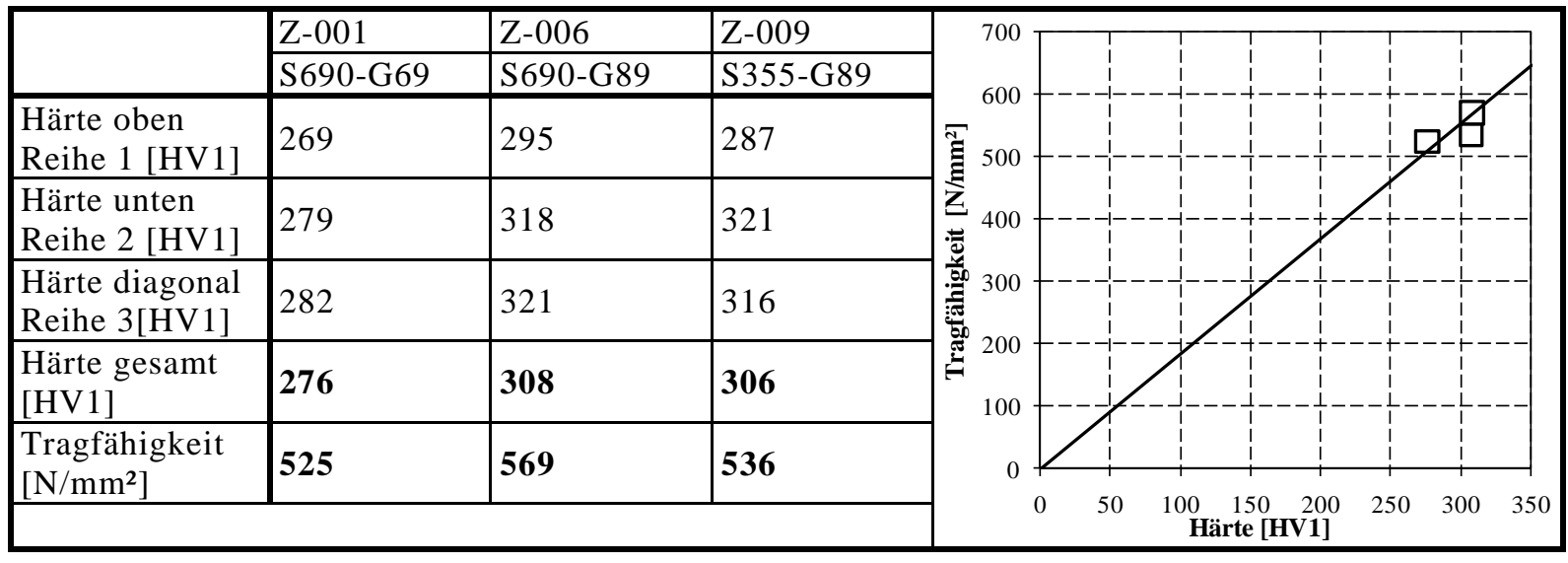

\subsection{Zusammenfassung}

Die Ergebnisse der durchgeführten Versuche [Zusatzversuche, 2008] zeigen, dass durch den gewählten vollmechanisierten Fertigungsprozess und die aufwändig hergestellte Prüfkörperform (ohne Nahtanfangs- und Nahtendbereich) eine deutlich geringere Streuung der Versuchsergebnisse im Vergleich zu den in [FOSTA P652, 2008] durchgeführten Untersuchungen (Kapitel 6) erzielt werden kann. Die Abweichungen der Tragfähigkeiten der jeweils nominell gleichen Versuchskörper liegen unterhalb von $2 \%$. Die Tragfähigkeiten sind somit sehr ähnlich.

Die Variation der Abkühlzeit $t_{8 / 5}$ von 7,5 s (Serie 1) auf $10 \mathrm{~s}$ und $15 \mathrm{~s}$ (Serie 2) hat gezeigt, dass die Abkühlbedingungen einen großen Einfluss auf die Tragfähigkeit haben. Langsame Abkühlzeiten können zu einer Abnahme der Tragfähigkeit führen.

Der Vergleich der Versuchsergebnisse der im Arbeitsfeld eines S690Q geschweißten Flankenkehlnahtverbindungen unter Verwendung eines Schweißzusatzwerkstoffs G69 mit denen unter Verwendung des höherfesteren Schweißzusatzwerkstoffes G89 geschweißten Versuchskörper zeigt eine Steigerung der Festigkeit zwischen dem Schweißzusatzwerkstoff G69 und G89 in Höhe von 9 \%. Dieser Unterschied findet sich auch in den durchgeführten Härtemessungen im Schweißgut der Verbindung wieder. Damit zeigt sich der deutliche Einfluss des Schweißzusatzwerkstoffs auf die Tragfähigkeit der Schweißverbindung. Die Steigerung der Tragfähigkeit ist aber deutlich geringer als die Steigerung der Zugfestigkeiten der eingesetzten Schweißzusatzwerkstoffe mit $17 \%$. Der Grund dafür ist darin zu sehen, dass der Grundwerkstoff die Festigkeit des Schweißgutes ebenfalls beeinflusst, da alle anderen Parameter identisch sind.

Der Vergleich der Versuchsergebnisse S355J2-G89 und S690-G89 zeigt, dass entgegen den bisherigen normativen Berechnungsansätzen die ermittelten maximalen Schweißnahtspannungen $\tau_{\text {II }}$, bedingt durch das Versagen bzw. den Bruch im Schweißgut, vor allem mit der Zugfestigkeit des Schweißzusatzwerkstoffs übereinstimmen, und damit die Tragfähigkeit der hier untersuchten Flankenkehlnahtverbindungen im Wesentlichen durch die Festigkeit des Schweißzusatzwerkstoffes bestimmt wird. Es zeigt sich aber auch, dass bei identischem Schweißzusatzwerkstoff (G89) ein Einfluss der Festigkeit des Grundwerkstoffs, hier S690Q und alternativ S355J2, vorhanden ist. 


\section{Untersuchungen an vollmechanisiert geschweißten Ver- suchskörpern}

\subsection{Einleitung}

In diesem Kapitel werden die Versuchsergebnisse, die im Rahmen des Forschungsvorhabens [FOSTA P812, 2011] ermittelt wurden, vorgestellt. Die Ziele dieses Forschungsvorhabens bauen auf den Ergebnissen der in Kapitel 6 [FOSTA P652, 2008] und Kapitel 7 [Zusatzversuche, 2008] vorgestellten Untersuchungen auf.

Die in Kapitel 6 ermittelten Tragfähigkeiten von Stecklaschenverbindungen mit dem Grundwerkstoff S690 und zugehörigem Schweißzusatzwerkstoff lagen unterhalb der Tragfähigkeiten nach den damals aktuellen Normen EN 1993-1-12 (2007). Die in Kapitel 7 vorgestellten Versuche an Überlappverbindungen bestätigten dieses Ergebnis. Zusätzlich wurden in Kapitel 7 Voruntersuchungen vorgestellt, bei denen durch Verwendung eines höherfesten Schweißzusatzwerkstoffs eine erhöhte Tragfähigkeit erreicht wurde.

Aufbauend auf den Ergebnisse, die in Kapitel 6 und 7 vorgestellt wurden, wurden im Rahmen des Forschungsvorhabens [FOSTA P812, 2011] weitere Untersuchungen geplant und durchgeführt. Innerhalb des Forschungsvorhabens [FOSTA P812, 2011], das in den nächsten Abschnitten vorgestellt wird, wurde detailliert und umfassend das Tragverhalten von Schweißverbindungen höherfester Baustähle S690Q und S700M analysiert.

Ziel des Projekts [FOSTA P812, 2011] ist die Ermittlung der Tragfähigkeit von Kehlnahtverbindungen höherfester Baustähle S690. Dazu wurden neben Tragfähigkeitsuntersuchungen auch metallurgische Untersuchungen durchgeführt. Der Schwerpunkt der Untersuchungen lag auf der Analyse des Einflusses der Festigkeit des Schweißzusatzwerkstoffs auf die Tragfähigkeit der Schweißverbindung höherfester Stähle S690.

\subsection{Versuchsprogramm}

Um mögliche Einflussparameter auf die Tragfähigkeit von Kehlnahtverbindungen eindeutig bestimmen zu können, wurden alle Versuchskörper unter den gleichen konstanten Bedingungen vollmechanisiert gefertigt. Zur Bestimmung der Tragfähigkeit der Naht wurden die Versuchskörper so dimensioniert, dass ein Versagen im Schweißgut und nicht im Grundwerkstoff auftrat. Die Überlappverbindungen mit Flankenkehlnähten und die Kreuzstöße mit Stirnkehlnähten sind in Tabelle 8.1 schematisch dargestellt. Für die Traglastversuche an Flankenkehlnahtverbindungen wurden jeweils zwei nominell gleiche Versuchskörper hergestellt. Für die Analyse der Kreuzstöße standen jeweils drei gleiche Versuchskörper zur Verfügung. Die Parameter der einzelnen Versuchskörper sind im Detail im Anhang aufgeführt, Tabelle 8.1 gibt einen Überblick.

Zur Analyse des Einflusses einzelner Parameter wurden Untersuchungen zur Traglastbestimmung in 7 Serien durchgeführt, vgl. Tabelle 8.1. Untersucht wurde immer der Einfluss dieser Parameter auf die Tragfähigkeit der Schweißverbindung.

In Serie 1 wurden Flankenkehlnahtverbindungen höherfester Stähle S690 verschiedener Stähle mit Schweißzusatzwerkstoffen zugehöriger Festigkeitsklassen verschiedener Hersteller kombiniert.

Um den Einfluss der Abkühlzeiten $t_{8 / 5}$ an Flankenkehlnahtverbindungen S690-G69 zu bestimmen, wurden in Serie 2 die Schweißparameter in Form von unterschiedlichen Abkühlzeiten $t_{8 / 5}$ innerhalb des Arbeitsfensters mit $\mathrm{t}_{8 / 5}=10 \mathrm{~s}$ und $\mathrm{t}_{8 / 5}=15 \mathrm{~s}$ (vgl. z. B. Abbildung 8.1) variiert.

Serie 3 variierte die Nahtlängen über das Verhältnis l/a an Schweißverbindungen gleicher Grundwerkstoffe S690 und zugehörigen Schweißzusatzwerkstoffen.

In Serie 4 wurde der Einfluss der Nahtdicke und der Blechdicke untersucht. Dazu wurden mehrlagige Verbindungen gleicher Grundwerkstoffe S690 und zugehöriger Schweißzusatzwerkstoffe von Flankenkehlnähten geprüft. 
Zur Bestimmung des Einflusses der Zugfestigkeit des Schweißzusatzwerkstoffs auf die Tragfähigkeit von Flankenkehlnahtverbindungen wurden in Serie 5 Versuchskörper mit dem Grundwerkstoff S690 geprüft, die mit Schweißzusatzwerkstoffen nominell nieder- oder höherfesten Schweißzusatzwerkstoffen (G46 oder G89) verschweißt waren.

In Serie 7 wurden ergänzende Flankenkehlnahtversuche mit dem Grundwerkstoff S460 und zugehörigem Schweißzusatzwerkstoff G46 durchgeführt, um die Tragfähigkeit im Vergleich mit den Versuchen mit dem Grundwerkstoff S690 zu bestimmen. Zusätzlich wurde der Einfluss der Vorwärmtemperatur ermittelt.

In Serie 6 wurden die Versuche an Kreuzstößen mit Stirnkehlnähten durchgeführt. Dazu wurde die Tragfähigkeit von Schweißverbindungen gleicher Grund- und zugehöriger Schweißzusatzwerkstoffe S690-G69 bestimmt. Außerdem wurde analog zur Serie 5 an Kreuzstößen die Festigkeit des Schweißzusatzwerkstoffs variiert. An einem Versuchskörper S690-G69 wurde der Winkel zwischen Naht und Kraftrichtung variiert.

Tabelle 8.1: Untersuchte Parameter

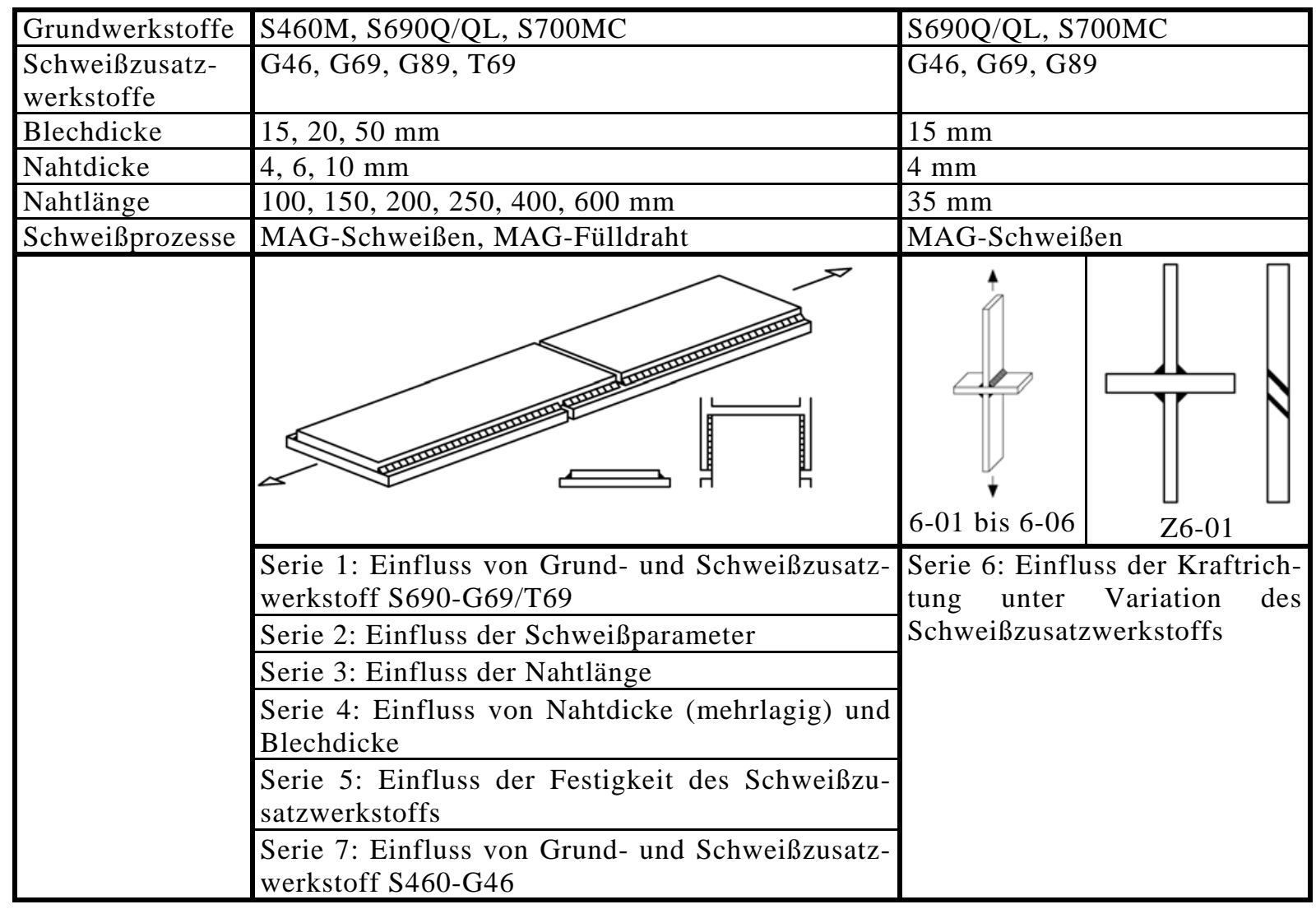

Darüberhinaus wurden für jede Versuchsserie Begleituntersuchungen für die Werkstoffkennwerte, metallografische Untersuchungen und Härtemessungen durchgeführt. In Einzelnen wurden Zugversuche am Grund- und Schweißzusatzwerkstoff durchgeführt. Da die Werkstoffkennwerte des Schweißzusatzwerkstoffs im verschweißten Zustand deutlich von der Art der Schweißung und der Geometrie abhängen, wurden sowohl Zugversuche mit Versuchskörpern aus Stumpfstoßverbindungen zur Bestimmung der normativen Festigkeit des reinen Schweißgutes als auch Zugversuche mit Versuchskörpern aus Kehlnahtverbindungen zur Bestimmung der Werkstoffkennwerte der Kehlnaht durchgeführt. Zusätzlich wurden Härtemessungen durchgeführt, um eine mögliche Korrelation zwischen Härte und Zugfestigkeit zu bestimmen. Weitere Details zum Versuchsprogramm sind in [FOSTA P812, 2011] aufgeführt. Die Kreuzstöße sind auch in [Breunig, 2011] diskutiert. 


\subsection{Verwendete Werkstoffe}

Für die Versuchskörper wurden als Grundwerkstoffe Stähle S690 nach DIN EN 10025-6 (2005), S700M nach DIN EN 10149-2 (1995) und S460M nach DIN EN 10025-4 (2005) verwendet, deren Zeugniskennwerte der mechanischen Eigenschaften in Tabelle 8.2 zusammengestellt sind. Für die verwendeten Schweißzusatzwerkstoffe sind die Normen und Bezeichnungen in Tabelle 8.3 aufgeführt, in Tabelle 8.4 sind die Zeugniskennwerte der mechanischen Eigenschaften zusammengestellt. Die Ergebnisse eigener Zugversuche am Grundwerkstoff und am reinen Schweißgut sind in Tabelle 8.5 und Tabelle 8.6 zusammengefasst. Die ermittelten Zugfestigkeiten beim reinen Schweißgut stimmen mit den Zeugniswerten gut überein (Abweichung ca. $3 \%$ ). Bei mehreren Zugversuchen wurden die Mittelwerte verwendet. In Anhang A.4.1 sind die chemischen Kennwerte der verwendeten Grund- und Schweißzusatzwerkstoffe aufgeführt.

Tabelle 8.2: Mechanische Eigenschaften der verwendeten Grundwerkstoffe gemäß Lieferzeugnis

\begin{tabular}{|c|c|c|c|c|c|c|c|c|c|c|c|c|}
\hline \multirow[t]{2}{*}{ Werkstoff } & \multirow{2}{*}{$\begin{array}{l}\text { Blech- } \\
\text { dicke } \\
{[\mathrm{mm}]}\end{array}$} & \multicolumn{2}{|c|}{$\begin{array}{l}\mathrm{R}_{\mathrm{eH}} \\
{\left[\mathrm{N} / \mathrm{mm}^{2}\right]}\end{array}$} & \multirow{2}{*}{\begin{tabular}{|l|}
$\mathrm{R}_{\mathrm{p} 0,2}$ \\
{$\left[\mathrm{~N} / \mathrm{mm}^{2}\right]$} \\
$\mathrm{Q}$ \\
\end{tabular}} & \multicolumn{2}{|c|}{$\begin{array}{l}\mathrm{R}_{\mathrm{m}} \\
{\left[\mathrm{N} / \mathrm{mm}^{2}\right]}\end{array}$} & \multicolumn{2}{|l|}{\begin{tabular}{|l}
$\mathrm{A}_{5}$ \\
{$[\%]$}
\end{tabular}} & \multicolumn{2}{|c|}{$\begin{array}{l}\mathrm{A}_{\mathrm{v}}[\mathrm{J}] \\
-40^{\circ} \mathrm{C}\end{array}$} & \multicolumn{2}{|c|}{$-60^{\circ} \mathrm{C}$} \\
\hline & & $\mathrm{Q}$ & $\mathrm{L}$ & & Q & $\mathrm{L}$ & Q & $\mathrm{L}$ & $\mathrm{L} \mathrm{O}$ & Q O & $\mathrm{LO}$ & QV \\
\hline S460M & 17,6 & - & 451 & - & 550 & 536 & 25,6 & 30,9 & 346 & & & \\
\hline S690QL1 & 15 & 807 & & - & 836 & & 16 & & & & 215 & \\
\hline S700MC & 15 & 931 & & 926 & 935 & & 15,0 & & 297 & & & \\
\hline S690Q & 20 & 793 & & - & 833 & & 17 & & & 156 & & \\
\hline S700MC & 20 & & & 821 & 860 & & 15,8 & & 234 & & & \\
\hline S690Q & 50 & 796 & & - & 849 & & 17 & & & & & 161 \\
\hline S700MC & 50 & & & 776 & 820 & & 20,8 & & 234 & & & \\
\hline
\end{tabular}

Tabelle 8.3: Verwendete Schweißzusatzwerkstoffe

\begin{tabular}{|l|l|l|l|}
\hline Schweißprozess & Norm & \multicolumn{2}{|l|}{ Bezeichnung } \\
\hline \multirow{3}{*}{135 (MAG) } & DIN EN ISO 14341 (2008) & G46 & G46 2C G4Si1/G46 4MG4Si1 \\
\cline { 2 - 4 } & DIN EN ISO 16834 (2007) & G69 & G69 6 M Mn4Ni1,5CrMo \\
\cline { 2 - 4 } & DIN EN ISO 16834(2007) & G89 & G89 6 M Mn4Ni2CrMo \\
\hline 136 (MAG-Fülldraht) & DIN EN ISO 18276 (2006) & T69 & T69 6 Mn2NiCrMo B M 3 H5 \\
\hline
\end{tabular}

Tabelle 8.4: Mechanische Eigenschaften der verwendeten Schweißzusatzwerkstoffe gemäß Lieferzeugnis

\begin{tabular}{|c|c|c|c|c|c|c|c|c|}
\hline \multicolumn{2}{|l|}{ Schweißzusatzwerkstoff } & $\begin{array}{l}\varnothing \\
{[\mathrm{mm}]}\end{array}$ & $\begin{array}{l}\mathrm{R}_{\mathrm{eH}} \\
{\left[\mathrm{N} / \mathrm{mm}^{2}\right]}\end{array}$ & $\begin{array}{l}\mathrm{R}_{\mathrm{p} 0,2} \\
{\left[\mathrm{~N} / \mathrm{mm}^{2}\right]}\end{array}$ & $\begin{array}{l}\mathrm{R}_{\mathrm{m}} \\
{\left[\mathrm{N} / \mathrm{mm}^{2}\right]}\end{array}$ & $\begin{array}{l}\mathrm{A}_{5} \\
{[\%]} \\
\end{array}$ & $\begin{array}{l}\mathrm{Av}[\mathrm{J}] \\
-40^{\circ} \mathrm{C} \\
\end{array}$ & $-60^{\circ} \mathrm{C}$ \\
\hline Union K56 $^{1)}$ & G46 & 1,2 & & 544 & 627 & 26,4 & 111 & 71 \\
\hline Union NiMoCr ${ }^{1)}$ & G69 & 1,2 & & 719 & 820 & 20,8 & 87 & 71 \\
\hline OK AristoRod 13.29 & G69 & 1,2 & 730 & & 800 & 19 & 55 & \\
\hline STEIN-Megafil $742 \mathrm{~B}^{1)}$ & T69 & 1,2 & & 775 & 834 & 22 & 65 & 53 \\
\hline Union X90 ${ }^{1)}$ & G89 & 1,2 & & 907 & 1028 & 29,6 & 74 & 63 \\
\hline
\end{tabular}

Tabelle 8.5: Ermittelte mechanische Eigenschaften der Schweißzusatzwerkstoffe

\begin{tabular}{|l|l|l|l|l|}
\hline Schweißzusatzwerkstoff & $\mathrm{R}_{\mathrm{eH}}\left[\mathrm{N} / \mathrm{mm}^{2}\right]$ & $\mathrm{R}_{\mathrm{p} 0,2}\left[\mathrm{~N} / \mathrm{mm}^{2}\right]$ & $\mathrm{R}_{\mathrm{m}}\left[\mathrm{N} / \mathrm{mm}^{2}\right]$ & $\mathrm{A}_{5}[\%]$ \\
\hline G46 - Union K56 & $582 / 583$ & & $599 / 605$ & $26,5 / 26,0$ \\
\hline G69A - NiMoCr & $729 / 748$ & & $798 / 798$ & $19,0 / 20,0$ \\
\hline G69B - OK AristoRod 13.29 & \multicolumn{5}{|l}{} \\
\hline G89 - Union X90 & Fehler & & $771 / 764$ & $17,5 / 18,5$ \\
\hline
\end{tabular}

Tabelle 8.6: Ermittelte mechanische Eigenschaften der Grundwerkstoffe

\begin{tabular}{|l|l|l|l|l|l|l|l|}
\hline \multirow{2}{*}{ Werkstoff } & \multirow{2}{*}{ Blechdicke } & \multicolumn{2}{l|}{$\mathrm{R}_{\mathrm{p} 0,2}\left[\mathrm{~N} / \mathrm{mm}^{2}\right]$} & \multicolumn{2}{l|}{$\mathrm{R}_{\mathrm{m}}\left[\mathrm{N} / \mathrm{mm}^{2}\right]$} & \multicolumn{2}{l|}{$\mathrm{A}_{5}[\%]$} \\
& & $\mathrm{Q}$ & $\mathrm{L}$ & $\mathrm{Q}$ & $\mathrm{L}$ & $\mathrm{Q}$ & $\mathrm{L}$ \\
\hline S690QL1 & 15 & 818 & $809 / 810$ & 856 & $847 / 850$ & 18,4 & $18,1 / 18,3$ \\
\hline DILLIMAX690T & 20 & 760 & & 811 & & & 19,6 \\
\hline DILLIMAX690E & 50 & & & & & & \\
\hline ALFORM 700M & 15 & & $838 / 890 / 898$ & & $860 / 904 / 910$ & & $18,5 / 17,3 / 18,5$ \\
\hline ALFORM700M & 20 & & 851 & & 876 & & 17,4 \\
\hline ALFORM700M & 50 & & & & & & \\
\hline S460M & 17,6 & 463 & 480 & 538 & 555 & 29,4 & 26,3 \\
\hline
\end{tabular}


Im Weiteren werden für die eingesetzten Werkstoffe Kurzbezeichnungen verwendet. Es wird zwischen Stahl S700A, S690B und S460 und bei den Schweißzusatzwerkstoffen zwischen G69A, G69B, T69, G46 und G89 unterschieden, Tabelle 8.7 erläutert diese Bezeichnungen.

Tabelle 8.7: Benennung der verwendeten Werkstoffe

\begin{tabular}{|l|l|l|l|l|}
\hline Schweißzusatzwerkstoff & Grundwerkstoff \\
\hline Produktbezeichnung & Kurzbezeichnung & Produktbezeichnung & Dicke & Kurzbezeichnung \\
\hline G46-Union K56 & G46 & S690QL1 & 15 & S690B \\
\hline G69-NiMoCr & G69A & DILLIMAX690T & 20 & S690B \\
\hline G69-OK AristoRod 13.29 & G69B & DILLIMAX690E & 50 & S690B \\
\hline STEIN-Megafil 742 B & T69 & ALFORM 700M & 15 & S700A \\
\hline G89 Union X90 & G89 & ALFORM700M & 20 & S700A \\
\hline & ALFORM700M & 50 & S700A \\
\cline { 2 - 5 } & S460M & 17,6 & S460 \\
\hline
\end{tabular}

\subsection{Herstellung der Versuchskörper und Versuchsdurchführung}

Für die Versuchskörper in Form von Überlappverbindungen und Kreuzstößen erfolgte der Zuschnitt der Bleche mittels Wasserstrahlschneiden, Plasmaschnitt und Brennschnitt. Die Herstellung der Versuchskörper erfolgte anhand von Schweißanweisungen nach DIN EN 1011-2 (2001), [SEW 088, 1993], [SEW 088 Beiblatt 1, 1993] und [SEW 088 Beiblatt 2, 1993], wie in Kapitel 2.5 beschrieben. Die Schweißparameter wurden rechnerisch ermittelt. In Vorversuchen wurden diese Parameter am Realbauteil erprobt. Aus den Vorgaben zur maximalen Nahtdicke erfolgten für die einlagigen Verbindungen folgende Schweißparameter:

Drahtvorschub DV = 8,5 m/min (10,5 m/min für Fülldraht)

Schweißgeschwindigkeit $\mathrm{v}=40 \mathrm{~cm} / \mathrm{min}$

Spannung $\mathrm{U}=28,9 \mathrm{~V}$

Strom I = $310 \mathrm{~A}$

Vorwärmtemperatur von $100^{\circ} \mathrm{C}$

Energieeintrag E von $\mathrm{E}=13,4 \mathrm{~kJ} / \mathrm{cm}$.

Die Schweißparameter wurden so festgelegt, dass sie rechnerisch für alle Werkstoffkombinationen der einlagigen Nähte und einer Blechdicke von $t=15 \mathrm{~mm}$ möglich waren. In Abbildung 8.1 ist das Arbeitsfenster dargestellt, in dem die Schweißparameter, wie in Kapitel 2.5 beschrieben, liegen.

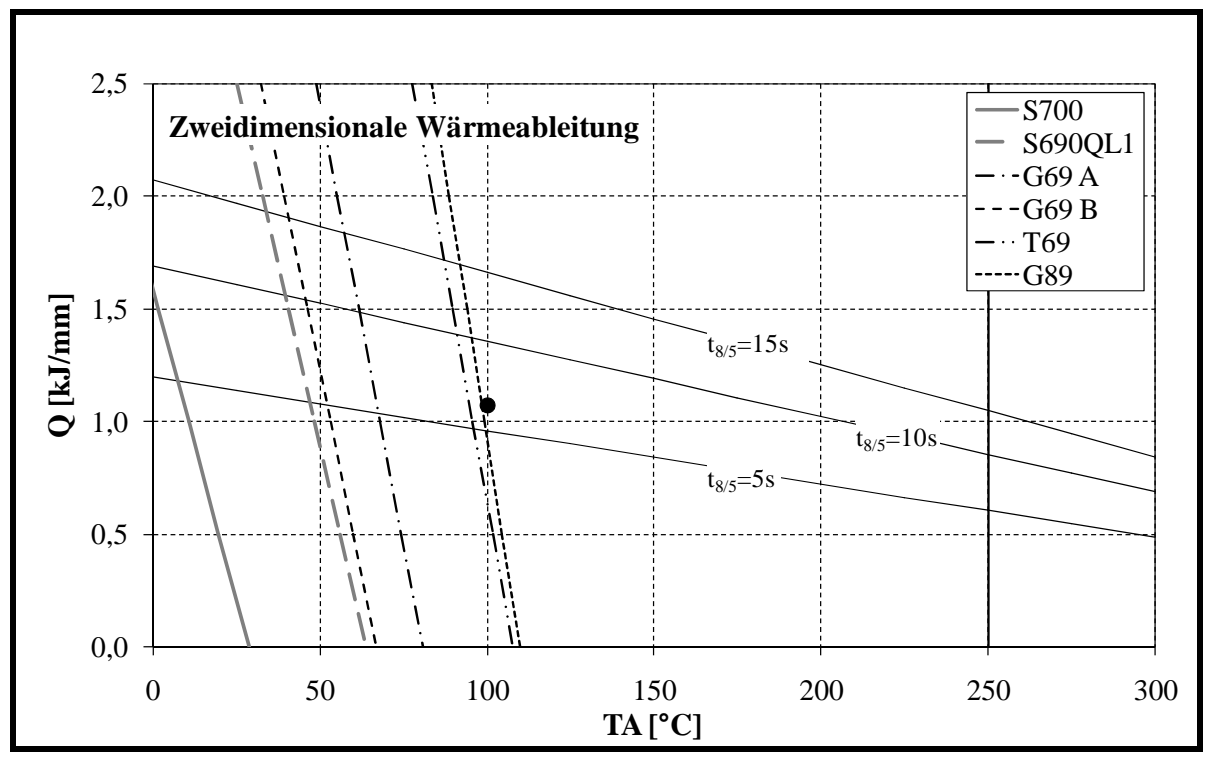

Abbildung 8.1: Arbeitspunkt und Arbeitsfenster für t $=15$ mm 
Alle Versuchskörper wurden vollmechanisiert unter Laborbedingungen geschweißt. Die gemessenen Schweißparameter Streckenenergie, Vorwärmtemperatur und Abkühlzeit $\mathrm{t}_{8 / 5}$ sind im Anhang aufgeführt. Weitere Angaben zur Herstellung der Versuchskörper sind in [FOSTA P812, 2011] zu finden.

Alle Versuchskörper wurden vor dem Versuch vermessen. Die theoretische Nahtdicke wurde mit einer Schweißnahtlehre entlang der Naht gemessen. Es wurde bei allen Versuchskörpern eine zerstörungsfreie Werkstoffprüfung mittels Sichtprüfung durchgeführt. Teilweise wurde bei den Versuchskörpern zusätzlich eine Magnetpulverprüfung durchgeführt. Dabei wurden keine Auffälligkeiten festgestellt. Die Versuche wurden als weggeregelte Zugversuche bis zum Bruchversagen der Schweißnaht durchgeführt. Bei etwa der Hälfte der erwarteten Bruchlast wurde der Versuch 5 Minuten lang angehalten. Anschließend wurde entlastet und dann bis zum Bruch belastet. Während des Versuchs wurden die Kraft und der Traversenweg gemessen. Wegmessungen erfolgten mit Hilfe von optischen Messungen und Wegaufnehmern. Zusätzlich wurden teilweise Dehnungsmessungen durchgeführt. Nach dem Bruch der Schweißnaht und dem Ausbau der Versuchskörper wurden die Bruchflächen vermessen. Bei den Flankenkehlnähten trat der Bruch stets in der Naht unter einem Winkel nahe $45^{\circ}$ ein, während die Kreuzstöße unter einem steileren Winkel von ca. 20 - 30 bezogen auf die Senkrechte versagten. Die Bruchfläche wurde auf einer aufgelegten Folie nachgezeichnet. Diese Abbildung der Bruchfläche wurde digitalisiert, und die Größe der Fläche wurde grafisch bestimmt.

Von jeder Werkstoffkombination mit einlagigen und mehrlagigen Flankenkehlnähten bei Überlappverbindungen und bei den Kreuzstößen mit Stirnkehlnähten wurde ein Makroschliff gefertigt. Die Schliffe wurden bei den Flankenkehlnahtverbindungen im ungebrochenen Teil des Versuchskörpers entnommen, wie Abbildung 8.2 für die Kleinprüfkörper mit einer Nahtlänge von $\mathrm{l}=100 \mathrm{~mm}$ zeigt (Schnitt A-A und B-B). Die Entnahme für die mehrlagigen Versuchskörper (Serie 4) erfolgte analog. Bei den langen Nähten (Serie 3) wurden keine Schliffe entnommen, da dieselben Werkstoffe und dieselben Schweißparameter wie bei den Kleinprüfkörpern (Serie 1) verwendet wurden. Aus jedem Kreuzstoß wurde ein zusätzlicher Versuchskörper als Makroschliff entnommen. Es wurden jeweils 3 Härtereihen an der Nahtoberfläche (Reihe 1), in der Nahtwurzel (Reihe 2) und durch den Nahtquerschnitt (Reihe 3) gelegt. Am deutlichsten zeigen sich die Unterschiede an der Nahtoberfläche (obere Reihe 1), so dass für Vergleiche jeweils diese Reihen herangezogen werden.
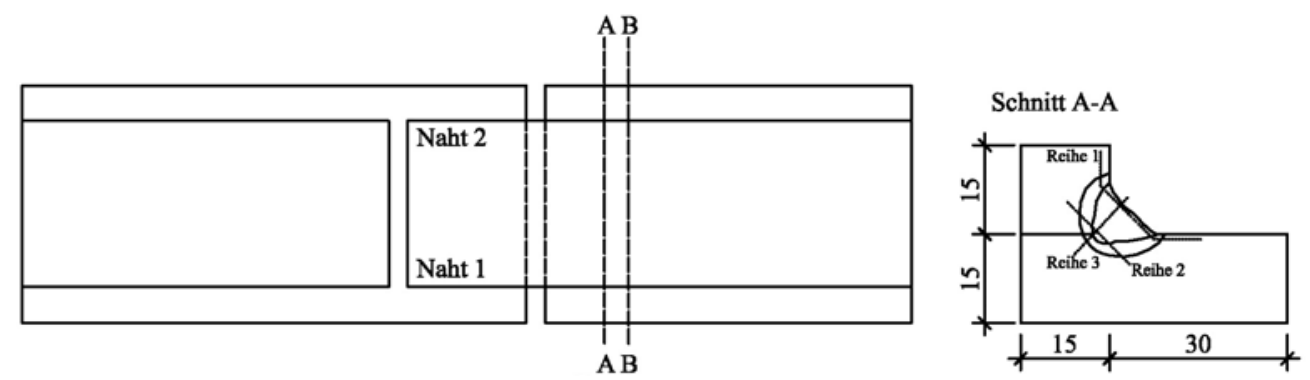

Abbildung 8.2: Entnahme der Makroschliffe von Kleinprüfkörpern - Lage der Härtereihen

\section{5 Überblick über die Versuchsergebnisse an Flankenkehlnahtverbin- dungen}

Um einen Überblick zu geben, sind in Tabelle 8.8 die Versuchsergebnisse aller durchgeführten Traglastversuche an Flankenkehlnahtverbindungen dargestellt. Für die untersuchten Kombinationen aus Grund- und Schweißzusatzwerkstoffen sind für jeden Versuch die gemessene maximale Prüfkraft, die ermittelte Bruchfläche und die Maximalspannung $\tau_{l l}$ aufgeführt, die sich als Quotient der gemessenen Größen Kraft und Bruchfläche ergibt. Das Versagen tritt bei allen Versuchen planmäßig in der Naht auf und nie im Grundwerkstoff. Für die statistische Auswertung konnten aufgrund der vollmechanisierten Schweißung alle Versuchsergebnisse verwendet werden. Alle Abkühlzeiten liegen aufgrund der vollmechanisierten Schweißung unter Laborbedingungen im Bereich von 4 - 15 Sekunden.

In den folgenden Abbildungen werden die Tragfähigkeiten der Versuche in Abhängigkeit der ermittelten Kennwerte für die Zugfestigkeit der Grund- und Schweißzusatzwerkstoffe dargestellt. Die Zeugniswerte werden nur verwendet, wenn keine eigenen Werte vorliegen. 
Tabelle 8.8: Versuchsergebnisse Flankenkehlnahtverbindungen

\begin{tabular}{|c|c|c|c|c|c|c|c|c|c|c|c|}
\hline$\dot{\mathrm{z}}$ & 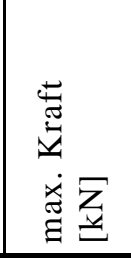 & 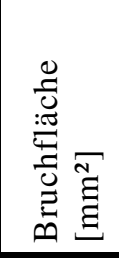 & 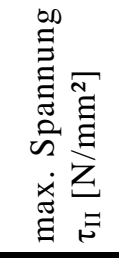 & $\dot{z}$ & 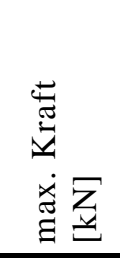 & 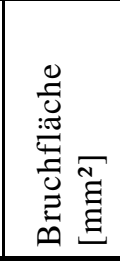 & 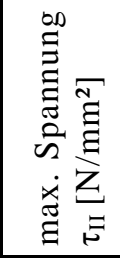 & $\dot{\mathrm{Z}}$ & 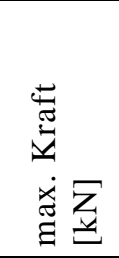 & 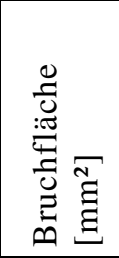 & 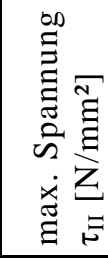 \\
\hline \multicolumn{4}{|c|}{ S700A-S700A-G69A } & \multicolumn{4}{|c|}{ S690B-S690B-G69B } & \multicolumn{4}{|c|}{ S690B-S690B-G69A } \\
\hline $1-01$ & 692,0 & 1329 & 521 & $1-09$ & 700,7 & 1240 & 565 & $1-07$ & 675,0 & 1230 & 549 \\
\hline Z1-01 & 658,3 & 1248 & 527 & $1-10$ & 657,6 & 1165 & 564 & $1-08$ & 720,7 & 1331 & 542 \\
\hline $1-02$ & 697,6 & 1331 & 524 & 3-03 & 1406,3 & 2535 & 555 & \multicolumn{4}{|c|}{ S460-S460-G46 } \\
\hline $2-01$ & 676,0 & 1337 & 505 & 3-04 & 1395,7 & 2527 & 552 & 7-01 & 678,4 & 1487 & 456 \\
\hline Z2-01 & 707,5 & 1409 & 502 & $3-07$ & 2825,3 & 5086 & 556 & Z7-01 & 682,8 & 1342 & 509 \\
\hline $2-02$ & 647,3 & 1269 & 510 & 3-08 & 2798,0 & 5029 & 556 & 7-02 & 702,0 & 1559 & 450 \\
\hline $2-03$ & 640,6 & 1306 & 490 & 3-11 & 4073,7 & 7530 & 541 & 7-03 & 688,5 & 1421 & 484 \\
\hline Z2-03 & 662,2 & 1358 & 488 & 3-12 & 4146,3 & 7634 & 543 & $7-04$ & 714,2 & 1490 & 479 \\
\hline $2-04$ & 647,2 & 1326 & 488 & 4-03 & 1676,9 & 3266 & 513 & \multicolumn{4}{|c|}{ S690B-S690B-G46 } \\
\hline 3-01 & Fehler & & & 4-04 & 1600,1 & 3089 & 518 & 5-05 & 699,8 & 1301 & 538 \\
\hline 3-02 & 1534,4 & 2867 & 535 & 4-07 & 3686,6 & 7382 & 499 & 5-06 & 715,2 & 1320 & 542 \\
\hline $3-05$ & 2662,4 & 4968 & 536 & 4-08 & 3756,1 & 7550 & 498 & \multicolumn{4}{|c|}{ S690B-S690B-G89 } \\
\hline $3-06$ & 2615,3 & 4881 & 536 & \multicolumn{4}{|c|}{ S700A-S700A-T69 } & 5-07 & 802,1 & 1306 & 614 \\
\hline 3-09 & 4363,2 & 8251 & 529 & $1-05$ & 668,6 & 1320 & 506 & 5-08 & 805,6 & 1295 & 622 \\
\hline $3-10$ & 4644,0 & 8767 & 530 & 1-06 & 692,0 & 1387 & 499 & \multicolumn{4}{|c|}{ S700A-S700A-G46 } \\
\hline 4-01 & 1720,0 & 3384 & 508 & \multicolumn{4}{|c|}{ S700A-S700A-G69B } & 5-01 & 663,0 & 1296 & 512 \\
\hline Z4-01 & 1680,4 & 3336 & 504 & $1-03$ & 702,3 & 1327 & 529 & Z5-01 & 655,3 & 1271 & 515 \\
\hline $4-02$ & 1749,9 & 3417 & 512 & $1-04$ & 645,8 & 1230 & 525 & 5-02 & 671,6 & 1325 & 507 \\
\hline 4-05 & 3721,3 & 8098 & 460 & \multicolumn{4}{|c|}{ S690B-S690B-T69 } & \multicolumn{4}{|c|}{ S700A-S700A-G89 } \\
\hline 4-06 & 3727,4 & 8147 & 457 & $1-11$ & 733,7 & 1363 & 538 & $5-03$ & 730,1 & 1283 & 569 \\
\hline Z4-06 & 3920,8 & 8663 & 453 & $1-12$ & 743,7 & 1393 & 534 & Z5-03 & 741,3 & 1282 & 578 \\
\hline & & & & & & & & 5-04 & 741,7 & 1285 & 577 \\
\hline
\end{tabular}

\subsection{Flankenkehlnahtverbindungen mit gleichen Grund- und zugehörigen Schweißzusatzwerkstoffen}

\subsubsection{Einfluss von Grund- und Schweißzusatzwerkstoff S690-G69/T69 (Serie 1) Untersuchungen zur Tragfähigkeit}

Zur Bestimmung der Tragfähigkeit von Flankenkehlnahtverbindungen höherfester Baustähle wurden in Serie 1 verschiedene Stähle und Schweißzusatzwerkstoffe gleicher Nenngüte aber unterschiedlicher Hersteller kombiniert. Als Grundwerkstoff für die Flankenkehlnahtverbindungen wurden zwei Stähle im typischen Kohlenstoffanwendungsbereich ausgewählt: ein vergüteter Stahl S690Q und ein thermomechanisch gewalzter Stahl S700MC. Außerdem wurden Massivdrähte von zwei verschiedenen Herstellern (G69A und G69B) sowie ein Fülldraht (T69) als Schweißzusatzwerkstoffe ausgewählt. Die Schweißparameter waren bei allen Versuchen gleich. Es wurde eine Streckenenergie von 13,4 kJ/cm, einer Vorwärmtemperatur von $100^{\circ} \mathrm{C}$ und einer Abkühlzeit t $8 / 5$ von $5 \mathrm{~s}$ vorgegeben.

Die Ergebnisse der 13 durchgeführten Versuche sind in Abbildung 8.3 dargestellt. Die Tragfähigkeiten sind als maximale Spannungen $\tau_{\text {II }}$ der verschiedenen Werkstoffkombinationen in Abhängigkeit der Zugfestigkeit des verwendeten Schweißzusatzwerkstoffs aufgetragen. Die Tragfähigkeiten liegen zwischen 499 - 565 N/mm², sie unterscheiden sich also um $13 \%$. Da die Versuchskörper nominell gleich sind, können alle 13 Versuchsergebnisse gemeinsam betrachtet werden. Die statistische Auswertung zeigt einen Mittelwert von 533 N/mm² und eine Standardabweichung von 20 N/mm².

Abbildung 8.4 zeigt die Spannungs-Verformungs-Linien für jede der sechs Materialkombinationen. Dargestellt ist die Spannung in Abhängigkeit der Relativverschiebung der Nahtenden, die mit Wegaufnehmern gemessen wurde. Für den Schweißzusatzwerkstoff G69A und B ist die Verformungsfä- 
higkeit bei Stahl A größer als bei Stahl B, für den Schweißzusatzwerkstoff T69 ist die Verformungsfähigkeit bei beiden Stählen gleich groß.

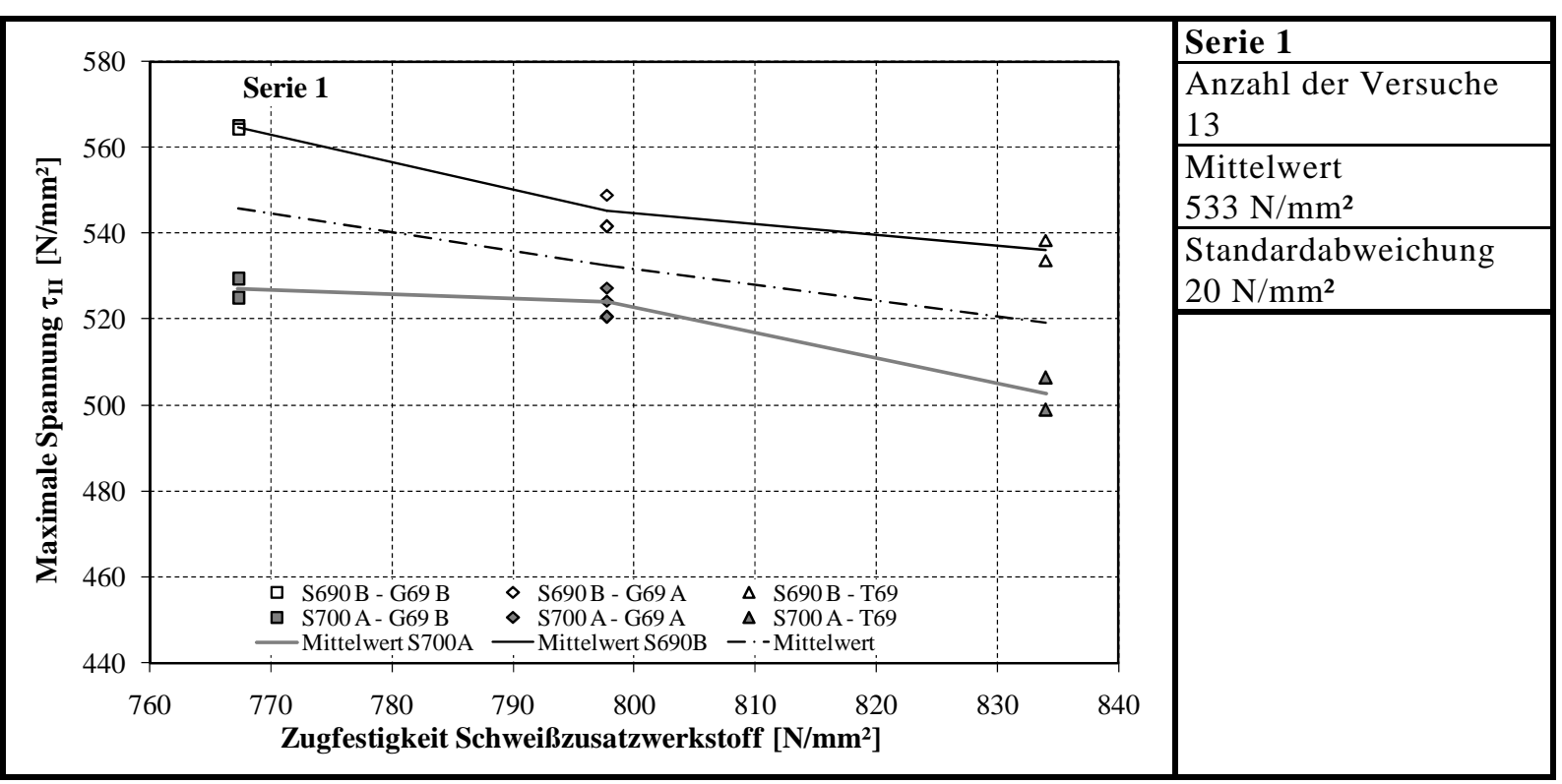

Abbildung 8.3: Einfluss von Grund- und Schweißzusatzwerkstoff S690-G69/T69 auf die Tragfähigkeit

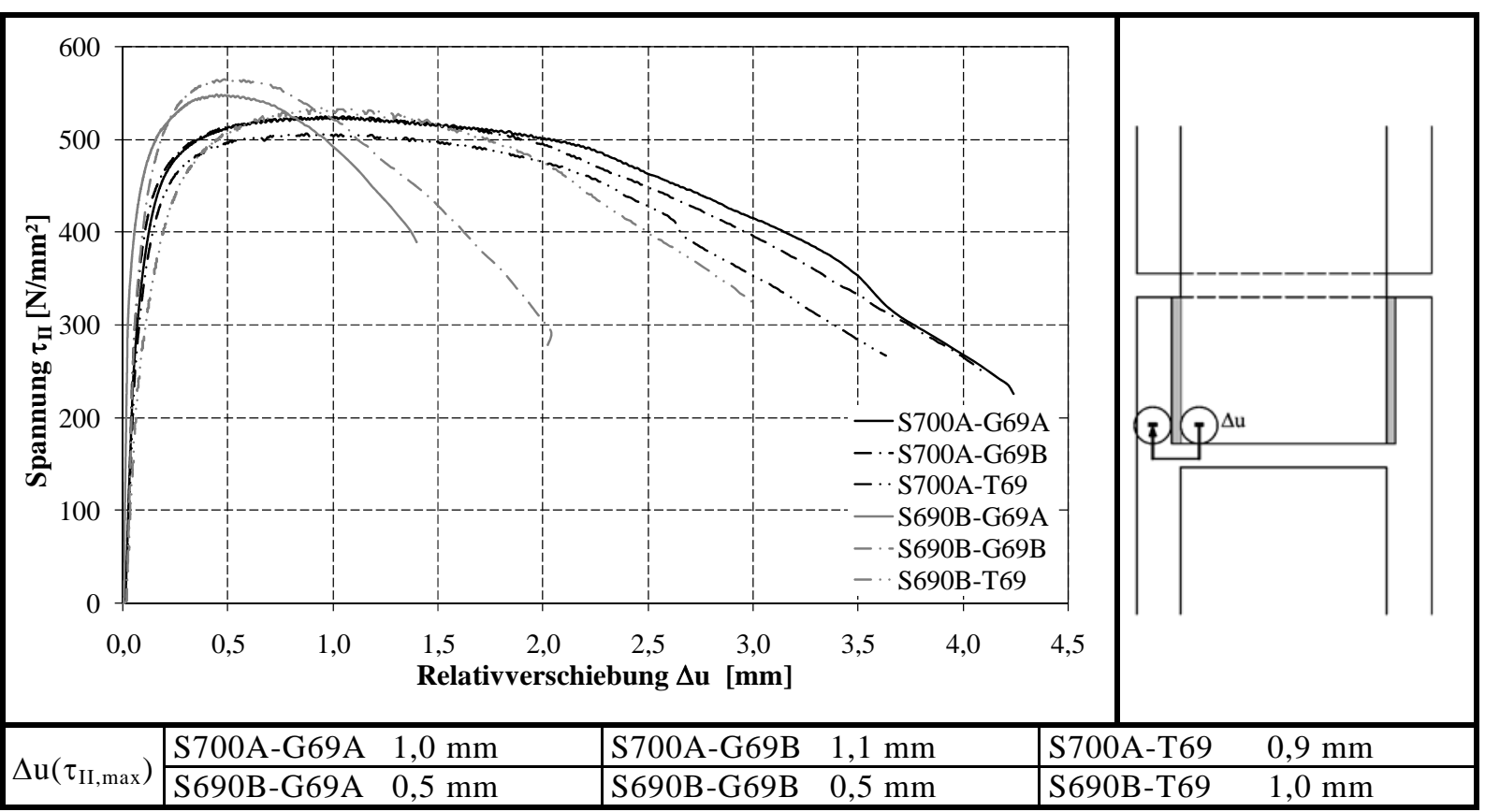

Abbildung 8.4: Einfluss von Grund- und Schweißzusatzwerkstoff S690-G69/T69 auf die Verformungsfähigkeit

Es ist deutlich sichtbar, dass die jeweils gleichen Versuchskörper sehr ähnliche Ergebnisse mit einer Streuung in den Tragfähigkeitswerten unterhalb von 2 \% liefern. Der Vergleich der Versuchsergebnisse in Abhängigkeit der verschiedenen Schweißzusatzwerkstoffe zeigt, dass die Tragfähigkeiten für den Stahl S690B für die verschiedenen Schweißzusatzwerkstoffe jeweils höher liegen als für Stahl S700A. Der thermomechanisch gewalzte Stahl S700A hatte stets eine geringere Tragfähigkeit als die Verbindungen mit dem vergüteten Stahl S690B. Der in Kapitel 2.2.5 beschriebene Unterschied zwischen diesen beiden Typen von Stählen kann als Erklärung für den Abfall der Tragfähigkeit dienen. Während der Stahl S690Q einen ähnlichen Legierungsgehalt hat wie die eingesetzten Schweißzusatzwerkstoffe, hat der Grundwerkstoff S700A einen deutlich geringeren Legierungsgehalt. Durch die Vermischung von Grund- und Schweißzusatzwerkstoff kommt es insgesamt in der Naht zu einem geringeren Legierungsgehalt, insbesondere Kohlenstoff, damit verringert sich die Tragfähigkeit der Naht. 


\section{Vergleich von Härtemessungen und Tragfähigkeit}

Die Ergebnisse der durchgeführten Härtemessungen werden in Abbildung 8.5 für den Stahl S700A in Kombination mit den verwendeten Schweißzusatzwerkstoffen G69A, G69B und T69 verglichen. Für den Stahl S700A zeigen sich für den Schweißzusatzwerkstoff A und B sehr ähnliche Härtewerte genau wie bei der Tragfähigkeit. Die Härtewerte für den Schweißzusatzwerkstoff T69 liegen um 8 \% höher, die Tragfähigkeit liegt jedoch um 4 \% niedriger als bei den Massivdrähten G69A und G69B. Die Unterschiede in der chemischen Zusammensetzung der Schweißzusatzwerkstoffe beeinflussen die Härtewerte, so dass eine Einordung zwischen Härte und Tragfähigkeit bei unterschiedlichen Schweißzusatzwerkstoffen gleicher Nenngüte nicht eindeutig möglich ist.

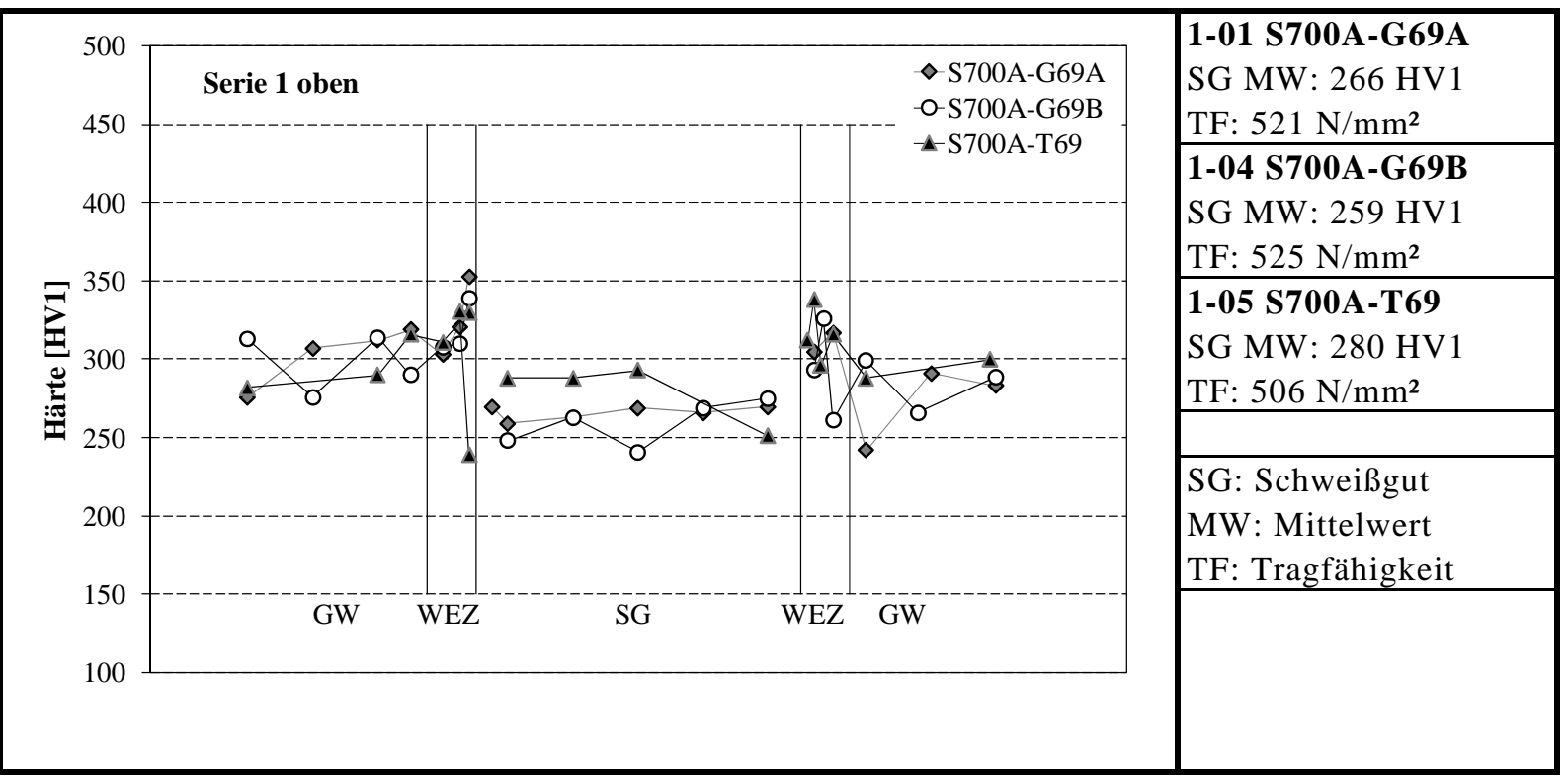

Abbildung 8.5: Vergleich der Härtewerte bei gleichen Grund- und verschiedenen Schweißzusatzwerkstoffen gleicher Nenngüte S700A-G69/T69

Abbildung 8.6 zeigt den Einfluss des Grundwerkstoffs (S690B und S700A) auf die Härte bei gleichem Schweißzusatzwerkstoff. Für den Schweißzusatzwerkstoff G69A liegen die Härtewerte von Stahl S690B höher als von Stahl S700A. Bei gleichbleibendem Schweißzusatzwerkstoff korreliert die Härte mit der Tragfähigkeit der Verbindung. Infolge der Vermischung zwischen Grund- und Schweißzusatzwerkstoff stellen sich unterschiedliche Härtewerte und dazu passende Tragfähigkeiten trotz eines gleichen Schweißzusatzwerkstoffs ein.

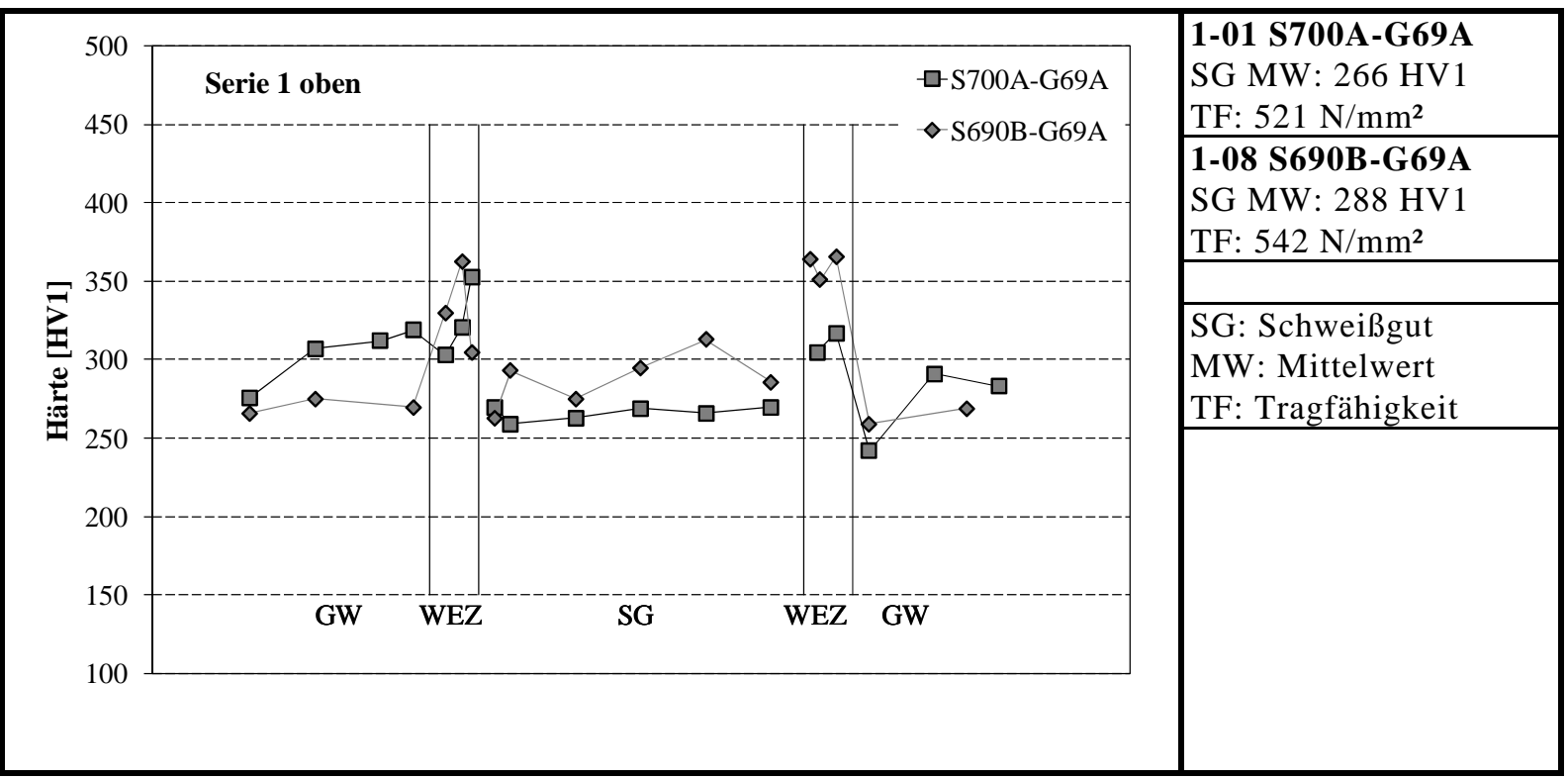

Abbildung 8.6: Vergleich der Härtewerte bei verschiedenen Grund- und gleichen Schweißzusatzwerkstoffen gleicher Nenngüte S700A/S690B-G69A 


\section{Fazit}

Ziel der Untersuchungen in Serie 1 war es, untere und obere Grenzen der Tragfähigkeit in Abhängigkeit der Werkstoffe zu ermitteln. Dieses Ziel konnte durch Untersuchungen mit den unterschiedlichen Werkstoffkombinationen gleicher Nenngüte erreicht werden. Es konnte ein Bereich der Tragfähigkeit von 499 - 565 N/mm² ermittelt werden. Durch die vollmechanisierte Schweißung war die Streuung bei gleichen Versuchskörpern sehr gering, so dass der Einfluss der untersuchten Parameter eindeutig identifiziert werden konnte.

Die Ergebnisse der Härtemessungen zeigen die gleiche Tendenz wie die Ergebnisse der Tragfähigkeitsuntersuchungen. Insbesondere bei gleichen Schweißzusatzwerkstoffen korrelieren die Tragfähigkeiten und die Härtewerte gut.

Es konnte gezeigt werden, dass sowohl der Grundwerkstoff als auch der Schweißzusatzwerkstoff die Tragfähigkeit und Härte der Schweißverbindung beeinflussen.

\subsubsection{Einfluss der Schweißparameter S700-G69A (Serie 2)}

\section{Untersuchungen zur Tragfähigkeit}

Um den Einfluss der Schweißparameter auf die Tragfähigkeit der Flankenkehlnahtverbindungen zu ermitteln, wurden Versuchskörper hergestellt, bei denen eine höhere Abkühlzeit t ${ }_{8 / 5}$ innerhalb des Arbeitsfensters gewählt wurde als bei den Versuchen der Serie 1. Diese Untersuchungen wurden an einer Werkstoffkombination aus Grund- und zugehörigem Schweißzusatzwerkstoff S700A-G69A durchgeführt. Es wurden 3 Versuche mit einer Vorwärmtemperatur von $175^{\circ} \mathrm{C}$ und einer Abkühlzeit $t_{8 / 5}=10 \mathrm{~s}$ und 3 Versuche mit einer Vorwärmtemperatur von $235^{\circ} \mathrm{C}$ und einer Abkühlzeit $\mathrm{t}_{8 / 5}=15 \mathrm{~s}$ durchgeführt. Die weiteren Schweißparameter wurden beibehalten, vgl. Anhang A.4.3.

Die Ergebnisse der 6 durchgeführten Versuche sind in Abbildung 8.7 gemeinsam mit 3 Versuchen der Serie 1 dieser Materialkombination (Vorwärmtemperatur $100^{\circ} \mathrm{C}$, Abkühlzeit $\mathrm{t}_{8 / 5}=5 \mathrm{~s}$ ) als Referenz dargestellt. Das Diagramm zeigt die maximalen Spannungen $\tau_{\text {II }}$ in Abhängigkeit der Vorwärmtemperatur. Der Vergleich zeigt, dass die Tragfähigkeit der Verbindungen mit zunehmender Vorwärmtemperatur von $524 \mathrm{~N} / \mathrm{mm}^{2}$ zu $489 \mathrm{~N} / \mathrm{mm}^{2}$ abnimmt. Die Abnahme liegt im Mittel bei einer Steigerung der Vorwärmtemperatur um $75^{\circ} \mathrm{C}$ bei $18 \mathrm{~N} / \mathrm{mm}^{2}$ (4\%). Bei einer Steigerung der Vorwärmtemperatur um $135^{\circ} \mathrm{C}$ liegt sie bei $35 \mathrm{~N} / \mathrm{mm}^{2}$ (7\%). Die Streuung der drei nominell gleichen Versuche ist jeweils sehr gering.

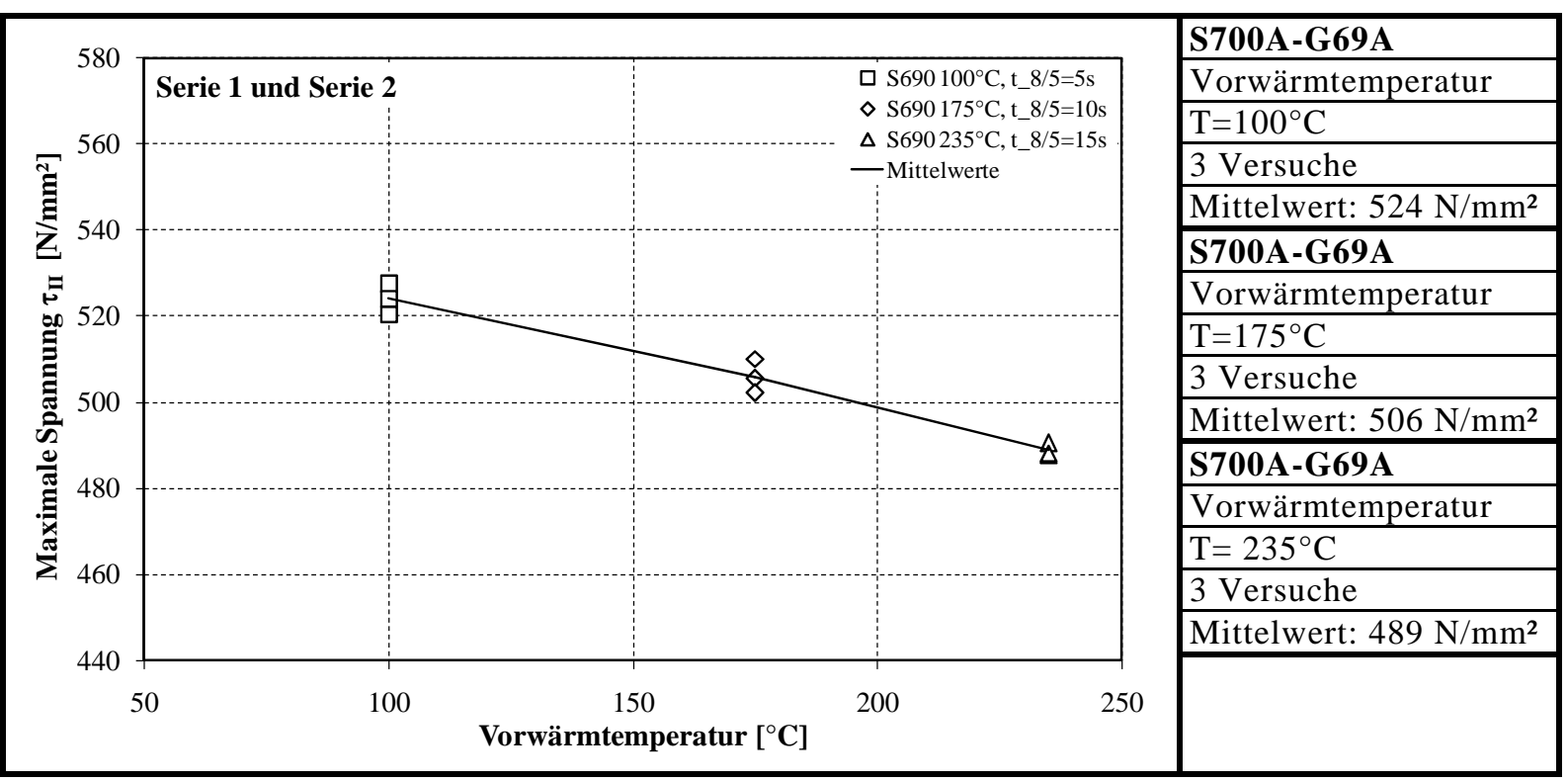

Abbildung 8.7: Einfluss der Vorwärmtemperatur auf die Tragfähigkeit

Abbildung 8.8 zeigt die Spannungs-Verformungs-Linien für die drei Vorwärmtemperaturen der Serien 1 und 2. Die Spannung $\tau_{\text {II }}$ ist in Abhängigkeit der Relativverschiebungen der Bleche zueinander am Nahtende dargestellt. Für alle drei Vorwärmtemperaturen bleibt ein ähnliches Verformungsverhalten 
erhalten. Die Verformung bei der maximalen Spannung wird mit zunehmender Vorwärmtemperatur größer.

Der in Kapitel 2.4.6 und 4.2.3 beschriebene Einfluss der Abkühlbedingungen wird hier deutlich. Da als einziger Parameter das Vorwärmen geändert wurde, kann dieser Einflussfaktor eindeutig identifiziert werden. Durch eine langsamere Abkühlung können sich Anteile aus Ferrit und Perlit bilden, während weniger Martensit entsteht. Damit kann es zu einer Erweichung kommen, die zu einem Verlust an Tragfähigkeit führt.

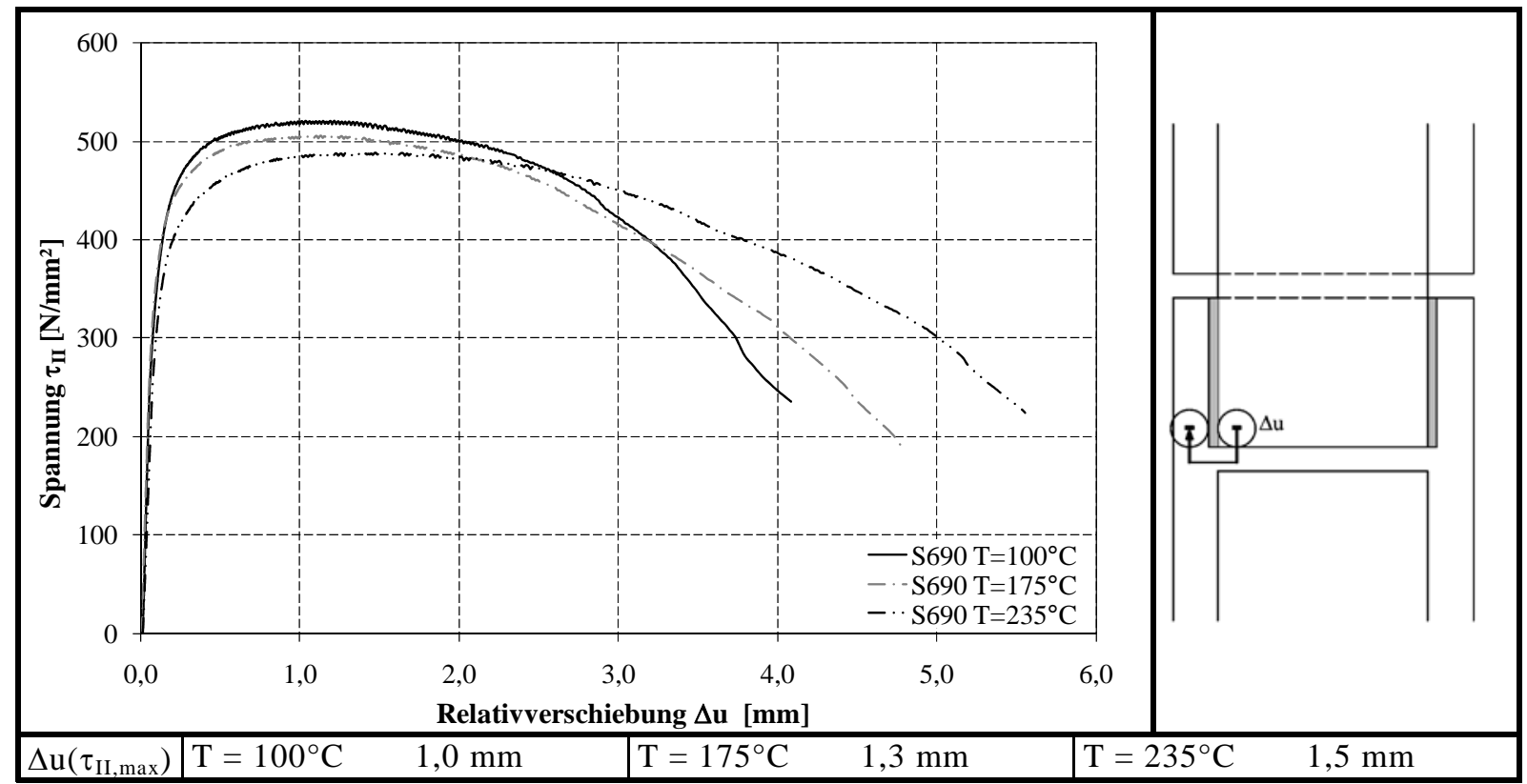

Abbildung 8.8: Einfluss der Vorwärmtemperatur S700A-G69A auf die Verformungsfähigkeit

\section{Vergleich von Härtemessungen und Tragfähigkeit}

An den Versuchskörpern 1-01, Z2-01 und 2-04 wurden Härtemessungen durchgeführt, um den Einfluss der Vorwärmtemperatur auf die Härte im Schweißgut zu ermitteln. Diese Härtemessungen sind in Abbildung 8.9 verglichen. Es zeigt sich, dass mit zunehmender Abkühlzeit $t_{8 / 5}$ die Härte im Schweißgut leicht abnimmt. Zudem bilden sich bei hohen Vorwärmtemperaturen geringere Härtespitzen in der WEZ. Die Abnahme der Härte mit zunehmender Vorwärmtemperatur liegt mit 4 - 6 \% im gleichen Bereich wie die Abnahme der Tragfähigkeit von 4 - 7 \%.

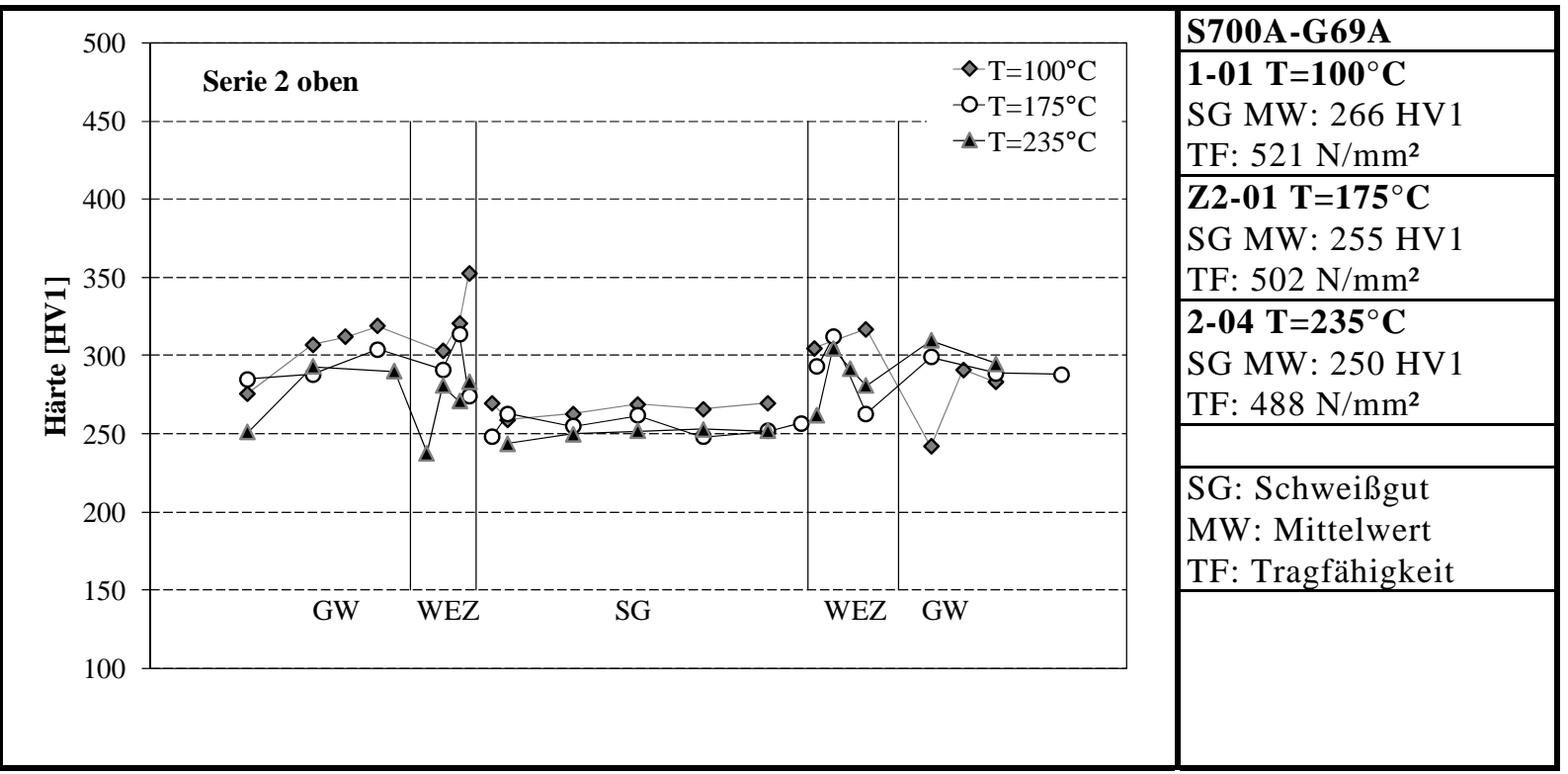

Abbildung 8.9: Vergleich der Härtewerte bei verschiedenen Vorwärmtemperaturen S700A-G69A 


\section{Fazit}

Insgesamt ergaben sich mit den unterschiedlichen Vorwärmtemperaturen und damit verbundenen Abkühlzeiten $\mathrm{t}_{8 / 5}$ Unterschiede in der Tragfähigkeit von 489 - $524 \mathrm{~N} / \mathrm{mm}^{2}$. Die Tragfähigkeit variiert also um $7 \%$ innerhalb des Arbeitsfensters. Diese Werte stellen untere und obere Grenzen der Tragfähigkeit im gesamten Arbeitsfenster dar. Für diesen Parameter Vorwärmtemperatur stimmen die Aussagen der Härtemessungen und der Tragfähigkeit gut überein.

\subsubsection{Einfluss der Nahtlänge S690-G69 (Serie 3)}

\section{Untersuchungen zur Tragfähigkeit}

In Serie 3 wurde der Einfluss der Nahtlänge auf die Tragfähigkeit untersucht. Hierzu wurde an zwei Werkstoffkombinationen von Grund- und Schweißzusatzwerkstoffen gleicher Nenngüte S700A-G69A und S690B-G69B die Nahtlänge über das Verhältnis Nahtlänge zu Nahtdicke l/a variiert. Die Schweißparameter entsprachen denen der Serie 1.

Die Ergebnisse der elf durchgeführten Versuche zeigt Abbildung 8.10 im Vergleich mit den Versuchsergebnissen der der Serie 1 dieser Werkstoffkombinationen. Dargestellt sind die maximalen Spannungen $\tau_{\text {II }}$ in Abhängigkeit der Nahtlänge.

Für die Werkstoffkombination S690B-G69B liegen die ermittelten Tragfähigkeiten für alle untersuchten Nahtlängen im Mittel zwischen 542 und 565 N/mm². Der Einfluss einer Änderung der Nahtlänge im Bereich von $1 / a=25$ bis $1 / a=150$ liegt bei etwa $4 \%$, wobei die Tragfähigkeit mit zunehmender Nahtlänge abnimmt. Für die Werkstoffkombination S700A-G69A zeigt sich kein eindeutiger Trend. Hier variiert die Tragfähigkeit im Streubereich von 2 \% zwischen 524 und 536 N/mm².

Die Abnahme der Tragfähigkeit mit zunehmender Nahtlänge ist eher gering und lässt sich nicht eindeutig feststellen. Es kann damit gezeigt werden, dass bis zu einer Nahtlänge von l/a = 150 keine wesentliche Abnahme der Tragfähigkeit auftritt.

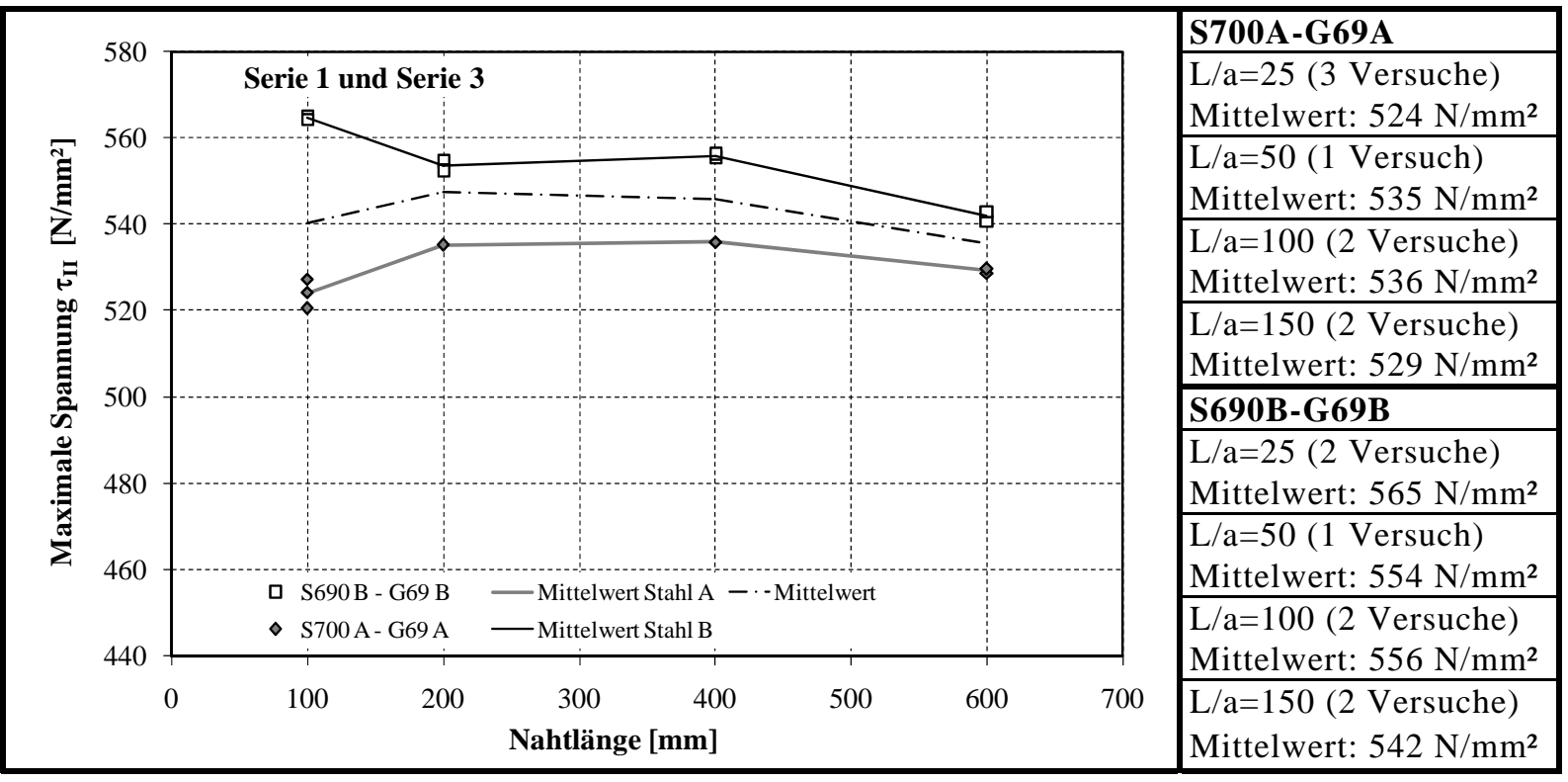

Abbildung 8.10: Einfluss der Nahtlänge auf die Tragfähigkeit

Abbildung 8.11 zeigt die Spannungs-Verformungs-Linien für die unterschiedlichen Nahtlängen der beiden Werkstoffkombinationen. Die Wegaufnehmer mussten vor Erreichen der Traglast abgenommen werden, um ihre Beschädigung zu vermeiden. Zusätzlich sind die Kurven für die Maschinenwege in Abhängigkeit der Spannungen in Abbildung 8.12 dargestellt. Der Maschinenweg zeigt, dass das Versagen der Versuchskörper kurz nach Erreichen der Maximallast auftritt. Tendenziell ergeben sich für alle untersuchten Nahtlängen beider Werkstoffkombinationen ähnliche Werte für die Verformungsfähigkeit bei der maximalen Spannung. 


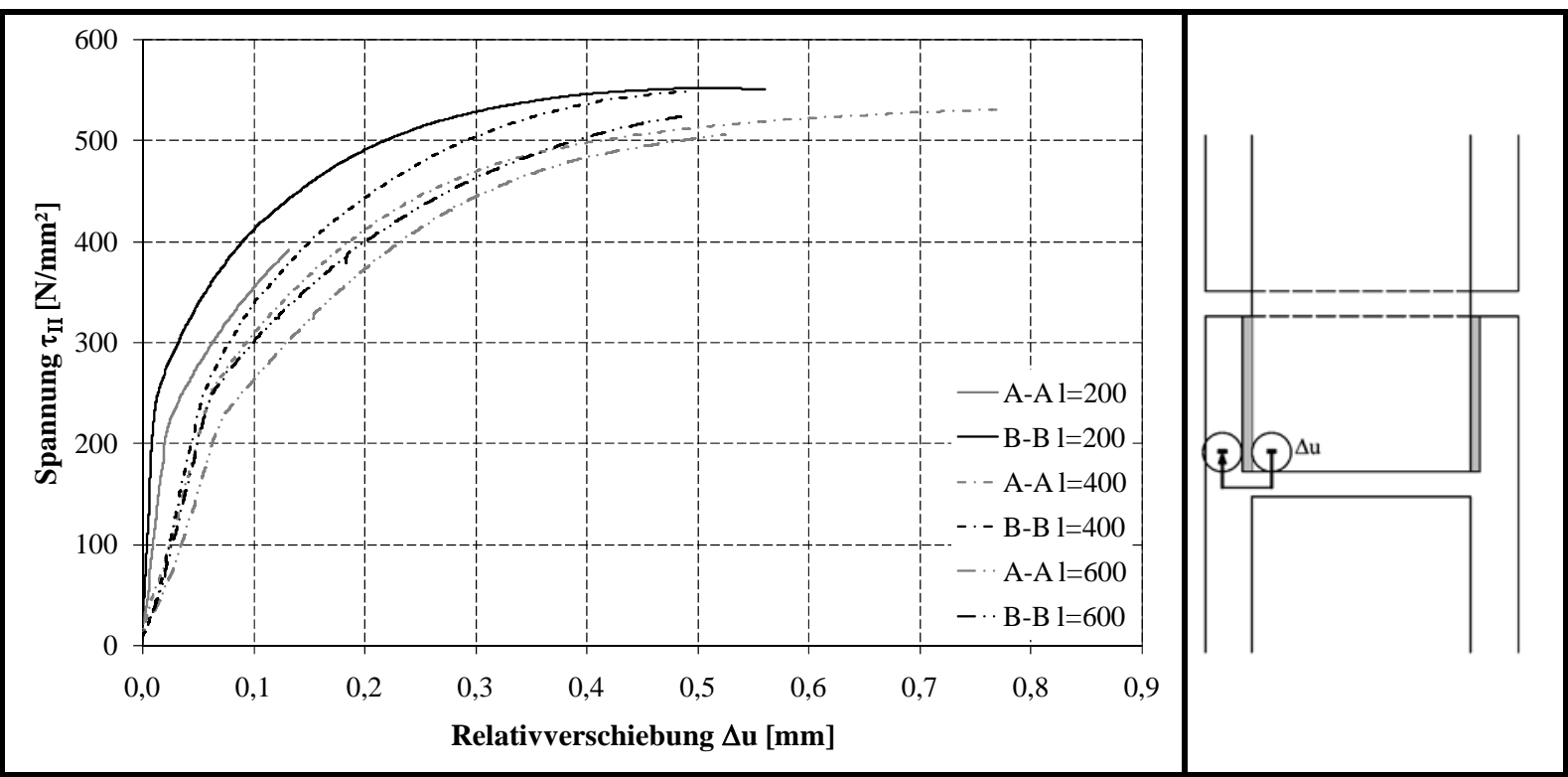

Abbildung 8.11: Einfluss der Nahtlänge auf die Verformungsfähigkeit (Relativverschiebung)

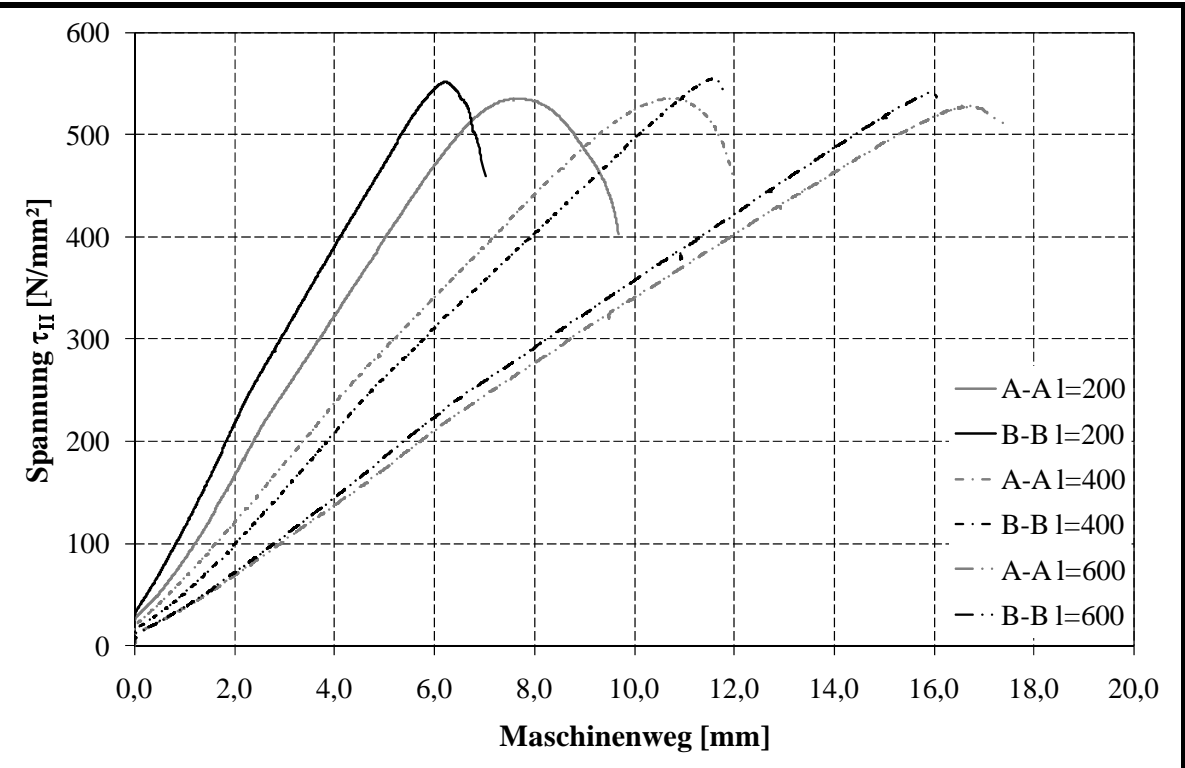

Abbildung 8.12: Einfluss der Nahtlänge auf die Verformungsfähigkeit (Maschinenweg)

\section{Härtemessungen und Vergleich mit der Tragfähigkeit}

Härtemessungen wurden an den Versuchskörpern der Serie 3 nicht durchgeführt, da die Versuchskörper mit den gleichen Parameter wie die Versuchskörper der Serie 1 geschweißt wurden. Es wird davon ausgegangen, dass eine ähnliche Nahtgeometrie und ähnliche Härtewerte entstehen. Abbildung 8.10 zeigt, dass für die Versuche der Serie 3 ähnliche Tragfähigkeiten wie in Serie 1 erreicht wurden.

\section{Fazit}

Die Versuchsergebnisse zeigen, dass für die Kehlnähte mit größerer Länge keine eindeutige, wesentliche Abnahme der Tragfähigkeit festgestellt wurde. Die Tragfähigkeiten streuen im Bereich von $4 \%$ bei gleichen Werkstoffkombinationen. 


\subsubsection{Einfluss von Nahtdicke (mehrlagig) und Blechdicke S690-G69 (Serie 4) Untersuchungen zur Tragfähigkeit}

Um den Einfluss der Mehrlagigkeit auf die Tragfähigkeit zu untersuchen, wurden mehrlagige Versuchskörper mit den Werkstoffkombinationenen S700A-G69A und S690B-G69B geprüft. Dazu wurden zum einen aus Blechen mit einer Dicke von $t=20 \mathrm{~mm}$ zweilagige (dreiraupige) Nähte mit einer Nahtdicke von $\mathrm{a}=6 \mathrm{~mm}$ gefertigt. Zum anderen wurden aus Blechen mit einer Blechdicke von $\mathrm{t}=$ $50 \mathrm{~mm}$ dreilagige (sechsraupige) Schweißverbindungen mit einer Nahtdicke von a $=10 \mathrm{~mm}$ hergestellt. Als Ergebnisse der zehn durchgeführten Versuche zeigt Abbildung 8.13 die maximalen Spannungen $\tau_{\text {II }}$ im Vergleich mit den entsprechenden Versuchsergebnissen der einlagigen Schweißverbindungen der Serie 1.

Für die Werkstoffkombination S700A - G69A liegen die Tragfähigkeiten zwischen 457 - 524 N/mm². Der Unterschied liegt somit bei 15 \%. Für die Werkstoffkombination S690B-G69B zeigen sich Unterschiede von $14 \%$ zwischen 565 und $498 \mathrm{~N} / \mathrm{mm}^{2}$. Es lässt sich somit eine Abnahme der Tragfähigkeit mit zunehmender Nahtdicke feststellen. Die Abnahme der Tragfähigkeit kann darauf beruhen, dass sich Vergütungseffekte durch das Überschweißen bei den mehrlagigen Nähten einstellen.

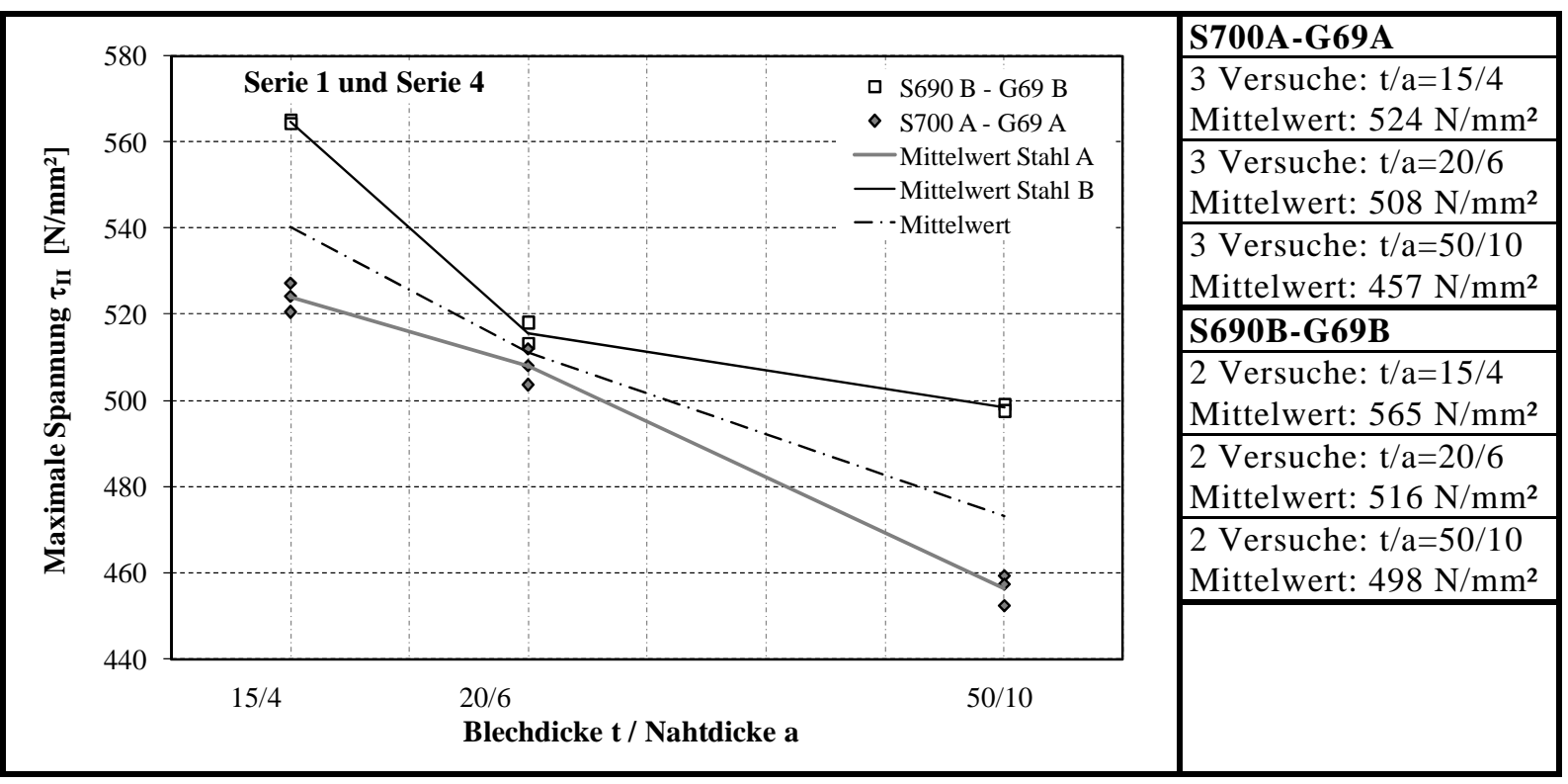

Abbildung 8.13: Einfluss der Blechdicke t und der Nahtdicke a auf die Tragfähigkeit

\section{Härtemessungen und Vergleich mit der Tragfähigkeit}

Analog zum Vergleich der Tragfähigkeiten werden in Abbildung 8.14 exemplarisch die Ergebnisse der durchgeführten Härtemessungen für die unterschiedlichen Nahtdicken bei gleicher Werkstoffkombination S700A-G69A dargestellt. Für den Stahl S700A (Abbildung 8.14) liegen die Härtewerte der mehrlagigen Nähte etwas höher als für die einlagigen Nähte. Insgesamt streuen die Werte, so dass sich eindeutige Tendenzen nicht ablesen lassen. Bei mehrlagigen Nähten ist das Schweißgut nicht homogen sondern auch durch die Schweißraupen als unterschiedliche Bereiche geprägt. Die Härtewerte wurden nahe der Oberfläche bestimmt und können die unterschiedlichen Bereiche der mehrlagigen Naht nicht abbilden, so dass geringere Härten, die durch das Vergüten entstehen, nicht berücksichtigt werden können. 


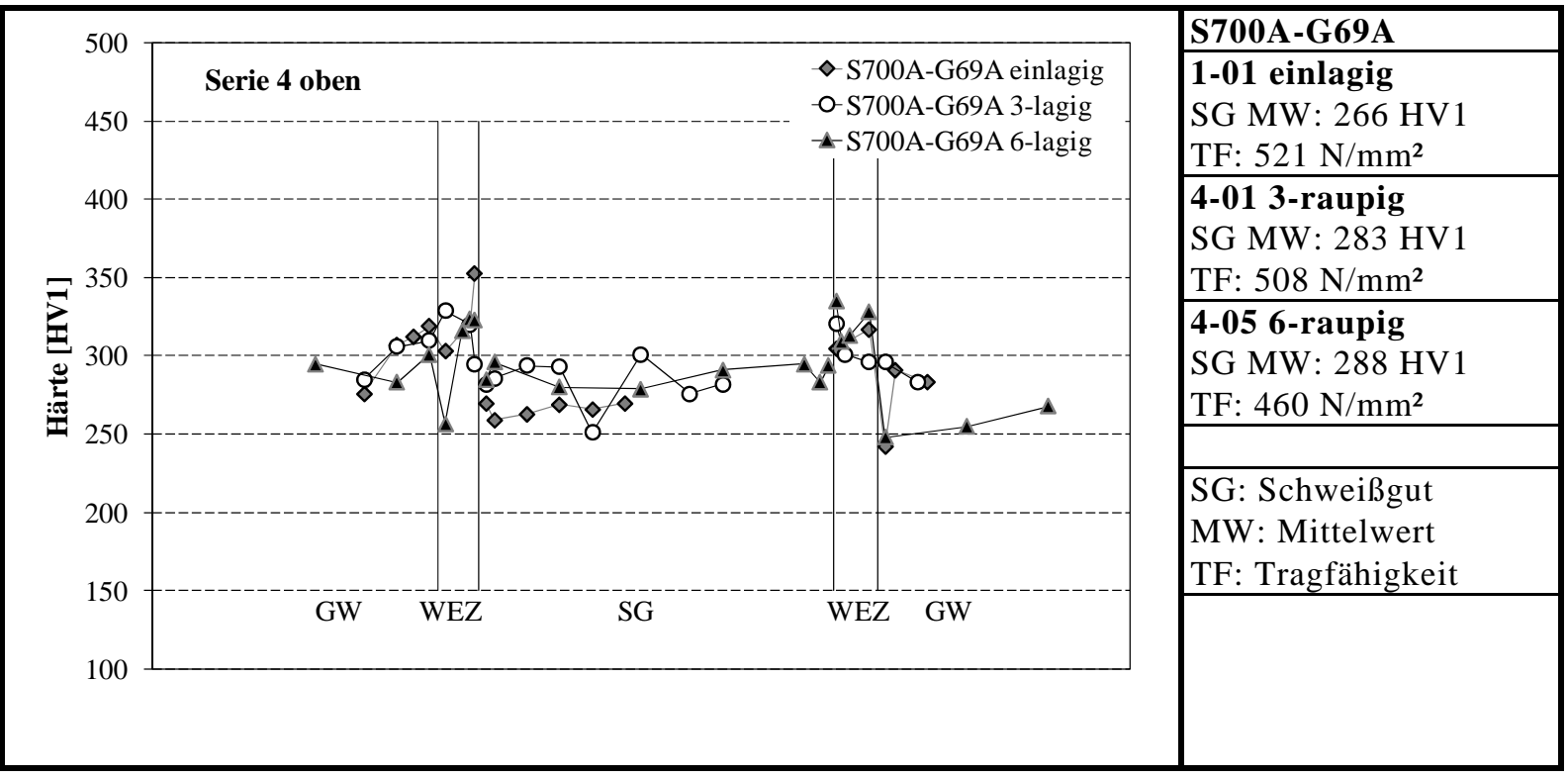

Abbildung 8.14: Vergleich der Härtewerte unterschiedlicher Nahtdicken S700A-G69A

\section{Fazit}

Insgesamt konnte mit den unterschiedlichen Naht- und Blechdicken ein Streuband von 457 $565 \mathrm{~N} / \mathrm{mm}^{2}$ ermittelt werden, um untere und obere Grenzen der Tragfähigkeit zu ermitteln. Infolge der vollmechanisierten Schweißung und der Versuchskörperform ergab sich eine sehr geringe Streuung bei gleichen Versuchskörpern, so dass der Einfluss der untersuchten Parameter eindeutig identifiziert werden konnte. Es zeigt sich eine Abnahme der Tragfähigkeit infolge von Auf- und Abmischungseffekten mit zunehmender Nahtdicke, wobei das Verformungsverhalten ähnlich bleibt.

\subsubsection{Statistische Auswertung S690-G69/T69}

Wie in Kapitel 4.5 beschrieben, wurden die beschriebenen Versuche gleicher Grundwerkstoffe und zugehöriger Schweißzusatzwerkstoffe mit dem Bemessungsmodell nach EN 1993-1-8 (2005) statistisch ausgewertet. Die statistische Auswertung wurde sowohl in Abhängigkeit der Zugfestigkeit des Grundwerkstoffs als auch in Abhängigkeit der Festigkeit des Schweißzusatzwerkstoffs durchgeführt.

Für die statistische Auswertung der Versuche gleicher Grund- und zugehöriger Schweißzusatzwerkstoffe S690-G/T69 muss berücksichtigt werden, dass die Streuung zwischen den nominell gleichen Versuchskörper sehr gering ist. In Abbildung 8.15 sind als Versuchsergebnisse die maximalen Spannungen $\tau_{\text {II }}$ aller Flankenkehlnahtverbindungen S690-G/T69 getrennt nach Serien dargestellt. Es zeigt sich, dass durch die unterschiedlichen Einflussparameter eine gezielte Streuung erzeugt wurde. Die beiden gleichen Versuchsergebnisse haben jeweils eine nahezu gleiche Tragfähigkeit, die unterschiedlichen Parameter der einzelnen Serien führen jedoch zu einer Streuung. In der statistische Auswertung wurde diese geringe Streuung der gleichen Versuche berücksichtigt, in dem für die Bestimmung der Fraktilenfaktoren $k_{n}$ und $k_{n, d}$ für die Widerstandsfunktion nur eine reduzierte Anzahl von Versuchen verwendet wird. Das heißt, dass nur jeweils ein Versuch von 2 identischen Versuchen für die Streuung berücksichtigt wird. Anstelle von 40 Versuchsergebnissen wird nur eine reduzierte Anzahl von 24 Versuchen berücksichtigt.

Mit der Werkstoffkombination S690 und einem Schweißzusatzwerkstoff mit der nominellen Streckgrenze $\mathrm{f}_{\mathrm{y}}=690 \mathrm{~N} / \mathrm{mm}^{2}$ wurden 40 Versuchsergebnisse ermittelt, die statistisch ausgewertet wurden, wie in Tabelle 8.9 zusammengefasst. 


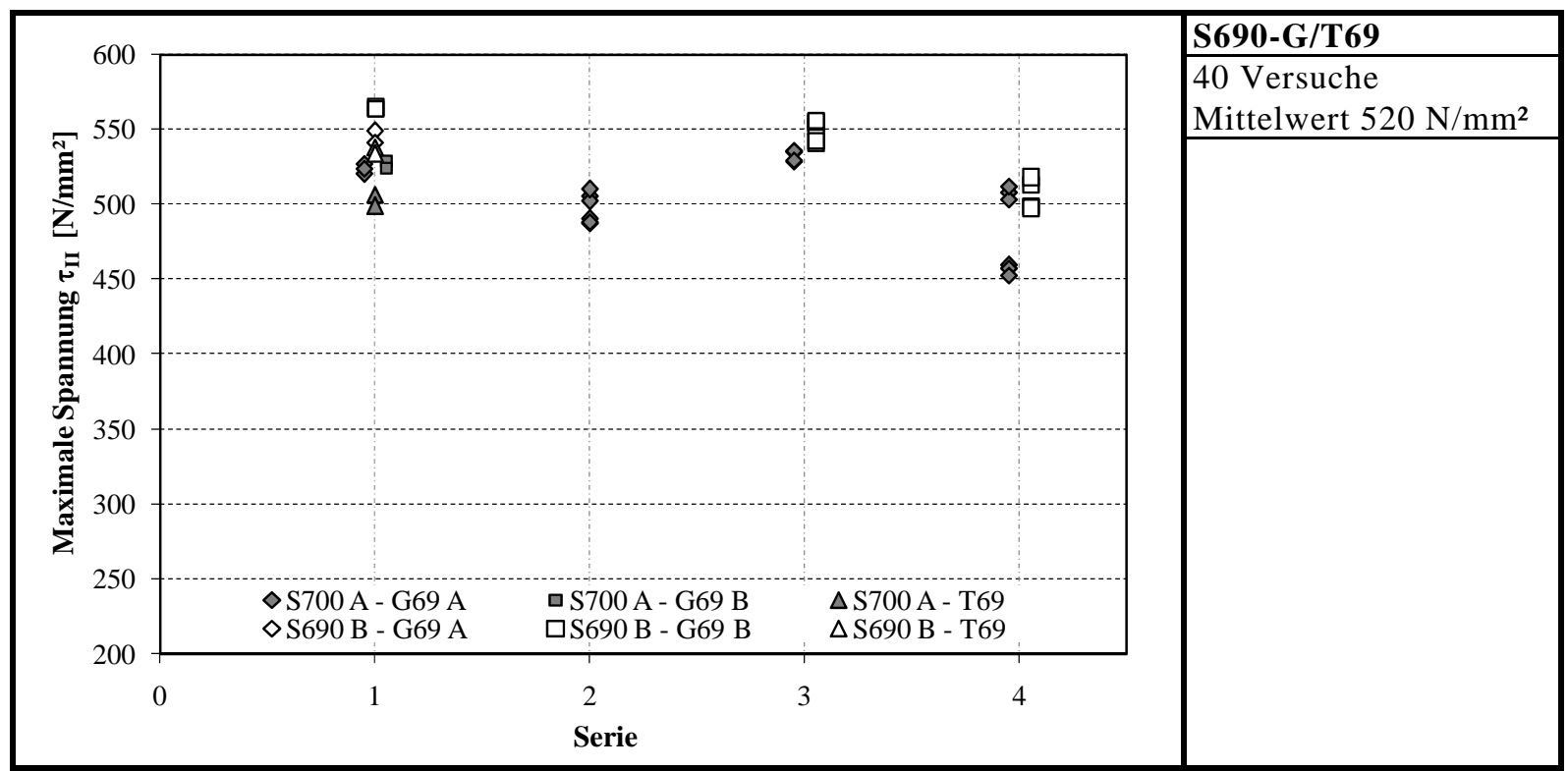

Abbildung 8.15: Darstellung aller Versuchsergebnisse S690-G/T69

Tabelle 8.9: Statistische Auswertung der Flankenkehlnahtverbindungen S690 bezogen auf die Festigkeit des Grundwerkstoffs (links) oder des Schweißzusatzwerkstoffs (rechts)

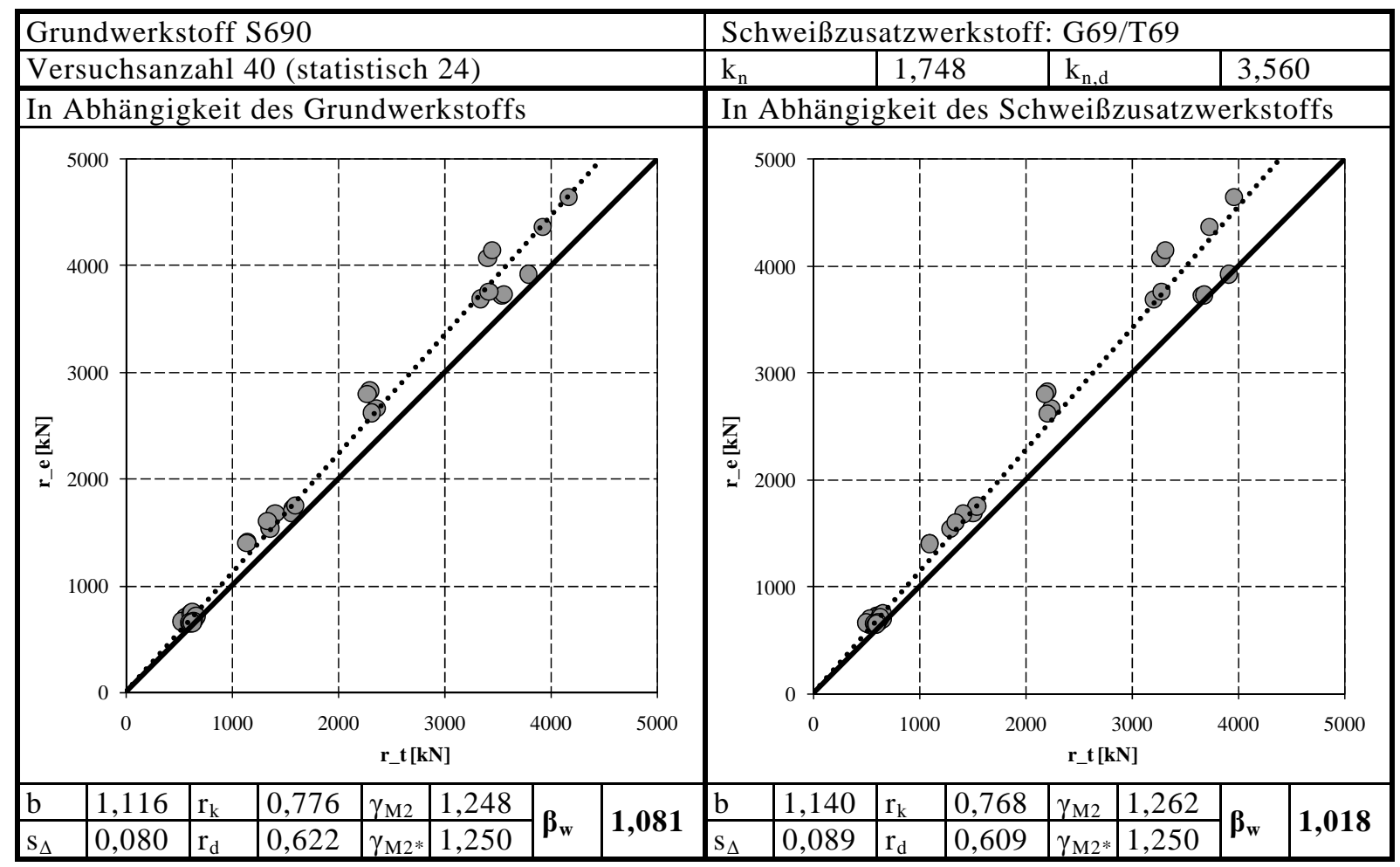

Einen Überblick über die Ergebnisse der statistischen Auswertung gibt Tabelle 8.10 für die Werkstoffkombination S690-G/T69. Für die Verbindungen S690-G/T69 zeigt der Beiwert $\beta_{w}>1,0$, dass die Festigkeit der Kehlnaht geringer als die Festigkeit des Schweißzusatzwerkstoffs ist.

Wie in Tabelle 8.9 angegeben, ergibt sich bei der statistischen Auswertung der Versuche eine Mittelwertabweichung von $\mathrm{b}=1,14$ bezogen auf die Auswertung in Abhängigkeit des Schweißzusatzwerkstoffs. Für die in Kapitel 6 beschriebenen Versuche zeigt sich in der statistischen Auswertung der Verbindungen S690-G/T69 eine Mittelwertabweichung von 1,12, vgl. Tabelle 6.9. Da diese Werte vergleichbar sind, wird die vorhandene Streuung der in Abbildung 8.15 abgebildeten Versuche als repräsentativ betrachtet. 
Tabelle 8.10: Bemessungsgrenzspannung für Flankenkehlnähte S690-G/T69

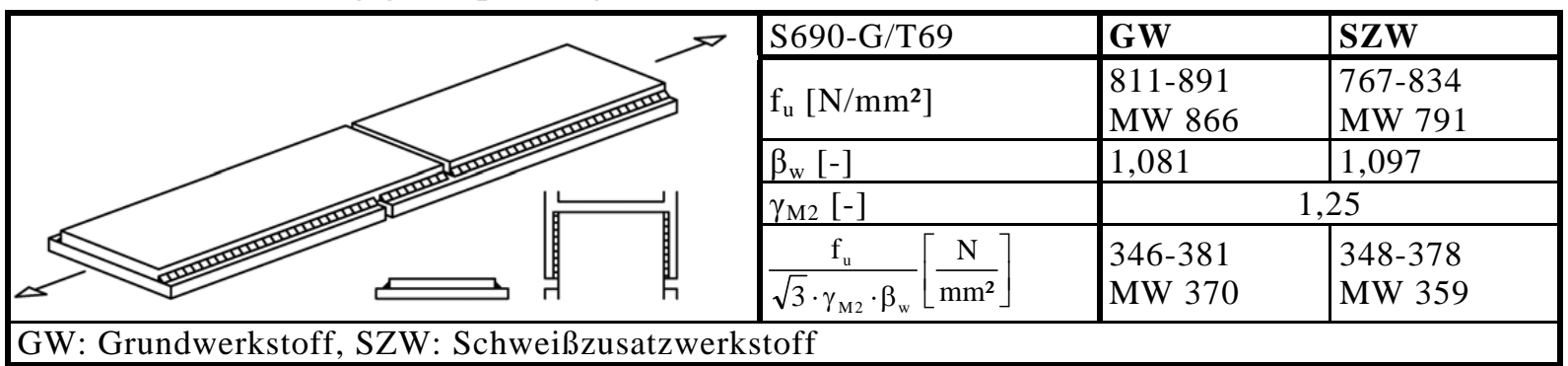

\subsubsection{Einfluss des Grund- und Schweißzusatzwerkstoffs S460-G46 (Serie 7) Untersuchungen zur Tragfähigkeit}

In Serie 7 wurden ergänzende Flankenkehlnahtversuche mit dem Grundwerkstoff S460M und zugehörigem Schweißzusatzwerkstoff G46 durchgeführt. Ziel dieser Versuche ist die Bestimmung der Tragfähigkeit sowie ein Vergleich mit den Versuchen der Werkstoffkombination S690-G69 (Serie 1) unter sonst gleichen Bedingungen. Außerdem werden die ermittelten Tragfähigkeiten mit den Versuchsergebnissen, die im Projekt [FOSTA P652, 2008] für die Verbindungen S460-G46 bestimmt wurden, verglichen. Zusätzlich wurden die Schweißparameter durch Änderung der Vorwärmtemperatur variiert, um den Einfluss dieses Parameters auch für die Verbindungen S460-G46 zu bestimmen und mit den Ergebnissen der Serie 2 zu vergleichen.

Zwei Versuche wurden aus Gründen der Vergleichbarkeit genau wie in Serie 1 mit einer Vorwärmtemperatur von $\mathrm{T}=100^{\circ} \mathrm{C}$ geschweißt. Bei zwei weiteren Versuchen betrug die Vorwärmtemperatur $\mathrm{T}=50^{\circ} \mathrm{C}$. Ein Versuch wurde praxisgerecht ohne Vorwärmen geschweißt. Die Streckenenergie war mit 13,4 kJ/cm bei allen Versuchen gleich. Der bei Raumtemperatur geschweißte Versuchskörper erreichte nur eine Abkühlzeit $t_{8 / 5}$ von 4 Sekunden. Dieser Versuch wird nicht für die Auswertung berücksichtigt. Die Vorwärmtemperatur von $50^{\circ} \mathrm{C}$ führte zu einer Abkühlzeit $t_{8 / 5}$ von 4,5 - 5 Sekunden.

Die Tragfähigkeiten der 5 Versuche liegen zwischen 450 - 509 N/mm². Der Vergleich der Versuchsergebnisse in Abhängigkeit der verschiedenen Vorwärmtemperaturen in Abbildung 8.16 zeigt eine Steigerung der maximalen Spannung $\tau_{\text {II }}$ mit geringer werdender Vorwärmtemperatur. Die Steigerung liegt im Mittel bei $29 \mathrm{~N} / \mathrm{mm}^{2}$ (6\%) bei einer Verringerung der Vorwärmtemperatur um $50^{\circ} \mathrm{C}$ und bei $56 \mathrm{~N} / \mathrm{mm}^{2}$ (12 \%) bei einer Verringerung der Vorwärmtemperatur um $80^{\circ} \mathrm{C}$.

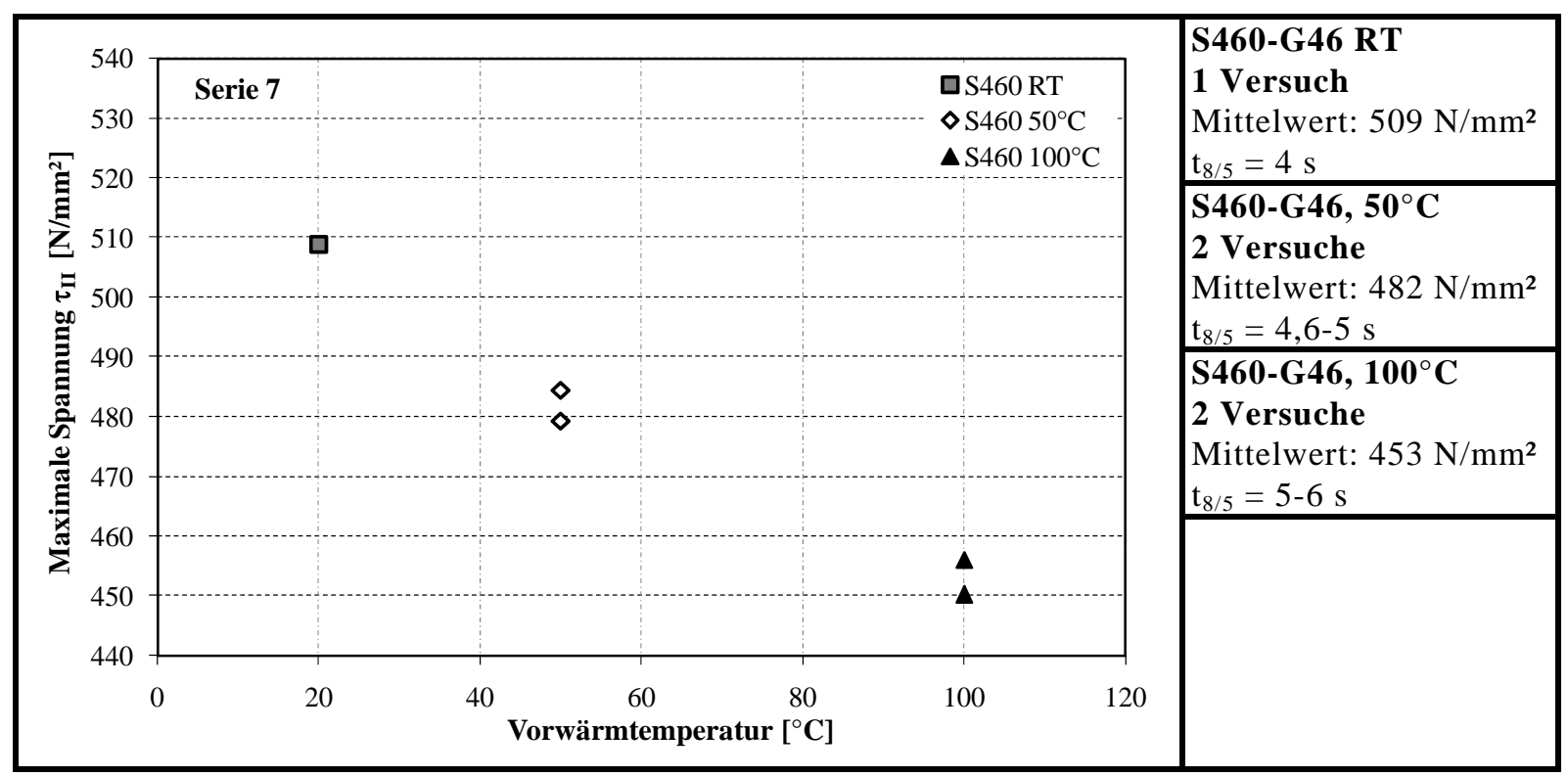

Abbildung 8.16: Einfluss der Vorwärmtemperatur auf die Tragfähigkeit S460-G46 
Abbildung 8.17 zeigt die Spannungs-Verformungs-Linien für die drei Vorwärmtemperaturen der Serie 7. Die Spannung $\tau_{\text {II }}$ ist in Abhängigkeit der Relativverschiebungen der Bleche zueinander am Nahtende dargestellt. Es zeigt sich ein sehr ähnliches Verformungsverhalten. Die Lage des Messaufnehmers von Versuch Z7-01 weicht von der Lage der anderen beiden Versuche ab, deshalb wird der Wert nicht angegeben.

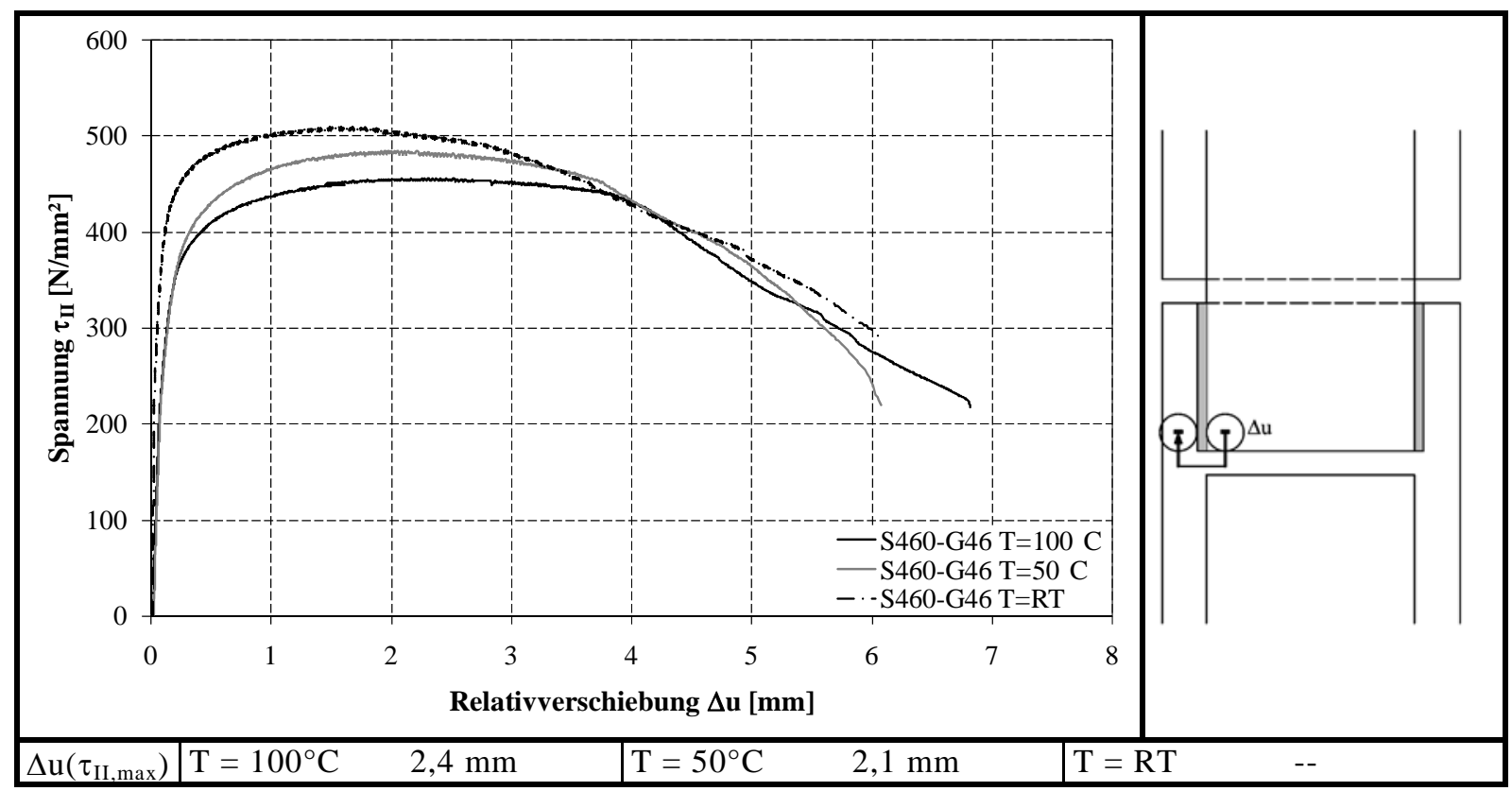

Abbildung 8.17: Einfluss der Vorwärmtemperatur auf die Verformungsfähigkeit S460-G46

Auch für Schweißverbindungen mit dem Grundwerkstoff S460 und zugehörigem Schweißzusatzwerkstoff konnte eindeutig der Einfluss der Abkühlbedingungen auf die Tragfähigkeit nachgewiesen werden.

\section{Härtemessungen und Vergleich mit der Tragfähigkeit}

Die Ergebnisse der durchgeführten Härtemessungen werden in Abbildung 8.18 für die verschiedenen Vorwärmtemperaturen der Verbindung S460-G46 dargestellt. Die Härtewerte unterscheiden sich um insgesamt $6 \%$. Sie wiederholen die Tendenzen der Tragfähigkeit, wobei die Unterschiede in der Tragfähigkeit bei insgesamt $12 \%$ liegen.

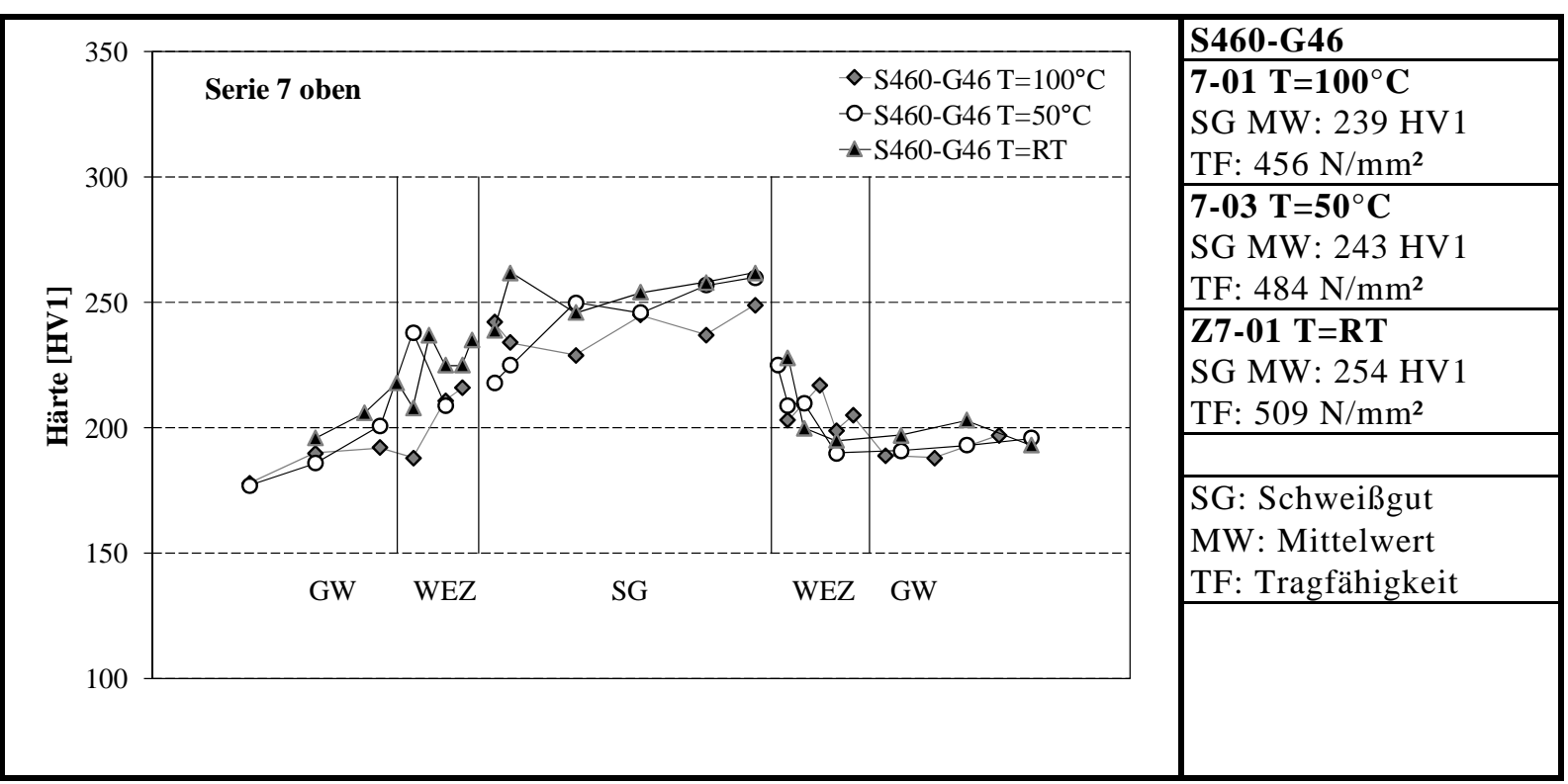

Abbildung 8.18: Vergleich der Härtewerte bei verschiedenen Vorwärmtemperaturen S460-G46 


\section{Fazit}

Die Versuchsergebnisse zeigen einen Einfluss der Vorwärmtemperatur auf die Tragfähigkeit der Verbindung. Bei den 4 Versuchen innerhalb des Arbeitsfensters ändern sich die Maximalspannungen im Mittel um 6 \%. Die Aussagen der Härtemessungen und der Tragfähigkeit stimmen gut überein.

\subsubsection{Diskussion der Versuchsergebnisse bei gleichen Grundwerkstoffen und zugehörigen Schweißzusatzwerkstoffen an Flankenkehlnähten}

Einfluss der Festigkeit von Grund- und Schweißzusatzwerkstoff

Zum Vergleich der Tragfähigkeiten der einlagigen Verbindungen S460-G46 und S690-G69 sind in Abbildung 8.19 als Versuchsergebnisse die maximalen Spannungen $\tau_{\text {II }}$ der Serien 1 und 7 in Abhängigkeit der Zugfestigkeit des Schweißzusatzwerkstoffs aufgetragen. Dabei handelt es sich um die einlagigen Verbindungen S690-G69 und S460-G46 gleicher Geometrie mit gleichen Schweißparametern (Vorwärmtemperatur $\mathrm{T}=100^{\circ} \mathrm{C}$ ). Zusätzlich sind für die Verbindungen S460-G46 die drei Versuchsergebnisse von Versuchen mit unterschiedlichen Vorwärmtemperaturen aufgeführt. Die Mittelwerte der vergleichbaren Versuche (mit gleicher Vorwärmtemperatur geschweißt) sind angegeben.

Es zeigt sich eine deutliche Steigerung der Mittelwerte von 18 \%. Die zugehörige Steigerung der Zugfestigkeit des Grundwerkstoffs liegt im Mittel bei $62 \%$ und die des Schweißzusatzwerkstoffs bei 33 \%. Die Steigerung ist somit nicht proportional zur Zugfestigkeit des Schweißzusatzwerkstoffs.

Wie in Kapitel 2.4.6 beschrieben, entsteht die höhere Tragfähigkeit der Verbindungen S460-G46 durch die Abkühlbedingungen der Kehlnaht im Vergleich zu den eingesetzten Werkstoffen. Bei den Schweißverbindungen S690 entsteht durch die Aufmischung von Grund- und Schweißzusatzwerkstoff ein Gefüge, das einen geringeren Legierungsgehalt hat als der eingesetzte Schweißzusatzwerkstoff. Dadurch verringert sich die Tragfähigkeit. Infolge der beschriebenen Unterschiede verringern sich die Unterschiede in der Tragfähigkeit im Vergleich zu den Festigkeitsunterschieden der eingesetzten Grund- und Schweißzusatzwerkstoffe.

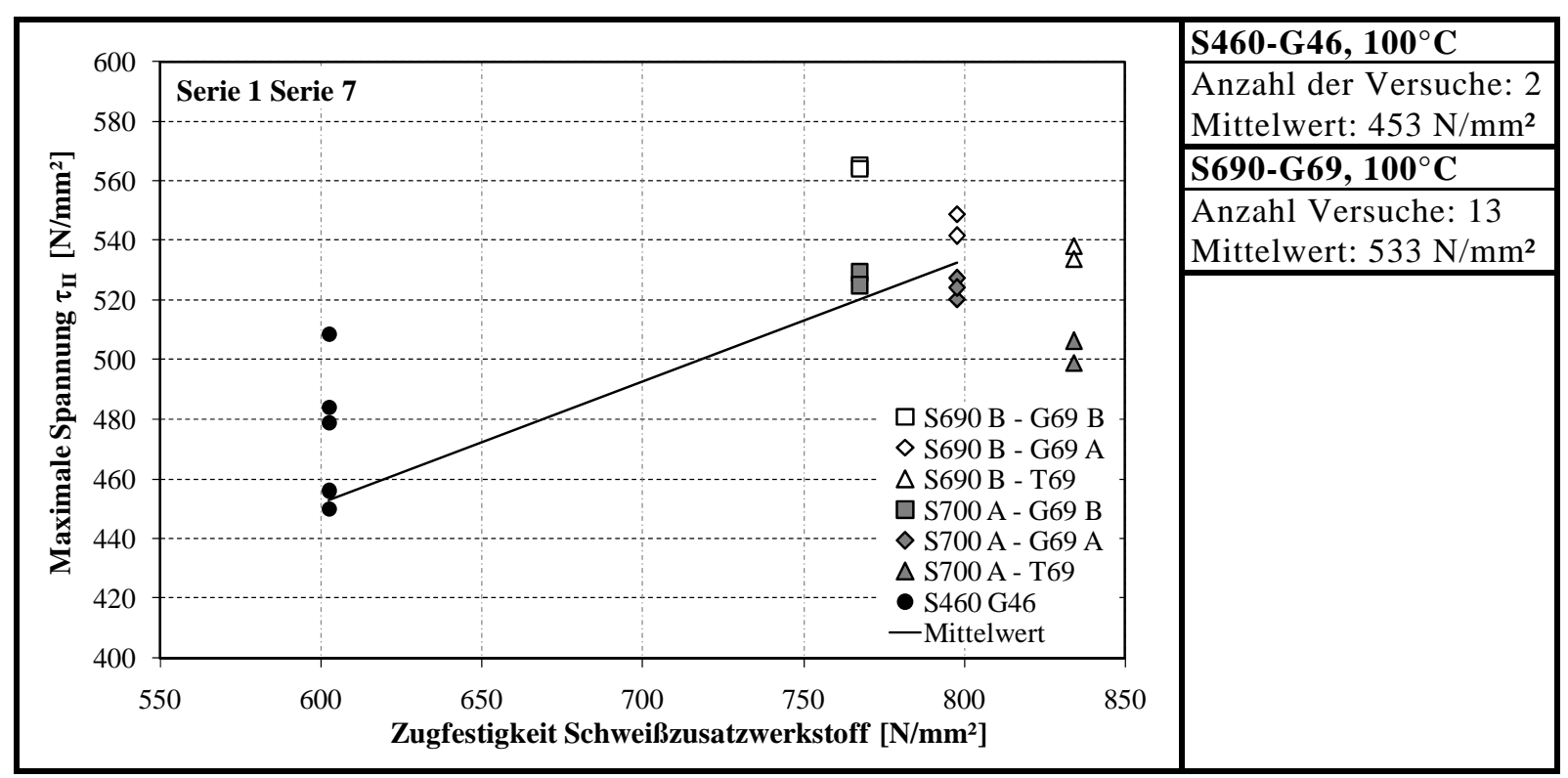

Abbildung 8.19: Vergleich der Tragfähigkeit S460-G46 und S690-G69

In Abbildung 8.20 ist der Vergleich der Spannungs-Verformungs-Linien für die Verbindung S460G46 und S690-G69 dargestellt. Es zeigt sich ein deutlicher Unterschied der Verformbarkeit für die unterschiedlichen Werkstoffkombinationen. Die Verformungen der Versuche der Werkstoffkombination S460-G46 sind deutlich größer als die Verformungen der Werkstoffkombination S690-G69. 


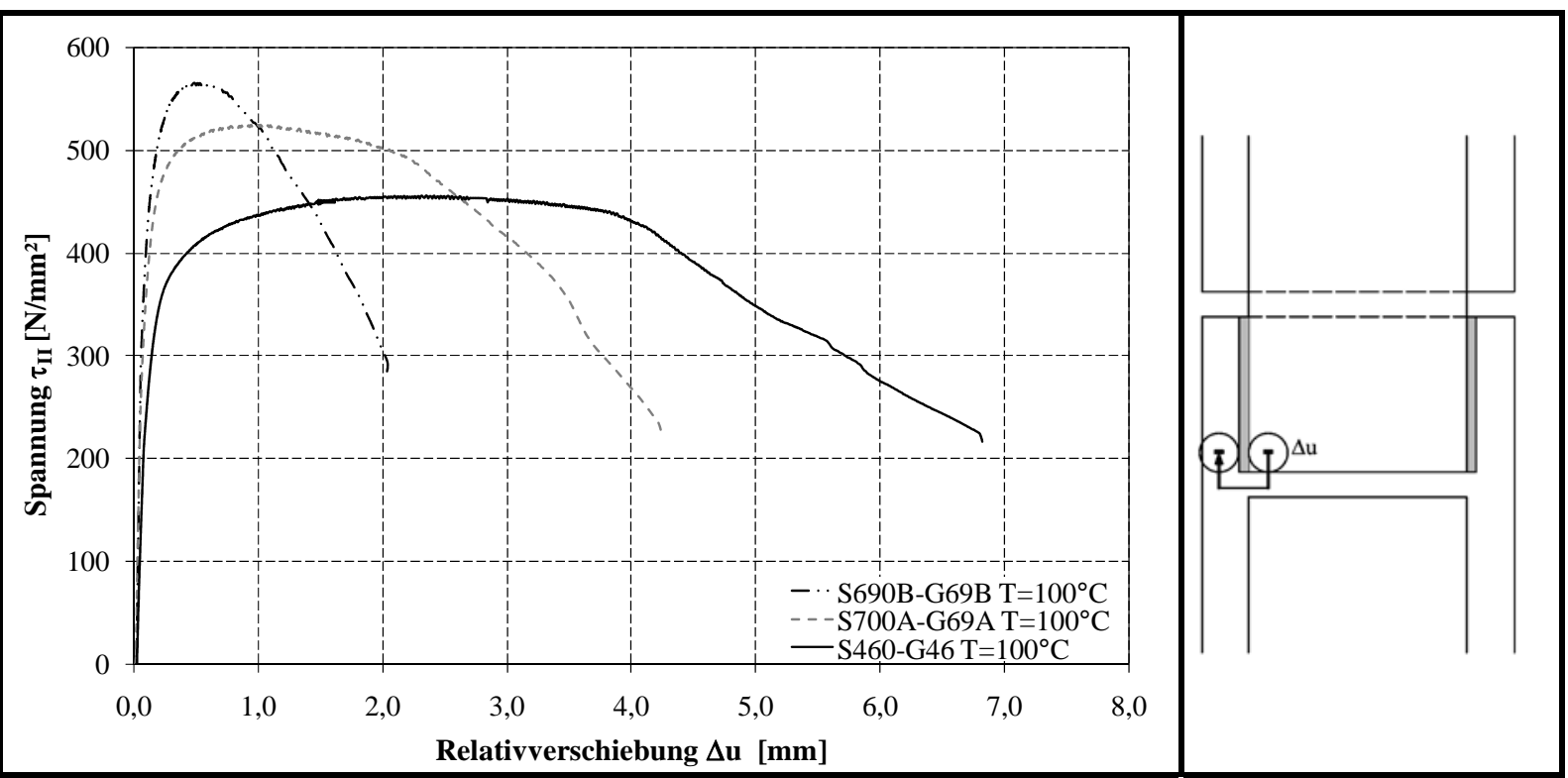

Abbildung 8.20: Vergleich der Verformungsfähigkeiten S460-G46 und S690-G69

Um den Einfluss von Werkstoffkombinationen unterschiedlicher Festigkeiten auf die Härte zu bestimmen, werden in Abbildung 8.21 die Härtewerte der Werkstoffverbindung S460-G46 und S690G69 verglichen. Die Versuche wurden unter gleichen Bedingungen hergestellt. Die Härtewerte wiederholen qualitativ die Aussagen der Tragfähigkeit.

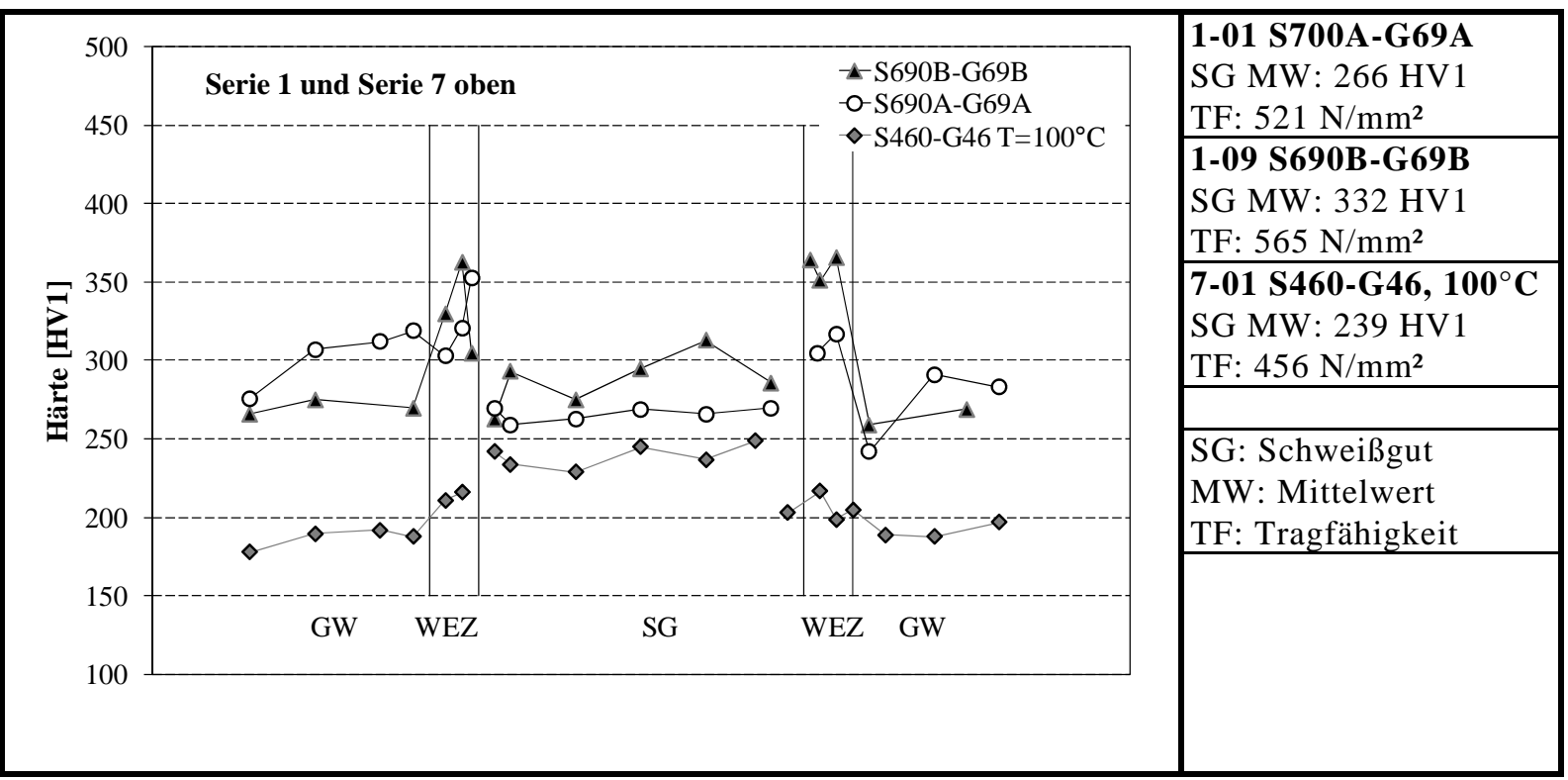

Abbildung 8.21: Vergleich der Härtewerte S460-G46 und S690-G69

\section{Einfluss der Vorwärmtemperatur}

Um den Einfluss der Vorwärmtemperatur zu vergleichen, sind in Abbildung 8.22 die Versuchsergebnisse der Serie 2 (S690-G69) mit denen der Serie 7 (S460-G46) aufgetragen. Ein direkter Vergleich ist nicht möglich, da unterschiedliche Vorwärmtemperaturen verwendet wurden. Es zeigen sich aber in beiden Fällen abnehmende Tragfähigkeiten mit zunehmender Vorwärmtemperatur. Bei geringeren Vorwärmtemperaturen und Verbindungen S460-G46 ist der Einfluss größer als bei höheren Vorwärmtemperaturen und S690-G69. 


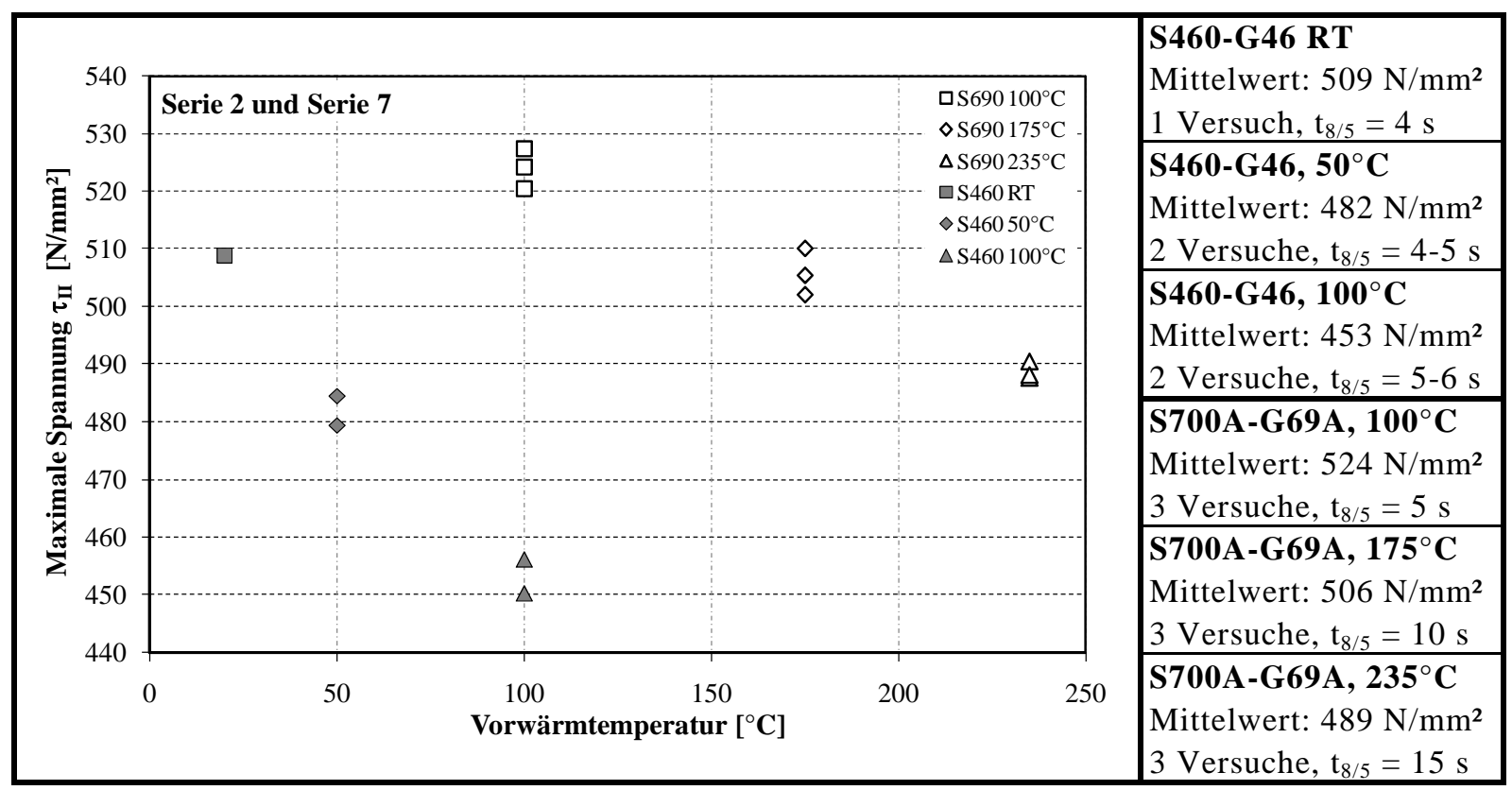

Abbildung 8.22: Einfluss der Vorwärmtemperatur S460-G46 und S690-G69

\section{Härte und Tragfähigkeit}

Tabelle 8.11 zeigt den Vergleich der Härtewerte als Mittelwerte, die in Kapitel 8.6.1 bis 8.6.6 dargestellt sind, mit den zugehörigen Tragfähigkeiten dieser Versuche. Das dargestellte Diagramm zeigt eine Ausgleichsgerade mit der Steigung 3,2/ $\sqrt{3}$, die eine gute Übereinstimmung liefert. Die Härtewerte korrelieren mit den Tragfähigkeiten, es sind aber Streuungen vorhanden.

Tabelle 8.11: Vergleich der Härtemessungen und Tragfähigkeiten von einlagigen Kehlnähten

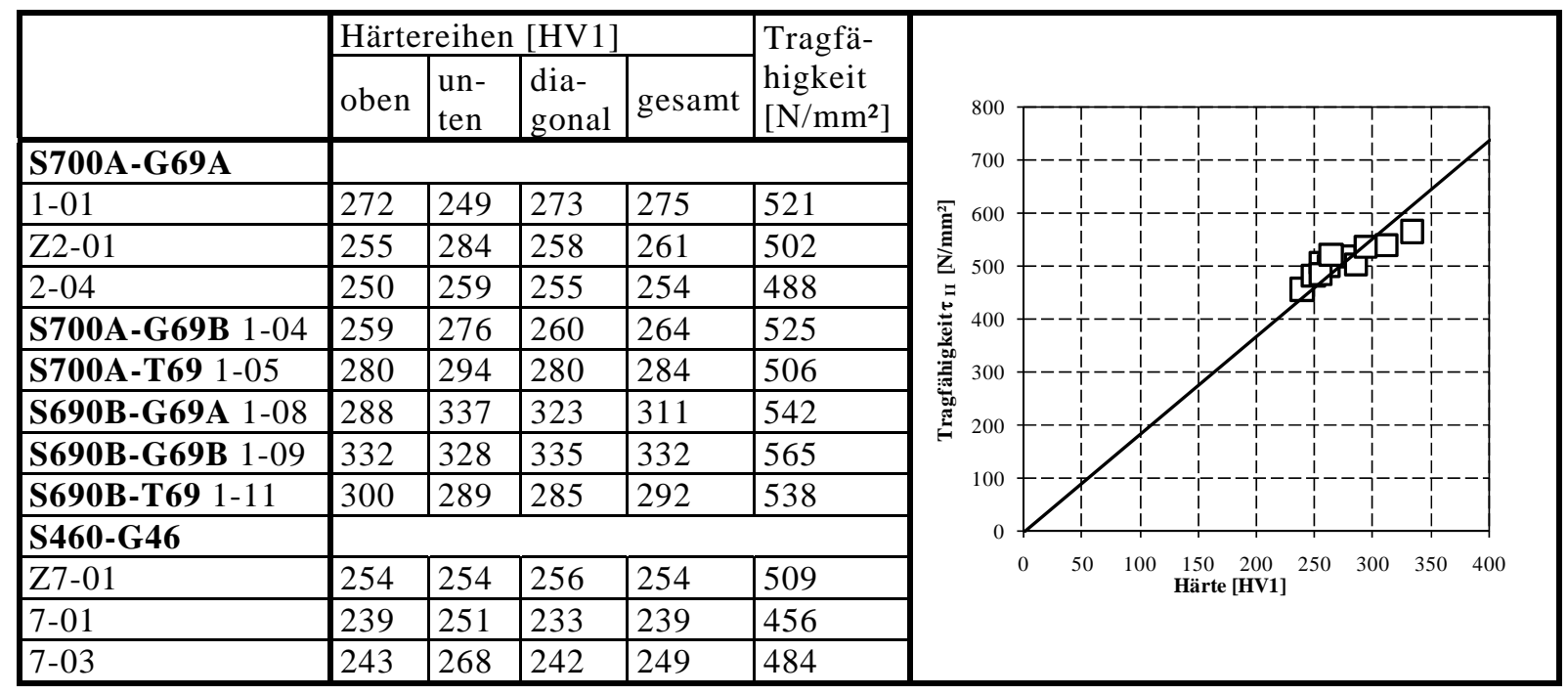

\subsubsection{Zusammenfassung}

In Kapitel 8.6 wurden Untersuchungen an Flankenkehlnähten mit gleichen Grund- und zugehörigen Schweißzusatzwerkstoffen vorgestellt. Als Werkstoffkombinationen wurden Schweißverbindungen S690B/S700A-G/T69 und S460M-G46 betrachtet. Durch die vollmechanisierte Schweißung konnte die Streuung bei gleichen Versuchskörpern sehr gering gehalten werden, so dass der Einfluss der untersuchten Parameter eindeutig aufgezeigt werden konnte.

Durch Variation der Werkstoffkombinationen gleicher Nenngüte S690-G/T/69 konnte das gewünschte Streuband erzielt werden, und damit konnten untere und obere Grenzen der Tragfähigkeit in Abhängigkeit der unterschiedlichen Werkstoffe gleicher Nenngüte ermittelt werden. In Abhängigkeit der 
möglichen Abkühlzeiten t $8 / 5_{5}$ wurde durch Variation der Vorwärmtemperatur die mögliche minimale und maximale Tragfähigkeit im Arbeitsfenster bestimmt.

Für den Parameter Nahtlänge konnte gezeigt werden, dass für die Kehlnähte mit dem Grundwerkstoff S690 und zugehörigem Schweißzusatzwerkstoff mit größerer Länge keine Abnahme der Tragfähigkeit auftritt.

Die Untersuchung des Parameters Naht- und Blechdicke hat gezeigt, dass die Tragfähigkeit mit zunehmender Nahtdicke abnimmt. Für die Werkstoffkombination S700A-G69A wurde eine Reduzierung der Tragfähigkeit um $15 \%$ von $524 \mathrm{~N} / \mathrm{mm}^{2}$ bei einlagigen Nähten zu $457 \mathrm{~N} / \mathrm{mm}^{2}$ bei sechsraupigen Nähten festgestellt. Für die Werkstoffkombination S690B-G69B verringerte sich die Tragfähigkeit von 565 N/mm² zu 498 N/mm² um 14 \% zwischen ein- und sechsraupigen Kehlnähten.

Der Vergleich der Tragfähigkeiten der Verbindungen S460-G46 und S690-G69 zeigt eine deutliche Steigerung der Mittelwerte von 18 \% bei sonst gleichen Bedingungen.

Der Vergleich von Härtewerten und Tragfähigkeiten korreliert. Die Tendenzen der Werte der Härtemessungen und der Tragfähigkeit stimmen gut überein. Die quantitativen Aussagen streuen.

\subsection{Flankenkehlnahtverbindungen mit gleichen Grundwerkstoffen und Variation der Schweißzusatzwerkstoffe (Serie 5)}

\subsubsection{Untersuchungen zur Tragfähigkeit}

Ziel der Untersuchungen in Serie 5 ist die Bestimmung des Einflusses der Zugfestigkeit des Schweißzusatzwerkstoffs auf die Tragfähigkeit von Flankenkehlnahtverbindungen. Dazu wurden Versuchskörper mit den Grundwerkstoffen S700A und S690B geprüft, die mit einem nominell niederfesten Schweißzusatzwerkstoff G46 oder mit einem nominell höherfesten Schweißzusatzwerkstoff G89 verschweißt waren. Die Schweißparameter waren bei allen Versuchen mit einer Streckenenergie von $13,4 \mathrm{~kJ} / \mathrm{cm}$, einer Vorwärmtemperatur von $100^{\circ} \mathrm{C}$ und einer Abkühlzeit $t_{8 / 5}$ von $5 \mathrm{~s}$ gleich vorgegeben.

Die Ergebnisse der zehn durchgeführten Versuche zeigt Abbildung 8.23 gemeinsam mit den neun Versuchen von Serie 1. Dargestellt sind die maximalen Spannungen $\tau_{\text {II }}$ in Abhängigkeit der Zugfestigkeit des Schweißzusatzwerkstoffs. Die Tragfähigkeiten liegen zwischen 507 - 618 N/mm². In Abbildung 8.23 zeigt sich eine Steigerung der Tragfähigkeit mit zunehmender Festigkeit des Schweißzusatzwerkstoffs. Die Steigerungen sind aber nicht proportional zur Zugfestigkeit des Schweißzusatzwerkstoffs.

Der Vergleich der Versuchsergebnisse von Versuchen mit gleichen und unterschiedlichen Schweißzusatzwerkstoffen zeigt deutlich den Einfluss des Grundwerkstoffs auf die Tragfähigkeit der Schweißverbindungen. Für alle Schweißzusatzwerkstoffe liegen die Tragfähigkeiten mit dem Grundwerkstoff S690B höher als die mit dem Grundwerkstoff S700A.

Für den Stahl S700A führt die 30 \% geringere Zugfestigkeit des reinen Schweißgutes zu einem Verlust an Tragfähigkeit von 2,5 \%. Die Steigerung der Zugfestigkeit von G69 nach G89 um 25 \% führt zu einer um 10 \% höheren Tragfähigkeit.

Für Stahl S690B führt die 30 \% geringere Zugfestigkeit des reinen Schweißgutes zu einem Verlust an Tragfähigkeit von 1\%. Die Steigerung der Zugfestigkeit von G69 nach G89 um 25 \% führt zu einer um $13 \%$ höheren Tragfähigkeit.

Auch der Vergleich der Tragfähigkeiten bei gleichem Grundwerkstoff und unterschiedlichen Schweißzusatzwerkstoffen zeigt den Einfluss des Grundwerkstoffs auf die Tragfähigkeit der Verbindungen. Bei einer Zunahme der Zugfestigkeit des Grundwerkstoffs von $12 \%$ von Stahl S700A zu Stahl S690B sinkt die Tragfähigkeit für den Schweißzusatzwerkstoff G46 um 6 \%, für G69 um 4 \% und für G89 um 7 \%. Die Unterschiede in den Festigkeiten der Schweißzusatzwerkstoffe führen nicht in gleichem Maße zu Steigerungen in der Tragfähigkeit, da die Festigkeit des Grundwerkstoffs die Tragfähigkeit ebenfalls beeinflusst. 


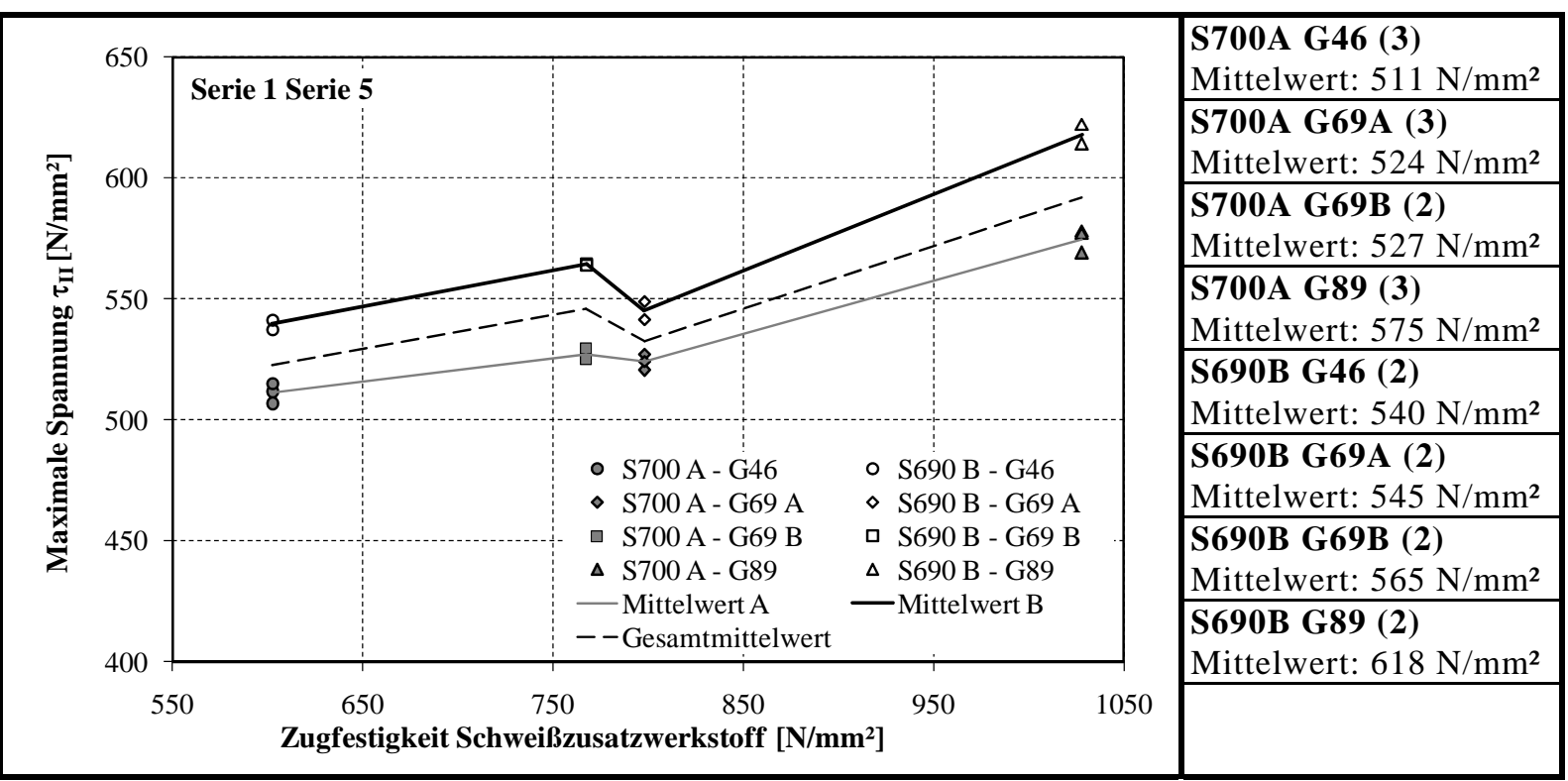

Abbildung 8.23: Einfluss der Festigkeit des Schweißzusatzwerkstoffs auf die Tragfähigkeit S690

Die dargestellten Spannungs-Verformungs-Linien in Abbildung 8.24 für Stahl S700A und für Stahl S690B in Abbildung 8.25 zeigen tendenziell, dass die Verformungsfähigkeit mit zunehmender Festigkeit des Schweißzusatzwerkstoffs geringer wird, es lassen sich aber keine deutlichen Effekte ablesen. Dargestellt sind jeweils die gegenseitigen Verschiebungen der Bleche am Nahtende.

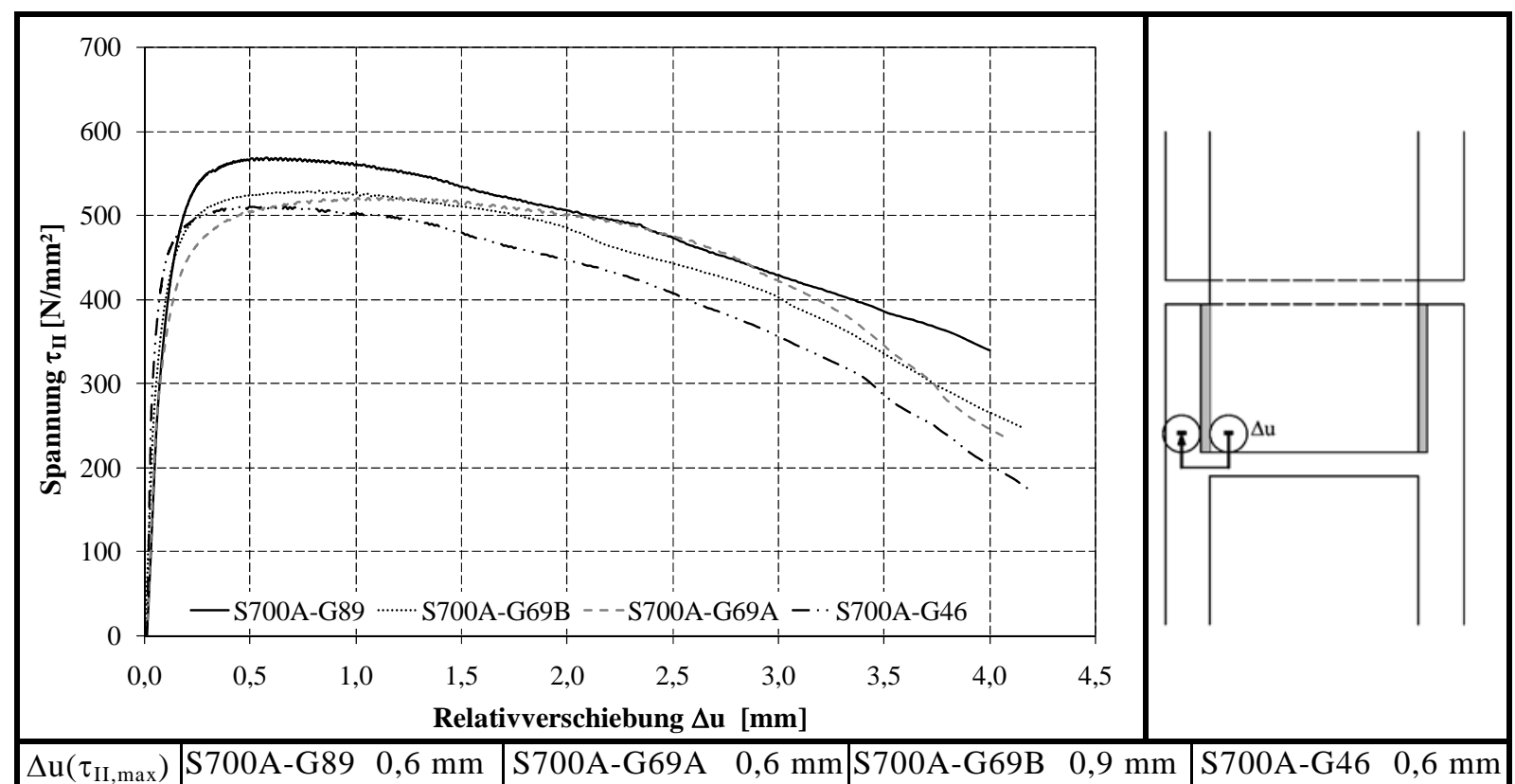

Abbildung 8.24: Einfluss der Festigkeit des Schweißzusatzwerkstoffs auf die Verformungsfähigkeit S700A 


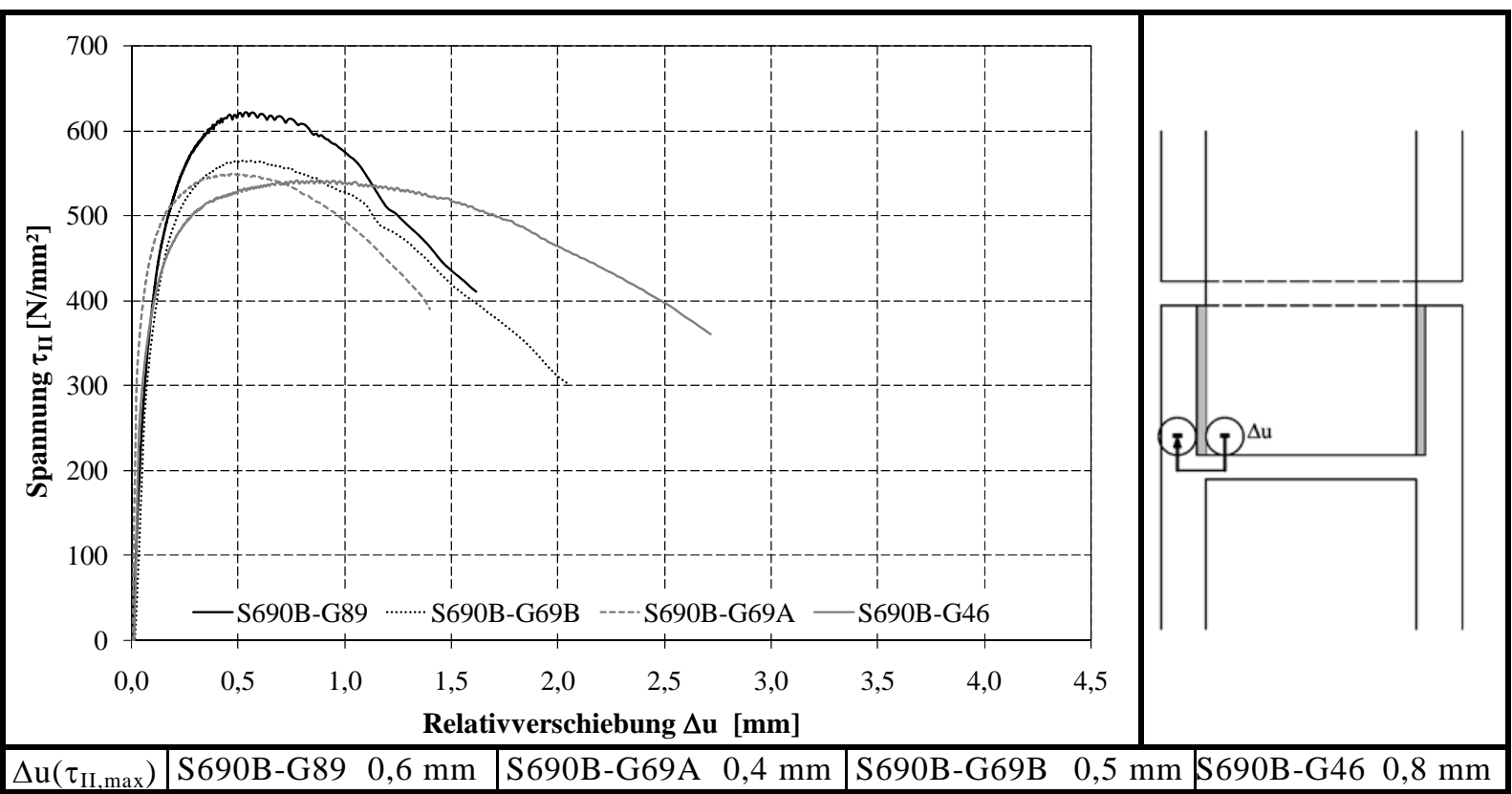

Abbildung 8.25: Einfluss der Festigkeit des Schweißzusatzwerkstoffs auf die Verformungsfähigkeit S690B

\subsubsection{Vergleich von Härtemessungen und Tragfähigkeit}

In Abbildung 8.26 werden die Ergebnisse der durchgeführten Härtemessungen für den Stahl S700A mit unterschiedlichen Schweißzusatzwerkstoffen verglichen. Abbildung 8.27 zeigt den Vergleich der Härtemessungen für den Stahl S690B und verschiedene Schweißzusatzwerkstoffe. Es werden die Schweißzusatzwerkstoffe G46, G69A, G69B und G89 verglichen.

Für beide Stähle zeigen sich für den Schweißzusatzwerkstoff G46 und G69 sehr ähnliche Härtewerte genau wie bei der Tragfähigkeit. Die Härtewerte für den Schweißzusatzwerkstoff G89 liegen für beide Stähle deutlich höher, genau wie die Tragfähigkeit.

Für die Härtewerte des Schweißgutes G89 zeigen sich große Unterschiede zwischen den ermittelten Härtewerten in der Naht.

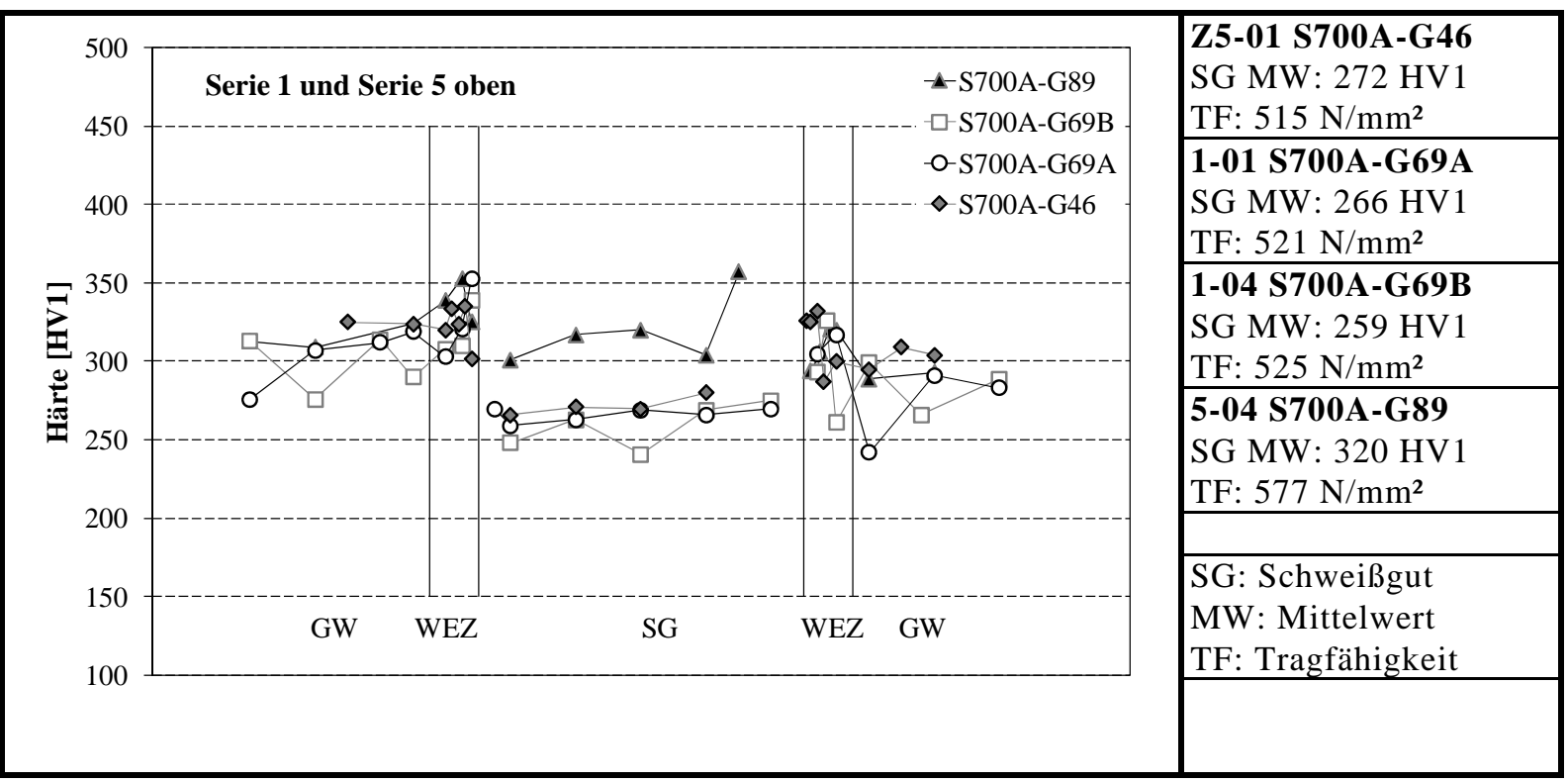

Abbildung 8.26: Vergleich der Härtewerte bei verschiedenen Schweißzusatzwerkstoffen S700A 


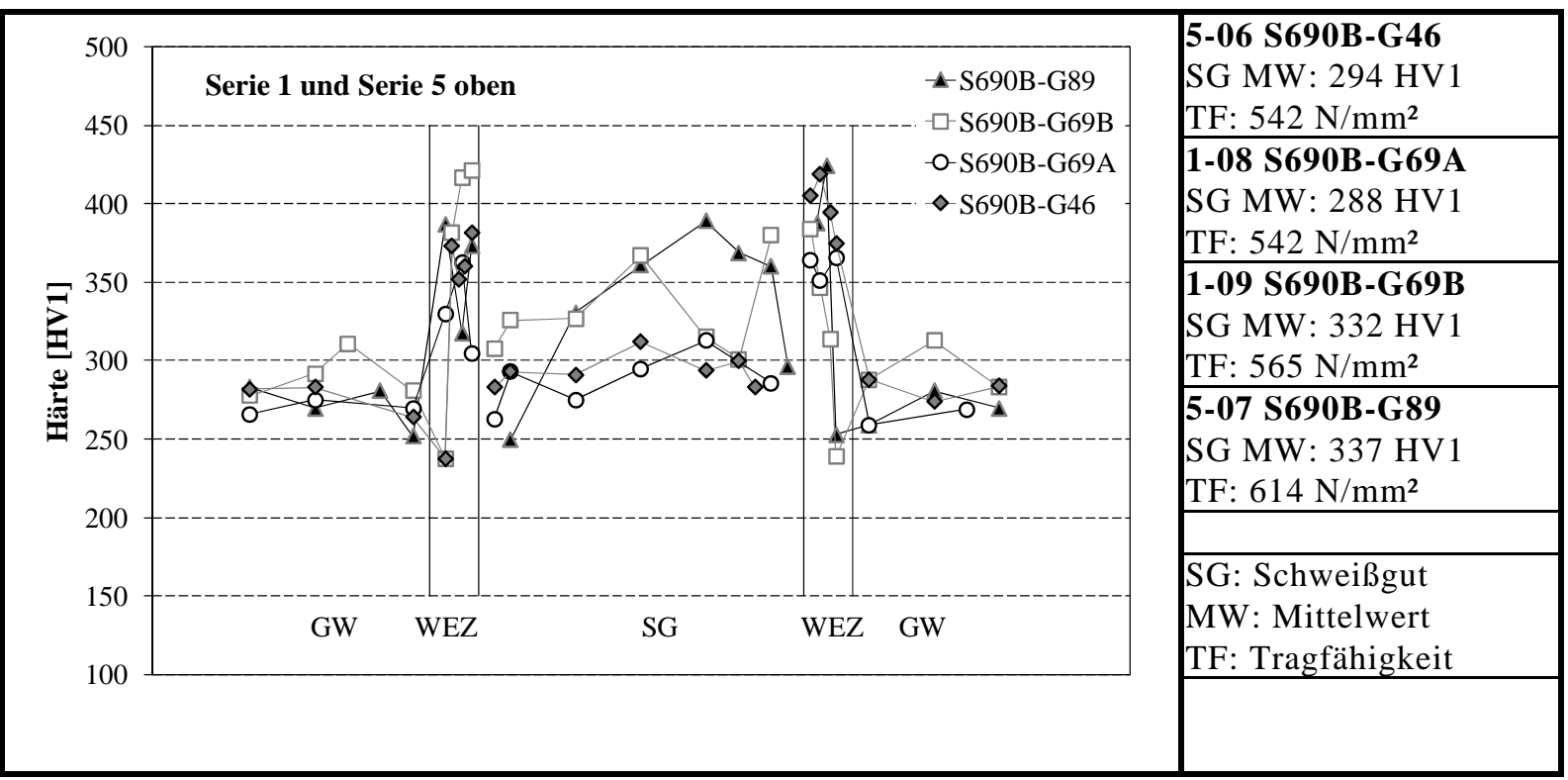

Abbildung 8.27: Vergleich der Härtewerte bei verschiedenen Schweißzusatzwerkstoffen S690B

Vergleicht man die Härtewerte von Verbindungen der beiden Stähle S690B und S700A mit einem Schweißzusatzwerkstoff G46 oder G89, so zeigt sich in beiden Fällen, dass die Härte von Verbindungen mit dem Stahl S690B höher als von Stahl S700A liegt. Bei gleichbleibendem Schweißzusatzwerkstoff korreliert die Härte mit der Tragfähigkeit der Verbindung.

\subsubsection{Fazit}

Insgesamt konnte der Einfluss der Festigkeit des Schweißzusatzwerkstoffs auf die Tragfähigkeit der Verbindung ermittelt werden. Durch die vollmechanisierte Schweißung konnte eine sehr geringe Streuung bei nominell gleichen Versuchskörpern erzielt werden. Die Härtemessungen wiederholen im Wesentlichen die Tendenzen der Tragfähigkeit. Insbesondere bei gleichen Schweißzusatzwerkstoffen korrelieren die Tragfähigkeiten und die Härtewerte gut. Durch den Vergleich von Verbindungen mit gleichen und unterschiedlichen Schweißzusatzwerkstoffen zeigt sich, dass auch der Grundwerkstoff eine große Rolle spielt und die Tragfähigkeit der Verbindung mitbestimmt.

Die Überfestigkeit des Schweißzusatzwerkstoffs führt zu einer Erhöhung der Tragfähigkeit, während die Unterfestigkeit des Schweißzusatzwerkstoffs nur zu einem geringen Verlust an Tragfähigkeit führt. 


\subsection{Untersuchungen an Kreuzstößen}

\subsection{1 Überblick über die Versuchsergebnisse}

Zur Ergänzung der Versuche an Flankenkehlnahtverbindungen wurden Versuche an Kreuzstößen mit Stirnkehlnähten durchgeführt. An diesen Kreuzstößen wurde neben der Bestimmung der Tragfähigkeit von Verbindungen gleicher Grund- und zugehöriger Schweißzusatzwerkstoffe S690-G69 zusätzlich der Einfluss der Festigkeit des Schweißzusatzwerkstoffs getestet. Außerdem wurde der Einfluss der Kraftrichtung auf die Tragfähigkeit bei Kreuzstößen untersucht.

Die Versuchsergebnisse aller durchgeführten Traglastversuche an Kreuzstößen sind in Tabelle 8.12 zusammengefasst. Für die untersuchten Werkstoffkombinationen sind jeweils die gemessenen maximalen Prüfkräfte, die Nahtfläche und als Quotient aus Kraft und Fläche die maximale Spannung dargestellt.

Bei den Kreuzstößen wird zwischen zwei Nahtflächen unterschieden. Zum einen wird die Bruchfläche $A_{B r u c h}$ und zum anderen die Nahtfläche $A_{E C}$ betrachtet. Als ermittelte Bruchfläche $A_{B r u c h}$ wird die nach dem Bruch ausgemessene Nahtfläche bezeichnet. Für die Nahtfläche $A_{E C}$ wird der Makroschliff betrachtet. In die Schweißnaht wird ein Dreieck eingeschrieben und dessen Höhe bestimmt. Als Nahtfläche $A_{E C}$ wird das Produkt aus dieser Höhe des einschreibbaren Dreiecks und der Nahtlänge ermittelt. Details zu den Unterschieden zwischen der Bruchfläche $A_{B r u c h}$ und der Nahtdicke $A_{E C}$ sind in Kapitel 3.3.2 beschrieben.

Als Quotient aus den Prüfkräften und den unterschiedlichen Nahtflächen $A_{B r u c h}$ oder $A_{E C}$ ergeben sich die dargestellten Spannungen $\sigma_{\mathrm{Bruch}}$ und $\sigma_{\mathrm{EC}}$.

Die Werkstoffkombinationen der einzelnen Versuche sind im Anhang zusammen mit den gemessenen Schweißparametern Streckenenergie, Vorwärmtemperatur und Abkühlzeit $t_{8 / 5}$ aufgeführt. Alle Abkühlzeiten lagen im Bereich von 4 - 15 Sekunden.

Tabelle 8.12: Versuchsergebnisse Kreuzstöße

\begin{tabular}{|l|l|l|l|l|l|l|l|l|l|l|l|}
\hline & \\
\end{tabular}


In den folgenden Abschnitten werden die Ergebnisse der experimentellen Untersuchungen zu Traglast und Härte der Kreuzstöße mit Stirnkehlnähten vorgestellt, grafisch dargestellt und diskutiert. Es sind sowohl die maximalen Spannungen als auch die Härte dokumentiert und bewertet. Die Tragfähigkeiten der Versuche werden in Abhängigkeit der ermittelten Kennwerte für die Zugfestigkeit der Grundwerkstoffe und der Schweißzusatzwerkstoffe dargestellt. Die Zeugniswerte werden nur verwendet, wenn keine eigenen Werte vorliegen. In allen Diagrammen werden die Stahlgüten genannt und nach Stahl S700A und S690B unterschieden. Bei den Schweißzusatzwerkstoffen wird zwischen G69A, G69B, G46 und G89 unterschieden. In Tabelle 8.7 sind diese Kurzbezeichnungen definiert.

\subsubsection{Untersuchungen zur Tragfähigkeit}

Untersuchungen zur Tragfähigkeit von Kreuzstößen mit Stirnkehlnähten wurden an Verbindungen gleicher Grund- und zugehöriger Schweißzusatzwerkstoffe sowie an Verbindungen mit über- und niederfesten Schweißzusatzwerkstoffen ermittelt. Dazu wurden die Stähle S700A und S690B und die Schweißzusatzwerkstoffe G69A, G69B, G46 und G89 verwendet.

Die Ergebnisse der zwölf durchgeführten Versuche im Vergleich mit den 7 Versuchen mit zugehörigem Schweißzusatzwerkstoff zeigt Abbildung 8.28 in Abhängigkeit der Bruchfläche und Abbildung 8.29 in Abhängigkeit der Nahtdicke $A_{\mathrm{EC}}$ nach EN 1993-1-8 (2005).

Die ermittelten Spannungen $\sigma_{\mathrm{Bruch}}$ und $\sigma_{\mathrm{EC}}$ unterscheiden sich für einige Versuchsergebnisse um bis zu $10 \%$. Obwohl die drei nominell gleichen Versuche aus einem Versuchskörper stammen, ergeben sich große Streuungen zwischen den Versuchsergebnissen der einzelnen Serien von bis zu 14 \% bezogen auf die Bruchfläche $A_{B r u c h}$ und bis zu 11 \% bezogen auf die Nahtfläche $A_{E C}$.

Die Betrachtung der Versuchsergebnisse mit gleichen Grund- und Schweißzusatzwerkstoffen S690 zeigt deutliche Unterschiede in der Tragfähigkeit zwischen den Werkstoffkombinationen S700AG69A und S690B-G69B im Bereich von 19 bzw. 22 \%.

Die Untersuchung des Einflusses der Festigkeit des Schweißzusatzwerkstoffs zeigt ein unterschiedliches Verhalten für die beiden Stähle. Bei Stahl S700A steigt die Tragfähigkeit mit zunehmender Festigkeit des Schweißzusatzwerkstoffs. Für Stahl S690B sind die Tragfähigkeiten der Verbindungen mit den Schweißzusatzwerkstoffen G46 und G89 größer als die Tragfähigkeiten mit G69B.

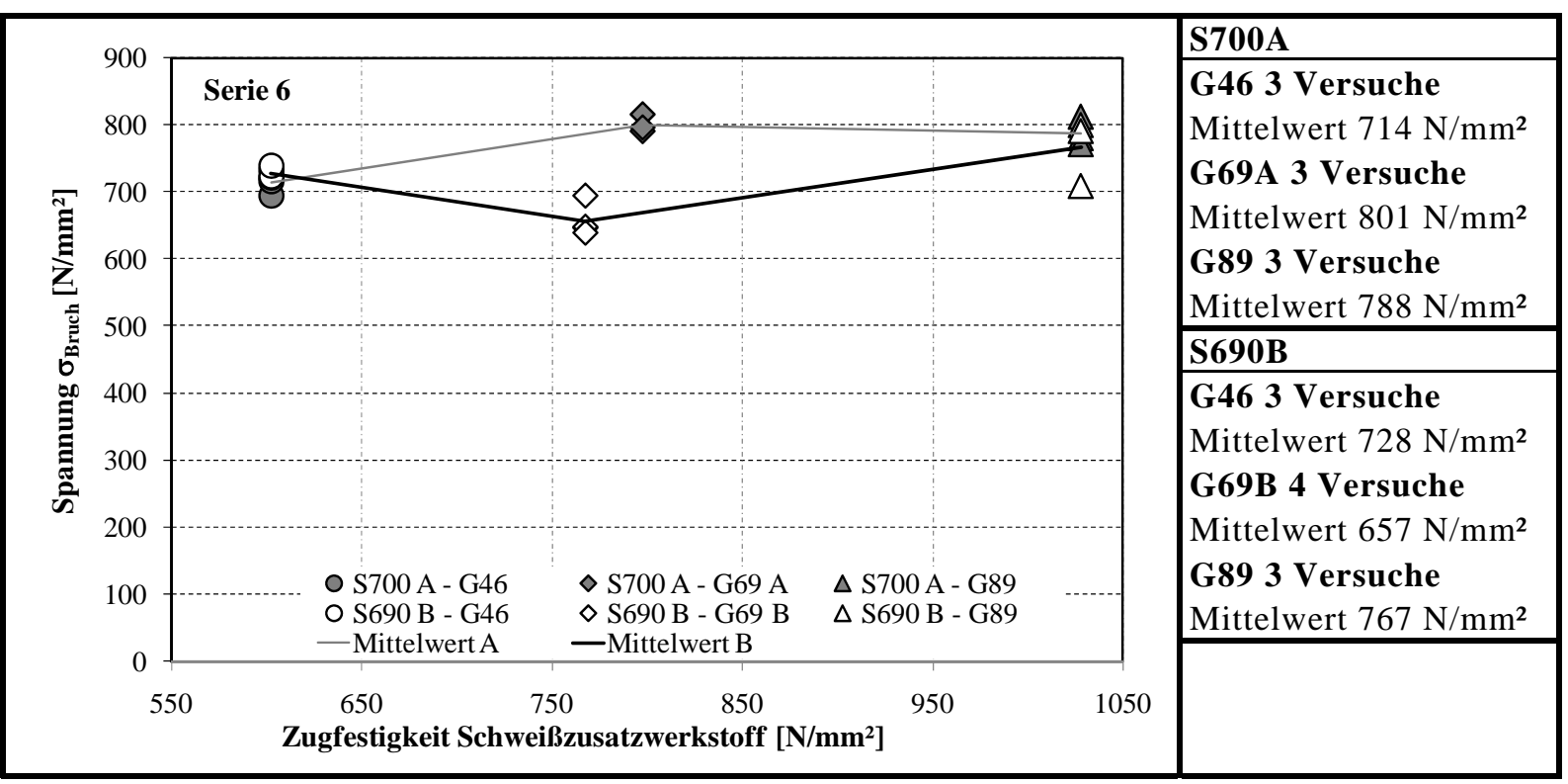

Abbildung 8.28: Einfluss der Festigkeit des Schweißzusatzwerkstoffs auf die Tragfähigkeit S690 (Aruch) 


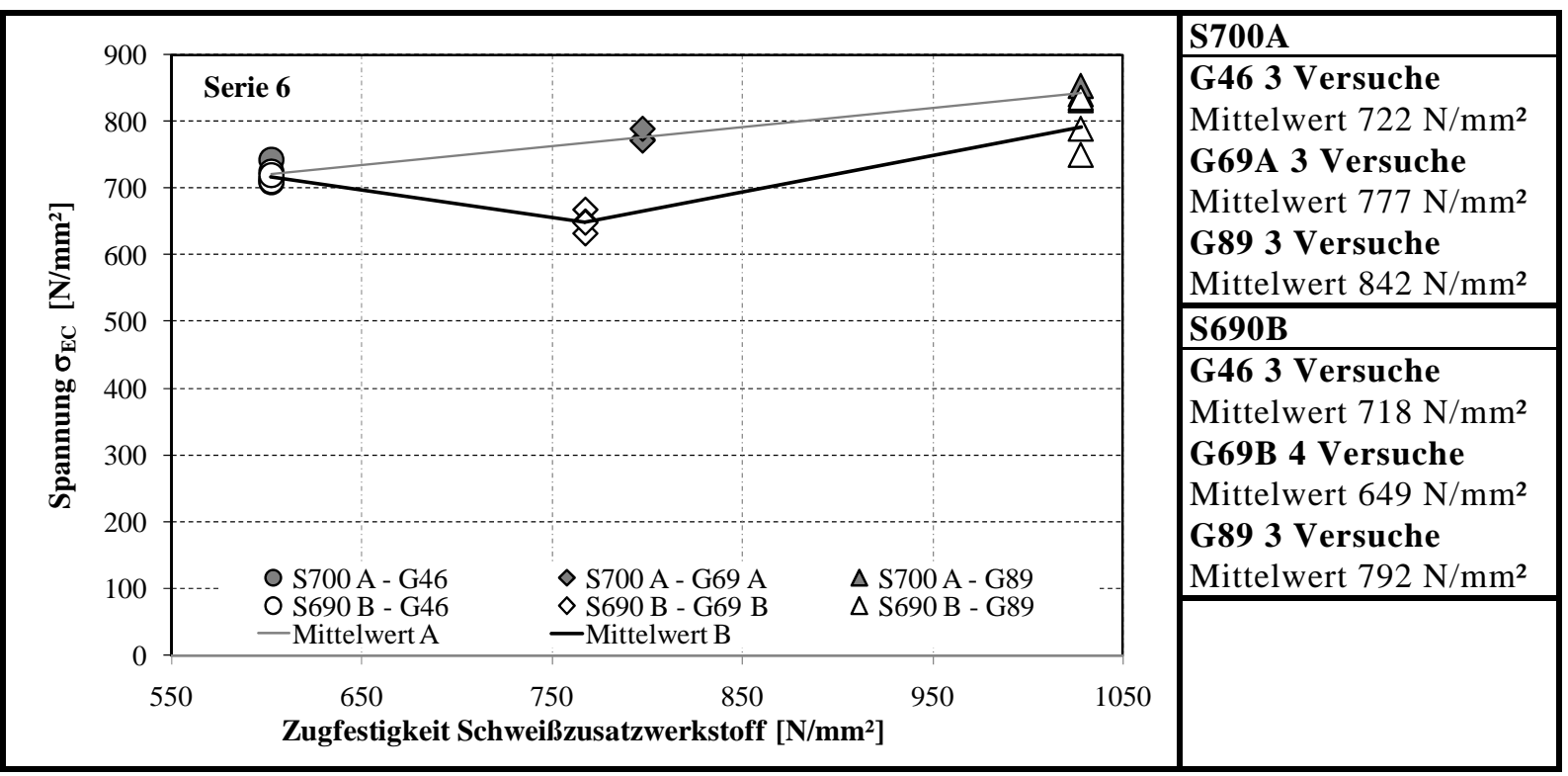

Abbildung 8.29: Einfluss der Festigkeit des Schweißzusatzwerkstoffs auf die Tragfähigkeit S690 ( $\left.\mathrm{A}_{\mathrm{EC}}\right)$

Das Versagen der Schweißnähte fand unter einem sehr steilen Winkel statt, so dass davon ausgegangen wird, dass die Bruchfläche teilweise in der Wärmeeinflusszone und nicht im Schweißgut verlief. In der Wärmeeinflusszone herrschen andere Festigkeitseigenschaften als im Schweißgut und im Grundwerkstoff. Damit lässt sich auch erklären, warum sich die Tragfähigkeiten nicht eindeutig in Abhängigkeit der zunehmenden Festigkeit des Schweißzusatzwerkstoffs ändern.

\subsubsection{Ergebnisse der Härtemessungen}

Für die beiden Versuchskörper der Werkstoffkombination S700A-G69A (6-01) und S690B-G69B (604) der Serie 6 wurde ein Makroschliff angefertigt, an dem Härtemessungen durchgeführt wurden, vgl. Abbildung 8.30 und Abbildung 8.31. Die Härtewerte der Werkstoffkombination S690B-G69B liegen mit 331 HV1 im Schweißgut deutlich höher (um 9 \%) als die von Materialkombination S700A-G69A mit 285 HV1, während die Tragfähigkeit um $22 \%$ niedriger liegt. Bei der Materialkombination S700A-G69A sind die Härtespitzen in der Wärmeeinflusszone deutlich geringer als bei der Werkstoffkombination S690B-G69B. Die Härtewerte des Schweißgutes sind etwa so groß wie die des Grundwerkstoffs. Bei der Werkstoffkombination B-B liegen die Härtewerte des Schweißgutes höher als die des Grundwerkstoffs. In der Wärmeeinflusszone gibt es Härtespitzen bis 430 HV1.

Vergleicht man die Härtewerte von Verbindungen der beiden Stähle S690B und S700A mit einem Schweißzusatzwerkstoff G46 oder G89 zeigt sich in beiden Fällen, dass die Härte von Verbindungen mit dem Stahl S690B höher als von Stahl S700A liegt. Für den Schweißzusatzwerkstoff G46 stimmen die Härtewerte mit den Ergebnissen der Härtemessungen der Flankenkehlnähte gut überein (vgl. Abbildung 8.26 und Abbildung 8.27). Für den Schweißzusatzwerkstoff G89 stimmen die Werte für den Grundwerkstoff S700A gut mit den in Abbildung 8.26 dargestellten Härtewerten an Flankenkehlnahtverbindungen überein. Für den Schweißzusatzwerkstoff G89 und dem Grundwerkstoff S690B liegen die Härtewerte beim Kreuzstoß (im Mittel 383 HV1) deutlich höher als bei der Flankenkehlnaht (im Mittel 337 HV1). Die Werte streuen aber stark.

In Abbildung 8.30 sind die durchgeführten Härtemessungen für die unterschiedlichen Schweißzusatzwerkstoffe und den Stahl S700A dargestellt. Für den Stahl S700A sind die Härtewerte für die Verbindungen mit gleicher Festigkeit des Schweißzusatzwerkstoffs etwas höher als die der Verbindungen mit niederfestem Schweißzusatzwerkstoff. Die Werte für den G89 liegen deutlich höher. Es gibt keine ausgeprägten Härtespitzen in der Wärmeeinflusszone. Für den Stahl S690B (vgl. Abbildung 8.31) liegen die Werte für den niederfesten Schweißzusatzwerkstoff niedriger als die mit zugehörigem Schweißzusatzwerkstoff. Die Werte für den höhefesten Schweißzusatzwerkstoff liegen etwas höher. In der Wärmeeinflusszone gibt es Härtespitzen. Die Härtewerte entsprechen nicht der Tragfähigkeit. 
Die Ergebnisse der durchgeführten Härtemessungen an Kreuzstößen (Abbildung 8.30 und Abbildung 8.31) entsprechen den Ergebnissen der Härtemessungen an Flankenkehlnähten (Abbildung 8.26 und Abbildung 8.27). Die eindeutigen Steigerungen der Tragfähigkeit bei zunehmender Festigkeit des Schweißzusatzwerkstoffs der Flankenkehlnähte (vgl. Abbildung 8.23) kann bei den Kreuzstößen nicht so eindeutig wiederholt werden, wie Abbildung 8.29 zeigt. Ein Grund dafür ist, dass die Bruchfläche im Randbereich zur Wärmeeinflusszone oder in der Wärmeeinflusszone lag und somit nicht nur durch den Schweißzusatzwerkstoff beeinflusst wurde.

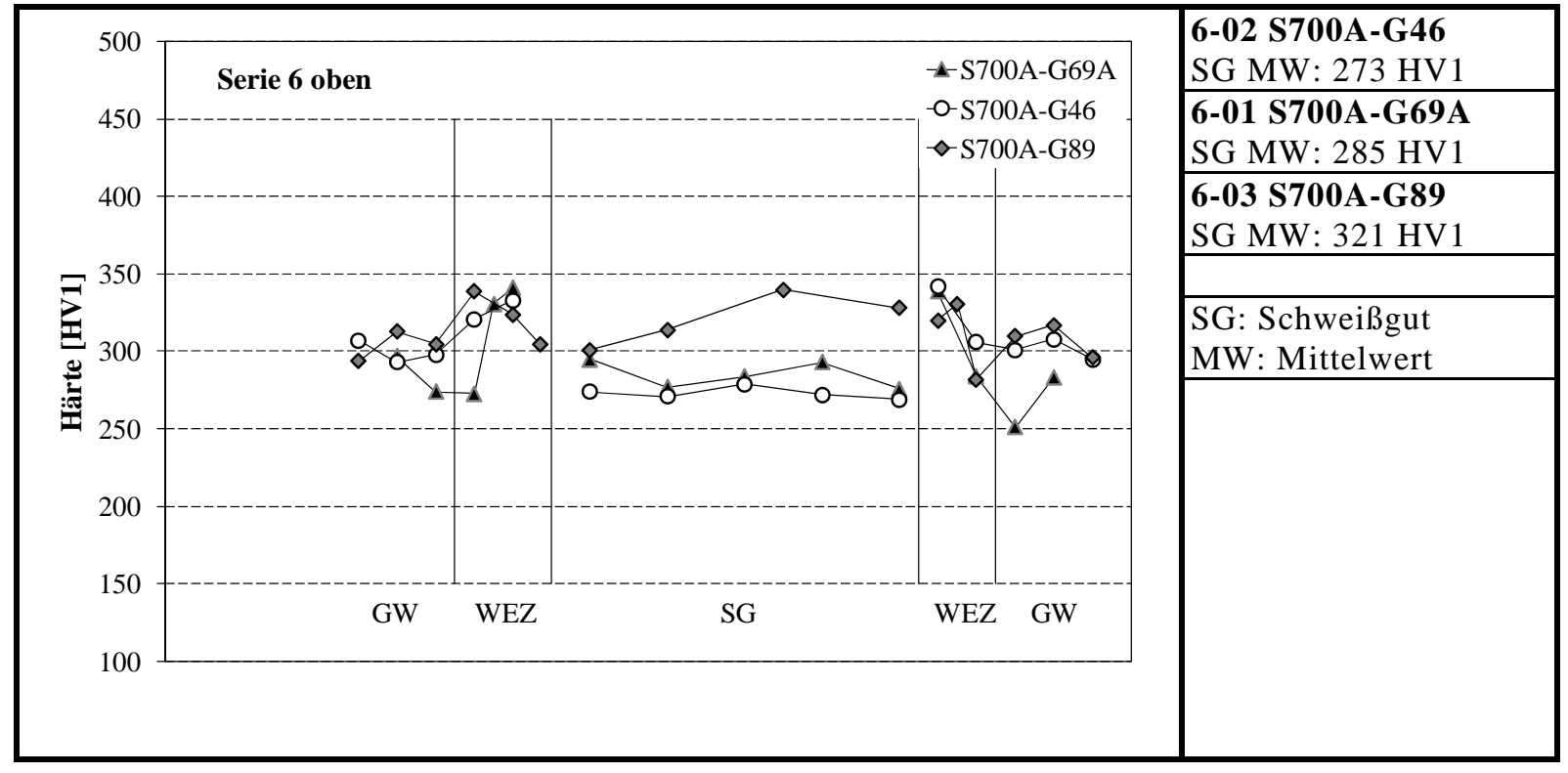

Abbildung 8.30: Vergleich der Härtewerte S700A mit verschiedenen Schweißzusatzwerkstoffen

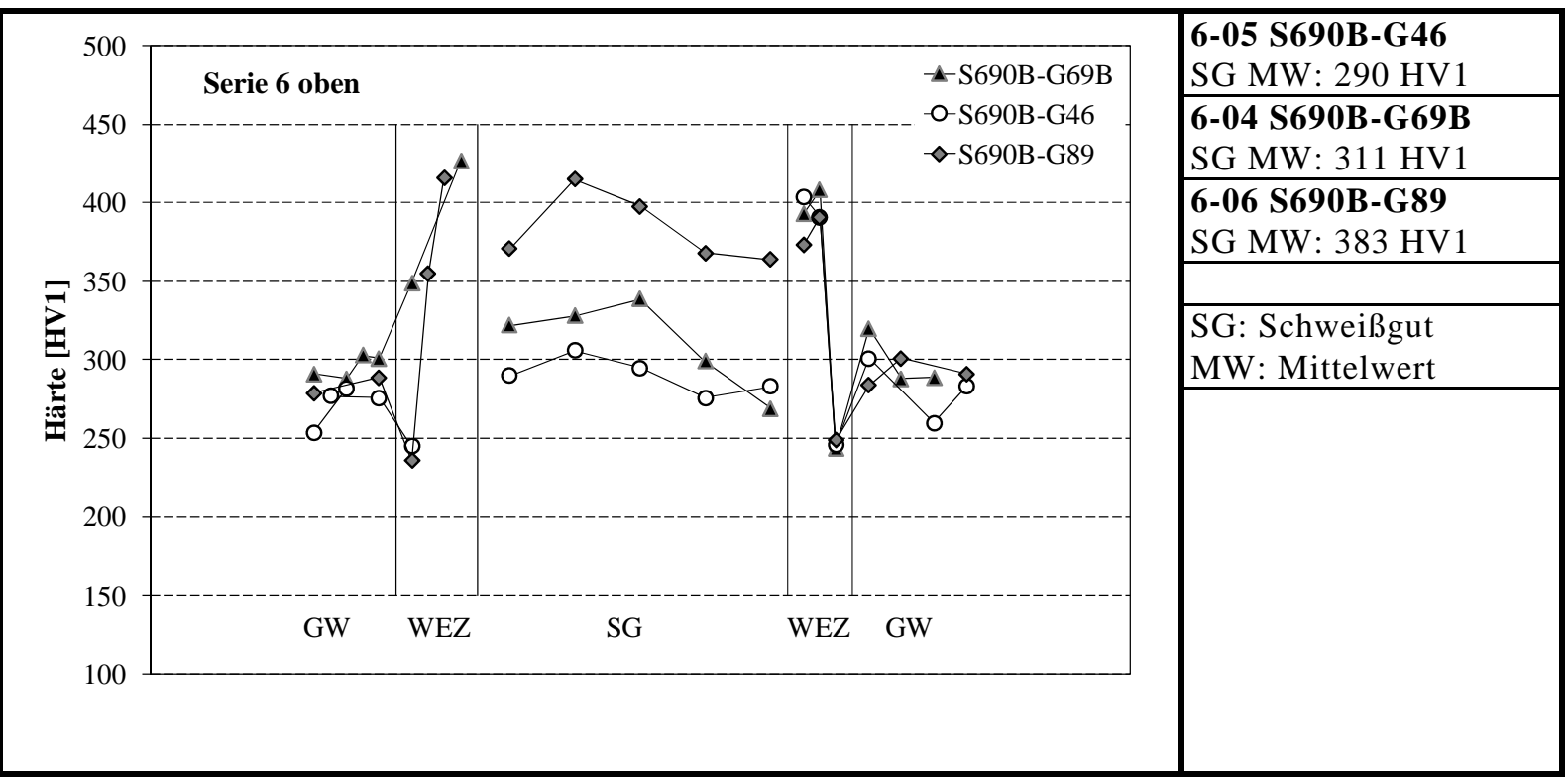

Abbildung 8.31: Vergleich der Härtewerte S690B mit verschiedenen Schweißzusatzwerkstoffen

\subsubsection{Einordnung in Bewertungsgruppen}

Die Maximalspannungen der Kreuzstöße sind nicht eindeutig mit der Festigkeit der Schweißzusatzwerkstoffe angestiegen. Um einen weiteren Grund dafür zu finden, wurde die Geometrie der Kreuzstöße betrachtet. Anhand der Makroschliffe wurden die Schweißverbindungen in die Bewertungsgruppen nach DIN EN ISO 5817 (2006) eingeordnet. Für die Einordnung in Bewertungsgruppen können die Aspekte der Decklagenunterwölbung, der Asymmetrie, des Kantenversatzes und des Spaltes eine Rolle spielen. 
Tabelle 8.13: Überblick über die Bewertungsgruppen nach DIN EN ISO 5817 (2006)

\begin{tabular}{|l|l|l|l|l|}
\hline & $\begin{array}{l}\text { Decklagenunter- } \\
\text { wölbung }\end{array}$ & Asymmetrie & Spalt & Kantenversatz \\
\hline $\begin{array}{l}\text { Bewer- } \\
\text { tungs- } \\
\text { gruppe }\end{array}$ & & & \\
\hline & $\mathrm{für} \mathrm{t}>3 \mathrm{~mm}$ & $\mathrm{für} \mathrm{t}>=0,5 \mathrm{~mm}$ & $\mathrm{für} \mathrm{t}>0,5 \mathrm{~mm}$ & $\mathrm{für}>3 \mathrm{~mm}$ \\
\hline D & $\mathrm{h} \leq 0,25 \mathrm{t} \leq 2 \mathrm{~mm}$ & $\mathrm{~h} \leq 2 \mathrm{~mm}+0,2 \mathrm{a}$ & $\mathrm{h} \leq 1 \mathrm{~mm}+0,3 \mathrm{a} \leq 4 \mathrm{~mm}$ & $\mathrm{~h} \leq 0,25 \mathrm{t} \leq 5 \mathrm{~mm}$ \\
\hline C & $\mathrm{h} \leq 0,1 \mathrm{t} \leq 1 \mathrm{~mm}$ & $\mathrm{~h} \leq 2 \mathrm{~mm}+0,15 \mathrm{a}$ & $\mathrm{h} \leq 0,5 \mathrm{~mm}+0,2 \mathrm{a} \leq 3 \mathrm{~mm}$ & $\mathrm{~h} \leq 0,15 \mathrm{t} \leq 4 \mathrm{~mm}$ \\
\hline B & $\mathrm{h} \leq 0,05 \mathrm{t} \leq 0,5 \mathrm{~mm}$ & $\mathrm{~h} \leq 1,5 \mathrm{~mm}+0,15 \mathrm{a}$ & $\mathrm{h} \leq 0,5 \mathrm{~mm}+0,1 \mathrm{a} \leq 2 \mathrm{~mm}$ & $\mathrm{~h} \leq 0,1 \mathrm{t} \leq 3 \mathrm{~mm}$ \\
\hline
\end{tabular}

Die Versuchskörper hatten nur einen geringen Spalt $(<1 \mathrm{~mm})$ und konnten hinsichtlich des Spaltes in Bewertungsgruppe B eingeordnet werden. Die Decklagenunterwölbung ist immer geringer als $\mathrm{h}=$ $1 \mathrm{~mm}$, danach ist also für alle Versuchskörper mindestens Bewertungsgruppe $\mathrm{C}$ erreicht. Wird die Asymmetrie betrachtet, erreichen alle Versuchskörper Bewertungsgruppe B bis auf zwei Nähte, die der Bewertungsgruppe $\mathrm{C}$ zuzuordnen sind.

Die Einordnung der Unregelmäßigkeit Kantenversatz ist in Tabelle 8.14 dargestellt. Die Versuche mit dem Grundwerkstoff S700A (6-01, 6-02 und 6-03) lassen sich in die Bewertungsgruppe C einordnen, während die Verbindungen mit den Stählen S690B nur Bewertungsgruppe D erreichen. Eine Ausnahme bildet Versuch 6-06/4, der die Bewertungsgruppe D nicht erreicht.

Ein großer Kantenversatz bedeutet für den Kreuzstoß, dass die Schweißnaht zusätzlich auf Biegung beansprucht wird. Diese zusätzliche Beanspruchung kann zu einem vorzeitigen Versagen führen. Darin ist die Erklärung zu sehen für die großen Streuungen der Versuchsergebnisse und die geringe Tragfähigkeit mit dem Stahl S690B. Die Versuchsergebnisse mit dem Grundwerkstoff S690B werden nicht weiter betrachtet.

Tabelle 8.14: Bestimmung der Bewertungsgruppen für den Kantenversatz für $t=15$ mm

\begin{tabular}{|c|c|c|c|c|c|}
\hline & Kantenversatz h & Bewertungsgruppe & & Kantenversatz h & Bewertungsgruppe \\
\hline $6-01 / 2$ & $1,9 \mathrm{~mm}$ & $\mathbf{C}$ & 6-04/2 & $2,9 \mathrm{~mm}$ & D \\
\hline $6-01 / 3$ & $1,8 \mathrm{~mm}$ & $\mathbf{C}$ & 6-04/3 & $3,3 \mathrm{~mm}$ & $\mathbf{D}$ \\
\hline $6-01 / 4$ & $1,9 \mathrm{~mm}$ & C & 6-04/4 & $3,7 \mathrm{~mm}$ & D \\
\hline $6-02 / 2$ & $1,5 \mathrm{~mm}$ & $\mathbf{B}$ & 6-04/5 & $3,7 \mathrm{~mm}$ & $\mathbf{D}$ \\
\hline $6-02 / 3$ & $1,8 \mathrm{~mm}$ & $\mathbf{C}$ & $6-05 / 2$ & $2,7 \mathrm{~mm}$ & $\mathbf{D}$ \\
\hline $6-02 / 4$ & $2,1 \mathrm{~mm}$ & $\mathbf{C}$ & $6-05 / 3$ & $2,4 \mathrm{~mm}$ & $\mathbf{D}$ \\
\hline $6-03 / 2$ & $1,8 \mathrm{~mm}$ & C & $6-05 / 4$ & $1,9 \mathrm{~mm}$ & $\mathbf{C}$ \\
\hline $6-03 / 3$ & $1,9 \mathrm{~mm}$ & $\mathbf{C}$ & 6-06/1 & $3,1 \mathrm{~mm}$ & $\mathbf{D}$ \\
\hline $6-03 / 4$ & $1,8 \mathrm{~mm}$ & $\mathbf{C}$ & $6-06 / 3$ & $2,4 \mathrm{~mm}$ & $\mathbf{D}$ \\
\hline & 6-06/4 & $4,1 \mathrm{~mm}$ & -- \\
\hline
\end{tabular}

\subsubsection{Statistische Auswertung}

Für eine statistische Auswertung der Versuche gleicher Grundwerkstoffe und zugehöriger Schweißzusatzwerkstoffe stehen nur 3 Versuchsergebnisse S700A-G69A zur Verfügung. Die Versuchsergebnisse der Werkstoffkombination S690B-G69B können aufgrund ihrer schlechten Bewertungsgruppe nicht verwendet werden. Auf eine statistische Auswertung mit nur 3 Versuchen wird an dieser Stelle verzichtet. 


\subsubsection{Einfluss der Kraftrichtung bei Kreuzstößen}

Zur Untersuchung des Einflusses der Kraftrichtung auf die Tragfähigkeit wurde an einem Kreuzstoß der Winkel zwischen Naht und Kraftrichtung variiert. Die Ergebnisse der durchgeführten 6 Versuche zeigen Abbildung 8.32 und Abbildung 8.33. Es zeigen sich deutliche Unterschiede zwischen den verschiedenen Bezugsflächen des schrägen Kreuzstoßes, bei dem geraden Kreuzstoß sind die Unterschiede gering. Der Tragfähigkeitsunterschied bezogen auf die $A_{\text {Bruch }}$ liegt bei $25 \%$.

Der Unterschied in den Spannungen bezogen auf unterschiedliche Nahtflächen zeigt deutlich den Einfluss der Bezugsfläche.

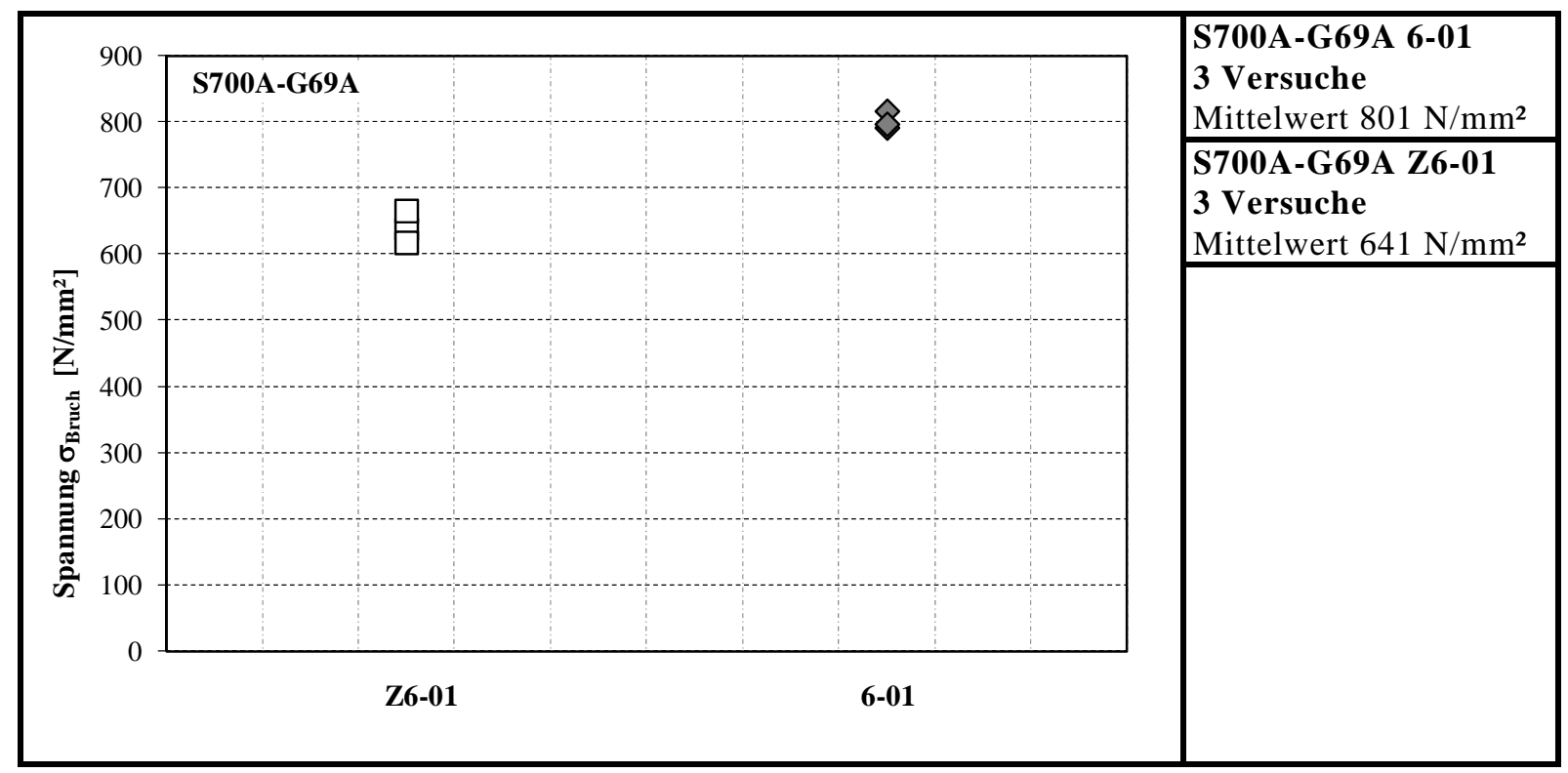

Abbildung 8.32: Einfluss der Kraftrichtung auf die Tragfähigkeit $\left(A_{\text {Bruch }}\right)$

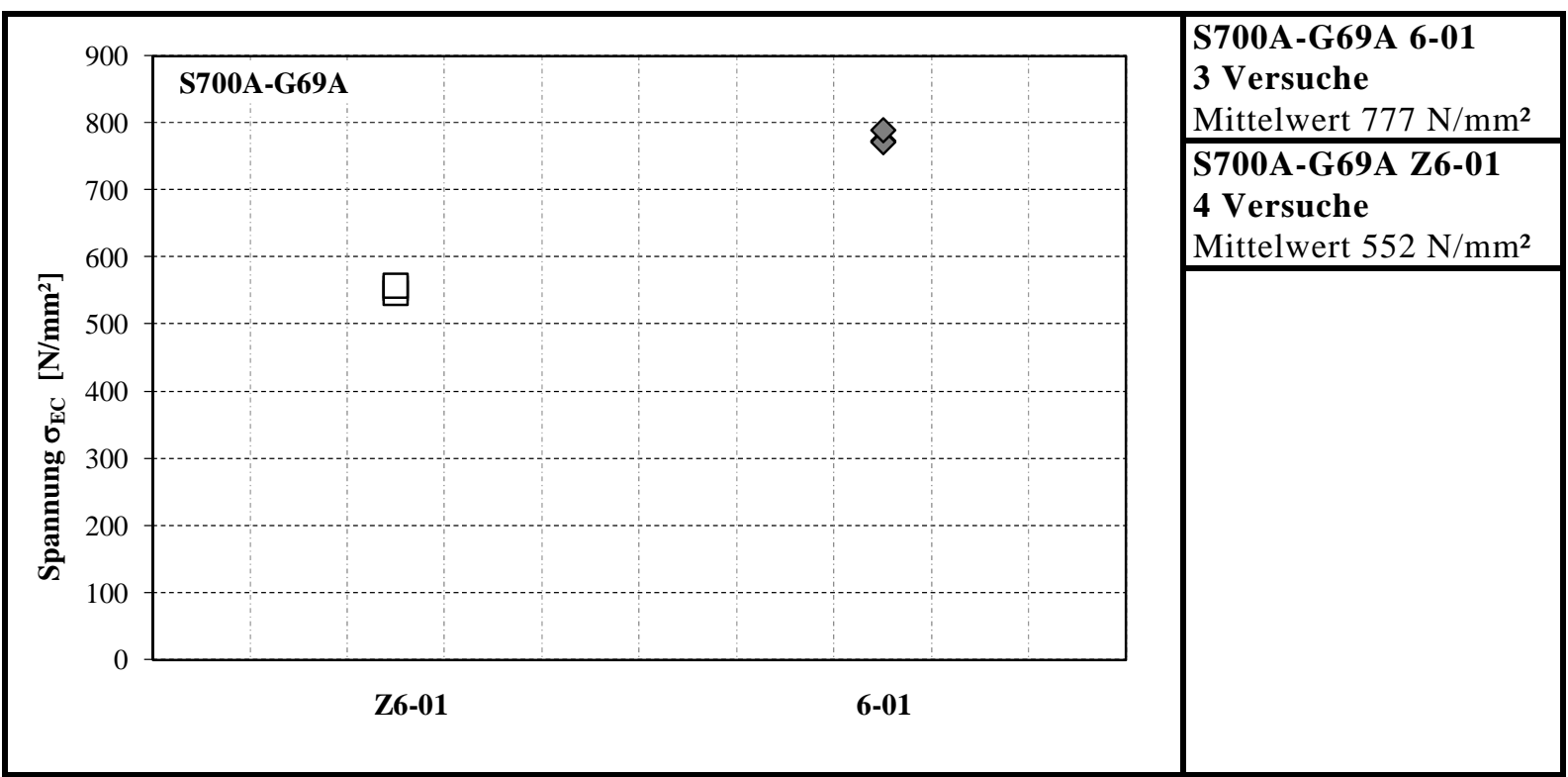

Abbildung 8.33: Einfluss der Kraftrichtung auf die Tragfähigkeit $\left(\mathrm{A}_{\mathrm{EC}}\right)$

An dem Versuchskörper der Materialkombination A-A (Z6-01), der schräg belastet wurde, wurden ebenfalls Härtemessungen durchgeführt. Der Vergleich der Härtemessungen bei gleichen Werkstoffen zeigt in Abbildung 8.34 sehr ähnliche Werte, da die beiden Versuchskörper gleich geschweißt wurden. 


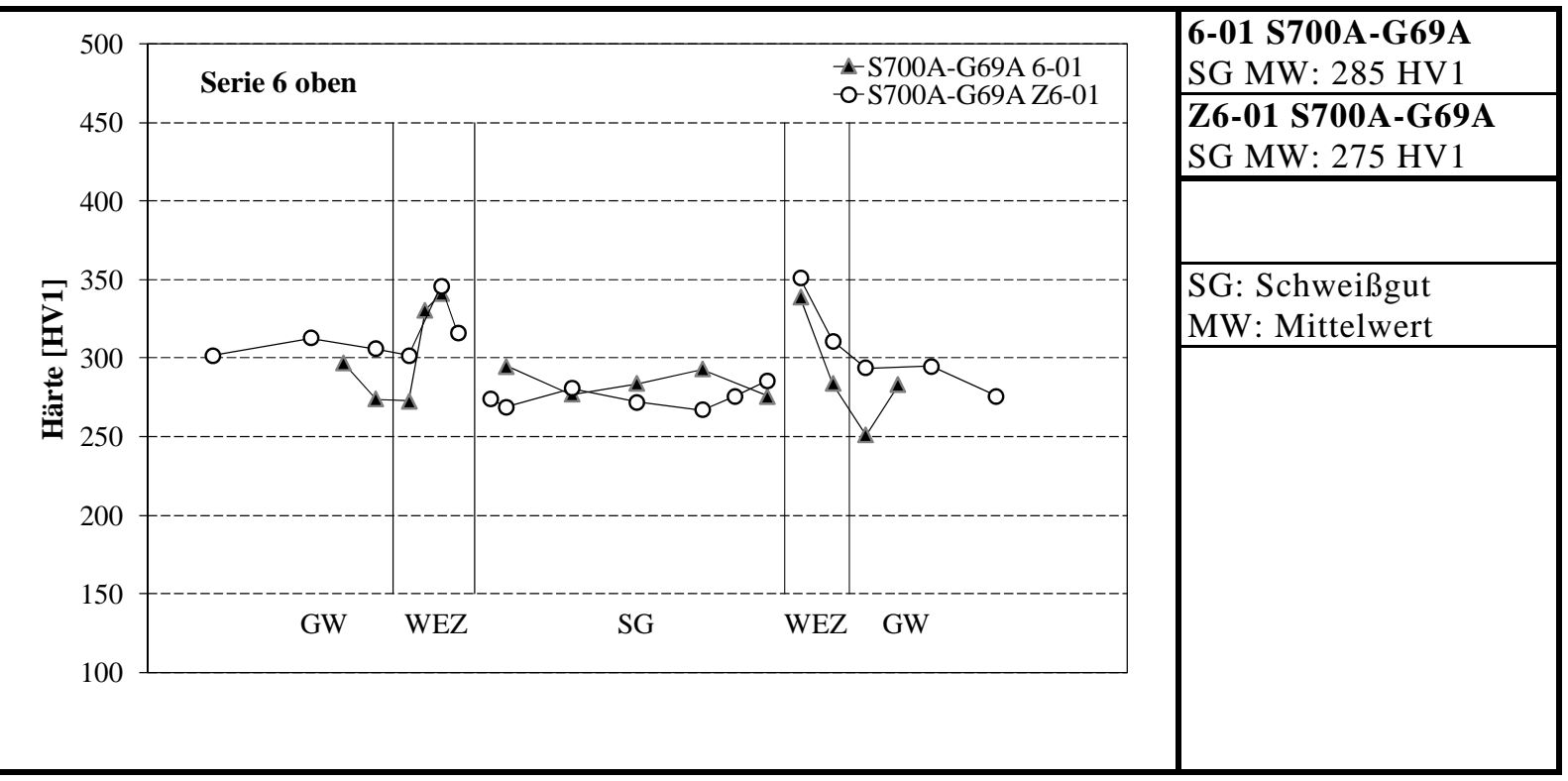

Abbildung 8.34: Vergleich der Härtewerte bei gleichen Grund- und Schweißzusatzwerkstoffen S690-G69

\subsubsection{Fazit}

Die Auswertung der Versuchsergebnisse von Kreuzstößen bezogen auf die unterschiedlichen Nahtflächen $A_{E C}$ und $A_{B r u c h}$ hat gezeigt, dass sich die ermittelten Spannungen $\sigma_{B r u c h}$ und $\sigma_{\mathrm{EC}}$ für einige Versuchsergebnisse um bis zu $10 \%$ unterscheiden.

Trotz der vollmechanisierten Schweißung ist die Streuung bei gleichen Versuchskörpern größer als bei den Flankenkehlnahtverbindungen. Zwischen den Ergebnissen der nominell gleichen Versuche wurden Unterschiede von bis zu $14 \%$ bezogen auf die Bruchfläche $A_{\text {Bruch }}$ und bis zu $11 \%$ auf die Nahtfläche $A_{E C}$ festgestellt.

Als Grund für die große Streuung der Versuchsergebnisse wird der Bruch unter einem sehr steilen Winkel vermutet, der damit einhergeht, dass der Bruch in der Wärmeeinflusszone erfolgt, wo keine definierten Bedingungen vorherrschen.

Zur Beurteilung der Versuchsergebnisse wurde die Geometrie der Versuchskörper in Bewertungsgruppen nach DIN EN ISO 5817 (2006) vorgenommen. Danach können die Versuche mit dem Grundwerkstoff S700A (6-01, 6-02 und 6-03) in die Bewertungsgruppe C eingeordnet werden, während sich für die Verbindungen mit den Stählen S690B nur Bewertungsgruppe D ergibt. Eine Ausnahme bildet Versuch 6-06/4, der die Bewertungsgruppe D nicht erreicht. Das ausschlaggebende Kriterium war dabei der Kantenversatz. Ein großer Kantenversatz führt zu einer zusätzlichen undefinierten Beanspruchung in der Naht, die zu einem vorzeitigen Versagen führen kann. Darin ist die Erklärung zu sehen für die großen Streuungen der Versuchsergebnisse mit dem Stahl S690B. Die Versuchsergebnisse mit dem Grundwerkstoff S690B werden nicht weiter betrachtet.

Die Untersuchung des Einflusses der Festigkeit des Schweißzusatzwerkstoffs für den Stahl S700A zeigt eine ansteigende Tragfähigkeit mit zunehmender Festigkeit des Schweißzusatzwerkstoffs. Die Härtemessungen ergeben ähnliche Tendenzen wie die Tragfähigkeitsuntersuchungen.

Die Zusatzversuche an Kreuzstößen mit schrägen Schweißnähten haben eindeutig gezeigt, dass die Kraftrichtung einen Einfluss auf die Tragfähigkeit hat. 


\subsection{Werkstoffuntersuchungen}

\subsubsection{Einleitung}

Um die Tragfähigkeit von Schweißverbindungen zu bestimmen, ist die Kenntnis der Zugfestigkeit der betrachteten Naht besonders wichtig.

Die im Materialprüfzeugnis angegebene Zugfestigkeit wird an Zugproben bestimmt, die aus einer Stumpfnaht entnommen werden. Die Festigkeit von Kehlnähten kann aufgrund von einer anderen Geometrie, anderen Abkühlbedingungen und anderen Schweißparametern davon abweichen. Die Zugfestigkeit von einlagigen Kehlnähten kann über Härtemessungen abgeschätzt werden, wie in Kapitel 2.6 beschrieben. Eine direkte Bestimmung der Zugfestigkeit an einachsigen Zugproben ist nicht möglich, da aus den einlagigen Kehlnähten keine Zugproben gefertigt werden können.

Es wurden Versuchskörper mit Stumpfnähten (Serie SN) und mehrlagigen Kehlnähten (Serie KN) vollmechanisiert geschweißt, aus denen jeweils ein Schliff für Härtemessungen sowie Rundzugproben (Prüfdurchmesser $\mathrm{d}=4 \mathrm{~mm}$, Außendurchmesser $\mathrm{D}=10 \mathrm{~mm}$ ) ausgearbeitet und geprüft wurden. Die Härtemessungen wurden waagerecht und senkrecht durch die Naht gelegt, um den Bereich, in dem die Probe entnommen wurde, zu erfassen. Abbildung 8.35 zeigt schematisch die Lage der Härtereihen und der Zugproben mit Prüfdurchmesser und Außendurchmesser.

Die Härtemessungen wurden miteinander und in Relation zu den ermittelten Zugfestigkeiten verglichen. Die Einordnung zu den Härtemessungen an den einlagigen (Serie 1, 5, 7) und mehrlagigen Versuchen (Serie KN und Serie 4) wird für die numerischen Berechnungen benötigt und somit in Kapitel 9 vorgestellt.
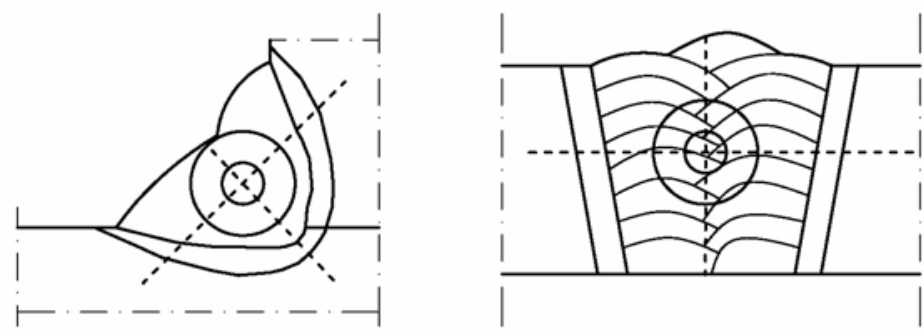

Abbildung 8.35: Entnahme der Zugproben und Härtemessungen

Die geprüften Werkstoffkombinationen der mehrlagigen Kehlnähte und das Prüfergebnis sind in Tabelle 8.15 zusammengestellt. Die beiden Versuche liefern jeweils sehr ähnliche Ergebnisse. Die Schweißparameter der mehrlagigen Kehlnähte entsprachen denen der Versuchskörper. Die Versuche aus Stumpfnähten (Serie SN) wurden normgerecht geschweißt, um die Zugfestigkeiten der Zeugnisse zu bestätigen. Die Ergebnisse der Zugversuche sind im Vergleich mit den Zeugniswerten nach Tabelle 8.4 und Tabelle 8.5 in der folgenden Tabelle 8.16 dargestellt. Die Zeugniswerte werden um bis zu $5 \%$ unterschritten, erfüllen jedoch die Anforderung der Norm.

In Tabelle 8.16 werden die Zugfestigkeiten der Stumpfnähte mit denen der mehrlagigen Kehlnähte der zugehörigen Grund- und Schweißzusatzwerkstoffe verglichen. Für die Verbindung S460-G46 kann eine deutliche Steigerung der Festigkeit um 12 - 14 \% zum reinen Schweißgut G46 festgestellt werden. Für die Werkstoffkombination S700A-G69A zeigt sich eine um $5 \%$ höhere Zugfestigkeit als beim reinen Schweißgut G69A. Für die Verbindung S690B-G69B ist die Zugfestigkeit um 12 - 15 \% höher als beim reinen Schweißgut G69B. 
Tabelle 8.15: Ermittelte mechanische Eigenschaften der Schweißverbindungen (Serie KN)

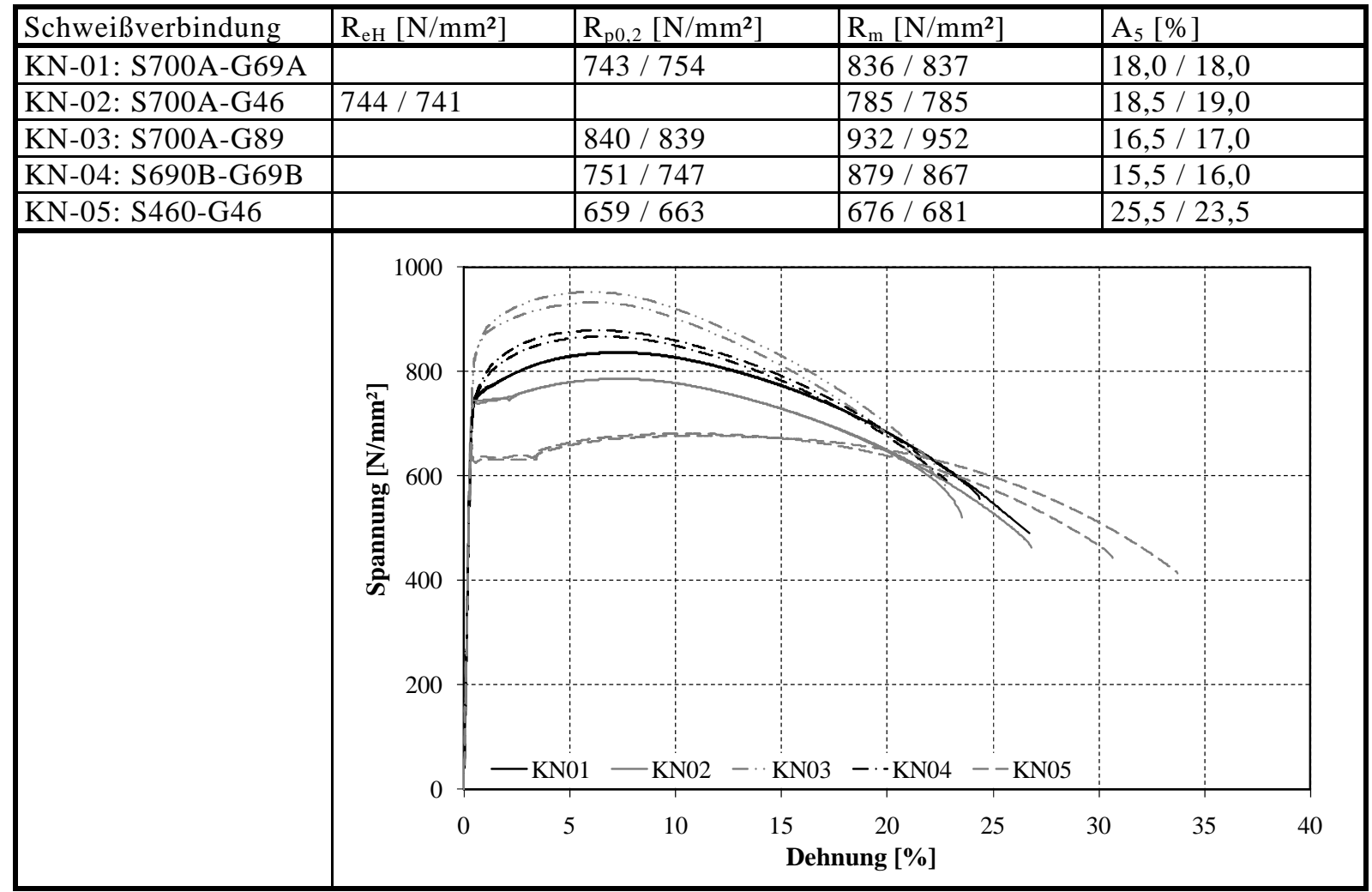

Tabelle 8.16: Vergleich der Zugfestigkeiten aus Stumpf- und Kehlnähten zugehöriger Grund- und Schweißzusatzwerkstoffe

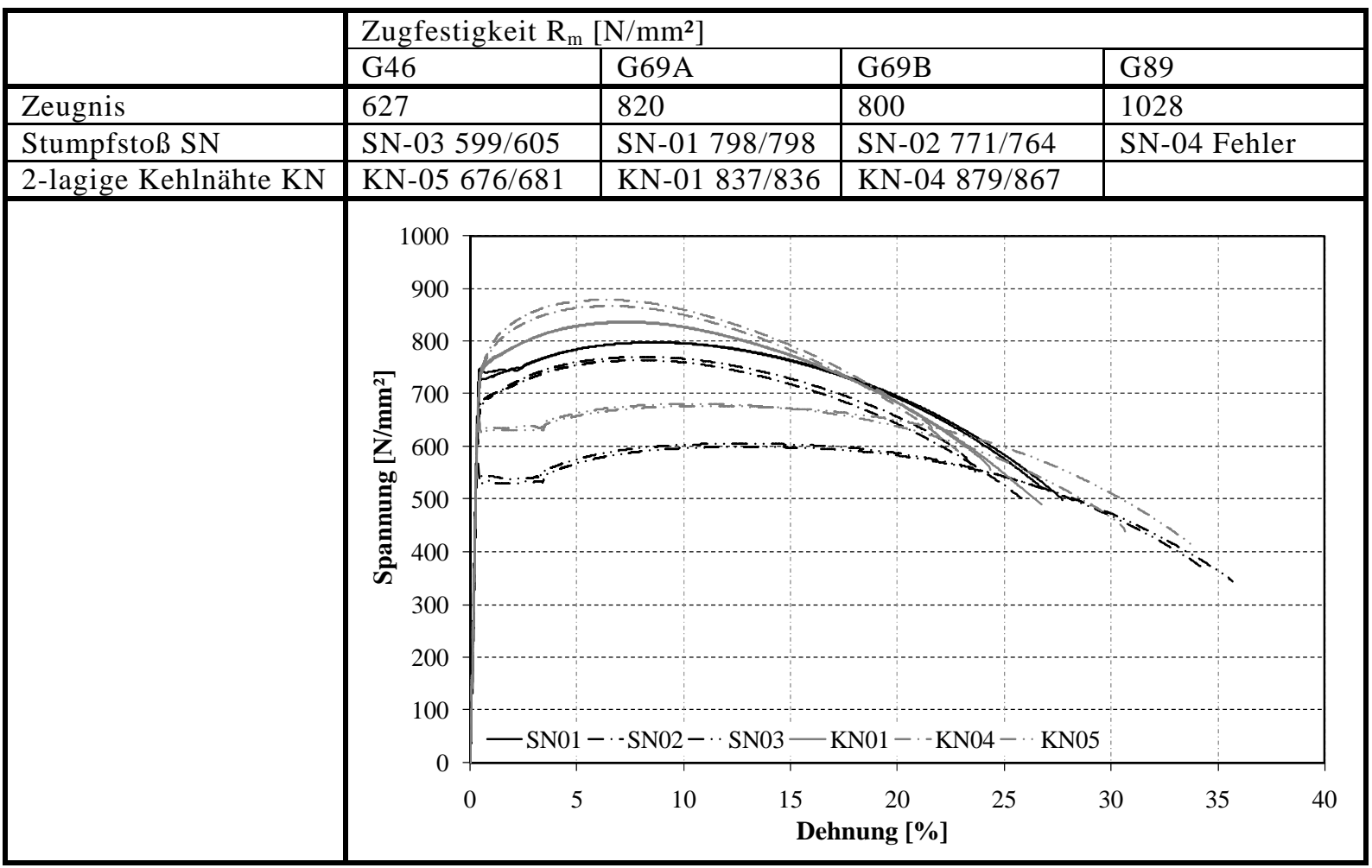

Es zeigt sich, dass sich für die Werkstoffkombination S700A-G69A an der mehrlagigen Kehlnaht KN01 um 5 \% höher Zugfestigkeiten ergeben als im reinen Schweißgut G69A. Für diese Werkstoffkombination konnte bei den mehrlagigen Kehlnähten eine ähnliche Zugfestigkeit festgestellt werden wie bei den Stumpfnähten. Die Einflussfaktoren der Abkühlbedingungen und der Aufmischung zwischen 
Grund- und Schweißzusatzwerkstoffen beeinflussen jeweils die Festigkeit so, dass beide Einflussfaktoren zusammen zu der gleichen Zugfestigkeit führen.

Für die Werkstoffkombination S690B-G69B ergeben sich in Schweißgut der mehrlagigen Kehlnaht KN-04 um 14 \% höhere Werte der Zugfestigkeit als im reinen Schweißgut G69B. Für diese Werkstoffkombination zeigt die höhere Festigkeit der Kehlnaht im Vergleich zur Stumpfnaht, dass die extremeren Abkühlbedingungen und der Einfluss des Grundwerkstoffs die Zugfestigkeit beeinflussen.

Für die Beurteilung der Schweißverbindungen mit dem Schweißzusatzwerkstoff G89 wurden Stumpfnahtverbindungen G89 und mehrlagige Kehlnähte der Werkstoffkombination S700A-G89 gefertigt. Eigene Werte für die Zugfestigkeit G89 fehlen, der Vergleich mit den Werten des Herstellers zeigt, dass die Werte des reinen Schweißgutes um 9 \% höher liegen. Der Vergleich der Zugfestigkeiten von der Stumpfnaht G89 SN-04 und der Kehlnaht KN-03 zeigt eine höhere Zugfestigkeit für die Stumpfnaht. Durch den Einfluss des Grundwerkstoffs, der im Vergleich zum Schweißzusatzwerkstoff eine geringere Zugfestigkeit hat, entsteht eine Kehlnaht deren Festigkeit geringer ist als die des eingesetzten Schweißzusatzwerkstoffs.

Für die Werkstoffkombination S460-G46 ergibt sich im Schweißgut der mehrlagigen Kehlnaht KN-05 eine um ca. 13 \% höhere Zugfestigkeit als im reinen Schweißgut G46 SN-03. Hier zeigt sich bei gleichem Grund- und Schweißzusatzwerkstoff der Einfluss der extremeren Abkühlbedingungen bei der Kehlnaht im Vergleich zur Stumpfnaht. Für das Schweißgut G46 ergibt der Vergleich mit der Werkstoffkombination S700A-G46 in der mehrlagigen Kehlnaht KN-02 um 20 \% höhere Zugfestigkeiten als im reinen Schweißgut G46. Vergleicht man die Zugfestigkeit der Kehlnahtverbindungen KN-01 und KN-05 mit gleichem Schweißzusatzwerkstoff G46 und unterschiedlichen Grundwerkstoffen S700A und S460, so zeigt sich ein deutlicher Einfluss des Grundwerkstoffs auf die Zugfestigkeit der Kehlnaht. Bei gleichem Schweißzusatzwerkstoff unterscheiden sich die Zugfestigkeiten um 16 \%.

\subsubsection{Vergleich von Härtemessungen und Zugfestigkeiten}

\section{Ergebnisse}

Aus den Versuchskörpern mit Stumpfnähten (Serie SN) und den Versuchskörpern mit mehrlagigen Kehlnähten (Serie KN) wurden neben den Zugproben zusätzlich Proben für Makroschliffe und Härtemessungen ausgearbeitet. Die Ergebnisse der Härtemessungen werden vorgestellt und mit den Ergebnissen der Zugversuche verglichen. Abbildung 8.36 zeigt die Härtemessungen am reinen Schweißgut für die Schweißzusatzwerkstoffe G46, G69A, G69B und G89.

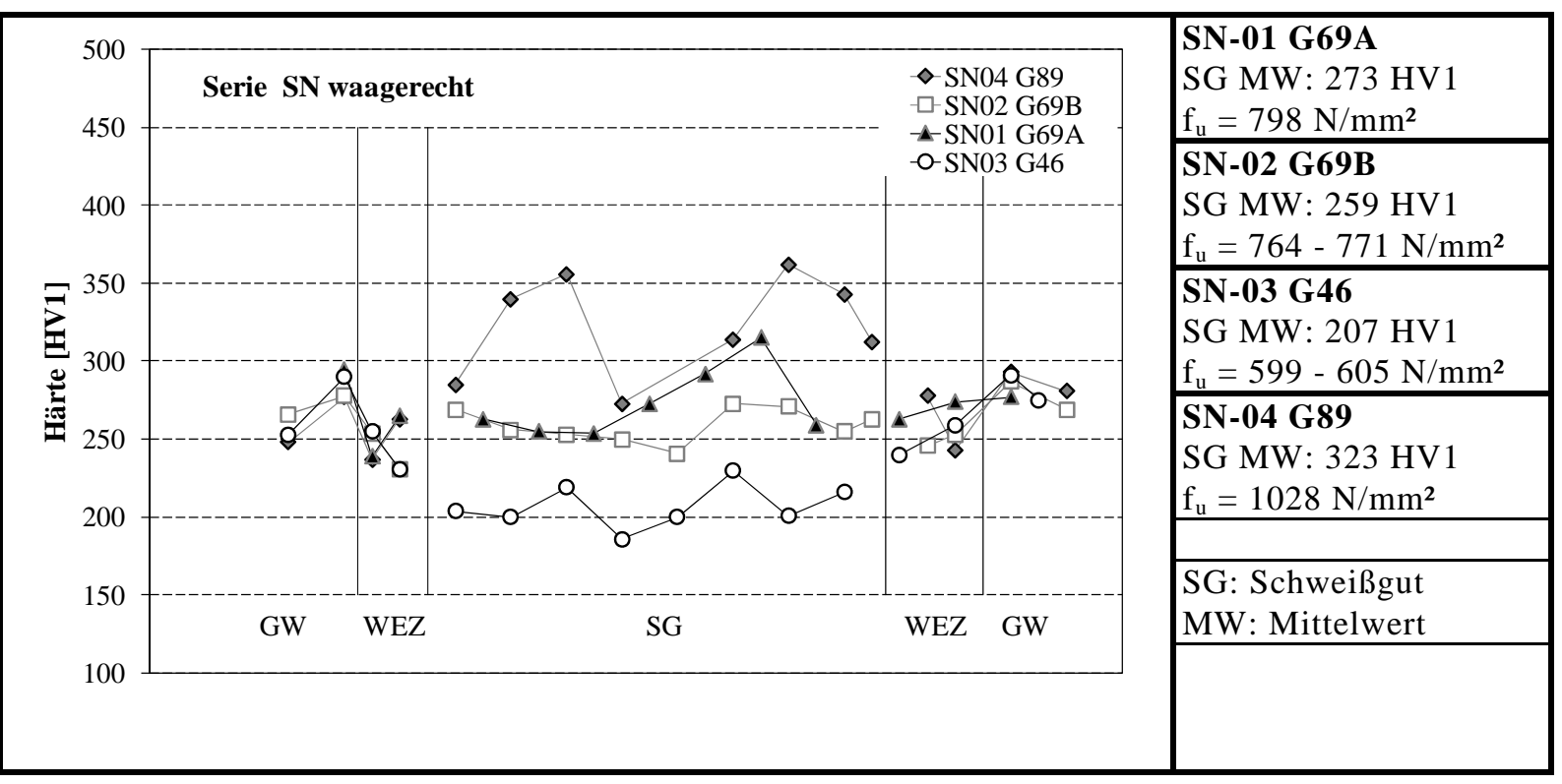

Abbildung 8.36: Vergleich der Härtewerte des reinen Schweißgutes (SN)

Die Härtewerte des Schweißguts G69A liegen um etwa 3-5 \% höher als die des Schweißguts G69B. Das entspricht dem Unterschied der Zugfestigkeiten der beiden Schweißzusatzwerkstoffe. Im Ver- 
gleich zum Schweißzusatzwerkstoff G69A wurden für den höherfesten Schweißzusatzwerkstoff G89 um $18 \%$ höher liegende Härtewerte als die Härtewerte von G69A festgestellt. Die Werte des Schweißzusatzwerkstoffs G89 streuen jedoch stark. Die zugehörige Zugfestigkeit liegt um 29 \% höher. Ein Vergleich der Schweißzusatzwerkstoffe G69A und G46 zeigt, dass die Härtewerte bei dem niederfesten Schweißzusatzwerkstoff G46 um 35 \% geringer sind als die Werte beim Schweißzusatzwerkstoff G69A, während der Abfall der Zugfestigkeit bei 32 \% liegt. Die Härtewerte und die Zugfestigkeit korrelieren bei den Stumpfnähten der Serie SN.

In Abbildung 8.37 werden die Härtewerte der Kehlnahtverbindungen mit den Grundwerkstoff S700A und verschiedenen Schweißzusatzwerkstoffen G46, G69A und G89 verglichen. Durch die Über- und Unterfestigkeit ergeben sich Unterschiede in der Härte von $+20 \%$ und -12 \%. Die zugehörigen Unterschiede in der Zugfestigkeit liegen bei $+12 \%$ und $-7 \%$.

Der Schwerpunkt der Traglastversuche mit zugehörigen Grund- und Schweißzusatzwerkstoffen lag auf der Untersuchung der Werkstoffkombinationen S700A-G69A, S690B-G69B und S460-G46. Diese wurden auch als Zugversuche und Härtemessungen aus mehrlagigen Kehlnähten analysiert, wie in Abbildung 8.38 dargestellt.

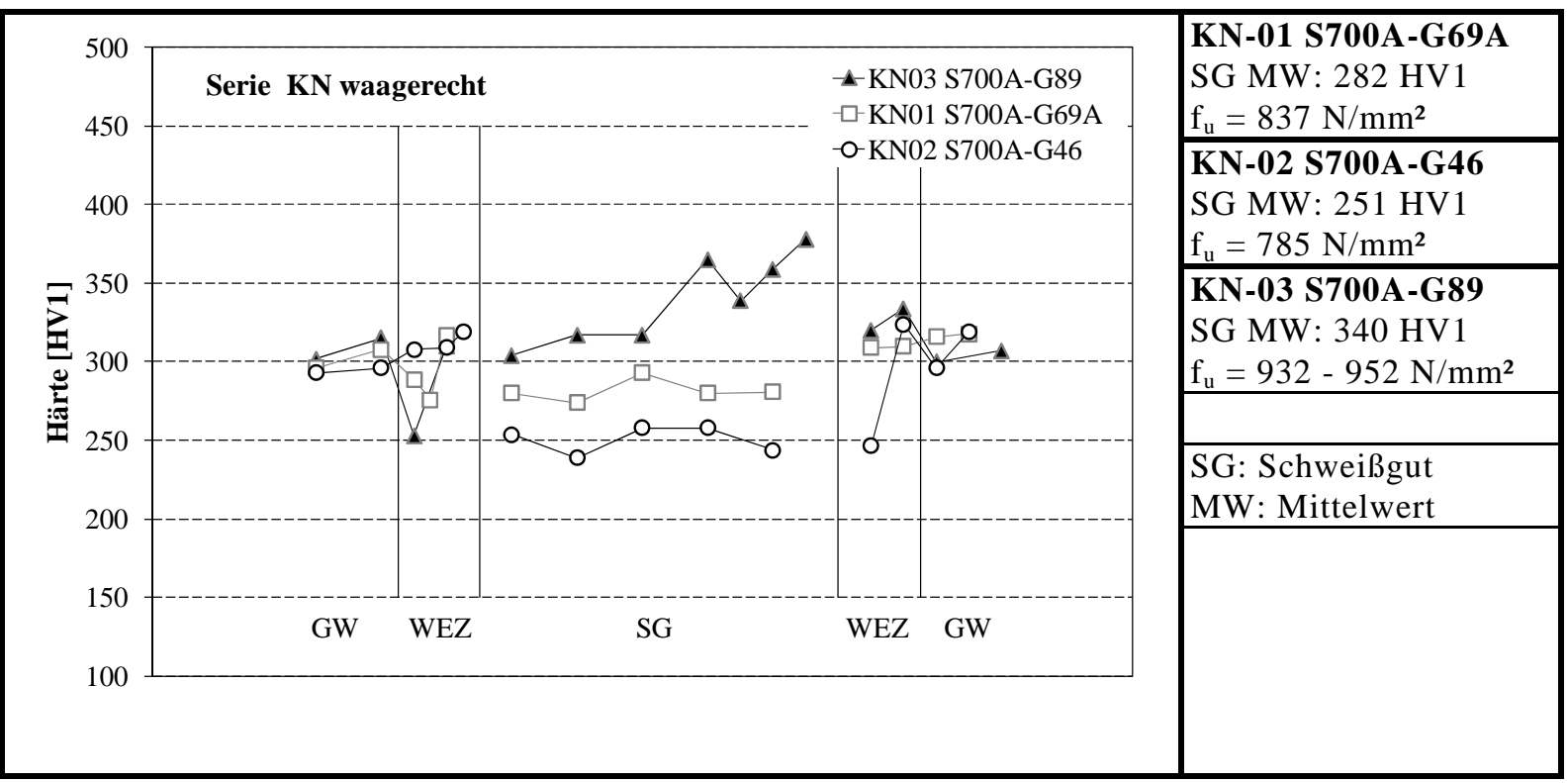

Abbildung 8.37: Vergleich der Härtewerte unterschiedlicher Schweißzusatzwerkstoff-Kombinationen

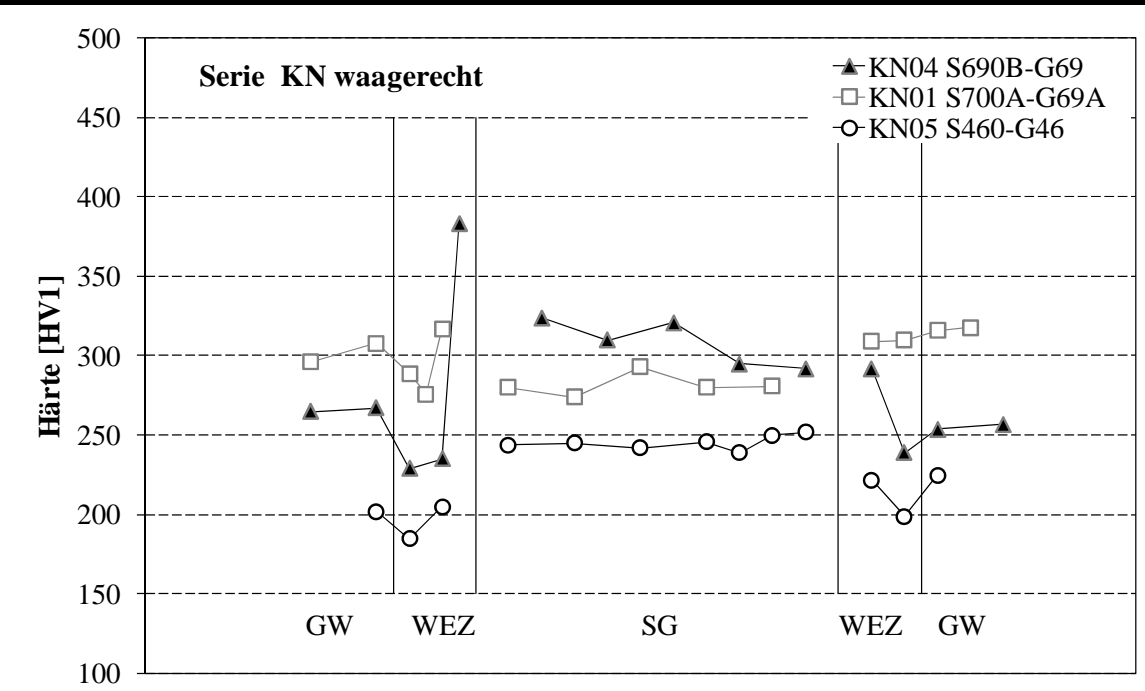

KN-01 S700A-G69A

SG MW: 282 HV1

$\mathrm{f}_{\mathrm{u}}=837 \mathrm{~N} / \mathrm{mm}^{2}$

KN-04 S690B-G69B

SG MW: 308 HV1

$\mathrm{f}_{\mathrm{u}}=867-879 \mathrm{~N} / \mathrm{mm}^{2}$

KN-05 S460-G46

SG MW: 245 HV1

$\mathrm{f}_{\mathrm{u}}=676-681 \mathrm{~N} / \mathrm{mm}^{2}$

SG: Schweißgut

MW: Mittelwert 
Die Härtewerte der Verbindung S460-G46 liegen deutlich niedriger als für die Verbindungen S690G69. Die Unterschiede der Härtewerte zwischen S460-G46 und S690A-G69A liegen bei 12 \%, während sich die Zugfestigkeiten um 22 - $24 \%$ unterscheiden. Im Vergleich von S460-G46 und G690BG69B zeigen sich Unterschiede in der Härte von $31 \%$ und in der Zugfestigkeit von 27 - 30 \%. Der Vergleich der beiden Werkstoffkombinationen S690-G69A und B zeigt einen Unterschied in der Härte von 9 \% und in den Zugfestigkeiten von 4 - 5 \%. Insgesamt lässt sich auch für die mehrlagigen Kehlnähte der Serie KN sagen, dass die Härtewerte und die Zugfestigkeit korrelieren.

Im Folgenden werden die in Abbildung 8.36 bis Abbildung 8.38 dargestellten Ergebnisse verglichen und interpretiert.

Für den Vergleich der Ergebnisse an Stumpfnähten für das reine Schweißgut und an mehrlagigen Kehlnähten stehen die Verbindungen S700A-G69A und S690B-G69B zur Verfügung. Es zeigt sich, dass sich für die Werkstoffkombination S700A-G69A an der mehrlagigen Kehlnaht KN-01 ähnliche Härtewerte (282 HV1) ergeben wie im reinen Schweißgut G69A (HV1 273). Im Mittel liegen die Werte der Kehlnaht um 3 \% höher, die Werte der Zugfestigkeit liegen um $5 \%$ höher. Für diese Werkstoffkombination konnte bei den mehrlagigen Kehlnähten eine ähnliche Zugfestigkeit festgestellt werden wie bei den Stumpfnähten. Für die Werkstoffkombination S690B-G69B ergeben sich in Schweißgut der mehrlagigen Kehlnaht KN-04 mit 308 HV1 höhere Härtewerte als im reinen Schweißgut G69B mit 259 HV1. Im Mittel liegen die Härtewerte der Kehlnaht um ca. 19 \% höher, die Werte der Zugfestigkeit liegen um 14 \% höher.

Für die Beurteilung der Schweißverbindungen mit dem Schweißzusatzwerkstoff G89 wurden Stumpfnahtverbindungen G89 und mehrlagige Kehlnähte der Werkstoffkombination S700A-G89 gefertigt. Für das Schweißgut G89 zeigen sich jeweils stark streuende Härtewerte, im Mittel liegen die Werte der Kehlnaht um 5 \% höher. Eigene Werte für die Zugfestigkeit G89 fehlen. Der Vergleich mit den Werten des Herstellers zeigt, dass die Werte des reinen Schweißgutes um 9 \% höher liegen.

Für die Werkstoffkombination S460-G46 ergeben sich im Schweißgut der mehrlagigen Kehlnaht KN05 mit 245 HV1 höhere Härtewerte als im reinen Schweißgut G46 SN-03 mit 207 HV1. Im Mittel liegen die Härtewerte der Kehlnaht um ca. 18 \% höher, die Werte der Zugfestigkeit liegen um ca. 13 \% höher. Für das Schweißgut G46 ergibt der Vergleich mit der Werkstoffkombination S700A-G46 in der mehrlagigen Kehlnaht höhere Härtewerte (251 HV1) als im reinen Schweißgut G46 (207 HV1). Im Mittel liegen die Werte der Kehlnaht um ca. 20 \% höher, die Werte der Zugfestigkeit liegen um ca. 30 \% höher.

\section{Fazit}

Ein Vergleich zwischen den Festigkeitsunterschieden und den Unterschieden in der Härte zeigt Abbildung 8.39. Es zeigt sich, dass die Zugfestigkeiten und Härtewerte korrelieren. Die Ausgleichsgerade hat eine Steigung von 3,2, wie in Kapitel 2.6 beschrieben. Einen Überblick über die ermittelten Härtewerte und Zugfestigkeiten gibt Tabelle 8.17.
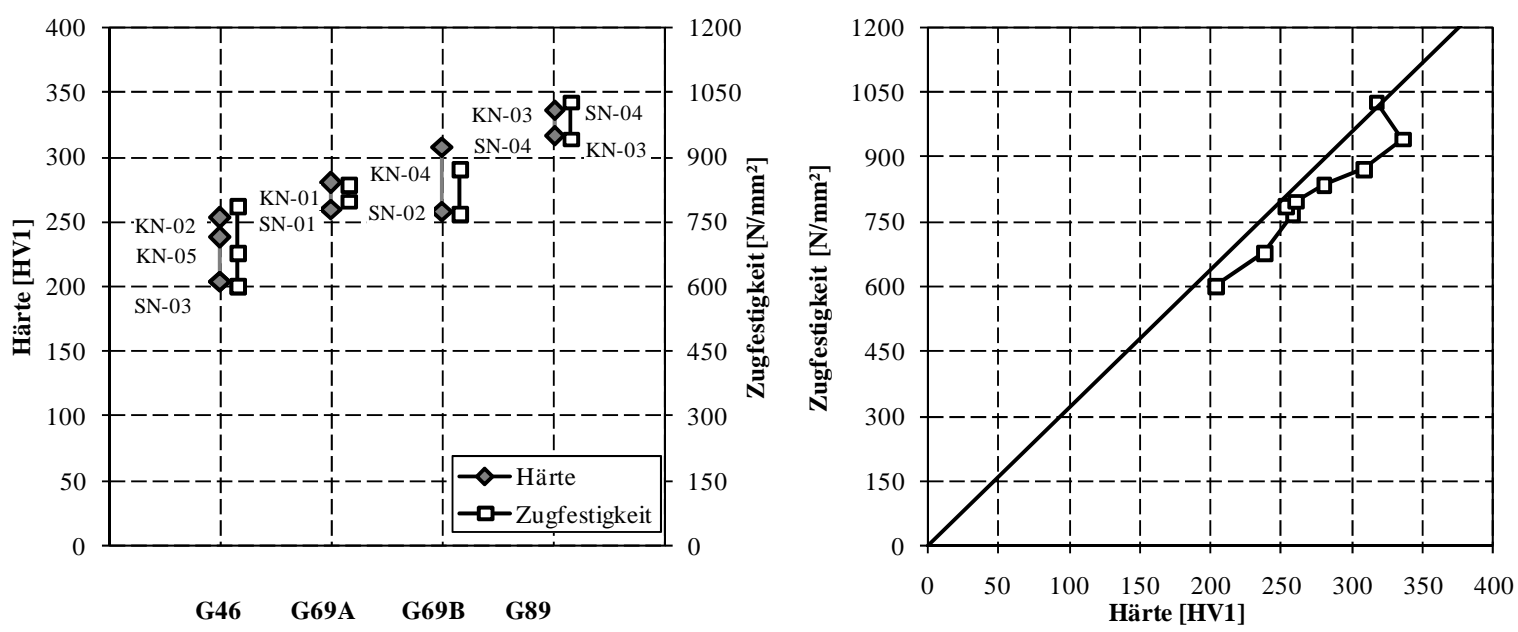

Abbildung 8.39: Vergleich von Härte und Zugfestigkeit der Serie KN und SN 
Tabelle 8.17: Vergleich der Zugfestigkeiten und Härtemessungen aus Stumpf- und Kehlnähten

\begin{tabular}{|c|c|c|}
\hline SN-01 G69A & KN-01 S700A-G69A & \\
\hline \begin{tabular}{|l|l|l} 
SG Mittelwert:273 HV1 & $\mathrm{f}_{\mathrm{u}}=798 \mathrm{~N} / \mathrm{mm}^{2}$ \\
\end{tabular} & SG Mittelwert:282 HV1 & $\mathrm{f}_{\mathrm{u}}=837 \mathrm{~N} / \mathrm{mm}^{2}$ \\
\hline SN-02 G69B & KN-04 S690B-G69B & \\
\hline \begin{tabular}{|l|l|l} 
SG Mittelwert:259 HV1 & $\mathrm{f}_{\mathrm{u}}=764-771 \mathrm{~N} / \mathrm{mm}^{2}$ \\
\end{tabular} & SG Mittelwert:308 HV1 & $\mathrm{f}_{\mathrm{u}}=867-879 \mathrm{~N} / \mathrm{mm}^{2}$ \\
\hline SN-03 G46 & KN-05 S460-G46 & \\
\hline \begin{tabular}{|l|l} 
SG Mittelwert:207 HV1 & $\mathrm{f}_{\mathrm{u}}=599-605 \mathrm{~N} / \mathrm{mm}^{2}$ \\
\end{tabular} & SG Mittelwert:245 HV1 & $f_{u}=676-681 \mathrm{~N} / \mathrm{mm}^{2}$ \\
\hline SN-04 G89 & KN-02 S700A-G46 & \\
\hline \begin{tabular}{|l|l|l} 
SG Mittelwert:323 HV1 & $\mathrm{f}_{\mathrm{u}}=1028 \mathrm{~N} / \mathrm{mm}^{2}$ \\
\end{tabular} & SG Mittelwert:251 HV1 & $\mathrm{f}_{\mathrm{u}}=785 \mathrm{~N} / \mathrm{mm}^{2}$ \\
\hline & KN-03 S700A-G89 & \\
\hline & SG Mittelwert:340 HV1 & $f_{u}=932-952 \mathrm{~N} / \mathrm{mm}^{2}$ \\
\hline
\end{tabular}

Die Werkstoffuntersuchungen haben gezeigt, dass sich die Zugfestigkeit vom reinen Schweißgut von der Zugfestigkeit der mehrlagigen Kehlnähte unterscheidet. Für die Verbindungen zugehöriger Grundund Schweißzusatzwerkstoffe hat der Vergleich von Härte und Zugfestigkeiten der Stumpfnähte (SN01, SN-02 und SN-03) und der Kehlnähte (KN-01, KN-04, KN-05) gezeigt, dass die Festigkeit der Kehlnaht in allen Fällen höher ist als die Festigkeit des Schweißzusatzwerkstoffs. Der Grund dafür liegt in den extremeren Abkühlbedingungen und die fehlenden Vergütungseffekte der Kehlnaht im Vergleich zur Stumpfnaht. Für die Schweißverbindungen S460-G46 ergeben sich bei den mehrlagigen Kehlnähten deutliche Überfestigkeiten im Vergleich mit Werten des reinen Schweißgutes im Bereich von 13 \%. Für die Schweißverbindungen S700A-G69A ergeben sich ähnliche Festigkeiten mit Unterschieden von $5 \%$. Die Verbindung S690B-G69B zeigt eine Zunahme der Festigkeit von reinem Schweißgut zur mehrlagigen Kehlnaht von $14 \%$. Daraus ergibt sich, dass die Unterschiede in den Festigkeiten der Kehlnähte kleiner sind als die Unterschiede in den Festigkeiten der Stumpfnähte. Der Vergleich der Festigkeitswerte G69A und G69B zeigt für die Stumpfnaht ähnliche Zugfestigkeiten, während für die mehrlagigen Kehlnähte die Festigkeit von G69B deutlich größer ist als für G69A.

Mit Hilfe den untersuchten Mischverbindungen (KN-02, KN-03) konnte ein deutlicher Einfluss des Grundwerkstoffs auf die Festigkeit und Härte der Verbindung nachgewiesen werden.

Die Ergebnisse der Zugversuche werden für die in Kapitel 9 beschriebenen numerischen Untersuchungen als Werkstoffkennwerte verwendet. Das gilt sowohl für die Stumpfnähte SN als auch für die Kehlnähte KN. Für Verbindungen bei denen keine Zugfestigkeiten vorliegen, werden mithilfe der Härtewerte Abschätzungen getroffen. 


\subsubsection{Vergleich der Werkstoff- und Traglastuntersuchungen}

Abbildung 8.40 wiederholt das Diagramm aus Abbildung 8.23, in dem die Tragfähigkeit in Abhängigkeit des reinen Schweißgutes dargestellt ist. Im Vergleich dazu ist im unteren Diagramm in Abbildung 8.40 die Tragfähigkeit der Flankenkehlnahtverbindungen in Abhängigkeit der Zugfestigkeit der mehrlagigen Kehlnähte (Serie KN) dargestellt. Es zeigt sich eine deutlich bessere Korrelation zwischen der Zugfestigkeit des Schweißguts (KN) und der Tragfähigkeit der einlagigen Verbindung. Dabei ist jedoch zu beachten, dass für die Mischverbindungen S690B-G46 und S690B-G89 die Werte der mehrlagigen Kehlnaht dieser Schweißzusatzwerkstoffe mit dem Stahl S700A verwendet wurden. Außerdem wird deutlich, dass sich die Zugfestigkeiten der mehrlagigen Kehlnähte zwischen G46 und G89 weniger unterscheiden als beim reinen Schweißgut. Aus diesem Umstand lassen sich auch die geringeren Unterschiede in der Tragfähigkeit erklären.

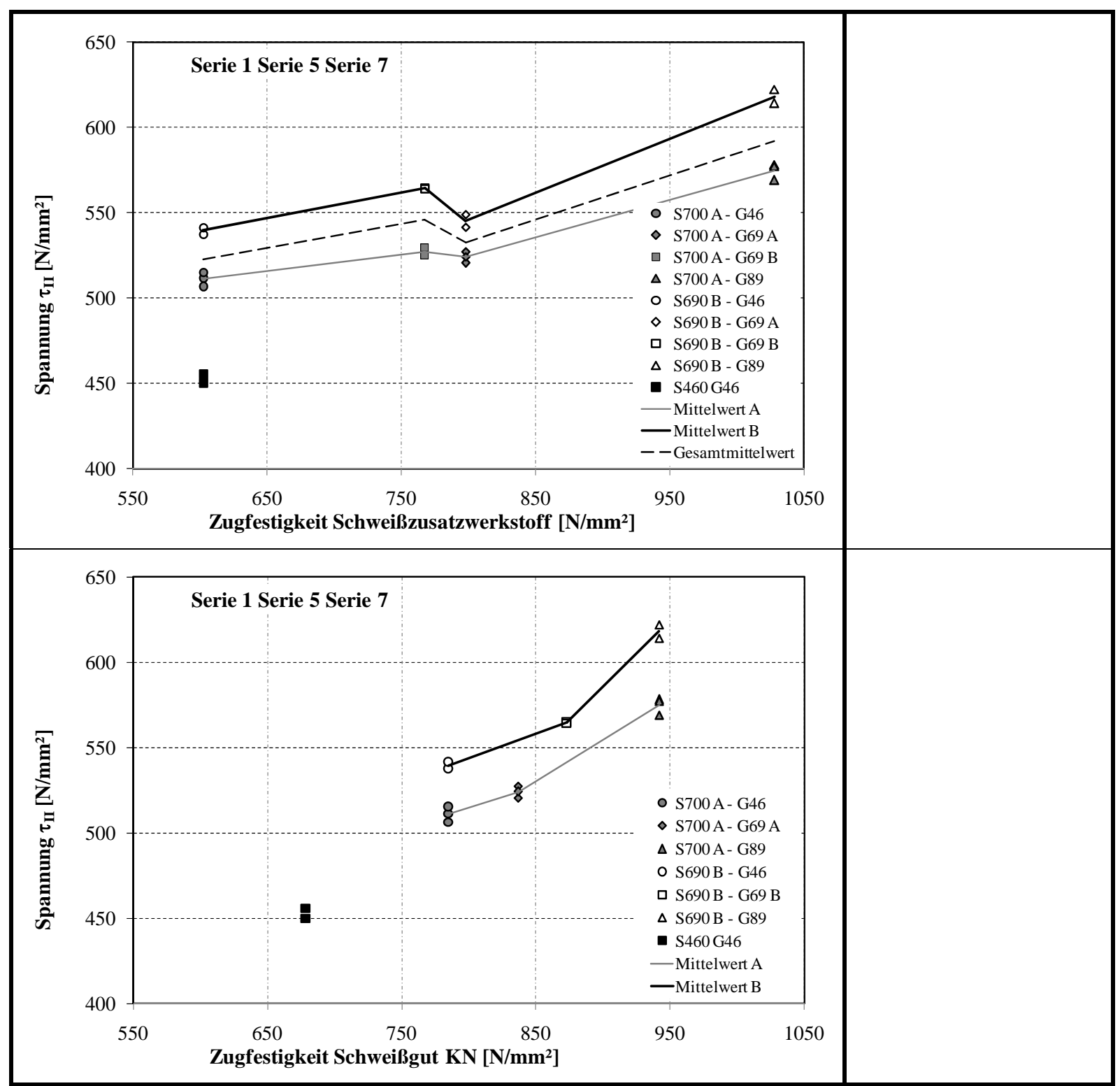

Abbildung 8.40: Ergebnisse der Flankenkehlnahtverbindungen S690 bezogen aus SN oder KN 


\subsection{Zusammenfassung}

In diesem Kapitel wurden die im Rahmen des FOSTA/AiF-Forschungsprojekts „Tragfähigkeit von Kehlnahtverbindungen höherfester Baustähle S690 im Stahlbau“ [FOSTA P812, 2011] durchgeführten experimentellen Untersuchungen beschrieben und analysiert. Im Einzelnen wurden Tragfähigkeitsuntersuchungen an Flanken- und Stirnkehlnähten mit den Grundwerkstoffen S690 und unterschiedlichen Schweißzusatzwerkstoffen, Flankenkehlnähte S460 sowie Werkstoffuntersuchungen vorgestellt.

Für Flankenkehlnahtverbindungen ergaben sich durch die Art der Versuchskörper sehr geringe Streuungen, die zu eindeutigen Ergebnissen führten. Die Versuchsergebnisse zeigen eine höhere Tragfähigkeit bei Schweißverbindungen mit dem Stahl S690 im Vergleich mit Verbindungen mit dem Stahl S460. Im Arbeitsfenster für die Schweißparameter ergibt sich für Schweißverbindungen S690 eine Abnahme der Tragfähigkeit von maximal $7 \%$. Der Einfluss der Nahtlänge auf die Tragfähigkeit ist gering. Bei den mehrlagigen Nähten ist eine Abnahme der Tragfähigkeit von bis zu $15 \%$ zu beobachten. Es konnte ein Einfluss der Festigkeit des Schweißzusatzwerkstoffs auf die Tragfähigkeit der Schweißverbindung höherfester Stähle S690 festgestellt werden. Bei Verwendung von niederfesten Schweißzusatzwerkstoffen wurde die Tragfähigkeit nur wenig geringer, durch Verwendung von höherfesten Schweißzusatzwerkstoffen konnte eine Steigerung der Tragfähigkeit festgestellt werden. Tabelle 8.18 fasst die Ergebnisse zusammen.

Tabelle 8.18: Zusammenfassung der Versuchsergebnisse von Flankenkehlnahtverbindungen

\begin{tabular}{|l|l|}
\hline Parameter & Einfluss \\
\hline $\begin{array}{l}\text { Stahlsorte und Schweiß- } \\
\text { zusatzwerkstoff }\end{array}$ & $\begin{array}{l}\text { S690-G69: bis zu 18 \% höhere Tragfähigkeit als S460-G46 bei gleichen } \\
\text { Bedingungen }\end{array}$ \\
\hline Schweißparameter & $\begin{array}{l}\text { S690-G69: Geringe Abnahme der Tragfähigkeit von ca. 7 \% im Anwen- } \\
\text { dungsbereich von DIN EN 1011-2 (2001) und [SEW 088, 1993] }\end{array}$ \\
\hline Nahtlänge & Geringe Abnahme (4 \%) der Tragfähigkeit bis L/a = 150 \\
\hline Naht-/ und Blechdicke & Abnahme der Tragfähigkeit um bis zu 15 \% \\
\hline $\begin{array}{l}\text { Schweißzusatzwerkstoff } \\
\text { (SZW) }\end{array}$ & $\begin{array}{l}\text { Steigerung der Tragfähigkeit bei zunehmender Festigkeit des SZW, } \\
\text { nur geringe Abnahme der Tragfähigkeit bei abnehmender Festigkeit }\end{array}$ \\
\hline Schweißprozess & Kaum Einfluss auf die Tragfähigkeit \\
\hline
\end{tabular}

Für die Kreuzstöße wurde die Festigkeit des Schweißzusatzwerkstoffs variiert. Trotz der vollmechanisierten Schweißung ist die Streuung bei gleichen Versuchskörpern größer als bei den Flankenkehlnahtverbindungen. Die Einordnung der Versuchskörper der Kreuzstöße in Bewertungsgruppen hat ergeben, dass die Verbindungen mit den Stählen S690B nur Bewertungsgruppe D erreichen. Es wird vermutet, dass diese Unregelmäßigkeiten die Tragfähigkeit beeinflussen. Die Versuchsergebnisse mit dem Grundwerkstoff S690B werden nicht weiter betrachtet.

Die Untersuchung des Einflusses der Festigkeit des Schweißzusatzwerkstoffs für Kreuzstöße mit dem Stahl S700A zeigt eine ansteigende Tragfähigkeit mit zunehmender Festigkeit des Schweißzusatzwerkstoffs. Die Härtemessungen ergeben ähnliche Tendenzen wie die Tragfähigkeitsuntersuchungen.

Die Testversuche an Kreuzstößen mit schrägen Schweißnähten haben eindeutig gezeigt, dass die Kraftrichtung einen Einfluss auf die Tragfähigkeit hat.

Die durchgeführten Werkstoffuntersuchungen haben gezeigt, dass die Festigkeit des reinen Schweißgutes sich deutlich von der Zugfestigkeit der mehrlagigen Kehlnaht unterscheidet. Insbesondere für den Schweißzusatzwerkstoff G46 wurde für die mehrlagige Kehlnaht eine Steigerung von 12 - 14 \% festgestellt. Mit Hilfe dieser Ergebnisse konnte eine gute Korrelation zwischen den Tragfähigkeiten der Kehlnahtverbindungen und den Zugfestigkeiten der Schweißzusatzwerkstoffe festgestellt werden. 


\section{Analyse des Tragverhaltens anhand numerischer Untersu- chungen}

\subsection{Einleitung}

Mit Hilfe von experimentellen Untersuchungen wurde die Tragfähigkeit von Schweißverbindungen bestimmt. Die zusätzlichen numerischen Untersuchungen wurden durchgeführt, um die Versuche nachzubilden. Mit den Ergebnissen der numerischen Untersuchungen kann das Tragverhalten der Schweißverbindungen analysiert werden.

\subsection{Beschreibung der numerischen Berechnungen}

\subsubsection{Allgemeines}

Im Folgenden werden das Vorgehen und die Ergebnisse der numerischen Untersuchungen der in Kapitel 8 vorgestellten Versuche, [FOSTA P812, 2011] präsentiert. Die numerischen Berechnungen wurden mit Hilfe der Methode der Finiten Elemente (FEM) durchgeführt. Es wurde das Finite-Elemente Programm Ansys 13.0 ${ }^{\circledR}$ [Ansys 13.0] verwendet. Für die Modellierung wurde das SOLID45-Element, ein achtknotiges Volumenelement mit drei Freiheitsgraden je Knoten verwendet.

Die Lasteinleitung und die Festhalterung, die im Versuch durch die Pressbacken der Prüfmaschine erfolgten, wurden im numerischen Modell als Auflagerbedingungen und als Randbedingungen der jeweiligen Knoten erfasst. Die Last wurde als Knotenverschiebung aufgebracht. Die Berechnung erfolgte weggesteuert. Die Berechnungen wurden unter Berücksichtigung nichtlinearer Geometrie (nlgeom) durchgeführt.

\subsubsection{Geometriemodellierung}

An Flankenkehlnahtverbindungen wurden insgesamt 54 Traglastversuche durchgeführt. Da jeweils zwei (oder drei) gleiche Versuchskörper gefertigt wurden, ergaben sich 19 verschiedene Werkstoffund Geometriekombinationen, von denen jeweils ein Makroschliff entnommen wurde.

Der Versuchskörper wurde anhand der tatsächlichen Abmessungen modelliert. Für die geometrische Modellierung ist neben den Außenabmessungen des Versuchskörpers die Modellierung der Schweißnaht besonders wichtig. Aus diesem Grund wurde die Nahtgeometrie anhand der Makroschliffe modelliert. Die Umrandung der Schweißnaht und der Wärmeeinflusszone wurde anhand des Makroschliffes multi-linear nachgebildet. Der Makroschliff und die numerische Modellierung sind beispielhaft in Abbildung 9.1 dargestellt.

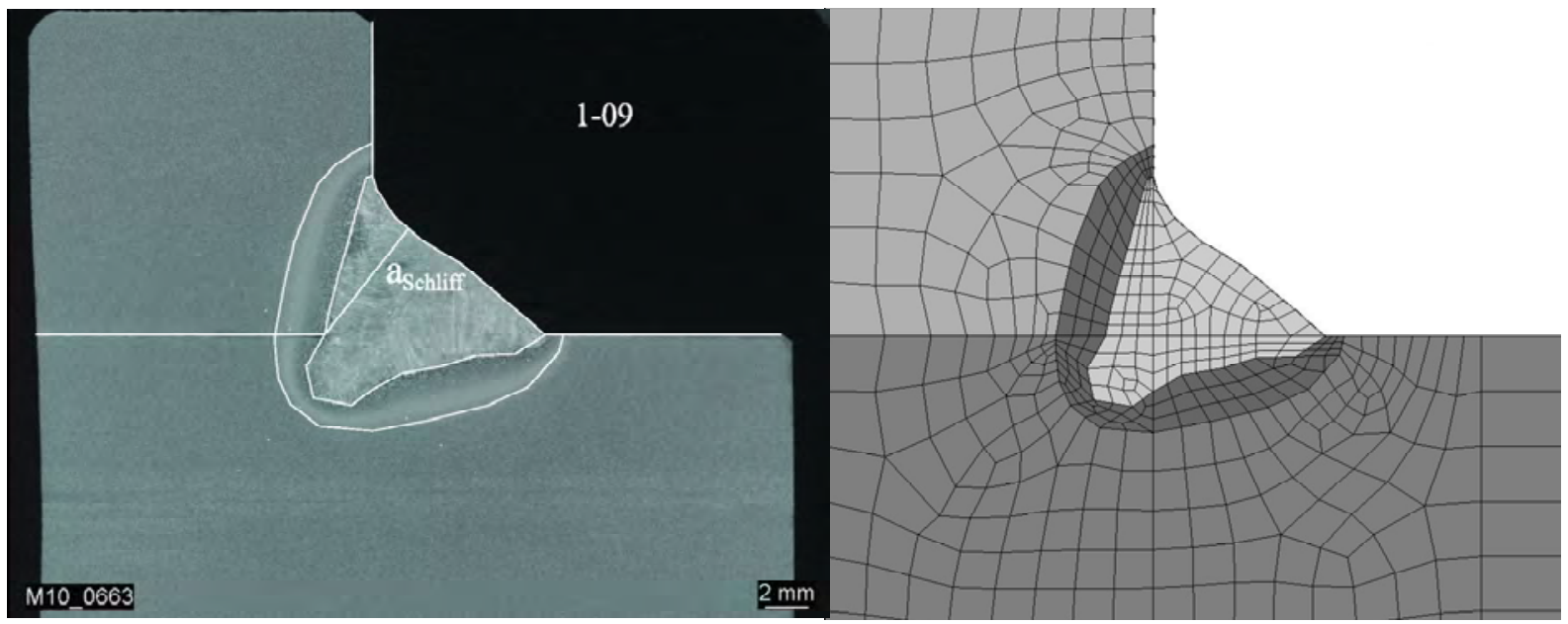

Abbildung 9.1: Modellierung der Makroschliffe in Ansys 13.0 ${ }^{\circledR}$ [Ansys 13.0] 


\subsubsection{Materialmodellierungen}

Die Modellierung des Materials erfolgte über ein multi-lineares isotropes Materialgesetz mit Verfestigung und unter Berücksichtigung der von-Mises-Plastizität (tb, miso). Für den Elastizitäts-Modul wurde der Wert $\mathrm{E}=210.000 \mathrm{~N} / \mathrm{mm}^{2}$ verwendet. Die Querdehnzahl wurde mit $v=0,3$ berücksichtigt. Die für die Modellierung erforderlichen Werkstoffkennwerte des Grundwerkstoffs und des Schweißzusatzwerkstoffs wurden mit eigenen Zugversuchen bestimmt (vgl. Tabelle 8.5 und 8.6). Die Werte der Abnahmeprüfzeugnisse wurden nur verwendet, wenn keine eigenen Werte vorlagen. Als Werkstoffkennwerte des Schweißzusatzwerkstoffs wurden sowohl Berechnungen mit den Werten aus dem reinen Schweißgut (SN, vgl. Tabelle 8.16) als auch mit den Werten aus den mehrlagigen Kehlnähten (KN, vgl. Tabelle 8.15) durchgeführt, wie in Kapitel 8.9 beschrieben. Aus Gründen der Übersichtlichkeit sind die verwendeten Werte für die technische Zugfestigkeit in Tabelle 9.1 zusammengefasst.

Tabelle 9.1: Verwendete Werkstoffkennwerte für Grund- und Schweißzusatzwerkstoffe (KN und SN)

\begin{tabular}{|l|l|l|l|}
\hline \multirow{2}{*}{ Grundwerkstoff } & Technische Spannung $\mathrm{f}_{\mathrm{u}}$ & Wahre Spannung $\mathrm{f}_{\mathrm{u}, \text { wahr }}$ \\
\hline & S690B $\mathrm{t}=15 \mathrm{~mm}$ & $848 \mathrm{~N} / \mathrm{mm}^{2}$ & $899 \mathrm{~N} / \mathrm{mm}^{2}$ \\
\cline { 2 - 4 } & S700A t $=15 \mathrm{~mm}$ & $904 \mathrm{~N} / \mathrm{mm}^{2}$ & $960 \mathrm{~N} / \mathrm{mm}^{2}$ \\
\cline { 2 - 4 } & S460 t $=17,6 \mathrm{~mm}$ & $538 \mathrm{~N} / \mathrm{mm}^{2}$ & $595 \mathrm{~N} / \mathrm{mm}^{2}$ \\
\hline \multirow{3}{*}{ Kchweißzusatzwerkstoff } & G69B & $771 \mathrm{~N} / \mathrm{mm}^{2}$ & $835 \mathrm{~N} / \mathrm{mm}^{2}$ \\
\hline & S690B-G69B KN04 & $879 \mathrm{~N} / \mathrm{mm}^{2}$ & $936 \mathrm{~N} / \mathrm{mm}^{2}$ \\
\cline { 2 - 4 } & S700A-G69A KN01 & $837 \mathrm{~N} / \mathrm{mm}^{2}$ & $898 \mathrm{~N} / \mathrm{mm}^{2}$ \\
\cline { 2 - 4 } & S460-G46 KN05 & $681 \mathrm{~N} / \mathrm{mm}^{2}$ & $750 \mathrm{~N} / \mathrm{mm}^{2}$ \\
\cline { 2 - 4 } & S700A-G46 KN02 & $785 \mathrm{~N} / \mathrm{mm}^{2}$ & $841 \mathrm{~N} / \mathrm{mm}^{2}$ \\
\cline { 2 - 4 } & S700A-G89KN03 & $932 \mathrm{~N} / \mathrm{mm}^{2}$ & $990 \mathrm{~N} / \mathrm{mm}^{2}$ \\
\hline
\end{tabular}

Abbildung 9.2 zeigt exemplarisch für die Werkstoffkennwerte von Versuch 1-09 (S690B-G69B) den Vergleich der ermittelten Kurven aus dem Zugversuch mit den gewählten Punkten für das multilineare Werkstoffgesetz mit technischen Spannungen und Dehnungen. Die Umrechnung von technischen Dehnungen $\varepsilon$ und Spannungen $\sigma$ in wahre Dehnungen $\varepsilon_{\text {wahr }}$ und Spannungen $\sigma_{\text {wahr }}$ erfolgte über folgende Berechnungen (vgl. z. B. [Gross et al., 2007]):

Wahre Dehnung: $\varepsilon_{\text {wahr }}=\ln (1+\varepsilon)$

Wahre Spannung: $\sigma_{\text {wahr }}=\sigma(1+\varepsilon)$

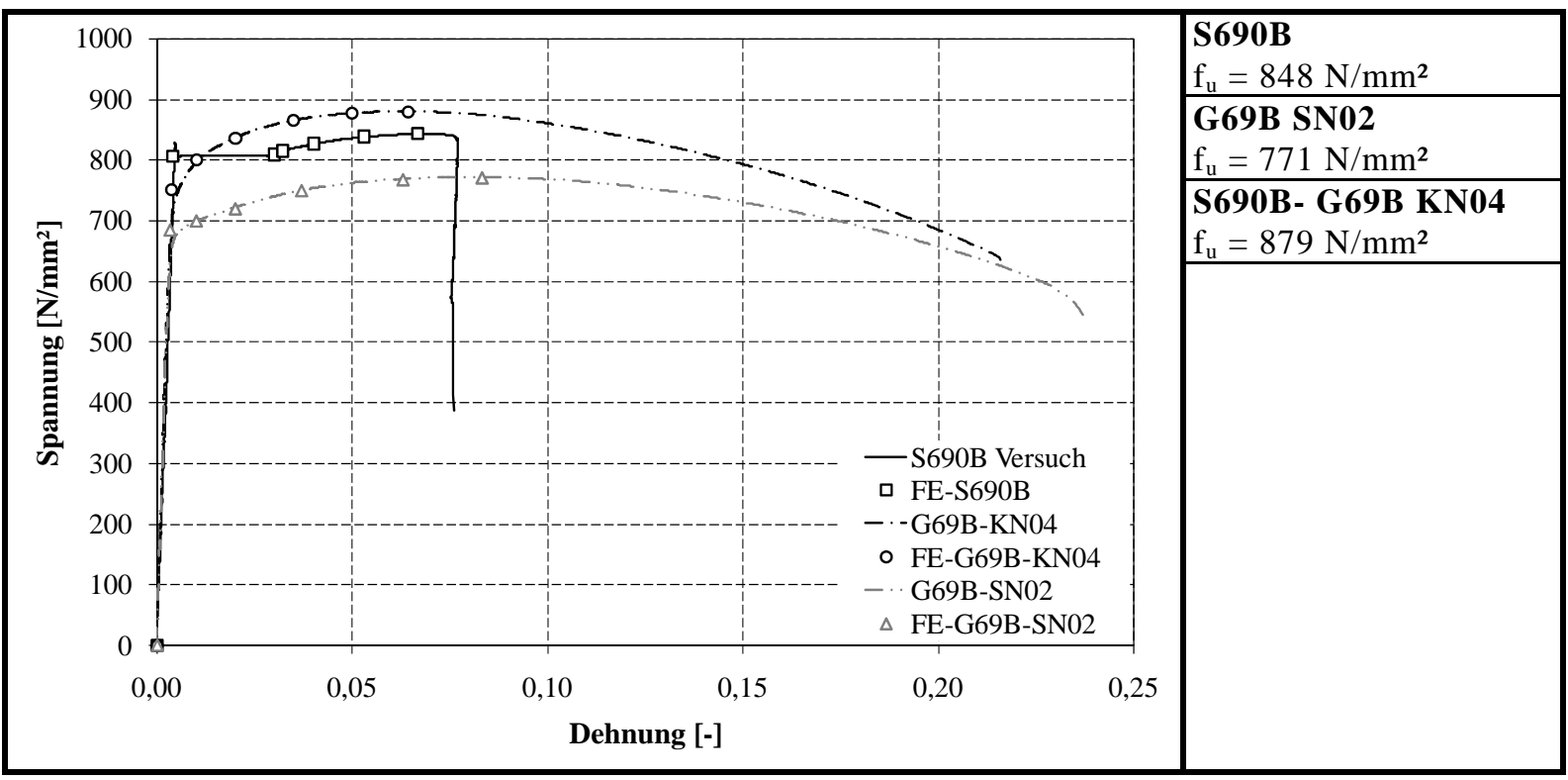

Abbildung 9.2: Multi-lineare technische Spannungs-Dehnungslinie S690B-G69B, G69B-KN und G69B-SN

Alternativ zu den Zugversuchen an mehrlagigen Kehlnähten kann die Zugfestigkeit von einlagigen Kehlnähten mithilfe von Härtemessungen abgeschätzt werden, wie in Kapitel 2.6 beschrieben. Dieses Vorgehen wurde auch in einem Beitrag zur numerischen Berechnung von Flankenkehlnähten S460 vorgestellt, [Hölbling et al., 2005]. 


\subsubsection{Vergleich von numerischen Ergebnissen und den Versuchsergebnisse der Flankenkehlnähte}

Als Versuchsergebnisse stehen Kraftmessungen zur Verfügung. Zusätzlich wurden Wegmessungen mit induktiven Wegaufnehmern an den Nahtenden durchgeführt, um die Relativverschiebungen der Nahtenden zu messen. Lokale Dehnungen wurden mit Dehnungsmessstreifen gemessen. Zusätzlich wurden optische Messungen durchgeführt, die Absolutverschiebungen in vertikaler Richtung gemessen haben. Die Lage der Messpunkte ist in Abbildung 9.3 abgebildet. Die Ergebnisse der numerischen Untersuchungen werden mit diesen Versuchsergebnissen verglichen. Der Vergleich bezieht sich jeweils auf die Kraft, die im Versuch gemessen wurde und die Kraft, die numerisch als Auflagerkraft errechnet wurde.
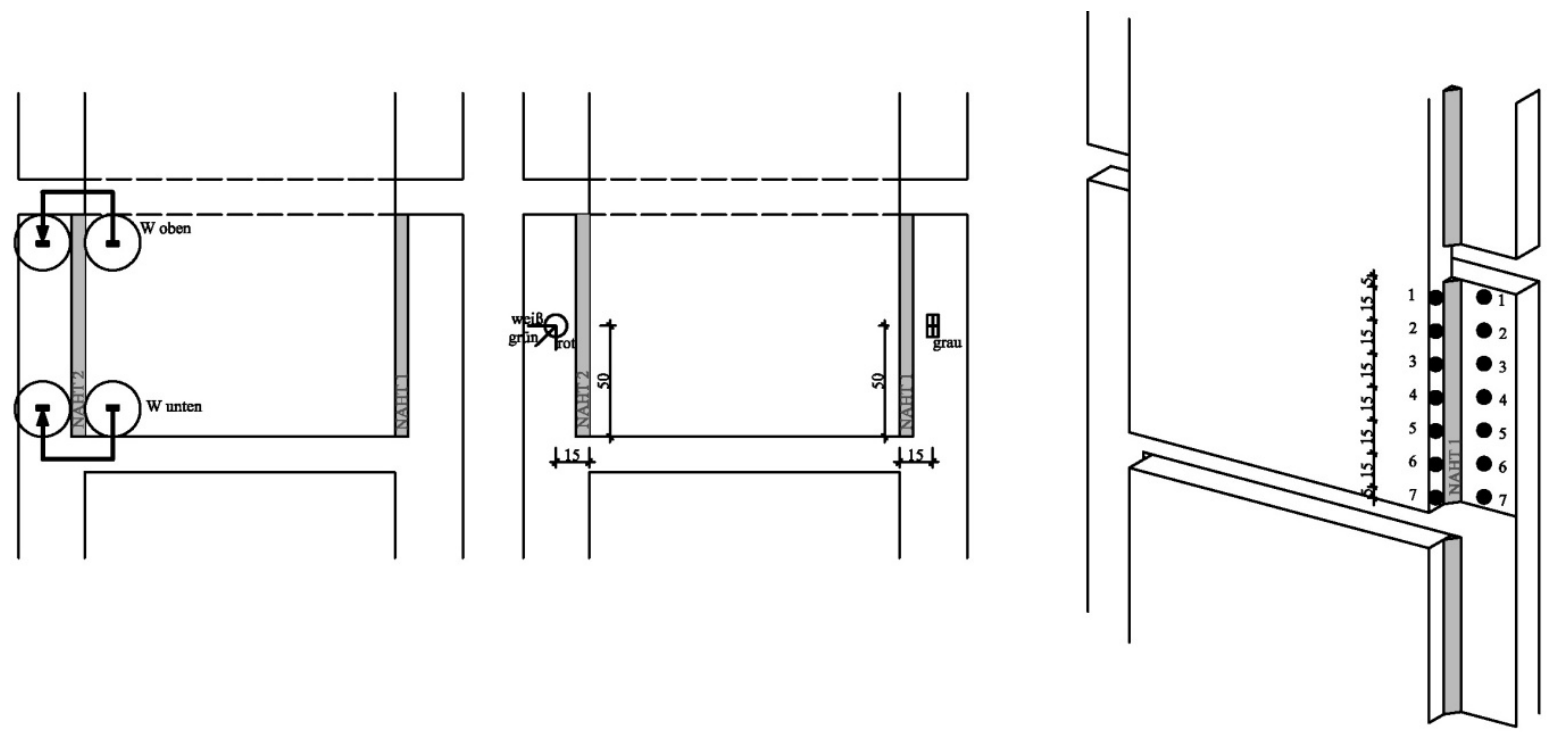

Abbildung 9.3: Messungen mit Wegaufnehmern, Dehnungsmessstreifen und optische Messungen

\subsection{Voruntersuchungen an Flankenkehlnahtverbindungen}

\subsubsection{Allgemeines}

Die Besonderheiten der numerischen Versuchsnachrechnungen werden im Folgenden im Allgemeinen und anhand der Nachrechnung eines Versuches (1-09) vorgestellt. Bei dem Versuchskörper 1-09 handelt es sich um eine Werkstoffkombination aus zwei gleichen Grundwerkstoffen S690B und einem zugehörigen Schweißzusatzwerkstoff G69B.

\subsubsection{Geometriemodellierung an Flankenkehlnahtverbindungen}

Zur Abbildung der Geometrie wurde der Versuchskörper anhand der tatsächlichen Abmessungen mithilfe des Makroschliffes modelliert, wie in Kapitel 9.2.2 beschrieben und in Abbildung 9.4 mit weißen Linien dargestellt. Im Makroschliff in Abbildung 9.4 ist zusätzlich die kleinste Nahtdicke aschliff eingezeichnet, an der die Schweißnaht folglich versagt.

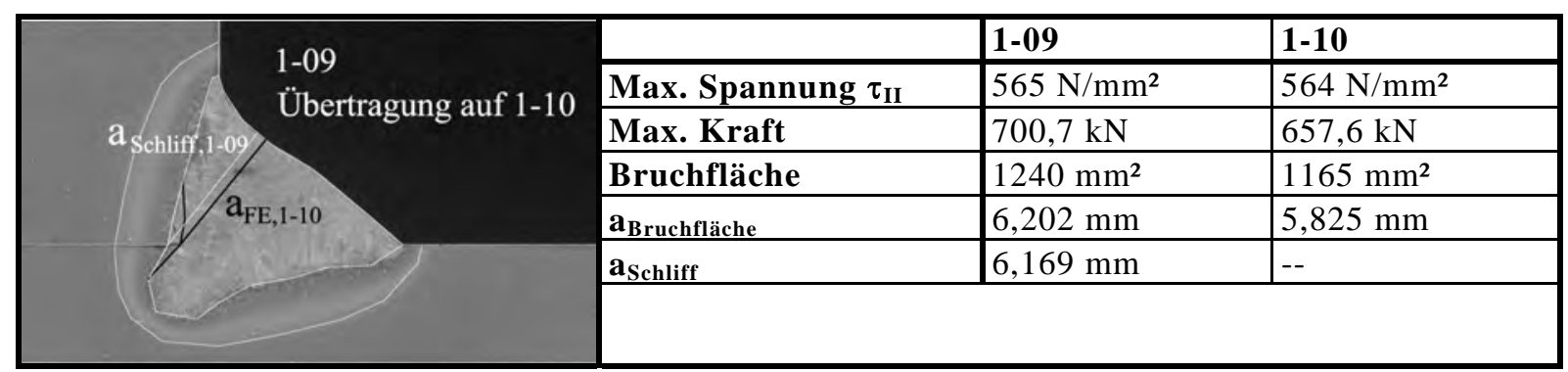

Abbildung 9.4: Übertragung der Geometrie auf einen anderen Versuchskörper gleicher Art 
Wie in Kapitel 8.5 angegeben, wurden bei den beiden gleichen Versuchskörpern jeweils sehr ähnliche Bruchspannungen ermittelt. Die maximalen Kräfte aus dem Versuch und die Bruchflächen unterscheiden sich jedoch, wie exemplarisch in Abbildung 9.4 für die beiden Versuche 1-09 und 1-10 zusammengefasst. Für den Versuchskörper 1-09 lässt sich aus dem Makroschliff eine Nahtdicke a $\mathrm{a}_{\text {schliff }}=$ 6,169 mm ermitteln, während die Rückrechnung nach Tabelle 8.8 oder Abbildung 9.4 aus der Bruchfläche einen Wert $\mathrm{a}_{\text {Bruchfläche }}=6,202 \mathrm{~mm}$ ergibt. Der Unterschied zwischen beiden Werten liegt bei $0,5 \%$. In diesem Fall entspricht die aus der Bruchfläche ermittelte Nahtdicke der Nahtdicke, die im Makroschliff bestimmt wurde.

Der Versuchskörper 1-10 ist nominell gleich mit dem Versuchskörper 1-09. D. h. er wurde aus den gleichen Werkstoffen mit den gleichen Schweißparametern gefertigt. Wie Tabelle 8.8 und Abbildung 9.4 zeigen, ergaben sich beim Versuch 1-10 mit $565 \mathrm{~N} / \mathrm{mm}^{2}$ die gleichen maximalen Spannungen wie im Versuch 1-09 mit 564 N/mm². Die maximalen Kräfte und die maximalen Bruchflächen unterscheiden sich jedoch. Vom Versuch 1-10 lag kein Makroschliff vor. Für die Modellierung der Nahtgeometrie des Versuches 1-10, wurde die Nahtdicke $\mathrm{a}_{\mathrm{FE}}$, die im numerischen Modell verwendet werden soll, aus der Bruchfläche zu a Bruchflache $=5,825 \mathrm{~mm}$ errechnet, vgl. Abbildung 9.4. Die Umsetzung für das numerische Modell ist in Abbildung 9.4 in schwarz dargestellt. Die entsprechenden Knoten wurden im numerischen Modell verschoben, so dass die kleinste Fläche (an der die Naht versagt) die Größe $\mathrm{a}_{\text {Bruchfläche }}$ hat. Der Wert $\mathrm{a}_{\text {Bruchfläche }}$ wurde im numerischen Modell als Nahtdicke verwendet und wird im Folgenden als $\mathrm{a}_{\mathrm{FE}}$ bezeichnet. Dieses Vorgehen ist möglich, da davon ausgegangen wird, dass sich die Form der Schweißnaht bei den beiden gleichen Versuchskörpern nicht unterscheidet. Die Unterschiede zwischen den Bruchflächen haben sich durch unterschiedliche Einbrandtiefen ergeben. Die Einbrandtiefe wird z. B. durch die Ebenheit der Bleche und den dadurch entstehenden Spalt beeinflusst. Die im Schliff ermittelte Nahtdicke kann nicht unbedingt über die gesamte Nahtdicke konstant angenommen werden.

Für die Versuche 1-09 und 1-10 wurden mit dieser Geometrie und den in Kapitel 9.2.3 beschriebenen Werkstoffkennwerten (KN-04 für die Schweißnaht und die WEZ, S690B für den Grundwerkstoff) numerische Berechnungen durchgeführt, die mit den Versuchsergebnissen verglichen werden.

In Abbildung 9.5 ist die Relativverschiebung am Nahtende in Abhängigkeit von der einwirkenden Kraft für Versuch und numerische Berechnung dargestellt. Für den Versuch 1-09 ergibt sich ein Unterschied der maximalen Kräfte zwischen Versuch und Berechnung von 0,3\% und damit eine sehr gute Übereinstimmung. Die Berechnungen für den Versuch 1-10 ergeben eine Abweichung in der Traglast von 1,9\% und damit eine gute Übereinstimmung.

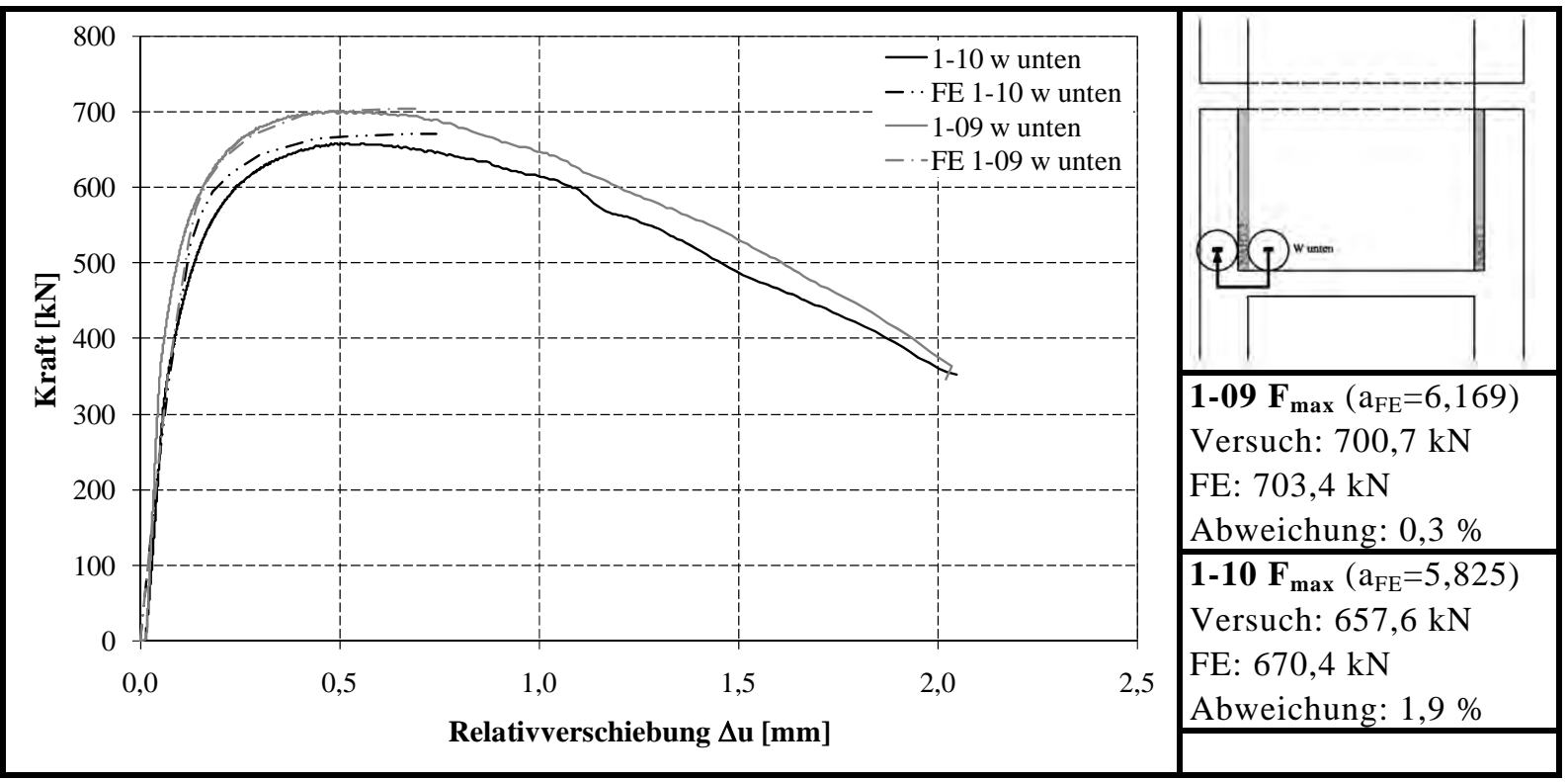

Abbildung 9.5: Vergleich der Verschiebung von Versuch und Simulation

Beim Versuch 1-09 wurden Dehnungen in der Nahtmitte mit Hilfe von Dehnungsmessstreifen und Messrosetten gemessen. In Abbildung 9.6 sind die Dehnungen in Nahtlängsrichtung (rot) und quer zur Naht (weiß) angegeben. Es zeigt sich, dass die Dehnungen direkt neben der Naht im elastischen Be- 
reich bleiben. Ein Fließen tritt nur in der Naht auf. Es wird der Vergleich der Dehnungen, die im Versuch 1-09 gemessen wurden und der Dehnungen, die berechnet wurden, gezeigt. Es zeigt sich eine gute Übereinstimmung zwischen Berechnung und Versuch. Im Experiment wurde bei Versuch 1-10 keine Dehnungen gemessen, die berechneten Dehnungen sind in Abbildung 9.6 dargestellt. Die beiden Berechnungen zeigen ähnliche Dehnungen bei dem beschriebenen Unterschied in der Kraft.

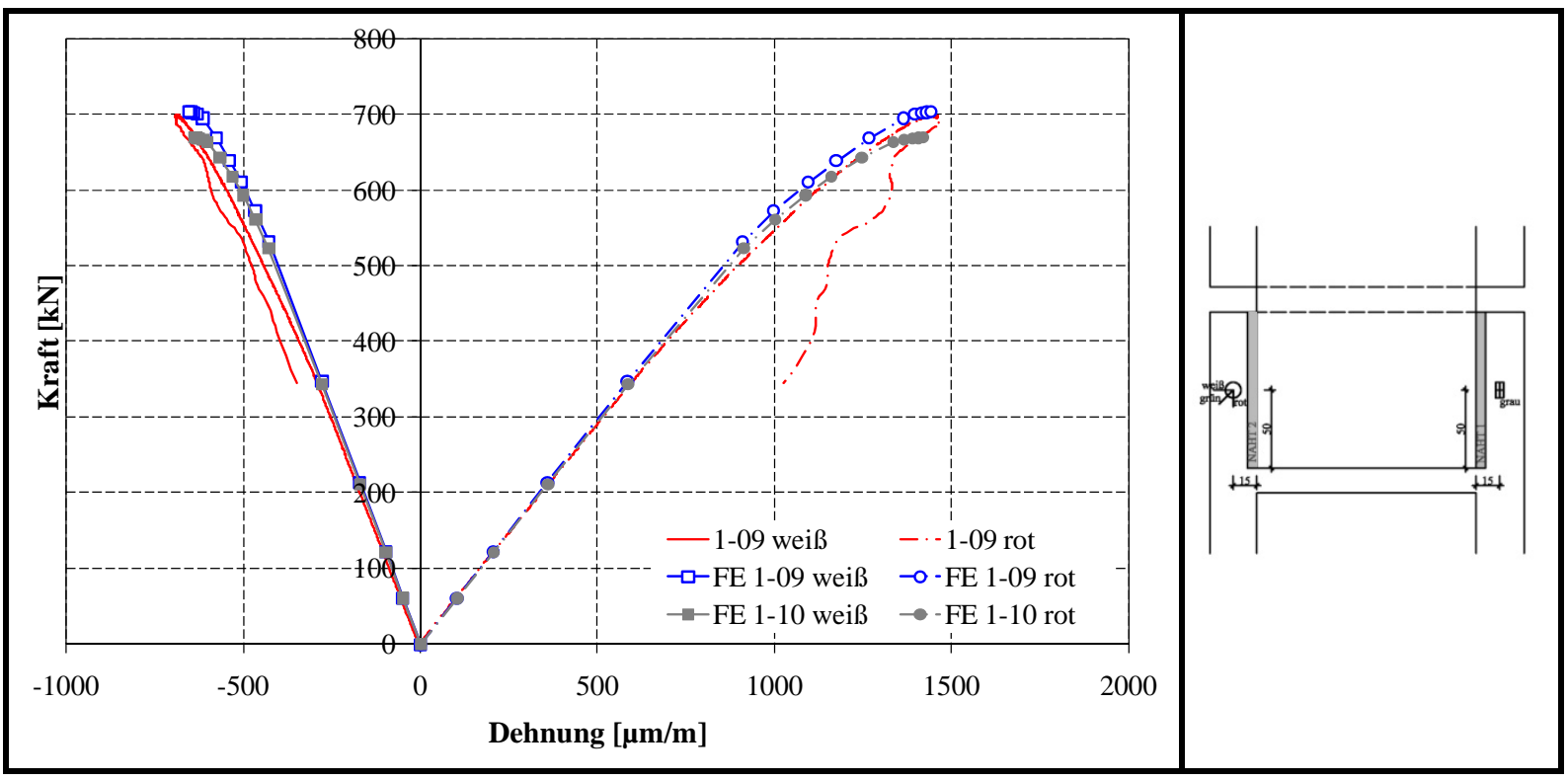

Abbildung 9.6: Vergleich der Dehnung von Versuch und Simulation

Die verglichenen maximalen Kräfte in der Berechnung und im Versuch zeigen, dass das Vorgehen zur Ermittlung der Geometrie mithilfe des Makroschliffes eine gute Übereinstimmung liefert. Es zeigt sich auch, dass eine „Übertragung“ der Bruchfläche auf die Geometrie des Makroschliffes mithilfe einer Verschiebung der Knoten zu sinnvollen Ergebnissen führt.

\subsubsection{Materialmodellierungen an Flankenkehlnahtverbindungen}

Ein besonders wichtiger Einflussfaktor bei den numerischen Berechnungen ist die Materialmodellierung. Anhand von Vergleichsberechnungen wird gezeigt, welchen Einfluss die Werkstoffmodellierung (vgl. Kapitel 9.2.3) auf die Tragfähigkeit der Verbindung hat. Es wurden Berechnungen mit Werkstoffkennwerten aus dem reinen Schweißgut (SN) als auch mit den Werten aus den mehrlagigen Kehlnähten (KN) durchgeführt. Weitere Vergleichsberechnungen wurden durchgeführt, um zu überprüfen, welchen Einfluss die Festigkeit der Wärmeeinflusszone auf die Tragfähigkeit der Verbindung hat. Die Geometrie für diese Berechnungen wurde anhand des Makroschliffes gebildet, wie in Kapitel 9.3.2 beschrieben.

Anhand der Geometrie des Versuches 1-09 wurden Berechnungen mit den Werkstoffkennwerten aus dem reinen Schweißgut (SN) als auch mit den Werten aus den mehrlagigen Kehlnähten (KN) durchgeführt. Die geometrische Modellierung mit der in 9.2.2 beschriebenen Geometrie des Makroschliffes war bei beiden Berechnungen gleich. Als Werkstoffkennwert wurde für die Grundwerkstoffe und die Wärmeeinflusszone jeweils die Spannungs-Dehnungs-Linie von S690B verwendet, lediglich die Werkstoffkennwerte im Schweißgut unterscheiden sich. Einen Vergleich zwischen Versuch und numerischen Berechnungen mit den beiden unterschiedlichen Werkstoffkennwerten zeigt Abbildung 9.7. Dargestellt ist die Relativverschiebung am Nahtende in Abhängigkeit von der einwirkenden Kraft. Es ergibt sich ein Unterschied der Kräfte aus den beiden Berechnungen in Höhe von 10,5 \%, während sich die Zugfestigkeiten als wahre Spannungen im Schweißgut um $12 \%$ unterscheiden $\left(835 \mathrm{~N} / \mathrm{mm}^{2}\right.$ $\rightarrow 936 \mathrm{~N} / \mathrm{mm}^{2}$ ). Der Unterschied der eingesetzten Zugfestigkeit spiegelt sich direkt im Unterschied der Tragfähigkeiten wider. Der Vergleich mit dem Versuchsergebnis zeigt deutlich, dass die Berechnung mit den Werkstoffkennwerten der mehrlagigen Kehlnaht (KN) eine bessere Übereinstimmung liefert als die mit denen der Stumpfnähte (SN). 


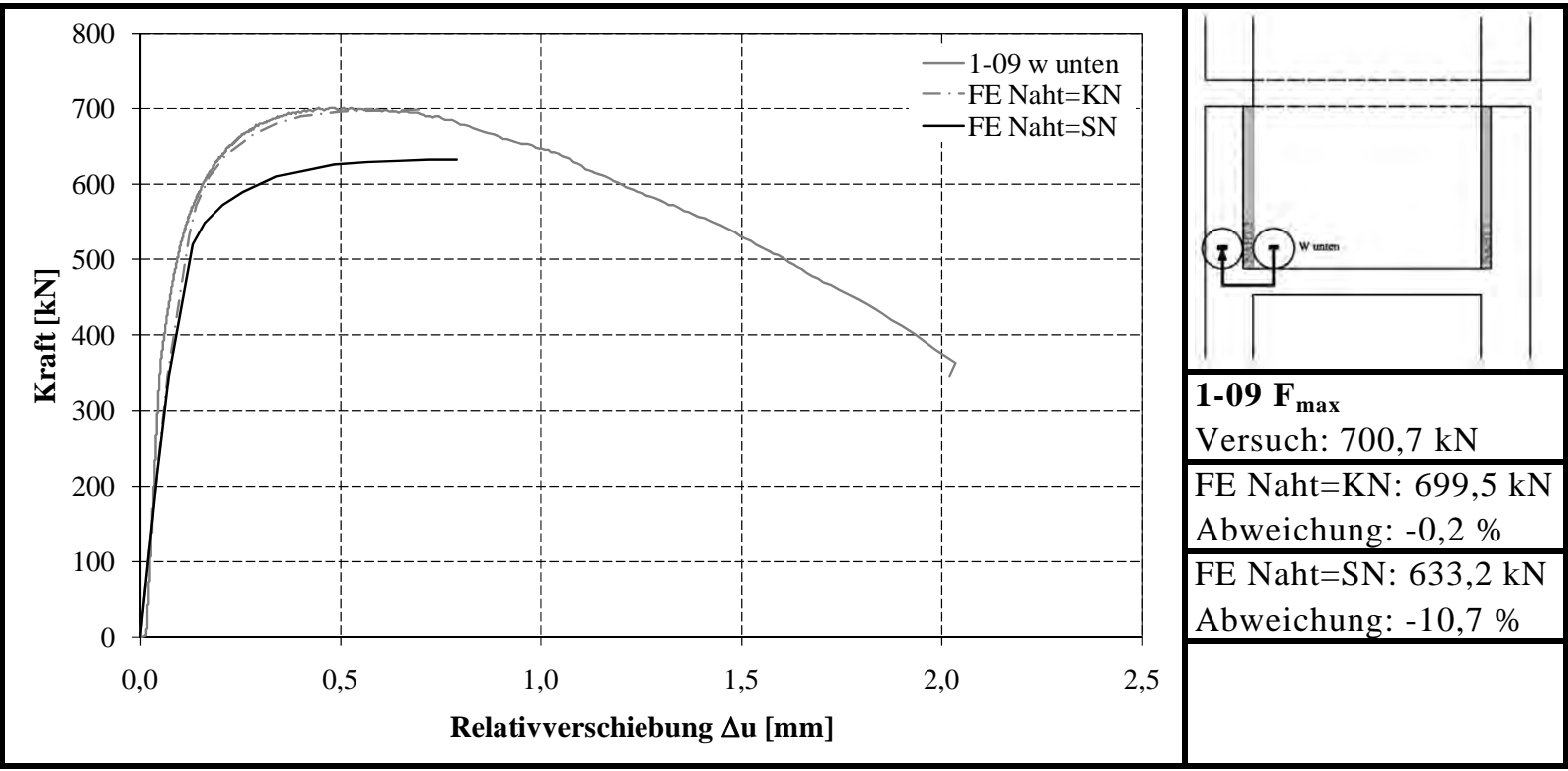

Abbildung 9.7:Einfluss der Festigkeit der Schweißnaht (KN/SN) auf die Tragfähigkeit der Verbindung

Weitere Vergleichsberechnungen wurden durchgeführt, um zu überprüfen, welchen Einfluss die Festigkeit der Wärmeeinflusszone auf die Tragfähigkeit der Verbindung hat.

Als Werkstoffkennwerte wurde für die Grundwerkstoffe die Spannungs-Dehnungs-Linie von S690B und für die Schweißnaht die Festigkeit der mehrlagigen Kehlnaht (KN04) verwendet. Für die Wärmeeinflusszone wurden entweder die wahre Spannungs-Dehnungs-Linie des Grundwerkstoffs (mit $f_{u, w a h r}$ $=899 \mathrm{~N} / \mathrm{mm}^{2}$ ) oder die des Schweißgutes $\left(\mathrm{mit}_{\mathrm{u}, \mathrm{wahr}}=936 \mathrm{~N} / \mathrm{mm}^{2}\right)$ verwendet. Einen Vergleich zwischen Versuch und numerischen Berechnungen mit den beiden unterschiedlichen Wärmeeinflusszonen zeigt Abbildung 9.8. Dargestellt ist die Relativverschiebung am Nahtende in Abhängigkeit von der einwirkenden Kraft. Es ergibt sich ein Unterschied der Kräfte aus den beiden Berechnungen bei gleicher Relativverschiebung in Höhe von $0,6 \%$ bei einem Unterschied in der Festigkeit $f_{u \text {,wahr }}$ von $4 \%$, während die Unterschiede zum Versuch ebenfalls sehr gering sind. Der Vergleich zeigt deutlich, dass für die Flankenkehlnaht die Festigkeit der Wärmeeinflusszone nur eine untergeordnete Rolle für die Tragfähigkeit der Verbindung spielt.

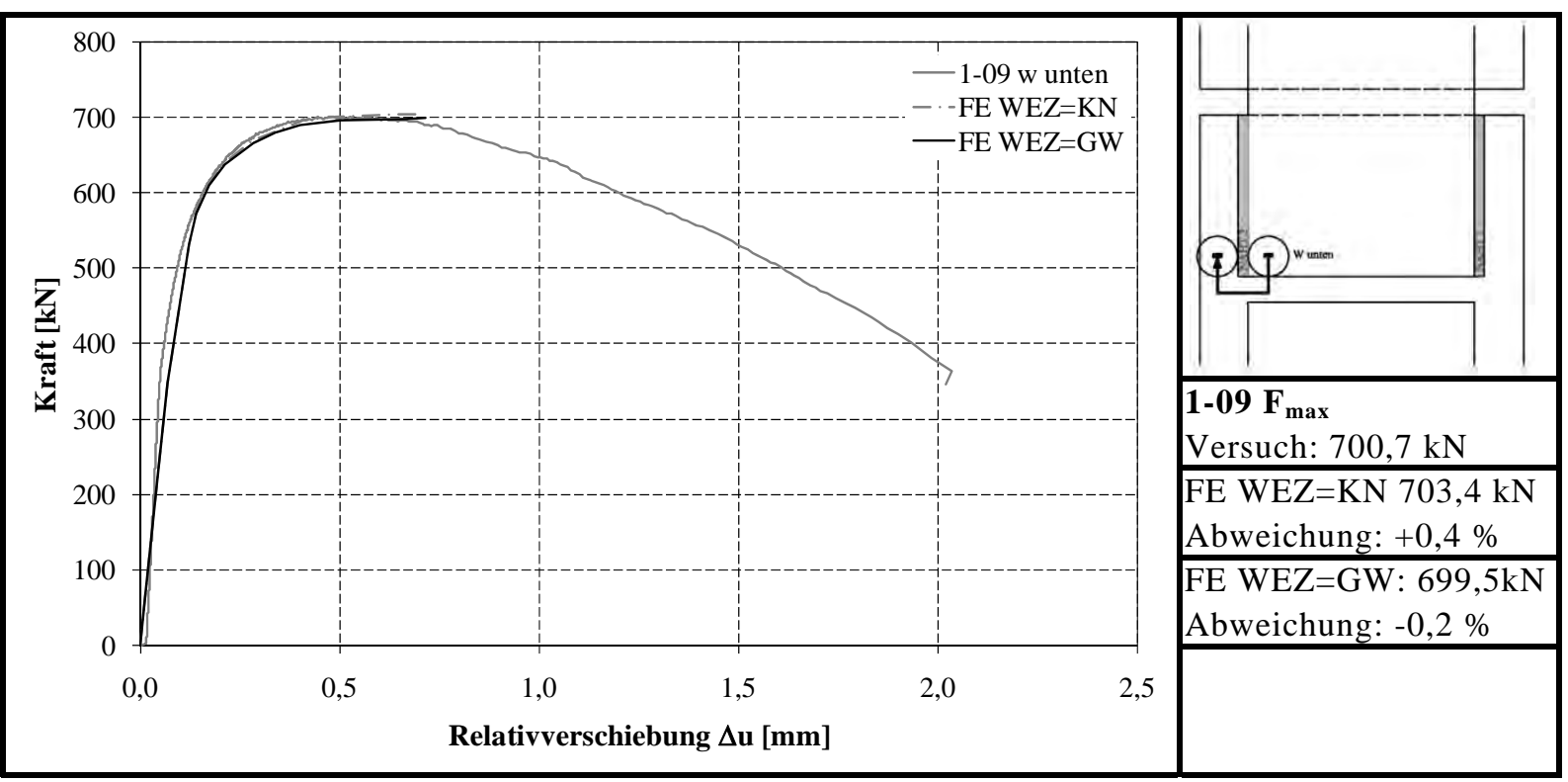

Abbildung 9.8:Einfluss der Festigkeit der Wärmeeinflusszone auf die Tragfähigkeit der Verbindung

Als weitere Untersuchung des Einflusses der Materialmodellierung auf die Tragfähigkeit wurden Berechnungen durchgeführt, bei denen die Schweißnaht, die Wärmeeinflusszone und der Grundwerkstoff mit dem gleichen Material abgebildet wurden. Es wurde zum einen für den ganzen Versuchskörper die Materialkennwerte des Grundwerkstoffs oder die des Schweißzusatzwerkstoffs (KN) sowie die des 
Schweißzusatzwerkstoffs (SN) verwendet. Im Vergleich dazu sind die oben genannten Berechnungen mit einer Kombination aus Grundwerkstoff und Schweißgut aus KN und SN dargestellt. Bei allen Berechnungen wurde die Wärmeeinflusszone mit den Werkstoffkennwerten der Schweißnaht modelliert. In Abbildung 9.9 werden die numerisch ermittelten Maximalkräfte im Vergleich mit der Versuchskraft dargestellt.

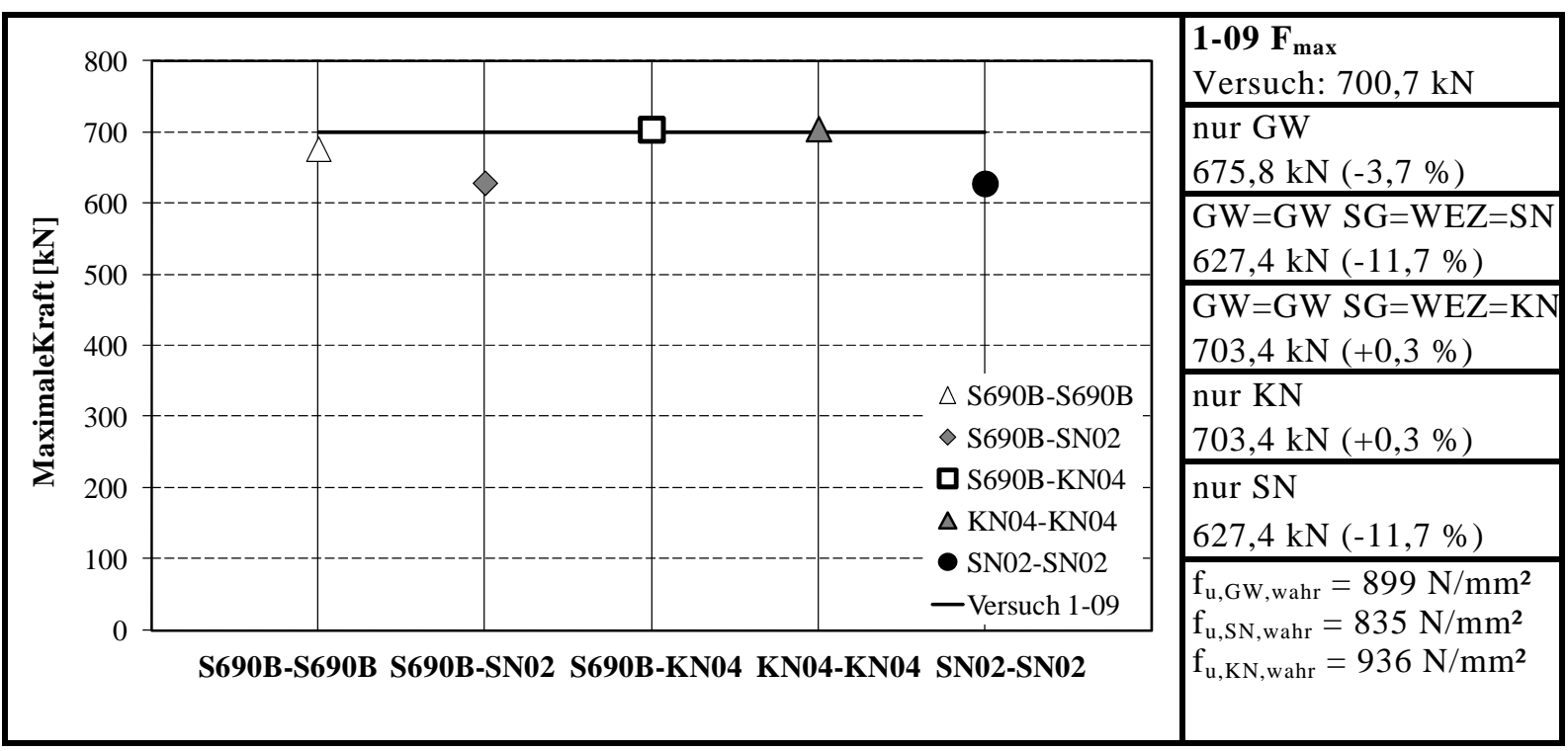

Abbildung 9.9:Einfluss der Materialkennwerte auf die Tragfähigkeit der Verbindung

Die Berechnungen mit dem Grundwerkstoff S690B und verschiedenen Festigkeiten der Schweißnaht zeigen den deutlichen Einfluss der Schweißnahtfestigkeit auf die Tragfähigkeit der Verbindung. Die Steigerung der Zugfestigkeit von $835 \mathrm{~N} / \mathrm{mm}^{2}$ auf $936 \mathrm{~N} / \mathrm{mm}^{2}$ (12 \%) führt zu einer Steigerung der Tragfähigkeit von 627,4 zu 703,4 kN (12 \%). Mit steigender Zugfestigkeit des Nahtwerkstoffs steigt die Tragfähigkeit der Verbindung in gleichem Maße. Die Berechnungen mit gleichem Nahtwerkstoff KN oder SN und verschiedenen Grundwerkstoffen zeigen in beiden Fällen, dass sich die Festigkeit des Grundwerkstoffs nicht auf die Tragfähigkeit der Verbindung auswirkt. Für den Nahtwerkstoff KN-04 ergibt sich bei verschiedenen Festigkeiten der Grundwerkstoffe (899 und 936 N/mm²) die gleiche Tragfähigkeit von 703,4 kN. Bei Verwendung der Nahtfestigkeit SN-02 ergibt sich für beide Festigkeiten der Grundwerkstoffe eine maximale Kraft von 627,4 kN.

Es wäre möglich, über die ermittelten Härtewerte in der Wärmeeinflusszone die Festigkeit der Wärmeeinflusszone abzuschätzen. Aufgrund des geringen Einflusses der Wärmeeinflusszone auf die Tragfähigkeit der Verbindung wird darauf verzichtet, den Bereich der Wärmeeinflusszone genauer zu modellieren.

Dieses Vorgehen lässt sich auch auf die anderen Werkstoffkombinationen übertragen, da gezeigt werden konnte, dass vor allem die Spannungs-Dehnungs-Linie, die für den Bereich der Naht verwendet wird, die Tragfähigkeit der Verbindung bestimmt. Eine Steigerung der Festigkeit in der Naht führt direkt zu einer höheren Tragfähigkeit in gleichem Maße. Der Grund dafür liegt im Versagen der Naht unter einem Winkel von $45^{\circ}$. Dort ist die Beeinflussung durch die Wärmeeinflusszone gering.

\subsubsection{Beschreibung des Tragverhaltens an Flankenkehlnahtverbindungen}

Bei den Berechnungen wurde immer festgestellt, dass ab Erreichen einer bestimmten Laststufe die resultierende Auflagerkraft, die an den Festhalterungen auftritt, bei zunehmenden Verschiebungen nicht mehr zunimmt. Werden weitere Verformungen aufgebracht, nehmen die Dehnungen zu, die Last wird jedoch nicht mehr gesteigert. Das Tragverhalten lässt sich so beschreiben, dass im Volumen der Schweißnaht die Vergleichsspannungen ihren gemäß der eingegebenen Spannungs-Dehnungs-Linie möglichen Maximalwert erreichen und damit die rechnerische Traglast erreicht ist. Abbildung 9.10 zeigt schematisch die Vergleichsspannungen und die Vergleichsdehnungen bei Erreichen dieser rechnerischen Traglast. Auch numerisch zeigt sich deutlich, dass die Naht am höchsten beansprucht ist, da es sich dabei um den kleinsten Querschnitt handelt. Das rechnerische Versagen ist, wie in den Versu- 
chen beobachtet, immer in der Naht aufgetreten. Abbildung 9.10 zeigt, dass sich lokal in der Naht die Dehnungen konzentrieren und dort entsprechend auch die höchsten Spannungen auftreten.

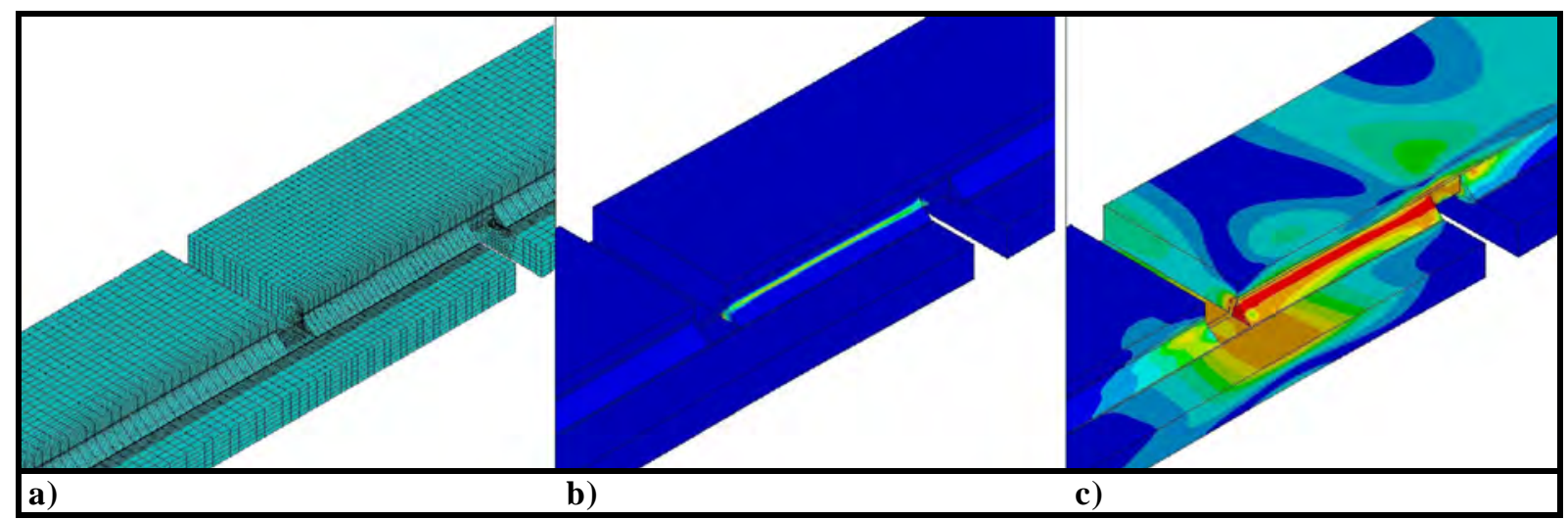

Abbildung 9.10: a) Modell, b) Vergleichsdehnung, c) Vergleichsspannung bei Erreichen der Traglast

Die Berechnungen werden in mehreren Lastschritten durchgeführt. Die folgende Abbildung 9.11 zeigt einen typischen Berechnungsverlauf, die Berechnung erfolgt in diesem Fall in 14 Schritten. In Abbildung 9.11 ist die Kraft-Verformungs-Linie angegeben, die Schritte 5, 8, 10, 12 und 14 sind gekennzeichnet. An diesen Lastschritten sind jeweils die Vergleichsdehnungen und die Vergleichsspannungen als Bilder in Abbildung 9.11 dargestellt. Sichtbare Dehnungen treten nur im Bereich der Naht auf. Die maximalen Vergleichsspannungen $\left(=\mathrm{f}_{\mathrm{u}, \text { wahr }}=936 \mathrm{~N} / \mathrm{mm}^{2}\right)$ treten ebenfalls im Bereich der Naht auf. Zusätzlich treten lokale Biegespannungen auf.

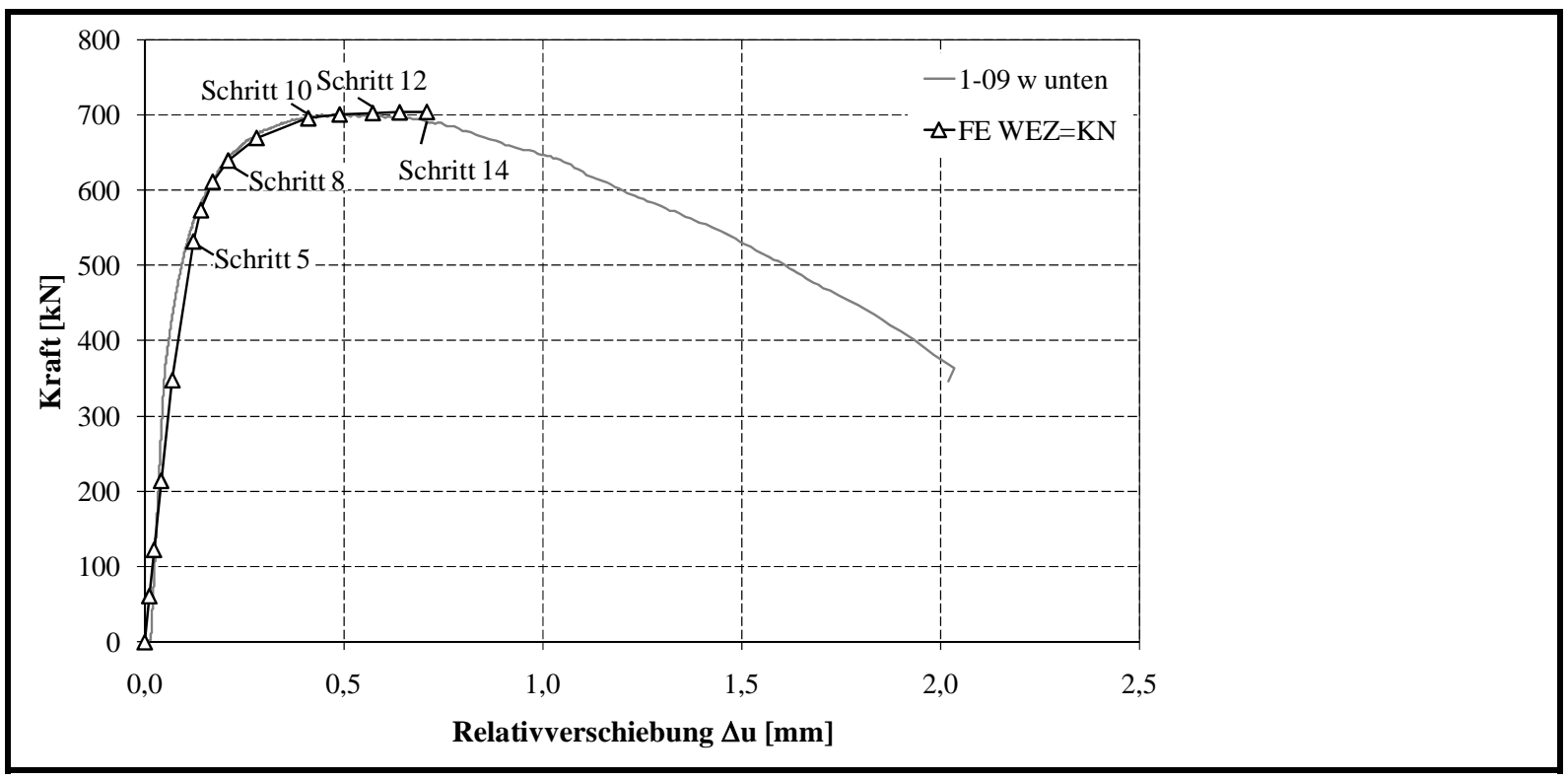

Abbildung 9.11: Exemplarische Darstellung der numerischen Berechnungsergebnisse 


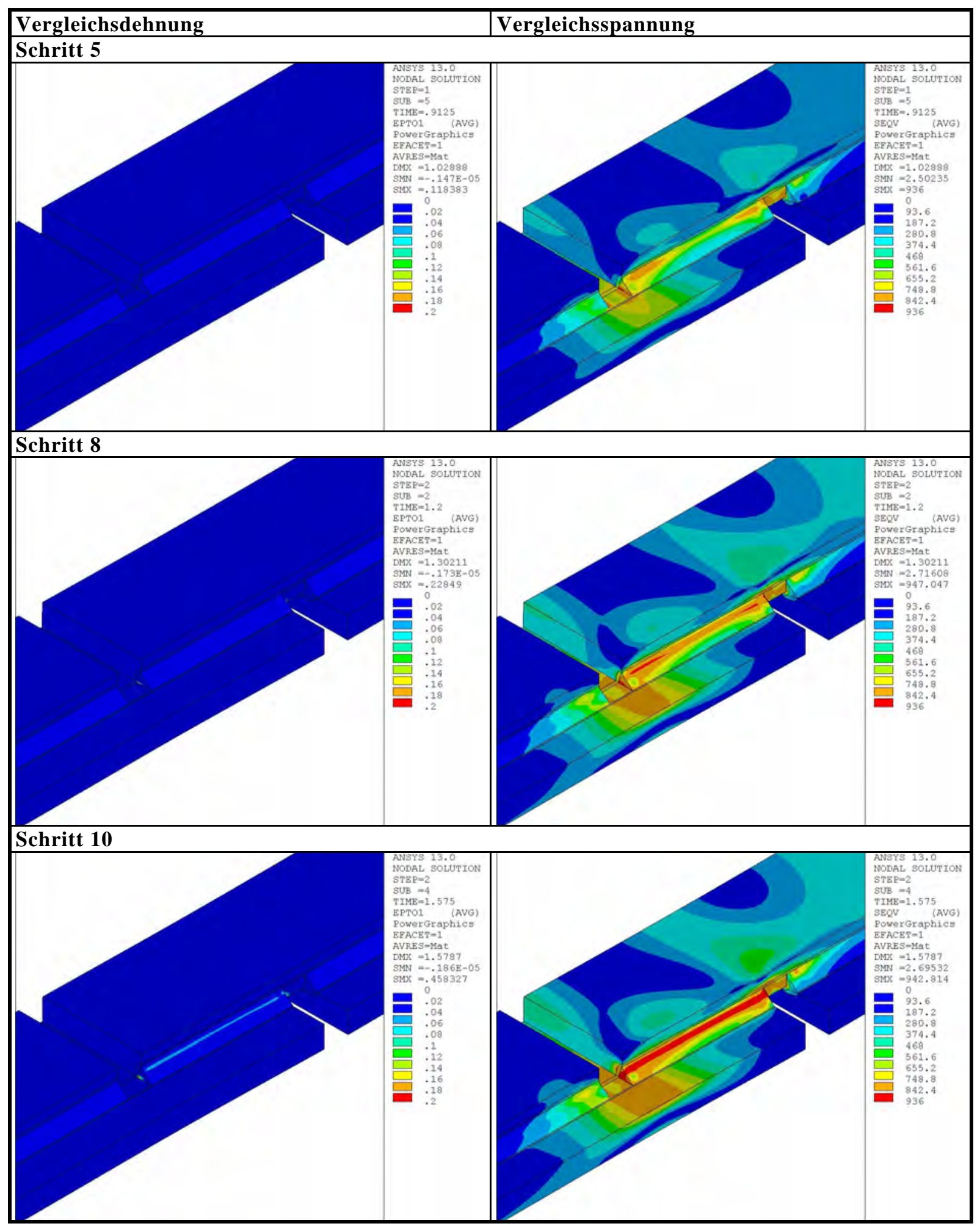

Abbildung 9.11 Fortsetzung: Exemplarische Darstellung der numerischen Berechnungsergebnisse 


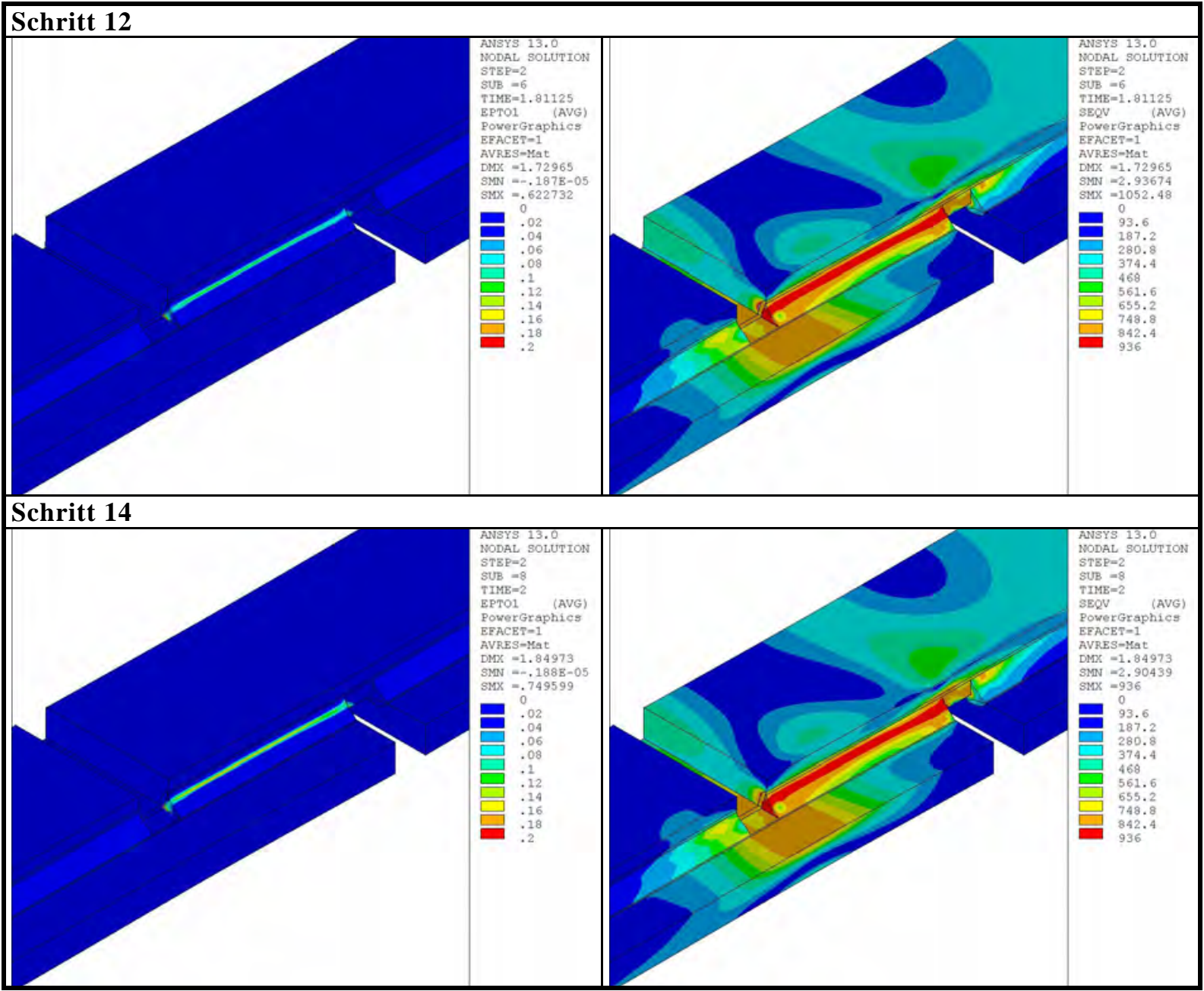

Abbildung 9.11 Fortsetzung: Exemplarische Darstellung der numerischen Berechnungsergebnisse

\subsubsection{Fazit der Voruntersuchungen an Flankenkehlnahtverbindungen}

Die Voruntersuchungen haben gezeigt, dass durch genaue Modellierung der Geometrie der Schweißnaht und genaue Kenntnis der Festigkeit der Schweißnaht die numerische Berechnung der Traglast möglich ist.

In den numerischen Berechnungen wurde festgestellt, dass die Berechnung mit der Zugfestigkeit, die an mehrlagigen Kehlnähten bestimmt wurde, die Tragfähigkeit des Versuches besser abbildet als mit der Zugfestigkeit aus dem reinen Schweißgut. Die Festigkeit der Schweißnaht kann nur mithilfe von Zugversuchen an mehrlagigen Kehlnähten bestimmt werden. Die Festigkeit der einlagigen Nähte ist nicht immer bekannt, wie in Kapitel 8.9 beschrieben.

Weitere Berechnungen haben gezeigt, dass die Festigkeit in der Wärmeeinflusszone nur einen sehr geringen Einfluss auf die Tragfähigkeit der Verbindung hat.

Die numerischen Betrachtungen zum Tragverhalten haben gezeigt, dass ein Plastizieren der Naht eintritt und die Kraft nicht weiter gesteigert werden kann, wenn die Vergleichsspannung in der Naht erreicht ist. Es tritt kein vorzeitiges Versagen auf.

Für alle weiteren Berechnungen werden die Werte aus der Spannungs-Dehnungs-Linie der mehrlagigen Kehlnähte verwendet. 


\subsection{Berechnungen zu Flankenkehlnahtverbindungen}

\subsubsection{Beschreibung des Vorgehens}

Für die numerischen Berechnungen wurde die Geometrie anhand der im Makroschliff bestimmten

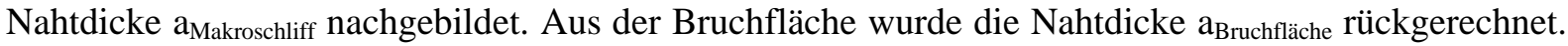
Teilweise unterscheiden sich die beiden beschriebenen Nahtdicken, so dass die Geometrie in der numerischen Berechnung geändert wurde, vgl. Kapitel 9.3.2, damit die Bruchfläche im Versuch mit der Nahtfläche in der numerischen Berechnung übereinstimmen.

Als Werkstoffkennwerte wurden die beschriebenen Ergebnisse der durchgeführten Zugversuche an Grundwerkstoffen und aus den Zugproben aus den Kehlnähten (KN) verwendet, wie in Kapitel 9.2.3 beschrieben.

Für die Einordnung der numerischen Ergebnisse werden die Ergebnisse der Härtemessungen verwendet.

\subsubsection{Einfluss von Grund- und Schweißzusatzwerkstoff S690-G69}

Es wurden numerische Berechnungen zur Nachrechnung der Versuche der Serie 1 (Kapitel 8.6.1) durchgeführt. Die Serie 1 umfasst Schweißverbindungen von verschiedenen Stählen und Schweißzusatzwerkstoffen gleicher Nenngüte verschiedener Hersteller.

Die Ergebnisse der durchgeführten Berechnungen sind im Vergleich mit den Versuchsergebnissen in Tabelle 9.2 dargestellt. Es sind jeweils die im Versuch maximal erreichten Kräfte und die aus der Bruchfläche rückgerechnete Nahtdicke $\mathrm{a}_{\text {Bruchfläche }}$ sowie die im Makroschliff bestimmte Nahtdicke $\mathrm{a}_{\text {Makroschliff }}$ angegeben.

Für die Modellierung der Geometrie wurde die Form der Naht aus dem Makroschliff verwendet mit einer Nahdicke $\mathrm{a}_{\mathrm{FE}}$, wie in Abbildung 9.4 dargestellt und in Kapitel 9.3.2 beschrieben. Teilweise unterscheiden sich die beiden beschriebenen Nahtdicken $\mathrm{a}_{\text {Bruchfläche }}$ und $\mathrm{a}_{\text {Makroschliff, }}$ so dass für die Geometrie für den Wert $\mathrm{a}_{\mathrm{FE}}$ in der numerischen Berechnung entweder $\mathrm{a}_{\text {Makroschliff oder }} \mathrm{a}_{\text {Bruchfläche verwendet }}$ wurde. Als Ergebnis der numerischen Berechnung ist die maximal erreichte Kraft angegeben.

In der letzten Spalte wird das berechnete Ergebnis durch den Vergleich mit dem Versuchsergebnis beurteilt. Es zeigt sich in allen Fällen, dass durch die Anpassung der Nahtdicke eine bessere Übereinstimmung mit dem Versuch erreicht werden kann.

Es zeigt sich auch, dass die Änderung der Nahtdicke in gleichem Maße zu einer Änderung der numerisch bestimmten Tragfähigkeit führt.

Tabelle 9.2: Berechnungen an Flankenkehlnähten gleicher Grund- und zugehöriger Schweißzusatzwerkstoffe (Serie 1)

\begin{tabular}{|l|l|l|l|l|l|l|l|l|l|}
\hline \multicolumn{2}{|l|}{ Versuch } & $\begin{array}{l}\text { Kraft } \\
{[\mathrm{kN}]}\end{array}$ & $\begin{array}{l}\text { Span- } \\
\text { nung } \\
{\left[\mathrm{N} / \mathrm{mm}^{2}\right]}\end{array}$ & $\begin{array}{l}\mathrm{a}_{\text {Makroschliff }} \\
\mathrm{a}_{\text {Bruchfläche }} \\
{[\mathrm{mm}]}\end{array}$ & $\begin{array}{l}\text { FE- } \\
\text { Berech- } \\
\text { nung }\end{array}$ & $\begin{array}{l}\text { Kraft } \\
{[\mathrm{kN}]}\end{array}$ & $\begin{array}{l}\mathrm{a}_{\mathrm{FE}} \\
{[\mathrm{mm}]}\end{array}$ & $\begin{array}{l}\mathrm{a}_{\mathrm{FE}} / \\
\mathrm{a}_{\text {Bruchfläche }}\end{array}$ & $\begin{array}{l}\text { Kraft Versuch/ } \\
\text { Kraft FE }\end{array}$ \\
\hline 1-01 & $\begin{array}{l}\text { S700A } \\
\text {-G69A }\end{array}$ & 692,0 & 521 & $\begin{array}{l}7,099 \\
6,646\end{array}$ & $\begin{array}{l}\text { S700A- } \\
\text { KN01 }\end{array}$ & $\begin{array}{l}765,4 \\
\mathbf{7 2 3 , 0}\end{array}$ & $\begin{array}{l}7,099 \\
\mathbf{6 , 6 4 6}\end{array}$ & $\begin{array}{l}1,068 \\
\mathbf{1 , 0 0 0}\end{array}$ & $\begin{array}{l}1,106 \\
\mathbf{1 , 0 4 5}\end{array}$ \\
\hline $1-04$ & $\begin{array}{l}\text { S700A } \\
\text {-G69B }\end{array}$ & 645,8 & 525 & $\begin{array}{l}6,348 \\
6,149\end{array}$ & $\begin{array}{l}\text { S700A- } \\
\text { KN04 }\end{array}$ & $\begin{array}{l}727,1 \\
\mathbf{7 0 7 , 0}\end{array}$ & $\begin{array}{l}6,348 \\
\mathbf{6 , 1 5 1}\end{array}$ & $\begin{array}{l}1,032 \\
\mathbf{1 , 0 0 0}\end{array}$ & $\begin{array}{l}1,126 \\
\mathbf{1 , 0 9 5}\end{array}$ \\
\hline $1-08$ & $\begin{array}{l}\text { S690B } \\
\text {-G69A }\end{array}$ & 720,7 & 542 & $\begin{array}{l}-- \\
6,654\end{array}$ & $\begin{array}{l}\text { S690B- } \\
\text { KN01 }\end{array}$ & $\mathbf{7 3 6 , 1}$ & $\mathbf{6 , 7 0 5}$ & $\mathbf{1 , 0 0 8}$ & $\mathbf{1 , 0 2 1}$ \\
\hline $1-09$ & $\begin{array}{l}\text { S690B } \\
\text {-G69B }\end{array}$ & 700,7 & 565 & $\begin{array}{l}6,169 \\
6,202\end{array}$ & $\begin{array}{l}\text { S690B- } \\
\text { KN04 }\end{array}$ & $\mathbf{7 0 3 , 5}$ & $\mathbf{6 , 1 6 9}$ & $\mathbf{0 , 9 9 5}$ & $\mathbf{1 , 0 0 4}$ \\
\hline
\end{tabular}

Für den Versuch 1-09 konnte eine sehr gute Übereinstimmung $(1,004)$ zwischen Berechnung und Versuch gefunden werden, die bereits in Kapitel 9.3.2 und 9.3.3 vorgestellt wurde. Ein Vergleich der Härtewerte der mehrlagigen Kehlnaht KN-04 und dem Versuchskörper 1-09 ist in Abbildung 9.12 dargestellt. Für die Verbindung S690B-G69B liegen die Härtewerte der einlagigen Naht 1-09 um 8 \% höher 
als die der mehrlagigen Naht, wobei die Werte stark streuen. Es wird davon ausgegangen, dass die Werte hinreichend gut übereinstimmen und die Werte der mehrlagigen Nähte für die numerische Berechnung der einlagigen Naht verwendet werden können.

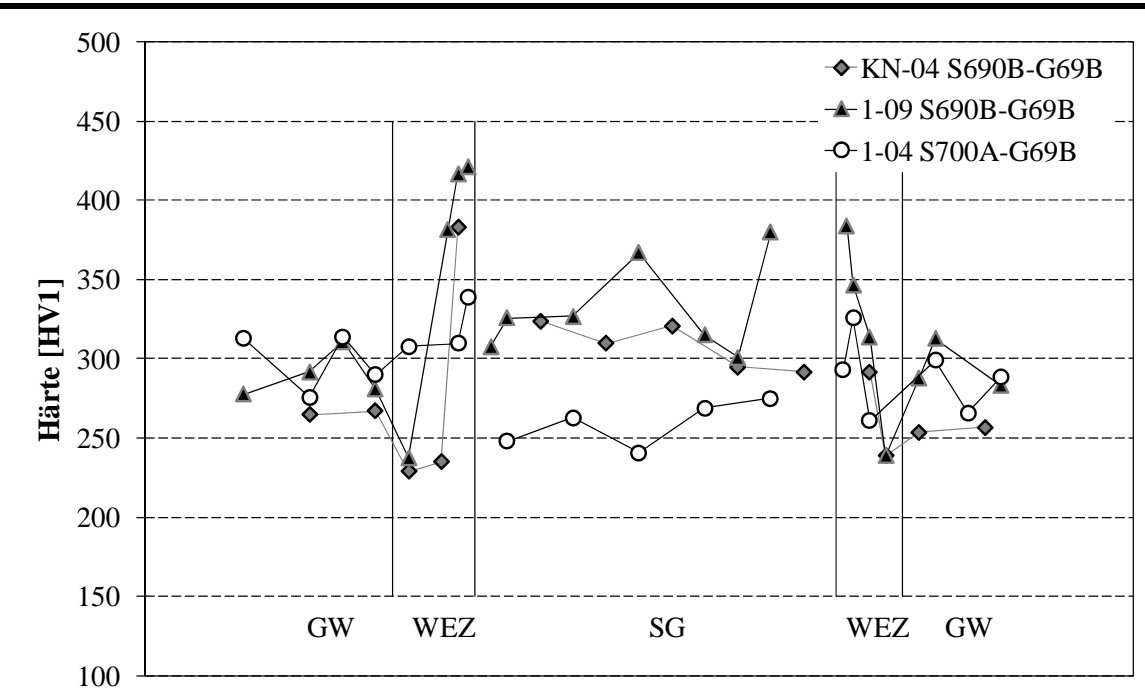

\begin{tabular}{|l|}
\hline KN-04 S690B-G69B \\
SG MW: 308 HV1 \\
\hline 1-09 S690B-G69B \\
SG MW: 332 HV1 \\
\hline 1-04 S700A-G69B \\
SG MW: 259 HV1 \\
\hline \\
\hline SG: Schweißgut \\
MW: Mittelwert \\
\hline
\end{tabular}

Abbildung 9.12: Härtemessung bei einlagigen Nähten und KN G69B

Die Nachrechnung des Versuchs 1-01 überschätzt das Versuchsergebnis um 4 \% bei gleicher Nahtfläche $a_{\text {Bruchfläche }}=a_{\mathrm{FE}}$ in der numerischen Berechnung. Der Vergleich der Härtewerte der mehrlagigen Kehlnaht KN-01 und der einlagigen Naht 1-01 in Abbildung 9.13 zeigt eine um 6 \% größere Härte im Schweißgut der mehrlagigen Naht, die sich in der Überschätzung der Tragfähigkeit von $4 \%$ widerspiegelt. Der Härteunterschied ist nahezu konstant über die ganze Nahtdicke. In den höheren Härtewerten für die mehrlagigen Nähte zeigen sich Vergütungseffekte, die zu einer größeren Festigkeit führen.

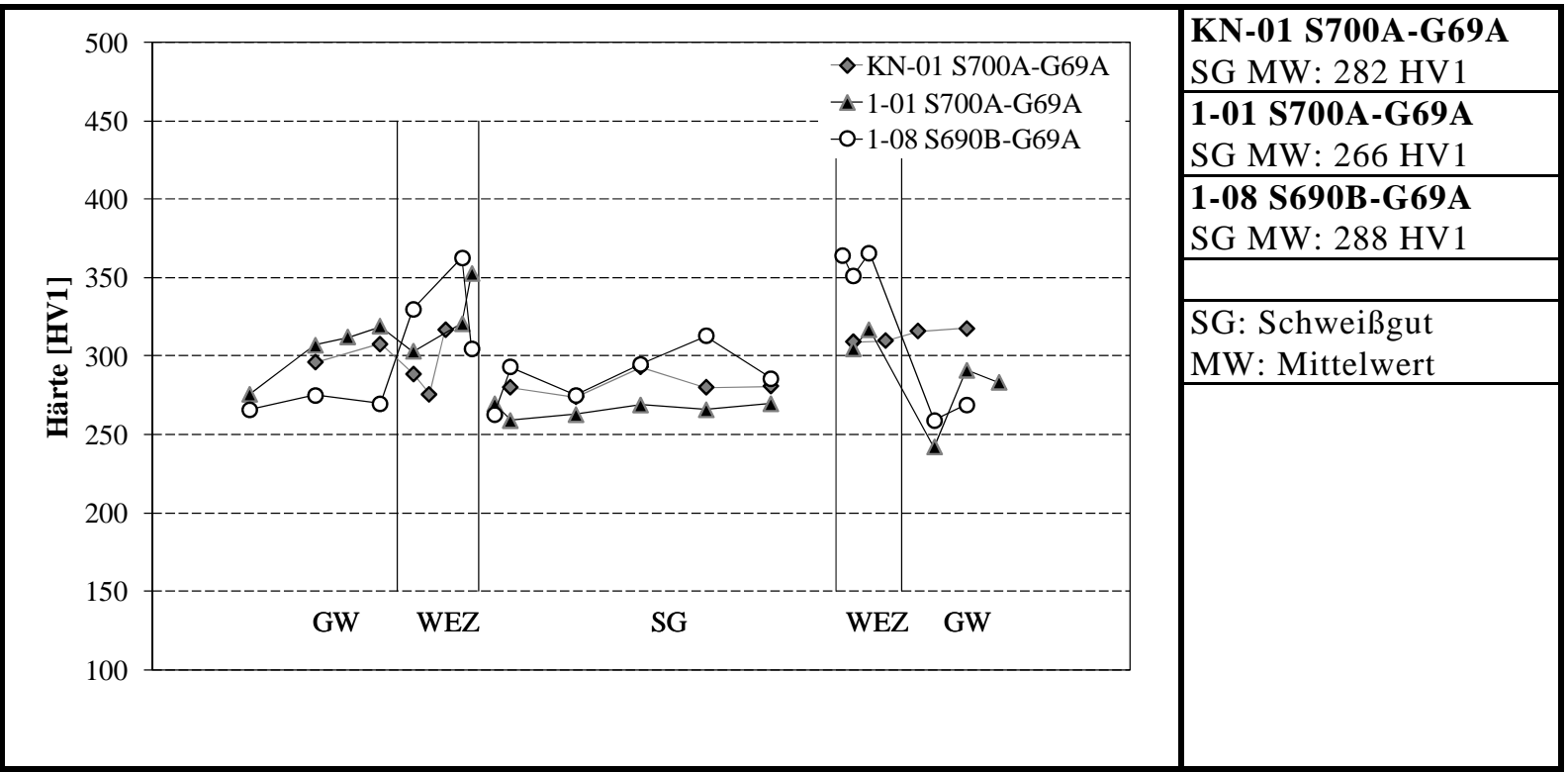

Abbildung 9.13: Härtemessung bei einlagigen Nähten und KN G69A

Bei der Nachrechnung der Versuche 1-04 und 1-08 wurden die Materialkennwerte KN-01 und KN-04 verwendet, bei denen der Schweißzusatzwerkstoff aber nicht der Grundwerkstoff mit den Versuchen übereinstimmt.

Das Berechnungsergebnis des Versuchs 1-08 stimmt sehr gut mit dem Versuchsergebnis überein. Die verwendete Materialkennlinie KN-01 wurde mit einem anderen Grundwerkstoff (S700A) hergestellt, der Vergleich der Härtewerte in Abbildung 9.13 zeigt jedoch eine sehr gute Übereinstimmung. Trotz 
des unterschiedlichen Grundwerkstoffs stimmen die Werte gut überein, so dass auch für diesen Versuchskörper (1-08) die Zugfestigkeit mit KN-01 abgeschätzt werden kann. Aus diesem Grund wird davon ausgegangen, dass die Festigkeiten ebenfalls gut übereinstimmen.

Für den Versuch 1-04 weicht das Berechnungsergebnis deutlich (um 9,5 \%) vom Versuchsergebnis ab. Abbildung 9.12 zeigt den Vergleich der Härtewerte zwischen der verwendeten mehrlagigen Kehlnaht KN-04 und der einlagigen Naht 1-04. Die Härtewerte sind trotz gleichem Schweißzusatzwerkstoffs bei der einlagigen Kehlnaht deutlich geringer sind als bei der mehrlagigen Kehlnaht. Der Einfluss des Grundwerkstoffs auf die Zugfestigkeit der Kehlnaht kann in diesem Fall nicht vernachlässigt werden. Die Härtewerte fallen infolge des anderen Grundwerkstoffs deutlich (ca. $20 \%$ ) ab. Somit wird die Festigkeit des Schweißgutes des Versuches 1-04 mit Werten von KN-04 überschätzt.

Die Ergebnisse der Härtemessungen und der numerischen Ergebnisse zeigen, dass beim Schweißzusatzwerkstoff G69B der Einfluss des Grundwerkstoffs deutlich größer ist als beim Schweißzusatzwerkstoff G69A.

Außerdem zeigt sich, dass beim Stahl S700A numerisch die Tragfähigkeit überschätzt wird, was daran liegt, dass die einlagigen Nähte eine Abminderung gegenüber der KN-Probe erfahren.

\subsubsection{Einfluss der Schweißparameter S690-G69}

In Serie 2 wurden an einer Werkstoffkombination aus Grund- und Schweißzusatzwerkstoff die Schweißparameter über die Vorwärmtemperatur $\mathrm{T}=175^{\circ} \mathrm{C}$ und $\mathrm{T}=235^{\circ} \mathrm{C}$ in Form von unterschiedlichen Abkühlzeiten $t_{8 / 5}$ innerhalb des Arbeitsfensters variiert. Diese Versuche sind in Kapitel 8.6.2 beschrieben. Die numerischen Berechnungen berücksichtigen die geometrischen Abmessungen der Versuche. Für die Materialkennwerte wurden die Werte der Probe $\mathrm{KN}-01$ verwendet, die bei einer Vorwärmtemperatur von $100^{\circ} \mathrm{C}$ geschweißt wurden.

In Tabelle 9.3 sind die Berechnungsergebnisse der Serie 2 mit dem Ergebnis der gleichen Werkstoffkombination der Serie 1 dargestellt. Es sind jeweils die im Versuch maximal erreichten Kräfte und die

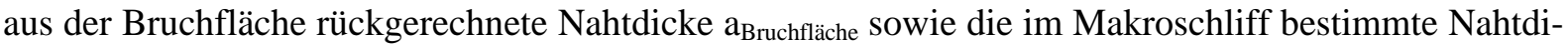
cke $\mathrm{a}_{\text {Makroschliff }}$ angegeben. Für die Modellierung der Nahtform wurde der Makroschliff verwendet, mit einer Nahdicke $\mathrm{a}_{\mathrm{FE}}$. Teilweise unterscheiden sich die beiden beschriebenen Nahtdicken $\mathrm{a}_{\text {Bruchfläche }}$ und

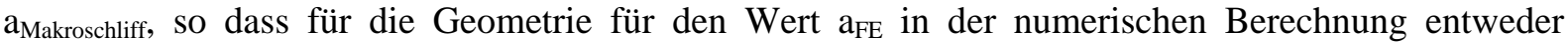

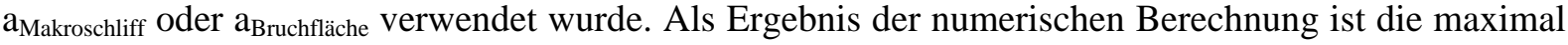
erreichte Kraft angegeben. In der letzten Spalte wird das berechnete Ergebnis durch den Vergleich mit dem Versuchsergebnis beurteilt. Es zeigt sich in allen Fällen, dass durch die Anpassung der Nahtdicke eine bessere Übereinstimmung mit dem Versuch erreicht werden kann. Auch hier führt die Änderung der Nahtdicke in gleichem Maße zu einer Änderung der numerisch bestimmten Tragfähigkeit.

Tabelle 9.3: Berechnungen an Flankenkehlnähten gleicher Grund- und zugehöriger Schweißzusatzwerkstoffe (Serie 2)

\begin{tabular}{|l|l|l|l|l|l|l|l|l|l|}
\hline \multicolumn{2}{|l|}{ Versuch } & $\begin{array}{l}\text { Kraft } \\
{[\mathrm{kN}]}\end{array}$ & $\begin{array}{l}\text { Span- } \\
\text { nung } \\
{\left[\mathrm{N} / \mathrm{mm}^{2}\right]}\end{array}$ & $\begin{array}{l}\mathrm{a}_{\text {Makroschliff }} \\
\mathrm{a}_{\text {Bruchlläche }}\end{array}$ & $\begin{array}{l}\text { FE- } \\
\text { Berech- } \\
\text { nung }\end{array}$ & $\begin{array}{l}\text { Kraft } \\
{[\mathrm{kN}]}\end{array}$ & $\begin{array}{l}\mathrm{a}_{\mathrm{FE}} \\
{[\mathrm{mm}]}\end{array}$ & $\begin{array}{l}\mathrm{a}_{\mathrm{FE}} / \\
\mathrm{a}_{\text {Bruchlläche }}\end{array}$ & $\begin{array}{l}\text { Kraft Versuch/ } \\
\text { Kraft FE }\end{array}$ \\
\hline \multirow{2}{*}{$1-01$} & $\begin{array}{l}\text { S700A } \\
\text {-G69A }\end{array}$ & 692,0 & 521 & $\begin{array}{l}7,099 \\
6,646\end{array}$ & $\begin{array}{l}\text { S700A- } \\
\text { KN01 }\end{array}$ & $\begin{array}{l}765,4 \\
\mathbf{7 2 3 , 0}\end{array}$ & $\begin{array}{l}7,099 \\
\mathbf{6 , 6 4 6}\end{array}$ & $\begin{array}{l}1,068 \\
\mathbf{1 , 0 0 0}\end{array}$ & $\begin{array}{l}1,106 \\
\mathbf{1 , 0 4 5}\end{array}$ \\
\hline Z2-01 & $\begin{array}{l}\text { S700A } \\
\text {-G69A }\end{array}$ & 707,5 & 502 & $\begin{array}{l}7,088 \\
7,045\end{array}$ & $\begin{array}{l}\text { S700A- } \\
\text { KN01 }\end{array}$ & $\mathbf{7 7 0 , 0}$ & $\mathbf{7 , 0 8 8}$ & $\mathbf{1 , 0 0 6}$ & $\mathbf{1 , 0 8 8}$ \\
\hline 204 & $\begin{array}{l}\text { S700B } \\
\text { 2-G69A }\end{array}$ & 647,2 & 488 & $\begin{array}{l}7,092 \\
6,629\end{array}$ & $\begin{array}{l}\text { S700A- } \\
\text { KN01 }\end{array}$ & $\begin{array}{l}774,3 \\
\mathbf{7 3 1 , 0}\end{array}$ & $\begin{array}{l}7,092 \\
\mathbf{6 , 6 2 9}\end{array}$ & $\begin{array}{l}1,070 \\
\mathbf{1 , 0 0 0}\end{array}$ & $\begin{array}{l}1,196 \\
\mathbf{1 , 1 2 9}\end{array}$ \\
\hline
\end{tabular}

Es zeigt sich in allen Fällen, dass durch die Anpassung der Nahtdicke eine bessere Näherung an das Versuchsergebnis erreicht werden kann. Es zeigt sich aber vor allem, dass die Berechnungen in allen Fällen die Versuchsergebnisse überschätzen. Bezogen auf die Nahtdicke ergibt sich numerisch in allen Fällen die gleiche Tragfähigkeit. Der Vergleich der Härtewerte in Abbildung 7.12 zeigt eine Abnahme der Härte in gleichem Maße wie die Abnahme der Tragfähigkeit. Zur numerischen Nachbildung der Versuche muss die Festigkeit der Naht KN abgemindert werden. Ein Grund dafür liegt darin, dass die 
Veränderung der Nahtfestigkeit infolge unterschiedlicher Vorwärmtemperaturen numerisch nicht berücksichtigt wurde.

\subsubsection{Einfluss des Grund- und Schweißzusatzwerkstoffs S460-G46}

Die Versuche der Serie 7 werden in Kapitel 8.6.6 beschrieben. Es handelt es sich um Versuchskörper mit dem Grundwerkstoff S460M mit zugehörigem Schweißzusatzwerkstoff G46. Zwei Versuche wurden aus Gründen der Vergleichbarkeit genau wie in Serie 1 mit einer Vorwärmtemperatur von $\mathrm{T}=100^{\circ} \mathrm{C}$ geschweißt. Zwei weitere Versuche wurden mit einer Vorwärmtemperatur von $\mathrm{T}=50^{\circ} \mathrm{C}$ geschweißt. Ein Versuch wurde praxisgerecht ohne Vorwärmen geschweißt.

Die numerischen Berechnungen berücksichtigen die geometrischen Abmessungen der Versuche. Für die Materialkennwerte wurden die Werte der Probe KN-05 verwendet, die bei einer Vorwärmtemperatur von $100^{\circ} \mathrm{C}$ geschweißt wurde. In Tabelle 9.4 sind die Berechnungsergebnisse der Serie 7 dargestellt. Es sind jeweils die im Versuch maximal erreichten Kräfte und die aus der Bruchfläche rückgerechnete Nahtdicke $a_{\text {Bruchfläche }}$ angegeben. Für die Modellierung der Geometrie wurde die Form des

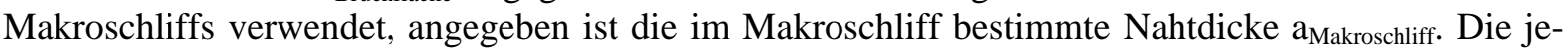
weils im numerischen Modell verwendete Nahtdicke $\mathrm{a}_{\mathrm{FE}}$ ist angegeben. Als Ergebnis der numerischen Berechnung ist die maximal erreichte Kraft angegeben.

Tabelle 9.4: Berechnungen an Flankenkehlnähten S460-G46 (Serie 7)

\begin{tabular}{|c|c|c|c|c|c|c|c|c|c|}
\hline Versu & & $\begin{array}{l}\text { Kraft } \\
{[\mathrm{kN}]}\end{array}$ & $\begin{array}{l}\text { Span- } \\
\text { nung } \\
{\left[\mathrm{N} / \mathrm{mm}^{2}\right]}\end{array}$ & $\begin{array}{l}a_{\text {Makroschliff }} \\
a_{\text {Bruchfläche }} \\
{[\mathrm{mm}]}\end{array}$ & $\begin{array}{l}\text { FE- } \\
\text { Berech- } \\
\text { nung }\end{array}$ & $\begin{array}{l}\text { Kraft } \\
{[\mathrm{kN}]}\end{array}$ & $\begin{array}{l}\mathrm{a}_{\mathrm{FE}} \\
{[\mathrm{mm}]}\end{array}$ & $\begin{array}{l}\mathrm{a}_{\mathrm{FE}} / \\
\mathrm{a}_{\mathrm{Bruchfläche}}\end{array}$ & $\begin{array}{l}\text { Kraft Versuch/ } \\
\text { Kraft FE }\end{array}$ \\
\hline 7-01 & $\begin{array}{l}\text { S460- } \\
\text { G46 }\end{array}$ & 678,4 & 456 & $\begin{array}{l}7,021 \\
7,437\end{array}$ & $\begin{array}{l}\text { S460- } \\
\text { KN-05 }\end{array}$ & $\begin{array}{l}633,1 \\
\mathbf{6 7 0 , 8}\end{array}$ & $\begin{array}{l}7,021 \\
7,437\end{array}$ & $\begin{array}{l}0,944 \\
\mathbf{1 , 0 0 0}\end{array}$ & $\begin{array}{l}0,933 \\
\mathbf{0 , 9 8 9}\end{array}$ \\
\hline 7-03 & $\begin{array}{l}\text { S460- } \\
\text { G46 }\end{array}$ & 688,5 & 484 & $\begin{array}{l}7,658 \\
6,709\end{array}$ & $\begin{array}{l}\text { S460- } \\
\text { KN-05 }\end{array}$ & \begin{tabular}{|l|}
694,3 \\
$\mathbf{6 4 4 , 8}$
\end{tabular} & $\begin{array}{l}7,658 \\
7,106\end{array}$ & $\begin{array}{l}1,078 \\
\mathbf{1 , 0 0 0}\end{array}$ & $\begin{array}{l}1,008 \\
\mathbf{0 , 9 3 7}\end{array}$ \\
\hline Z7-01 & $\begin{array}{l}\text { S460- } \\
\text { G46 }\end{array}$ & 682,8 & 509 & \begin{tabular}{|l|}
7,303 \\
7,106 \\
\end{tabular} & $\begin{array}{l}\text { S460- } \\
\text { KN-05 }\end{array}$ & $\begin{array}{l}661,0 \\
\mathbf{6 1 6 , 4}\end{array}$ & $\begin{array}{l}7,303 \\
6,709 \\
\end{array}$ & $\begin{array}{l}1,089 \\
\mathbf{1 , 0 0 0}\end{array}$ & $\begin{array}{l}0,968 \\
\mathbf{0 , 9 0 3}\end{array}$ \\
\hline
\end{tabular}

Für den Versuch 7-01 konnte eine sehr gute Übereinstimmung $(0,989)$ gefunden werden. Ein Vergleich der Härtewerte der mehrlagigen Kehlnaht KN-05 und dem Versuchskörper 7-01 in Abbildung 9.14 zeigt, dass die Härtewerte der einlagigen Naht mit denen der mehrlagigen Naht übereinstimmen, so dass davon ausgegangen wird, dass die Festigkeiten gleich sind. Es zeigen sich sehr gleichmäßige Bedingungen im Vergleich der Härtewerte zwischen mehr- und einlagigen Kehlnähte.

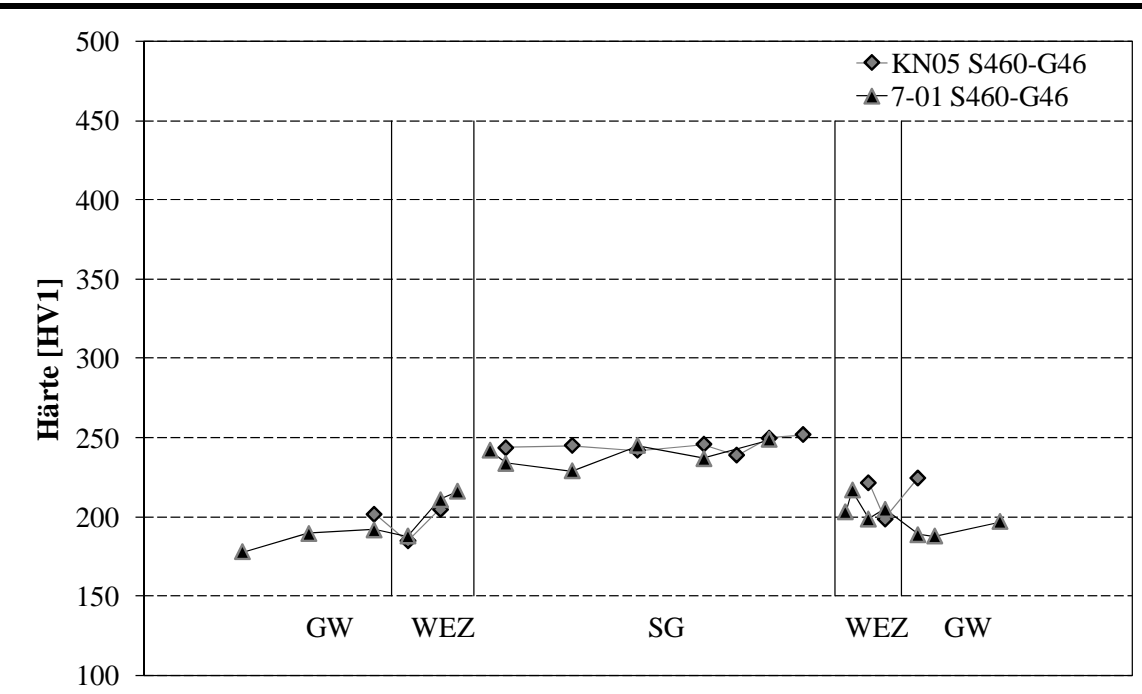

KN-05 S460-G46

SG MW:245 HV1

7-01 S460-G46

SG MW:239 HV1

SG: Schweißgut

MW: Mittelwert 
Die Nachrechnungen der Versuche 7-03 und Z7-01 unterschätzen die Versuchsergebnisse, weil die Veränderung der Nahtfestigkeit infolge unterschiedlicher Vorwärmtemperaturen numerisch nicht berücksichtigt wurde. Diese Änderung der Nahtfestigkeit zeigt sich auch in dem Vergleich der zugehörigen Härtewerte in Abbildung 8.18.

\subsubsection{Tragverhalten bei gleichen Grundwerkstoffen und Variation der Schweiß- zusatzwerkstoffe}

Die Versuche der Serie 5 sind in Kapitel 8.7 beschrieben. Die Serie 5 umfasst Schweißverbindungen mit gezielten Über- und Unterfestigkeiten im Schweißgut durch Verwendung eines nieder- oder höherfesten Schweißzusatzwerkstoffs.

Zur numerischen Nachrechnung der Versuche wurde die Geometrie anhand der vorhandenen Makroschliffe nachgebildet. Als Werkstoffkennwerte wurden die beschriebenen Ergebnisse der durchgeführten Zugversuche an Grundwerkstoffen und aus den Zugproben aus den Kehlnähten (KN) verwendet, wie in Kapitel 9.2.2 und 9.2.3 beschrieben. Allerdings standen nur Ergebnisse von Kehlnähten mit dem Grundwerkstoff S700A zur Verfügung. Einen Überblick über die durchgeführten Berechnungen im Vergleich mit den Versuchsergebnissen gibt Tabelle 9.5.

Tabelle 9.5: Berechnungen an Flankenkehlnähten gleicher Grund- und verschiedener Schweißzusatzwerkstoffe

\begin{tabular}{|l|l|l|l|l|l|l|l|l|l|}
\hline Versuch & $\begin{array}{l}\text { Kraft } \\
{[\mathrm{kN}]}\end{array}$ & $\begin{array}{l}\text { Span- } \\
\text { nung } \\
{\left[\mathrm{N} / \mathrm{mm}^{2}\right]}\end{array}$ & $\begin{array}{l}\mathrm{a}_{\text {Makroschliff }} \\
\mathrm{a}_{\text {Bruchfläche }}[\mathrm{mm}]\end{array}$ & $\begin{array}{l}\text { FE- } \\
\text { Berech- } \\
\text { nung }\end{array}$ & $\begin{array}{l}\text { Kraft } \\
{[\mathrm{kN}]}\end{array}$ & $\begin{array}{l}\mathrm{a}_{\mathrm{FE}} \\
{[\mathrm{mm}]}\end{array}$ & $\begin{array}{l}\mathrm{a}_{\mathrm{FE}} / \\
\mathrm{a}_{\text {Bruchfläche }}\end{array}$ & $\begin{array}{l}\text { Kraft Versuch/ } \\
\text { Kraft FE }\end{array}$ \\
\hline Z5-01 & $\begin{array}{l}\text { S700A } \\
-\mathrm{G} 46\end{array}$ & 655,3 & 515 & $\begin{array}{l}6,444 \\
6,357\end{array}$ & $\begin{array}{l}\text { S700A- } \\
\text { KN02 }\end{array}$ & 655,0 & 6,444 & 1,014 & 1,000 \\
\hline $5-04$ & $\begin{array}{l}\text { S700A } \\
\text {-G89 }\end{array}$ & 741,7 & 577 & $\begin{array}{l}6,488 \\
6,424\end{array}$ & $\begin{array}{l}\text { S700A- } \\
\text { KN03 }\end{array}$ & 773,9 & 6,488 & 1,010 & 1,043 \\
\hline $5-06$ & $\begin{array}{l}\text { S690B } \\
\text {-G46 }\end{array}$ & 715,2 & 542 & $\begin{array}{l}6,910 \\
6,602\end{array}$ & $\begin{array}{l}\text { S690B- } \\
\text { KN02 }\end{array}$ & $\begin{array}{l}708,4 \\
\mathbf{6 8 0 , 0}\end{array}$ & $\begin{array}{l}6,910 \\
\mathbf{6 , 6 0 2}\end{array}$ & $\begin{array}{l}1,047 \\
\mathbf{1 , 0 0 0}\end{array}$ & $\begin{array}{l}0,991 \\
\mathbf{0 , 9 5 1}\end{array}$ \\
\hline $5-07$ & $\begin{array}{l}\text { S690B } \\
\text {-G89 }\end{array}$ & 802,1 & 614 & $\begin{array}{l}6,640 \\
6,531\end{array}$ & $\begin{array}{l}\text { S690B- } \\
\text { KN03 }\end{array}$ & 802,4 & 6,640 & 1,006 & 1,006 \\
\hline
\end{tabular}

Für den Versuch Z5-01 konnte eine sehr gute Übereinstimmung $(1,000)$ gefunden werden. Eine Betrachtung der Härtewerte in Abbildung 9.15 zeigt jedoch, dass die Härtewerte der einlagigen Naht (Z501) um ca. 8 \% höher liegen als bei der mehrlagigen Kehlnaht KN-02. Dabei streuen die Werte von KN-02.

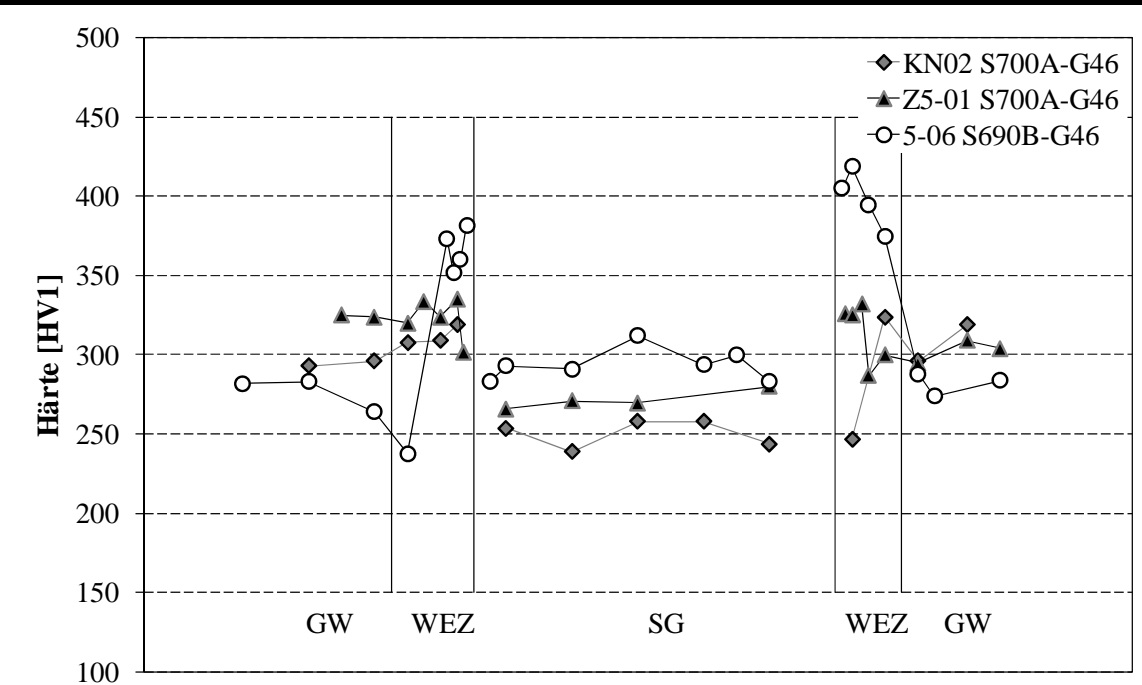

KN-02 S700A-G46

SG MW: 251 HV1

Z5-01 S700-G46

SG MW: 272 HV1

5-06 S690B-G46

SG MW: 294 HV1

SG: Schweißgut

MW: Mittelwert 
Die Nachrechnung des Versuchs 5-04 überschätzt das Versuchsergebnis um $4 \%$. Der Vergleich der Härtewerte der mehrlagigen Kehlnaht KN-03 und der einlagigen Naht 5-04 ist in Abbildung 9.16 dargestellt. Für die Verbindung S690-G89 zeigen sich Unterschiede im Bereich von 9 \%, wobei die Werte sich innerhalb der Naht stark ändern. Eindeutige Tendenzen lassen sich nicht ablesen.

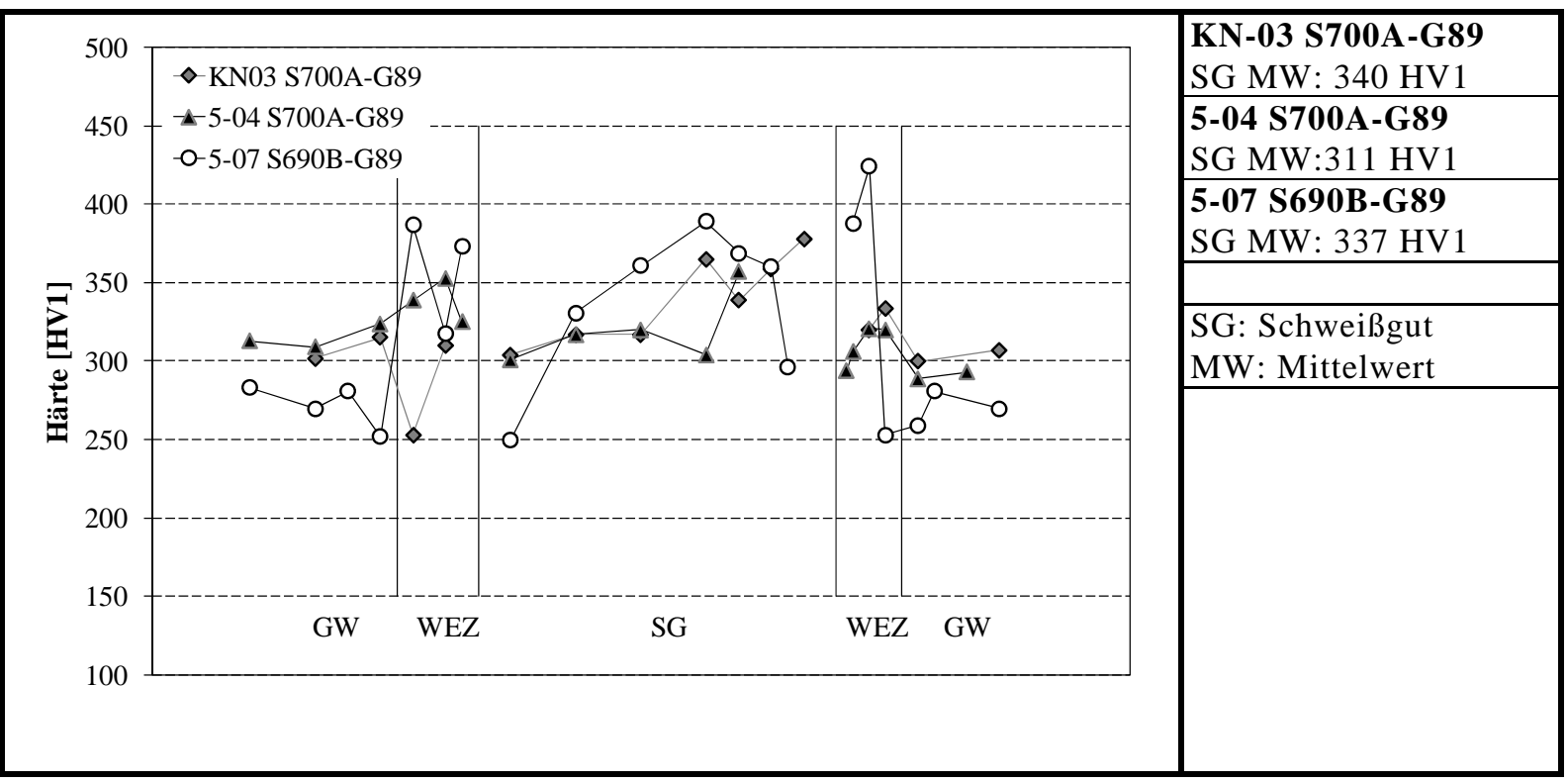

Abbildung 9.16: Härtemessung bei ein- und mehrlagigen Nähten: G89

Bei der Nachrechnung der Versuche 5-06 und 5-07 wurden die Materialkennwerte KN-02 und KN-03 verwendet, bei denen der Schweißzusatzwerkstoff aber nicht der Grundwerkstoff mit den Versuchen übereinstimmt.

Das Berechnungsergebnis des Versuchs 5-06 unterschätzt das Versuchsergebnis um 5 \%. Die verwendete Materialkennlinie KN-02 wurde mit dem anderen Grundwerkstoff S700A hergestellt. Der Vergleich der Härtewerte in Abbildung 9.15 zeigt, dass die Härtewerte deutlich oberhalb (17 \%) der Werte der KN-Probe liegen. Diese größeren Härtewerte deuten auf die höhere Festigkeit der Schweißnaht hin.

Für den Versuch 5-07 stimmen Berechnungsergebnis und Versuchsergebnis sehr gut überein. Abbildung 9.16 zeigt im Vergleich der Mittelwerte der Härtewerte zwischen der verwendeten mehrlagigen Kehlnaht KN-03 und der einlagigen Naht 5-07 eine sehr gute Übereinstimmung. Es ist jedoch zu beachten, dass die Härtewerte der Probe KN-03 im Bereich der Naht stark streuen.

\subsubsection{Parameterstudie zum Einfluss der Nahtlänge S690-G69}

Zum Einfluss der Nahtlänge wurde eine Parameterstudie durchgeführt. Auf Basis der Nahtgeometrie von Versuch 1-09 wurde bei ansonsten gleichen Parametern die Nahtlänge variiert. Die so ermittelten maximalen Kräfte sind für die Verhältnisse Nahtlänge L zu Nahtdicke a von 25, 50, 100 und 150 in Abbildung 9.17 dargestellt. Das Versagen tritt ein, wenn in der Naht die Vergleichsspannung erreicht wird. Man kennt einen linearen Anstieg der Tragfähigkeit mit der Nahtlänge. Die beiden Parameter korrelieren, d. h. es stellen sich keine anderen Effekte ein. Auch in den Versuchen wurde keine Abnahme der Tragfähigkeit mit zunehmender Länge festgestellt. Es ist also davon auszugehen, dass die Naht plastiziert und so Spannungsspitzen am Nahtanfang und Nahtende abgebaut werden können. 


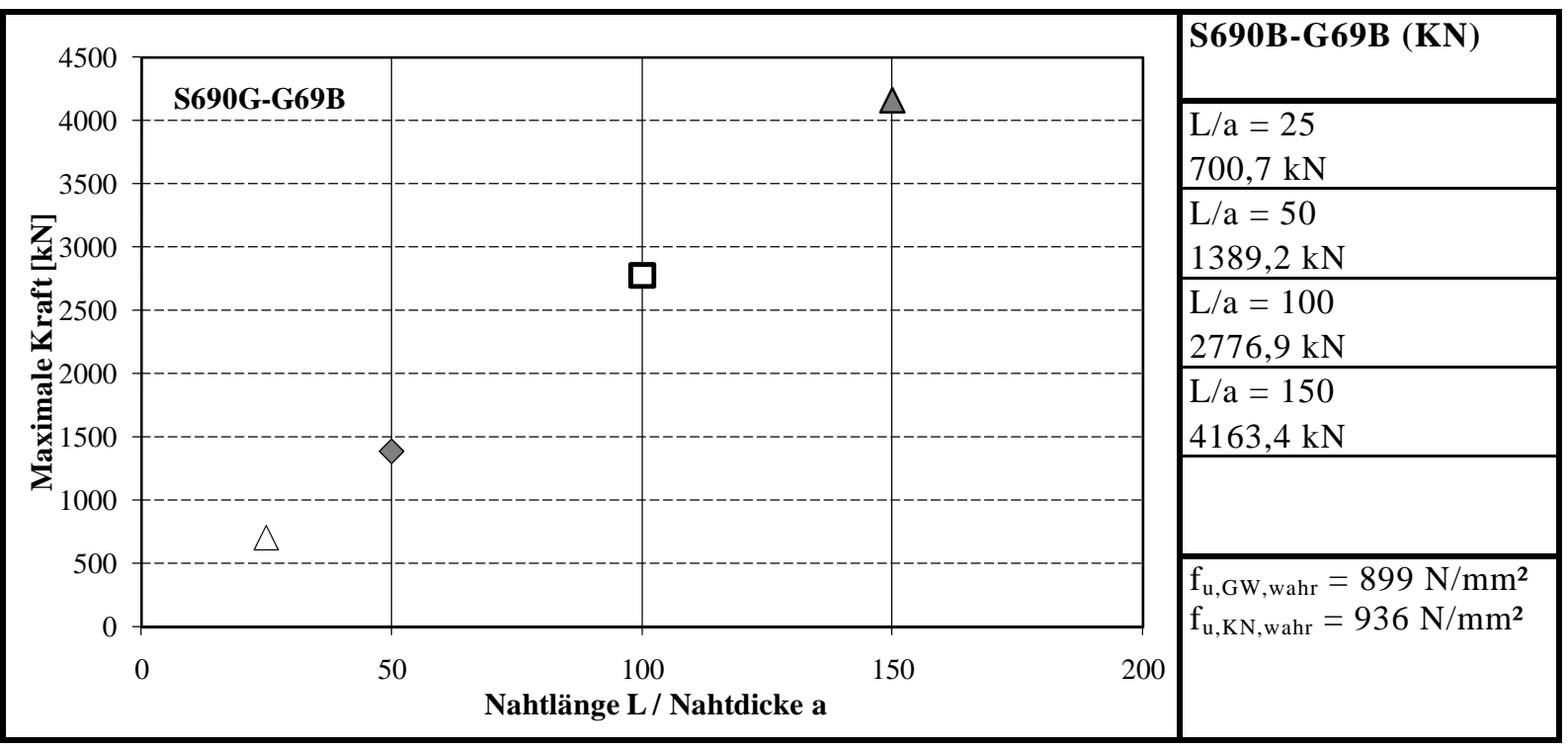

Abbildung 9.17:Einfluss der Nahtlänge auf die Tragfähigkeit der Verbindung

\subsubsection{Parameterstudie zum Einfluss von Nahtdicke (mehrlagig) und Blechdicke S690-G69}

Bei den mehrlagigen Kehlnähten stellen sich durch das Überschweißen Vergütungseffekte ein, die dazu führen können, dass in der Naht unterschiedliche Festigkeiten vorzufinden sind.

Zusätzlich wurden bei den Versuchen der Serie 4 Grundwerkstoffe anderer Blechdicken verwendet. Durch die größeren Blechdicken ändern sich die Schweißparameter, so dass sich für die Festigkeit in der mehrlagigen Naht Unterschiede zu den Festigkeiten der KN-Proben ergeben können,

Aus diesem Grund wird auf Berechnungen zum Einfluss der Blechdicke verzichtet.

\subsubsection{Zusammenfassung Flankenkehlnahtverbindungen}

In diesem Kapitel wurden die Versuchsnachrechnungen an einlagigen Flankenkehlnahtverbindungen vorgestellt.

Die numerisch berechneten Traglasten stimmen gut mit den Traglasten aus den Versuchen überein.

Bei den Versuchen mit dem Grundwerkstoff S700A, bei denen die Festigkeit der Schweißnaht über die KN-Proben mit diesem Grundwerkstoff und den entsprechenden Schweißzusatzwerkstoffen bestimmt wurde (1-01, Z5-01 und 5-06), sind Abweichungen kleiner als 4,5 \% zu beobachten. Mithilfe der durchgeführten Härtemessungen konnten die Abweichungen erklärt werden.

Bei den Versuchen mit dem Grundwerkstoff S690B stellen sich Abweichungen bis zu 5 \% ein, die immer über die Härtewerte begründet werden konnten. Dabei handelte es sich um Versuche, von deren Werkstoffkombinationen nicht die zugehörigen Zugproben aus Kehlnähten vorhanden waren (1-08, 506, 5-07). Nur im Versuch 1-09 lagen die entsprechenden Werkstoffkennwerte aus der mehrlagigen Kehlnaht vor, da lag die Übereinstimmung bei 1,00.

Bei dem Versuch mit dem Grundwerkstoff S460 und einer Vorwärmtemperatur t $=100^{\circ} \mathrm{C}$ konnte eine sehr gute Übereinstimmung festgestellt werden.

Bei den Versuchen der Serie 2 und 7, bei denen die Vorwärmtemperatur geändert wurde, hatte die Vorwärmtemperatur einen Einfluss auf die Tragfähigkeit. Dieser Parameter wurde numerisch nicht abgebildet, weil die verwendeten Werkstoffkennwerte aus Versuchen mit einer anderen Vorwärmtemperatur stammten. Über die Ergebnisse der Härtemessungen konnten diese Unterschiede erklärt werden. 


\subsection{Berechnungen zu Kreuzstößen}

Im Rahmen einer Diplomarbeit [Breunig, 2011] wurden numerische Berechnungen zu Kreuzstößen durchgeführt.

Exemplarisch dargestellt ist das Berechnungsergebnis von Versuch 6-01/4. Bei diesem Versuch handelt es sich um die Werkstoffkombination S700A-G69A. Numerisch wurden die Werkstoffkennwerte nach Tabelle 9.1 mit dem Grundwerkstoff S700A $\left(f_{u, w a h r}=960 \mathrm{~N} / \mathrm{mm}^{2}\right)$ und KN-01 $\left(\mathrm{f}_{\mathrm{u} \text {,wahr }}=\right.$ $898 \mathrm{~N} / \mathrm{mm}^{2}$ ) verwendet. Das numerische Modell ist gemeinsam mit den Vergleichsdehnungen beim Erreichen der Traglast in Abbildung 9.18 dargestellt. Das numerische Modell berücksichtigt die Geometrie der Nähte und auch die Exzentrizitäten im Versuchskörper, wie den Kantenversatz und den Spalt. Im Rahmen der Diplomarbeit [Breunig, 2011] wurde numerisch ein Einfluss der Exzentrizitäten auf die Tragfähigkeit nachgewiesen. Die Exzentrizitäten führen dazu, dass beide Nähte nicht gleichzeitig versagen.

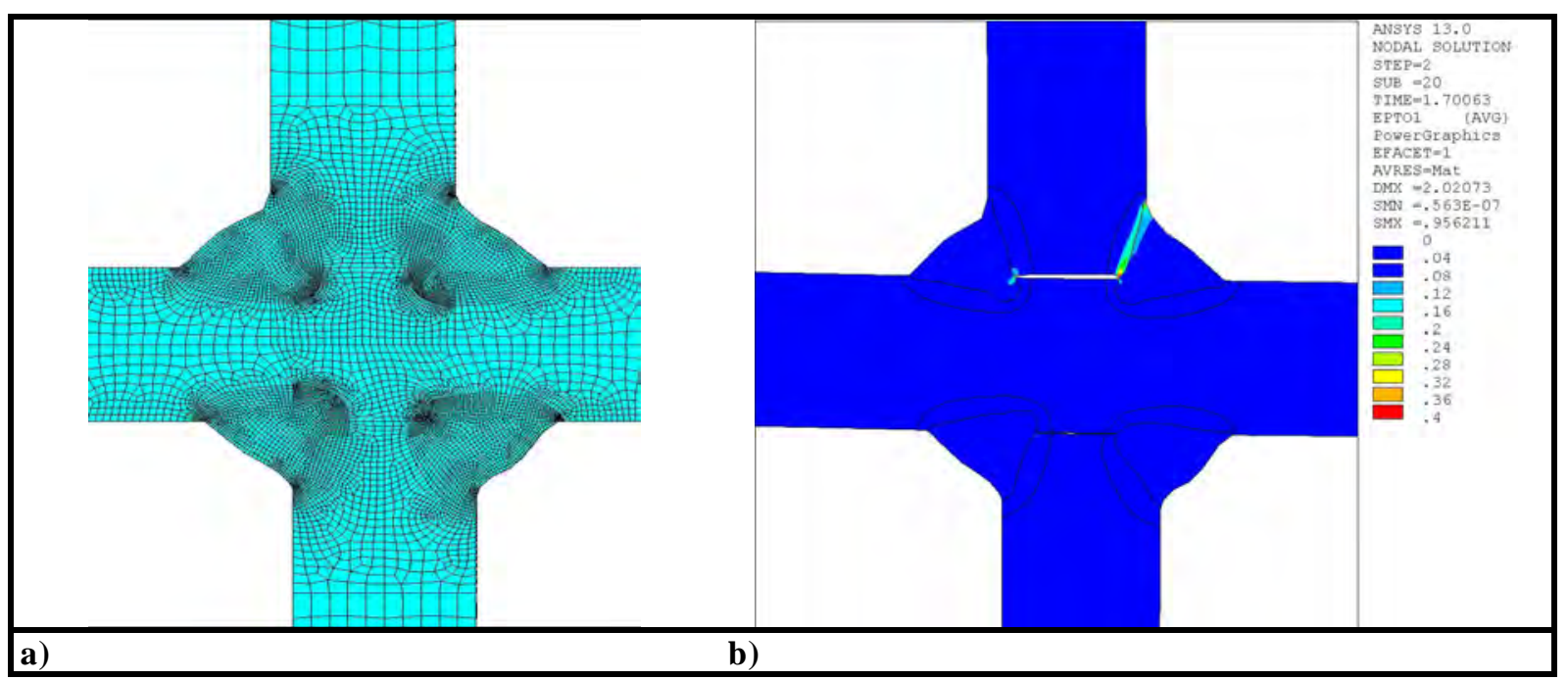

Abbildung 9.18: a) Modell, b) Vergleichsdehnung bei Erreichen der Traglast

In Abbildung 9.18 b) zeigt die Darstellung der Vergleichsdehnung das Versagen der Naht. Durch die Dehnung zeigt sich die Versagensfläche, die sehr steil ist und im Bereich der Wärmeeinflusszone verläuft. Man erkennt auch, dass die beiden Nähte nicht gleichzeitig versagen.

Numerisch konnte gezeigt werden, dass das Versagen bei den Kreuzstößen nicht wie bei den Flankenkehlnähten unter einem Winkel von $45^{\circ}$ eintritt, sondern dass sich ein steilerer Winkel ergibt. Ein Versagen in der Wärmeeinflusszone kann also nicht ausgeschlossen werden. Das Werkstoffverhalten in der Wärmeeinflusszone variiert stark. Aus diesem Grund wird in dieser Arbeit darauf verzichtet, die Versuche an Kreuzstößen numerisch nachzubilden.

\subsection{Zusammenfassung und Fazit}

Es wurden numerische Untersuchungen durchgeführt, um das Tragverhalten der Versuche zu erklären.

Berechnungen haben gezeigt, dass es möglich ist, mithilfe des Makroschliffes und der Bruchfläche die Geometrie des Versuchskörpers sinnvoll abzubilden.

Mit weiteren numerischen Untersuchungen konnte der Einfluss der Werkstofffestigkeiten geklärt werden. Variationen in der Festigkeit des Grundwerkstoffs, der Wärmeeinflusszone und der Naht haben ergeben, dass nur die Festigkeit des Schweißnahtvolumens die numerische Tragfähigkeit der Flankenkehlnahtverbindung beeinflusst, während die Festigkeit des Grundwerkstoffs und der Wärmeeinflusszone nur eine untergeordnete Rolle spielen.

Zur werkstofftechnischen Abbildung der Naht standen die Ergebnisse von Zugversuchen am reinen Schweißgut und Zugversuche, die aus mehrlagigen Kehlnähten gefertigt wurden, zur Verfügung. Die Berechnungen mit diesen unterschiedlichen Zugfestigkeiten haben eindeutig ergeben, dass mit der 
Zugfestigkeit aus der Kehlnaht Traglasten erreicht werden, die denen aus dem Versuch sehr ähnlich sind.

Die numerischen Betrachtungen zum Tragverhalten haben gezeigt, dass ein Plastizieren der Naht eintritt. Die Kraft kann nicht weiter gesteigert werden, wenn die Vergleichsspannung in der Naht erreicht ist. Der Vergleich der Kraft-Verformungs-Linien zeigt, dass das Tragverhalten gut abgebildet wird. Es tritt kein vorzeitiges Versagen durch Dehnungsüberschreitungen auf.

Zusammenfassend lässt sich sagen, dass durch genaue Modellierung der Geometrie der Schweißnaht und genaue Kenntnis der Festigkeit im Volumen der Naht die numerische Berechnung der Traglast möglich ist.

Als allgemeine Schlussfolgerung zur numerischen Modellierung von Kehlnahtverbindungen lässt sich festhalten, dass die Tragfähigkeit der Kehlnahtverbindung bestimmt werden kann, in dem für die Werkstoffkennwerte der Naht anstelle der Festigkeit des Grundwerkstoffs die Kehlnahtfestigkeit verwendet wird. Ohne genauere Angaben kann diese Kehlnahtfestigkeit als Quotient aus der Festigkeit des eingesetzten Schweißzusatzwerkstoffs dividiert durch den zugehörigen Beiwert $\beta_{\mathrm{w}}$ bestimmt werden. 


\section{Entwicklung eines Bemessungsvorschlags}

\subsection{Allgemeines}

In diesem Kapitel werden die bisher vorgestellten Untersuchungen zusammengeführt und auf Grundlage der Ergebnisse ein Bemessungsvorschlag entwickelt.

Um einen Vergleich der Versuchsergebnisse der verschiedenen Versuchsserien zu ermöglichen, werden die Werkstoffe, die für die Versuche verwendeten wurden, in die heute gültigen Normen eingeordnet.

Die Versuchsergebnisse, die in Kapitel 6 bis 8 vorgestellt wurden, haben unterschiedliche Versuchskörperformen und wurden mit unterschiedlichen Verfahren gefertigt. In diesem Kapitel wird daher beurteilt, inwieweit die Versuchsergebnisse der einzelnen Projekte vergleichbar sind. Die verwendeten Ergebnisse wurden im Rahmen des FOSTA/AiF-Forschungsprojekts „Wirtschaftliche Schweißverbindungen höherfester Baustähle“ [FOSTA P652, 2008], außerdem als eigene Zusatzuntersuchungen zu diesem Projekt [Zusatzversuche, 2008] und als Anschlussprojekt im Rahmen des FOSTA/AiFForschungsprojekts „Tragfähigkeit von Kehlnahtverbindungen höherfester Baustähle S690 im Stahlbau“ [FOSTA P812, 2011] ermittelt.

Diese eigenen Versuche und die in Kapitel 5 vorgestellten bekannten Versuche aus der Literatur mit gleichen Grund- und Schweißzusatzwerkstoffen werden gemeinsam statistisch ausgewertet. Daraus wird ein Verbesserungsvorschlag entwickelt für die Tragfähigkeit von Kehlnähten mit gleichen Grund- und zugehörigen Schweißzusatzwerkstoffen für die Grundwerkstoffe S460 und S690 nach EN 1993-1-8 (2005) und EN 1993-1-12 (2007).

Darauf aufbauend wird ein eigener Bemessungsansatz vorgestellt, der die Tragfähigkeit in Abhängigkeit von Grund- und Schweißzusatzwerkstoff beurteilt.

\subsection{Einordnung der verwendeten Werkstoffe}

Die für die Versuche aus der Literatur und für die eigenen Versuche verwendeten Werkstoffe werden an dieser Stelle zusammengefasst und in die heute gültigen Normen eingeordnet. In Tabelle 10.1 sind die verwendeten Werkstoffe aufgeführt. Für jeden Grund- und Schweißzusatzwerkstoff sind die normative Mindeststreckgrenze und der zulässige Bereich für die Zugfestigkeit angegeben.

Für die Stähle erfolgt eine Einordnung mit Hilfe von Streckgrenze und Zugfestigkeit nach DIN EN 10025-2 (2005), DIN EN 10025-4 (2005), DIN EN 10025-6 (2005) und DIN EN 10149-2 (1995). Die Schweißzusatzwerkstoffe werden nach DIN EN ISO 14341 (2008), DIN EN ISO 16834 (2007), DIN EN ISO 17632 (2008), DIN EN ISO 18276 (2006), DIN EN ISO 2560 (2006) und DIN EN 757 (1997) eingeordnet.

Anhand dieser Angaben werden die verwendeten Grundwerkstoffe in Tabelle 10.2 und die verwendeten Schweißzusatzwerkstoffe in Tabelle 10.3 der verschiedenen Versuchsserien eingeordnet.

Tabelle 10.1: Bereiche für die Streckgrenze und Zugfestigkeit der betrachteten Werkstoffe

\begin{tabular}{|c|c|c|c|c|c|c|}
\hline Grundwerkstoff & $\begin{array}{l}\text { Dicke } \\
{[\mathrm{mm}]}\end{array}$ & \begin{tabular}{|l} 
Streck- \\
grenze \\
{$\left[\mathrm{N} / \mathrm{mm}^{2}\right]$}
\end{tabular} & $\begin{array}{l}\text { Zugfes- } \\
\text { tigkeit } \\
{\left[\mathrm{N} / \mathrm{mm}^{2}\right]}\end{array}$ & $\begin{array}{l}\text { Schweißzusatz- } \\
\text { werkstoff }\end{array}$ & $\begin{array}{l}\begin{array}{l}\text { Streck- } \\
\text { grenze } \\
{\left[\mathrm{N} / \mathrm{mm}^{2}\right]}\end{array} \\
\end{array}$ & $\begin{array}{l}\text { Zugfestig- } \\
\text { keit } \\
{\left[\mathrm{N} / \mathrm{mm}^{2}\right]}\end{array}$ \\
\hline \multirow{2}{*}{ S355 } & $t \leq 16$ & 355 & \multirow{2}{*}{$510-680$} & G42 / E42 & 420 & $500-640$ \\
\hline & $16<\mathrm{t} \leq 40$ & 345 & & G46 / T46 / E46 & 460 & $530-680$ \\
\hline \multirow{4}{*}{$\mathrm{S} 460 \mathrm{M} / \mathrm{ML}$} & $t \leq 16$ & 460 & \multirow{2}{*}{$540-720$} & E55 & 550 & $640-820$ \\
\hline & $16<\mathrm{t} \leq 40$ & 440 & & G69 / T69 & 690 & $770-940$ \\
\hline & $40<\mathrm{t} \leq 63$ & 430 & $530-710$ & G89 & 890 & $940-1180$ \\
\hline & $63<t \leq 80$ & 410 & $510-690$ & & & \\
\hline S690Q/QL/QL1 & $t \leq 50$ & 690 & $770-940$ & & & \\
\hline \multirow{2}{*}{ S700 } & $t \leq 8$ & 700 & $750-950$ & & & \\
\hline & $8<\mathrm{t} \leq 16$ & 680 & $750-950$ & & & \\
\hline
\end{tabular}


Tabelle 10.2: Einordnung der Grundwerkstoffe der Versuchsserien

\begin{tabular}{|c|c|c|c|}
\hline Grundwerkstoff & Streckgrenze & Zugfestigkeit & Heutige Be- \\
\hline Herkunft & {$\left[\mathrm{N} / \mathrm{mm}^{2}\right]$} & {$\left[\mathrm{N} / \mathrm{mm}^{2}\right]$} & zeichnung \\
\hline $\begin{array}{l}{[\text { Kob et al., 1987] } t=10 \mathrm{~mm}} \\
\mathrm{t}=20 \mathrm{~mm}, \mathrm{t}=30 \mathrm{~mm}\end{array}$ & \begin{tabular}{|l|}
$375-379$ \\
$339-387$ \\
\end{tabular} & \begin{tabular}{|l}
$550-555$ \\
$535-552$ \\
\end{tabular} & S355J0 \\
\hline$[$ Kob et al., 1987] t=10, $20 \mathrm{~mm}$ & $774-812$ & $826-854$ & S690Q \\
\hline$[$ Niemi, 1988] $\mathrm{t}=8 \mathrm{~mm}$ & $618-660$ & $738-759$ & S640*) \\
\hline $\begin{array}{l}{[\text { Saal, 2005] t }=20,30 \mathrm{~mm}, 31 \mathrm{~mm}} \\
\mathrm{t}=53 \mathrm{~mm} \\
\mathrm{t}=77 \mathrm{~mm}\end{array}$ & $\begin{array}{l}538-559 \\
515 \\
514 \\
\end{array}$ & $\begin{array}{l}614-671 \\
620 \\
652\end{array}$ & S460ML \\
\hline $\begin{array}{l}{[\text { Background D.03, 1990] } \mathrm{t}=10 \mathrm{~mm},} \\
\mathrm{t}=20 \mathrm{~mm} / \mathrm{t}=28 \mathrm{~mm} / \mathrm{t}=40 \mathrm{~mm}\end{array}$ & - & $\begin{array}{l}613 \\
702-725\end{array}$ & S460 \\
\hline [Collin, Johansson, 2005] W, t $=10 \mathrm{~mm}$ & 789 & 833 & S690QL \\
\hline [Collin, Johansson, 2005] D, t = $10 \mathrm{~mm}$ & 779 & 827 & S650MC \\
\hline [FOSTA P652, 2008] S355J2 t = $15 \mathrm{~mm}$ & 422 & 577 & S355J2 \\
\hline $\begin{array}{l}\text { [FOSTA P652, 2008] S460M t }=15 \mathrm{~mm} \\
\text { S460ML t }=50 \mathrm{~mm}\end{array}$ & $\begin{array}{l}466 \\
505 \\
\end{array}$ & $\begin{array}{l}621 \\
568 \\
\end{array}$ & $\begin{array}{l}\text { S460M } \\
\text { S460ML }\end{array}$ \\
\hline $\begin{array}{l}{[\text { FOSTA P652, 2008] S690Q } \mathrm{t}=15 \mathrm{~mm}} \\
\text { S690Q } \mathrm{t}=20 \mathrm{~mm} \\
\text { S690QL } \mathrm{t}=50 \mathrm{~mm}\end{array}$ & $\begin{array}{l}830 \\
832 \\
797 \\
\end{array}$ & $\begin{array}{l}871 \\
876 \\
871 \\
\end{array}$ & $\begin{array}{l}\text { S690Q } \\
\text { S690Q } \\
\text { S690QL } \\
\end{array}$ \\
\hline [Zusatzversuche, 2008] S690Q t = $20 \mathrm{~mm}$ & 767 & 797 & S690Q \\
\hline [Zusatzversuche, 2008] S355J2 t = $15 \mathrm{~mm}$ & 422 & 577 & S355J2 \\
\hline [FOSTA P812, 2011] S460M t $=17,6 \mathrm{~mm}$ & 463 & 538 & S460M \\
\hline $\begin{array}{l}\text { [FOSTA P812, 2011] S690QL1 } \mathrm{t}=15 \mathrm{~mm} \\
\text { S690Q } \mathrm{t}=20 \mathrm{~mm} \\
\text { S690Q } \mathrm{t}=50 \mathrm{~mm}\end{array}$ & $\begin{array}{l}809 \\
760 \\
796\end{array}$ & $\begin{array}{l}848 \\
811 \\
849\end{array}$ & $\begin{array}{l}\text { S690QL1 } \\
\text { S690Q } \\
\text { S690Q }\end{array}$ \\
\hline $\begin{array}{l}\text { [FOSTA P812, 2011] S700MC t }=15 \mathrm{~mm} \\
\mathrm{t}=20 \mathrm{~mm} \\
\mathrm{t}=50 \mathrm{~mm}\end{array}$ & $\begin{array}{l}876 \\
851 \\
796\end{array}$ & \begin{tabular}{|l|}
891 \\
876 \\
849 \\
\end{tabular} & $\begin{array}{l}\text { S700MC } \\
\text { S700MC } \\
\text { S700MC }\end{array}$ \\
\hline
\end{tabular}

Tabelle 10.3: Einordnung der Schweißzusatzwerkstoffe der Versuchsserien

\begin{tabular}{|c|c|c|c|}
\hline Schweißzusatzwerkstoff & Streckgrenze & Zugfestigkeit & Heutige Bezeich- \\
\hline Herkunft & {$\left[\mathrm{N} / \mathrm{mm}^{2}\right]$} & {$\left[\mathrm{N} / \mathrm{mm}^{2}\right]$} & nung \\
\hline \multirow{2}{*}{ [Kob et al., 1987] } & 503 & 590 & G42 \\
\hline & 480 & 577 & G42 \\
\hline \multirow{2}{*}{ [Niemi, 1988] } & 637 & 739 & E55 \\
\hline & 767 & 849 & -- \\
\hline [Saal, 2005] & 582 & 618 & T46 \\
\hline [Background D.03, 1990] & 550 & 620 & E55*) \\
\hline [Collin, Johansson, 2005] W & 619 & 758 & -- \\
\hline [Collin, Johansson, 2005] D & 462 & 548 & E42 \\
\hline \multirow{5}{*}{ [FOSTA P652, 2008] } & 487 & 583 & G42 \\
\hline & $\begin{array}{l}547 \\
525\end{array}$ & $\begin{array}{l}639 \\
595\end{array}$ & G46 \\
\hline & 568 & 602 & T46 \\
\hline & 724 & 836 & G69 \\
\hline & 738 & 794 & T69 \\
\hline \multirow{2}{*}{ [Zusatzversuche, 2008] } & 724 & 836 & G69 \\
\hline & 931 & 981 & G89 \\
\hline \multirow[t]{4}{*}{ [FOSTA P812, 2011] } & $\begin{array}{l}739 \\
682\end{array}$ & $\begin{array}{l}798 \\
767\end{array}$ & G69 \\
\hline & 583 & 602 & G46 \\
\hline & 775 & 834 & T69 \\
\hline & 907 & 1028 & G89 \\
\hline
\end{tabular}




\subsection{Beurteilung der Versuchsergebnisse der Flankenkehlnähte}

\subsubsection{Einleitung}

In den Kapiteln 6, 7 und 8 sind eigene Versuche zur Bestimmung der Tragfähigkeit von Flankenkehlnahtverbindungen vorgestellt worden. Im Folgenden wird beurteilt, in wieweit die Ergebnisse der Traglastuntersuchungen vergleichbar sind.

\subsubsection{Flankenkehlnahtverbindungen gleicher Grund- und zugehöriger Schweiß- zusatzwerkstoffe}

Die Ergebnisse der Untersuchungen an Schweißverbindungen mit gleichen Grund- und zugehörigen Schweißzusatzwerkstoffen sind in Abbildung 10.1 dargestellt. In Abhängigkeit der nominellen Mindestzugfestigkeit der Grundwerkstoffe nach Tabelle 10.1 sind die maximalen Spannungen $\tau_{\text {II }}$ aufgetragen.

Für die Verbindungen mit dem Grundwerkstoff S460 und zugehörigem Schweißzusatzwerkstoff zeigt Abbildung 10.1 den Vergleich der Versuchsergebnisse an manuell hergestellten Versuchskörpern (P652 S460 G/T/E46) mit den Versuchsergebnissen an vollmechanisiert geschweißten Versuchskörpern (P812 S460-G46). Für beide Arten von Versuchskörpern ergeben sich die gleichen Mittelwerte. Infolge einer unterschiedlichen Anzahl an Versuchen und Unterschieden in den untersuchten Parametern ist ein Vergleich der Streuung schwierig. Es wird davon ausgegangen, dass die Maximalspannungen vergleichbar sind.

Für den Vergleich der Verbindungen mit dem Grundwerkstoff S690 und zugehörigem Schweißzusatzwerkstoff stehen Versuchsergebnisse von manuell hergestellten Versuchskörpern (P652 S690G/T69) und von vollmechanisiert geschweißten Versuchskörpern aus zwei Projekten (P812 S690G/T69 und Zusatzversuche S690-G69) zur Verfügung. Die Mittelwerte der Versuchsergebnisse aus dem Projekt P812 liegen um ca. 4 \% höher als die Ergebnisse des Vorgängerprojekts P652. Die Versuchsergebnisse der vollmechanisiert geschweißten Versuchskörper aus beiden Projekten sind sehr ähnlich. Auch hier wird angenommen, dass die Versuchsergebnisse vergleichbar sind.

Für die statistische Auswertung wird davon ausgegangen, dass die Versuche trotz unterschiedlicher Fertigung und Versuchskörperform vergleichbar sind. Das heißt, alle Versuche werden als Grundgesamtheit für die statistische Auswertung herangezogen. Betrachtet man die Mittelwerte aller Verbindungen S460-G/T/E46 mit allen Verbindungen S690-G/T69, so lässt sich eine Steigerung der Tragfähigkeit von S460 zu S690 von etwa $11 \%$ feststellen.

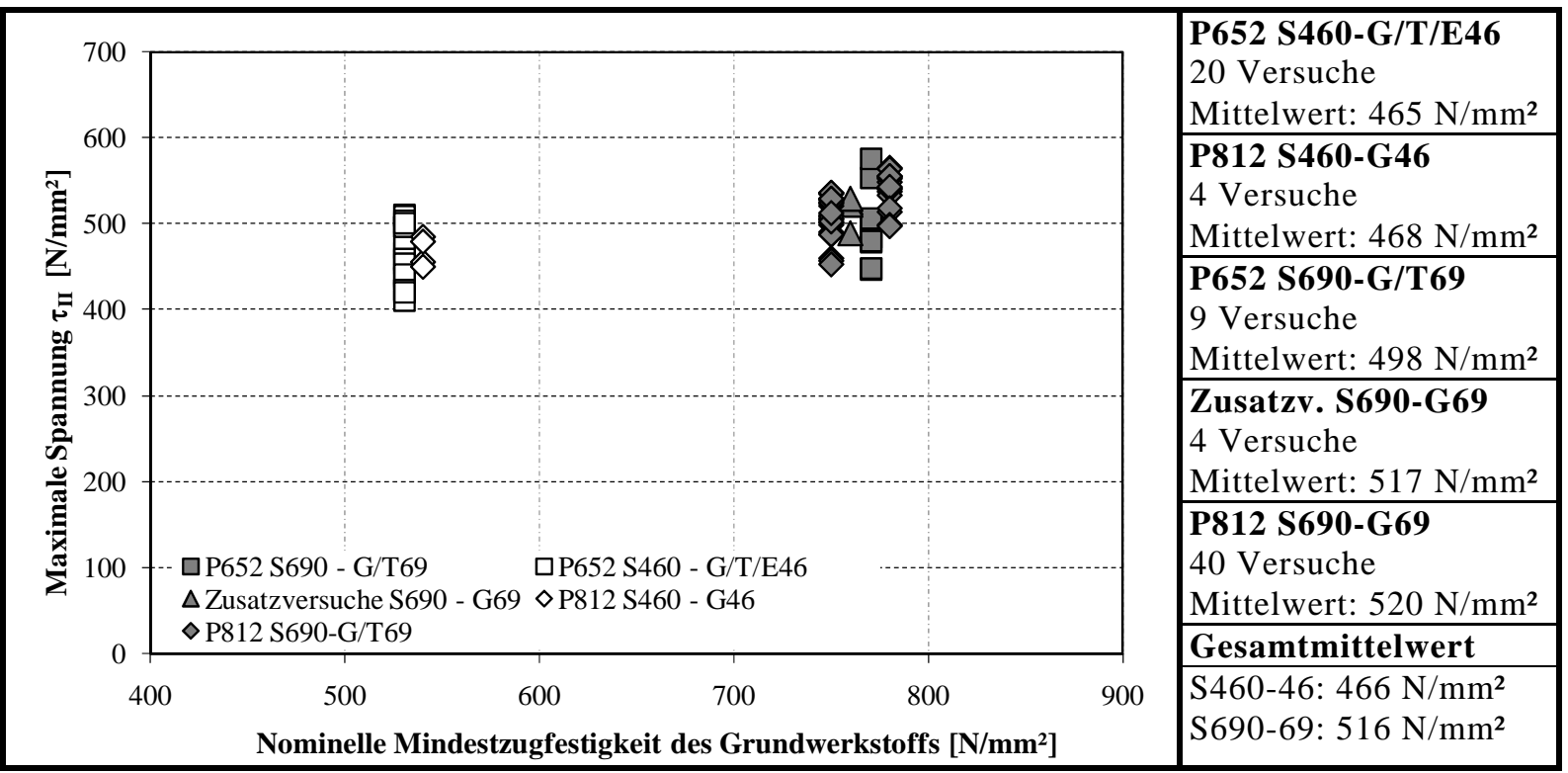

Abbildung 10.1: Vergleich der Ergebnisse der Flankenkehlnahtverbindungen gleicher Grund- und zugehöriger Schweißzusatzwerkstoffe S460 und S690 


\subsubsection{Flankenkehlnahtverbindungen gleicher Grund- und unterschiedlicher Schweißzusatzwerkstoffe}

Für die Flankenkehlnahtverbindungen mit Schweißzusatzwerkstoffen, die eine andere Festigkeit als die Grundwerkstoffe besitzen, wird ermittelt, in wieweit die Ergebnisse der verschiedenen Projekte (vgl. Kapitel 6 bis 8) zu gleichen Ergebnissen geführt haben. Da Versuche mit nominell gleichen Grundwerkstoffen verglichen werden, sind die maximalen Spannungen $\tau_{\text {II }}$ von insgesamt 16 Versuchen in Abbildung 10.2 in Abhängigkeit der nominellen Mindestzugfestigkeit des Schweißzusatzwerkstoffs dargestellt.

Für die Verbindungen mit dem Grundwerkstoff S690 und dem Schweißzusatzwerkstoff G46 steht nur ein Versuchsergebnis an manuell hergestellten Versuchskörpern (P652 S690-G46) und fünf Versuchsergebnisse an vollmechanisiert hergestellten Versuchskörpern (P812 S690-G46) zur Verfügung. Die Mittelwerte der maximalen Spannungen $\tau_{\text {II }}$ aus dem Projekt P812 liegen deutlich höher (ca. $15 \%$ ) als die Tragfähigkeiten im Vorgängerprojekt P652. Eine Beurteilung der Vergleichbarkeit ist aufgrund der geringen Versuchsanzahl schwierig.

Für die Verbindungen mit dem Grundwerkstoff S690 und dem Schweißzusatzwerkstoff G89 stehen vollmechanisiert geschweißte Versuchskörper aus zwei Projekten zur Verfügung. Aus dem Projekt P812 stehen mit der Werkstoffkombination S690-G89 drei Versuche mit einlagigen Nähten und dem Stahl S700A und zwei Versuche mit einlagigen Nähten und dem Grundwerkstoff S690B zur Verfügung. Aus den Zusatzversuchen mit der Werkstoffkombination S700A-G89 sind zwei Versuche mit einlagigen Nähten und zwei Versuche mit 2-lagigen (3-raupigen) Nähten vorhanden.

Der Vergleich der Mittelwerte der 5 Versuchsergebnisse aus dem Projekt P812 und der 4 Versuchsergebnisse der Zusatzversuche zeigt für die Versuchsergebnisse aus dem Projekt P812 um ca. 6 \% höhere Tragfähigkeiten. Vergleicht man jedoch in beiden Projekten jeweils nur die Versuchsergebnisse von Versuchen mit einlagigen Nähten und dem Grundwerkstoff S700A, ergeben sich ähnliche Mittelwerte von $572 \mathrm{~N} / \mathrm{mm}^{2}$ (Zusatzversuche) und $575 \mathrm{~N} / \mathrm{mm}^{2}$ (P812). Damit sieht man, dass die Ergebnisse vergleichbar sind.

Abweichungen, die sich zwischen den Versuchsergebnissen der verschiedenen Projekte feststellen lassen, treten vor allem zwischen den manuell und den vollmechanisiert geschweißten Versuchskörpern auf. So weisen die manuell gefertigten Versuchskörper eine geringere Tragfähigkeit auf als die vollmechanisiert geschweißten Versuchskörper. Im Weiteren wird dennoch auf diese Versuche zurückgegriffen.

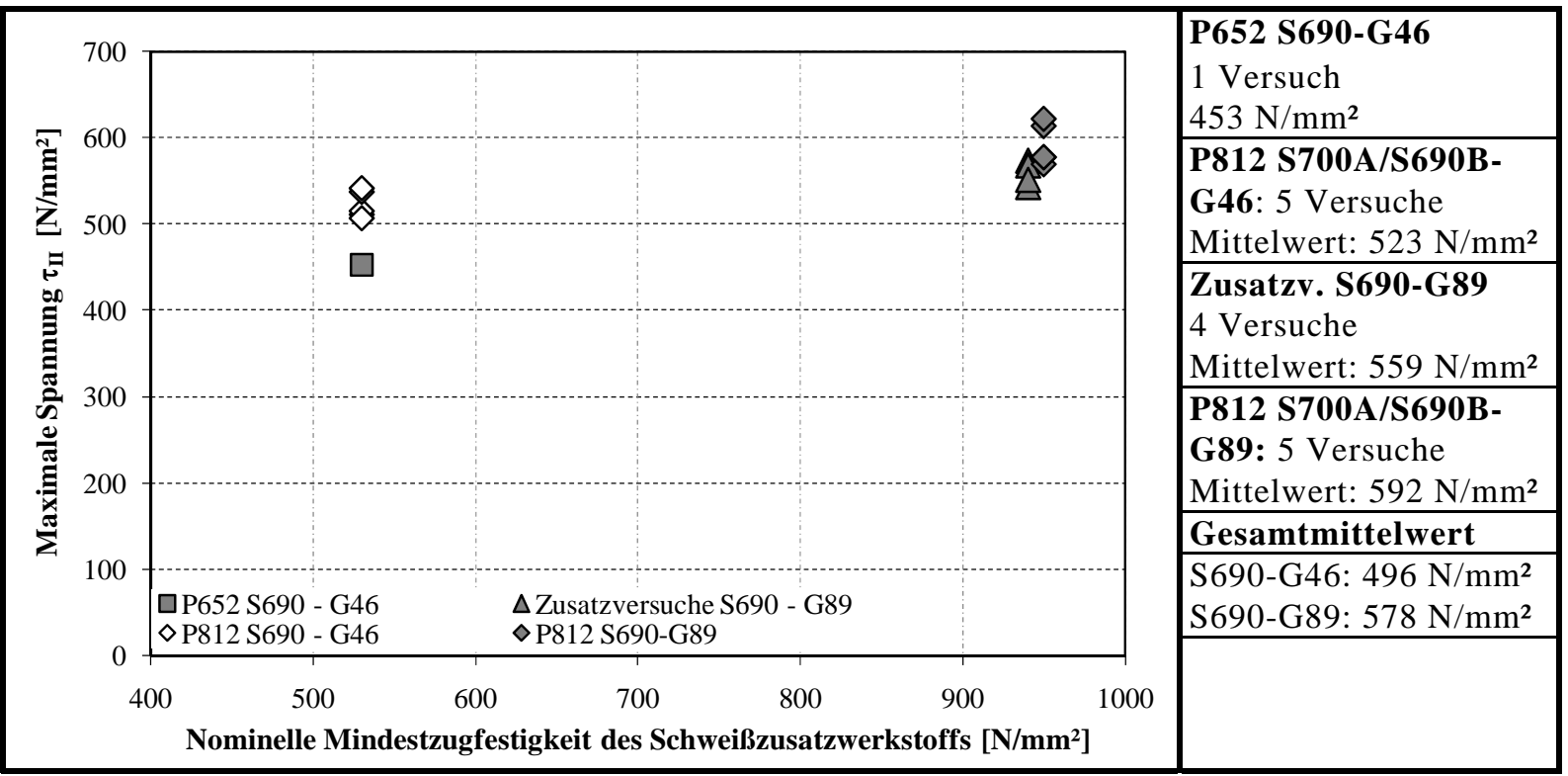

Abbildung 10.2: Vergleich der Ergebnisse der Flankenkehlnahtverbindungen unterschiedlicher Grundund Schweißzusatzwerkstoffe S460 und S690 


\subsection{Beurteilung der Versuchsergebnisse der Kreuzstöße}

\subsubsection{Einleitung}

Die Versuchsergebnisse an Kreuzstößen, die in den Kapiteln 6 und 8 vorgestellt wurden, werden in diesem Abschnitt analysiert. Das Ziel dieser Analyse ist eine Einschätzung, ob die Versuche gemeinsam ausgewertet werden können. Die Versuchsergebnisse werden in Abhängigkeit der Nahtfläche $A_{E C}$ verglichen. Ein Vergleich auf Basis der Nahtfläche $A_{\text {Bruch }}$ ist nicht möglich, da diese Werte nicht für alle Serien zur Verfügung stehen.

\subsubsection{Kreuzstöße gleicher Grund- und zugehöriger Schweißzusatzwerkstoffe}

In Abbildung 10.3 werden die in Kapitel 6 und 8 vorgestellten Ergebnisse von Kreuzstößen gleicher Grund- und zugehöriger Schweißzusatzwerkstoffe verglichen. Dargestellt sind die maximalen Spannungen $\sigma_{\mathrm{EC}}$ in Abhängigkeit der nominellen Zugfestigkeit des Grundwerkstoffs. Für die Verbindung S690-G69 sind Versuchsergebnisse an manuell und vollmechanisiert hergestellten Versuchskörpern vorhanden. Es zeigen sich große Streuungen der einzelnen Versuchsserien. Der Vergleich der Mittelwerte zeigt deutliche Unterschiede. Für die Werkstoffkombination S460-G46 steht nur eine Versuchsserie zur Verfügung. Betracht man die Mittelwerte der Verbindungen S460-46 und S690-69, so lässt sich für die untersuchten Werkstoffe eine Steigerung von 6 \% von S460 zu S690 feststellen.

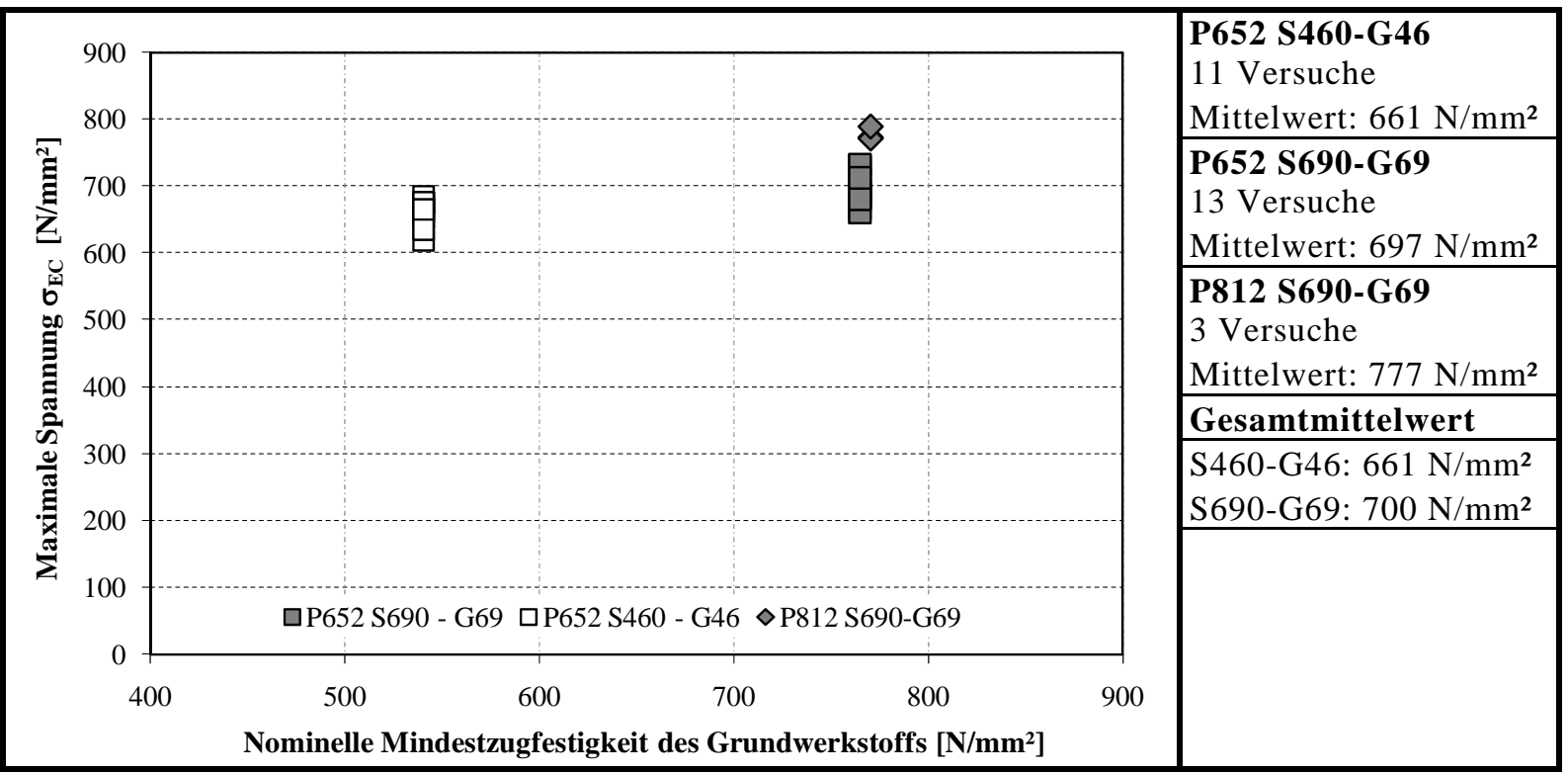

Abbildung 10.3: Vergleich der Ergebnisse der Kreuzstöße S460 und S690 


\subsubsection{Kreuzstöße gleicher Grund- und unterschiedlicher Schweißzusatzwerk- stoffe}

In Abbildung 10.4 ist der Vergleich der Versuchsergebnisse von Kreuzstößen gleicher Grund- und unterschiedlicher Schweißzusatzwerkstoffe dargestellt. Der Vergleich erfolgt in Abhängigkeit der nominellen Mindestzugfestigkeit des Schweißzusatzwerkstoffs, da die nominelle Mindestzugfestigkeit des Grundwerkstoffs bei allen Versuchen gleich ist. Für die Verbindungen der Werkstoffkombination S690-G46 unterscheiden sich die Mittelwerte der maximalen Spannungen $\sigma_{\mathrm{EC}}$ um etwa $8 \%$. Für die Verbindungen mit dem Schweißzusatzwerkstoff G89 steht nur eine Versuchsserie zur Verfügung.

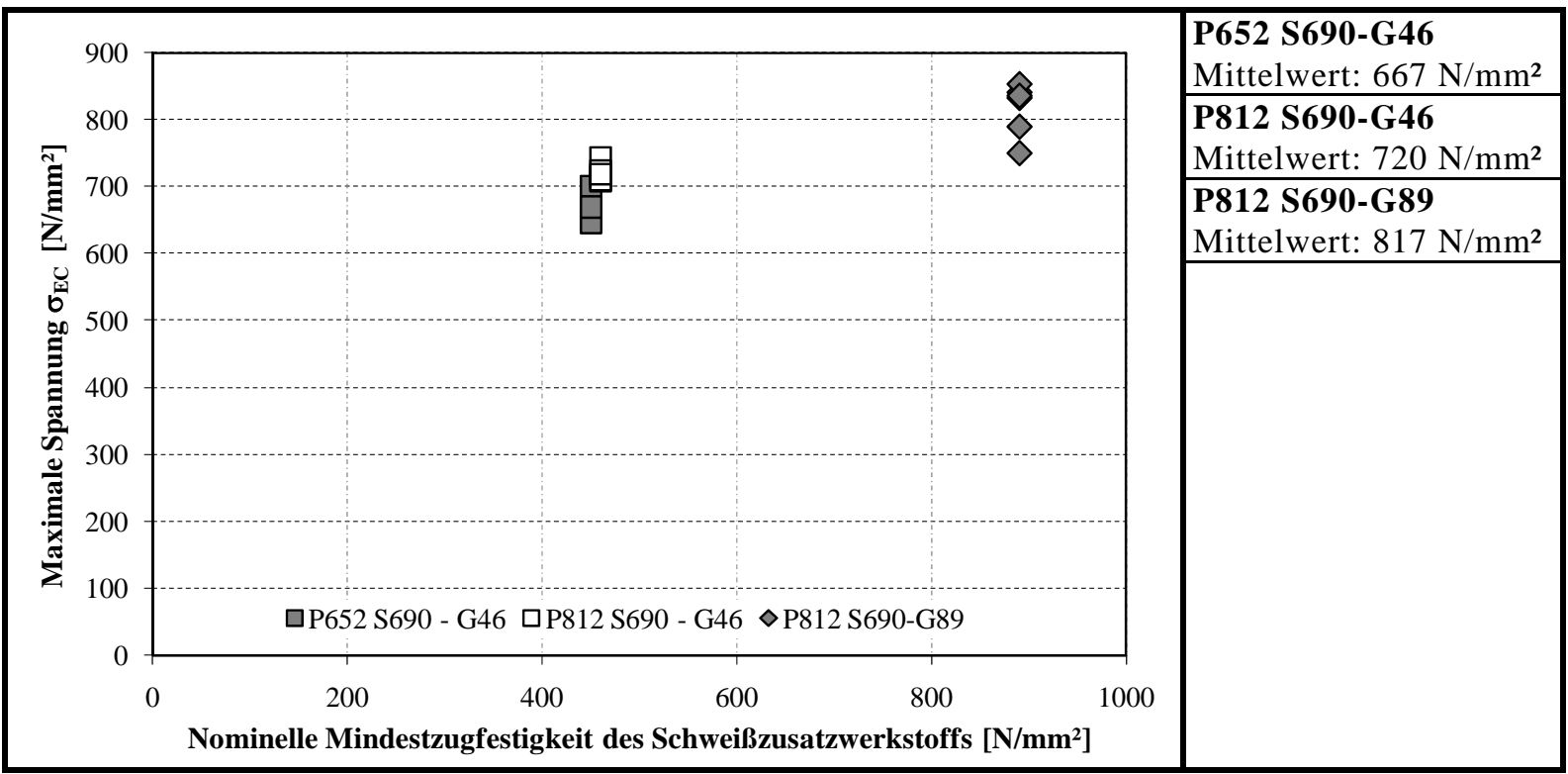

Abbildung 10.4: Vergleich der Ergebnisse der Stirnkehlnahtverbindungen S460 und S690

\subsubsection{Fazit für die Vergleichbarkeit der Ergebnisse an Kreuzstößen}

Für die Kreuzstöße zeigt der Vergleich der Versuchsergebnisse der unterschiedlichen Projekte, dass die Tragfähigkeiten stark streuen, so dass eine eindeutige Festlegung zur Vergleichbarkeit der Ergebnisse schwierig ist. Außerdem ist die Anzahl von Versuchen sehr gering. Die großen Unterschiede deuten darauf hin, dass der Bezug auf die Nahtfläche $A_{E C}$ nicht sinnvoll ist und zu großen Abweichungen führt. Ein Vergleich auf Basis der Nahtfläche $A_{\text {Bruch }}$ ist nicht möglich, da diese Werte nicht für alle Serien zur Verfügung stehen. 


\subsection{Vorschlag für das bestehende Bemessungsmodell}

\subsubsection{Auswertung für Flankenkehlnahtverbindungen}

Die bisherige Bestimmung der normativen Tragfähigkeit nach EN 1993-1-8 (2005) und EN 1993-1-12 (2007) besonders für S690 beruht auf wenigen Versuchen. Mithilfe der vorgestellten Versuche ist es nun möglich, die normative Tragfähigkeit neu zu bestimmen. Auf der Grundlage dieser statistischen Auswertung lässt sich der Beiwert $\beta_{\mathrm{w}}$ neu herleiten. Der in der Norm angegebene Beiwert $\beta_{\mathrm{w}}$ kann dann durch den in dieser Arbeit identifizierten Wert ersetzt werden. Die dazu durchgeführte statistische Auswertung umfasst alle Versuche mit gleichen Grund- und zugehörigen Schweißzusatzwerkstoffen. Daraus wird ein Vorschlag für das bestehende Bemessungsmodell nach EN 1993-1-8 (2005) gemacht.

Tabelle 10.4 fasst die dazu verwendeten Versuchsergebnisse der Kapitel 5, 6, 7 und 8 zusammen. Es stehen 58 Versuchsergebnisse mit dem Grundwerkstoff S460 und zugehörigem Schweißzusatzwerkstoff zur Verfügung. Außerdem werden 53 Versuche mit der Werkstoffkombination S690-G/T69 herangezogen. Angegeben sind jeweils die Anzahl der Versuche und die Werkstoffkennwerte der eingesetzten Grund- und Schweißzusatzwerkstoffe. Als Ergebnis der statistischen Auswertung dieser Einzelserien ist der ermittelte Beiwert $\beta_{\mathrm{w}}$ für die Auswertung in Bezug auf den Grund- oder Schweißzusatzwerkstoff angegeben.

Durch die vollmechanisierte Schweißung unter Laborbedingung der in Kapitel 8 vorgestellten Versuche wurden jeweils Versuchspaare erzeugt, deren Ergebnisse sehr ähnlich sind und die damit eine sehr geringe Streuung aufweisen. In der Realität werden entsprechend geringe Abweichungen nicht zu erreichen sein. Daher wird die geringe Streuung der vollmechanisiert hergestellten Versuchskörper in der statistischen Auswertung so berücksichtigt, dass die Anzahl der Versuche reduziert wurde. Zwar wurden für die statistische Auswertung alle Versuche berücksichtigt, die erforderlichen Fraktilenfaktoren $k_{n}$ und $k_{n, d}$ für die Widerstandsfunktion wurden jedoch für eine reduzierte Versuchsanzahl bestimmt. Die Reduzierung erfolgt nach folgender Regel: Es wurde jeweils ein Versuch von zwei Versuchen oder bei drei gleichen Versuchen wurden nur zwei Versuche berücksichtigt. Die statistisch verwendete Anzahl von Versuchen ist in Tabelle 10.4 in Klammern angegeben.

Tabelle 10.4: Versuche an Flankenkehlnähten gleicher Grund- und zugehöriger Schweißzusatzwerkstoffe

\begin{tabular}{|c|c|c|c|c|c|c|c|}
\hline & & \multicolumn{3}{|c|}{ Grundwerkstoff } & \multicolumn{3}{|c|}{ Schweißzusatzwerkstoff } \\
\hline \multirow{2}{*}{ Herkunft } & \multirow{2}{*}{ Anzahl } & \multirow{2}{*}{$\begin{array}{l}\mathrm{f}_{\mathrm{u}} \\
{\left[\mathrm{N} / \mathrm{mm}^{2}\right]}\end{array}$} & \multirow{2}{*}{$\beta_{\mathrm{w}}$} & $\mathrm{f}_{\mathrm{u}}$ & \multirow{2}{*}{$\begin{array}{l}\mathrm{f}_{\mathrm{u}} \\
{\left[\mathrm{N} / \mathrm{mm}^{2}\right]}\end{array}$} & \multirow{2}{*}{$\beta_{\mathrm{w}}$} & $\mathrm{f}_{\mathrm{u}}$ \\
\hline & & & & $\sqrt{3} \cdot \gamma_{\mathrm{M} 2} \cdot \beta_{\mathrm{w}}$ & & & $\sqrt{3} \cdot \gamma_{\mathrm{M} 2} \cdot \beta_{\mathrm{w}}$ \\
\hline \multicolumn{8}{|l|}{ S460-G/T/E46 } \\
\hline [Saal, 2005] & 34 & 614-671 & 0,788 & MW 370 & 618 & 0,775 & 369 \\
\hline [FOSTA P652, 2008] & 20 & $568-621$ & 0,842 & MW 338 & $590-639$ & 0,935 & MW 309 \\
\hline [FOSTA P812, 2011] & $4(2)$ & 538 & -- & -- & 602 & -- & -- \\
\hline \multicolumn{8}{|l|}{ S690-G/T69 } \\
\hline [FOSTA P652, 2008] & 9 & $871-876$ & 1,238 & MW 325 & 794-836 & 1,097 & MW 322 \\
\hline [Zusatzversuche, 2008] & $4(2)$ & 797 & -- & -- & 836 & -- & -- \\
\hline [FOSTA P812, 2011] & $40(24)$ & $811-891$ & 1,081 & MW 370 & $767-834$ & 1,018 & MW 359 \\
\hline
\end{tabular}

Die Ergebnisse der statistischen Auswertung sind in Tabelle 10.5 und Tabelle 10.6 dargestellt.

Mit der Werkstoffkombination S690 und einem Schweißzusatzwerkstoff mit der nominellen Streckgrenze $\mathrm{f}_{\mathrm{y}}=690 \mathrm{~N} / \mathrm{mm}^{2}$ standen 53 Versuchsergebnisse zur Verfügung. Als Ergebnis der statistischen Auswertung ergibt sich ein Beiwert $\beta_{\mathrm{w}}$ von 1,1. Dieser Beiwert ist ungünstiger als der Wert nach EN 1993-1-12 (2007) $\left(\beta_{\mathrm{w}}=1,0\right)$, aber günstiger als der Beiwert nach DIN EN 1993-1-12/NA (2011) $\left(\beta_{\mathrm{w}}=\right.$ $1,2)$.

Für den Stahl S460 mit passendem Schweißzusatzwerkstoff konnten 58 Versuche statistisch ausgewertet werden, wie in Tabelle 10.6 dargestellt ist. Der Beiwert $\beta_{w}$ ist deutlich günstiger als die Beiwert nach EN 1993-1-8 (2005) $\left(\beta_{\mathrm{w}}=1,0\right)$ und entspricht dem Beiwert nach DIN EN 1993-1-8/NA (2010) $\left(\beta_{\mathrm{w}}=0,85\right)$. 
Tabelle 10.5: Statistische Auswertung aller Flankenkehlnahtverbindungen S690 bezogen auf die Festigkeit des Grundwerkstoffs (links) oder des Schweißzusatzwerkstoffs (rechts)

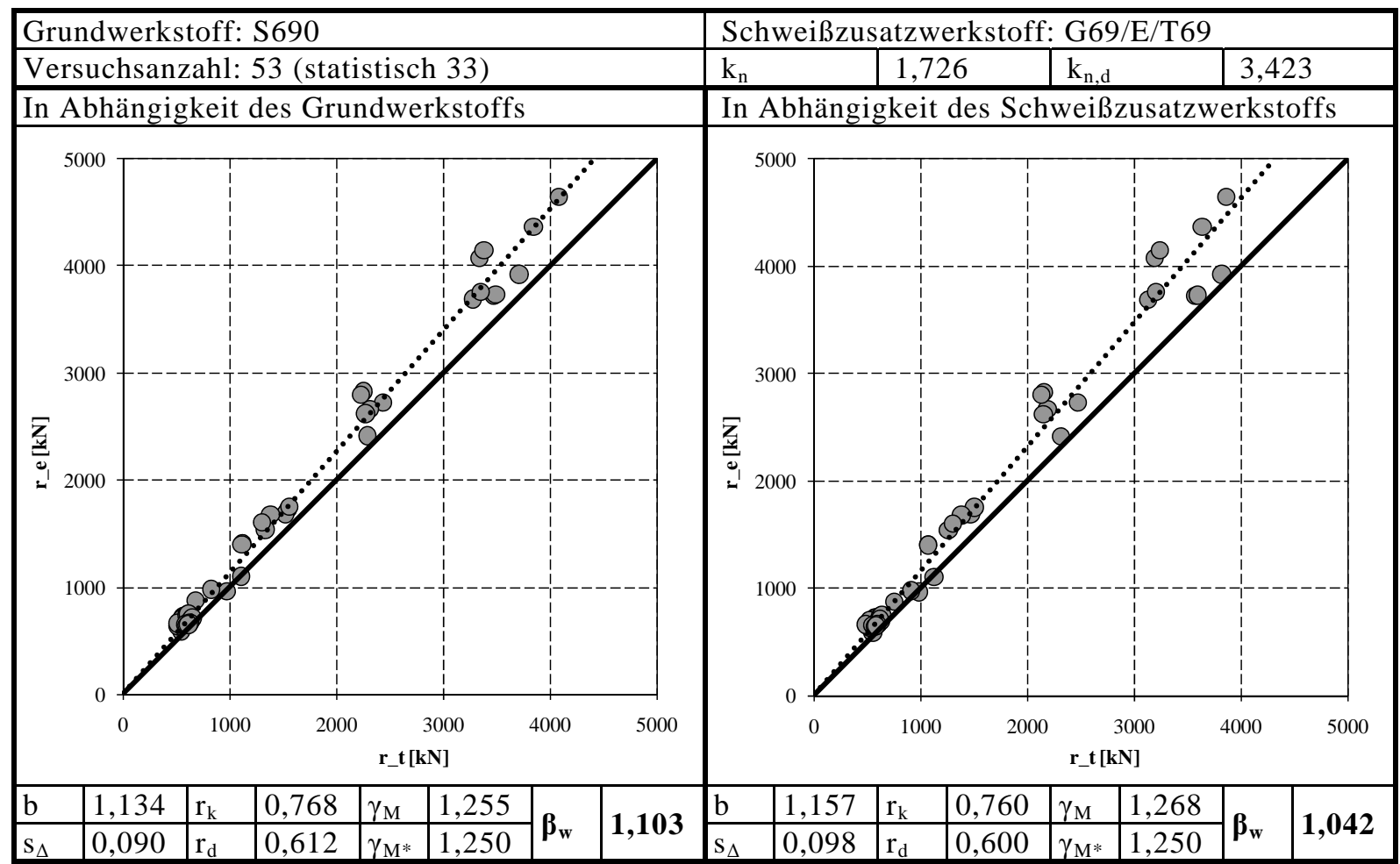

Tabelle 10.6: Statistische Auswertung aller Flankenkehlnahtverbindungen S460 bezogen auf die Festigkeit des Grundwerkstoffs (links) oder des Schweißzusatzwerkstoffs (rechts)

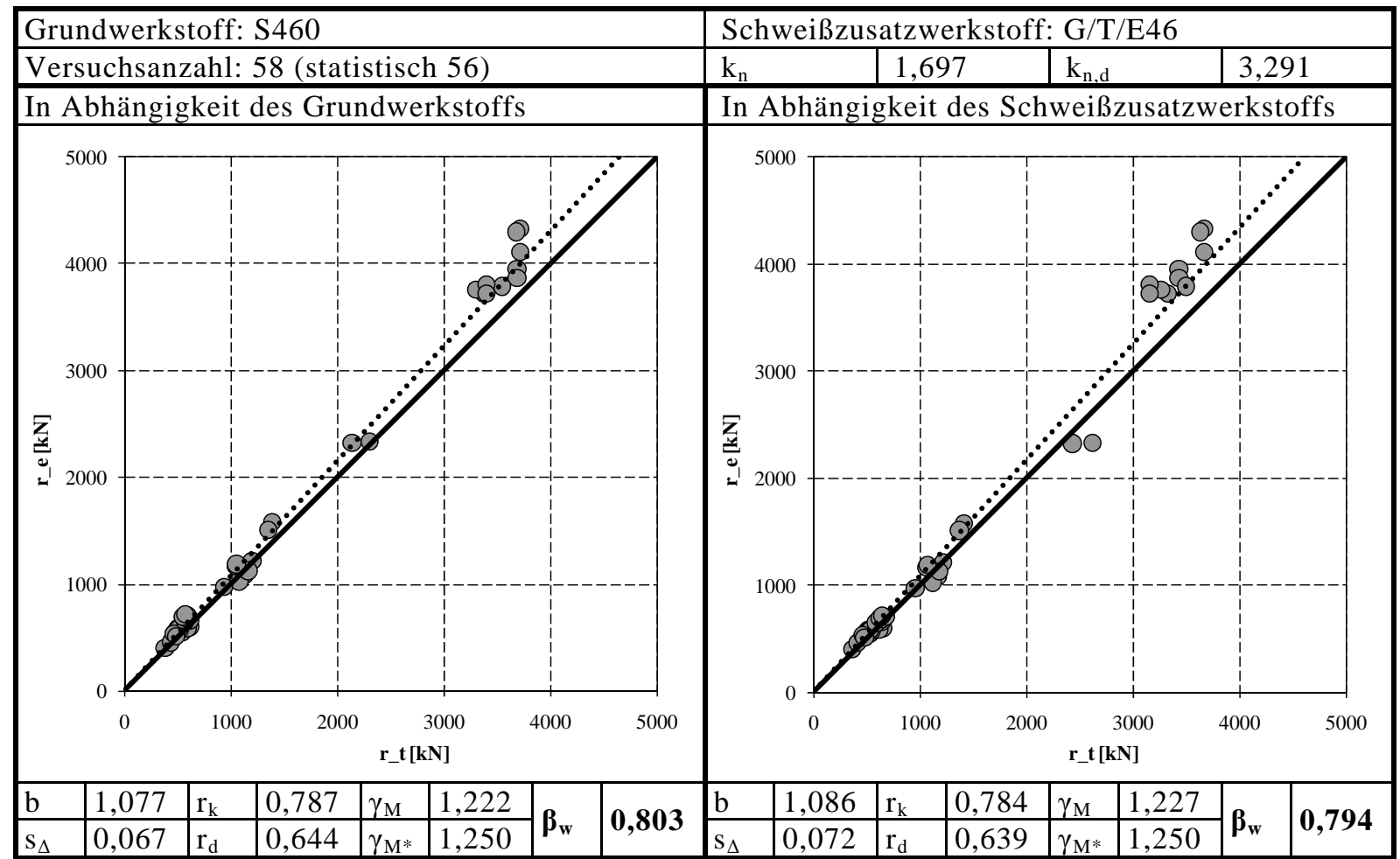


Die Ergebnisse der statistischen Auswertung werden in Tabelle 10.7 für die Werkstoffkombination S460-G/T/E46 und S690-G/T69 verglichen. Aufgrund der großen Anzahl von Versuchen ergeben sich für die Auswertung in Abhängigkeit von Grund- und Schweißzusatzwerkstoffen sehr ähnliche Bemessungsgrenzspannungen von 356 und $360 \mathrm{~N} / \mathrm{mm}^{2}$ für die Verbindung S460-G/T/E46 und 361 und 355 $\mathrm{N} / \mathrm{mm}^{2}$ für die Verbindung S690-G/T69.

Für die Verbindungen S460-G/T/E46 zeigt der Beiwert $\beta_{\mathrm{w}}<1,0$, dass die Festigkeit der Kehlnaht höher als die Festigkeit des Schweißzusatzwerkstoffs liegt. Für die Verbindungen mit dem S690-G/T69 liegt die Festigkeit der Kehlnaht unterhalb der Festigkeit des Schweißzusatzwerkstoffs. Dies wird mit dem Beiwert $\beta_{w}>1,0$ angegeben. Infolge der unterschiedlichen Überfestigkeiten bzw. Unterfestigkeiten verringern sich die Unterschiede in der Tragfähigkeit zwischen S460-G/T/E46 und S690-G/T69. Der Vergleich zwischen den Verbindungen S460-G/T/E46 und S690-G/T69 zeigt demnach eine geringe Steigerung der Bemessungsgrenzspannung. Die Gründe dafür sind in Kapitel 4.2.3 beschrieben.

Tabelle 10.7: Bemessungsgrenzspannung für Flankenkehlnähte

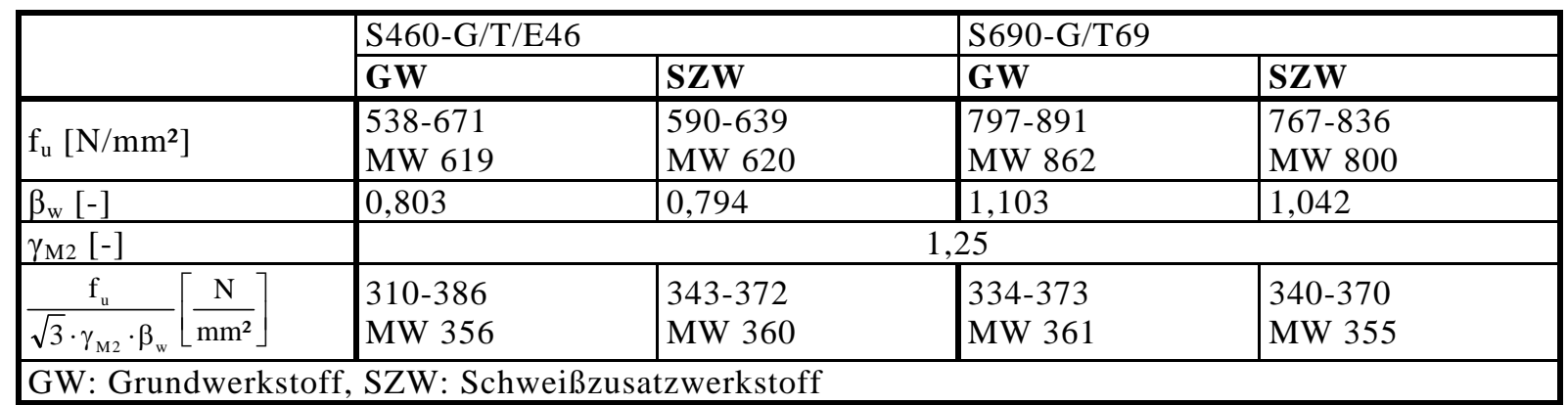

In Abbildung 10.5 sind die beschriebenen Versuchsergebnisse zusammen mit den ermittelten Bemessungsgrenzspannungen dargestellt. Es ergeben sich sehr ähnliche Bemessunggrenzspannungen. Die Versuchsergebnisse zeigen, dass es bei Betrachtung der Mittelwerte eine Steigerung der Tragfähigkeit um 7 \% von $480 \mathrm{~N} / \mathrm{mm}^{2}$ für S460-G/T/E46 auf $516 \mathrm{~N} / \mathrm{mm}^{2}$ für S690-G/T/E69 zu beobachten ist. Aufgrund der größeren Streuung und der geringeren Anzahl von Versuchen für die Verbindungen S690-G/T69 ist diese Steigerung in den Bemessungswerten mit $1 \%$ deutlich geringer.

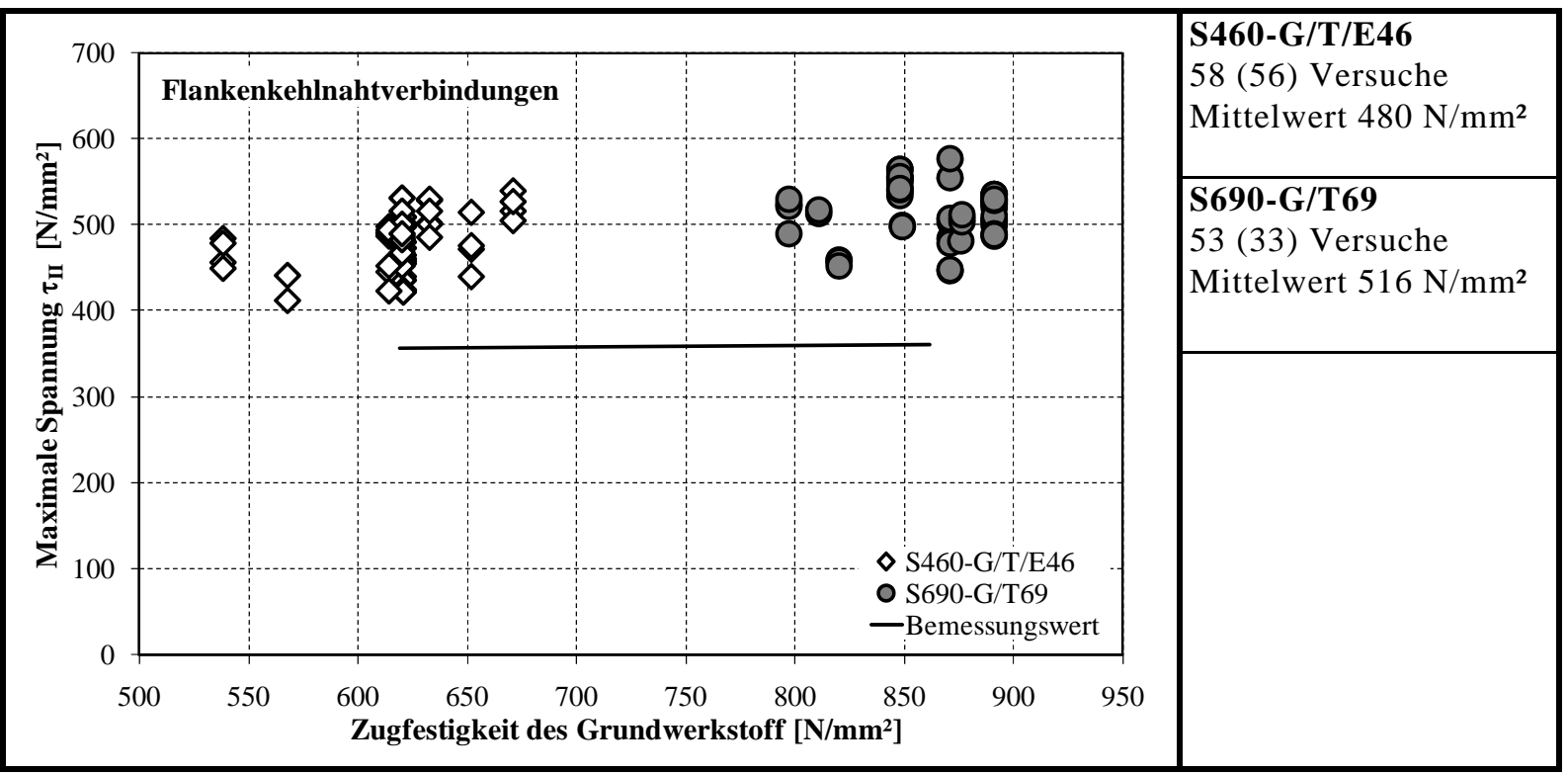

Abbildung 10.5: Vergleich der Ergebnisse der Flankenkehlnahtverbindungen S460-G/T/E46 und S690-G/T69 


\subsubsection{Auswertung für Kreuzstöße}

Durch statistische Auswertung alle Versuche gleicher Grundwerkstoffe und zugehöriger Schweißzusatzwerkstoffe ist eine Beurteilung der Tragfähigkeit der Schweißverbindungen möglich. Tabelle 10.8 fasst die vorhandenen Versuchsergebnisse der Kapitel 5, 6, 7 und 8 zusammen. Eine gemeinsame statistische Auswertung ist für die Verbindungen S460-G46 nicht möglich, da nur eigene Versuche zur Verfügung stehen. Auch der Vergleich der Versuchsergebnisse der Kreuzstöße der Werkstoffkombination S690-G69 ist zu hinterfragen, da von den in Kapitel 8 vorgestellten Versuchsergebnissen gleicher Grund- und Schweißzusatzwerkstoffe nur 3 Versuchsergebnisse verwendet werden können.

Mit der Werkstoffkombination S690 und Schweißzusatzwerkstoffen mit der nominellen Streckgrenze $\mathrm{f}_{\mathrm{y}}=690 \mathrm{~N} / \mathrm{mm}^{2}$ standen 16 Versuchsergebnisse von Kreuzstößen zur Verfügung, vgl. Tabelle 10.9. Der ermittelte Beiwert $\beta_{\mathrm{w}}=0,92$ ist günstiger als der ermittelte Wert für die Flankenkehlnahtverbindungen.

Tabelle 10.8: Versuche an Stirnkehlnähten gleicher Grund- und zugehöriger Schweißzusatzwerkstoffe

\begin{tabular}{|c|c|c|c|c|c|c|c|c|c|}
\hline \multirow{3}{*}{ Herkunft } & \multirow{3}{*}{$\begin{array}{l}\text { Naht- } \\
\text { fläche }\end{array}$} & \multirow{3}{*}{$\begin{array}{l}\text { An- } \\
\text { zahl }\end{array}$} & \multirow{3}{*}{$\begin{array}{l}\text { Mittel- } \\
\text { werte }\end{array}$} & \multicolumn{3}{|c|}{ Grundwerkstoff } & \multicolumn{3}{|c|}{ Schweißzusatzwerkstoff } \\
\hline & & & & \multirow{2}{*}{$\begin{array}{l}\mathrm{f}_{\mathrm{u}} \\
{\left[\mathrm{N} / \mathrm{mm}^{2}\right]}\end{array}$} & \multirow[t]{2}{*}{$\beta_{\mathrm{w}}$} & $\mathrm{f}_{\mathrm{u}}$ & \multirow{2}{*}{\begin{tabular}{|l|}
$\mathrm{f}_{\mathrm{u}}$ \\
{$\left[\mathrm{N} / \mathrm{mm}^{2}\right]$}
\end{tabular}} & \multirow[t]{2}{*}{$\beta_{\mathrm{w}}$} & $\mathrm{f}_{\mathrm{u}}$ \\
\hline & & & & & & $\overline{\sqrt{2} \cdot \gamma_{\mathrm{M} 2} \cdot \beta_{\mathrm{w}}}$ & & & $\overline{\sqrt{2} \cdot \gamma_{\mathrm{M} 2} \cdot \beta_{\mathrm{w}}}$ \\
\hline \multicolumn{10}{|l|}{ S460-G46 } \\
\hline $\begin{array}{ll}\text { [FOSTA } & \text { P652, } \\
2008] & \end{array}$ & $\mathrm{A}_{\mathrm{EC}}$ & 11 & 661 & 621 & 0,688 & MW 511 & 639 & 0,708 & 511 \\
\hline \multicolumn{10}{|l|}{ S690-G69 } \\
\hline $\begin{array}{ll}\text { [FOSTA } & \text { P652, } \\
\text { 2008] } & \end{array}$ & $A_{E C}$ & 13 & 697 & 871 & 0,917 & 537 & 836 & 0,880 & 537 \\
\hline \multirow{2}{*}{$\begin{array}{ll}\text { [FOSTA } & \text { P812, } \\
\text { 2011] } & \end{array}$} & $\mathrm{A}_{\mathrm{EC}}$ & \multirow{2}{*}{3} & 801 & \multirow{2}{*}{891} & & & \multirow{2}{*}{798} & & \\
\hline & $\mathrm{A}_{\text {Bruch }}$ & & 777 & & & & & & \\
\hline
\end{tabular}

Tabelle 10.9: Statistische Auswertung aller Stirnkehlnahtverbindungen S690 bezogen auf die Festigkeit des Grundwerkstoffs (links) oder des Schweißzusatzwerkstoffs (rechts)

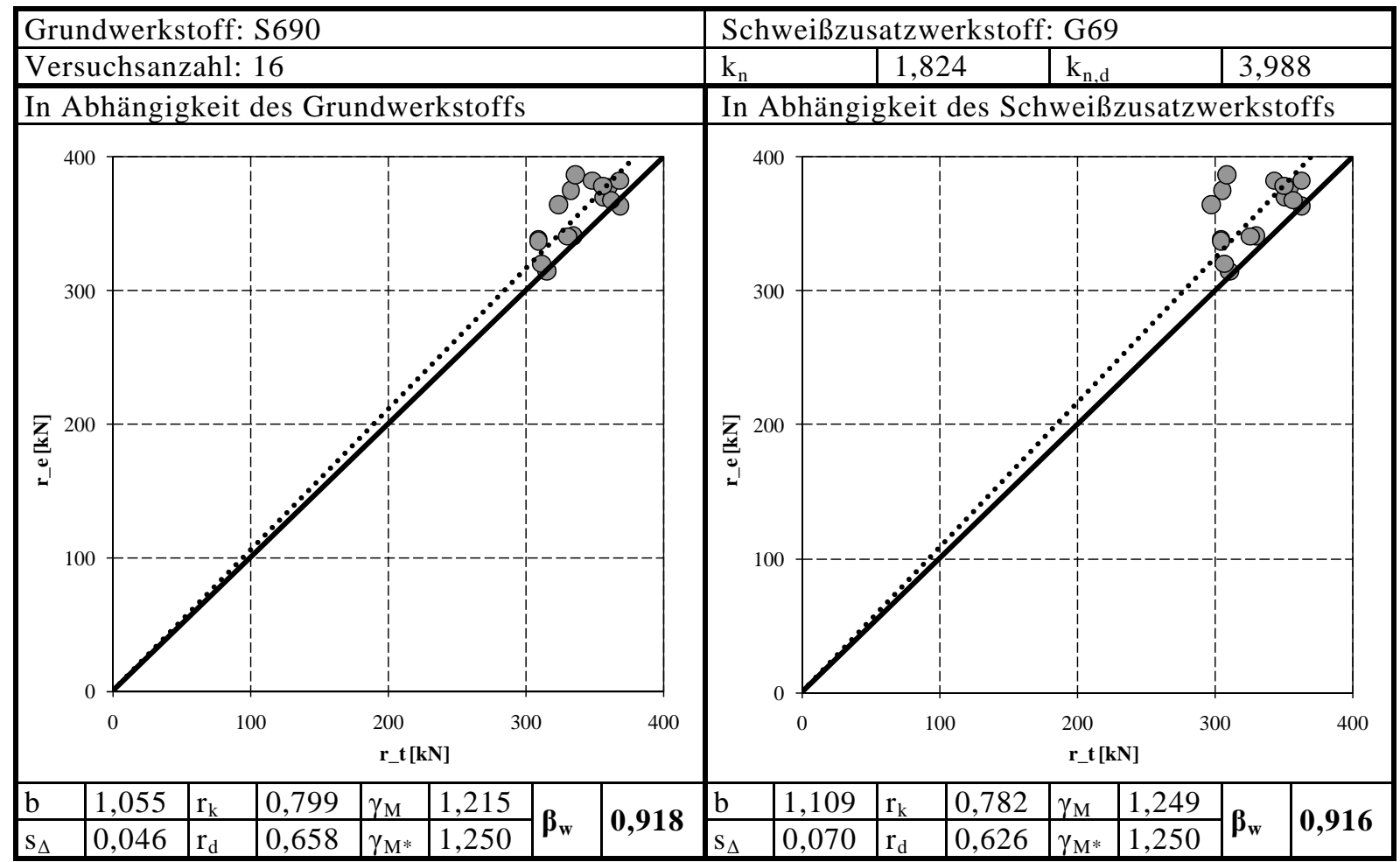




\subsubsection{Vorschlag für die Bemessung nach DIN EN 1993}

Da festgestellt wurde, dass längsbeanspruchte Kehlnähte eine geringere Tragfähigkeit als Stirnkehlnähte haben, werden für die Auswertung nur Flankenkehlnähte berücksichtigt. Aus der Auswertung nach Tabelle 10.5 und Tabelle 10.6 werden die in Abbildung 10.6 gegebenen Beiwerte für die Anwendung von EN 1993-1-8 (2005) und EN 1993-1-12 (2007) vorgeschlagen. Für die Verbindungen mit dem Grundwerkstoff S460 und zugehörigem Schweißzusatzwerkstoff wird ein Beiwert $\beta_{\mathrm{w}}=0,85$ vorgeschlagen. Die Schweißverbindungen mit dem Grundwerkstoff S690 sollten mit einem Beiwert $\beta_{\mathrm{w}}=1,10$ dimensioniert werden. Mit diesen Beiwerten ergeben sich die Bemessungsgrenzspannungen $\tau_{\mathrm{Rd}}=293 \mathrm{~N} / \mathrm{mm}^{2}$ für S460 und $\tau_{\mathrm{Rd}}=323 \mathrm{~N} / \mathrm{mm}^{2}$ für S690. Damit unterscheiden sich die Bemessungsgrenzspannungen um $10 \%$. In Abbildung 10.6 sind die Versuchsergebnisse im Vergleich zu den Bemessungsgrenzspannungen dargestellt. Im Vergleich mit den bisherigen Werten nach EN 1993-1-8 (2005) mit $\beta_{\mathrm{w}}=1,0$ ergibt sich für die Schweißverbindungen S460 eine Verbesserung der normativen Tragfähigkeit. Für die Schweißverbindungen S690 wurden geringere Tragfähigkeiten als nach EN 1993-1-12 (2007) mit $\beta_{\mathrm{w}}=1,0$ festgestellt.

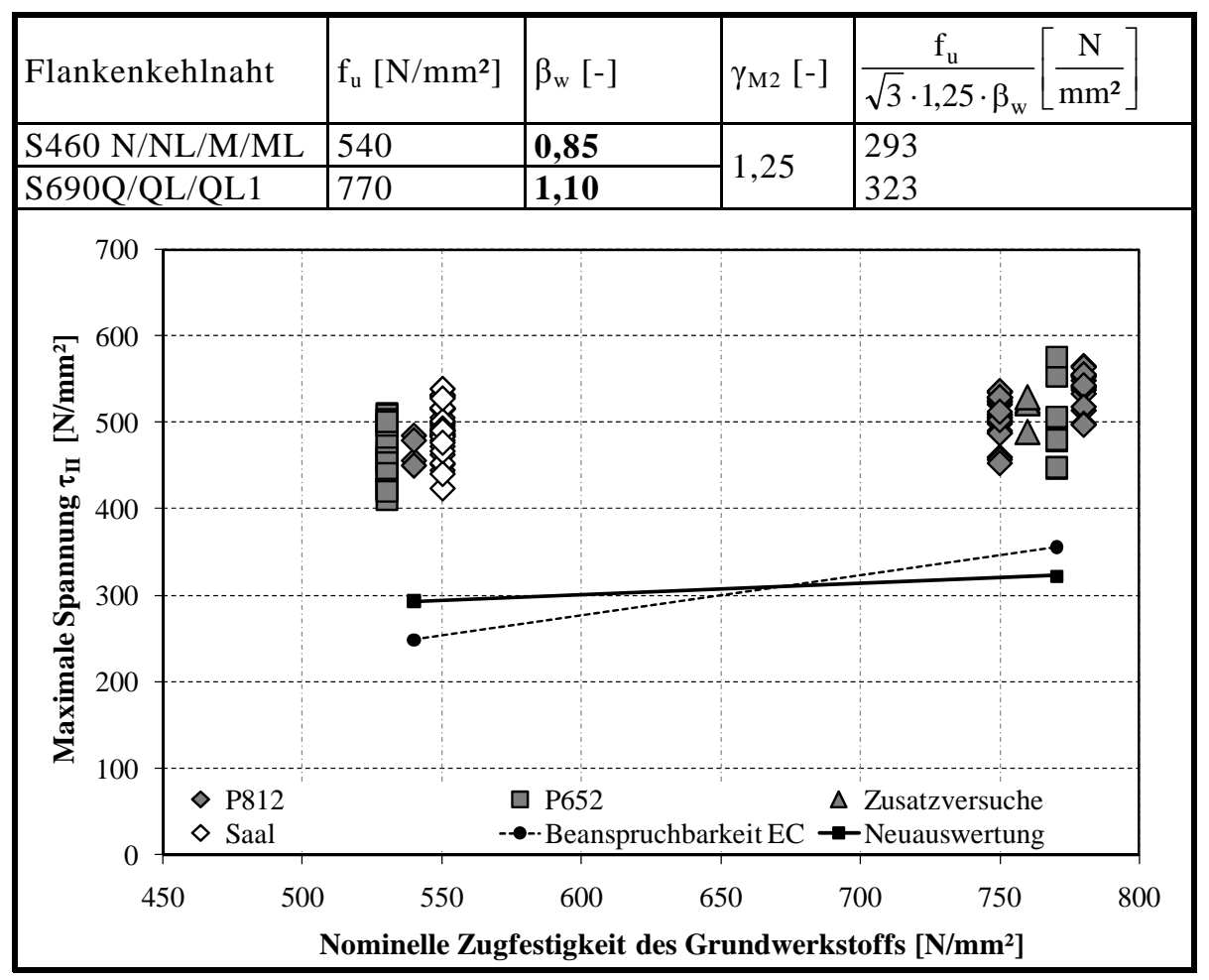

Abbildung 10.6: Vorschlag $\beta_{w}$-Werte für Flankenkehlnahtverbindungen S460 und S690 nach EN 1993-1-8 (2005) und EN 1993-1-12 (2007)

Für alle vorgestellten Untersuchungen lag die Tragfähigkeit von Kreuzstößen mit Stirnkehlnähten höher als die Tragfähigkeit von Flankenkehlnahtverbindungen. Auf der sicheren Seite liegend können die ermittelten Beiwerte $\beta_{w}$ auch für Stirnkehlnahtverbindungen verwendet werden. 


\subsection{Bestimmung der Tragfähigkeit in Abhängigkeit von Grund- und Schweißzusatzwerkstoff}

\subsubsection{Herleitung des Bemessungsansatzes}

Die in Kapitel 9 vorgestellten numerischen Untersuchungen haben gezeigt, dass die Festigkeit in der Schweißnaht vorwiegend die Tragfähigkeit von Flankenkehlnähten bestimmt. In diesem Abschnitt wird aufgezeigt, wie sich diese Festigkeit der Naht in Abhängigkeit der Festigkeit von Grund- und Schweißzusatzwerkstoffen bestimmen lässt.

Eine Betrachtung der Tragfähigkeit von Kehlnahtverbindungen gleicher Grund- und zugehöriger Schweißzusatzwerkstoffe zeigt eine abweichende Festigkeit der Kehlnaht im Vergleich zur Festigkeit der eingesetzten Werkstoffe. Diese Kehlnahtfestigkeit wird mithilfe des Beiwertes $\beta_{\mathrm{w}}$ in der statistischen Auswertung ermittelt. Eine Überfestigkeit ist durch einen Beiwert $\beta_{\mathrm{w}}<1,0$ gekennzeichnet. Diese Überfestigkeit entsteht durch die Abkühlverhältnisse von Kehlnähten, die sich deutlich von den Verhältnissen beim Schweißen von reinen Stumpfnähten unterscheiden. Die Festigkeit dieser Stumpfnähte liegt als Werkstoffkennwert allen normativen Nachweisen, vgl. Kapitel 3.4.3, zugrunde. Die Überfestigkeiten werden vor allem durch den Schweißzusatzwerkstoff geprägt. Die Auswertung im vorherigen Kapitel 10.5, vgl. Abbildung 10.6, zeigt für die Verbindungen S460-G/T/E46 mit dem Beiwert $\beta_{w}=0,85$, dass eine Überfestigkeit von 1/0,85 = 1,18 vorherrscht. Im Gegensatz dazu konnte bei den Verbindungen S690-G/T69 keine Überfestigkeit festgestellt werden. Im Vergleich zur Festigkeit des Grundwerkstoffs muss die Festigkeit der Kehlnähte auf 1/1,10 = 0,91 abgemindert werden. Bei Schweißverbindungen mit Grund- und Schweißzusatzwerkstoffen unterschiedlicher Festigkeit wird die Tragfähigkeit der Verbindung neben den beschriebenen Abkühlverhältnissen durch die Vermischung der Werkstoffe bestimmt.

Durch den Vergleich von Versuchsreihen, in denen bei gleichem Schweißzusatzwerkstoff nur der Grundwerkstoff variiert wird, soll im Weiteren dieses Mischungsverhältnis bestimmt werden. Auf Basis dieses Mischungsverhältnisses wird ein Bemessungsansatz vorgestellt. Anschließend wird dieser Bemessungsansatz mithilfe der statistischen Auswertung für alle Schweißzusatzwerkstoffe bestätigt.

Betrachtet werden zwei Versuchsreihen A und B, bei denen in der Versuchsreihe A Grund- und Schweißzusatzwerkstoffe nominell gleicher Festigkeit $\left(\mathrm{f}_{\mathrm{u}, \mathrm{GWA}} \approx \mathrm{f}_{\mathrm{u}, \mathrm{SZWA}}\right)$ verwendet werden, während in der anderen Versuchsreihe B ein Grundwerkstoff mit einer anderen Festigkeit als der des Schweißzusatzwerkstoffs $\left(\mathrm{f}_{\mathrm{u}, \mathrm{GWB}} \neq \mathrm{f}_{\mathrm{u}, \mathrm{SZWA}}\right)$ verwendet wird.

Die Gleichungen (10.1) oder (10.2) für die Versuchsreihe A mit gleichen Grund- und Schweißzusatzwerkstoffen wurden in Anlehnung an die Bemessungsgleichung nach EN 1993-1-8 (2005) gewählt. Im Gegensatz zum Nachweis nach EN 1993-1-8 (2005) wird dabei sowohl die Zugfestigkeit des Grundwerkstoffs $\mathrm{f}_{\mathrm{u}, \mathrm{GWA}}$ als auch die Zugfestigkeit des Schweißzusatzwerkstoffs $\mathrm{f}_{\mathrm{u} \text {,SZWA }}$ (Werte des reinen Schweißgutes) verwendet. Die Koeffizienten x und y berücksichtigen dabei das Mischungsverhältnis. Die Bemessungsgrenzspannung $\tau_{\mathrm{II}, \mathrm{A}}$ (für Flankenkehlnähte) oder $\sigma_{\mathrm{u}, \mathrm{A}}$ (für Kreuzstöße) und der Beiwert $\beta_{\mathrm{wA}}$ sind aus den statistischen Auswertungen in Abhängigkeit des Grundwerkstoff und / oder des Schweißzusatzwerkstoffs bekannt. Für die Anwendung von Gleichungen (10.1) oder (10.2) wird gefordert, dass für beide Auswertungen etwa gleiche Werte berechnet werden.

$$
\begin{aligned}
& \text { Für Flankenkehlnähte } \tau_{\mathrm{II}, \mathrm{A}}=\frac{\mathrm{x} \cdot \mathrm{f}_{\mathrm{u}, \mathrm{GWA}}+\mathrm{y} \cdot \mathrm{f}_{\mathrm{u}, \mathrm{SZWA}}}{\sqrt{3} \cdot \beta_{\mathrm{wA}} \cdot \gamma_{\mathrm{M} 2}} \\
& \text { Für Kreuzstöße } \sigma_{\mathrm{u}, \mathrm{A}}=\frac{\mathrm{x} \cdot \mathrm{f}_{\mathrm{u}, \mathrm{GWA}}+\mathrm{y} \cdot \mathrm{f}_{\mathrm{u}, \mathrm{SZWA}}}{\sqrt{2} \cdot \beta_{\mathrm{wA}} \cdot \gamma_{\mathrm{M} 2}}
\end{aligned}
$$

Die Gleichungen (10.3) und (10.4) für die Tragfähigkeit der Versuchsreihe B mit unterschiedlichen Festigkeiten von Grund- und Schweißzusatzwerkstoff verwenden die Bemessungsgrenzspannung $\tau_{\mathrm{II}, \mathrm{B}}$ (für Flankenkehlnähte) oder $\sigma_{\mathrm{u}, \mathrm{B}}$ (für Kreuzstöße) dieser Serie. Diese Bemessungsgrenzspannungen sind aus den statistischen Auswertungen in Abhängigkeit vom Grundwerkstoff und vom Schweißzusatzwerkstoff bekannt. 
Die Überfestigkeit durch die Abkühlverhältnisse bei Kehlnähten (vgl. Kapitel 4.2.3) werden durch den Beiwert $\beta_{\mathrm{wA}}$ der Versuchsreihe A berücksichtigt. Wie oben beschrieben, wird davon ausgegangen, dass die Überfestigkeit vorwiegend durch den Schweißzusatzwerkstoff und die Geometrie bestimmt wird. Aus diesem Grund muss diese Überfestigkeit auch bei Verwendung eines anderen Grundwerkstoffs $\left(f_{u, G W B}\right)$ bei gleichem Schweißzusatzwerkstoff $\left(f_{u, S Z W A}\right)$ vorhanden sein. Die Festigkeitsänderung der Verbindung, die durch die Vermischung unterschiedlicher Werkstoffe beeinflusst wird, wird über die Festigkeitswerte des Grundwerkstoffs $\mathrm{f}_{\mathrm{u}, \mathrm{GWB}}$, des Schweißzusatzwerkstoffs $\mathrm{f}_{\mathrm{u}, \mathrm{SZWA}}$ und mithilfe der Unbekannten $\mathrm{x}$ und y berücksichtigt.

$$
\begin{aligned}
& \text { Für Flankenkehlnähte } \tau_{\mathrm{II}, \mathrm{B}}=\frac{\mathrm{x} \cdot \mathrm{f}_{\mathrm{u}, \mathrm{GWB}}+\mathrm{y} \cdot \mathrm{f}_{\mathrm{u}, \mathrm{SZWA}}}{\sqrt{3} \cdot \beta_{\mathrm{wA}} \cdot \gamma_{\mathrm{M} 2}} \\
& \text { Für Kreuzstöße } \sigma_{\mathrm{u}, \mathrm{B}}=\frac{\mathrm{x} \cdot \mathrm{f}_{\mathrm{u}, \mathrm{GWB}}+\mathrm{y} \cdot \mathrm{f}_{\mathrm{u}, \mathrm{SZWA}}}{\sqrt{2} \cdot \beta_{\mathrm{wA}} \cdot \gamma_{\mathrm{M} 2}}
\end{aligned}
$$

Es entsteht ein Gleichungssystem mit zwei Gleichungen und zwei Unbekannten, das sich lösen lässt: Für Flankenkehlnähte (10.1) und (10.3) und für Stirnkehlnähte (10.3) und (10.4). Die Unbekannten x und y sind dabei die Anteile von Grund- und Schweißzusatzwerkstoff an der Festigkeit der Kehlnahtverbindung. Theoretisch muss sich die Summe aus x und y zu 1 ergeben, also $100 \%$. Aufgrund von Abweichungen, die sich in der statistischen Auswertung ergeben, weicht das Ergebnis von $100 \%$ leicht ab.

Das Vorgehen wird am Beispiel der Flankenkehlnahtverbindungen nach [Kob et al., 1987] in Tabelle 10.10 verdeutlicht. Die Ergebnisse der statistischen Auswertung stammen aus Tabelle 5.2. Dieses Beispiel wurde ausgewählt, da es sich um eine große Anzahl von Versuchen handelt und die Unterschiede zwischen den eingesetzten Grundwerkstoffen besonders groß sind, während bei allen Versuchen der gleiche Schweißzusatzwerkstoff verwendet wurde. Außerdem liefert die statistische Auswertung in diesem Fall in Abhängigkeit des Grundwerkstoffs ähnliche Ergebnisse wie die Auswertung in Abhängigkeit des Schweißzusatzwerkstoffs. Es wird der Beiwert $\beta_{w}$ in Abhängigkeit des Schweißzusatzwerkstoffs verwendet, da dieser, wie oben beschrieben, die Tragfähigkeit des Schweißzusatzwerkstoffs definiert. Aus Tabelle 10.10 folgt, dass die Festigkeit des Grundwerkstoffs mit ca. 22 \% zu berücksichtigen ist, während die Festigkeit des Schweißzusatzwerkstoffs mit 79 \% eingeht.

Tabelle 10.10: Beispiel für die Berechnung der Nahtfestigkeit in Abhängigkeit der eingesetzten Grund-

\begin{tabular}{|c|c|c|c|c|c|c|c|c|c|}
\hline \multicolumn{5}{|c|}{ S355J0-G42 } & \multicolumn{5}{|c|}{ S690Q-G42 } \\
\hline \multicolumn{2}{|c|}{$\mathrm{f}_{\mathrm{u}}\left[\mathrm{N} / \mathrm{mm}^{2}\right]$} & \multirow[b]{2}{*}{$\beta_{\mathrm{w}}[-]$} & \multirow{2}{*}{$\frac{\mathrm{f}_{\mathrm{u}}}{\sqrt{3} \cdot \gamma_{\mathrm{M} 2} \cdot \beta_{\mathrm{w}}}$} & \multirow{2}{*}[\frac{\mathrm{N}}{\mathrm{mm}^{2}}]{} & \multicolumn{2}{|c|}{$\mathrm{f}_{\mathrm{u}}\left[\mathrm{N} / \mathrm{mm}^{2}\right]$} & \multirow[b]{2}{*}{$\beta_{\mathrm{w}}[-]$} & \multirow{2}{*}{$\frac{f_{u}}{\sqrt{3} \cdot \gamma_{M 2} \cdot \beta_{w}}$} & \multirow{2}{*}[\frac{\mathrm{N}}{\mathrm{mm}^{2}}]{} \\
\hline GW A & SZW A & & & & GW B & SZW A & & & \\
\hline MW 551 & 590 & 0,853 & $\left.319^{*}\right)$ & & MW 846 & 577 & 0,762 & $\left.350^{*}\right)$ & \\
\hline \multicolumn{5}{|c|}{$\tau_{\mathrm{II}, \mathrm{A}}=\frac{\mathrm{x} \cdot \mathrm{f}_{\mathrm{u}, \mathrm{GWA}}+\mathrm{y} \cdot \mathrm{f}_{\mathrm{u}, \mathrm{SZWA}}}{\sqrt{3} \cdot \beta_{\mathrm{wA}} \cdot \gamma_{\mathrm{M} 2}}$} & \multicolumn{5}{|c|}{$\tau_{\mathrm{II}, \mathrm{B}}=\frac{\mathrm{x} \cdot \mathrm{f}_{\mathrm{u}, \mathrm{GWB}}+\mathrm{y} \cdot \mathrm{f}_{\mathrm{u}, \mathrm{SZWA}}}{\sqrt{3} \cdot \beta_{\mathrm{wA}} \cdot \gamma_{\mathrm{M} 2}}$} \\
\hline \multicolumn{5}{|c|}{$319=\frac{x \cdot 551+y \cdot 590}{\sqrt{3} \cdot 0,853 \cdot 1,25}$} & \multicolumn{5}{|c|}{$350=\frac{x \cdot 846+y \cdot 577}{\sqrt{3} \cdot 0,853 \cdot 1,25}$} \\
\hline \multicolumn{5}{|c|}{ Ergebnis: $x=0,224$ und $y=0,790$} & & & & $\gamma_{\mathrm{M} 2}=$ & 1,25 \\
\hline
\end{tabular}
und Schweißzusatzwerkstoffe für Flankenkehlnähte nach [Kob et al., 1987] und Tabelle 5.2

Für Kreuzstöße ergibt sich ein analoges Vorgehen, das in Tabelle 10.11 vorgestellt wird. Dazu werden die Ergebnisse der statistischen Auswertung von Versuchen an Kreuzstößen mit Stirnkehlnähten nach [Kob et al., 1987] (Kapitel 5.3.3) unter Berücksichtigung der Bruchfläche $A_{\text {Bruch verwendet. Die Er- }}$ gebnisse der statistischen Auswertung stammen aus Tabelle 5.3. Das in Tabelle 10.11 berechnete Ergebnis zeigt, dass die Festigkeit des Grundwerkstoffs mit ca. 26 \% zu berücksichtigen ist, während die Festigkeit des Schweißzusatzwerkstoffs mit 76 \% eingeht. 
Tabelle 10.11: Beispiel für die Berechnung der Nahtfestigkeit in Abhängigkeit der eingesetzten Grundund Schweißzusatzwerkstoffe für Stirnkehlnähte nach [Kob et al., 1987] und Tabelle 5.4

\begin{tabular}{|c|c|c|c|c|c|c|c|c|c|}
\hline \multicolumn{5}{|c|}{ S355J0-G42 } & \multicolumn{5}{|c|}{ S690Q-G42 } \\
\hline \multicolumn{2}{|c|}{$\mathrm{f}_{\mathrm{u}}\left[\mathrm{N} / \mathrm{mm}^{2}\right]$} & \multirow[b]{2}{*}{$\beta_{\mathrm{w}}[-]$} & $\mathrm{f}_{\mathrm{u}}$ & \multirow{2}{*}{$\frac{\mathrm{N}}{\mathrm{mm}^{2}}$} & \multicolumn{2}{|c|}{$\mathrm{f}_{\mathrm{u}}\left[\mathrm{N} / \mathrm{mm}^{2}\right]$} & \multirow[b]{2}{*}{$\beta_{\mathrm{w}}[-]$} & $\mathrm{f}_{\mathrm{u}}$ & \multirow{2}{*}{$\frac{\mathrm{N}}{\mathrm{mm}^{2}}$} \\
\hline GW A & SZW A & & $\sqrt{2} \cdot \gamma_{\mathrm{M} 2} \cdot \beta_{\mathrm{w}}$ & & GW B & SZW A & & $\sqrt{2} \cdot \gamma_{\mathrm{M} 2} \cdot \beta_{\mathrm{w}}$ & \\
\hline MW 543 & 590 & 0,803 & $\left.416^{*}\right)$ & & MW 833 & 577 & 0,707 & $\left.462^{*}\right)$ & \\
\hline \multicolumn{5}{|c|}{$\sigma_{\mathrm{u}, \mathrm{A}}=\frac{\mathrm{x} \cdot \mathrm{f}_{\mathrm{u}, \mathrm{GWA}}+\mathrm{y} \cdot \mathrm{f}_{\mathrm{u}, \mathrm{SZWA}}}{\sqrt{2} \cdot \beta_{\mathrm{wA}} \cdot \gamma_{\mathrm{M} 2}}(10.2)$} & \multicolumn{5}{|c|}{$\sigma_{\mathrm{u}, \mathrm{B}}=\frac{\mathrm{x} \cdot \mathrm{f}_{\mathrm{u}, \mathrm{GWB}}+\mathrm{y} \cdot \mathrm{f}_{\mathrm{u}, \mathrm{SZWA}}}{\sqrt{2} \cdot \beta_{\mathrm{wA}} \cdot \gamma_{\mathrm{M} 2}}$} \\
\hline \multicolumn{5}{|c|}{$416=\frac{x \cdot 543+y \cdot 590}{\sqrt{2} \cdot 0,803 \cdot 1,25}$} & \multicolumn{5}{|c|}{$462=\frac{x \cdot 833+y \cdot 577}{\sqrt{2} \cdot 0,803 \cdot 1,25}$} \\
\hline \multicolumn{5}{|c|}{ Ergebnis: $x=0,258$ und $y=0,763$} & & & & $\left.{ }^{*}\right)$ mit $\gamma_{\mathrm{M} 2}$ & 1,25 \\
\hline
\end{tabular}

Als Ergebnis der Berechnungen wird vorgeschlagen, für die Bemessungsgleichung die Festigkeit von Grund- und Schweißzusatzwerkstoff wie folgt zu berücksichtigen:

$$
\begin{aligned}
& \text { Für Flankenkehlnähte } \tau_{\mathrm{II}}=\frac{0,25 \cdot \mathrm{f}_{\mathrm{u}, \mathrm{GW}}+0,75 \cdot \mathrm{f}_{\mathrm{u}, \mathrm{SZW}}}{\sqrt{3} \cdot \beta_{\mathrm{w}} \cdot \gamma_{\mathrm{M} 2}} \\
& \text { Für Kreuzstöße } \sigma_{\mathrm{u}}=\frac{0,25 \cdot \mathrm{f}_{\mathrm{u}, \mathrm{GW}}+0,75 \cdot \mathrm{f}_{\mathrm{u}, \mathrm{SZW}}}{\sqrt{2} \cdot \beta_{\mathrm{w}} \cdot \gamma_{\mathrm{M} 2}}
\end{aligned}
$$

Diese Bemessungsgleichung wird in den nächsten Kapiteln für die verschiedenen Schweißzusatzwerkstoffe angewendet, indem die in Kapitel 5-8 vorgestellten Versuche mit der Bemessungsgleichung nach Gleichung (10.5) oder (10.6) statistisch ausgewertet werden.

\subsubsection{Vorstellung der verwendeten Versuchsergebnisse}

Die Bemessung in Abhängigkeit des Schweißzusatzwerkstoffs erlaubt die Betrachtung von Versuchsergebnissen mit Grundwerkstoffen unterschiedlicher Festigkeit. Betrachtet werden Schweißzusatzwerkstoffe mit den nominellen Streckgrenzen $\mathrm{f}_{\mathrm{y}}=420 \mathrm{~N} / \mathrm{mm}^{2}, 460 \mathrm{~N} / \mathrm{mm}^{2}, 550 \mathrm{~N} / \mathrm{mm}^{2}, 690 \mathrm{~N} / \mathrm{mm}^{2}$ und $890 \mathrm{~N} / \mathrm{mm}^{2}$. Die für die einzelnen Schweißzusatzwerkstoffe zur Verfügung stehenden Versuche werden in Tabelle 10.12 aufgeführt. Die Festigkeit, die sich aus dem Mischungsverhältnis von Schweißzusatzwerkstoff und Grundwerkstoff ergibt, ist jeweils mit angegeben. Die Versuchsergebnisse mit nominell gleichen Schweißzusatzwerkstoffen werden gemeinsam statistisch ausgewertet, um jeweils den Beiwert $\beta_{\mathrm{w}}$ zu bestimmen.

Für Schweißverbindungen mit dem Schweißzusatzwerkstoff der nominellen Streckgrenze $\mathrm{f}_{\mathrm{y}}=420 \mathrm{~N} / \mathrm{mm}^{2}$ können 105 Versuche mit zugehörigem Grundwerkstoff als auch mit Grundwerkstoffen höherer Festigkeit betrachtet werden.

Die zur Verfügung stehenden Versuche mit einem Schweißzusatzwerkstoff der nominellen Streckgrenze $\mathrm{f}_{\mathrm{y}}=460 \mathrm{~N} / \mathrm{mm}^{2}$ stammen von Versuchskörpern mit den Grundwerkstoffen S355, S460 und S690. Die meisten Versuche sind Verbindungen mit Grundwerkstoffen zugehöriger Festigkeit. Zwei Versuche mit einer geringeren Festigkeit des Grundwerkstoffs und sieben Versuche mit Grundwerkstoffen höherer Festigkeit werden verwendet.

Der Schweißzusatzwerkstoff mit einer Streckgrenze von $\mathrm{f}_{\mathrm{y}}=550 \mathrm{~N} / \mathrm{mm}^{2}$ ist im Bauwesen in Deutschland nicht üblich. Versuche aus der Literatur werden dennoch ausgewertet, um einen Hinweis darauf zu erhalten, welche Tragfähigkeit sich „zwischen“ den Tragfähigkeiten der Schweißzusatzwerkstoffe der Festigkeitsklassen 46 und 69 einstellt. Es werden auch die Versuche nach [Background D.03, 1990] verwendet, obwohl die Zugfestigkeit des Schweißzusatzwerkstoffs die Mindestzugfestigkeit um $3 \%$ unterschreitet.

Zur Bestimmung der Tragfähigkeit von Flankenkehlnähten mit einem Schweißzusatzwerkstoff der nominellen Festigkeit $\mathrm{f}_{\mathrm{y}}=690 \mathrm{~N} / \mathrm{mm}^{2}$ stehen 55 Versuchsergebnisse zur Verfügung, von denen nur zwei Versuche nominell unterschiedliche Festigkeiten im Grund- und Schweißzusatzwerkstoff haben. 
Um den Einfluss des Schweißzusatzwerkstoffs mit einer nominellen Streckgrenze von $\mathrm{f}_{\mathrm{y}}=890 \mathrm{~N} / \mathrm{mm}^{2}$ auf die Tragfähigkeit von Flankenkehlnähten zu bestimmen, stehen nur Versuche mit unterschiedlichen Grund- und Schweißzusatzwerkstoffen zur Verfügung. Davon hat ein Versuch den Grundwerkstoff S355 und die anderen den Grundwerkstoff S690.

Tabelle 10.12: Versuche an Flankenkehlnähten verschiedener Grund- und Schweißzusatzwerkstoffe

\begin{tabular}{|c|c|c|c|c|c|c|}
\hline \multirow{2}{*}{\begin{tabular}{|l} 
Herkunft \\
\end{tabular}} & \multirow[t]{2}{*}{ Anzahl } & \multirow{2}{*}{\multicolumn{2}{|c|}{\begin{tabular}{|l} 
Grundwerkstoff \\
$\mathrm{f}_{\mathrm{u}, \mathrm{GW}}\left[\mathrm{N} / \mathrm{mm}^{2}\right]$ \\
\end{tabular}}} & \multirow{2}{*}{\multicolumn{2}{|c|}{$\begin{array}{l}\text { Schweißzusatzwerkstoff } \\
\mathrm{f}_{\mathrm{u}, \mathrm{Szw}}\left[\mathrm{N} / \mathrm{mm}^{2}\right] \\
\end{array}$}} & \multirow{2}{*}{$\begin{array}{l}0,25 \cdot f_{\mathrm{u}, \mathrm{GW}} \\
+0,75 \cdot \mathrm{f}_{\mathrm{u}, \mathrm{SZW}}\end{array}$} \\
\hline & & & & & & \\
\hline \multicolumn{7}{|l|}{ S355-G42 } \\
\hline [Kob et al., 1987] & 50 & S355J0 & MW 551 & G42 & 590 & 580 \\
\hline [FOSTA P652, 2008] & 4 & S355J2 & 557 & G42 & 583 & 577 \\
\hline \multicolumn{7}{|l|}{ S460-G42 } \\
\hline [FOSTA P652, 2008] & 2 & S460M & 621 & G42 & 583 & 593 \\
\hline [Collin, Johansson, 2005] & 10 & S650MC & 827 & E42 & 548 & 618 \\
\hline \multicolumn{7}{|l|}{ S690Q-G42 } \\
\hline [Kob et al., 1987] & 39 & S690Q & MW 846 & G42 & 577 & 644 \\
\hline \multicolumn{7}{|l|}{ S355-G46 } \\
\hline [FOSTA P652, 2008] & 2 & S355J2 & 557 & G46 & 639 & 619 \\
\hline \multicolumn{7}{|l|}{ S460-G/T/E46 } \\
\hline [Saal, 2005] & 34 & S460ML & MW 631 & T46 & 618 & 621 \\
\hline [FOSTA P652, 2008] & 20 & $\mathrm{~S} 460 \mathrm{M} / \mathrm{ML}$ & MW 616 & G/E/T46 & MW 626 & 624 \\
\hline [FOSTA P812, 2011] & $4(2)$ & S460M & 538 & G46 & 602 & 586 \\
\hline \multicolumn{7}{|l|}{ S690-G46 } \\
\hline [FOSTA P652, 2008] & 1 & S690Q & 871 & G46 & 639 & 697 \\
\hline [FOSTA P812, 2011] & $3(2)$ & S700M & 891 & G46 & 602 & 674 \\
\hline [FOSTA P812, 2011] & $2(1)$ & S690Q & 848 & G46 & 602 & 664 \\
\hline \multicolumn{7}{|l|}{ S460-„E55“ } \\
\hline [Background D.03, 1990] & 13 & S460 & MW 672 & E55 & 620 & 633 \\
\hline \multicolumn{7}{|l|}{ „S640“-E55 } \\
\hline [Niemi, 1988] & 14 & S640 & MW 743 & E55 & 739 & 740 \\
\hline \multicolumn{7}{|l|}{ S460-G69 } \\
\hline [FOSTA P652, 2008] & 2 & S460M & 621 & G69 & 836 & 782 \\
\hline \multicolumn{7}{|l|}{ S690-G/T/69 } \\
\hline [FOSTA P652, 2008] & 9 & S690Q/QL & MW 872 & G/T69 & MW 827 & 838 \\
\hline [Zusatzversuche, 2008] & $4(2)$ & S690QL & 797 & G69 & 836 & 826 \\
\hline [FOSTA P812, 2011] & $24(16)$ & S700M & MW 877 & G/T69 & MW 796 & 816 \\
\hline [FOSTA P812, 2011] & $16(8)$ & S690Q & MW 849 & G/T69 & MW 783 & 800 \\
\hline \multicolumn{7}{|l|}{ S355J2-G89 } \\
\hline [Zusatzversuche, 2008] & 1 & S355J2 & 557 & G89 & 981 & 875 \\
\hline \multicolumn{7}{|l|}{ S690-G89 } \\
\hline [Zusatzversuche, 2008] & $4(2)$ & S690QL & 797 & G89 & 981 & 935 \\
\hline [FOSTA P812, 2011] & $3(2)$ & S700M & 891 & G89 & 1028 & 994 \\
\hline [FOSTA P812, 2011] & $2(1)$ & S690Q & 848 & G89 & 1028 & 983 \\
\hline
\end{tabular}




\subsubsection{Statistische Auswertung der Versuche für die verschiedenen Schweißzu- satzwerkstoffe}

\section{Allgemeines}

Die in Tabelle 10.12 zusammengestellten Versuche wurden getrennt statistisch ausgewertet, da sich für jeden Schweißzusatzwerkstoff ein anderer Beiwert $\beta_{w}$ einstellt. Als Bemessungsgleichung dient der Vorschlag nach Gleichung (10.5). Das heißt, die Versuchsergebnisse werden unter Berücksichtigung der Festigkeiten von Grund- und Schweißzusatzwerkstoffen statistisch ausgewertet. Die detaillierte statistische Auswertung ist in Anhang A.5 für die einzelnen Schweißzusatzwerkstoffe aufgeführt. In diesem Abschnitt werden die Ergebnisse der Auswertung erläutert und bewertet. Die Ergebnisse sind jeweils auf die nominelle Zugfestigkeit der Grundwerkstoffe bezogen.

\section{Schweißverbindungen mit Schweißzusatzwerkstoffen der Festigkeitsklasse 42}

Für die Schweißverbindungen mit dem Schweißzusatzwerkstoff der nominellen Streckgrenze $\mathrm{f}_{\mathrm{y}}=420 \mathrm{~N} / \mathrm{mm}^{2}$ wurde ein Beiwert $\beta_{\mathrm{w}}=0,890$ ermittelt. In Abbildung 10.7 sind die Versuchsergebnisse mit den Schweißzusatzwerkstoffen G42 und E42 gemeinsam mit den Bemessungswerten in Abhängigkeit der nominellen Zugfestigkeit der Grundwerkstoffe aufgetragen. Man sieht die Steigerung der Tragfähigkeit in Abhängigkeit vom eingesetzten Grundwerkstoff. Der Bemessungsansatz zeigt eine gute Übereinstimmung mit den Versuchsergebnissen. Die Variation der Grundwerkstoffe wird gut abgebildet.

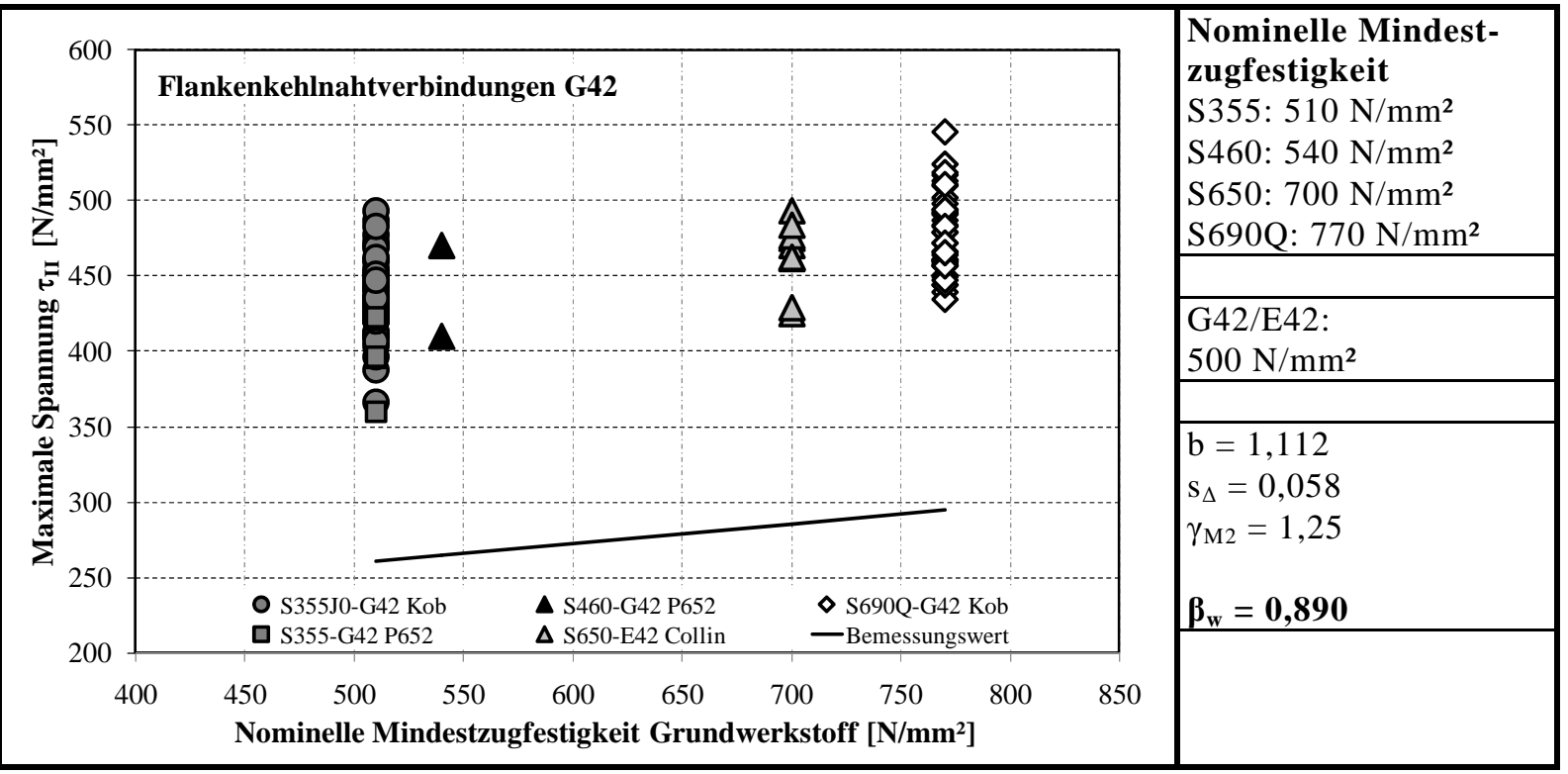

Abbildung 10.7: Versuchsergebnisse der Festigkeitsklasse 42 im Vergleich mit den Bemessungswerten 
Schweißverbindungen mit Schweißzusatzwerkstoffen der Festigkeitsklasse 46

Alle Versuchsergebnisse mit den Schweißzusatzwerkstoffen G46, T46 und E46 sind in Abbildung 10.8 gemeinsam mit den Bemessungswerten in Abhängigkeit der nominellen Mindestzugfestigkeit der Grundwerkstoffe dargestellt. Man sieht die Steigerung der Tragfähigkeit in Abhängigkeit vom eingesetzten Grundwerkstoff. Für die Verbindungen mit dem Grundwerkstoff S690 stehen nur wenige Versuchsergebnisse zur Verfügung, die stark streuen. Als Tendenz kann das Bemessungskonzept auch für diese Versuche bestätigt werden. Als Ergebnis der statistischen Auswertung wurde für Schweißverbindungen mit Schweißzusatzwerkstoffen der Festigkeitsklasse 46 ein Beiwert $\beta_{w}=0,847$ ermittelt, vorgeschlagen wird ein Beiwert $\beta_{\mathrm{w}}=0,85$.

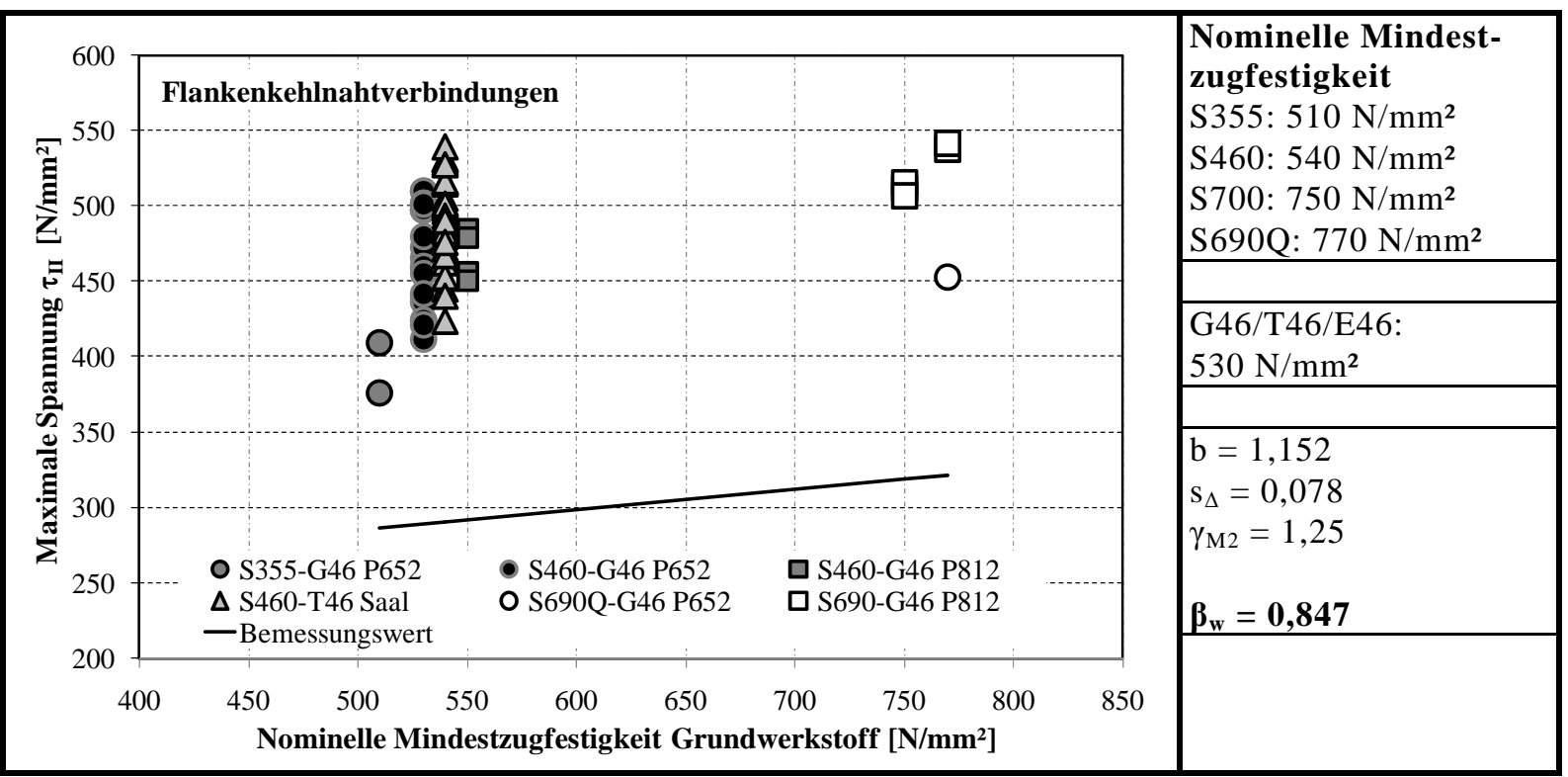

Abbildung 10.8: Versuchsergebnisse der Festigkeitsklasse 46 im Vergleich mit den Bemessungswerten

\section{Schweißverbindungen mit Schweißzusatzwerkstoffen der Festigkeitsklasse 55}

Für die Schweißverbindungen mit dem Schweißzusatzwerkstoff E55 wurde ein Beiwert $\beta_{\mathrm{w}}$ von $\beta_{\mathrm{w}}=$ 0,961 ermittelt. Da die einzelnen Serien stark streuen (vgl. Kapitel 5), weist auch die gemeinsame Auswertung eine etwas größere Streuung auf, die jedoch deutlich geringer ist als die der Einzelserien. Abbildung 10.9 zeigt die Versuchsergebnisse gemeinsam mit den Bemessungswerten bezogen auf die nominelle Zugfestigkeit der Grundwerkstoffe. Vorgeschlagen wird ein Beiwert $\beta_{\mathrm{w}}$ von $\beta_{\mathrm{w}}=0,96$.

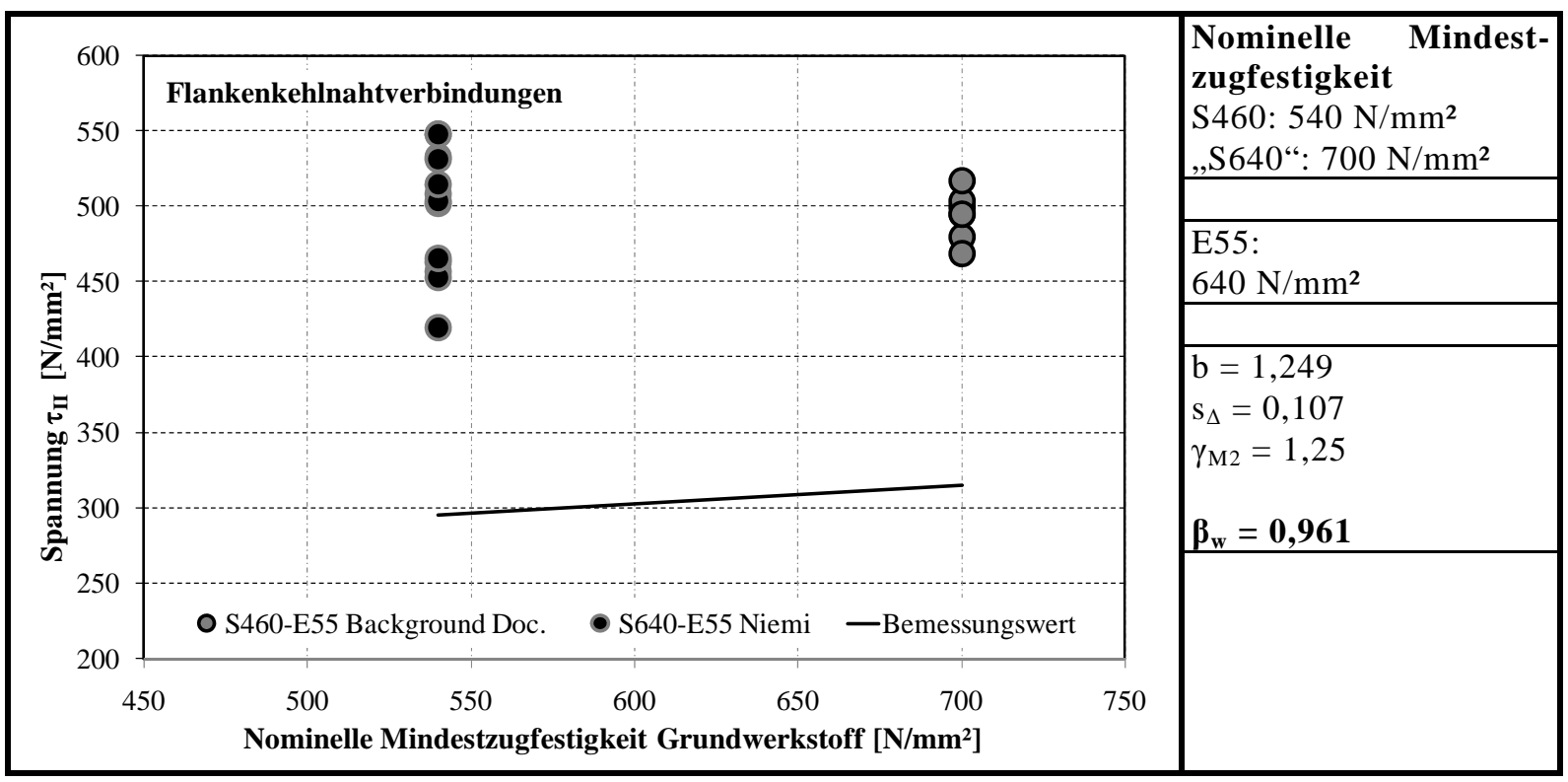

Abbildung 10.9: Versuchsergebnisse der Festigkeitsklasse 55 im Vergleich mit den Bemessungswerten 
Schweißverbindungen mit Schweißzusatzwerkstoffen der Festigkeitsklasse 69

Zur Bestimmung des Beiwertes $\beta_{\mathrm{w}}$ von Schweißverbindungen mit den Schweißzusatzwerkstoffen G69 und T69 stehen nur zwei Versuche mit unterschiedlichen Festigkeiten des Grund- und Schweißzusatzwerkstoffs (S460-G69) zur Verfügung. Durch die geringe Anzahl von Versuchen können hier nur Tendenzen aufgezeigt werden. Aus der statistischen Auswertung ergibt sich ein Beiwert $\beta_{\mathrm{w}}=1,087$, vorgeschlagen wird ein Beiwert $\beta_{\mathrm{w}}=1,09$. Aufgetragen sind die Versuchsergebnisse gemeinsam mit den Bemessungswerten in Abbildung 10.10 .

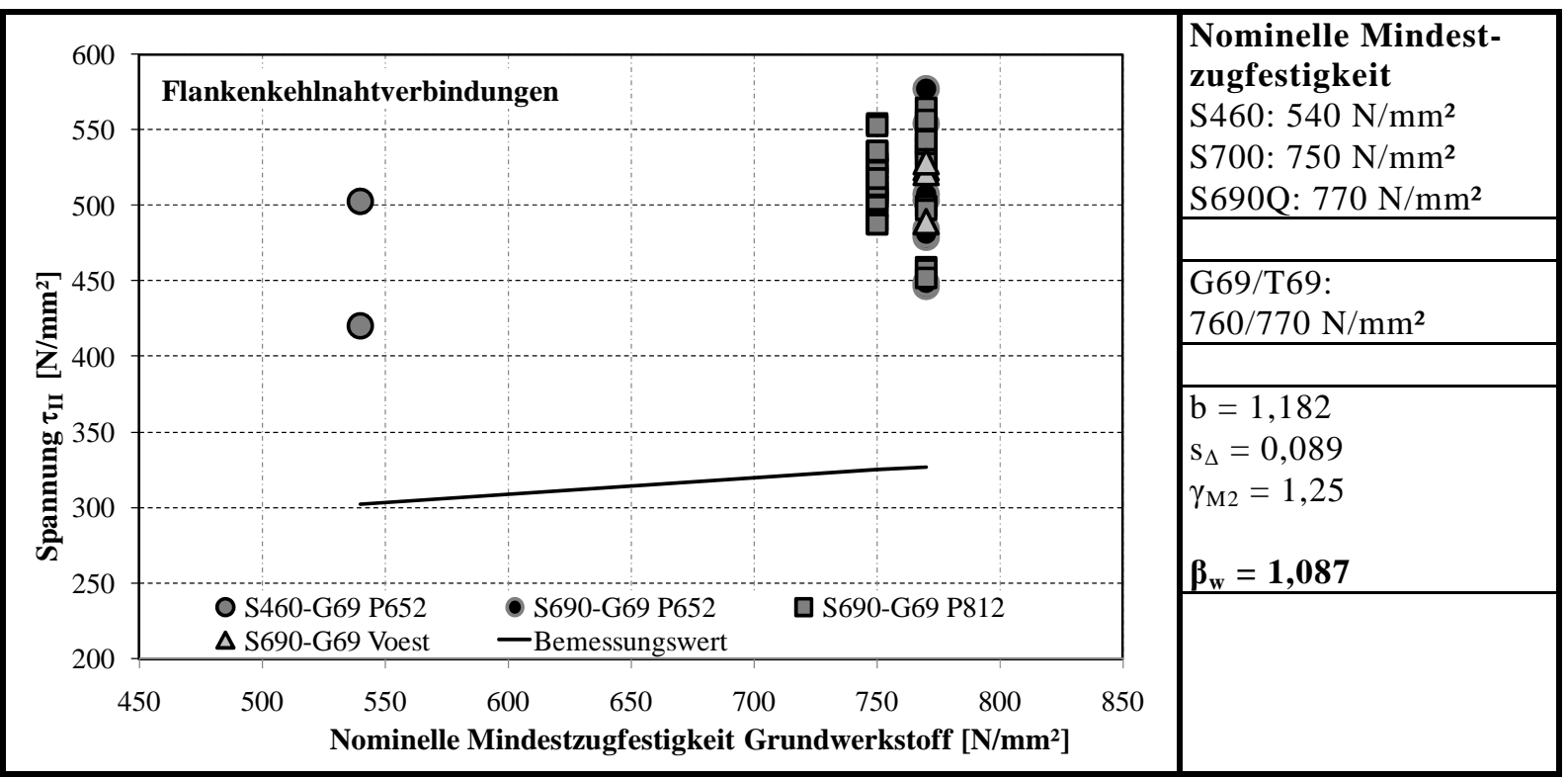

Abbildung 10.10: Versuchsergebnisse der Festigkeitsklasse 69 im Vergleich mit den Bemessungswerten

Schweißverbindungen mit Schweißzusatzwerkstoffen der Festigkeitsklasse 89

Die Tragfähigkeit von Schweißverbindungen mit dem Schweißzusatzwerkstoff G89 wurde nur an Verbindungen mit verschiedenen Grund- und Schweißzusatzwerkstoffen bestimmt. Da für die statistische Auswertung nur 10 Versuche (statistisch 5 Versuche, vgl. Tabelle 10.12) berücksichtigt werden konnten, ergibt sich ein großer Abstand zwischen Bemessungsgrenzspannung und Versuch. Statistisch ergab sich ein Beiwert $\beta_{w}=1,186$, vorgeschlagen wird ein Beiwert $\beta_{w}=1,19$. In Abbildung 10.11 sieht man die Steigerung der Tragfähigkeit in Abhängigkeit vom eingesetzten Grundwerkstoff. Auch für den Versuch mit dem Grundwerkstoff S355 lässt sich der Bemessungsansatz gut anwenden.

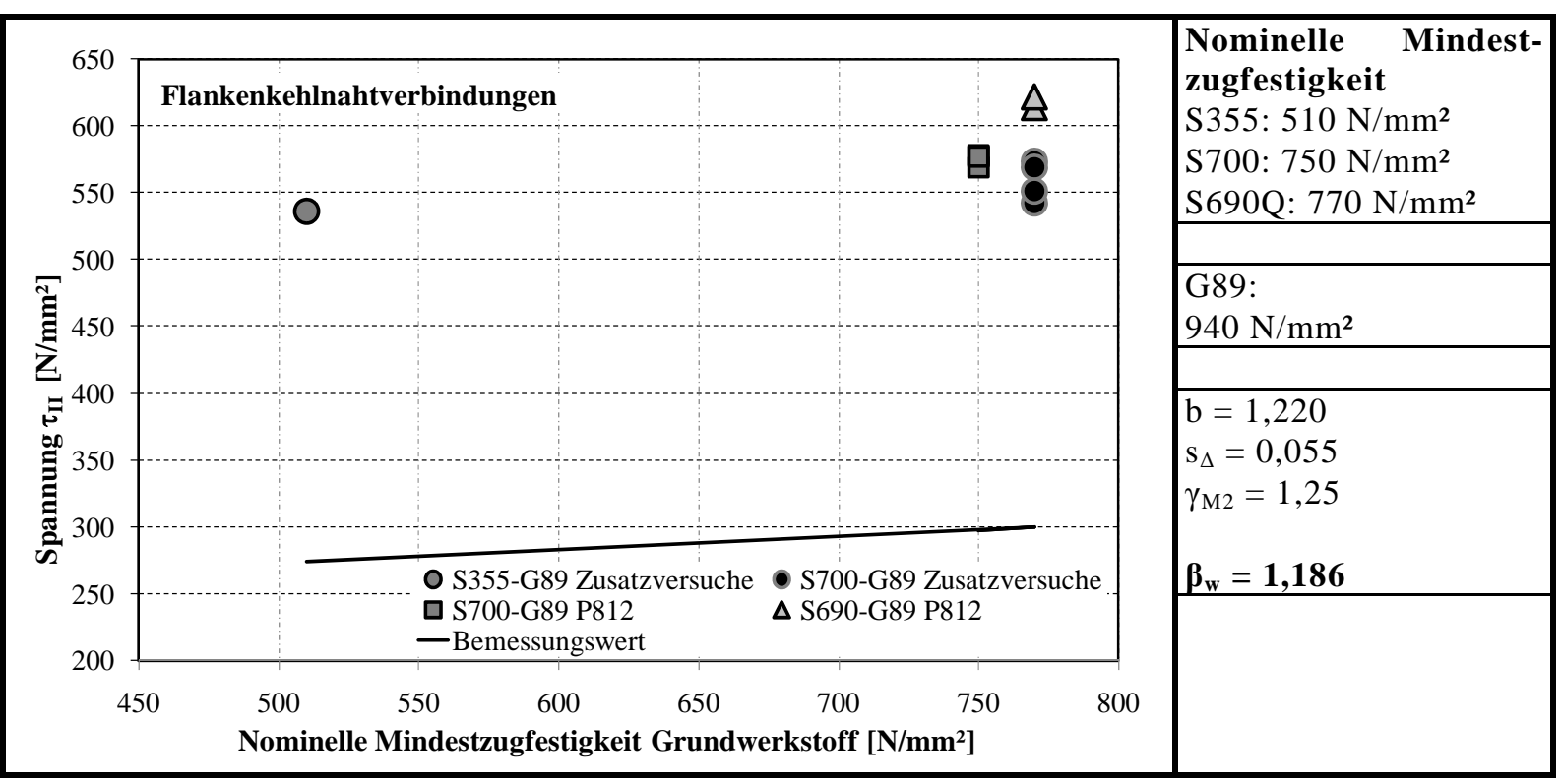

Abbildung 10.11: Versuchsergebnisse der Festigkeitsklasse 89 im Vergleich mit den Bemessungswerten 


\subsubsection{Bemessungsvorschlag für Flankenkehlnähte in Abhängigkeit des Schweiß- zusatzwerkstoffs}

In Tabelle 10.13 wird der entwickelte Bemessungsvorschlag zusammengefasst. Für die verschiedenen Grund- und Schweißzusatzwerkstoffe werden jeweils die Bemessungsgrenzspannungen $\tau_{\text {Rd }}$ nach Gleichung (10.5) bestimmt. Mit den gewählten Annahmen kann nun die Festigkeit von Grund- und Schweißzusatzwerkstoff berücksichtigt werden.

Tabelle 10.13: Bemessungsvorschlag für Flankenkehlnahtverbindungen

\begin{tabular}{|c|c|c|c|c|c|c|c|}
\hline Stahlsorte & S355 & & & S460 & & S700 & S690 \\
\hline & JR/JO/J2 & N/NL & M/ML & N/NL/M/ML & Q/QL/QL1 & MC & Q/QL/QL1 \\
\hline $\mathrm{f}_{\mathrm{y}}\left[\mathrm{N} / \mathrm{mm}^{2}\right]$ & 355 & 355 & 355 & 460 & 460 & 700 & 690 \\
\hline $\mathrm{f}_{\mathrm{u}}\left[\mathrm{N} / \mathrm{mm}^{2}\right]$ & 510 & 490 & 470 & 540 & 570 & 750 & 770 \\
\hline G42/E42 & $\beta_{w}=0,89$ & $420 \mathrm{~N} /$ & $m^{2} f_{u}=$ & $0 \mathrm{~N} / \mathrm{mm}^{2}$ & & & \\
\hline$\tau_{\mathrm{Rd}}\left[\mathrm{N} / \mathrm{mm}^{2}\right]$ & 261 & 258 & 256 & 265 & 269 & 292 & 295 \\
\hline G46/E46/T46 & $\beta_{w}=0,85$ & $460 \mathrm{~N} /$ & $\mathbf{m}^{2} \mathbf{f}_{u}=$ & $0 \mathrm{~N} / \mathrm{mm}^{2}$ & & & \\
\hline$\tau_{\mathrm{Rd}}\left[\mathrm{N} / \mathrm{mm}^{2}\right]$ & 285 & 283 & 280 & 289 & 293 & 318 & 321 \\
\hline E55 & $\beta_{w}=0,96$ & $550 \mathrm{~N} /$ & $\mathbf{m}^{2} \mathbf{f}_{u}=$ & $0 \mathrm{~N} / \mathrm{mm}^{2}$ & & & \\
\hline$\tau_{\mathrm{Rd}}\left[\mathrm{N} / \mathrm{mm}^{2}\right]$ & 292 & 290 & 287 & 296 & 300 & 321 & 324 \\
\hline G69/T69 & $\beta_{w}=1,09$ & $690 \mathrm{~N} /$ & $\mathbf{m}^{2} \mathbf{f}_{u}=$ & $0 \mathrm{~N} / \mathrm{mm}^{2}$ & & & \\
\hline$\tau_{\mathrm{Rd}}\left[\mathrm{N} / \mathrm{mm}^{2}\right]$ & 299 & 297 & 295 & 302 & 305 & 324 & 326 \\
\hline G89 & $\beta_{w}=1,19$ & $890 \mathrm{~N} /$ & $\mathbf{m}^{2} \mathbf{f}_{u}=$ & $0 \mathrm{~N} / \mathrm{mm}^{2}$ & & & \\
\hline$\tau_{\mathrm{Rd}}\left[\mathrm{N} / \mathrm{mm}^{2}\right]$ & 323 & 321 & 319 & 326 & 329 & 346 & 348 \\
\hline
\end{tabular}

In Abbildung 10.12 und Abbildung 10.13 sind die ermittelten Bemessungsgrenzspannungen für Flankenkehlnähte grafisch dargestellt. In Abbildung 10.12 sind die Bemessungsgrenzspannungen für die verschiedenen Stahlgüten in Abhängigkeit der nominellen Festigkeit des Schweißzusatzwerkstoffs aufgetragen, während Abbildung 10.13 die Bemessungsgrenzspannungen für die einzelnen Schweißzusatzwerkstoffe in Abhängigkeit der nominellen Festigkeit des Grundwerkstoffs zeigt.

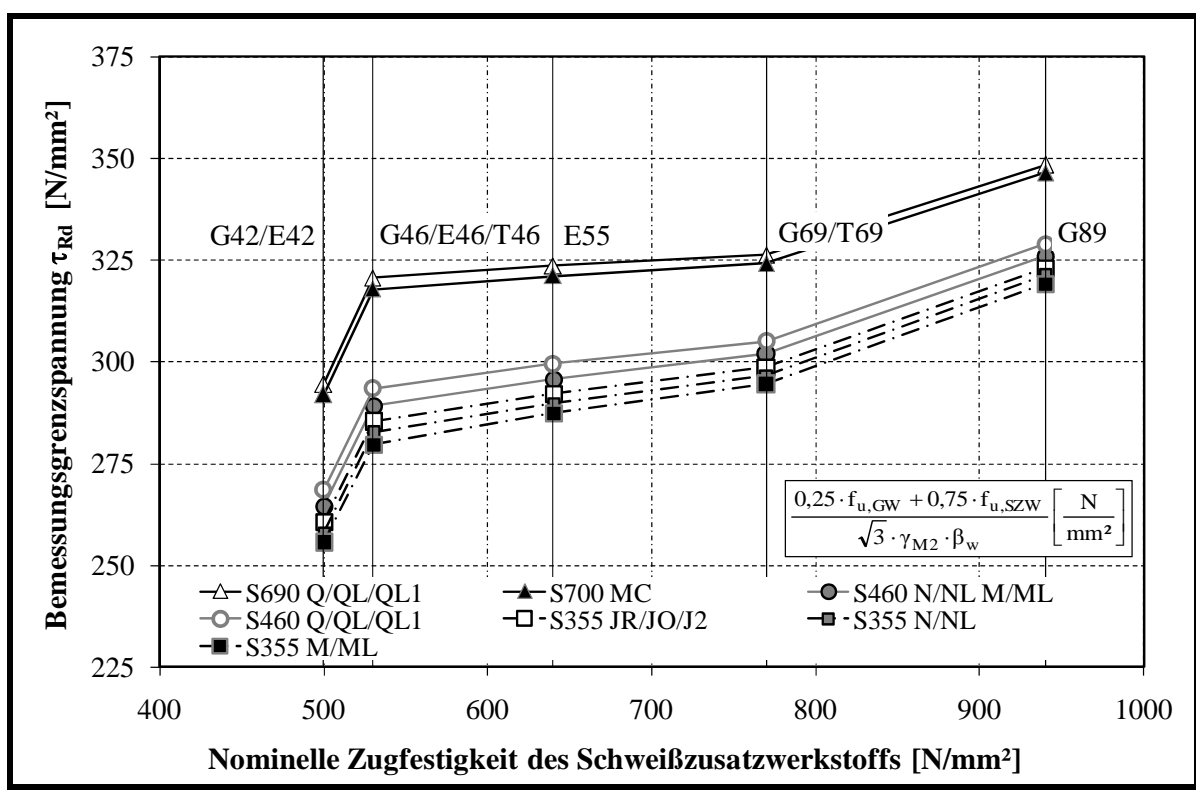

Abbildung 10.12: Darstellung des Bemessungsvorschlags für Flankenkehlnahtverbindungen in Abhängigkeit der Festigkeit des Schweißzusatzwerkstoffs 


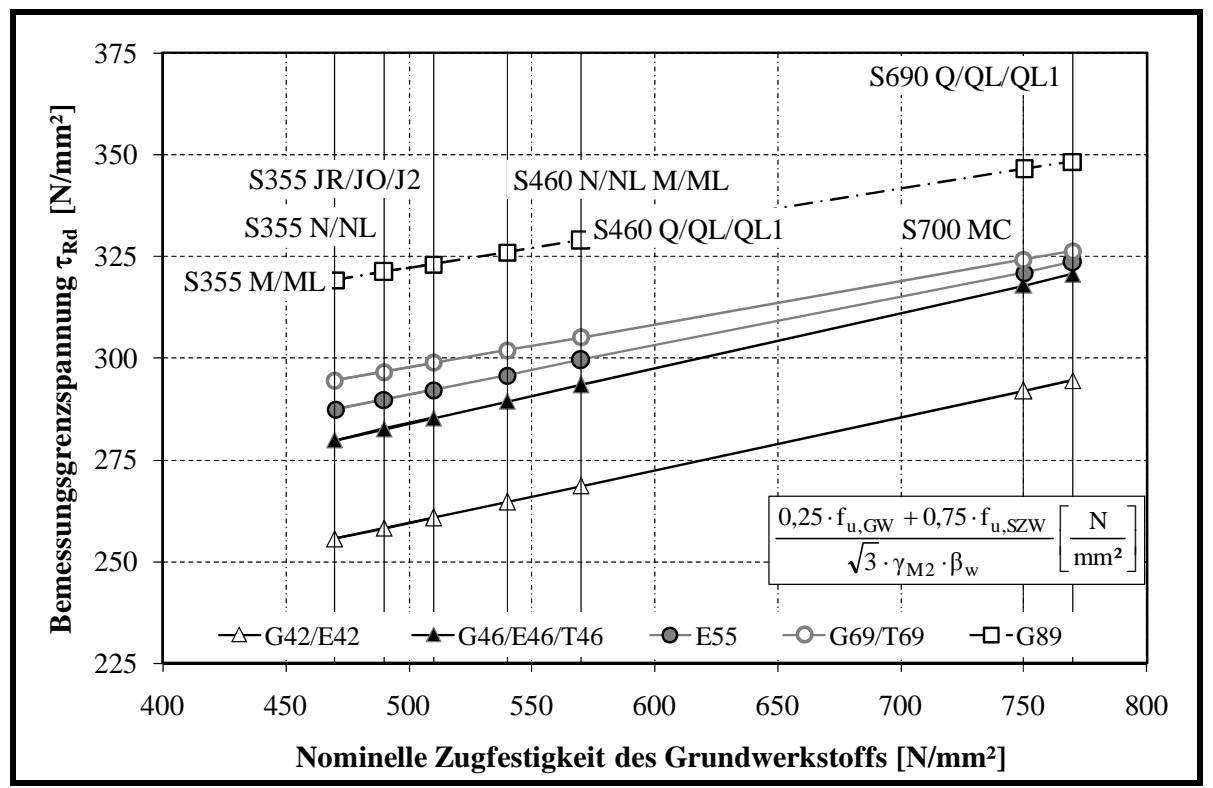

Abbildung 10.13: Darstellung des Bemessungsvorschlags für Flankenkehlnahtverbindungen in Abhängigkeit der Festigkeit des Grundwerkstoffs

Um den vorgestellten Bemessungsansatz für Flankenkehlnahtverbindungen zu beurteilen, werden nun alle Versuche mit den vorgeschlagenen Beiwerten $\beta_{\mathrm{w}}$ und der neuen Bemessungsgleichung ausgewertet, wie in Tabelle 10.14 dargestellt. Mit einer Mittelwertabweichung von $b=1,17$ und einem Teilsicherheitsbeiwert $\gamma_{\mathrm{M}^{*}}=1,23<1,25$ zeigt sich, dass die Bemessungsgleichung sehr gute Übereinstimmungen liefert.

Tabelle 10.14: Statistische Auswertung aller Flankenkehlnahtverbindungen

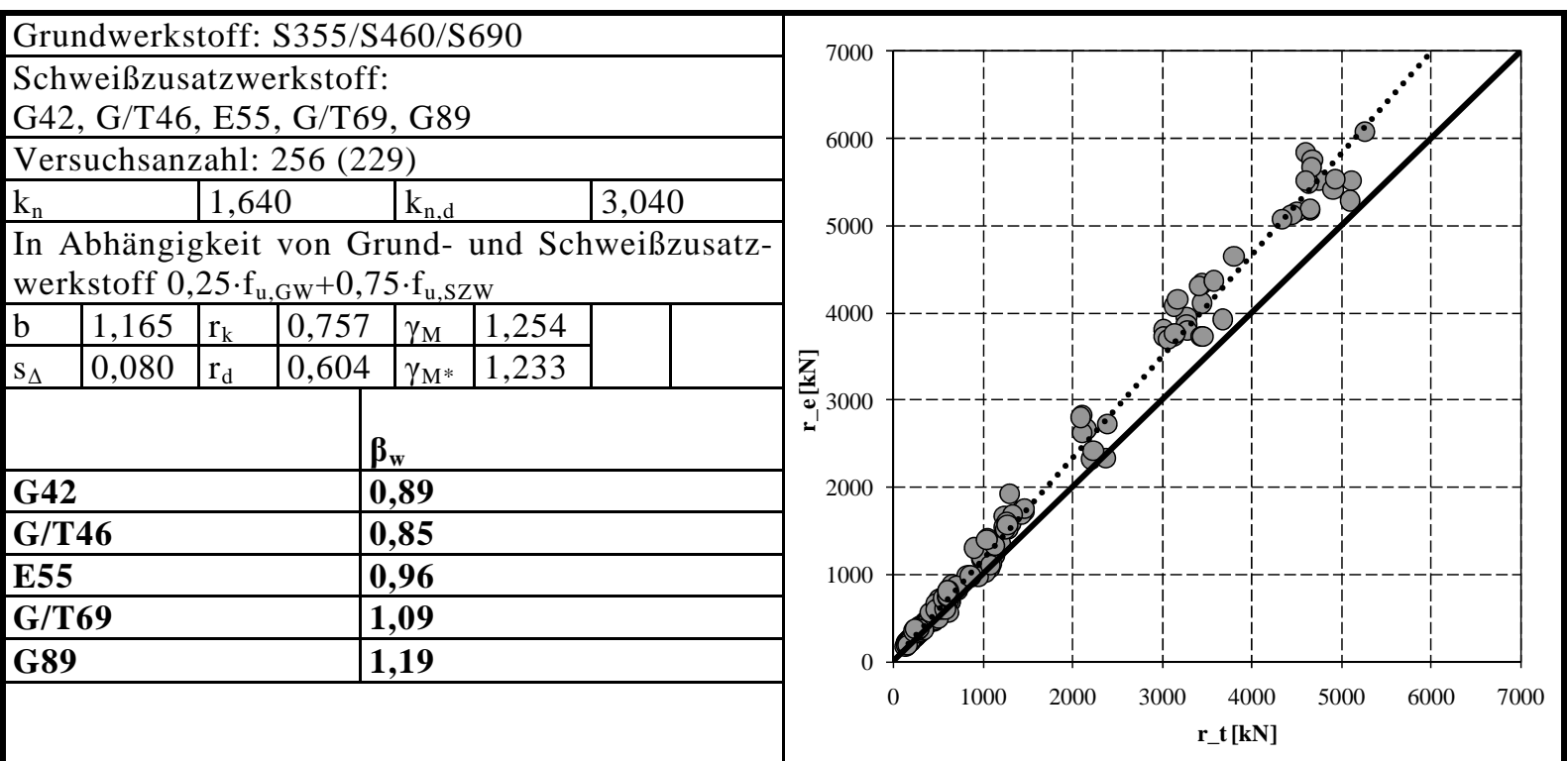

In Abbildung 10.14 sind die Versuchsergebnisse im Vergleich mit den Bemessungsgrenzspannungen dargestellt. Auf der Abszisse aufgetragen ist die Bemessungsgrenzspannung, ermittelt aus den nominellen Festigkeiten der Grund- und Schweißzusatzwerkstoffe im Verhältnis 25 \% des Grundwerkstoffs und $75 \%$ des Schweißzusatzwerkstoffs unter Berücksichtigung des jeweiligen Beiwertes $\beta_{\mathrm{w}}$. Aufgrund der unterschiedlichen Anzahl von Versuchen (vgl. Kapitel 10.6.3) variiert der Abstand zwischen den Versuchsergebnissen und den jeweiligen Bemessungswerten. Es zeigt sich aber eine gute Übereinstimmung zwischen Versuchsergebnissen und Bemessungsgleichung. 


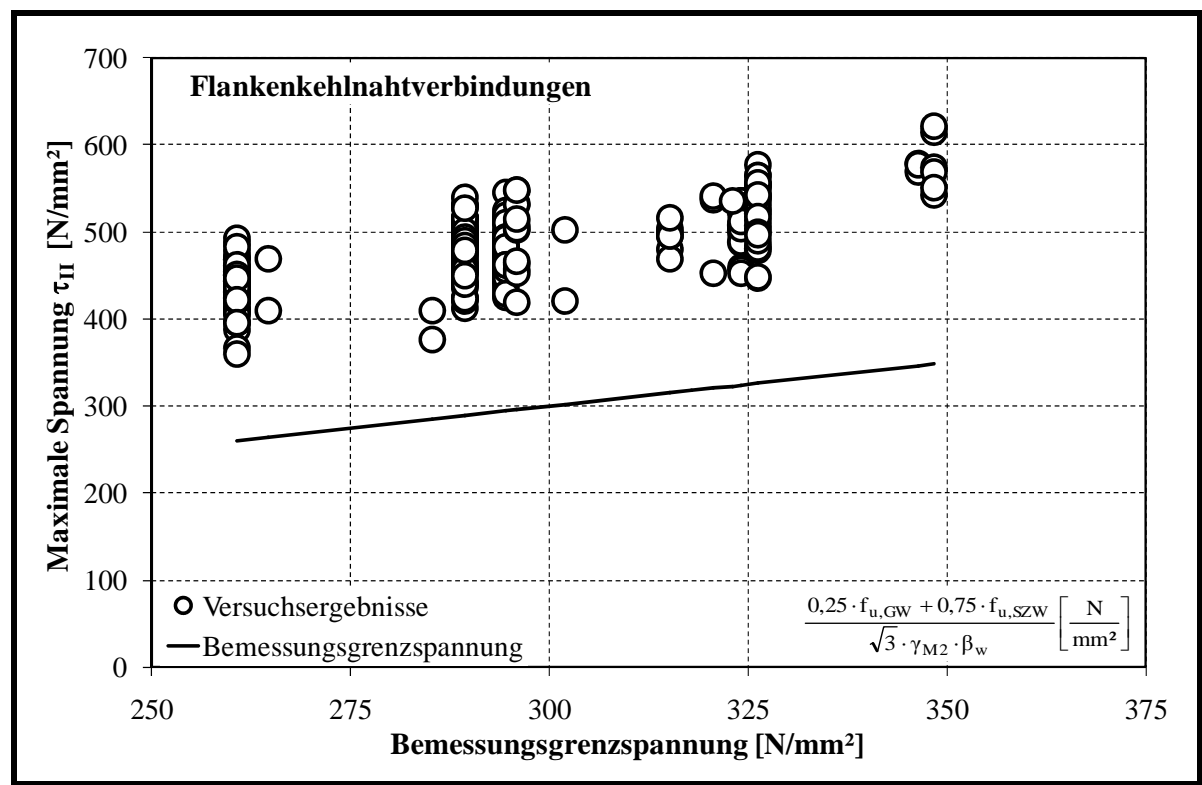

Abbildung 10.14: Versuchsergebnisse und Bemessungsvorschlag für Flankenkehlnahtverbindungen

\subsubsection{Bemessungsvorschlag für Kreuzstöße in Abhängigkeit des Schweißzusatz- werkstoffs}

Für die Kreuzstöße wird kein weiterer Bemessungsvorschlag gemacht, da die Ergebnisse sehr stark streuen und nur wenige Ergebnisse vorliegen. Die wenigen Ergebnisse konnten nicht gemeinsam ausgewertet werden, da die Ergebnisse auf den zwei unterschiedlichen Nahtflächen $A_{E C}$ und $A_{B r u c h}$ beruhen.

Außerdem ist, wie in Kapitel 4 erläutert, eine genaue Bestimmung der maximalen Spannung für Kreuzstöße aus den Versuchsergebnissen sehr schwierig. Neben der Bruchfläche und der maximalen Kraft muss der Bruchwinkel berücksichtigt werden, weil die Kraft über diesen Winkel aufgeteilt werden muss. Da der Kreuzstoß zwei Nähte mit unterschiedlichen Bruchwinkeln hat, muss zusätzlich die Kraft auf die beiden Nähte aufgeteilt werden. Da dieses Vorgehen praktisch nicht umsetzbar ist, muss auf Vereinfachungen zurückgegriffen werden. In Kapitel 4 wurde gezeigt, dass der Ansatz, die Kräfte unter einem Winkel von $45^{\circ}$ senkrecht und waagerecht aufzuteilen, fehlerbehaftet ist. Der Fehler ist abhängig von der Ausbildung der Naht. Dieser Ansatz liegt jedoch immer auf der sicheren Seite.

In dieser Arbeit werden die in Kapitel 10.6.4 für Flankenkehlnähte hergeleiteten Beiwerte $\beta_{\mathrm{w}}$ für die Bemessung von Kreuzstößen angewendet. Die vorhandenen Versuchsergebnisse werden in Abbildung 10.15 mit den so bestimmten Bemessungsgrenzspannungen verglichen. Es zeigt sich ein ausreichender Abstand zwischen den Versuchsergebnissen und den Bemessungswerten. Auf der Abszisse aufgetragen ist die Bemessungsgrenzspannung, ermittelt aus den nominellen Festigkeiten der Grund- und Schweißzusatzwerkstoffe im Verhältnis $25 \%$ des Grundwerkstoffs und 75 \% des Schweißzusatzwerkstoffs unter Berücksichtigung des jeweiligen Beiwertes $\beta_{\mathrm{w}}$. Es zeigt sich aber auch, dass der Abstand zwischen den Versuchsergebnissen und den Bemessungswerten sehr ungleichmäßig ist. Dies deutet darauf hin, dass der Bemessungsansatz für Kreuzstöße noch Reserven enthält. Auf der sicheren Seite liegend kann dieser Bemessungsvorschlag also für Stirnkehlnähte verwendet werden. 


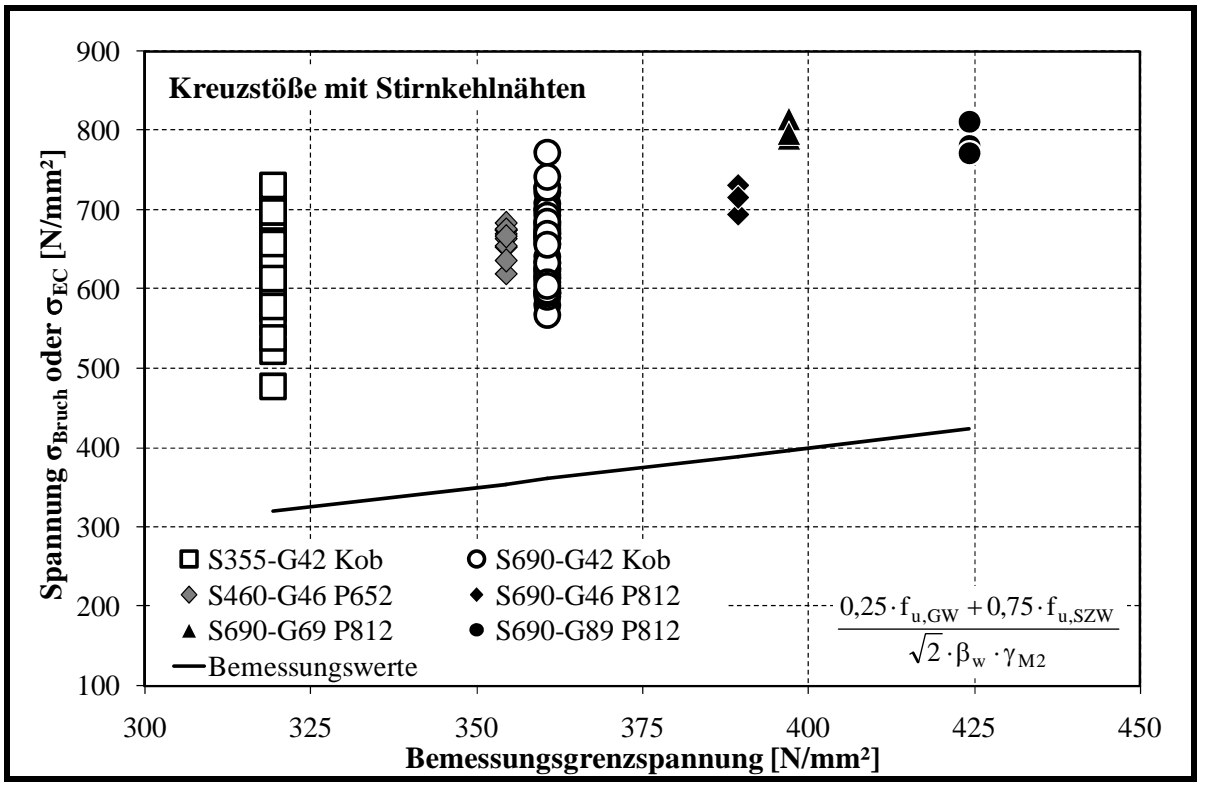

Abbildung 10.15: Versuchsergebnisse und Bemessungsvorschlag für Kreuzstöße

\subsection{Beurteilung des Bemessungsansatzes}

\subsubsection{Vergleich mit dem bisherigem Modell nach EN 1993-1-8 und 1-12}

In den beiden folgenden Diagrammen werden die erreichten Bemessungsgrenzspannungen des bestehenden Konzepts nach Eurocode und den neu ermittelten Beiwerten (Abbildung 10.6) mit den Bemessungsgrenzspannungen des neuen Ansatzes verglichen (Tabelle 10.13). Die Diagramme bauen auf Abbildung 10.12 auf. Aus Gründen der Übersichtlichkeit werden die Werte für den Stahl S690 in Abbildung 10.16 und die Werte für die Stähle S460 und S355 in Abbildung 10.17 dargestellt. In Abbildung 10.16 ist außerdem ist die Regelung nach EN 1993-1-12 (2007) dargestellt, nach der Verbindungen mit dem Grundwerkstoff S690 und Schweißzusatzwerkstoffen geringerer Festigkeit geregelt sind, wie in Kapitel 3, Tabelle 3.6 dargestellt. Dabei ist für Verbindungen S690Q-G69 der Nationale Anhang DIN EN 1993-1-12/NA (2011) berücksichtigt, der für diese Verbindungen einen Beiwert $\beta_{\mathrm{w}}=$ 1,2 anstelle von $\beta_{\mathrm{w}}=1,0$ (EN 1993-1-12 (2007)) fordert.

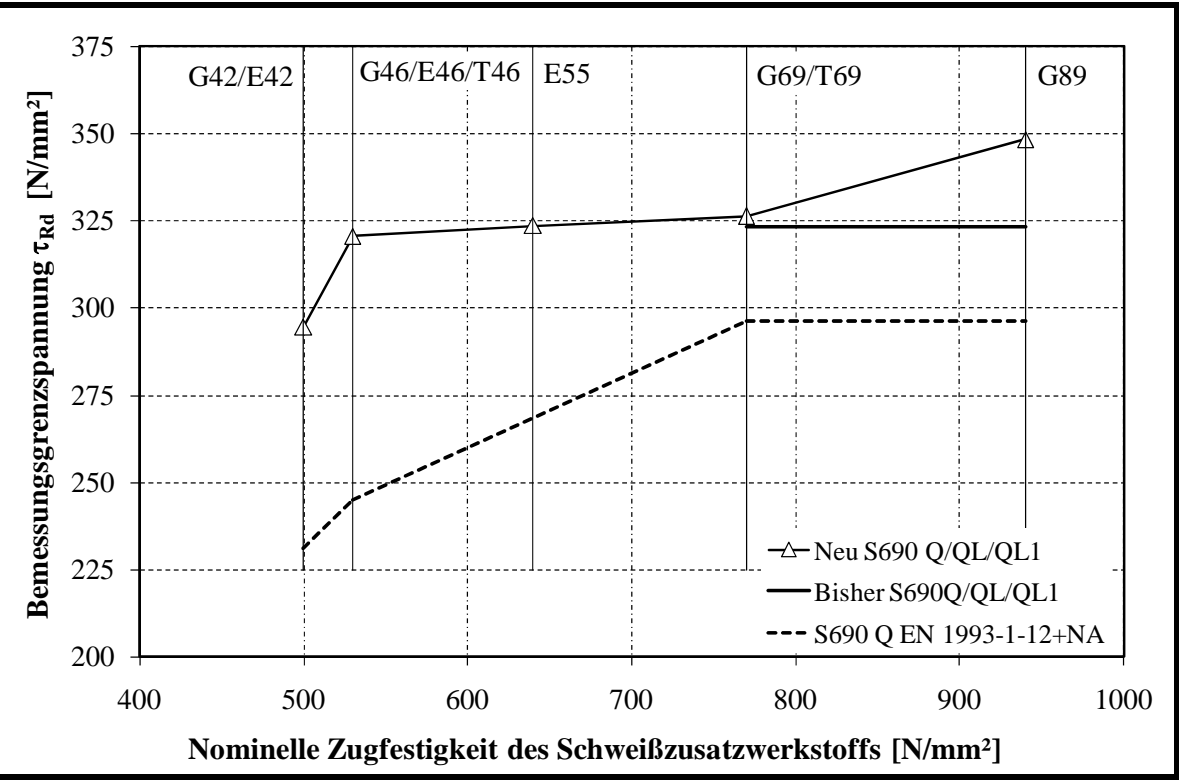

Abbildung 10.16: Vergleich beider Bemessungsansätze für den Stahl S690 


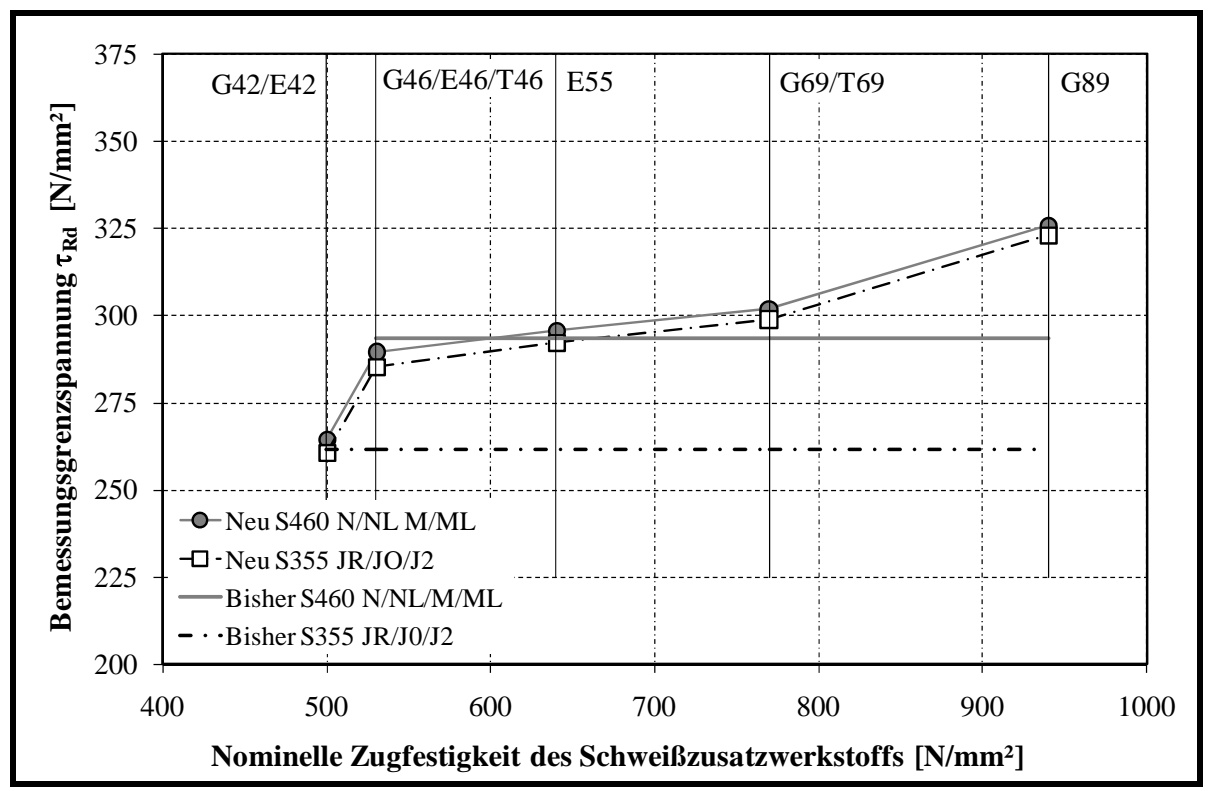

Abbildung 10.17: Vergleich beider Bemessungsansätze für Stähle S355 und S460

Für den Ansatz nach EN 1993-1-8 (2005) ergeben sich unabhängig vom Schweißzusatzwerkstoff konstante Werte (= horizontale Linien). Dabei sind jedoch nur Schweißzusatzwerkstoffe erlaubt, deren Festigkeit mindestens der Festigkeit des Grundwerkstoffs entspricht. Der Ansatz nach EN 1993-1-12 (2007) und DIN EN 1993-1-12/NA (2011) zeigt ein uneinheitliches Bild. Für Verbindungen mit niederfesten Schweißzusatzwerkstoffen und dem Grundwerkstoff S690 ergeben sich geringere Bemessungsgrenzspannungen als bei gleichem Schweißzusatzwerkstoff und Grundwerkstoffen S460 oder S355.

Für den neuen Bemessungsansatz sind sowohl Schweißzusatzwerkstoffe geringerer sowie höherer Festigkeit als die des Grundwerkstoffs möglich.

Für die Grundwerkstoffe S355, S460 und S690 ergeben sich bei den Verbindungen gleicher Grundund Schweißzusatzwerkstoffe ähnliche Bemessungsgrenzspannungen bei beiden Verfahren. Bei Verwendung von höherfesten Schweißzusatzwerkstoffen können bei dem neuen Ansatz jedoch höhere Bemessungsgrenzspannungen als nach dem Verfahren nach EN 1993-1-8 (2005) in Anspruch genommen werden.

Der Vorteil des neuen Bemessungsansatzes besteht darin, dass unterschiedliche Festigkeiten für Grund- und Schweißzusatzwerkstoff berücksichtigt werden können. Damit sind zum einen Verbindungen mit Schweißzusatzwerkstoffen niedrigerer Festigkeit nun möglich. Zum anderen können höhere Bemessungsgrenzspannungen infolge höherer Festigkeiten des Schweißzusatzwerkstoffs in Anspruch genommen werden.

\subsubsection{Vergleich des Bemessungsansatzes mit weiteren bestehenden Bemessungs- gleichungen}

In Kapitel 3 wurden verschiedene in Europa und international bestehende Bemessungsmodelle verglichen und beurteilt. Dabei stellte sich heraus, dass lediglich die schwedische Norm BSK 99 (2003) neben der Festigkeit des Grundwerkstoffs die Festigkeit Schweißzusatzwerkstoffs berücksichtigt. Zur Bestimmung der Bemessungsgrenzspannung wird bei den Festigkeiten des Schweißzusatzwerkstoffs unterschieden, ob die Festigkeit des Schweißzusatzwerkstoffs höher oder niedriger als die Festigkeit des Grundwerkstoffs ist. Eine weitere Unterscheidung für unterschiedliche Schweißzusatzwerkstoffe findet nicht statt. Gefordert wird ein Sicherheitsbeiwert $\gamma_{M}=1$,2. Gültig ist dieser Bemessungsansatz nur für Stähle bis zu einer nominellen Streckgrenze von $\mathrm{f}_{\mathrm{y}}=690 \mathrm{~N} / \mathrm{mm}^{2}$. 
Aus Gleichung (3.10) bis (3.13) folgt folgende Gleichung zur Bestimmung der Bemessungsgrenzspannung für Flankenkehlnahtverbindungen:

$\sigma_{E d}=\frac{F_{E d}}{a_{w} \cdot l} \leq 0,6 \cdot f_{w d}$

Wenn $\mathrm{f}_{\mathrm{uk}, \mathrm{SZW}}>\mathrm{f}_{\mathrm{uk}, \mathrm{GW}}$ :

$f_{w d}=0,9 \cdot \frac{\sqrt{f_{u k, G W} \cdot f_{u k, S z W}}}{1,0 \cdot 1,2}$, zusätzlich Nachweis des Schenkels z mit $f_{u k,}$, ̈ür $f_{u k}<f_{w d}$

oder wenn $\mathrm{f}_{\mathrm{uk}, \mathrm{SzW}}<\mathrm{f}_{\mathrm{uk}, \mathrm{GW}}$ :

$\mathrm{f}_{\mathrm{wd}}=\frac{0,9 \cdot \mathrm{f}_{\mathrm{uk}, \mathrm{szW}}}{1,0 \cdot 1,2}$

Mit

$\mathrm{f}_{\mathrm{uk}, \mathrm{GW}}$ : charakteristische Zugfestigkeit des Grundwerkstoffs

$\mathrm{f}_{\mathrm{uk}, \mathrm{szw}}$ : Zugfestigkeit des Schweißzusatzwerkstoffes

Die Versuche, die, wie in Kapitel 10.6.4 beschrieben, für die statistische Auswertung mit dem neuen Bemessungsvorschlag verwendet wurden, werden nun mit dem Modell nach BSK 99 (2003) erneut statistisch ausgewertet (vgl. Tabelle 10.15), um die beiden Ansätze zu vergleichen. Mit der Mittelwertabweichung von $b=1,369$ zeigt sich eine deutlich größere Streuung als bei dem neuen Bemessungsansatz mit $b=1,165$.

Tabelle 10.15: Statistische Auswertung aller Flankenkehlnahtverbindungen nach BSK 99 (2003)

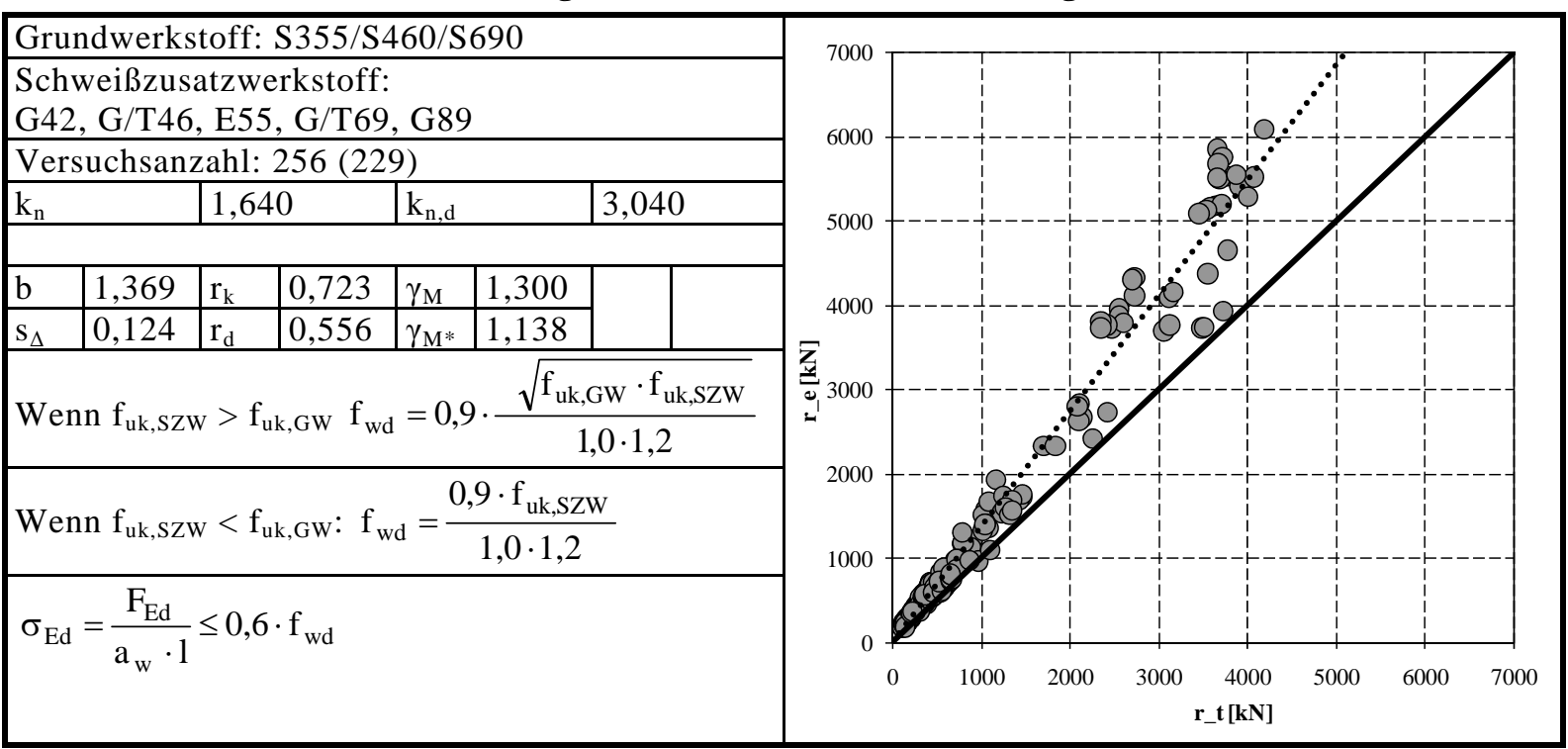

In Abbildung 10.18 sind die Versuchsergebnisse im Vergleich mit den Bemessungsgrenzspannungen nach BSK 99 (2003) dargestellt. Es zeigt sich, dass der Abstand zwischen Versuchen und Bemessungswert bei höheren Festigkeiten immer geringer wird. Darin zeigt sich die große Streuung, im Bereich geringer Festigkeiten liefert das Bemessungsmodell geringe Abweichungen, während im Bereich hoher Festigkeiten die Abweichungen immer größer werden. 


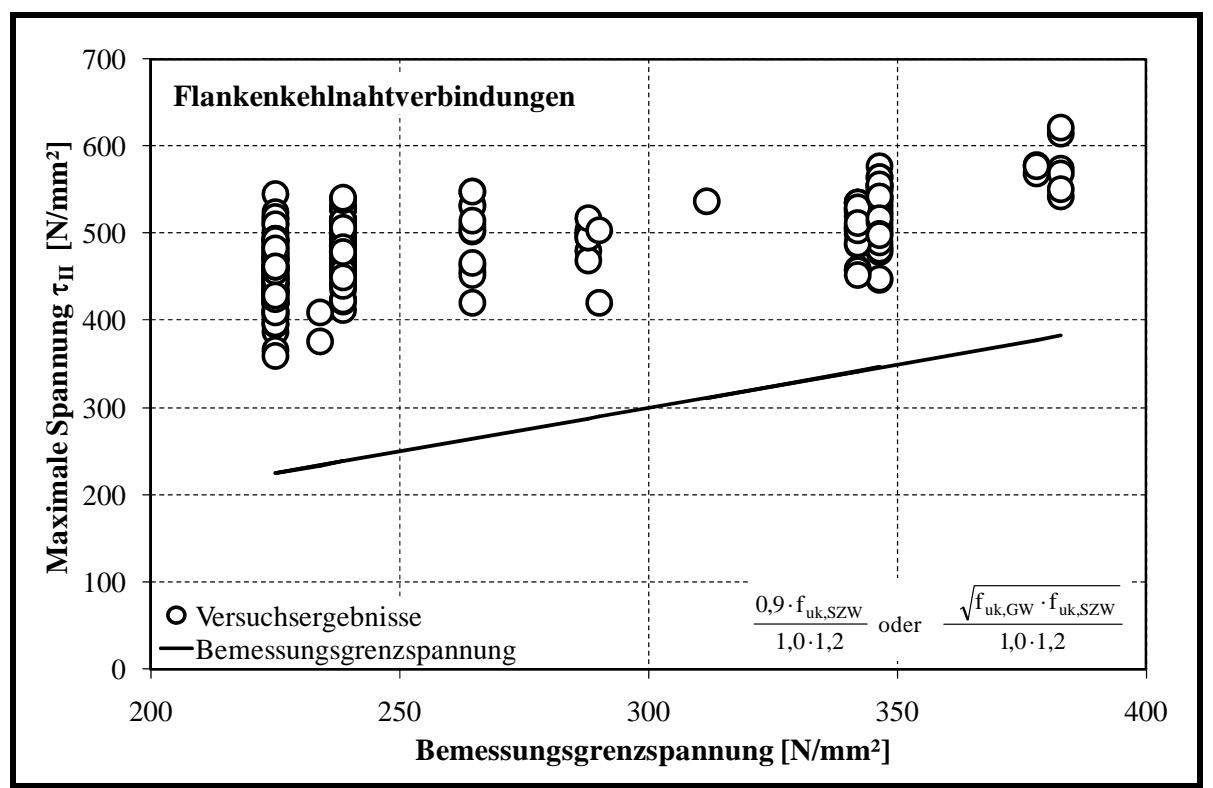

Abbildung 10.18: Versuchsergebnisse und Bemessungsvorschlag für Flankenkehlnahtverbindungen BSK 99 (2003)

In Abbildung 10.19 werden die Bemessungsgrenzspannungen für Flankenkehlnähte des neuen Modells mit dem Bemessungsgrenzspannungen nach BSK 99 (2003) verglichen. Für Schweißverbindungen mit Grund- und Schweißzusatzwerkstoffen nomineller Streckgrenzen $\mathrm{f}_{\mathrm{y}} \leq 460 \mathrm{~N} / \mathrm{mm}^{2}$ liefert der Schwedische Ansatz deutlich konservativere Bemessungsgrenzspannungen als der neu entwickelte Bemessungsansatz. Für Schweißverbindungen mit Grund- und Schweißzusatzwerkstoffen höherer Streckgrenzen liefert der Ansatz deutlich höhere Bemessungsgrenzspannungen.

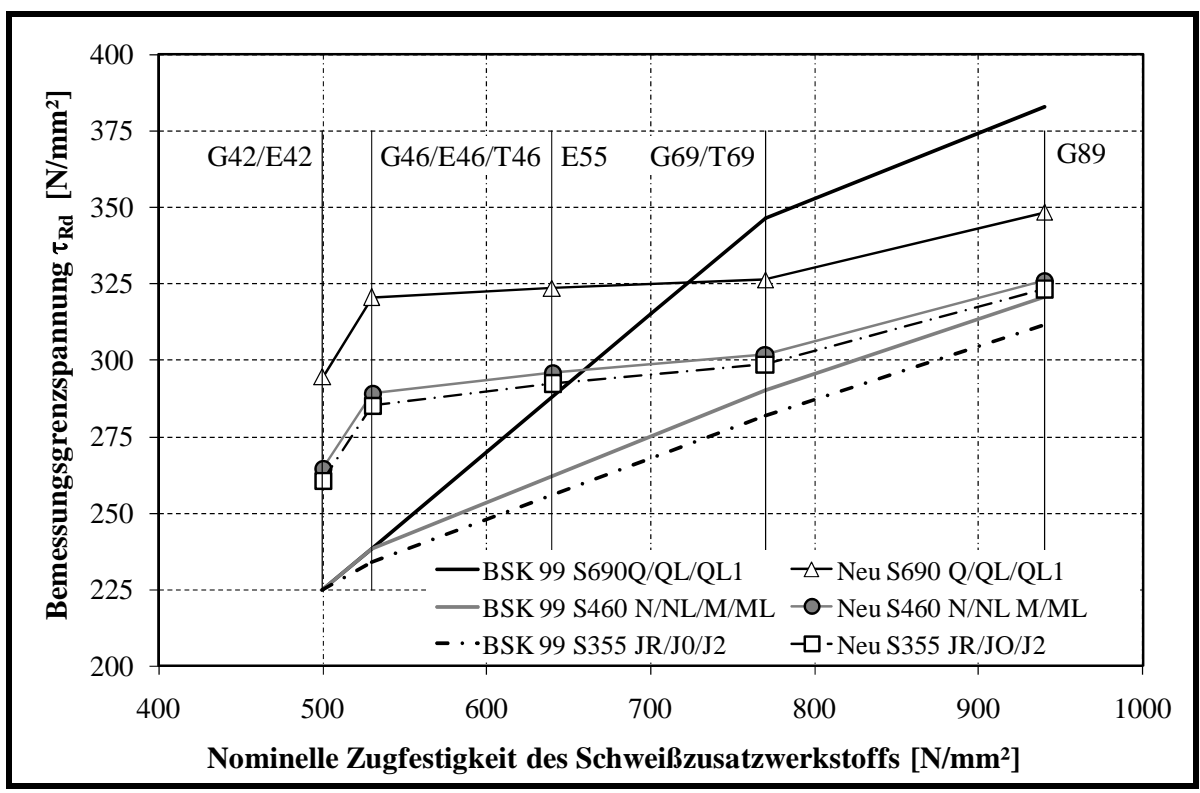

Abbildung 10.19: Vergleich des neuen Bemessungsmodells mit dem Bemessungsmodell nach BSK 99 (2003) 


\subsection{Zusammenfassung}

In diesem Kapitel wurden die bisher vorgestellten Untersuchungen zusammengeführt und daraus ein Bemessungsvorschlag entwickelt.

Die in dieser Arbeit vorgestellten Versuche wurden als Grundgesamtheit für die statistische Auswertung verwendet. Daraus wurde ein Vorschlag entwickelt, die Tragfähigkeit von Kehlnähten im bestehenden Bemessungsmodell nach EN 1993-1-8 (2005) und EN 1993-1-12 (2007) zu bestimmen. Dieser Vorschlag wird durch die Beiwerte $\beta_{\mathrm{w}}$ für die höherfesten Baustähle S460 und S690 bestimmt, wie in Tabelle 10.16 zusammengefasst.

Tabelle 10.16: Bemessungsvorschlag für Kehlnahtverbindungen nach EN 1993-1-8 (2005) und EN 1993-112 (2007)

\begin{tabular}{|l|l|l|l|}
\hline Stahlgüte & Beiwert $\beta_{\mathrm{w}}[-]$ & \multicolumn{1}{l|}{$\begin{array}{l}\text { Bemessungsgleichung für } \\
\text { Flankenkehlnähte }\end{array}$} \\
\hline S460 N/NL/M/ML & $\mathbf{0 , 8 5}$ & $\frac{\mathrm{f}_{\mathrm{u}}}{\sqrt{3} \cdot \gamma_{\mathrm{M} 2} \cdot \beta_{\mathrm{w}}}$ & $\frac{\mathrm{f}_{\mathrm{u}}}{\sqrt{2} \cdot \gamma_{\mathrm{M} 2} \cdot \beta_{\mathrm{w}}}$ \\
\hline S690Q/QL/QL1 & $\mathbf{1 , 1 0}$ &
\end{tabular}

Darauf aufbauend wurde ein eigener Bemessungsvorschlag entwickelt, der die Tragfähigkeit in Abhängigkeit von Grund- und Schweißzusatzwerkstoff beurteilt. Es wurde aufgezeigt, wie sich die Festigkeit der Naht in Abhängigkeit der Festigkeit von Grund- und Schweißzusatzwerkstoffen bestimmen lässt. Dazu wurde die Bemessungsgleichung nach EN 1993-1-8 (2005) modifiziert, so dass die Festigkeiten von Grund- und Schweißzusatzwerkstoff in der Bemessungsgleichung berücksichtigt werden. Mit dieser modifizierten Gleichung wurden dann die Beiwerte $\beta_{w}$ in Abhängigkeit der Festigkeit des Schweißzusatzwerkstoffs bestimmt, wie in Tabelle 10.17 dargestellt.

Tabelle 10.17: Modifizierter Bemessungsvorschlag für Kehlnahtverbindungen

\begin{tabular}{|c|c|c|c|}
\hline $\begin{array}{l}\text { Schweißzusatz- } \\
\text { werkstoff }\end{array}$ & Beiwert $\beta_{\mathrm{w}}[-]$ & $\begin{array}{l}\text { Bemessungsgleichung für } \\
\text { Flankenkehlnähte }\end{array}$ & Stirnkehlnähte \\
\hline G42/E42 & $\mathbf{0 , 8 9}$ & \multirow{4}{*}{$\frac{0,25 \cdot \mathrm{f}_{\mathrm{u}, \mathrm{GW}}+0,75 \cdot \mathrm{f}_{\mathrm{u}, \mathrm{SZW}}}{\sqrt{3} \cdot \gamma_{\mathrm{M} 2} \cdot \beta_{\mathrm{w}}}$} & \multirow{4}{*}{$\frac{0,25 \cdot \mathrm{f}_{\mathrm{u}, \mathrm{GW}}+0,75 \cdot \mathrm{f}_{\mathrm{u}, \mathrm{SZW}}}{\sqrt{2} \cdot \gamma_{\mathrm{M} 2} \cdot \beta_{\mathrm{w}}}$} \\
\hline G46/E46/T46 & 0,85 & & \\
\hline G69/T69 & 1,09 & & \\
\hline G89 & 1,19 & & \\
\hline
\end{tabular}

Es wurde gezeigt, dass mit diesem Bemessungsansatz für alle betrachteten Werkstoffkombinationen eine gute Übereinstimmung zwischen Versuch und Bemessungsgrenzspannung erreicht werden konnte.

Als Ergebnis des Normenvergleichs in Kapitel 3 wurde festgestellt, dass lediglich die Schwedische Norm BSK 99 (2003) und EN 1993-1-12 (2007) neben der Festigkeit des Grundwerkstoffs die Festigkeit Schweißzusatzwerkstoffs berücksichtigen und einen Schweißzusatzwerkstoff geringerer Festigkeit zulassen. Abschließend wurden die Versuche mit dem Modell nach BSK 99 (2003) und dem neuen Bemessungsansatz verglichen. Für Schweißverbindungen mit Grund- und Schweißzusatzwerkstoffen nomineller Streckgrenzen $\mathrm{f}_{\mathrm{y}} \leq 460 \mathrm{~N} / \mathrm{mm}^{2}$ liefert der schwedische Ansatz deutlich konservativere Bemessungsgrenzspannungen als der neu entwickelte Bemessungsansatz.

Im Vergleich mit den Versuchsergebnissen zeigte sich deutlich, dass der Abstand zwischen Versuchen und Bemessungswert nach Schwedischer Norm bei höheren Festigkeiten immer geringer wird. Diese Unterschiede werden auch in der großen Streuung des Schwedischen Modells deutlich.

Im Gegensatz dazu ergibt sich mit dem neuen Ansatz ein etwa gleichbleibender Abstand zwischen Versuch und Bemessungsgrenzspannung, der sich auch in einer geringen Streuung in der statistischen Auswertung widerspiegelt. 


\section{Zusammenfassung und Ausblick}

\subsection{Zusammenfassung}

In dieser Arbeit wurde die Tragfähigkeit von Kehlnahtverbindungen höherfester Baustähle experimentell und numerisch untersucht. Ziel dieser Arbeit war die Entwicklung eines realitätsnahen, zeitgemäßen Bemessungsmodells zur Bestimmung der Tragfähigkeit von Kehlnahtverbindungen höherfester Baustähle S460 und S690.

Beeinflusst wird die Tragfähigkeit zum einen durch die verwendeten Werkstoffe und zum anderen durch die Wärmebehandlung beim Schweißen. Deshalb wurden einleitend in Kapitel 2 die Herstellung von höherfesten Baustählen sowie das Verhalten der höherfesten Baustähle beim Schweißen und die Charakteristik der Schweißverbindung erklärt. Es wurde eine Möglichkeit vorgestellt, die Festigkeit in der Naht mit Hilfe von Härtemessungen zu beurteilen, da Härte und Zugfestigkeit korrelieren. Diese Erläuterungen dienten als Grundlage für die Interpretation und Erklärung der Versuchsergebnisse in den folgenden Kapiteln.

Um einen Überblick über die bestehenden Regelungen zu geben, wurden in Kapitel 3 die bestehenden Europäischen und Internationalen Bemessungsregeln zur Ermittlung der Tragfähigkeit von Kehlnahtverbindungen verglichen und die Besonderheiten erläutert.

In den bisher geltenden Normen wird für die Tragfähigkeit der Schweißnaht nur die Festigkeit des Grundwerkstoffs oder des Schweißzusatzwerkstoffs berücksichtigt, während gleichzeitig für den Schweißzusatzwerkstoff mindestens die gleiche Festigkeit wie die des Grundwerkstoffs gefordert wird. Nur in der Schwedischen Norm und nach EN 1993-1-12 (2007) für höherfeste Stähle mit $\mathrm{f}_{\mathrm{y}}>$ $460 \mathrm{~N} / \mathrm{mm}^{2}$ ist eine Berücksichtigung von niederfesten Schweißzusatzwerkstoffen möglich.

Für normalfeste Baustähle S235 und S355 ist die Vereinfachung der Verwendung von nur einer Festigkeit sinnvoll, da die zugehörigen Schweißzusatzwerkstoffe immer eine höhere Festigkeit als die Grundwerkstoffe haben. Für höherfeste Stähle S460 und S690 kann es auch sinnvoll sein, Schweißzusatzwerkstoffe mit geringerer oder auch höherer Festigkeit einzusetzen. Dazu müssen die Bemessungsregeln neben der Festigkeit des Grundwerkstoffs zusätzlich die Festigkeit des Schweißzusatzwerkstoffes aufnehmen. Es wurde gezeigt, dass die bestehenden Normen die Besonderheiten von höherfesten Baustählen nicht ausreichend berücksichtigen.

Der Vergleich der Nachweisformate zeigte außerdem, dass in den meisten Normen die Tragfähigkeiten von Kreuzstößen höher sind als die von Flankenkehlnähten. Der Vergleich der normativen Tragfähigkeiten zeigte insbesondere für höherfeste Baustähle große Unterschiede, so dass eine systematische Untersuchung notwendig war.

Als Einleitung und Hintergrund zur Beurteilung der experimentellen Untersuchungen wurde im vierten Kapitel das Tragverhalten von Kehlnähten hinsichtlich ihrer Werkstoffeigenschaften beschrieben. Für Flanken- und Stirnkehlnähte wurden die theoretische Spannungsverteilung in der Naht und die Ermittlung der Spannungen in den Versuchen hergeleitet. Es konnte gezeigt werden, dass der theoretische Bruchwinkel für Kreuzstöße mit 20 - 35 immer steiler ist als für Flankenkehlnahtverbindungen mit $45^{\circ}$. Die Problematik der Bestimmung der Spannungen bei Kreuzstößen und ein vereinfachtes Vorgehen wurden aufgezeigt.

Zur Bestimmung der Tragfähigkeit von Kehlnahtverbindungen der höherfesten Baustähle wurde neben eigenen Versuchen auf bereits bestehende Versuchsergebnisse zurückgegriffen. Im Rahmen von Kapitel 5 wurden deshalb bestehende bisher unabhängige Versuchsreihen von Flankenkehlnaht- und Stirnkehlnahtverbindungen aus der Literatur zusammengestellt, systematisch ausgewertet und so die Tragfähigkeit bestimmt. Diese bestehenden Versuche behandelten vorwiegend Werkstoffkombinationen mit Schweißzusatzwerkstoffen, deren Festigkeiten von der des Grundwerkstoffs abwichen. Sie unterschieden sich von den Werkstoffkombinationen der eigenen Untersuchungen und ergänzten diese da- 
mit. Es konnte eindeutig der Einfluss sowohl von Grund- als auch von Schweißzusatzwerkstoff festgestellt werden. Der Vergleich der Tragfähigkeiten zeigte somit, dass das bestehende Bemessungsmodell nach EN 1993-1-8 (2005) die unterschiedlichen Festigkeiten der Schweißzusatzwerkstoffe nicht ausreichend berücksichtigt. Für die Tragfähigkeitsunterschiede zwischen Flanken- und Stirnkehlnähten wurde festgestellt, dass die Tragfähigkeit von Stirnkehlnähten immer höher als die Tragfähigkeit von Flankenkehlnähten liegt. Diese Steigerung der Tragfähigkeit ist jedoch bei den einzelnen Serien sehr unterschiedlich. Insgesamt wurde festgestellt, dass für Schweißverbindungen höherfester Baustähle nur sehr wenige Daten bekannt sind.

Da in der Literatur nur wenige Versuchsdaten an Schweißverbindungen höherfester Baustähle bekannt sind, wurden eigene experimentelle Untersuchungen [FOSTA P652, 2008] an praxisgerechten Handschweißungen höherfester Stähle S460 und S690 durchgeführt, die in Kapitel 6 beschrieben und ausgewertet sind. Die Versuchsergebnisse zeigten nur geringfügig höhere Tragfähigkeiten bei Schweißverbindungen mit dem Stahl S690 im Vergleich mit Verbindungen mit dem Stahl S460.

Für Flankenkehlnahtverbindungen konnte festgestellt werden, dass die Parameter Nahtdicke, Blechdicke, Schweißprozess und Schweißprozessparameter keinen bedeutenden Einfluss auf die Tragfähigkeit ausüben. Für die Parameter Festigkeit des Grundwerkstoffs (Stahlsorte) und des Schweißzusatzwerkstoffs ergaben sich jedoch folgende Tendenzen: Mit zunehmender Festigkeit sowohl des Grund- als auch des Schweißzusatzwerkstoffs ist im Allgemeinen eine Tragfähigkeitssteigerung der Schweißverbindung zu erwarten. Die starke Streuung der Ergebnisse infolge der Handschweißung bei verschiedenen Fertigern machte eine eindeutige Feststellung von Einflüssen schwierig.

Für Kreuzstöße mit Stirnkehlnähten wurde für die Parameter Festigkeit des Grundwerkstoffs (Stahlsorte) und des Schweißzusatzwerkstoffs tendenziell mit zunehmender Festigkeit sowohl des Grundals auch des Schweißzusatzwerkstoff eine Tragfähigkeitssteigerung der Schweißverbindung festgestellt. Auch hier wurden die einzelnen Einflüsse durch die starke Streuung der Ergebnisse infolge der Handschweißung bei verschiedenen Fertigern verdeckt.

Die in Kapitel 6 ermittelten Tragfähigkeiten der Versuchskörper mit dem Grundwerkstoff S690 lagen unterhalb der Tragfähigkeiten nach der damals aktuellen Norm EN 1993-1-12 (2007). Im Anschluss an das in Kapitel 6 vorgestellte Vorhaben wurden erste Testversuche [Zusatzversuche, 2008] an Schweißverbindungen mit dem Grundwerkstoff S690 durchgeführt, um die Tragfähigkeiten der in Kapitel 6 vorgestellten Versuche mit dem Grundwerkstoff S690 zu bestätigen oder eine erhöhte Tragfähigkeit zu erreichen (vgl. Kapitel 7). Dazu wurde gezielt eine mögliche Verbesserung der Tragfähigkeit infolge einer vollmechanisierten Schweißung, einer aufwändig hergestellten Prüfkörperform und Verwendung höherfester Schweißzusatzwerkstoffe untersucht. Die Ergebnisse der durchgeführten Versuche [Zusatzversuche, 2008] zeigten durch den gewählten vollmechanisierten Fertigungsprozess und die aufwändig hergestellte Prüfkörperform (ohne Nahtanfangs- und Nahtendbereich) eine deutlich geringere Streuung der Versuchsergebnisse im Vergleich zu den in [FOSTA P652, 2008] durchgeführten Untersuchungen. Durch Verwendung eines höherfesten Schweißzusatzwerkstoffs konnte eine Steigerung der Tragfähigkeit um $8 \%$ erreicht werden. Es konnte gezeigt werden, dass entgegen den bisherigen normativen Berechnungsansätzen die ermittelten maximalen Schweißnahtspannungen $\tau_{\mathrm{II}}$, bedingt durch das Versagen bzw. den Bruch im Schweißgut, vor allem von der Zugfestigkeit des Schweißzusatzwerkstoffs abhängen. Zusätzlich hat der Vergleich der Versuchsergebnisse S355J2-G89 und S690-G89 gezeigt, dass bei gleichem Schweißzusatzwerkstoff infolge von unterschiedlichen Grundwerkstoffen unterschiedliche Tragfähigkeiten zu beobachten sind. Insgesamt konnte der Einfluss von Grund- und Schweißzusatzwerkstoff auf die Tragfähigkeit nachgewiesen werden, wobei die die Festigkeit des Schweißzusatzwerkstoffes den größeren Anteil hat.

Aufbauend auf den bisher vorgestellten Ergebnissen wurden weitere Untersuchungen [FOSTA P812, 2011] an vollmechanisiert geschweißten Versuchen mit Kehlnähten zum Tragverhalten von Schweißverbindungen höherfester Baustähle durchgeführt und ausgewertet (vgl. Kapitel 8). Im Einzelnen wurden Tragfähigkeitsuntersuchungen an Flanken- und Stirnkehlnahtverbindungen mit den Grundwerkstoffen S690Q und S700M und unterschiedlichen Schweißzusatzwerkstoffen, an Flankenkehlnahtverbindungen S460 sowie Werkstoffuntersuchungen vorgestellt. 
Aufgrund der vollmechanisiert hergestellten Versuchskörper ergaben sich sehr geringe Streuungen für die Flankenkehlnahtverbindungen. Durch die experimentelle Untersuchung der Parameter Nahtdicke, Nahtlänge und Schweißparameter konnte die Tragfähigkeit von Flankenkehlnahtverbindungen mit dem Grundwerkstoff S690 und zugehörigem Schweißzusatzwerkstoff bestimmt werden. Während der Einfluss der Nahtlänge auf die Tragfähigkeit gering ist, wurde bei den mehrlagigen Nähten eine Abnahme der Tragfähigkeit von bis zu $15 \%$ zu beobachtet. Vergleichend wurden Versuche mit dem Grundwerkstoff S460 betrachtet. Die Versuchsergebnisse zeigten eine höhere Tragfähigkeit bei Schweißverbindungen mit dem Stahl S690 im Vergleich mit Verbindungen mit dem Stahl S460.

Als weiterer Parameter wurde der Einfluss von Unter- und Überfestigkeiten des Schweißzusatzwerkstoffs auf die Tragfähigkeit der Verbindung S690 ermittelt. Bei Verwendung von niederfesten Schweißzusatzwerkstoffen wurde die Tragfähigkeit nur wenig geringer, durch Verwendung von höherfesten Schweißzusatzwerkstoffen konnte eine Steigerung der Tragfähigkeit festgestellt werden.

An allen Versuchskörpern wurden zusätzlich Härtemessungen durchgeführt, die jeweils ähnliche Tendenzen wie die Tragfähigkeitsuntersuchungen ergaben.

Die Tragfähigkeit von Kreuzstößen wurde an Verbindungen gleicher und unterschiedlicher Schweißzusatzwerkstoffe ermittelt. Trotz der vollmechanisierten Schweißung war die Streuung bei gleichen Versuchskörpern größer als bei den Flankenkehlnahtverbindungen. Der Bruchwinkel war sehr steil, so dass die Bruchfläche zum Teil durch die Wärmeeinflusszone verlief, in der keine eindeutigen Bedingungen vorliegen. Zusätzlich hat die Einordnung der Versuchskörper in Bewertungsgruppen für die Verbindungen mit dem Grundwerkstoff S690Q nur Bewertungsgruppe D ergeben. Es wird vermutet, dass diese Unregelmäßigkeiten die Tragfähigkeit beeinflussen. Somit wurden diese Versuchsergebnisse nicht weiter bei der Auswertung berücksichtigt. Die Untersuchung des Einflusses der Festigkeit des Schweißzusatzwerkstoffs für Kreuzstöße mit dem Stahl S700M zeigte eine ansteigende Tragfähigkeit mit zunehmender Festigkeit des Schweißzusatzwerkstoffs.

Die durchgeführten Werkstoffuntersuchungen haben gezeigt, dass die Festigkeit des reinen Schweißgutes sich deutlich von der Zugfestigkeit der mehrlagigen Kehlnaht unterscheidet. Insbesondere für den Schweißzusatzwerkstoff G46 wurde für die mehrlagige Kehlnaht eine Steigerung von 12 - 14 \% festgestellt.

Zusätzliche numerische Untersuchungen wurden durchgeführt, um die Versuche nachzubilden. Es konnte gezeigt werden, dass durch eine genaue Modellierung der Geometrie der Schweißnaht und genaue Kenntnis der Festigkeit der Naht die numerische Berechnung der Traglast möglich ist.

Zur werkstofftechnischen Abbildung der Naht standen die Ergebnisse von Zugversuchen am reinen Schweißgut und Zugversuche, die aus mehrlagigen Kehlnähten gefertigt wurden, zur Verfügung. Die Berechnungen mit diesen unterschiedlichen Zugfestigkeiten haben eindeutig ergeben, dass mit der Zugfestigkeit aus der Kehlnaht Traglasten erreicht werden, die denen aus dem Versuch sehr ähnlich sind. Mithilfe der durchgeführten Härtemessungen an den Versuchskörpern und den Körpern für die Zugversuche konnten die numerischen Ergebnisse eingeordnet werden.

Zum Tragverhalten konnte durch den Vergleich der Kraft-Verformungs-Linien gezeigt werden, dass das Tragverhalten gut abgebildet wird. Die numerischen Berechnungen haben ergeben, dass ein Plastizieren in der gesamten Naht mit dem Erreichen der maximalen Vergleichsspannung eintritt. Die Kraft kann nicht weiter gesteigert werden, wenn die Vergleichsspannung in der Naht erreicht ist. Somit tritt Versagen ein, wenn die maximale Vergleichsspannung in der Naht erreicht wird. Ein vorzeitiges Versagen durch lokale Dehnungsüberschreitungen kann damit ausgeschlossen werden.

Aufbauend auf den experimentellen und numerischen Untersuchungen und im Zusammenhang mit der statistischen Auswertung wurden die bisher vorgestellten Untersuchungen zusammengeführt und daraus ein Bemessungsvorschlag entwickelt.

Die in dieser Arbeit vorgestellten Versuche wurden als Grundgesamtheit für die statistische Auswertung verwendet. Daraus wurde ein Vorschlag entwickelt, die Tragfähigkeit von Kehlnähten im bestehenden Bemessungsmodell nach EN 1993-1-8 (2005) und EN 1993-1-12 (2007) zu bestimmen. Dieser 
Vorschlag wurde durch die Beiwerte $\beta_{\mathrm{w}}$ für die höherfesten Baustähle S460 und S690 bestimmt, wie in Tabelle 11.1 zusammengefasst.

Tabelle 11.1: Bemessungsvorschlag für Kehlnahtverbindungen nach EN 1993-1-8 (2005) und EN 1993-112 (2007)

\begin{tabular}{|l|l|l|l|}
\hline Stahlgüte & Beiwert $\beta_{\mathrm{w}}[-]$ & \multicolumn{2}{|l|}{ Bemessungsgleichung für } \\
Flankenkehlnähte
\end{tabular}

Darauf aufbauend wurde ein eigener Bemessungsvorschlag entwickelt, der die Tragfähigkeit in Abhängigkeit von Grund- und Schweißzusatzwerkstoff beurteilt. Es wurde aufgezeigt, wie sich die Festigkeit der Naht in Abhängigkeit der Festigkeit von Grund- und Schweißzusatzwerkstoffen bestimmen lässt. Dazu wurde die Bemessungsgleichung nach EN 1993-1-8 (2005) modifiziert, so dass die Festigkeit von Grund- und Schweißzusatzwerkstoff in der Bemessungsgleichung berücksichtigt wird. Mit dieser modifizierten Gleichung wurden dann die Beiwerte $\beta_{\mathrm{w}}$ in Abhängigkeit der Festigkeit des Schweißzusatzwerkstoffs bestimmt, wie in Tabelle 11.2 dargestellt.

Tabelle 11.2: Modifizierter Bemessungsvorschlag für Kehlnahtverbindungen

\begin{tabular}{|c|c|c|c|c|c|}
\hline $\begin{array}{l}\text { Schweißzusatz- } \\
\text { werkstoff }\end{array}$ & Beiwert $\beta_{\mathrm{w}}[-]$ & $\begin{array}{l}\text { Bemessungsgleichung } \\
\text { Flankenkehlnähte }\end{array}$ & & Stirnkehlnähte & \\
\hline G42/E42 & 0,89 & \multirow{4}{*}{$\frac{0,25 \cdot \mathrm{f}_{\mathrm{u}, \mathrm{GW}}+0,75 \cdot \mathrm{f}_{\mathrm{u}, \mathrm{SZW}}}{\sqrt{3} \cdot \gamma_{\mathrm{M} 2} \cdot \beta_{\mathrm{w}}}$} & \multirow{4}{*}{$\frac{\mathrm{N}}{\mathrm{mm}^{2}}$} & \multirow{4}{*}{$\frac{0,25 \cdot \mathrm{f}_{\mathrm{u}, \mathrm{GW}}+0,75 \cdot \mathrm{f}_{\mathrm{u}, \mathrm{SZW}}}{\sqrt{2} \cdot \gamma_{\mathrm{M} 2} \cdot \beta_{\mathrm{w}}}$} & \multirow[b]{2}{*}{$\mathrm{N}$} \\
\hline G46/E46/T46 & 0,85 & & & & \\
\hline G69/T69 & 1,09 & & & & $\overline{\mathrm{mm}^{2}}$ \\
\hline G89 & 1,19 & & & & \\
\hline
\end{tabular}

Es konnte gezeigt werden, dass mit diesem Bemessungsansatz für alle betrachteten Werkstoffkombinationen eine gute Übereinstimmung zwischen Versuch und Bemessungsgrenzspannung erreicht werden konnte.

Im Vergleich mit dem bisherigen Ansatz nach EN 1993-1-8 (2005) besteht der Vorteil des neuen Bemessungsansatzes darin, dass unterschiedliche Festigkeiten für Grund- und Schweißzusatzwerkstoff berücksichtigt werden können. Damit sind zum einen Verbindungen mit Schweißzusatzwerkstoffen niedrigerer Festigkeit nun möglich. Zum anderen können höhere Bemessungsgrenzspannungen infolge höherer Festigkeiten des Schweißzusatzwerkstoffs in Anspruch genommen werden. Im Gegensatz dazu erlaubt der bisherige Ansatz nur Schweißzusatzwerkstoffe, deren Festigkeit mindestens der Festigkeit des Grundwerkstoffs entspricht.

Für die Grundwerkstoffe S355, S460 und S690 ergaben sich bei den Verbindungen gleicher Grundund Schweißzusatzwerkstoffe ähnliche Bemessungsgrenzspannungen bei beiden Verfahren. Bei Verwendung von höherfesten Schweißzusatzwerkstoffen können bei dem neuen Ansatz also höhere Bemessungsgrenzspannungen als nach dem Verfahren nach EN 1993-1-8 (2005) in Anspruch genommen werden.

Zusätzlich wurde der neue Bemessungsansatz mit Werten der Schwedischen Norm BSK 99 (2003) verglichen, da neben EN 1993-1-12 (2007) nur diese Norm eine Möglichkeit gibt, neben der Festigkeit des Grundwerkstoffs die Festigkeit des Schweißzusatzwerkstoffs zu berücksichtigen.

Für Schweißverbindungen mit Grund- und Schweißzusatzwerkstoffen nomineller Streckgrenzen $\mathrm{f}_{\mathrm{y}} \leq$ $460 \mathrm{~N} / \mathrm{mm}^{2}$ liefert der Schwedische Ansatz deutlich konservativere Bemessungsgrenzspannungen als der neu entwickelte Bemessungsansatz. Im Vergleich mit den Versuchsergebnissen zeigte sich jedoch, dass der Abstand zwischen Versuchsergebnissen und Bemessungsgrenzspannung nach Schwedischer Norm bei höheren Festigkeiten immer geringer wird. Diese Unterschiede werden auch in der großen Streuung mit dem Schwedischen Modell deutlich.

Im Gegensatz dazu ergibt sich mit dem neuen Ansatz eine gute Übereinstimmung zwischen Versuch und Bemessungsgrenzspannung, der sich auch in einer geringen Streuung in der statistischen Auswertung widerspiegelt. 


\subsection{Beschreibung der Tragfähigkeit von Kehlnähten}

Mithilfe der durchgeführten Untersuchungen kann das Tragverhalten von Kehlnahtverbindungen höherfester Baustähle bestimmt werden.

Im Bemessungsansatz nach EN 1993-1-8 (2005) wird für Verbindungen gleicher Grund- und Schweißzusatzwerkstoffe die Festigkeit der Kehlnaht mit Hilfe eines Beiwertes $\beta_{\mathrm{w}}$ im Verhältnis zur Festigkeit des Grundwerkstoffs bestimmt.

Da bei Verbindungen gleicher Grund- und Schweißzusatzwerkstoffe die Festigkeiten der eingesetzten Werkstoffe übereinstimmen, bestimmt der Beiwert $\beta_{\mathrm{w}}$ indirekt auch das Verhältnis zwischen der Festigkeit der Kehlnaht und der Festigkeit des Schweißzusatzwerkstoffs. Die Untersuchungen haben gezeigt, dass die Festigkeit von Kehlnähten von der Festigkeit des Schweißzusatzwerkstoffs abweicht, weil die Abkühlbedingungen anders sind und geringere Vergütungseffekte auftreten.

Wenn der Beiwert $\beta_{\mathrm{w}}<1,0$ ist, herrscht in der Kehlnaht eine Überfestigkeit gegenüber der Festigkeit des Schweißzusatzwerkstoffs. Diese Überfestigkeit konnte für Schweißverbindungen mit dem Schweißzusatzwerkstoff G46 festgestellt werden. Für die Schweißverbindungen mit dem Schweißzusatzwerkstoff G69 wurde eine Festigkeit ermittelt, die geringer als die Festigkeit des Schweißzusatzwerkstoffs ist. Für die unterschiedlichen Schweißzusatzwerkstoffe ist die Abweichung in der Festigkeit verschieden.

Neben den Abkühlbedingungen und den Vergütungseffekten ist die Aufmischung mit dem Grundwerkstoff insbesondere bei unterschiedlichen Festigkeiten zwischen Grund- und Schweißzusatzwerkstoff zu berücksichtigen. Dies konnte als weiterer Einflussfaktor auf die Festigkeit der Schweißnaht gezeigt werden. Es wurde ein plausibler Ansatz vorgestellt, die Festigkeit des Grundwerkstoffs mit $25 \%$ zu berücksichtigen, während die Festigkeit des Schweißzusatzwerkstoffs mit 75 \% die Festigkeit der Schweißverbindung beeinflusst.

Für die Flankenkehlnahtverbindungen wurde ein Vorschlag gemacht, die Tragfähigkeit zu bestimmen, der eine sehr gute Übereinstimmun liefert. Für die Stirnkehlnahtverbindungen hat sich gezeigt, dass die Tragfähigkeit in den meisten Fällen höher liegt als die Tragfähigkeit der Flankenkehlnahtverbindungen. Es wurde jedoch festgestellt, dass die theoretische Spannungsermittlung für die Kreuzstöße sehr aufwändig ist und sich versuchstechnisch nicht umsetzen lässt. Aus diesem Grund wurde vorgeschlagen, auf der sicheren Seite, den neuen Bemessungsansatz auch für Stirnkehlnahtverbindungen anzuwenden. 


\subsection{Ausblick}

In dieser Arbeit wurde die Tragfähigkeit von Flanken- und Stirnkehlnahtverbindungen bestimmt. Der Bemessungsansatz liefert eine sehr gute Übereinstimmung für die Flankenkehlnähte, während er für die Kreuzstöße auf der sicheren Seite liegt.

Es wurde in dieser Arbeit experimentell gezeigt, dass das Versagen von Kreuzstößen teilweise in der Wärmeeinflusszone lag. Die Werkstoffkennwerte in der Wärmeeinflusszone können nicht experimentell bestimmt werden. Um das Tragverhalten von Kreuzstößen genauer zu bestimmen sind numerischen Untersuchungen notwendig. Mithilfe von Schweißsimulationen (vgl. z. B. [Hildebrand, 2008]) könnte die Festigkeit in der Wärmeeinflusszone bestimmt werden. Insbesondere könnte geklärt werden, in wieweit eine Steigerung der Festigkeit in der Naht durch Verwendung von im Vergleich zum Grundwerkstoff höherfesten Schweißzusatzwerkstoffen die Festigkeit in der Wärmeeinflusszone erhöht. Weitere Untersuchungen an Kreuzstößen könnten die Änderung der Tragfähigkeit durch Fertigungsqualitäten, die über die Bewertungsgruppen bestimmt werden, ermitteln.

Der vorgestellte Bemessungsansatz berücksichtigt die Unterschiede in der Festigkeit zwischen Grundund Schweißzusatzwerkstoff. In weiteren Untersuchungen könnte dieser Ansatz auf Basis von Versuchen oder auch numerischen Berechnungen auf Verbindungen mit zwei unterschiedlichen Grundwerkstoffen erweitert werden.

Der Bemessungsansatz nach EN 1993-1-8 (2005) gilt auch für nicht durchgeschweißte Stumpfnähte (z. B. DHY-Nähte). Diese Verbindungen wurden im Rahmen dieser Arbeit nicht untersucht. Bekannte Versuche [FOSTA P652, 2008] deuten darauf hin, dass die Tragfähigkeit dieser Nähte höher ist als die Tragfähigkeit der Flankenkehlnähte. 


\section{Schrifttum}

\subsection{Normen und Richtlinien}

AISC 360-05 (2005): ANSI/AISC 360-05: Specification for structural steel buildings: American Institute of steel construction, Inc, Chicago 2005

[ARiLi Stahl, 2001]: Änderungen und Ergänzungen der Anpassungsrichtlinie Stahlbau - Ausgabe Dezember 2001- Deutsches Institut für Bautechnik (DIBT), Berlin

[AWS D1.1, 2004]: AWS D1.1/D1.1M:2004 An American National Standard Structural Welding Code - Steel, $19^{\text {th }}$ Edition, American Welding Society, 2003

[Beuth-Kommentare, 1998]: Beuth-Kommentare Stahlbauten, Erläuterungen zu DIN 18800 Teil 1 bis Teil 4, 3. Auflage 1998, Beuth Verlag GmbH Berlin Köln, Ernst und Sohn Berlin

BSK 99 (2003): BSK 99: Swedish regulations for steel structures, National boarding of housing, building and planning, 2003. http://www.boverket.se/Global/Webbokhandel/Dokument /2003/ swedish_regulations_for_steel_BSK99.pdf, Stand 28.02.2011

CAN/NSA S16.1-94 (2006): Limit states design of steel structures, Canadian Standard Association, November 2006

DIN 4100 (1968): DIN 4100: Geschweißte Stahlbauten mit vorwiegend ruhender Belastung; Berechnung und bauliche Durchbildung. DIN Deutsches Institut für Normung e. V., Berlin, Ausgabe 1968-12

DIN 18800-1 (2008): Stahlbauten, Teil 1: Bemessung und Konstruktion, Deutsches DIN Deutsches Institut für Normung e. V., Ausgabe 2008-11

DIN 18800-1 (1990): Stahlbauten, Teil 1: Bemessung und Konstruktion, DIN Deutsches Institut für Normung e. V., Ausgabe 1990-11

DIN EN 440 (1994): DIN EN 440: Schweißzusätze - Drahtelektroden und Schweißgut zum MetallSchutzgasschweißen von unlegierten Stählen und Feinkornstählen - Einteilung; Deutsche Fassung EN 440:1994, DIN Deutsches Institut für Normung e. V., Ausgabe 1994-11

DIN EN 757 (1997): DIN EN 757: Umhüllte Stabelektroden zum Lichtbogenhandschweißen von hochfesten Stählen - Einteilung, DIN Deutsches Institut für Normung e. V., Berlin, Ausgabe 1997-05

DIN EN 758 (1997): DIN EN 758: Schweißzusätze - Fülldrahtelektroden zum Metall- Lichtbogenschweißen mit und ohne Schutzgas von unlegierten Stählen und Feinkornstählen - Einteilung, DIN Deutsches Institut für Normung e. V., Berlin, Ausgabe 1997-05

DIN EN 876 (1995): DIN EN 876: Zerstörende Prüfung von Schweißverbindungen an metallischen Werkstoffen, Längszugversuch am Schweißgut in Schmelzschweißverbindungen, DIN Deutsches Institut für Normung e. V., Berlin, Ausgabe 1995-10

DIN EN 1011-2 (2001): DIN EN 1011-2: Schweißen - Empfehlungen zum Schweißen metallischer Werkstoffe - Teil 2: Lichtbogenschweißen von ferritischen Stählen, DIN Deutsches Institut für Normung e. V., Berlin, Ausgabe 2001-05

DIN EN 1990 (2002): DIN EN 1990: Eurocode: Grundlagen der Tragwerksplanung, DIN Deutsches Institut für Normung e. V., Berlin, Ausgabe 2002-04

EN 1993-1-1 (2005): Eurocode 3: Bemessung und Konstruktion von Stahlbauten - Teil 1-1: Allgemeine Bemessungsregeln und Regeln für den Hochbau; Deutsche Fassung EN 1993-1-1:2005 + AC:2009, DIN Deutsches Institut für Normung e. V., Berlin, Ausgabe 2010-12

EN 1993-1-8 (2005): Eurocode 3: Bemessung und Konstruktion von Stahlbauten - Teil 1-8: Bemessung von Anschlüssen, Deutsche Fassung EN 1993-1-8:2005 + AC:2009, DIN Deutsches Institut für Normung e. V., Berlin, Ausgabe 2010-12 
DIN EN 1993-1-8/NA (2010): Nationaler Anhang - National festgelegte Parameter - Eurocode 3: Bemessung und Konstruktion von Stahlbauten - Teil 1-8: Bemessung von Anschlüssen, DIN Deutsches Institut für Normung e. V., Berlin, Ausgabe 2010-12

EN 1993-1-12 (2007): Eurocode 3: Bemessung und Konstruktion von Stahlbauten - Teil 1-12: Zusätzliche Regeln zur Erweiterung von EN 1993 auf Stahlgüten bis S700, Deutsche Fassung EN 1993-112:2007 + AC:2009, DIN Deutsches Institut für Normung e. V., Berlin, Ausgabe 2010-12

DIN EN 1993-1-12/NA (2011): Nationaler Anhang - National festgelegte Parameter - Eurocode 3: Bemessung und Konstruktion von Stahlbauten - Teil 1-12: Zusätzliche Regeln zur Erweiterung von EN 1993 auf Stahlgüten bis S700, DIN Deutsches Institut für Normung e. V., Berlin, Ausgabe 201108

DIN EN 10002-1 (2001): DIN EN 10002-1: Metallische Werkstoffe - Zugversuch - Teil 1: Prüfverfahren bei Raumtemperatur, DIN Deutsches Institut für Normung e. V., Berlin, Ausgabe 2001-12

DIN EN 10025 (2005): Warmgewalzte Erzeugnisse aus Baustählen. DIN Deutsches Institut für Normung e. V., 2005

DIN EN 10025-2 (2005): DIN EN 10025-2: Warmgewalzte Erzeugnisse aus Baustählen - Teil 2: Technische Lieferbedingungen für unlegierte Baustähle; DIN Deutsches Institut für Normung e. V., Ausgabe 2005-04

DIN EN 10025-4 (2005): DIN EN 10025-4: Warmgewalzte Erzeugnisse aus Baustählen - Teil 4: Technische Lieferbedingungen für thermomechanisch gewalzte schweißgeeignete Feinkornbaustähle; Deutsches Institut für Normung e.V. (DIN), Ausgabe 2005-04

DIN EN 10025-6 (2005): DIN EN 10025-6: Warmgewalzte Erzeugnisse aus Baustählen - Teil 6: Technische Lieferbedingungen für Flacherzeugnisse aus Stählen mit höherer Streckgrenze im vergüteten Zustand; DIN Deutsches Institut für Normung e. V., Ausgabe 2005-02

DIN EN 10149-2 (1995): DIN EN 10149-2: Warmgewalzte Flacherzeugnisse aus Stählen mit hoher Streckgrenze zum Kaltumformen - Teil 2: Lieferbedingungen für thermomechanisch gewalzte Stähle; DIN Deutsches Institut für Normung e. V., Ausgabe 1995-02

DIN EN 14532-1 (2005): DIN EN 14532-1: Schweißzusätze - Prüfverfahren und Qualitätsanforderungen - Teil 1: Grundprüfungen und Konformitätsbewertung von Schweißzusätzen für Stahl, Nickel und Nickellegierungen; Deutsche Fassung EN 14532-1:2004; DIN Deutsches Institut für Normung e. V., Berlin, Ausgabe 2005-02

DIN EN ISO 2560 (2006): DIN EN ISO 2560: Schweißzusätze - Umhüllte Stabelektroden zum Lichtbogenhandschweißen von unlegierten Stählen und Feinkornstählen - Einteilung, DIN Deutsches Institut für Normung e. V., Berlin, Ausgabe 2006-03

DIN EN ISO 5817 (2006): Schweißen -Schmelzschweißverbindungen an Stahl, Nickel, Titan und deren Legierungen (ohne Strahlschweißen) - Bewertungsgruppen von Unregelmäßigkeiten (ISO 5817:2003 + Cor. 1:2006); Deutsche Fassung EN ISO 5817:2003 + AC:2006, DIN Deutsches Institut für Normung e. V., Berlin, Ausgabe 2006-10

DIN EN ISO 14341 (2008): DIN EN ISO 14341: Schweißzusätze - Drahtelektroden und Schweißgut zum Metall-Schutzgasschweißen von unlegierten Stählen und Feinkornstählen - Einteilung (ISO 14341:2002); Deutsche Fassung EN ISO 14341:2008. DIN Deutsches Institut für Normung e. V., Berlin, Ausgabe 2008-08

DIN EN ISO 15792-1 (2008): Schweißzusätze -Prüfverfahren - Teil 1: Prüfverfahren für Prüfstücke zur Entnahme von Schweißgutproben an Stahl, Nickel, Nickellegierungen (ISO 15792-1:2000); Deutsche Fassung EN ISO 5817:2008, DIN Deutsches Institut für Normung e. V., Berlin, Ausgabe 200808

DIN EN ISO 16834 (2007): DIN EN ISO 16834: Schweißzusätze - Drahtelektroden, Drähte, Stäbe und Schweißgut zum Schutzgasschweißen von hochfesten Stählen - Einteilung. DIN Deutsches Institut für Normung e. V., Ausgabe 2007-05 
DIN EN ISO 17632 (2008): DIN EN ISO 17632: Schweißzusätze - Fülldrahtelektroden zum MetallLichtbogenschweißen mit und ohne Schutzgas von unlegierten Stählen und Feinkornstählen - Einteilung (ISO 17632:2004); Deutsche Fassung EN ISO 17632:2008. DIN Deutsches Institut für Normung e. V., Berlin, Ausgabe 2008-08

DIN EN ISO 18265 (2004): DIN EN ISO 18265: Metallische Werkstoffe - Umwertung von Härtewerten (ISO 18265:2003) Deutsche Fassung EN ISO 18265:2003, DIN Deutsches Institut für Normung e. V., Berlin, Ausgabe 2004-02

DIN EN ISO 18276 (2006): DIN EN ISO 18276: Schweißzusätze - Fülldrahtelektroden zum MetallLichtbogenschweißen mit und ohne Schutzgas von hochfesten Stählen - Einteilung, DIN Deutsches Institut für Normung e. V., Berlin, Ausgabe 2006-09

HSC (2000): Handbook of Steel Construction, Seventh Edition, Canadian Institute of Steel Construction, 2000

ISO 5951 (1980): ISO 5951: Stahlblech, warm gewalzt, mit höherer Streckgrenze und verbesserter Formbarkeit, Ausgabe 1980-09

[SEW 088, 1993]: SEW 088: Schweißgeeignete Feinkornbaustähle; Richtlinien für die Verarbeitung, besonders für das Schmelzschweißen, Stahlinstitut VDEh, Düsseldorf, 4. Ausgabe 1993-10

[SEW 088 Beiblatt 1, 1993]: SEW 088, Beiblatt 1:Schweißgeeignete Feinkornbaustähle, Richtlinien für die Verarbeitung, besonders für das Schmelzschweißen- Kaltrisssicherheit beim Schweißen; Ermittlung angemessener Mindestvorwärmtemperaturen, 4. Ausgabe 1993-10

[SEW 088 Beiblatt 2, 1993]: SEW 088, Beiblatt 2: Schweißgeeignete Feinkornbaustähle, Richtlinien für die Verarbeitung, besonders für das Schmelzschweißen, Ermittlung von Abkühlzeiten $t_{8 / 5}$ zur Kennzeichnung von Schweißtemperaturzyklen, 4. Ausgabe 1993-10

SIA 263 (2003): SIA 263: Stahlbau, Schweizerischer Ingenieur- und Architektenverein, Zürich 2003.

[Zulassung Z-30-1.1]: Allgemeine bauaufsichtliche Zulassung Z-30.1-1: Flacherzeugnisse aus hochfestem schweißgeeigneten Feinkornbaustahl S690QL1 und die daraus hergestellten Bauteile. Gültig bis 31. Januar 2010

[Zulassung Z-30.2-5]: Allgemeine bauaufsichtliche Zulassung Z-30.2-5: Langerzeugnisse aus warmgewalzten schweißgeeigneten Feinkornbaustählen im thermomechanisch gewalzten Zustand HISTAR 355/355L und HISTAR 460/460L. Gültig bis 30. Juni 2010

\subsection{Höherfeste Baustähle und Schweißen}

[Dilthey, 2005]: Dilthey, U.: Schweißtechnische Fertigungsverfahren 2 - Verhalten der Werkstoffe beim Schweißen; 3., bearbeitete Auflage, Springer-Verlag Berlin Heidelberg New York, 2005

[Dorn et al., 1986]: Dorn, L.; Hobbacher, A.; Schulze, G.; Weyland F.: Schweißen von Baustählen und hochfesten Feinkorn-Baustählen, Kontakt \& Studium Maschinenbau, Band 147, Expert Verlag, Sindelfingen, 2006

[Fahrenwaldt, Schuler, 2009]: Fahrenwaldt H. J.; Schuler V.: Praxiswissen Schweißtechnik, Werkstoffe, Prozesse, Fertigung; 3., aktualisierte Auflage, Vieweg + Teubner, GWV Fachverlage GmbH, Wiesbaden, 2009

[Hildebrand, 2008]: Hildebrand, J.: Numerische Schweißsimulation : Bestimmung von Temperatur, Gefüge und Eigenspannung an Schweißverbindungen aus Stahl- und Glaswerkstoffen, Dissertation, Schriftenreihe des Instituts für Konstruktiven Ingenieurbau, Universitätsverlag Bauhaus-Universität Weimar, Heft 018, 2008

[Schröter, 2007] Schröter F.: Stähle für den Stahlbau - Anwendung moderner Baustähle und Neuerungen im Regelwerk. In: Kuhlmann, U. (Hrsg.): Stahlbau-Kalender 2007, Ernst \& Sohn, 2007, S. 245316 
[Schulze, 2010]: Schulze, G.: Die Metallurgie des Schweißens, Eisenwerkstoffe - Nichteisenmetallische Werkstoffe, 4., neubearbeitete Auflage, Springer-Verlag Berlin Heidelberg, 2010

\subsection{Schweißverbindungen}

[Background D.03, 1990]: Background Documentation Document D.03 Annex D, Evaluations of test results on welded connections made from FeE 460 in order to obtain strength functions and suitable model factors, April 1990

[Bornscheuer, Feder, 1966]: Bornscheuer, W.; Feder, D: Traglastversuche an Laschenverbindungen aus St 37 mit Flanken- und Stirnkehlnähten, Schweißen + Schneiden Jahrgang 18, 1966, Heft 7, S. 305-308

[Breunig, 2011]: Breunig, S.: Numerische Untersuchungen zur Tragfähigkeit von Stirnkehlnahtverbindungen in Abhängigkeit des Schweißzusatzwerkstoffs, Universität Stuttgart, Institut für Konstruktion und Entwurf, Diplomarbeit, Nr. 2011-9X

[Callele et al., 2005]: Callele, L. J., Grondin, G. Y., Driver, R. G.: Strength and behaviour of multiorientation fillet weld connections, Structural Engineering Report No. 255, University of Alberta, 2005

[Collin, Johansson, 2005]: Collin, P.; Johansson, B.: Design of welds in high strength steel. In: Proceedings of the $4^{\text {th }}$ European Conference on Steel and Composite Structures, Maastricht, Volume C., S. 4.10-89-4.10-98, 2005

[Deng et al., 2003]: Deng, K., Grondin, G. Y., Driver, R. G.: Effect of loading angle on the behaviour of fillet welds, Structural Engineering Report No. 251, University of Alberta, Edmonton, Alberta, Canada, 2003

[Feder, 1966]: Feder, D.: Spannungsoptische und theoretische Untersuchungen zur Kraftverteilung in Kehlnahtlaschenverbindungen, Dissertation, 1966, Stuttgart

[Feder, Werner, 1977]: Feder, D.; Werner, G.: Ansätze zur Traglastberechnung von Schweißverbindungen des Stahlbaus, Schweißen + Schneiden Jahrgang 29, 1977, Heft 4, S. 125-132

[Fischer, Wenk, 1986]: Fischer, M.; Wenk, P.: Traglastversuche an statisch belasteten langen Längskehlnähten, Stahlbau, 1986, Heft 7, S. 193-199

[FOSTA P652, 2008]: Abschlussbericht: AiF-Vorhaben-Nr. 14195 BG (2007) Wirtschaftliche Schweißverbindungen höherfester Baustähle, Arbeitsgemeinschaft industrieller Forschungsvereinigungen e.V. (AiF), FOSTA P652, Düsseldorf, August 2008

[FOSTA P812, 2011]: Abschlussbericht: AiF-Vorhaben-Nr. 16097 Tragfähigkeit von Kehlnahtverbindungen höherfester Baustähle S690 im Stahlbau, Arbeitsgemeinschaft industrieller Forschungsvereinigungen e.V. (AiF), FOSTA P812, Laufzeit 01.06.2009-31.05.2011, Entwurf Stand 29.08.2011

[Gilbert et al., 2002]: Gilbert, Y., Grondin, G. Y., Driver, R., G.; Kennedy, L.: Strength of transverse fillet welds made with filler metals without specified toughness, Final report, University of Alberta, Edmonton Alberta, Canada, 2002

[Gresnigt, 1989]: Gresnigt, A. M,: The $\beta$ formula for the design of fillet welds. Welding in the World, Vol. 27, No. 5/6, S. 122-127, 1989

[Gresnigt, 2002]: Gresnigt, A. M.: Update on design rules for fillet welds. In: Proceedings of the 3rd European Conference on Steel Structures, Coimbra - Portugal, pp. 919-927, 2002

[Hölbling et al., 2005]: Hölbling, W.; Muller, G.; Saal, H.: Tragverhalten von Kehlnahtverbindungen von höherfesten Feinkornbaustählen. In: Stahlbau 74 (2005), Heft 1, S. 1-8

[Hölbling, 2008]: Hölbling, W.: Die statische Tragfähigkeit teilweise durchgeschweißter Nähte in Stahl, Dissertation, Berichte der Versuchsanstalt für Stahl, Holz und Steine, Karlsruher Institut für Technologie, Folge 5, Heft 20, 2008 
[Holzer et al., 2010]: Holzer, S., Kranz, B.; Nies, H.; Röw, N.; Scherer F.: Bemessung von Schweißnahtanschlüssen nach DIN EN 1993-1-8. In: Stahlbau 79 (2010), Heft 11, S. 827-834

[IIW, 1976]: International Institute of Welding (IIW) "Design rules for arc welded connections in steel submitted to static loads”. Welding in the World, Vol. 14, No. 5/6, S. 132-149, 1976

[Klöppel, Petri, 1966]: Klöppel, K.; Petri, R.: Versuche zur Ermittlung der Tragfähigkeit von Kehlnähten , Der Stahlbau, 1966, Heft 1, S. 9-25

[Klöppel, Petri, 1969]: Klöppel, K.; Petri, R.: Statische Versuche und Dauerversuche mit geschweißten Stabanschlüssen, Der Stahlbau, 1969, Heft 5, S. 129-140

[Kob et al., 1987]: Kob P., Harre W., Schmidt H.: Einfluß geometrischer Verhältnisse auf die Tragfähigkeit statisch beanspruchter Kehlnahtverbindungen; Otto-Graf-Institut; Forschungs- und Materialprüfanstalt Baden-Württemberg, Schriftenreihe Heft 78, Stuttgart 1987

[Krebs, 1990]: Krebs, H.: Untersuchungen zum Bruchverhalten von Schweißverbindungen aus Feinkornbaustählen; Fortschritts-Berichte VDI-Reihe 18 Nr. 83, Düsseldorf, VDI-Verlag 1990

[Kuhlmann et al., 2006]: Kuhlmann U., Dürr, A., Bergmann, J. Thumser R.: Forschungsvorhaben P 620 - Effizienter Stahlbau aus höherfesten Stählen unter Ermüdungsbeanspruchung, Verlag und Vertriebsgesellschaft mbH, Düsseldorf, 2006

[Müsch, 1999]: Müsch, K.: Untersuchungen zum Einfluss örtlicher Festigkeitsunterschiede (Mismatch) auf das Versagensverhalten von Schweißverbindungen aus höherfesten Feinkornbaustählen; Berichte aus dem Institut für Eisenhüttenkunde, Band 1799, Shaker Verlag, Dissertation, 1998, Aachen

[Niemi, 1988]: Niemi, E.: Tests on fillet weld joints with high strength steel specimens, Lappeenranta, 1988, ISBN 951-763-550-8

[Ng et al., 2005]: Ng, A. K. F.; Driver, R. G.; Grondin, G. Y., 2002: Behaviour of transverse fillet welds, Structural Engineering Report No. 245, University of Alberta, Edmonton, Alberta, Canada, 2002

[Saal, 2005]: Saal, H.; Hölbling W.: Zusammenfassung der Ergebnisse von Versuchen an Kehlnahtprüfkörpern aus HISTAR460/460L hergestellt im Blockgussverfahren, Bericht Nr. 042119-2, 2005

[Snijder et al., 1988]: Snijder, H.H., Ungermann, D., Stark, J.W.B, Sedlacek, G., Bijlaard, F.S.K, Hemmert-Halswick, A.: Evaluation of test results on welded connections in order to obtain strength functions and suitable model factors, Background Documentation, Document 6.05, October 1988

[Staier, 2010]: Staier, K.: Vergleich und Beurteilung bestehender Bemessungsmodelle zur Tragfähigkeit von Flankenkehlnahtverbindungen höherfester Baustähle, Universität Stuttgart, Institut für Konstruktion und Entwurf, Diplomarbeit, Nr. 2010-22X

[Volz, 2009]: Volz, M.: Die statische Tragfähigkeit teilweise durchgeschweißter Nähte in Stahl, Dissertation, Berichte der Versuchsanstalt für Stahl, Holz und Steine, Karlsruher Institut für Technologie, Folge 5, Heft 22, 2009

[Wenk, 1988]: Wenk, P.: Traglastuntersuchungen an vorwiegend ruhend beanspruchten langen Flankenkehlnähten, Dissertation, 1988, Dortmund

[Werner, Bornscheuer, 1978]: Werner, G.; Bornscheuer, W.: Bewertung der Festigkeit vorwiegend ruhend beanspruchter Kehlnahtschweißverbindungen, Schweißen + Schneiden Jahrgang 30, 1978, Heft 8, S. 287-290

[Zusatzversuche, 2008]: Kuhlmann, U.; Günther, H.-P.; Rasche C.: Versuche zur Bestimmung der Tragfähigkeit von Flankenkehlnahtverbindungen höherfester Baustähle S690Q. Versuchsbericht, Universität Stuttgart, Institut für Konstruktion und Entwurf, Mitteilung Nr. 2008-21X, 2008, unveröffentlicht 


\subsection{Programme und Sonstiges}

[Ansys 13.0]: ANSYS Release 13.0, Ansys Inc., Canonsburg, USA

[Gross et al., 2007]: Gross D., Hauger W., Wriggers P.: Technische Mechanik Band 4: Hydromechanik, Elemente der Höheren Mechanik, Numerische Methoden, Springer-Verlag Berlin Heidelberg, 2007

[Schnell et al., 1998]: Schnell W., Gross D., Hauger W.: Technische Mechanik Band 2: Elastostatik, 6. Auflage, Springer-Verlag Berlin Heidelberg, 1998 


\section{A Anhang}

\section{A.1 Auswertung bekannter Versuchsreihen der Literatur}

\section{A.1.1 Statistisches Auswerteverfahren nach DIN EN 1990 (2002)}

Im Folgenden werden die durchgeführten Schritte der statistischen Auswertung nach DIN EN 1990 (2002) Anhang D vorgestellt. In Anhang D wird das Vorgehen bei versuchsgestützter Bemessung erläutert. Es wird die statistische Bestimmung eines Widerstandsmodells vorgestellt.

\section{Schritt 1: Entwicklung eines Bemessungsmodells}

Festlegung eines Widerstandsmodells $r_{t}=g_{r t}(\underline{X})$ für die charakteristische Tragfähigkeit

Widerstandsmodell nach Eurocode für Flankenkehlnähte $r_{t}\left(A_{B r u c h}, f_{u}\right)=\frac{f_{u}}{\sqrt{3} \cdot \beta_{w}} \cdot A_{\text {Bruch }}$

Widerstandsmodell nach Eurocode für Kreuzstöße $r_{t}\left(A_{B r u c h}, f_{u}\right)=\frac{f_{u}}{\sqrt{2} \cdot \beta_{w}} \cdot A_{\text {Bruch }}$

Basisvariable:

$\mathrm{f}_{\mathrm{u}, \mathrm{GW}} \quad$ Zugfestigkeit des Grundwerkstoff

$\mathrm{f}_{\mathrm{u}, \mathrm{SZw}} \quad$ Zugfestigkeit des Schweißzusatzwerkstoffs

$A_{\text {Bruch }}$ Bruchfläche der Schweißnaht

Schritt 2: Vergleich der experimentellen und theoretischen Werte

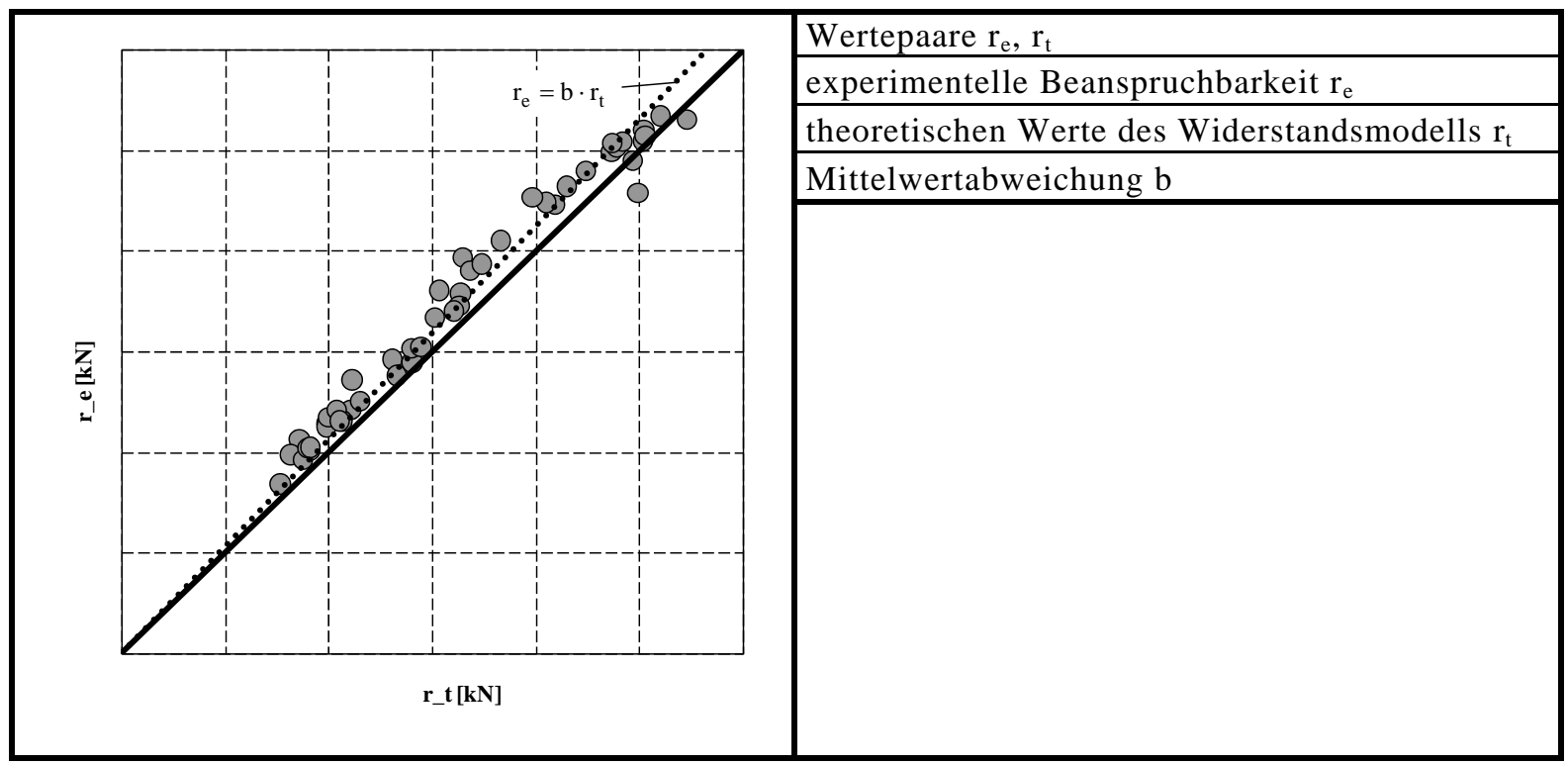

Abbildung A.1: Vergleich der experimentellen und theoretischen Werte

\section{Schritt 3: Schätzung der Mittelwertkorrektur b}

Widerstandsfunktion $\mathrm{r}=\mathrm{b} \cdot \mathrm{r}_{\mathrm{t}} \cdot \delta$

Mittelwertabweichung $\mathrm{b}=\frac{\Sigma \mathrm{r}_{\mathrm{e}} \cdot \mathrm{r}_{\mathrm{t}}}{\Sigma \mathrm{r}_{\mathrm{t}}^{2}}$

Theoretische Widerstandsfunktion $\mathrm{r}_{\mathrm{m}}=\mathrm{b} \cdot \mathrm{r}_{\mathrm{t}}\left(\underline{\mathrm{X}}_{\mathrm{m}}\right)=\mathrm{b} \cdot \mathrm{g}_{\mathrm{rt}} \cdot\left(\underline{\mathrm{X}}_{\mathrm{m}}\right) \cdot \delta$ 
Schritt 4: Schätzung des Variationskoeffizienten der Streugröße $\delta$

Streugröße $\delta_{\mathrm{i}}=\frac{\mathrm{r}_{\mathrm{ei}}}{\mathrm{b} \cdot \mathrm{r}_{\mathrm{ti}}}$

Variationskoeffizient $\mathrm{V}_{\delta}$ für die Streugröße $\delta \mathrm{V}_{\delta}=\sqrt{\exp \left(\mathrm{s}_{\Delta}^{2}\right)-1}$

mit $\Delta_{\mathrm{i}}=\ln \left(\delta_{\mathrm{i}}\right)$

$\bar{\Delta}=\frac{1}{\mathrm{n}} \sum_{\mathrm{i}=1}^{\mathrm{n}} \Delta_{\mathrm{i}}$

$\mathrm{s}_{\Delta}^{2}=\frac{1}{\mathrm{n}-1} \sum_{\mathrm{i}=1}^{\mathrm{n}}\left(\Delta_{\mathrm{i}}-\bar{\Delta}\right)^{2}$

\section{Schritt 5: Verträglichkeitsprüfung}

\section{Schritt 6: Bestimmung der Variationskoeffizienten $\mathrm{V}_{\mathrm{Xi}}$ der Basisvariablen}

Die Variationskoeffizienten $\mathrm{V}_{\mathrm{Xi}}$ müssen durch Vorinformationen bestimmt werden, wenn der Versuchsumfang nicht repräsentativ ist, was in der Regel vorliegt.

$\mathrm{V}_{\mathrm{ABruch}}=0,1 ; \mathrm{V}_{\mathrm{fu}}=0,07$

\section{Schritt 7: Bestimmung des charakteristischen Wertes $\mathbf{r}_{\mathbf{k}}$ der Widerstandsfunktion}

Widerstandsfunktion $\mathrm{r}=\mathrm{b} \cdot \mathrm{r}_{\mathrm{t}}=\mathrm{b} \cdot\left\{\mathrm{X}_{1} \times \mathrm{X}_{2} \ldots . . \mathrm{X}_{\mathrm{j}}\right\} \cdot \delta$

Mittelwert $\mathrm{E}(\mathrm{r})=\mathrm{b} \cdot\left\{\mathrm{E}\left(\mathrm{X}_{1}\right) \times \mathrm{E}\left(\mathrm{X}_{2}\right) \ldots . . \mathrm{E}\left(\mathrm{X}_{\mathrm{j}}\right)\right\}=\mathrm{b} \cdot \mathrm{g}_{\mathrm{rt}}\left(\underline{\mathrm{X}}_{\mathrm{m}}\right)$

Variationskoeffizient $\mathrm{V}_{\mathrm{r}}^{2}=\left(\mathrm{V}_{\delta}^{2}+1\right)\left[\prod_{\mathrm{i}=1}^{\mathrm{j}}\left(\mathrm{V}_{\mathrm{xi}}^{2}+1\right)\right]-1, \mathrm{~V}_{\mathrm{rt}}^{2}=\sum_{\mathrm{i}=1}^{\mathrm{j}} \mathrm{V}_{\mathrm{xi}}^{2}$

Für Versuchsanzahl $\mathrm{n}<100$ lautet der charakteristische Wert der Widerstandsfunktion

$$
\begin{aligned}
& \mathrm{r}_{\mathrm{k}}=\mathrm{b} \cdot \mathrm{g}_{\mathrm{R}}\left(\underline{X}_{\mathrm{m}}\right) \exp \left(-\mathrm{k}_{\infty} \cdot \alpha_{\mathrm{rt}} \cdot \mathrm{Q}_{\mathrm{rt}}-\mathrm{k}_{\mathrm{n}} \cdot \alpha_{\delta} \cdot \mathrm{Q}_{\delta}-0,5 \cdot \mathrm{Q}^{2}\right) \\
& \text { Mit } \mathrm{Q}_{\mathrm{rt}}=\sigma_{\ln (\mathrm{rt})}=\sqrt{\ln \left(\mathrm{V}_{\mathrm{rt}}^{2}+1\right)} \\
& \mathrm{Q}_{\delta}=\sigma_{\ln (\delta)}=\sqrt{\ln \left(\mathrm{V}_{\delta}^{2}+1\right)} \\
& \mathrm{Q}=\sigma_{\ln (\mathrm{r})}=\sqrt{\ln \left(\mathrm{V}_{\mathrm{r}}^{2}+1\right)} \\
& \alpha_{\mathrm{rt}}=\frac{\mathrm{Q}_{\mathrm{rt}}}{\mathrm{Q}} \\
& \alpha_{\delta}=\frac{\mathrm{Q}_{\delta}}{\mathrm{Q}} \\
& \mathrm{k}_{\mathrm{n}} \text { Fraktilenfaktor für den charakteristischen Wert aus } \\
& \text { Tabelle A.1 für „, } \mathrm{V}_{\mathrm{x}} \text { unbekannt“ } \\
& \mathrm{k}_{\infty} \text { Wert des Fraktilenfaktors } \mathrm{k}_{\mathrm{n}} \text { für } \mathrm{n} \rightarrow \infty\left[\mathrm{k}_{\infty}=1,64\right] \\
& \alpha_{\mathrm{rt}} \text { Wichtungsfaktor für } \mathrm{Q}_{\mathrm{rt}} \text { und } \alpha_{\delta} \text { Wichtungsfaktor für } \mathrm{Q}_{\delta}
\end{aligned}
$$


Tabelle A.1: Werte $k_{n}$ für charakteristische Werte (5 \%-Fraktile)

\begin{tabular}{|l|l|l|l|l|l|l|l|l|l|l|l|}
\hline $\mathrm{n}$ & 1 & 2 & 3 & 4 & 5 & 6 & 8 & 10 & 20 & 30 & $\infty$ \\
\hline $\mathrm{V}_{\mathrm{x}}$ bekannt & 2,31 & 2,01 & 1,89 & 1,83 & 1,80 & 1,77 & 1,74 & 1,72 & 1,68 & 1,67 & 1,64 \\
\hline $\mathrm{V}_{\mathrm{x}}$ unbekannt & - & - & 3,37 & 2,63 & 2,33 & 2,18 & 2,00 & 1,92 & 1,76 & 1,73 & 1,64 \\
\hline
\end{tabular}

\section{Schritt 8: Bemessungswert der Widerstandsfunktion bei beschränkter Versuchsanzahl}

$\mathrm{r}_{\mathrm{d}}=\mathrm{b} \cdot \mathrm{g}_{\mathrm{rt}}\left(\underline{\mathrm{X}}_{\mathrm{m}}\right) \exp \left(-\mathrm{k}_{\mathrm{d}, \infty} \cdot \alpha_{\mathrm{rt}} \cdot \mathrm{Q}_{\mathrm{rt}}-\mathrm{k}_{\mathrm{d}, \mathrm{n}} \cdot \alpha_{\delta} \cdot \mathrm{Q}_{\delta}-0,5 \cdot \mathrm{Q}^{2}\right)$

$\mathrm{k}_{\mathrm{d}, \mathrm{n}}$ Fraktilenfaktor für den Bemessungswert aus

Tabelle A.2 für „, $\mathrm{V}_{\mathrm{x}}$ unbekannt“

$\mathrm{k}_{\mathrm{d}, \infty}$ Wert des Fraktilenfaktors $\mathrm{k}_{\mathrm{d}, \mathrm{n}}$ für $\mathrm{n} \rightarrow \infty\left[\mathrm{k}_{\mathrm{d}, \infty}=3,04\right]$

$\alpha_{\text {rt }}$ Wichtungsfaktor für $\mathrm{Q}_{\mathrm{rt}}$ und $\alpha_{\delta}$ Wichtungsfaktor für $\mathrm{Q}_{\delta}$

Tabelle A.2: Werte $k_{\mathrm{d}, \mathbf{n}}$ für Bemessungswert für Tragfähigkeitsnachweise

\begin{tabular}{|l|l|l|l|l|l|l|l|l|l|l|l|}
\hline $\mathrm{n}$ & 1 & 2 & 3 & 4 & 5 & 6 & 8 & 10 & 20 & 30 & $\infty$ \\
\hline $\mathrm{V}_{\mathrm{x}}$ bekannt & 4,36 & 3,77 & 3,56 & 3,44 & 3,37 & 3,33 & 3,27 & 3,23 & 3,16 & 3,13 & 3,04 \\
\hline $\mathrm{V}_{\mathrm{x}}$ unbekannt & - & - & - & 11,4 & 7,85 & 6,36 & 5,07 & 4,51 & 3,64 & 3,44 & 3,04 \\
\hline Anmerkung 1 1 Diese Tabelle beruht auf der Annahme, dass der Bemessungswert dem Produkt \\
$\begin{array}{l}\alpha_{\mathrm{R}} \cdot \beta=0,8 \cdot 3,8=3,04 \\
\text { etwa } 0,1 \%\end{array}$ \\
entspricht und X normalverteilt ist. Die Unterschreitungswahrscheinlichkeit ist \\
\hline
\end{tabular}

\section{Schritt 9: Bestimmung des Teilsicherheitsbeiwertes}

$$
\gamma_{M}=\frac{r_{k}}{r_{d}}
$$

\section{Schritt 10: Verwendung zusätzlicher Vorinformationen}

Korrektur des Teilsicherheitsbeiwertes für den Fall, dass Mittelwerte der Basisvariabeln nicht als Nennwerte sondern als charakteristischen bzw. Fraktilwerte in die Tragfähigkeitsgleichung eingehen.

$$
\mathrm{r}_{\mathrm{k}}=\eta_{\mathrm{k}} \cdot \mathrm{r}_{\mathrm{em}}
$$

mit Mittelwert $r_{\text {em }}=\frac{r_{\text {ei }}}{b}$

$\eta_{\mathrm{k}}=0,9 \cdot \exp \left(-2,0 \cdot \mathrm{V}_{\mathrm{t}}-0,5 \cdot \mathrm{V}_{\mathrm{t}}^{2}\right)$

$\eta_{\mathrm{k}}$ Reduktionsfaktor zur Berücksichtigung von Vorinformationen bei mehreren Versuchen hier Korrektur Zugfestigkeit des Grundmaterials mit $\mathrm{V}_{\mathrm{t}}=\mathrm{V}_{\mathrm{fu}}$

Korrigierter Sicherheitsbeiwert $\gamma_{M^{*}}=\frac{r_{k^{*}}}{r_{d}}=\frac{\eta_{k} / b}{r_{d}}=\frac{\eta_{k} / b}{r_{k}} \gamma_{M}$ 


\section{A.1.2 Kehlnahtverbindungen höherfester Stähle S690 mit niederfestem Schweiß- zusatzwerkstoff}

Im Folgenden werden die Versuchsergebnisse nach [Kob et al., 1987] vorgestellt. In Tabelle A.3 sind die mechanischen Kennwerte der verwendeten Grundwerkstoffe zusammengestellt. Die Festigkeiten wurden an Normzugversuchen der Grundwerkstoffe und aus Stumpfnähten, also dem reinen Schweißgut ermittelt. Es wurden Mittelwerte gebildet, wenn mehrere Ergebnisse von Zugversuchen vorlagen. Über die Vorwärmtemperatur oder andere Schweißparameter werden in [Kob et al., 1987] keine Angaben gemacht.

Tabelle A.3: Mechanische Eigenschaften der verwendeten Grundwerkstoffe nach [Kob et al., 1987]

\begin{tabular}{|l|l|l|l|l|l|l|l|l|l|l|l|l|l|}
\hline & \multicolumn{9}{l|}{ Werkstoff S355J0+N } & \multicolumn{3}{l|}{ Werkstoff S690Q } \\
\hline Blech-Nr. & 1 & & 2 & 3 & 4 & 5 & 6 & 7 & 8 & 9 & 10 \\
\hline $\begin{array}{l}\text { Blechdicke } \\
{[\mathrm{mm}]}\end{array}$ & 10 & \multicolumn{3}{|l|}{20} & & 30 & 10 & 20 & \\
\hline & $\mathrm{L}$ & $\mathrm{Q}$ & $\mathrm{L}$ & $\mathrm{Q}$ & $\mathrm{Q}$ & $\mathrm{Q}$ & $\mathrm{Q}$ & $\mathrm{L}$ & $\mathrm{Q}$ & $\mathrm{L}$ & $\mathrm{Q}$ & $\mathrm{Q}$ \\
\hline $\mathrm{R}_{\mathrm{eH}}\left[\mathrm{N} / \mathrm{mm}^{2}\right]$ & 379 & 375 & 373 & 387 & 339 & 362 & 359 & 359 & 774 & 799 & 811 & 812 \\
\hline $\mathrm{R}_{\mathrm{m}}\left[\mathrm{N} / \mathrm{mm}^{2}\right]$ & 555 & 550 & 535 & 545 & 552 & 550 & 551 & 544 & 826 & 838 & 852 & 854 \\
\hline $\mathrm{A}_{5}[\%]$ & 29,3 & 28,1 & 31,3 & 31,1 & 28,6 & 28,7 & 28,6 & 33,2 & 16,9 & 18,0 & 15,8 & 15,5 \\
\hline
\end{tabular}

Bei den Flankenkehlnähten wurden als Versuchsergebnisse die maximale Kraft während des Versuches und die nach dem Versuch ausgemessene Bruchfläche angegeben. Daraus ergibt sich als Quotient von Kraft und Bruchfläche die maximale Spannung $\tau_{\text {II. }}$ Für die Flankenkehlnähte sind in Tabelle A.4 die Versuchsergebnisse S355-G42 zusammengefasst. Tabelle A.5 zeigt die statistische Auswertung dieser Versuche. Die Versuchsergebnisse der Flankenkehlnahtverbindungen der Werkstoffkombination S690Q-G42 sind in Tabelle A.6 vorgestellt, während in Tabelle A.7 die Ergebnisse der statistischen Auswertung der Versuche dargestellt ist. 
Tabelle A.4: Versuchsergebnisse der Flankenkehlnahtverbindungen S355J0-G42 nach [Kob et al., 1987]

\begin{tabular}{|c|c|c|c|c|c|c|c|c|c|c|c|c|c|c|}
\hline & \multicolumn{4}{|c|}{ Grundwerkstoff } & \multicolumn{6}{|c|}{ S355J0+N, vgl. Tabelle A.3 } \\
\hline & & & & & \multicolumn{4}{|c|}{$\begin{array}{l}\text { Schweißzusatzwerkstof- } \\
\text { fe und Schweißprozesse }\end{array}$} & \multicolumn{6}{|c|}{$\begin{array}{l}\text { MAG-Schweißen: SG } 2 \text { M21, Carbofil } 1 \\
\mathrm{f}_{\mathrm{v}}=503 \mathrm{~N} / \mathrm{mm}^{2}, \mathrm{f}_{\mathrm{u}}=590 \mathrm{~N} / \mathrm{mm}^{2}\end{array}$} \\
\hline & & & & & \multicolumn{4}{|c|}{ Blechdicke } & \multicolumn{6}{|c|}{$10,20 \mathrm{~mm}$} \\
\hline & & & & & \multicolumn{4}{|c|}{ Nahtdicke } & \multicolumn{6}{|c|}{$3 ; 4,5 ; 5 ; 5,5 ; 6 ; 7 ; 9 ; 10 \mathrm{~mm}$} \\
\hline & & & & & \multicolumn{4}{|c|}{ Nahtlänge l } & \multicolumn{6}{|c|}{$60 \mathrm{~mm}$} \\
\hline 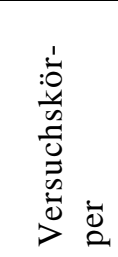 & $\mid \begin{array}{l}z \\
\bar{y} \\
\frac{\tilde{g}}{n}\end{array}$ & 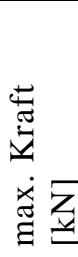 & 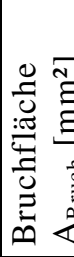 & 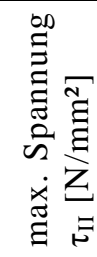 & 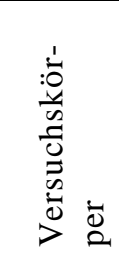 & 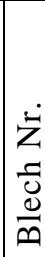 & 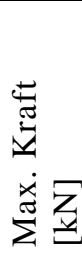 & 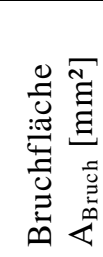 & 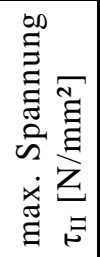 & 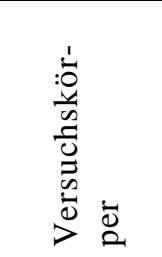 & $\begin{array}{l}\vec{z} \\
\bar{y} \\
\frac{\tilde{g}}{n}\end{array}$ & 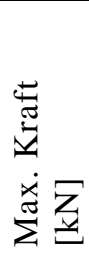 & 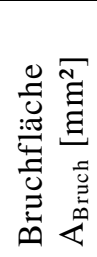 & 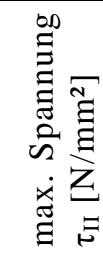 \\
\hline $1 / \mathrm{S} 5-1$ & 1 & 229 & 497 & 460 & $1 / \mathrm{S} 7-5$ & 3 & 387 & 872 & 444 & $1 / \mathrm{S} 9-5$ & 5 & 491 & 1237 & 397 \\
\hline $1 / \mathrm{S} 5-2$ & 1 & 234 & 530 & 442 & $1 /$ S7-6 & 3 & 272 & 558 & 487 & $1 / \mathrm{S} 9-6$ & 5 & 458 & 1250 & 367 \\
\hline $1 / \mathrm{S} 5-3$ & 1 & 234 & 509 & 460 & $1 / \mathrm{S} 7-7$ & 3 & 213 & 431 & 493 & $1 / \mathrm{S} 9-7$ & 5 & 305 & 726 & 420 \\
\hline $1 / \mathrm{S} 5-4$ & 1 & 225 & 497 & 453 & $1 / \mathrm{S} 7-8$ & 3 & 235 & 501 & 469 & $1 / \mathrm{S} 9-8$ & 5 & 345 & 818 & 422 \\
\hline $1 / \mathrm{S} 5-5$ & 1 & 235 & 521 & 451 & $1 /$ S7-9 & 3 & 198 & 409 & 483 & $1 / \mathrm{S} 9-9$ & 5 & 192 & 441 & 435 \\
\hline $1 / \mathrm{S} 5-6$ & 1 & 242 & 557 & 434 & $1 / \mathrm{S} 8-1$ & 4 & 480 & 1126 & 427 & $1 / \mathrm{S} 9-10$ & 5 & 204 & 452 & 451 \\
\hline $1 / \mathrm{S} 5-7$ & 1 & 231 & 536 & 431 & $1 / \mathrm{S} 8-2$ & 4 & 447 & 1051 & 425 & 1/S9E-1 & 5 & 515 & 1267 & 407 \\
\hline $1 / \mathrm{S} 6-1$ & 1 & 292 & 657 & 445 & $1 / \mathrm{S} 8-3$ & 4 & 449 & 1031 & 435 & 1/S9E-2 & 5 & 499 & 1186 & 421 \\
\hline $1 / \mathrm{S} 6-2$ & 1 & 250 & 580 & 432 & $1 / \mathrm{S} 8-4$ & 4 & 453 & 997 & 455 & 1/S9E-3 & 5 & 504 & 1198 & 420 \\
\hline $1 / \mathrm{S} 6-3$ & 1 & 276 & 669 & 413 & $1 / \mathrm{S} 8-5$ & 4 & 358 & 823 & 435 & $1 / \mathrm{S} 9 \mathrm{E}-4$ & 5 & 509 & 1212 & 420 \\
\hline $1 / \mathrm{S} 6-4$ & 1 & 289 & 706 & 410 & $1 / \mathrm{S} 8-6$ & 4 & 303 & 704 & 431 & $1 / \mathrm{S} 9 \mathrm{E}-5$ & 5 & 508 & 1187 & 428 \\
\hline $1 / \mathrm{S} 6-5$ & 1 & 201 & 426 & 472 & $1 / \mathrm{S} 8-7$ & 4 & 242 & 523 & 462 & 1/S9E-6 & 5 & 464 & 1079 & 430 \\
\hline $1 / \mathrm{S} 6-6$ & 1 & 202 & 457 & 442 & $1 / \mathrm{S} 8-8$ & 4 & 231 & 529 & 436 & $1 / \mathrm{S} 9 \mathrm{E}-7$ & 5 & 334 & 760 & 439 \\
\hline 1/S7-1 & 3 & 394 & 825 & 477 & 1/S9-1 & 5 & 535 & 1305 & 410 & 1/S9E-8 & 5 & 341 & 806 & 423 \\
\hline $1 /$ S7-2 & 3 & 380 & 842 & 452 & $1 / \mathrm{S} 9-2$ & 5 & 521 & 1264 & 412 & $1 / \mathrm{S} 9 \mathrm{E}-9$ & 5 & 168 & 386 & 436 \\
\hline $1 / \mathrm{S} 7-3$ & 3 & 361 & 768 & 470 & 1/S9-3 & 5 & 510 & 1263 & 404 & 1/S9E-10 & 5 & 205 & 458 & 447 \\
\hline $1 / \mathrm{S} 7-4$ & 3 & 410 & 916 & 448 & $1 / \mathrm{S} 9-4$ & 5 & 531 & 1369 & 388 & & & & & \\
\hline
\end{tabular}

Tabelle A.5: Statistische Auswertung der Flankenkehlnahtverbindungen S355J0-G42 bezogen auf die Festigkeit des Grundwerkstoffs (links) oder des Schweißzusatzwerkstoffs (rechts)

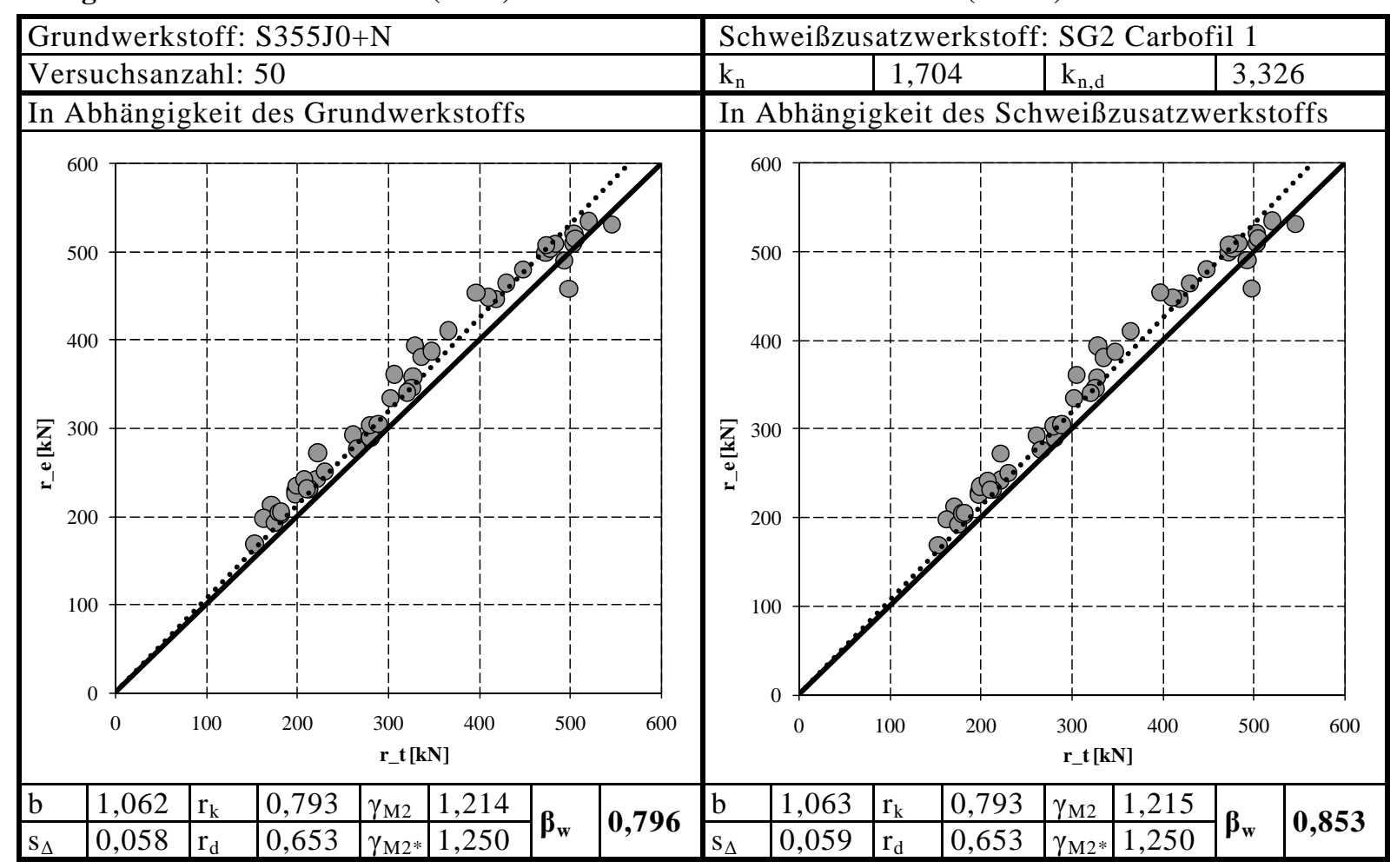


Tabelle A.6: Versuchsergebnisse der Flankenkehlnahtverbindungen S690Q-G42 nach [Kob et al., 1987]

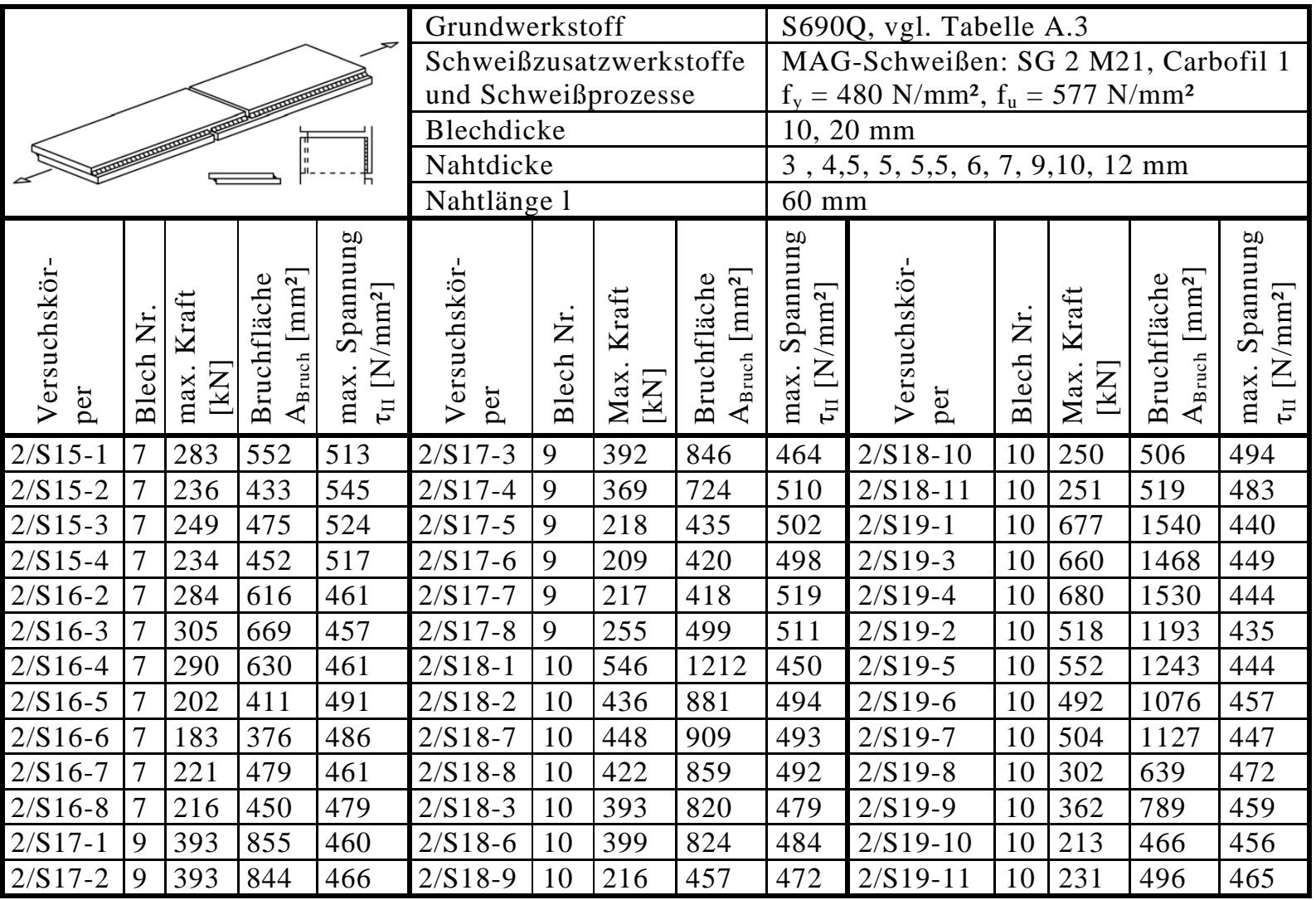

Tabelle A.7: Statistische Auswertung der Flankenkehlnahtverbindungen S690Q-G42 bezogen auf die Festigkeit des Grundwerkstoffs (links) oder des Schweißzusatzwerkstoffs (rechts)

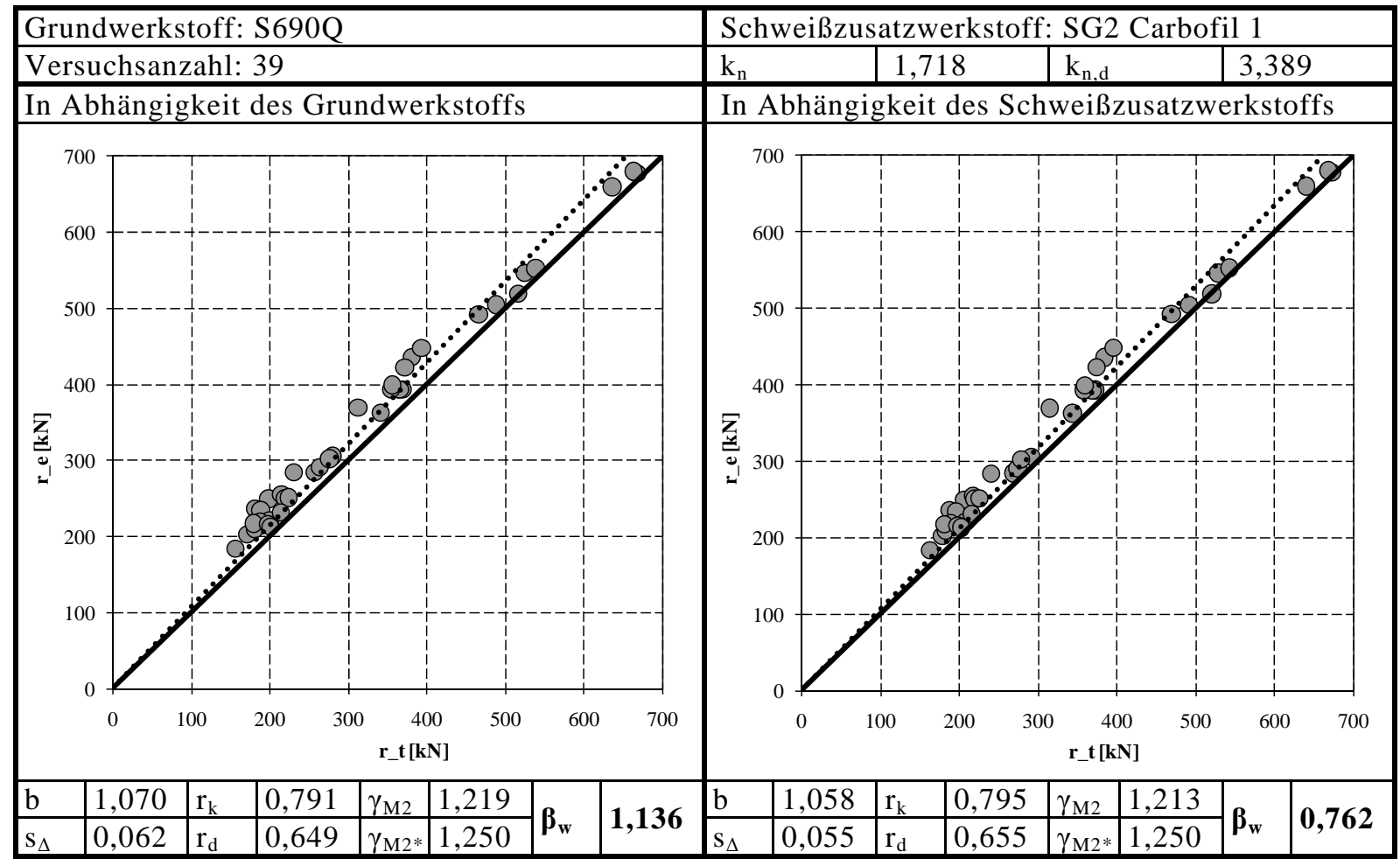


Bei den Kreuzstößen wurden als Versuchsergebnisse die maximale Kraft während des Versuches und die nach dem Versuch ausgemessene Bruchfläche $A_{\text {Bruch }}$ angegeben. Außerdem wurde die Nahtdicke $A_{E C}$ bestimmt. Daraus ergeben sich als Quotient von Kraft und Fläche jeweils die maximalen Spannungen. Der Bruch verlief stets in der Naht, der Bruchwinkel war sehr steil.

Die Tragfähigkeitsuntersuchungen an Stirnkehlnähten mit dem Grundwerkstoff S355J0+N und die ermittelten Versuchsergebnisse sind in Tabelle A.8 zusammengestellt. 38 Versuche mit Stirnkehlnähten wurden statistisch ausgewertet, wie in Tabelle A.9 in Abhängigkeit der Nahtfläche $A_{E C}$ und in Tabelle A.10 in Abhängigkeit der Bruchfläche $\mathrm{A}_{\text {Bruch }}$ zusammengefasst.

Tabelle A.8: Versuchsergebnisse der Kreuzstöße S355J0-G42 nach [Kob et al., 1987]

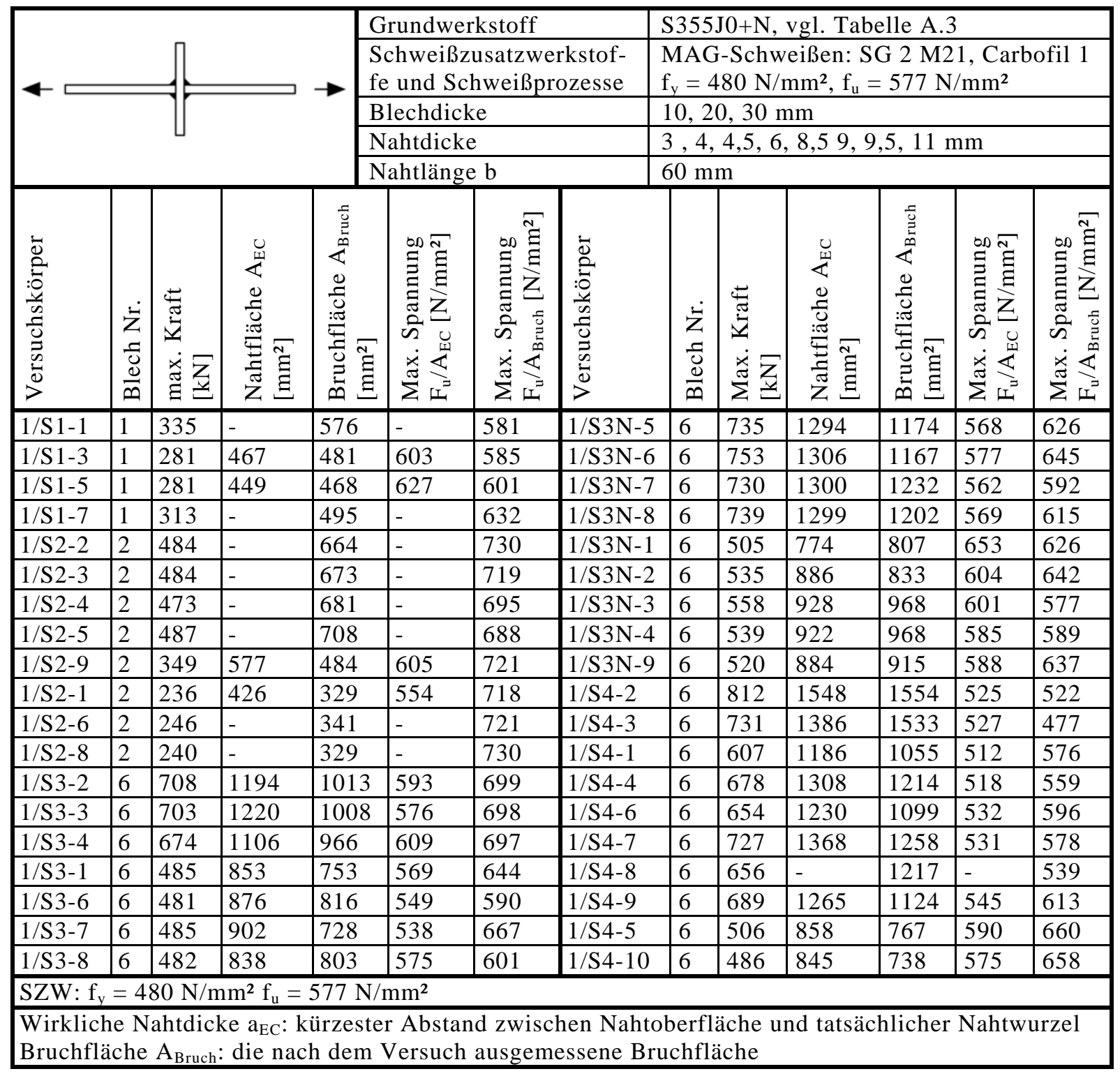


Tabelle A.9: Statistische Auswertung der Kreuzstöße S355J0-G42, Nahtfläche $A_{\mathrm{EC}}$ bezogen auf die Festigkeit des Grundwerkstoffs (links) oder des Schweißzusatzwerkstoffs (rechts)

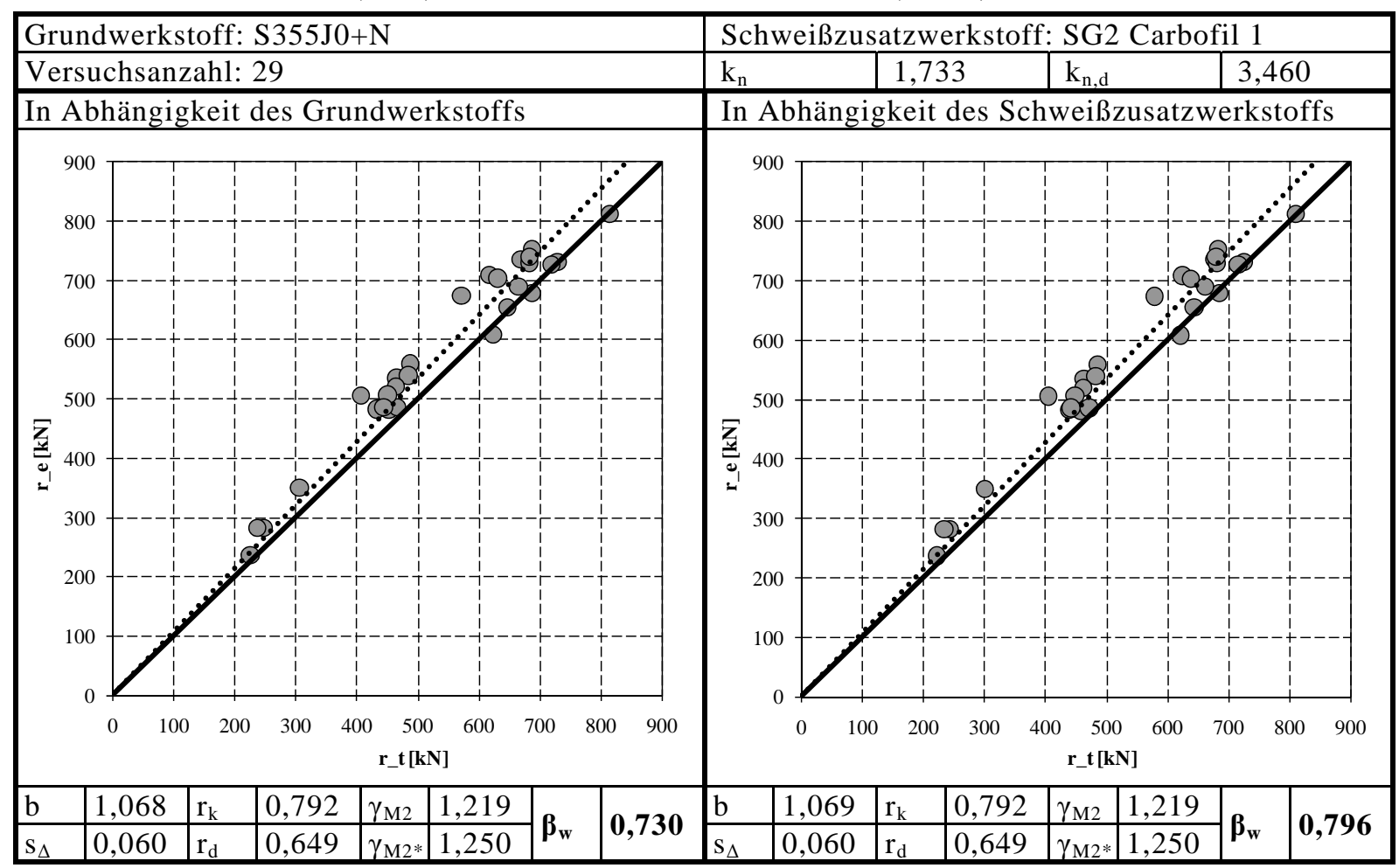

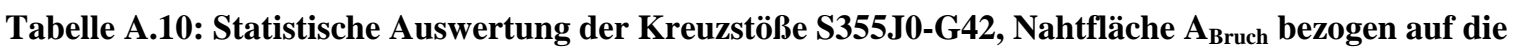
Festigkeit des Grundwerkstoffs (links) oder des Schweißzusatzwerkstoffs (rechts)

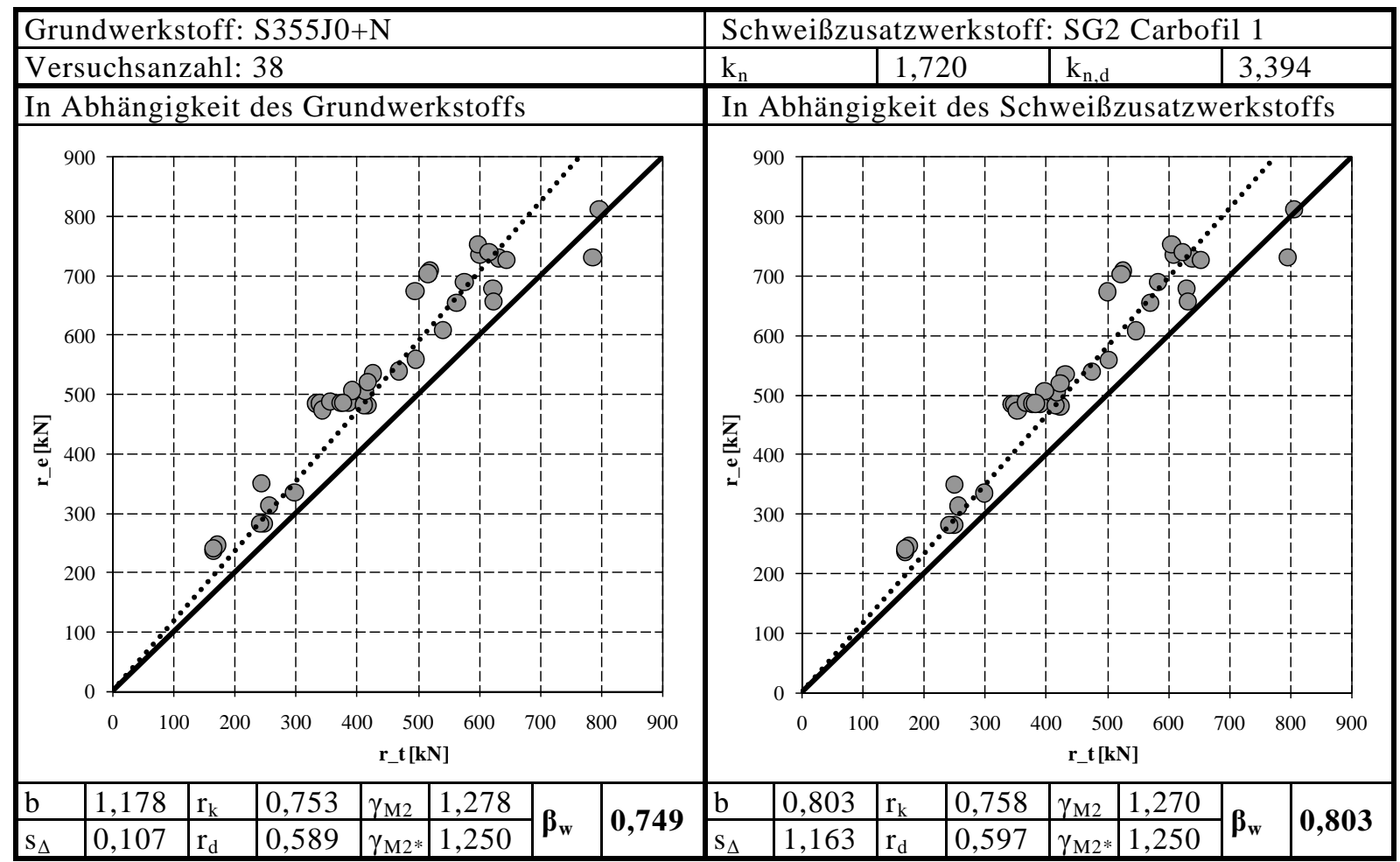


Die Versuchsergebnisse von 42 Versuchen an Stirnkehlnähten mit dem Grundwerkstoff S690 und dem Schweißzusatzwerkstoff G42 sind in Tabelle A.11 zusammengestellt. Die statistische Auswertung in Abhängigkeit der Bruchfläche $A_{B r u c h}$ ist in Tabelle A.12 und in Abhängigkeit der Nahtfläche $A_{E C}$ in Tabelle A.13 zusammengefasst.

Tabelle A.11: Versuchsergebnisse der Kreuzstöße S690Q-G42 nach [Kob et al., 1987]

\begin{tabular}{|c|c|c|c|c|c|c|c|c|c|c|c|c|c|}
\hline \multirow{5}{*}{\multicolumn{2}{|c|}{$\leftarrow$}} & \multirow{5}{*}{$\pi$} & & \multicolumn{4}{|c|}{ Grundwerkstoff } & \multicolumn{6}{|c|}{ S690Q, vgl. Tabelle A.3 } \\
\hline & & & & \multicolumn{4}{|c|}{$\begin{array}{l}\text { Schweißzusatzwerkstof- } \\
\text { fe und Schweißprozesse }\end{array}$} & \multicolumn{6}{|c|}{$\begin{array}{l}\text { MAG-Schweißen: SG } 2 \text { M21, Carbofil } 1 \\
\mathrm{f}_{\mathrm{y}}=480 \mathrm{~N} / \mathrm{mm}^{2}, \mathrm{f}_{\mathrm{u}}=577 \mathrm{~N} / \mathrm{mm}^{2}\end{array}$} \\
\hline & & & & \multicolumn{4}{|c|}{ Blechdicke } & \multicolumn{6}{|c|}{$10,20 \mathrm{~mm}$} \\
\hline & & & & \multicolumn{4}{|c|}{ Nahtdicke } & \multicolumn{6}{|c|}{$3,5,6,8,9,10,5 \mathrm{~mm}$} \\
\hline & & & & \multicolumn{4}{|c|}{ Nahtlänge b } & \multicolumn{6}{|c|}{$60 \mathrm{~mm}$} \\
\hline 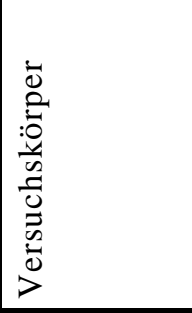 & \begin{tabular}{|l|}
$\vec{z}$ \\
$\bar{y}$ \\
$\frac{0}{m}$ \\
\end{tabular} & 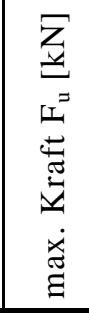 & 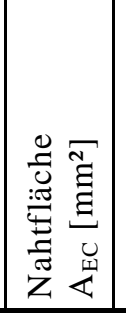 & 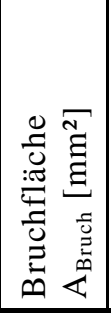 & 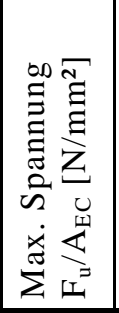 & 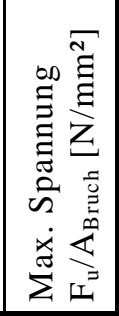 & 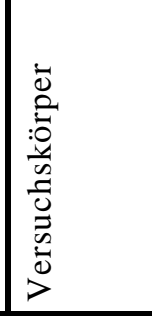 & $\begin{array}{l}\dot{\mathrm{z}} \\
\frac{1}{v} \\
\frac{\Delta}{n}\end{array}$ & 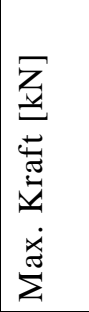 & 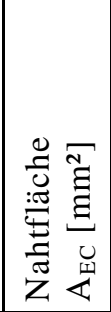 & 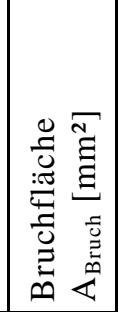 & 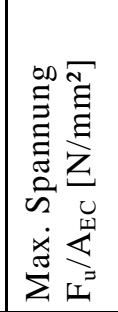 & 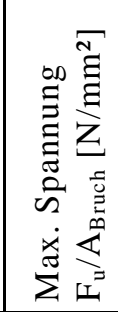 \\
\hline $2 / \mathrm{S} 10 \mathrm{~N}-1$ & 7 & 323 & 475 & 444 & 680 & 727 & 2/S12-5 & 8 & 360 & 496 & 525 & 725 & 685 \\
\hline $2 / \mathrm{S} 10 \mathrm{~N}-2$ & 7 & 323 & 499 & 462 & 647 & 699 & 2/S12-6 & 8 & 327 & 477 & 489 & 686 & 669 \\
\hline $2 / \mathrm{S} 10 \mathrm{~N}-3$ & 7 & 260 & 456 & 367 & 570 & 708 & 2/S12-8 & 8 & 326 & 550 & 524 & 593 & 622 \\
\hline $2 / \mathrm{S} 10 \mathrm{~N}-4$ & 7 & 322 & 480 & 446 & 671 & 722 & $2 / \mathrm{S} 13-5$ & 8 & 594 & 975 & 961 & 609 & 618 \\
\hline $2 / \mathrm{S} 10 \mathrm{~N}-6$ & 7 & 339 & 493 & 495 & 687 & 684 & $2 / \mathrm{S} 13-6$ & 8 & 607 & 1051 & 1047 & 577 & 579 \\
\hline 2/S10N-7 & 7 & 264 & 367 & 342 & 720 & 773 & 2/S13-7 & 8 & 548 & 975 & 894 & 562 & 613 \\
\hline 2/S10NN-1 & 7 & 265 & 417 & 396 & 636 & 669 & 2/S13-8 & 8 & 600 & 1050 & 1008 & 571 & 595 \\
\hline 2/S10NN-2 & 7 & 267 & 423 & 422 & 632 & 633 & 2/S13-9 & 8 & 580 & 993 & 1022 & 584 & 568 \\
\hline $2 / \mathrm{S} 10 \mathrm{NN}-3$ & 7 & 246 & 435 & 369 & 565 & 666 & $2 / \mathrm{S} 13-2$ & 8 & 456 & 795 & 729 & 573 & 625 \\
\hline $2 / \mathrm{S} 10 \mathrm{NN}-4$ & 7 & 256 & 438 & 409 & 584 & 625 & $2 / \mathrm{S} 13-3$ & 8 & 466 & 792 & 727 & 588 & 641 \\
\hline $2 / \mathrm{S} 10 \mathrm{NN}-5$ & 7 & 265 & 423 & 431 & 627 & 616 & $2 / \mathrm{S} 13-4$ & 8 & 465 & 795 & 734 & 585 & 634 \\
\hline 2/S11NN-1 & 7 & 442 & 768 & 653 & 575 & 677 & 2/S13-1 & 8 & 379 & 682 & 622 & 556 & 609 \\
\hline $2 / \mathrm{S} 11 \mathrm{NN}-2$ & 7 & 447 & 735 & 673 & 608 & 664 & 2/S14-5 & 8 & 740 & 1303 & 1253 & 568 & 590 \\
\hline 2/S11NN-3 & 7 & 404 & 678 & 609 & 596 & 663 & 2/S14-6 & 8 & 744 & 1344 & 1250 & 553 & 595 \\
\hline 2/S11NN-4 & 7 & 434 & 756 & 645 & 574 & 672 & 2/S14-7 & 8 & 740 & 1316 & 1242 & 562 & 595 \\
\hline 2/S11NN-5 & 7 & 449 & 788 & 683 & 570 & 657 & 2/S14-8 & 8 & 648 & 1169 & 1082 & 554 & 599 \\
\hline 2/S12-4 & 8 & 542 & 849 & 774 & 639 & 701 & 2/S14-9 & 8 & 632 & 1138 & 1046 & 555 & 604 \\
\hline 2/S12-7 & 8 & 558 & 840 & 767 & 665 & 728 & 2/S14-1 & 8 & 427 & 760 & 650 & 561 & 656 \\
\hline 2/S12-9 & 8 & 548 & 869 & 791 & 631 & 693 & 2/S14-2 & 8 & 432 & 760 & 673 & 568 & 641 \\
\hline $2 / \mathrm{S} 12-1$ & 8 & 333 & 457 & 489 & 729 & 681 & $2 / \mathrm{S} 14-3$ & 8 & 441 & 792 & 693 & 557 & 637 \\
\hline $2 / \mathrm{S} 12-2$ & 8 & 332 & 458 & 448 & 725 & 741 & $2 / \mathrm{S} 14-4$ & 8 & 433 & 712 & 648 & 608 & 668 \\
\hline
\end{tabular}


Tabelle A.12: Statistische Auswertung der Kreuzstöße S690Q-G42, Nahtfläche $A_{\mathrm{EC}}$ bezogen auf die Festigkeit des Grundwerkstoffs (links) oder des Schweißzusatzwerkstoffs (rechts)

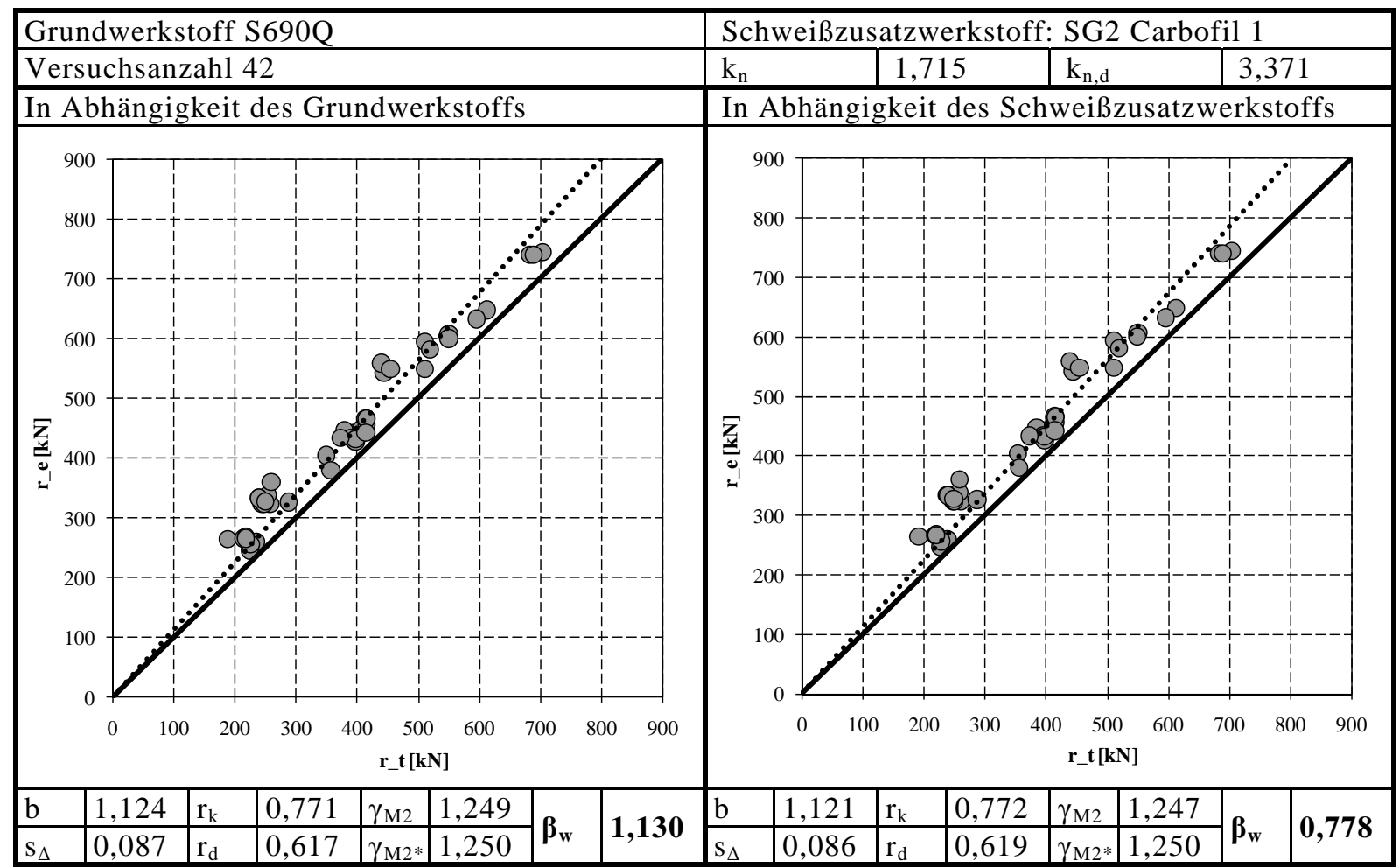

Tabelle A.13: Statistische Auswertung der Kreuzstöße S690Q-G42, Nahtfläche $A_{\text {Bruch }}$ bezogen auf die Festigkeit des Grundwerkstoffs (links) oder des Schweißzusatzwerkstoffs (rechts)

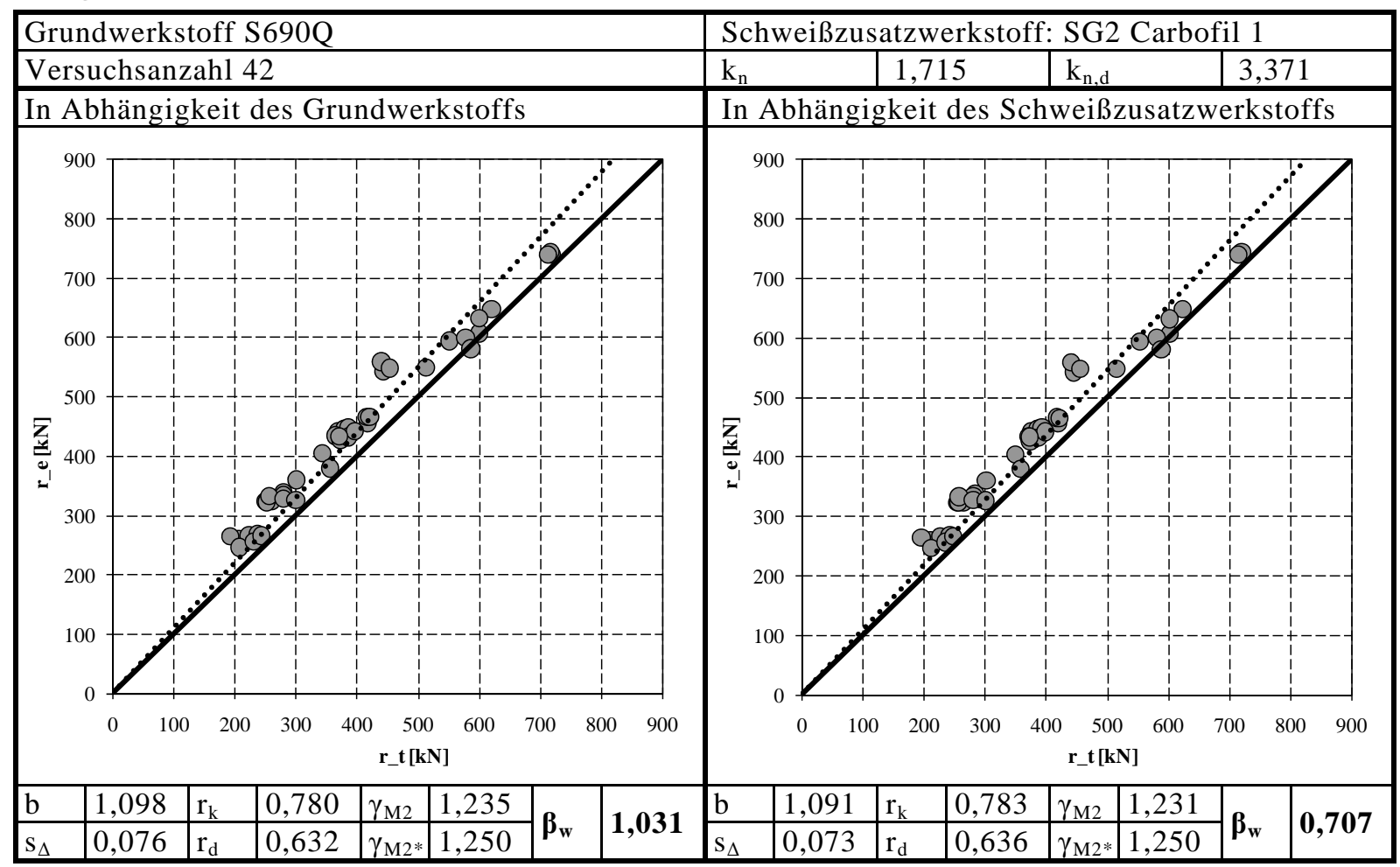




\section{A.1.3 Kehlnahtverbindungen höherfester Stähle HSF 640 mit höherfestem Schweißzusatzwerkstoff}

Im Folgenden werden die Versuchsergebnisse nach [Niemi, 1988] vorgestellt. Die mechanischen Eigenschaften der verwendeten Grundwerkstoffe sind in Tabelle A.14 zusammengestellt. Über die Vorwärmtemperatur oder andere Schweißparameter werden in [Niemi, 1988] keine Angaben gemacht.

Tabelle A.14: Mechanische Eigenschaften der verwendeten Grundwerkstoffe nach [Niemi, 1988]

\begin{tabular}{|c|c|c|c|c|c|c|c|c|}
\hline \multicolumn{9}{|c|}{ Werkstoff HSF 640 Blechdicke 8 mm } \\
\hline Blech-Nr. & 1 & 2 & 3 & 4 & 5 & 6 & 7 & 8 \\
\hline $\mathrm{R}_{\mathrm{eH}}\left[\mathrm{N} / \mathrm{mm}^{2}\right]$ & 643 & 643 & 660 & 641 & 633 & 639 & 618 & 631 \\
\hline $\mathrm{R}_{\mathrm{m}}\left[\mathrm{N} / \mathrm{mm}^{2}\right]$ & 748 & 746 & 759 & 744 & 746 & 744 & 746 & 738 \\
\hline $\mathrm{A}_{5}[\%]$ & 25 & 27 & 23 & 25 & 25 & 25 & 25 & 26 \\
\hline
\end{tabular}

Bei den Flankenkehlnähten wurden als Versuchsergebnisse die maximale Kraft während des Versuches und die nach dem Versuch ausgemessene Bruchfläche angegeben. Die Spannung $\tau_{\mathrm{u}}$ ergibt sich als Quotient von maximaler Kraft und Bruchfläche. Über den Bruchwinkel wurde nichts angegeben.

Die Versuchsergebnisse an Flankenkehlnähten sind in Tabelle A.15 aufgeführt. Die angegebenen Blechnummern beziehen sich jeweils auf das obere und untere Blech. 14 Versuche mit Flankenkehlnähten wurden getrennt nach Schweißzusatzwerkstoff statistisch ausgewertet, vgl. Tabelle A.16 und Tabelle A.17.

Tabelle A.15: Versuchsergebnisse der Flankenkehlnahtverbindungen nach [Niemi, 1988]

\begin{tabular}{|c|c|c|c|c|c|c|c|c|c|c|c|}
\hline & & & & \multicolumn{3}{|c|}{ Grundwerkstoff } & \multicolumn{5}{|c|}{ HSF 640, vgl. Tabelle A.14 } \\
\hline & & & & \multicolumn{3}{|c|}{$\begin{array}{l}\text { Schweißzusatz- } \\
\text { werkstoffe und } \\
\text { Schweißprozesse }\end{array}$} & \multicolumn{5}{|c|}{$\begin{array}{l}\text { MAG-Schweißen: OK Autrod } 13.12 \\
\mathrm{f}_{\mathrm{y}}=767 \mathrm{~N} / \mathrm{mm}^{2}, \mathrm{f}_{\mathrm{u}}=849 \mathrm{~N} / \mathrm{mm}^{2} \\
\text { E-Hand-Schweißen: } \\
\text { OK } 74.78 \mathrm{f}_{\mathrm{y}}=637 \mathrm{~N} / \mathrm{mm}^{2}, \mathrm{f}_{\mathrm{u}}=739 \mathrm{~N} / \mathrm{mm}^{2}\end{array}$} \\
\hline & & & & \multicolumn{3}{|c|}{ Nahtdicke } & \multicolumn{5}{|c|}{$3 \mathrm{~mm}$} \\
\hline & & & & \multicolumn{3}{|c|}{ Nahtlänge l } & \multicolumn{5}{|c|}{$50,100,150,200,300,400 \mathrm{~mm}$} \\
\hline $\begin{array}{l}\text { Ver- } \\
\text { suchs- } \\
\text { körper }\end{array}$ & $\begin{array}{l}\text { Blech } \\
\text { Nr. }\end{array}$ & $\begin{array}{l}\text { Max. } \\
\text { Kraft } \\
{[\mathrm{kN}]}\end{array}$ & \multicolumn{2}{|c|}{$\begin{array}{l}\text { Bruchfläche } \\
{\left[\mathrm{mm}^{2}\right]}\end{array}$} & $\begin{array}{l}\tau_{\mathrm{u}} \\
{\left[\mathrm{N} / \mathrm{mm}^{2}\right]}\end{array}$ & & & $\begin{array}{l}\text { Blech } \\
\text { Nr. }\end{array}$ & $\begin{array}{l}\text { Max. } \\
\text { Kraft } \\
{[\mathrm{kN}]}\end{array}$ & $\begin{array}{l}\text { Bruchfläche } \\
{\left[\mathrm{mm}^{2}\right]}\end{array}$ & $\begin{array}{l}\tau_{\mathrm{u}} \\
{\left[\mathrm{N} / \mathrm{mm}^{2}\right]}\end{array}$ \\
\hline A1-a & $5 / 3$ & 248 & \multicolumn{2}{|l|}{412} & 602 & & & $5 / 3$ & 178 & 371 & 480 \\
\hline A2-a & $5 / 3$ & 486 & \multicolumn{2}{|l|}{832} & 584 & & & $6 / 3$ & 360 & 768 & 469 \\
\hline A3-a & $5 / 4$ & 723 & \multicolumn{2}{|l|}{1250} & 578 & & & $4 / 4$ & 617 & 1234 & 500 \\
\hline A4-a & $6 / 4$ & 907 & \multicolumn{2}{|l|}{1587} & 572 & & & $4 / 4$ & 809 & 1634 & 495 \\
\hline A5-a & $8 / 8$ & 1350 & \multicolumn{2}{|l|}{2815} & 480 & & & $8 / 8$ & 980 & 1946 & 504 \\
\hline A6-a1 & $8 / 8$ & 1650 & \multicolumn{2}{|l|}{3364} & 490 & & b1 & $8 / 8$ & 1350 & 2729 & 495 \\
\hline A6-a2 & $7 / 7$ & 1444 & \multicolumn{2}{|l|}{2651} & 545 & & $\mathrm{~b} 2$ & $7 / 7$ & 1324 & 2561 & 517 \\
\hline \multicolumn{6}{|c|}{ SZW: $\mathrm{f}_{\mathrm{y}}=767 \mathrm{~N} / \mathrm{mm}^{2} \mathrm{f}_{\mathrm{u}}=849 \mathrm{~N} / \mathrm{mm}^{2}$} & \multicolumn{6}{|c|}{$\mathrm{SZW}: \mathrm{f}_{\mathrm{v}}=637 \mathrm{~N} / \mathrm{mm}^{2} \mathrm{f}_{\mathrm{u}}=739 \mathrm{~N} / \mathrm{mm}^{2}$} \\
\hline
\end{tabular}


Tabelle A.16: Statistische Auswertung der Flankenkehlnahtverbindungen mit Überfestigkeit bezogen auf die Festigkeit des Grundwerkstoffs (links) oder des Schweißzusatzwerkstoffs (rechts)

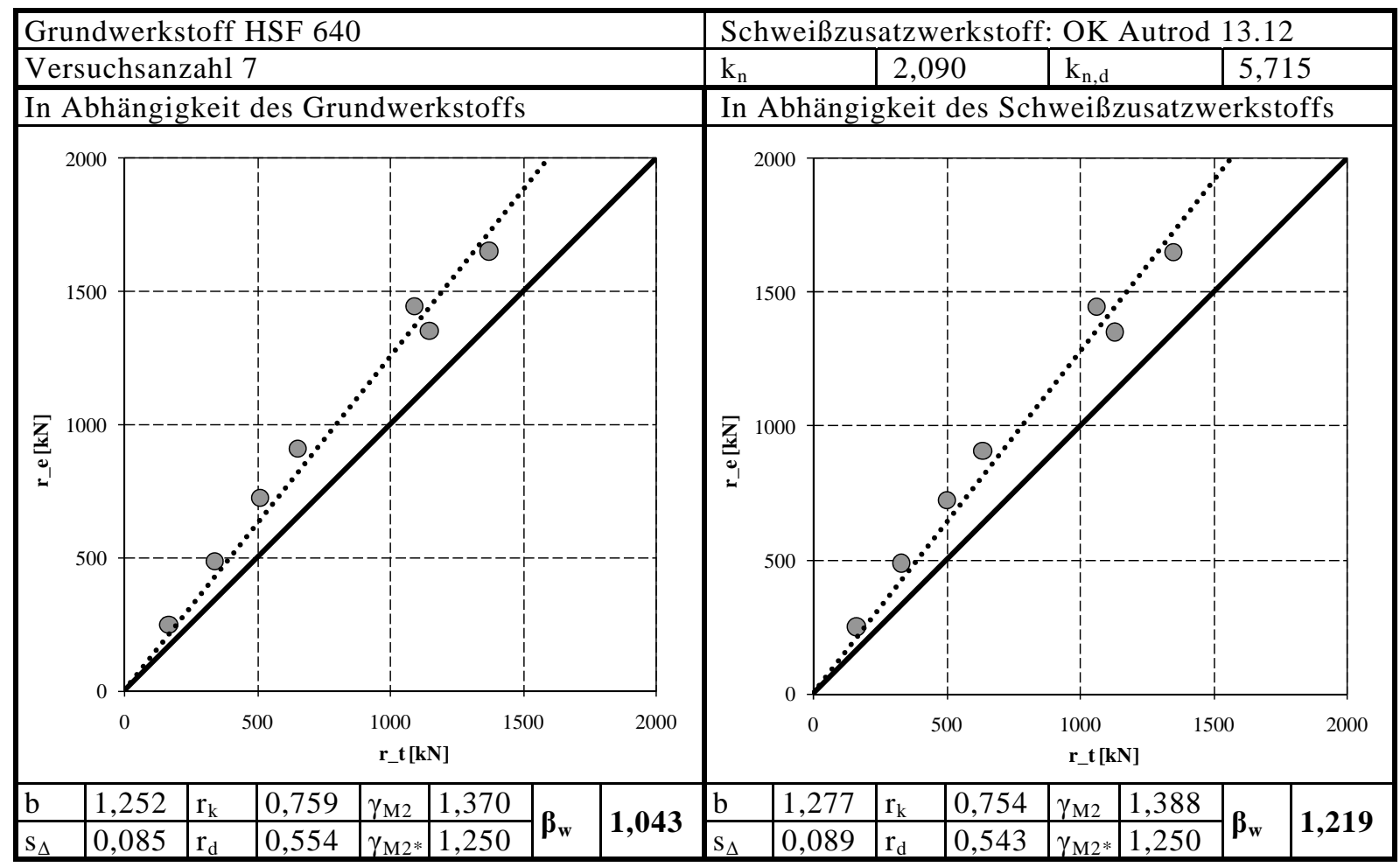

Tabelle A.17: Statistische Auswertung der Flankenkehlnahtverbindungen mit zugehörigem Schweißzusatzwerkstoff bezogen auf die Festigkeit des Grundwerkstoffs (links) oder des Schweißzusatzwerkstoffs (rechts)

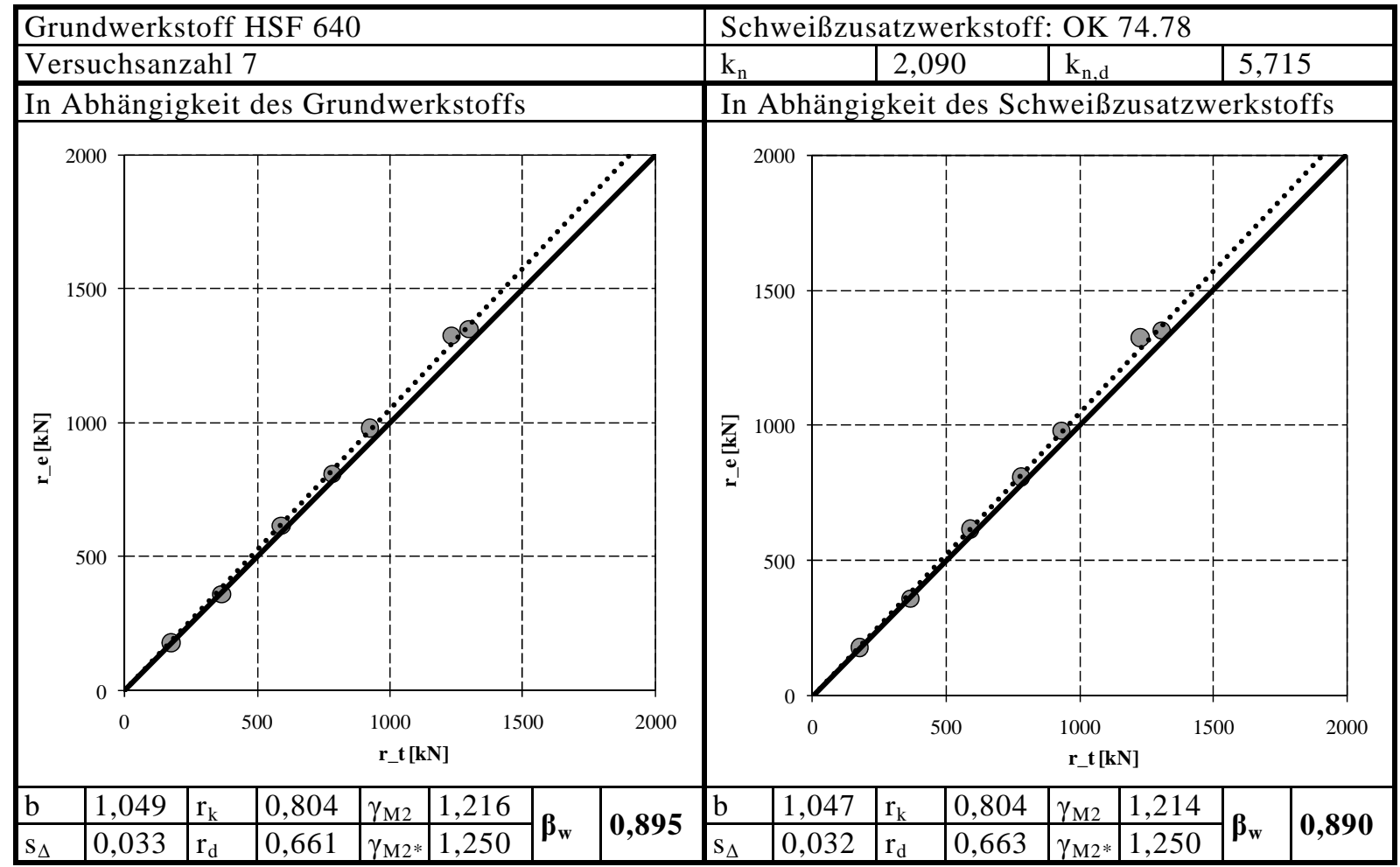


Die Versuchsergebnisse von 12 Stirnkehlnahtverbindungen sind in Tabelle A.18 zusammengestellt. Die Bruchfläche wurde nach dem Versuch ausgemessen. Der Bruchwinkel ist mit $15^{\circ}$ angegeben. Die angegebenen Spannungen ergeben sich aus der maximalen Kraft im Versuch, dividiert durch die nach dem Bruch ermittelte Bruchfläche. Die Versuche wurden getrennt nach Schweißzusatzwerkstoff statistisch ausgewertet, vgl. Tabelle A.19 und Tabelle A.20.

Tabelle A.18: Versuchsergebnisse der Stirnkehlnahtverbindungen nach [Niemi, 1988]

\begin{tabular}{|c|c|c|c|c|c|c|c|c|c|}
\hline \multirow{4}{*}{$\triangleleft$} & & \multirow{4}{*}{$\rightarrow$} & \multicolumn{3}{|c|}{ Grundwerkstoff } & \multicolumn{4}{|c|}{ HSF 640,vgl. Tabelle A.14 } \\
\hline & & & \multicolumn{3}{|c|}{$\begin{array}{l}\text { Schweißzusatzwerkstoffe } \\
\text { und Schweißprozesse }\end{array}$} & \multicolumn{4}{|c|}{$\begin{array}{l}\text { MAG-Schweißen: OK Autrod } 13.12 \\
\mathrm{f}_{\mathrm{y}}=767 \mathrm{~N} / \mathrm{mm}^{2}, \mathrm{f}_{\mathrm{u}}=849 \mathrm{~N} / \mathrm{mm}^{2} \\
\text { E-Hand-Schweißen: OK } 74.78 \\
\mathrm{f}_{\mathrm{y}}=637 \mathrm{~N} / \mathrm{mm}^{2}, \mathrm{f}_{\mathrm{u}}=739 \mathrm{~N} / \mathrm{mm}^{2}\end{array}$} \\
\hline & & & \multicolumn{3}{|c|}{ Nahtdicke } & \multicolumn{4}{|l|}{$3 \mathrm{~mm}$} \\
\hline & & & \multicolumn{3}{|c|}{ Nahtlänge b } & \multicolumn{4}{|c|}{$50,75,100,125,150,200 \mathrm{~mm}$} \\
\hline $\begin{array}{l}\text { Ver- } \\
\text { suchskör } \\
\text { per }\end{array}$ & $\begin{array}{l}\text { Blech } \\
\text { Nr. }\end{array}$ & $\begin{array}{l}\max . \\
\text { Kraft } \\
{[\mathrm{kN}]}\end{array}$ & $\begin{array}{l}\text { Bruch- } \\
\text { fläche } \\
\mathrm{A}_{\mathrm{B}} \\
{\left[\mathrm{mm}^{2}\right]}\end{array}$ & $\begin{array}{l}\text { Max. } \\
\text { Span- } \\
\text { nung } \\
\mathrm{F}_{\mathrm{u}} / \mathrm{A}_{\mathrm{Bruch}} \\
{\left[\mathrm{N} / \mathrm{mm}^{2}\right]}\end{array}$ & $\begin{array}{l}\text { Ver- } \\
\text { suchskör } \\
\text { per }\end{array}$ & $\begin{array}{l}\text { Blech } \\
\text { Nr. }\end{array}$ & $\begin{array}{l}\text { Max. } \\
\text { Kraft } \\
{[\mathrm{kN}]}\end{array}$ & $\begin{array}{l}\text { Bruch- } \\
\text { fläche } \\
\mathrm{A}_{\mathrm{B}} \\
{\left[\mathrm{mm}^{2}\right]}\end{array}$ & $\begin{array}{l}\text { Max. } \\
\text { Span- } \\
\text { nung } \\
\mathrm{F}_{\mathrm{u}} / \mathrm{A}_{\mathrm{Bruch}} \\
{\left[\mathrm{N} / \mathrm{mm}^{2}\right]}\end{array}$ \\
\hline$\overline{\mathrm{B} 1-\mathrm{a}}$ & $5 / 3$ & 158 & 247 & 640 & B1-b & $5 / 3$ & 137 & 224 & 612 \\
\hline B2-a & $5 / 2$ & 234 & 357 & 655 & B2-b & $4 / 2$ & 226 & 373 & 606 \\
\hline B3-a & $5 / 2$ & 320 & 488 & 656 & B3-b & $5 / 2$ & 312 & 584 & 534 \\
\hline B4-a & $6 / 2$ & 342 & 579 & 591 & B4-b & $6 / 2$ & 399 & 593 & 673 \\
\hline B5-a & $6 / 2$ & 418 & 707 & 591 & B5-b & $4 / 2$ & 487 & 772 & 631 \\
\hline $\mathrm{B} 6-\mathrm{a}$ & $4 / 2$ & 643 & 942 & 683 & B6-b & $4 / 2$ & 701 & 1070 & 655 \\
\hline \multicolumn{5}{|c|}{ SZW: $\mathrm{f}_{\mathrm{y}}=767 \mathrm{~N} / \mathrm{mm}^{2} \mathrm{f}_{\mathrm{u}}=849 \mathrm{~N} / \mathrm{mm}^{2}$} & \multicolumn{5}{|c|}{ SZW: $\mathrm{f}_{\mathrm{v}}=637 \mathrm{~N} / \mathrm{mm}^{2} \mathrm{f}_{\mathrm{u}}=739 \mathrm{~N} / \mathrm{mm}^{2}$} \\
\hline
\end{tabular}

Tabelle A.19: Statistische Auswertung der Stirnkehlnahtverbindungen mit Überfestigkeit bezogen auf die Festigkeit des Grundwerkstoffs (links) oder des Schweißzusatzwerkstoffs (rechts)

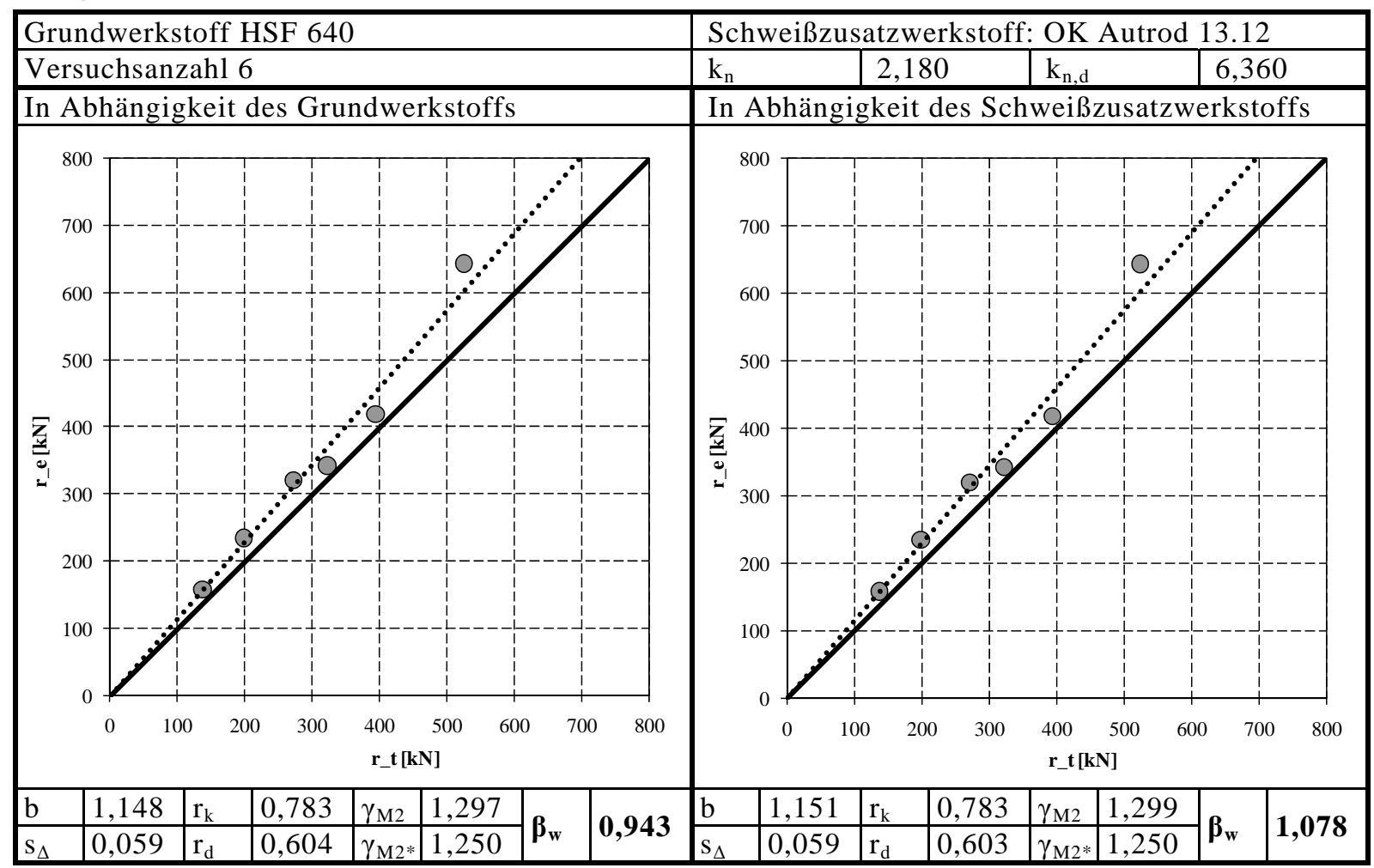


Tabelle A.20: Statistische Auswertung der Stirnkehlnahtverbindungen mit zugehörigem Schweißzusatzwerkstoff bezogen auf die Festigkeit des Grundwerkstoffs (links) oder des Schweißzusatzwerkstoffs (rechts)

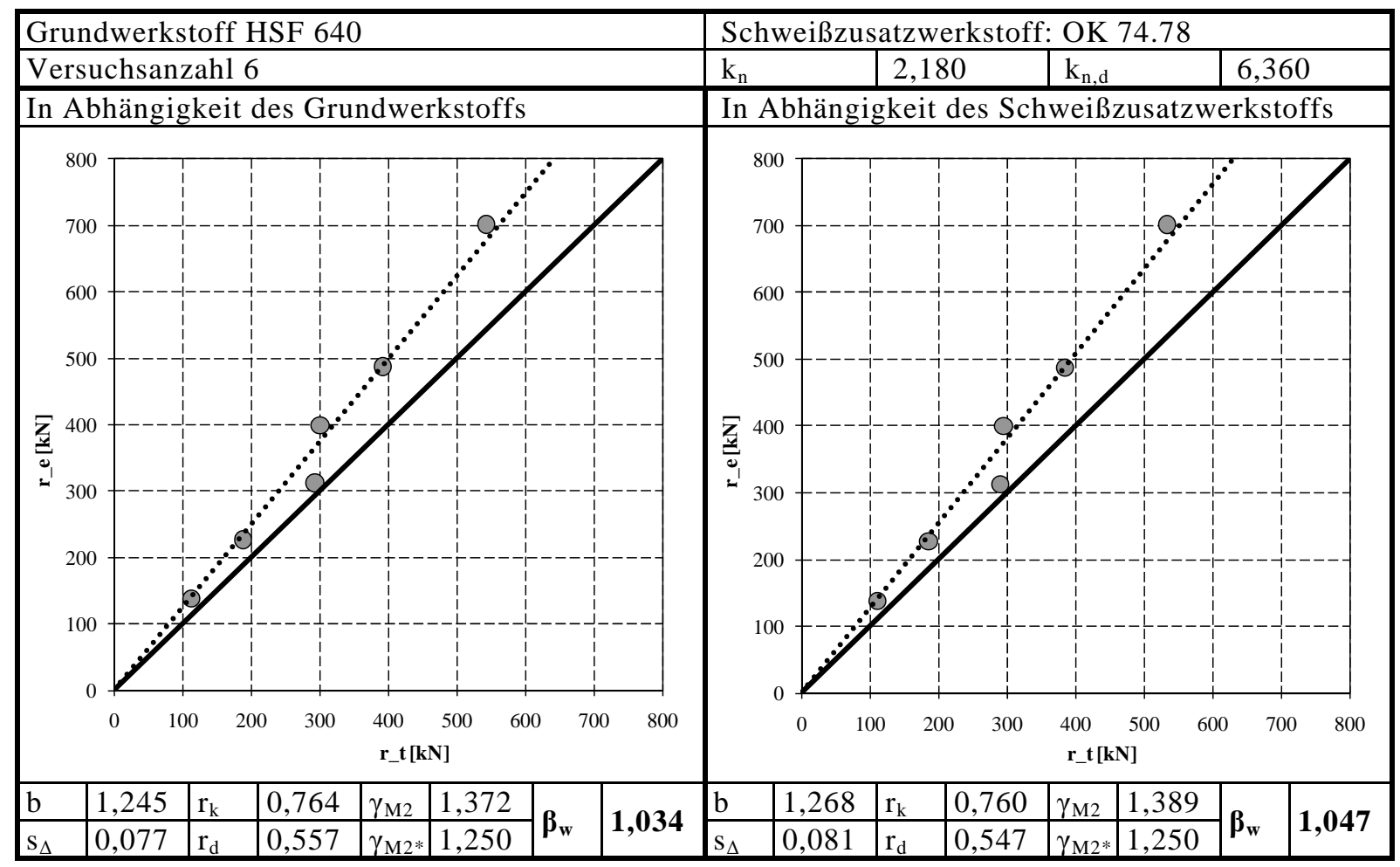




\section{A.1.4 Kehlnahtverbindungen S460 mit zugehörigem Schweißzusatzwerkstoff}

Im Folgenden werden die Versuchsergebnisse nach [Saal, 2005] vorgestellt.

Tabelle A.21 fasst die verwendeten Grundwerkstoffe zusammen. Alle betrachteten Versuche wurden mit demselben zugehörigen Schweißzusatzwerkstoff T46 geschweißt, dessen Festigkeit teilweise etwas niedriger als die der verwendeten Grundwerkstoffe lag. Alle Versuchskörper wurden ohne Vorwärmen geschweißt, Versuch S8B bildet eine Ausnahme, der auf $150^{\circ} \mathrm{C}$ vorgewärmt wurde.

Tabelle A.21: Mechanische Eigenschaften der verwendeten Grundwerkstoffe nach [Saal, 2005]

\begin{tabular}{|l|l|l|l|l|l|}
\hline \multirow{2}{*}{ Werkstoff } & $\begin{array}{l}\text { Blechdicke } \\
{[\mathrm{mm}]}\end{array}$ & \multicolumn{4}{|l|}{ Mechanische Eigenschaften } \\
\cline { 3 - 6 } & $\mathrm{Nr}$. & $\mathrm{R}_{\mathrm{eH}}\left[\mathrm{N} / \mathrm{mm}^{2}\right]$ & $\mathrm{R}_{\mathrm{m}}\left[\mathrm{N} / \mathrm{mm}^{2}\right]$ & $\mathrm{A}_{5}[\%]$ \\
\hline Histar 460L HD 400 x 237 & 30,2 & 1 & 538 & 633 & 22,0 \\
\hline Histar 460L HD 400 x 421 & 52,6 & 2 & 515 & 620 & 21,0 \\
\hline Histar 460L HD 360 x 147 & 19,8 & 3 & 538 & 614 & 23,6 \\
\hline HL 1100A & 31 & 4 & 559 & 671 & 22,0 \\
\hline W14 x 426 & 77,1 & 5 & 514 & 652 & 21,3 \\
\hline
\end{tabular}

Bei den Flankenkehlnähten wurden als Versuchsergebnisse die maximale Kraft während des Versuches und die nach dem Versuch ausgemessene Bruchfläche angegeben. Daraus ergibt sich als Quotient von Kraft und Bruchfläche die maximale Spannung $\tau_{\text {III }}$. Alle Versuchskörper haben zwei Nähte, als Bruchfläche wurde die Bruchfläche der kleineren von beiden Nähten doppelt verwendet. Für die Flankenkehlnähte sind in Tabelle A.22 die Versuchsergebnisse S460-T46 zusammengefasst. Tabelle A.23 zeigt die statistische Auswertung dieser Versuche.

Tabelle A.22: Versuchsergebnisse der Flankenkehlnahtverbindungen nach [Saal, 2005]

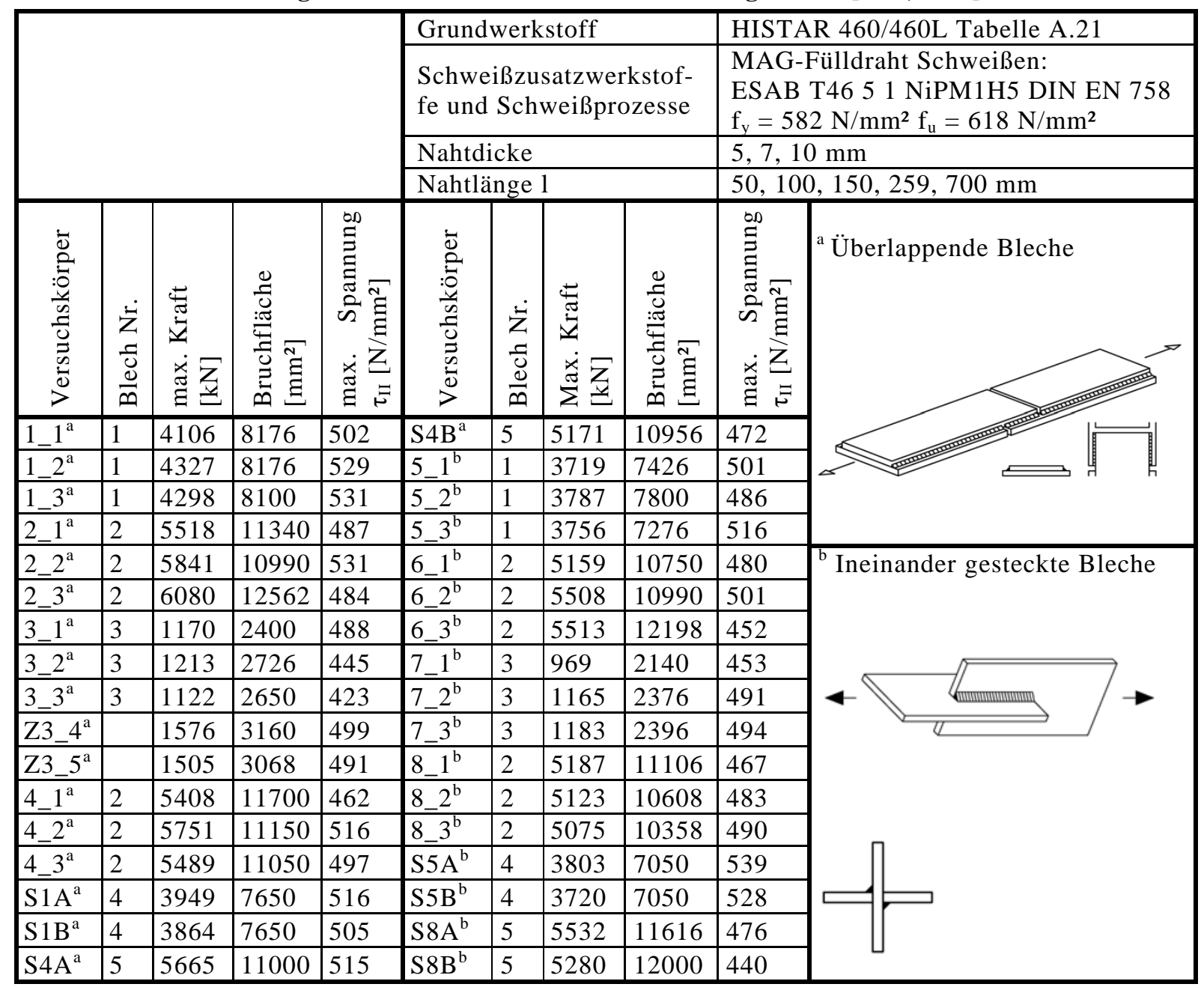


Tabelle A.23: Statistische Auswertung der Flankenkehlnahtverbindungen bezogen auf die Festigkeit des Grundwerkstoffs (links) oder des Schweißzusatzwerkstoffs (rechts)

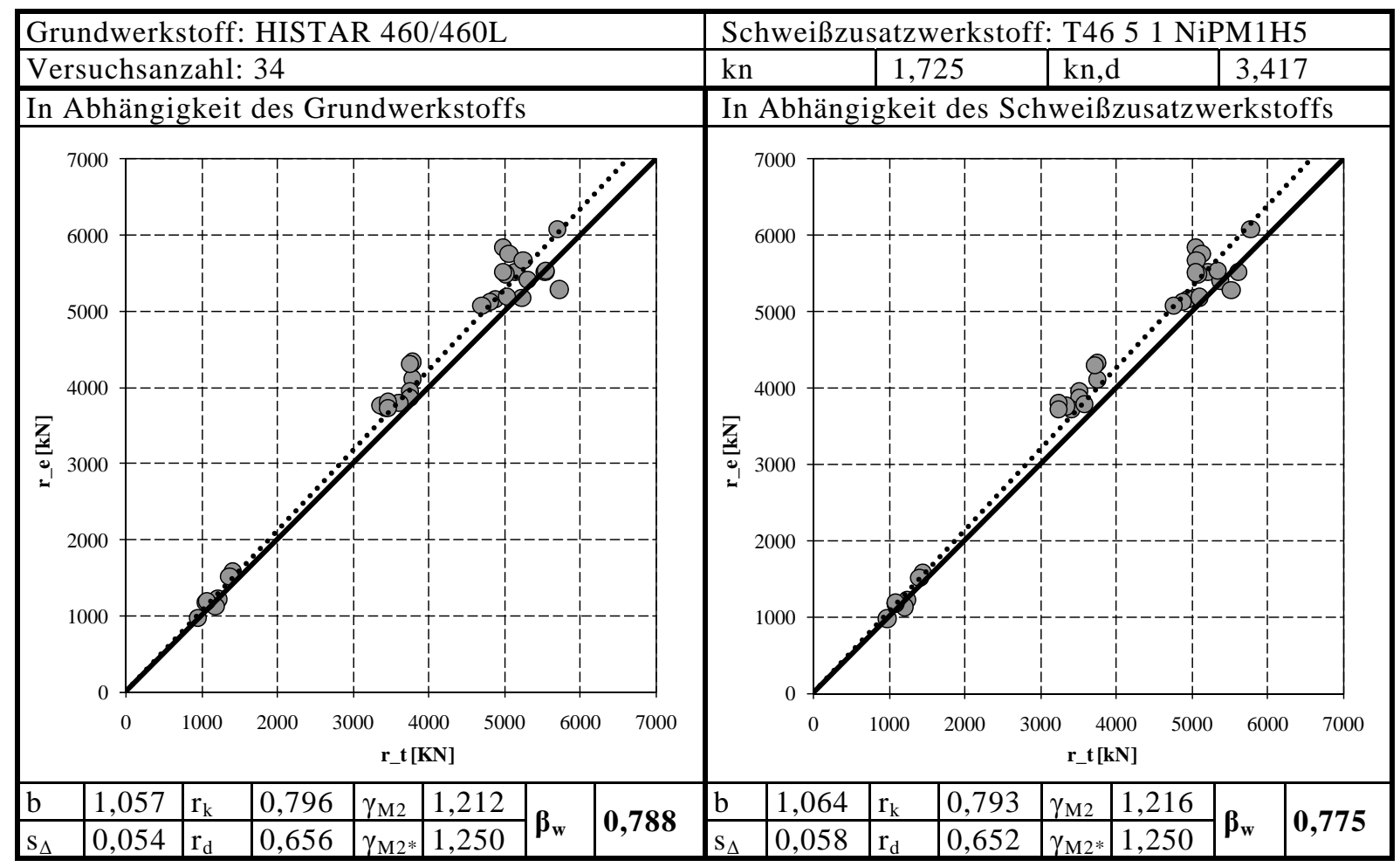




\section{A.1.5 Kehlnahtverbindungen höherfester Stähle S460 mit höherfestem Schweiß- zusatzwerkstoff}

Im Folgenden werden die Versuchsergebnisse nach [Background D.03, 1990] vorgestellt. Die verwendeten Grundwerkstoffe sind in Tabelle A.24 zusammengefasst. Informationen über Schweißparameter sind nicht angegeben.

Tabelle A.24: Mechanische Eigenschaften der verwendeten Grundwerkstoffe nach [Background D.03, 1990]

\begin{tabular}{|l|l|l|l|l|}
\hline Werkstoff & 1 & 2 & 3 & 4 \\
\hline Blechdicke [mm] & 28 & 10 & 20 & 40 \\
\hline Mechanische Eigenschaften $\mathrm{R}_{\mathrm{m}}\left[\mathrm{N} / \mathrm{mm}^{2}\right]$ & 702 & 613 & 715 & 725 \\
\hline
\end{tabular}

Im Folgenden werden 13 Versuchsergebnisse an Flankenkehlnähten S460-E55 beschrieben, vgl. Tabelle A.25. Es handelt sich dabei um zwei verschiedene Überlappverbindungen. Die Bruchflächen wurden aus den angegebenen Werten über die Zugfestigkeit des Grundwerkstoffs zurückgerechnet. 13 Versuche mit Flankenkehlnähten wurden statistisch ausgewertet, wie in Tabelle A.26 zusammengefasst.

Tabelle A.25: Versuchsergebnisse Flankenkehlnahtverbindungen nach [Background D.03, 1990]

\begin{tabular}{|c|c|c|c|c|c|c|c|c|c|}
\hline Grund & $\overline{\mathrm{ksto}}$ & & & & $\overline{F e E ~} 4$ & vgl. & $\overline{\text { Tabelle }}$ & & \\
\hline Schwe & dsat & toffe u & Schwei & ozesse & Conar & $G$ & $=550$ & $m^{2} f_{u}=$ & $0 \mathrm{~N} / \mathrm{mm}^{2}$ \\
\hline Nahtdi & & & & & Nicht & ege & & & \\
\hline Nahtlä & & & & & Nicht & ege & & & \\
\hline 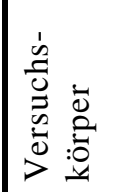 & $\begin{array}{l}\vec{z} \\
\bar{v} \\
\frac{d}{n}\end{array}$ & 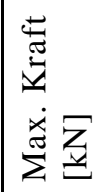 & 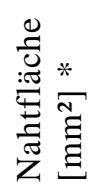 & 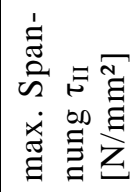 & 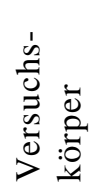 & 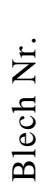 & 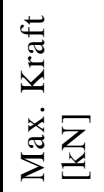 & 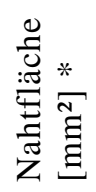 & 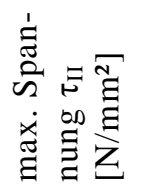 \\
\hline $2-1^{\text {a) }}$ & 1 & 828 & 1650 & 502 & $5-1^{b)}$ & $\overline{2}$ & 342 & 642 & 533 \\
\hline $2-2^{\text {a) }}$ & 1 & 560 & 1101 & 509 & $5-2^{b)}$ & 2 & 364 & 685 & 531 \\
\hline $2-9^{\text {a) }}$ & 2 & 357 & 770 & 463 & $5-3^{b)}$ & 3 & 876 & 1738 & 504 \\
\hline $2-10^{\text {a) }}$ & 2 & 365 & 799 & 457 & $5-4^{b)}$ & 3 & 979 & 2161 & 453 \\
\hline $6-1^{\text {a) }}$ & 1 & 652 & 1552 & 420 & $5-5^{b)}$ & 1 & 1298 & 2370 & 548 \\
\hline $6-5^{\text {a) }}$ & 2 & 1924 & 3510 & 548 & $5-6^{b)}$ & 1 & 1669 & 3244 & 515 \\
\hline & & & & & $5-7^{b)}$ & 4 & 1736 & 3725 & 466 \\
\hline
\end{tabular}


Tabelle A.26: Statistische Auswertung Flankenkehlnahtverbindungen bezogen auf die Festigkeit des Grundwerkstoffs (links) oder des Schweißzusatzwerkstoffs (rechts)

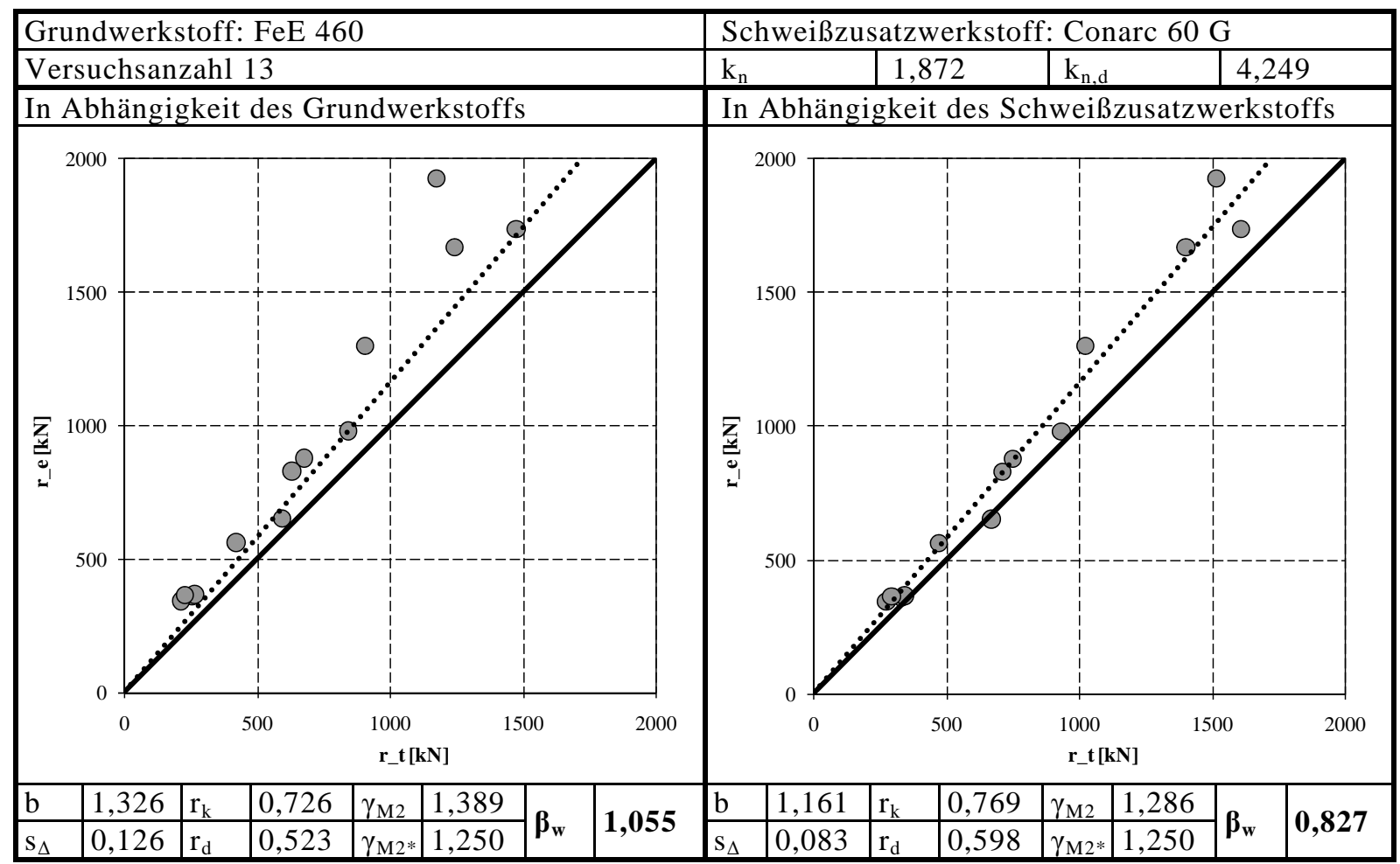

Die Versuchsergebnisse von 16 Kreuzstößen sind in Tabelle A.27 zusammengestellt. Die Bruchflächen wurden aus den angegebenen Werten zurückgerechnet. Die statistische Auswertung ist in Tabelle A.28 gegeben.

Tabelle A.27: Versuchsergebnisse Stirnkehlnahtverbindungen nach [Background D.03, 1990]

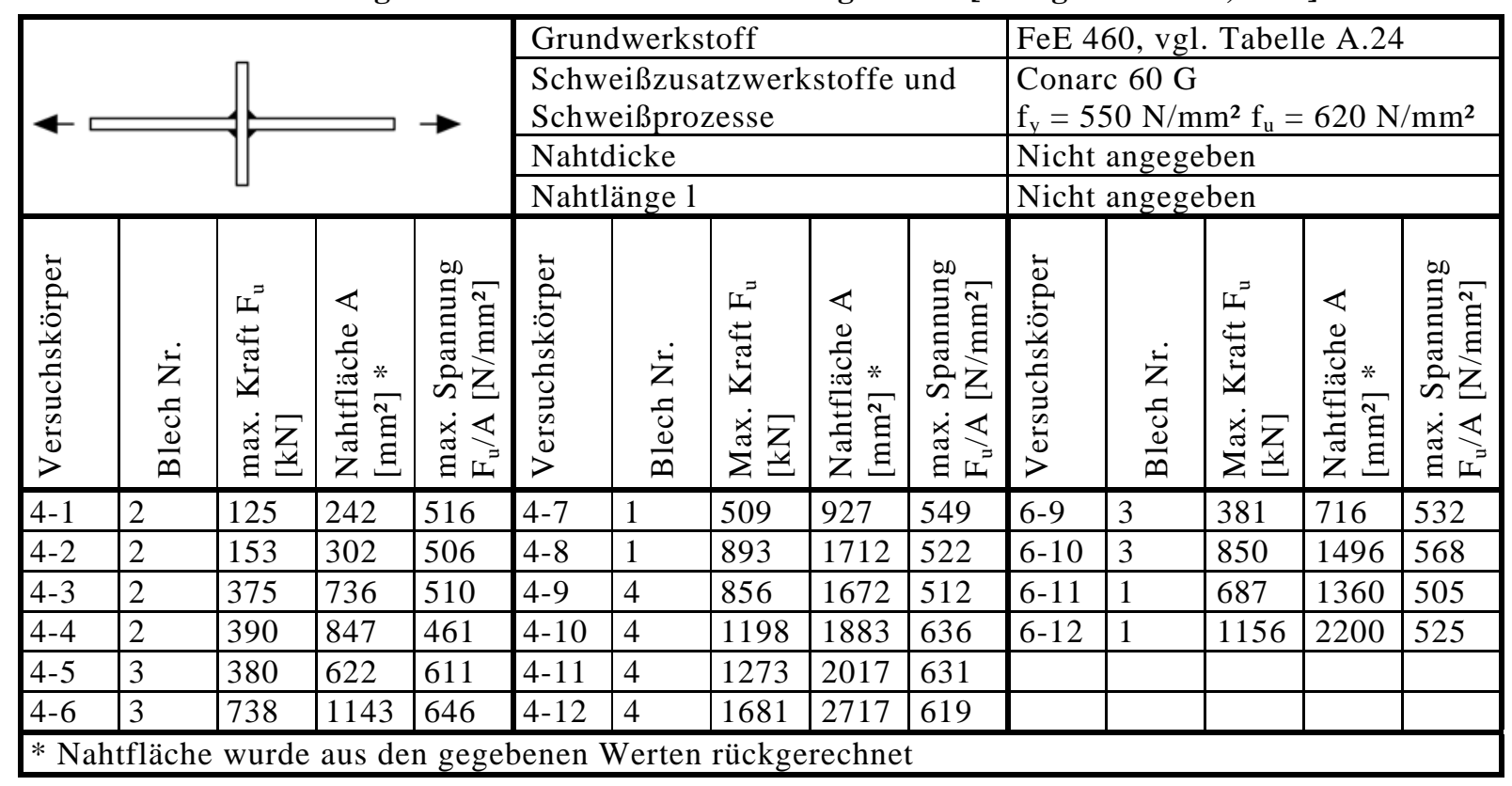


Tabelle A.28: Statistische Auswertung der Stirnkehlnahtverbindungen bezogen auf die Festigkeit des Grundwerkstoffs (links) oder des Schweißzusatzwerkstoffs (rechts)

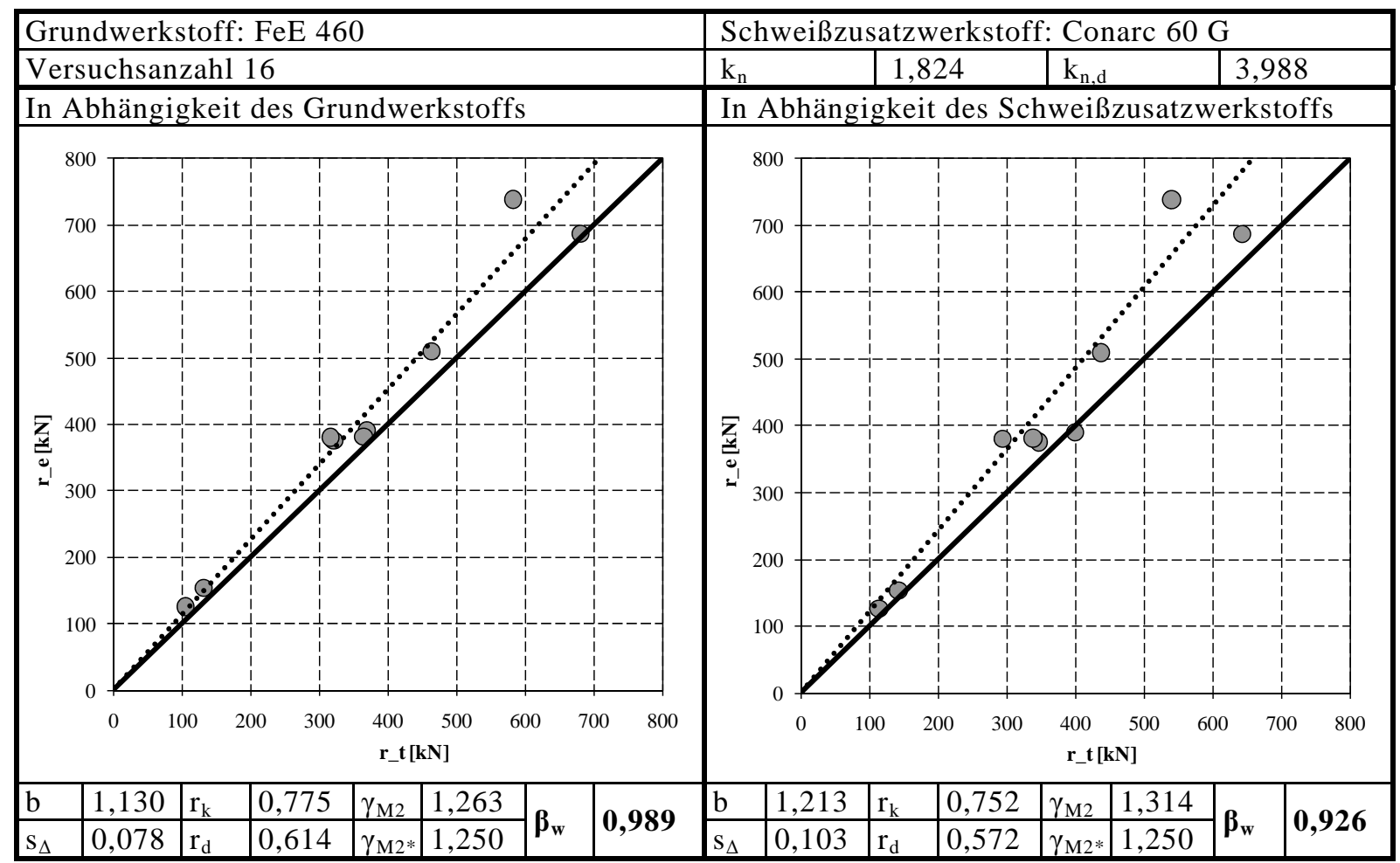




\section{A.1.6 Kehlnahtverbindungen höherfester Stähle S690 und niederfestem Schweißzusatzwerkstoff}

In diesem Abschnitt werden die Versuchsergebnisse nach [Collin, Johansson, 2005] vorgestellt. Diese Versuche wurden statistisch neu ausgewertet. Die verwendeten Grundwerkstoffe sind in Tabelle A.29 aufgeführt.

Tabelle A.29: Mechanische Eigenschaften der verwendeten Grundwerkstoffe nach [Collin, Johansson, 2005]

\begin{tabular}{|l|l|l|l|l|l|}
\hline Werkstoff & Blechdicke & \multicolumn{4}{|l|}{ Mechanische Eigenschaften } \\
\cline { 3 - 6 } & {$[\mathrm{mm}]$} & $\mathrm{Nr}$. & $\mathrm{R}_{\mathrm{eH}}\left[\mathrm{N} / \mathrm{mm}^{2}\right]$ & $\mathrm{R}_{\mathrm{m}}\left[\mathrm{N} / \mathrm{mm}^{2}\right]$ & $\mathrm{A}_{5}[\%]$ \\
\hline Weldox 700 E & 10 & 1 & 789 & 833 & 15 \\
\hline Domex 650 MC & 10 & 2 & 779 & 827 & 18 \\
\hline
\end{tabular}

Die vorliegenden 15 Versuchsergebnisse an Flankenkehlnähten sind in Tabelle A.30 zusammengestellt. Die Bruchfläche wurde nach dem Versuch ausgemessen. Da Schweißzusatzwerkstoffe unterschiedlicher Festigkeit verwendet wurden, erfolgt die statistische Auswertung getrennt, vgl. Tabelle A.31 und Tabelle A.32.

Tabelle A.30: Versuchsergebnisse nach [Collin, Johansson, 2005]

\begin{tabular}{|c|c|c|c|c|c|c|c|c|c|c|c|c|c|c|}
\hline & & & & & Grund & werl & kstoff & & $\begin{array}{l}\text { Welc } \\
\text { Tabe }\end{array}$ & $\begin{array}{l}\times 700 \\
\text { A. } 29\end{array}$ & $\mathrm{E}, \mathrm{Dc}$ & $\operatorname{mex} 6$ & $50 \mathrm{MC}$ & \\
\hline & & & & & $\begin{array}{l}\text { Schwe } \\
\text { stoffe } \\
\text { prozes }\end{array}$ & $\mathrm{u}$ & $\begin{array}{l}\text { satzw } \\
\text { d Sc }\end{array}$ & eiß- & $\begin{array}{l}\text { E-Hal } \\
\text { OK } 4 \\
\mathrm{f}_{\mathrm{y}}=4 \\
\text { OK } 7 \\
\mathrm{f}_{\mathrm{y}}=6 \\
\end{array}$ & $\begin{array}{l}\text { hd }(111 \\
8.00 \\
62 \mathrm{~N} / \mathrm{m} \\
5.75: \\
19 \mathrm{~N} / \mathrm{m}\end{array}$ & $\begin{array}{l}: \\
m^{2} f_{u} \\
m^{2} f_{u} \\
\end{array}$ & $\begin{array}{l}=548 \\
=758\end{array}$ & $\begin{array}{l}\mathrm{N} / \mathrm{mm} \\
\mathrm{N} / \mathrm{mm}\end{array}$ & \\
\hline & & & & & \begin{tabular}{|l|l} 
Nahtd \\
\end{tabular} & cke & & & $3 ; 6$ & & & & & \\
\hline & & & & & Nahtl: & nge & & & $50 ; 60$ & & & & & \\
\hline 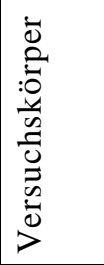 & $\begin{array}{l}\vec{z} \\
\bar{u} \\
\frac{\tilde{u}}{n}\end{array}$ & 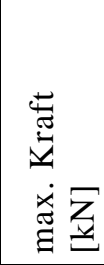 & 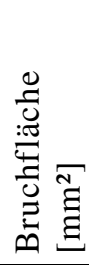 & 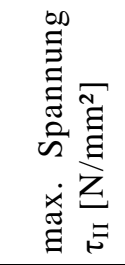 & 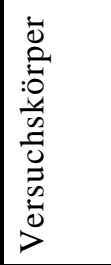 & $\begin{array}{l}\dot{z} \\
\overline{0} \\
\frac{0}{n} \\
\end{array}$ & 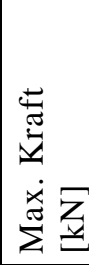 & 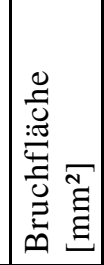 & 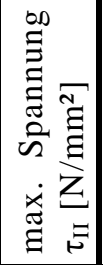 & 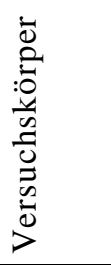 & $\begin{array}{l}\dot{z} \\
\bar{u} \\
\frac{0}{n}\end{array}$ & 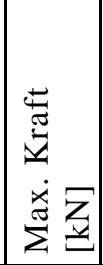 & 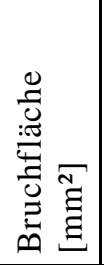 & 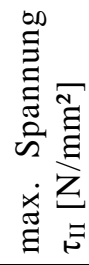 \\
\hline WL-1 & 1 & 472 & 890 & 531 & DL-1 & 2 & 333 & 701 & 476 & $\overline{\text { DL-6 }}$ & 2 & 573 & 1162 & 493 \\
\hline WL-2 & 1 & 465 & 925 & 503 & DL-2 & 2 & 343 & 730 & 469 & DL-7 & 2 & 581 & 1219 & 477 \\
\hline WL-3 & 1 & 461 & 862 & 535 & DL-3 & 2 & 338 & 732 & 462 & DL-8 & 2 & 525 & 1086 & 483 \\
\hline WL-4 & 1 & 433 & 877 & 494 & DL-4 & 2 & 318 & 749 & 425 & DL-9 & 2 & 571 & 1236 & 462 \\
\hline WL-5 & 1 & 453 & 886 & 511 & DL-5 & 2 & 337 & 785 & 429 & DL-10 & 2 & 584 & 1264 & 462 \\
\hline & & & & & $\mathrm{ZW}$ & $1-$ & & 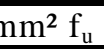 & 54 & $/ \mathrm{mm}^{2}$ & & & & \\
\hline
\end{tabular}


Tabelle A.31: Statistische Auswertung der Flankenkehlnahtverbindungen WL bezogen auf die Festigkeit des Grundwerkstoffs (links) oder des Schweißzusatzwerkstoffs (rechts)

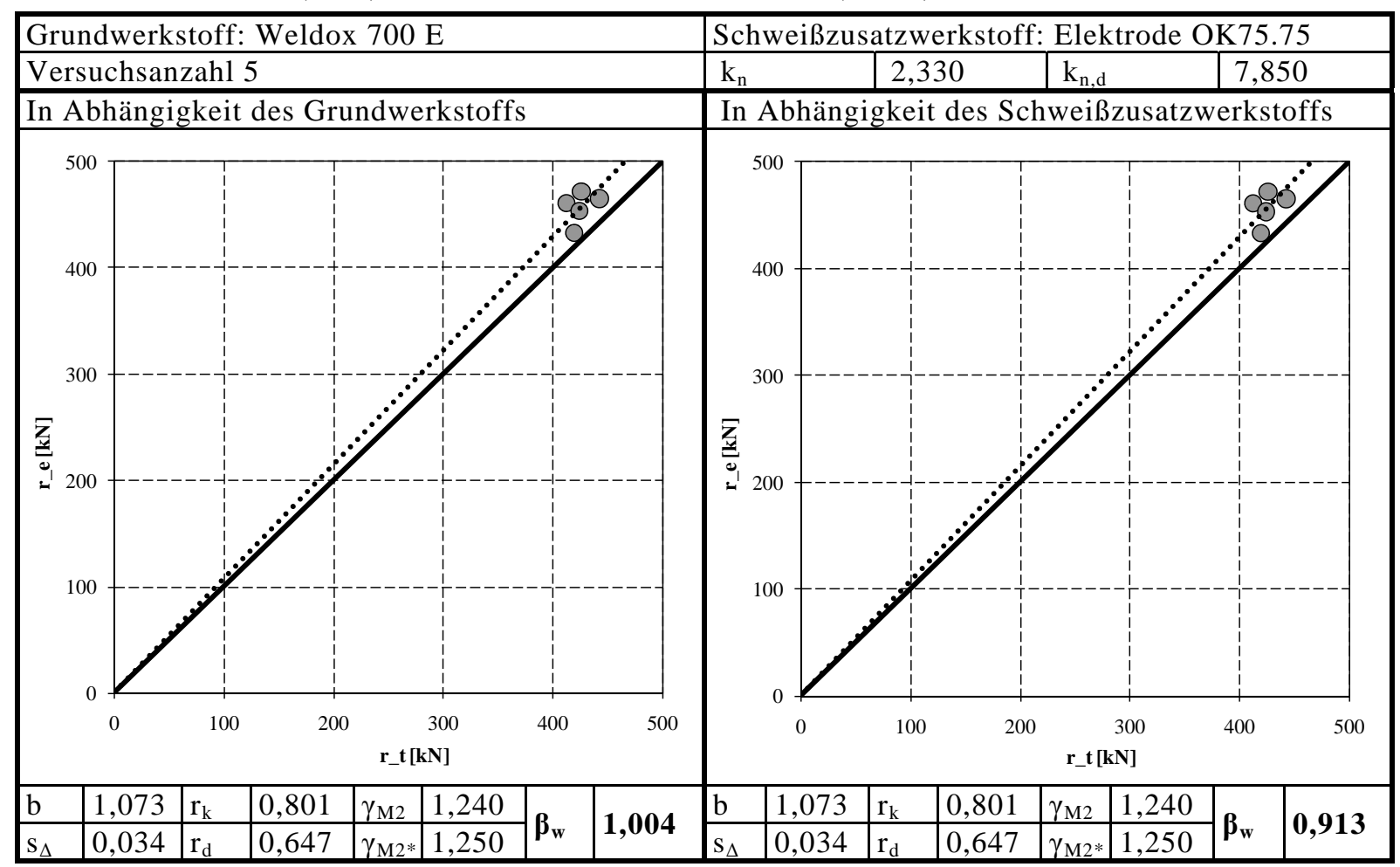

Tabelle A.32: Statistische Auswertung der Flankenkehlnahtverbindungen DL bezogen auf die Festigkeit des Grundwerkstoffs (links) oder des Schweißzusatzwerkstoffs (rechts)

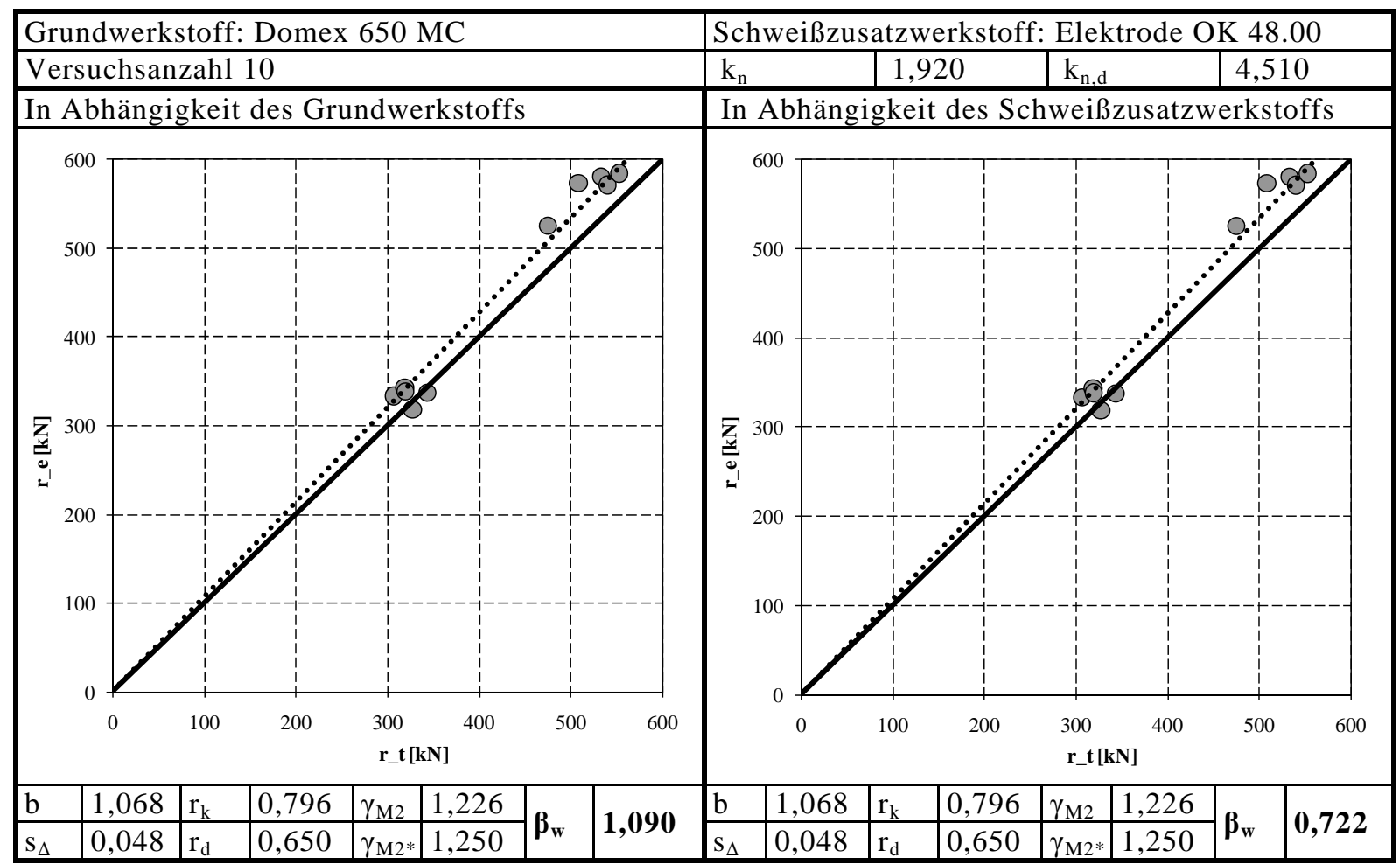


Es stehen 12 Versuchsergebnisse an Stirnkehlnähten mit überlappenden Blechen zur Verfügung (Tabelle A.33), die getrennt nach Schweißzusatzwerkstoff ausgewertet wurden (Tabelle A.34 und Tabelle A.35). Die Bruchfläche wurde nach dem Versuch ausgemessen.

Tabelle A.33: Versuchsergebnisse der Stirnkehlnahtverbindungen nach [Collin, Johansson, 2005]

\begin{tabular}{|c|c|c|c|c|c|c|c|c|c|c|c|c|c|c|}
\hline \multirow{4}{*}{$\triangleleft \overline{\text { 貝 }}$} & \multirow{2}{*}{$\overline{7}$} & \multirow{4}{*}{$\rightarrow$} & \multicolumn{4}{|c|}{ Grundwerkstoff } & \multicolumn{8}{|c|}{ Weldox 700 E, Domex 650 MC, Tabelle A.29 } \\
\hline & & & \multicolumn{4}{|c|}{$\begin{array}{l}\text { Schweißzusatzwerkstoffe } \\
\text { und Schweißprozesse }\end{array}$} & \multicolumn{8}{|c|}{$\begin{array}{l}\text { E-Hand }(111) \text { : } \\
\text { OK 48.00 } \mathrm{f}_{\mathrm{y}}=462 \mathrm{~N} / \mathrm{mm}^{2} \mathrm{f}_{\mathrm{u}}=548 \mathrm{~N} / \mathrm{mm}^{2} \\
\text { OK 75.75: } \mathrm{f}_{\mathrm{y}}=619 \mathrm{~N} / \mathrm{mm}^{2} \mathrm{f}_{\mathrm{u}}=758 \mathrm{~N} / \mathrm{mm}^{2}\end{array}$} \\
\hline & & & & tdicke & & & \multicolumn{8}{|c|}{$4 ; 6$} \\
\hline & & & \multicolumn{4}{|c|}{ Nahtlänge l } & \multicolumn{8}{|c|}{$50 ; 60$} \\
\hline 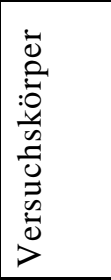 & 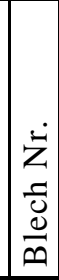 & 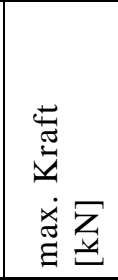 & 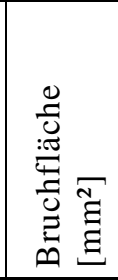 & 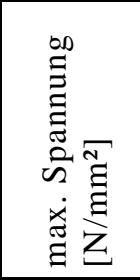 & 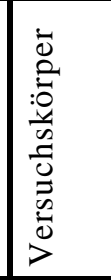 & $\begin{array}{l}\dot{z} \\
\tilde{u} \\
\stackrel{\Xi}{n} \\
\end{array}$ & 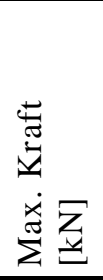 & 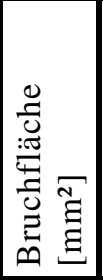 & 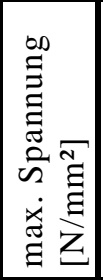 & 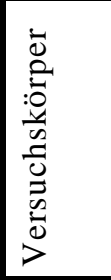 & $\left|\begin{array}{c}\dot{z} \\
\frac{c}{\tilde{U}} \\
\frac{\Theta}{n}\end{array}\right|$ & 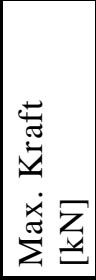 & 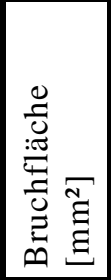 & 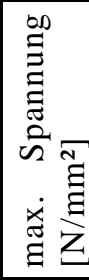 \\
\hline WT-1 & 1 & 575 & 617 & 932 & DT-1 & 2 & 470 & 548 & 857 & DT-6 & 2 & 593 & 719 & 825 \\
\hline WT-2 & 1 & 401 & 469 & 855 & DT-2 & 2 & 447 & 579 & 772 & DT-7 & 2 & 571 & 705 & 811 \\
\hline WT-3 & 1 & 445 & 450 & 988 & DT-3 & 2 & 407 & 500 & 814 & & & & & \\
\hline WT-4 & 1 & 431 & 429 & 1005 & DT-4 & 2 & 422 & 535 & 789 & & & & & \\
\hline WT-5 & 1 & 466 & 489 & 953 & DT-5 & 2 & 431 & 542 & 796 & & & & & \\
\hline $\begin{array}{l}\text { SZW: } \\
\text { N/mm² }\end{array}$ & & $\overline{519}$ & $\overline{\mathrm{mm}^{2}}$ & $=75$ & SZW & $=$ & 402 & $\mathrm{~m}^{2}$ & -52 & न & & & & \\
\hline
\end{tabular}

Tabelle A.34: Statistische Auswertung der Stirnkehlnahtverbindungen WT bezogen auf die Festigkeit des Grundwerkstoffs (links) oder des Schweißzusatzwerkstoffs (rechts)

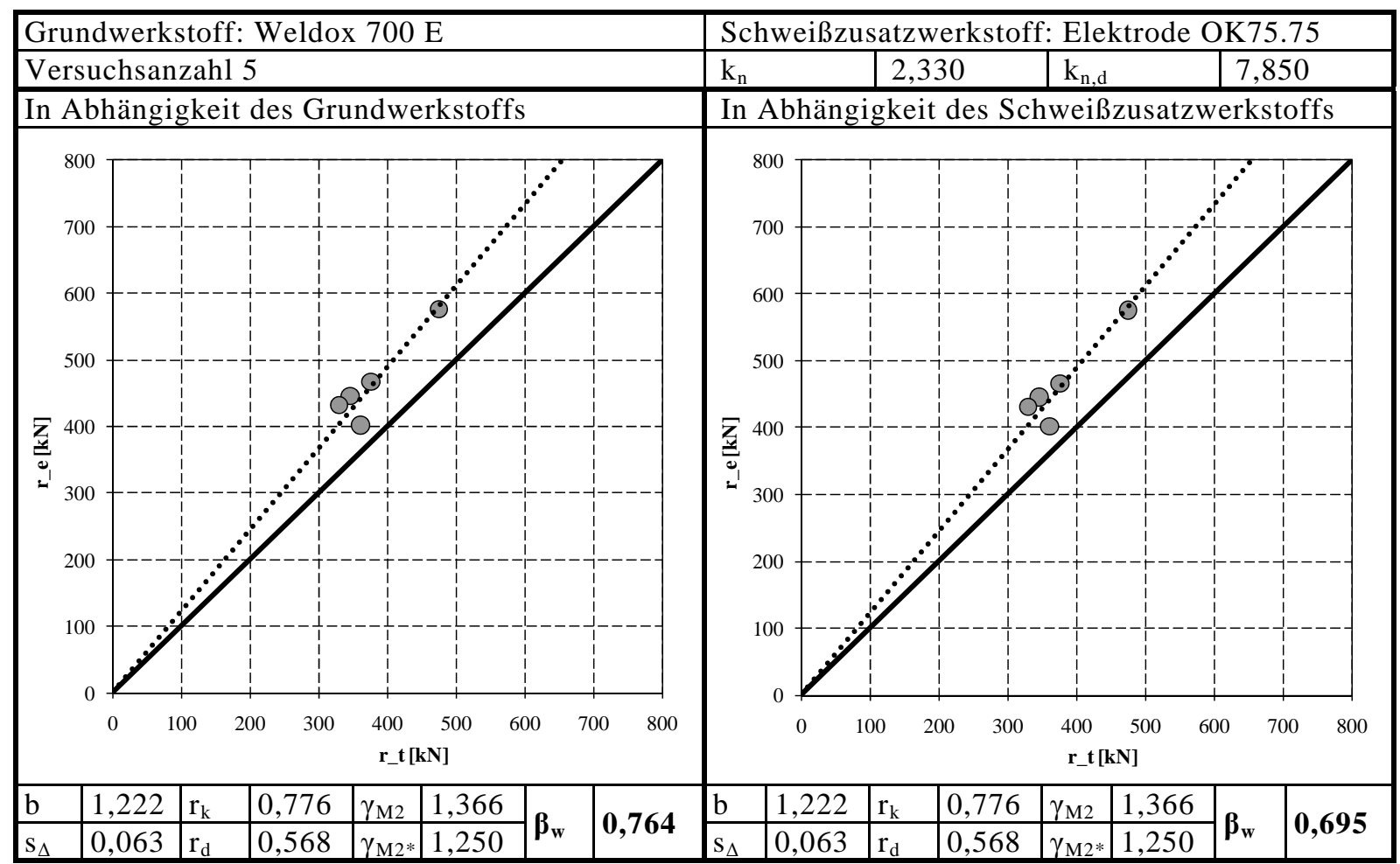


Tabelle A.35: Statistische Auswertung der Stirnkehlnahtverbindungen DT bezogen auf die Festigkeit des Grundwerkstoffs (links) oder des Schweißzusatzwerkstoffs (rechts)

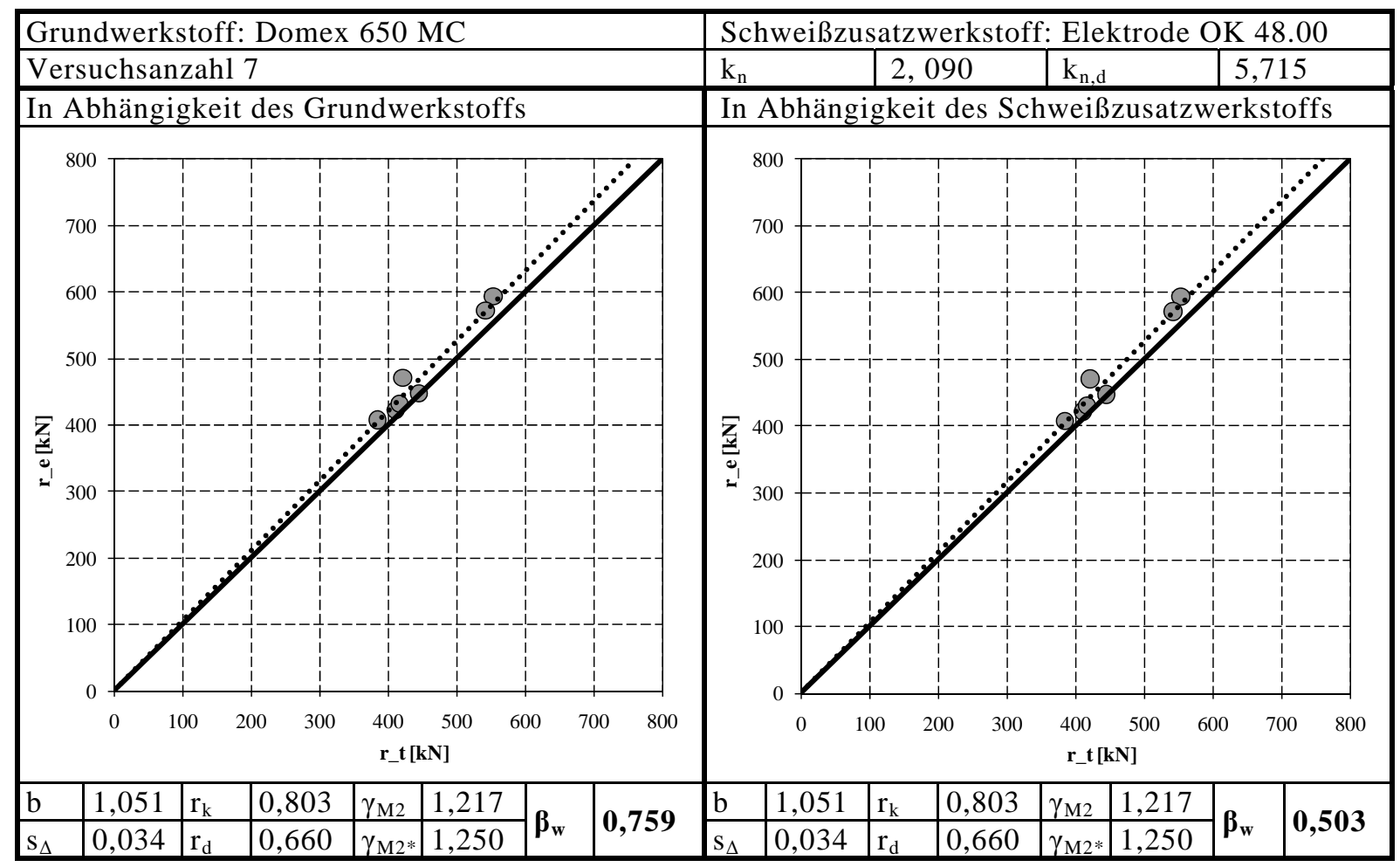




\section{A.2 Überblick Schweißverbindungen nach [FOSTA P652, 2008]}

\section{A.2.1 Chemische Kennwerte der verwendeten Grund- und Schweißzusatzwerk- stoffe}

Tabelle A.36: Chemische Zusammensetzung der verwendeten Grundwerkstoffe gemäß Lieferzeugnis in [\%] nach [FOSTA P652, 2008]

\begin{tabular}{|c|c|c|c|c|c|c|c|c|c|c|}
\hline Werkstoff & Blechdicke & $\mathrm{C}$ & Si & $\mathrm{Mn}$ & $\mathrm{P}$ & $S$ & $A l_{\text {ges }}$ & $\mathrm{N}$ & \multicolumn{2}{|l|}{$\mathrm{Cu}$} \\
\hline S355J2 & 15 & 0,163 & 0,469 & 1,56 & 0,010 & 0,0007 & 0,041 & 0,0039 & \multicolumn{2}{|l|}{0,30} \\
\hline S460M & 15 & 0,031 & 0,38 & 1,62 & 0,007 & 0,0010 & 0,038 & 0,0042 & \multicolumn{2}{|l|}{0,18} \\
\hline S690Q & 15 & 0,170 & 0,190 & 0,900 & 0,013 & 0,0010 & 0,100 & 0,0050 & \multicolumn{2}{|c|}{0,020} \\
\hline S355J2 & 20 & 0,20 & 0,44 & 1,39 & 0,015 & 0,003 & 0,041 & 0,008 & \multicolumn{2}{|c|}{0,000} \\
\hline S460M & 20 & 0,034 & 0,33 & 1,55 & 0,0069 & 0,001 & 0,036 & 0,0058 & \multicolumn{2}{|l|}{ 工 } \\
\hline S690Q & 20 & 0,160 & 0,230 & 0,890 & 0,011 & 0,0010 & - & - & \multicolumn{2}{|l|}{-} \\
\hline S355J2 & 50 & 0,17 & 0,55 & 1,51 & 0,017 & 0,001 & 0,052 & 0,008 & \multicolumn{2}{|c|}{0,045} \\
\hline S460ML & 50 & 0,07 & 0,38 & 1,57 & 0,012 & 0,001 & 0,043 & 0,005 & \multicolumn{2}{|c|}{0,160} \\
\hline S690QL & 50 & 0,13 & 0,37 & 1,07 & 0,011 & 0,001 & 0,032 & 0,006 & \multicolumn{2}{|c|}{0,700} \\
\hline Werkstoff & Blechdicke & Mo & $\overline{\mathrm{Ni}}$ & $\mathrm{Cr}$ & $\mathrm{V}$ & $\mathrm{Nb}$ & $\overline{\mathrm{Ti}}$ & B & $\mathrm{CE}$ & CET \\
\hline S355J2 & 15 & 0,007 & 0,035 & 0,033 & 0,000 & 0,000 & 0,009 & - & 0,45 & 0,34 \\
\hline S460M & 15 & 0,00 & 0,16 & 0,18 & 0,003 & 0,040 & 0,014 & 0,0001 & 0,36 & 0,22 \\
\hline S690Q & 15 & 0,200 & 0,030 & 0,320 & 0,000 & 0,029 & 0,004 & 0,0025 & 0,43 & 0,30 \\
\hline S355J2 & 20 & 0,000 & 0,00 & 0,00 & 0,000 & 0,000 & 0,000 & 0,0000 & 0,43 & 0,37 \\
\hline S460M & 20 & - & - & 0,16 & - & 0,042 & 0,013 & - & 0,32 & 0,20 \\
\hline S690Q & 20 & 0,400 & - & 0,340 & - & - & - & - & 0,46 & 0,31 \\
\hline S355J2 & 50 & 0,015 & 0,06 & 0,04 & 0,001 & 0,001 & 0,003 & 0,0001 & 0,44 & 0,33 \\
\hline S460ML & 50 & 0,140 & 0,42 & 0,03 & 0,001 & 0,019 & 0,008 & 0,0001 & 0,40 & 0,26 \\
\hline S690QL & 50 & 0,400 & 1,22 & 0,16 & 0,000 & 0,016 & 0,02 & 0,0000 & 0,55 & 0,35 \\
\hline
\end{tabular}

Tabelle A.37: Chemische Zusammensetzung der Schweißzusatzwerkstoffe gemäß Lieferzeugnis in [\%] nach [FOSTA P652, 2008]

\begin{tabular}{|c|c|c|c|c|c|c|c|c|c|c|c|c|}
\hline Schweißzusatzwer & toff & C & Si & $\mathrm{Mn}$ & $\mathrm{P}$ & $\mathrm{S}$ & $\mathrm{Al}$ & $\mathrm{Cu}$ & Mo & $\mathrm{Ni}$ & $\mathrm{Cr}$ & V \\
\hline Union K52 $^{1)}$ & G42 & 0,09 & 0,60 & 1,20 & 0,009 & 0,016 & 0,005 & 0,19 & 0,024 & 0,077 & 0,055 & $<0,001$ \\
\hline Union K56 $^{1)}$ & G46 & 0,082 & 0,77 & 1,45 & 0,011 & 0,015 & 0,004 & 0,12 & 0,021 & 0,049 & 0,067 & 0,008 \\
\hline OKAristoRod12.63 & G46 & 0,074 & 0,95 & 1,68 & 0,013 & 0,013 & 0,005 & 0,05 & & 0,02 & 0,03 & \\
\hline 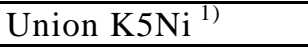 & G50 & 0,071 & 0,51 & 1,05 & 0,07 & 0,009 & 0,005 & 0,13 & 0,038 & 1,24 & 0,09 & $<0,001$ \\
\hline UnionMoNi $^{1)}$ & G62 & 0,11 & 0,51 & 1,13 & 0,010 & 0,014 & 0,009 & 0,12 & 0,37 & 0,90 & 0,15 & $<0,001$ \\
\hline Union NiMoCr ${ }^{1)}$ & G69 & 0,10 & 0,45 & 1,26 & 0,004 & 0,014 & 0,007 & 0,10 & 0,49 & 1,33 & 0,27 & $<0,001$ \\
\hline Union RV 71 2) & T46 & 0,050 & 0,43 & 1,14 & 0,020 & 0,009 & 0,014 & & & & & \\
\hline UnionMV NiMoCr & T69 & 0,053 & 0,45 & 1,30 & 0,010 & 0,51 & & & 0,42 & 2,09 & & \\
\hline PhoenixSHNi2K70 & E46 & 0,06 & 0,27 & 1,35 & & & & & 0,45 & 1,95 & & \\
\hline
\end{tabular}




\section{A.2.2 Versuchskörpertypen}

Tabelle A.38: Versuchskörpertyp 1 - Flankenkehlnahtverbindung, [FOSTA P652, 2008]

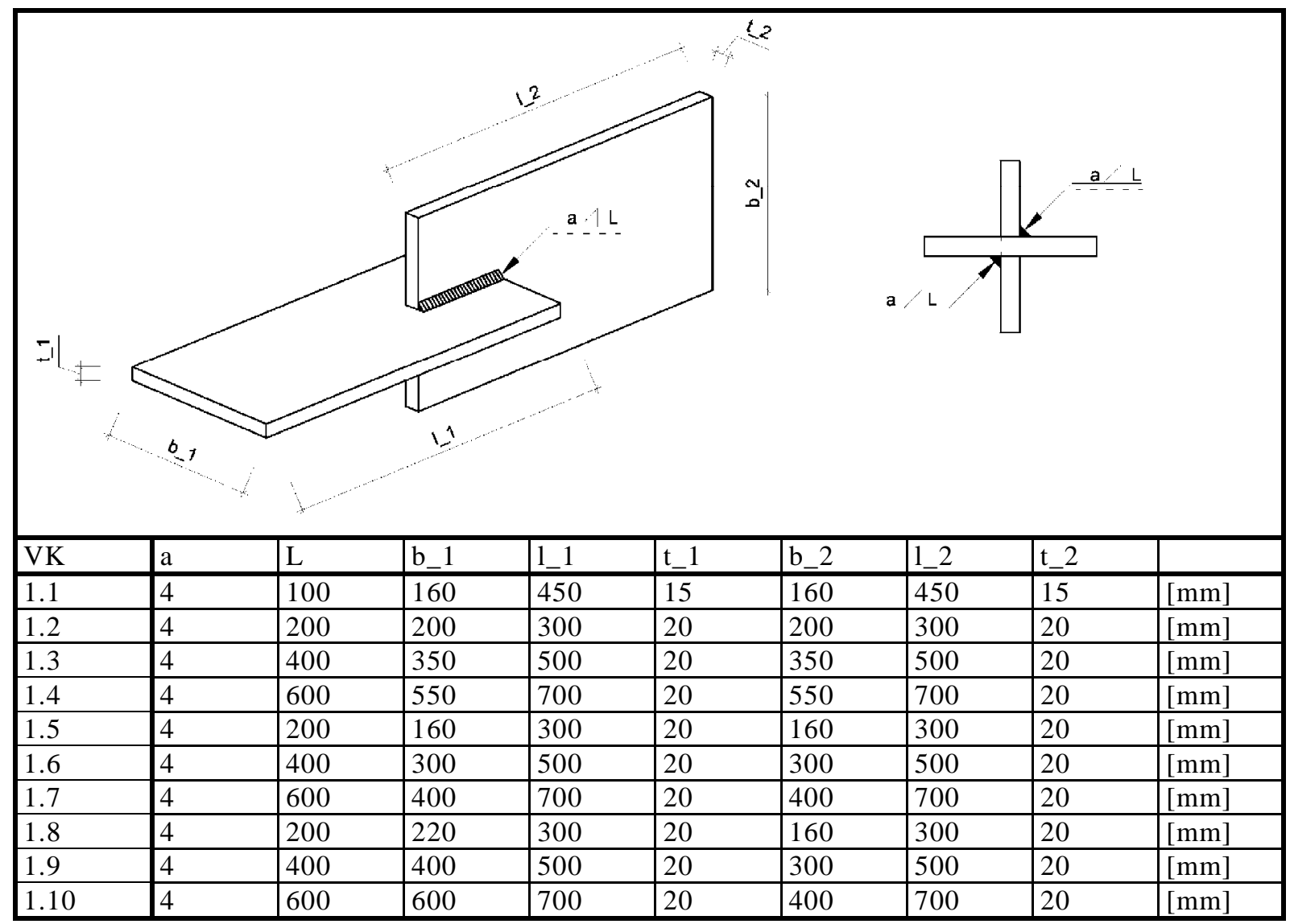

Tabelle A.39: Versuchskörpertyp 2 - Übergreifungsstoß, [FOSTA P652, 2008]

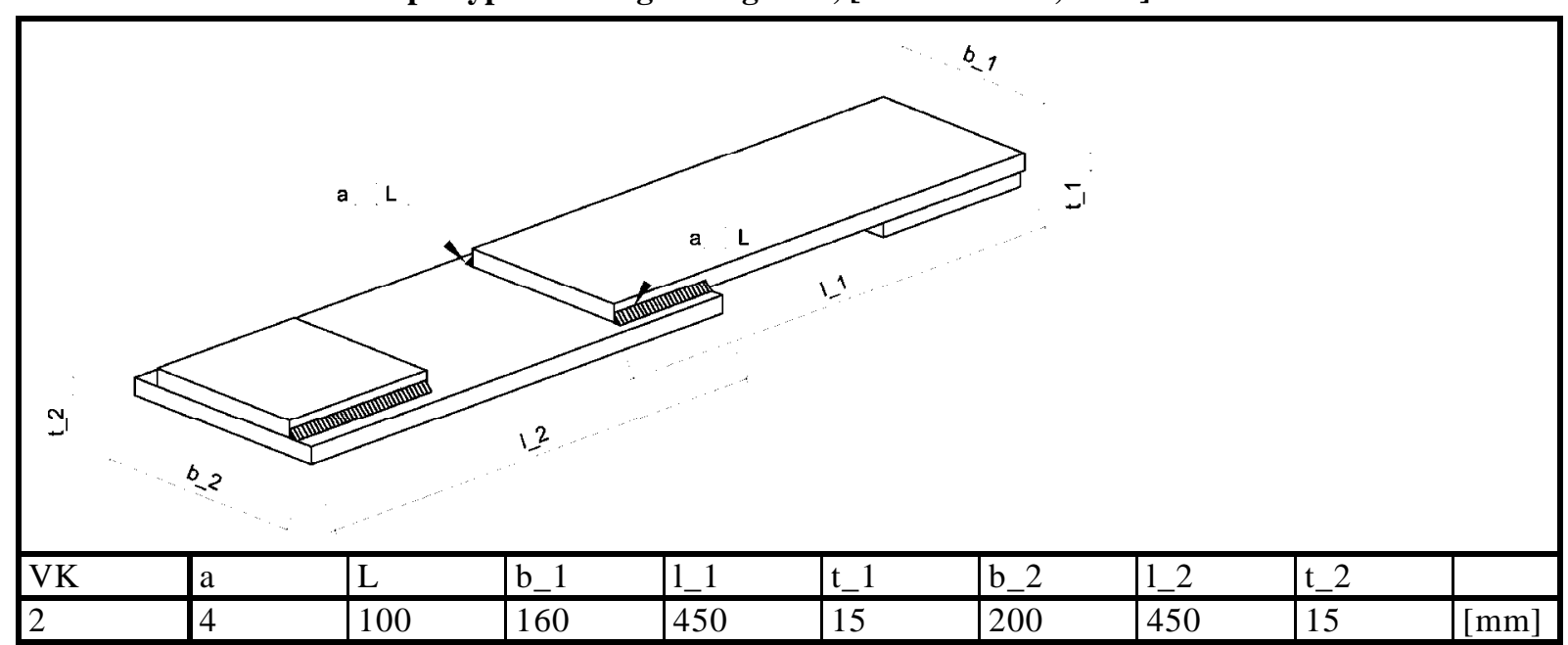


Tabelle A.40: Versuchskörpertyp 3 - Flankenkehlnahtverbindung, [FOSTA P652, 2008]

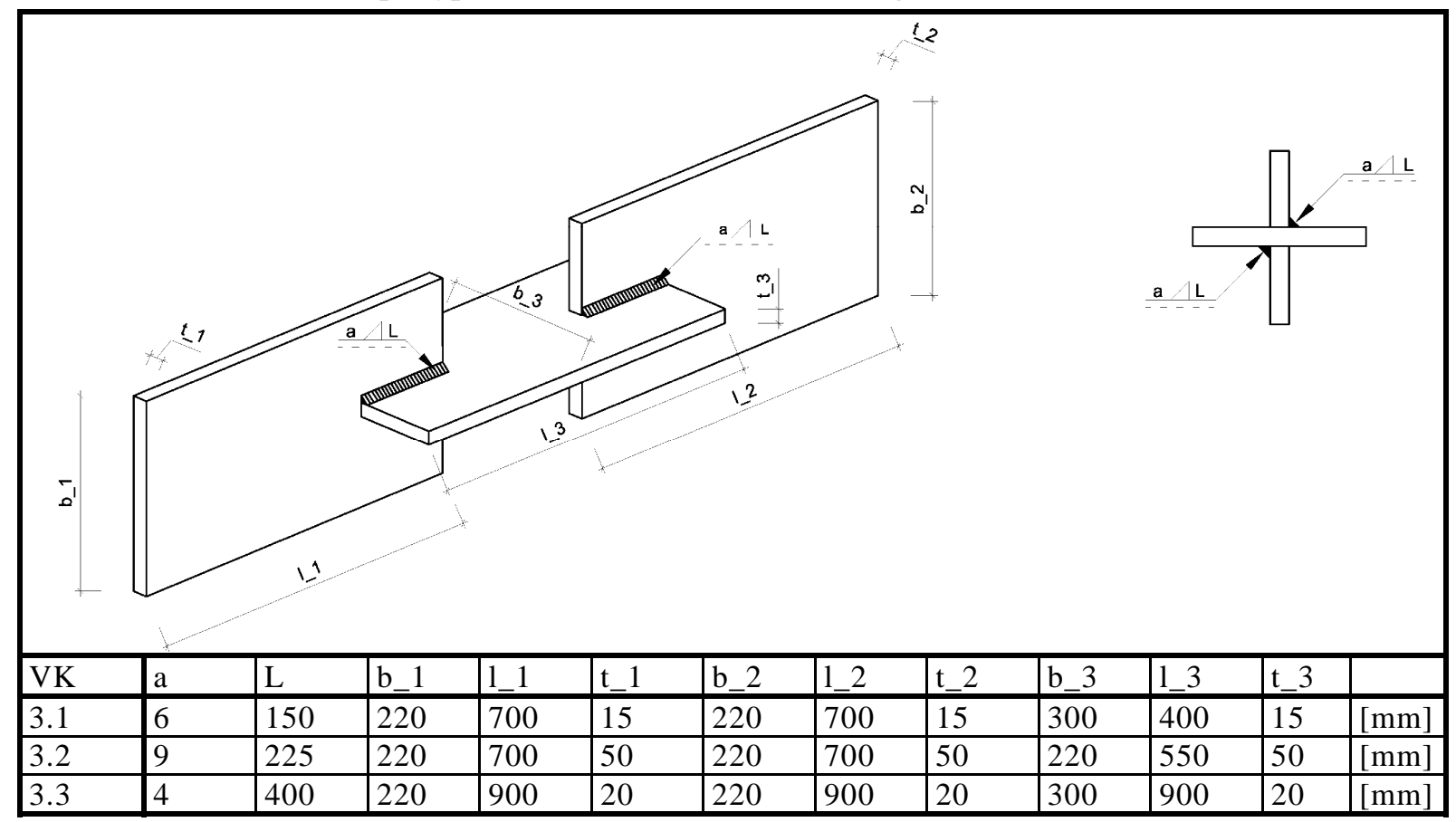

Tabelle A.41: Versuchskörpertyp 4 - Kreuzstoß, [FOSTA P652, 2008]

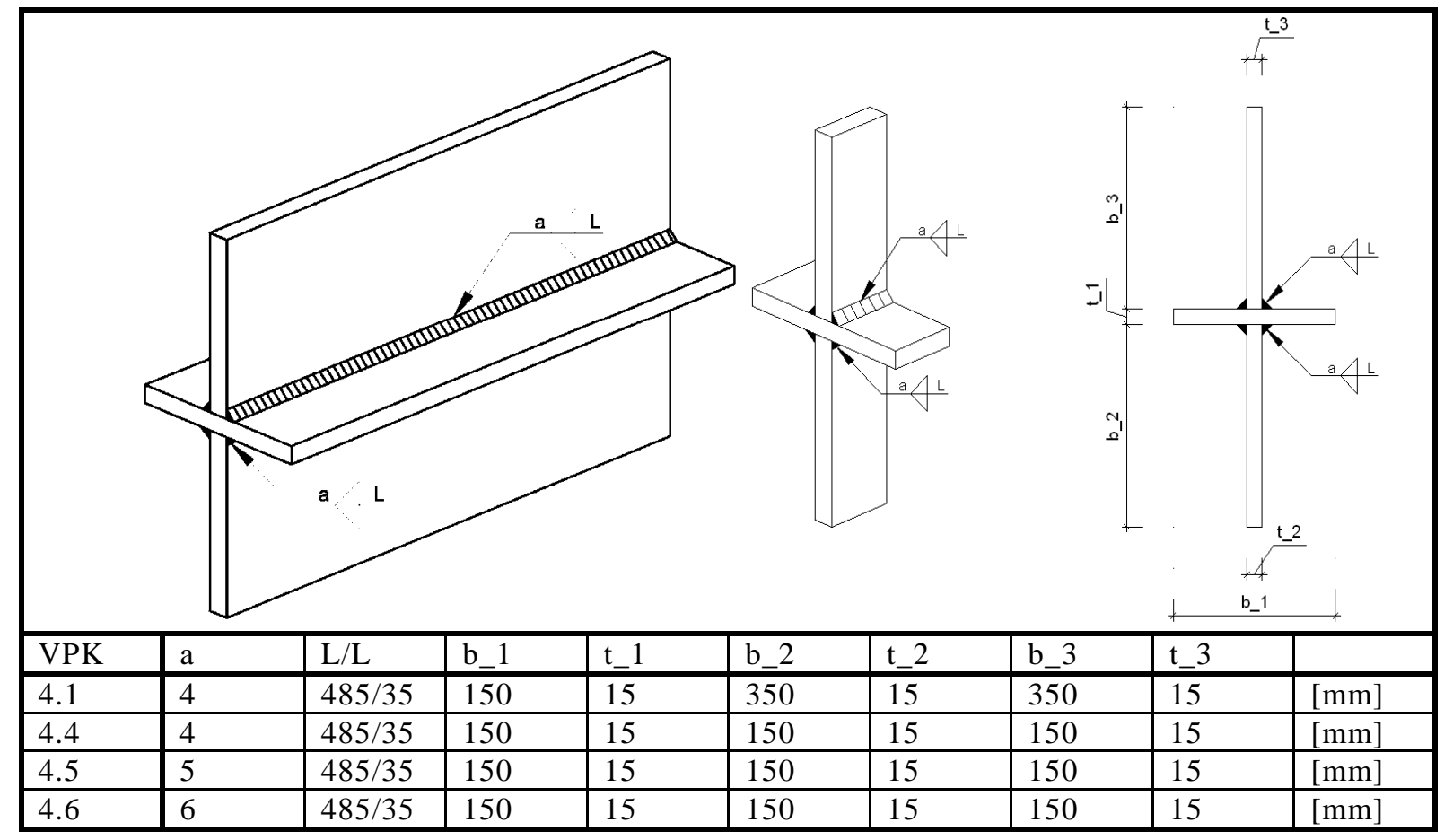




\section{A.2.3 Material und Schweißparameter der Flankenkehlnahtverbindungen}

Tabelle A.42: Durchgeführte Untersuchungen an Flankenkehlnahtverbindungen, [FOSTA P652, 2008]

\begin{tabular}{|c|c|c|c|c|c|c|c|c|c|c|c|c|c|}
\hline$\sum_{z}^{L}$ & 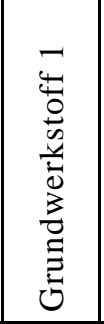 & 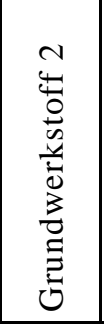 & 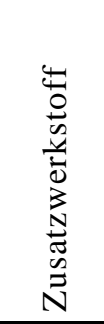 & 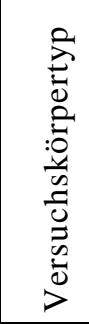 & 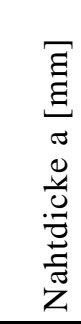 & 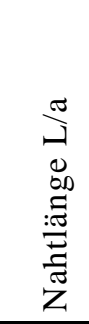 & 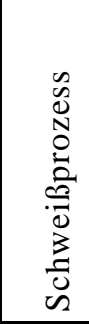 & 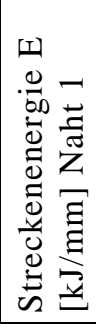 & 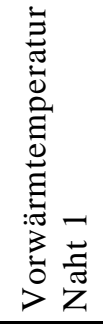 & 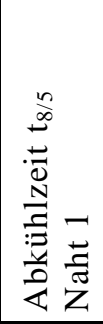 & 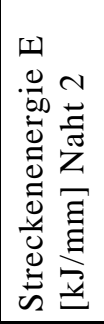 & 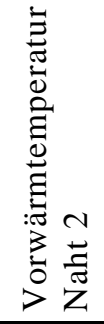 & 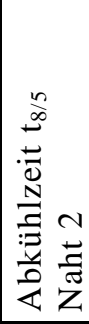 \\
\hline $1-001$ & S460 & \begin{tabular}{|l|}
$S 460$ \\
\end{tabular} & G46 & 1.1 & 4 & 25 & 135 & 1,52 & 20 & 5,3 & 1,45 & 47 & 5,2 \\
\hline $1-002$ & \begin{tabular}{|l|} 
S460 \\
\end{tabular} & S460 & G46 & 1.1 & 4 & 25 & 135 & 1,51 & 20 & 5,2 & 1,43 & 34 & 5,5 \\
\hline $1-004$ & S460 & S460 & G46 & 1.1 & 4 & 25 & 135 & 1,47 & 20 & 5,3 & 1,54 & 36 & 5,9 \\
\hline 1-005 & S460 & \begin{tabular}{|l|} 
S460 \\
\end{tabular} & G46B & 1.1 & 4 & 25 & 135 & 1,49 & 20 & 4,8 & 1,65 & 45 & 6,3 \\
\hline $1-006$ & S460 & S460 & G46B & 1.1 & 4 & 25 & 135 & 1,66 & 20 & 5,7 & 1,64 & 48 & 6,0 \\
\hline 1-009 & S460 & S460 & G46 & 1.1 & 4 & 25 & 135 & 1,62 & 20 & 5,8 & & 31 & \\
\hline 1-011 & \begin{tabular}{|l|} 
S460 \\
\end{tabular} & S460 & G46 & 1.1 & 4 & 25 & 135 & 1,51 & 20 & 5,1 & 1,46 & 30 & 6,2 \\
\hline $1-012$ & S460 & S460 & G46 & 1.1 & 4 & 25 & 135 & 1,44 & 20 & 4,0 & 1,53 & 50 & 6,2 \\
\hline 1-013 & S460 & S460 & G46 & 1.1 & 4 & 25 & 135 & 1,45 & 20 & 5,7 & 1,65 & 60 & 7,0 \\
\hline 1-015 & \begin{tabular}{|l|}
5460 \\
\end{tabular} & \begin{tabular}{|l|} 
S460 \\
\end{tabular} & G46 & 1.1 & 4 & 25 & 135 & 1,68 & 20 & 6,0 & 1,77 & 50 & 6,7 \\
\hline 1-016 & S460 & S460 & G46 & 1.1 & 4 & 25 & 135 & 1,76 & 20 & 6,0 & 1,87 & 52 & 7,2 \\
\hline $1-017$ & \begin{tabular}{|l|}
$S 460$ \\
\end{tabular} & S460 & E46 & 1.1 & 4 & 25 & 111 & 2,06 & 20 & & 1,63 & 50 & \\
\hline 1-019 & S460 & S460 & E46 & 1.1 & 4 & 25 & 111 & 2,68 & 20 & & 2,20 & 58 & \\
\hline $1-020^{2)}$ & \begin{tabular}{|l|}
$S 460$ \\
\end{tabular} & \begin{tabular}{|l|} 
S460 \\
\end{tabular} & E46 & 1.1 & 4 & 25 & 111 & & 20 & & & 60 & \\
\hline $1-021^{2)}$ & S460 & \begin{tabular}{|l|} 
S460 \\
\end{tabular} & E46 & 1.1 & 4 & 25 & 111 & & 20 & & & 68 & \\
\hline $1-022$ & S460 & S460 & T46 & 1.1 & 4 & 25 & 136 & 1,38 & 20 & & 1,49 & 45 & \\
\hline $1-024$ & \begin{tabular}{|l|} 
S460 \\
\end{tabular} & S460 & T46 & 1.1 & 4 & 25 & 136 & 1,57 & 20 & & 1,60 & 42 & \\
\hline $1-025^{2)}$ & S460 & S460 & T46 & 1.1 & 4 & 25 & 136 & 1,16 & 20 & & 1,54 & 70 & \\
\hline $1-026^{2)}$ & S460 & \begin{tabular}{|l|} 
S460 \\
\end{tabular} & T46 & 1.1 & 4 & 25 & 136 & 1,53 & 20 & & 1,41 & 81 & \\
\hline $1-027^{3)}$ & S460 & S460 & G46 & 1.2 & 4 & 50 & 135 & 1,38 & 20 & 4,5 & 1,42 & 40 & 4,5 \\
\hline $1-029^{3)}$ & S460 & S460 & G46 & 1.2 & 4 & 50 & 135 & 1,42 & 20 & & 1,62 & 70 & 6,5 \\
\hline $1-030^{3)}$ & S460 & S460 & G46 & 1.3 & 4 & 100 & 135 & 1,33 & 20 & & 1,36 & 33 & 5,2 \\
\hline $1-031^{3)}$ & S460 & S460 & G46 & 1.3 & 4 & 100 & 135 & 1,48 & 20 & 6,0 & 1,41 & 60 & 6,2 \\
\hline $1-032^{3)}$ & \begin{tabular}{|l|}
$S 460$ \\
\end{tabular} & S460 & G46 & 1.4 & 4 & 150 & 135 & 1,40 & 20 & 4,8 & 1,36 & 29 & 4,5 \\
\hline $1-035^{5)}$ & \begin{tabular}{|l|}
$S 460$ \\
\end{tabular} & \begin{tabular}{|l|} 
S460 \\
\end{tabular} & G46 & 3.2 & 9 & 25 & 135 & 1,84 & 80 & 8,4 & 1,84 & 77 & 7,6 \\
\hline $1-036^{5)}$ & \begin{tabular}{|l|}
$S 460$ \\
\end{tabular} & \begin{tabular}{|l|} 
S460 \\
\end{tabular} & G46 & 3.2 & 9 & 25 & 135 & 1,33 & 80 & 5,9 & 1,33 & 83 & 6,6 \\
\hline $1-037^{5)}$ & \begin{tabular}{|l|}
$S 460$ \\
\end{tabular} & S460 & G46 & 3.1 & 6 & 25 & 135 & 1,04 & 61 & 4,7 & 1,21 & 78 & 6,4 \\
\hline 1-038 & S460 & S460 & G46 & 3.1 & 6 & 25 & 135 & 2,11 & 20 & 9,7 & 2,04 & 100 & 11,3 \\
\hline $1-039^{2)}$ & \begin{tabular}{|l|}
$S 460$ \\
\end{tabular} & \begin{tabular}{|l|} 
S460 \\
\end{tabular} & G46 & 2 & 4 & 25 & 135 & 1,35 & 20 & 4,6 & 1,09 & 30 & 7,4 \\
\hline $1-040$ & \begin{tabular}{|l|} 
S460 \\
\end{tabular} & S460 & G46 & 2 & 4 & 25 & 135 & 1,32 & 20 & 4,9 & 1,33 & 30 & 6,4 \\
\hline 1-041 & S355 & S355 & G42 & 1.1 & 4 & 25 & 135 & 1,26 & 25 & 4,6 & 1,58 & 84 & 6,0 \\
\hline 1-042 & S355 & S355 & G42 & 1.1 & 4 & 25 & 135 & 1,25 & 25 & 0,0 & 1,44 & 79 & 6,1 \\
\hline 1-043 & S355 & S355 & G42 & 1.1 & 4 & 25 & 135 & 1,40 & & 4,7 & 1,38 & & \\
\hline 1-044 & S355 & S355 & G42 & 1.1 & 4 & 25 & 135 & 1,32 & 20 & 6,2 & 1,40 & 32 & 6,9 \\
\hline $1-045$ & S690 & S690 & G69 & 1.1 & 4 & 25 & 135 & 1,46 & 82 & 6,6 & 1,36 & 100 & 6,0 \\
\hline $1-047^{2)}$ & S690 & S690 & G69 & 1.1 & 4 & 25 & 135 & 1,74 & 100 & 9,7 & 2,10 & 120 & 12,0 \\
\hline $1-049^{1)}$ & S690 & S690 & G69 & 1.1 & 4 & 25 & 135 & 0,87 & 90 & 4,3 & 0,86 & 90 & 2,9 \\
\hline $1-050^{1)}$ & S690 & \begin{tabular}{|l|} 
S690 \\
\end{tabular} & G69 & 1.1 & 4 & 25 & 135 & 0,65 & 84 & 3,0 & 0,72 & 93 & 2,5 \\
\hline 1-052 & S690 & S690 & T69 & 1.1 & 4 & 25 & 136 & 1,44 & 102 & 6,8 & 1,41 & 104 & 6,9 \\
\hline $1-054$ & S690 & S690 & T69 & 1.1 & 4 & 25 & 136 & 1,42 & 95 & 6,1 & 1,54 & 108 & 5,7 \\
\hline $1-055^{3)}$ & S690 & S690 & G69 & 1.5 & 4 & 50 & 135 & 1,09 & 84 & 4,9 & 1,46 & 97 & 6,1 \\
\hline $1-057^{3)}$ & S690 & S690 & G69 & 1.5 & 4 & 50 & 135 & 1,26 & 90 & 5,0 & 1,21 & 105 & 4,3 \\
\hline $1-058^{3)}$ & S690 & S690 & G69 & 1.6 & 4 & 100 & 135 & 1,30 & 84 & 5,8 & 1,22 & 90 & 4,1 \\
\hline $1-059^{3)}$ & S690 & \begin{tabular}{|l|} 
S690 \\
\end{tabular} & G69 & 1.6 & 4 & 100 & 135 & 1,34 & 110 & 5,0 & 1,38 & 104 & 4,9 \\
\hline $1-060^{3)}$ & S690 & S690 & G69 & 1.7 & 4 & 150 & 135 & 1,21 & 90 & 5,4 & 1,57 & 79 & 5,4 \\
\hline
\end{tabular}


Tabelle A.42 Fortsetzung: Durchgeführte Untersuchungen an Flankenkehlnahtverbindungen, [FOSTA P652, 2008]

\begin{tabular}{|c|c|c|c|c|c|c|c|c|c|c|c|c|c|}
\hline$\sum_{\text {乙 }}^{\sum^{1}}$ & 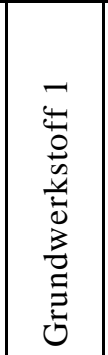 & 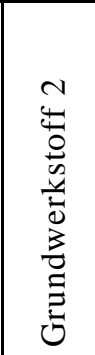 & 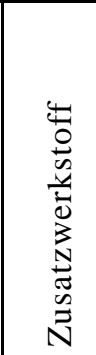 & 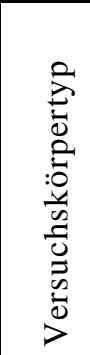 & 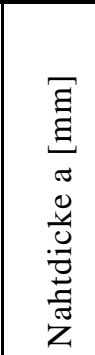 & 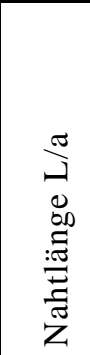 & 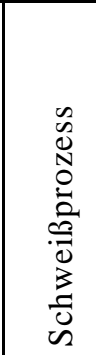 & 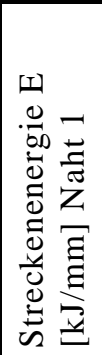 & 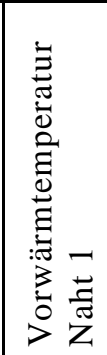 & 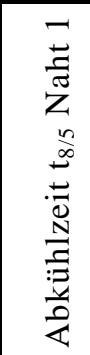 & 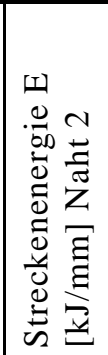 & 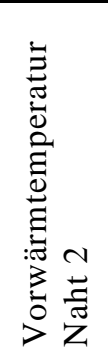 & 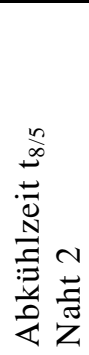 \\
\hline $1-062^{3)}$ & S690 & S690 & G69 & 1.7 & 4 & 150 & 135 & 1,51 & 95 & 5,3 & 1,52 & 104 & 6,0 \\
\hline $1-063$ & S690 & S690 & G69 & 3.1 & 6 & 25 & 135 & 2,42 & 102 & 15,1 & 2,08 & 108 & 10,3 \\
\hline $1-064$ & S690 & S690 & G69 & 3.1 & 6 & 25 & 135 & 2,15 & 85 & 12,5 & 2,16 & 100 & 12,1 \\
\hline $1-065^{5)}$ & \begin{tabular}{|l|} 
S690 \\
\end{tabular} & S690 & G69 & 3.2 & 9 & 25 & 135 & 1,69 & 88 & 7,8 & 1,69 & 88 & 8,5 \\
\hline $1-066^{2) 5)}$ & S690 & S690 & G69 & 3.2 & 9 & 25 & 135 & 1,46 & 100 & 7,1 & 1,45 & 94 & 6,0 \\
\hline $1-067$ & S690 & S690 & G69 & 2 & 4 & 25 & 135 & 1,12 & 94 & 5,2 & 1,12 & 80 & 5,3 \\
\hline $1-068$ & S690 & S690 & G69 & 2 & 4 & 25 & 135 & 1,51 & 98 & 4,0 & 1,38 & 93 & 4,1 \\
\hline $1-069$ & S460 & S460 & G42 & 1.1 & 4 & 25 & 135 & 1,54 & 20 & 4,8 & 1,46 & 53 & 5,2 \\
\hline $1-071$ & S460 & S460 & G42 & 1.1 & 4 & 25 & 135 & 1,34 & 20 & 5,2 & 1,39 & 83 & \\
\hline $1-072$ & S460 & S460 & G69 & 1.1 & 4 & 25 & 135 & 1,56 & 92 & 7,4 & 1,57 & 91 & 7,5 \\
\hline 1-074 & S460 & S460 & G69 & 1.1 & 4 & 25 & 135 & 1,58 & 77 & 6,0 & 1,80 & 57 & 8,2 \\
\hline $1-075$ & S355 & S355 & G46 & 1.1 & 4 & 25 & 135 & 1,21 & 26 & 5,5 & 1,29 & 56 & 5,1 \\
\hline $1-076$ & S355 & S355 & G46 & 1.1 & 4 & 25 & 135 & 1,58 & 20 & 6,2 & 1,57 & 90 & 7,4 \\
\hline $1-077$ & S690 & S690 & G46 & 1.1 & 4 & 25 & 135 & 1,11 & 91 & 5,8 & 1,13 & 100 & 5,1 \\
\hline $1-079^{4)}$ & S690 & S690 & G46 & 1.1 & 4 & 25 & 135 & 1,51 & 85 & 6,4 & 1,83 & 110 & 9,1 \\
\hline $1-080$ & S690 & S690 & G62 & 1.1 & 4 & 25 & 135 & 1,27 & 97 & 4,5 & 1,41 & 96 & 6,2 \\
\hline $1-081$ & S690 & S690 & G62 & 1.1 & 4 & 25 & 135 & 1,28 & 90 & 4,8 & 1,35 & 100 & \\
\hline $1-082^{3)}$ & S690 & S690 & G62 & 1.6 & 4 & 100 & 135 & 1,39 & 74 & 5,3 & 1,44 & 90 & 5,1 \\
\hline $1-083^{3)}$ & S690 & S690 & G62 & 1.6 & 4 & 100 & 135 & 1,42 & 100 & 4,3 & & 96 & 4,6 \\
\hline $1-084$ & S690 & S690 & G62 & 3.2 & 9 & 25 & 135 & 1,51 & 83 & 8,5 & 1,38 & 92 & 6,3 \\
\hline $1-085$ & S690 & S690 & G62 & 3.2 & 9 & 25 & 135 & 1,62 & 80 & 10,2 & 1,70 & 95 & 9,5 \\
\hline $1-086$ & S355 & S460 & G42 & 1.1 & 4 & 25 & 135 & 1,45 & 20 & 5,1 & 1,45 & 50 & 5,4 \\
\hline $1-088$ & S355 & S460 & G42 & 1.1 & 4 & 25 & 135 & 1,26 & 20 & 5,1 & 1,33 & 70 & 5,2 \\
\hline $1-089$ & S355 & S460 & G46 & 1.1 & 4 & 25 & 135 & 1,52 & 20 & 5,9 & 1,48 & 50 & 5,4 \\
\hline $1-091$ & S355 & S460 & G46 & 1.1 & 4 & 25 & 135 & 1,62 & 20 & 6,3 & 1,65 & 100 & 7,3 \\
\hline 1-092 & S355 & S690 & G42 & 1.1 & 4 & 25 & 135 & 1,23 & 82 & 5,2 & 1,39 & 102 & 5,3 \\
\hline $1-094$ & S355 & S690 & G42 & 1.1 & 4 & \begin{tabular}{|l|}
25 \\
\end{tabular} & 135 & \begin{tabular}{|l|}
1,37 \\
\end{tabular} & 85 & 7,2 & & 110 & \\
\hline $1-095$ & S355 & S690 & G50 & 1.1 & 4 & 25 & 135 & 1,44 & 83 & 7,8 & 1,29 & 100 & 5,7 \\
\hline $1-097$ & S355 & S690 & G50 & 1.1 & 4 & 25 & 135 & 1,44 & 95 & 0,0 & 1,43 & 105 & 7,6 \\
\hline $1-098$ & S355 & S690 & G69 & 1.1 & 4 & 25 & 135 & 1,39 & 90 & 6,8 & 1,47 & 96 & 7,3 \\
\hline $1-100$ & S355 & S690 & G69 & 1.1 & 4 & 25 & 135 & 1,37 & 85 & 6,6 & 1,39 & 89 & 7,6 \\
\hline $1-101$ & S460 & S690 & G46 & 1.1 & 4 & 25 & 135 & 1,47 & 95 & 6,3 & 1,52 & 83 & 7,3 \\
\hline $1-103$ & S460 & S690 & G46 & 1.1 & 4 & 25 & 135 & 1,38 & 80 & 6,0 & 1,60 & 100 & 7,9 \\
\hline $1-104$ & S460 & S690 & G62 & 1.1 & 4 & 25 & 135 & 1,72 & 93 & 8,5 & 1,60 & 100 & 7,5 \\
\hline $1-106$ & S460 & S690 & G62 & 1.1 & 4 & 25 & 135 & 1,41 & 100 & 6,8 & 1,61 & 120 & 8,6 \\
\hline $1-107$ & S460 & S690 & G69 & 1.1 & 4 & 25 & 135 & 1,87 & 77 & 8,1 & 1,61 & 100 & 8,2 \\
\hline $1-109$ & S460 & S690 & G69 & 1.1 & 4 & 25 & 135 & 1,25 & 85 & 6,0 & 1,26 & 60 & 6,6 \\
\hline $1-110$ & S355 & S690 & G42 & 3.2 & 9 & 25 & 135 & 1,43 & 85 & 6,9 & 1,43 & 97 & 7,1 \\
\hline $1-111$ & S355 & S690 & G42 & 3.2 & 9 & 25 & 135 & 1,50 & 89 & 8,2 & 1,41 & 75 & 7,6 \\
\hline $1-112^{1)}$ & S355 & S690 & G42 & 1.1 & 4 & 25 & 135 & 0,81 & 95 & 2,5 & 0,89 & 101 & 2,9 \\
\hline $1-113$ & S355 & S690 & G42 & 1.1 & 4 & 25 & 135 & 1,23 & 90 & 6,2 & 1,31 & 60 & 5,9 \\
\hline $1-118$ & S355 & S690 & G69 & 1.8 & 4 & 50 & 135 & 1,40 & 80 & 6,1 & 1,50 & 98 & 6,7 \\
\hline $1-119$ & S355 & S690 & G69 & 1.8 & 4 & 50 & 135 & 1,51 & 89 & 5,4 & 1,49 & 105 & 5,3 \\
\hline $1-120$ & S355 & S690 & G69 & 1.9 & 4 & 100 & 135 & 1,39 & 90 & 5,3 & 1,72 & 90 & 7,1 \\
\hline $1-121$ & S355 & S690 & G69 & 1.9 & 4 & 100 & 135 & 1,51 & 86 & 3,7 & 1,54 & 86 & 6,4 \\
\hline $1-123$ & S355 & S690 & G69 & 1.10 & 4 & 150 & 135 & 1,55 & 90 & 5,7 & 1,57 & 85 & 6,6 \\
\hline
\end{tabular}


Tabelle A.42 Fortsetzung: Durchgeführte Untersuchungen an Flankenkehlnahtverbindungen, [FOSTA P652, 2008]

\begin{tabular}{|c|c|c|c|c|c|c|c|c|c|c|c|c|c|}
\hline 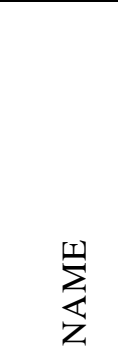 & 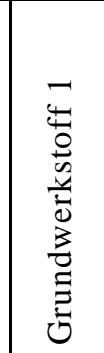 & 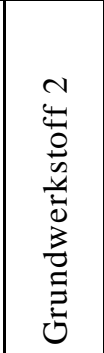 & 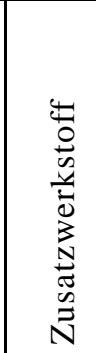 & 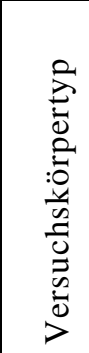 & 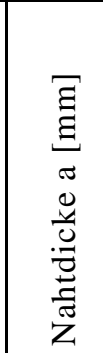 & 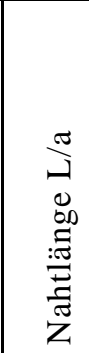 & 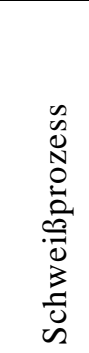 & 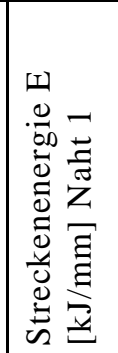 & 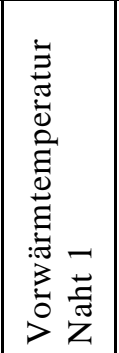 & 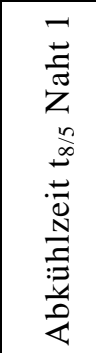 & 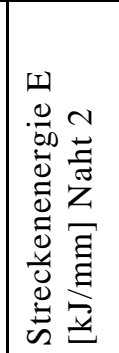 & 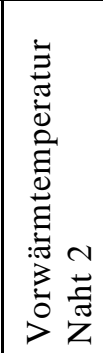 & 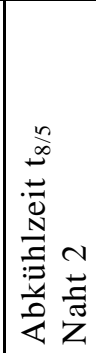 \\
\hline $1-146$ & 5690 & S690 & G69 & 3.3 & 4 & 100 & 135 & 1,42 & 101 & 7,5 & 1,52 & 105 & 6,8 \\
\hline $1-147^{6)}$ & S690 & S690 & G69 & 3.3 & 4 & 100 & 135 & 1,38 & 100 & 5,4 & 1,50 & 95 & 7,4 \\
\hline \multicolumn{14}{|c|}{$\begin{array}{l}\text { Diese Versuche wurden nicht für die statistische Auswertung verwendet, } \\
\text { 1) da die Abkühlzeit t }{ }_{8 / 5} \text { nicht im Bereich } 4-11 \text { Sekunden } \\
\text { 2) da die Schweißparameter nicht im Arbeitsfenster liegen, oder } \\
\text { 3) da es sich um Druckversuche handelt. } \\
\text { 4) Werte nicht plausibel, vermutlich Messfehler } \\
\text { 5) Bei den mehrlagigen Nähten ist für die Streckenenergie der Mittelwert aus allen Lagen gebildet, es } \\
\text { wird die geringste Vorwärmtemperatur aller Lagen angegeben und die Abkühlzeit } t_{8 / 5} \text { der letzten } \\
\text { Lage. }\end{array}$} \\
\hline
\end{tabular}

\section{A.2.4 Material und Schweißparameter der Kreuzstöße}

Tabelle A.43: Durchgeführte Untersuchungen an Kreuzstößen, [FOSTA P652, 2008]

\begin{tabular}{|c|c|c|c|c|c|c|c|c|c|c|c|c|c|c|c|c|}
\hline 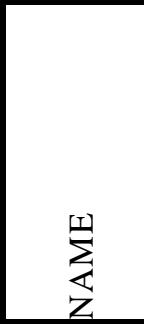 & 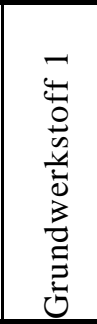 & 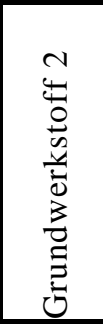 & 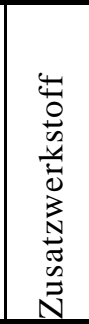 & 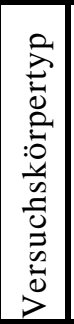 & 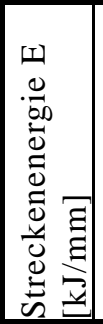 & 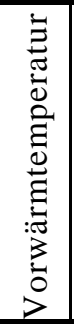 & 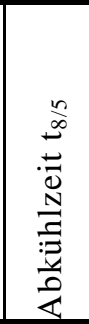 & 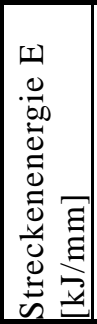 & 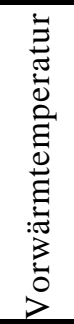 & 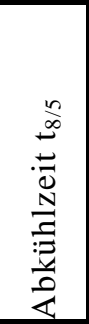 & 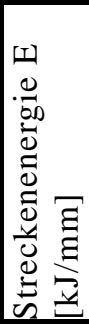 & 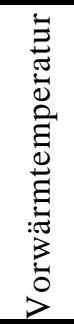 & 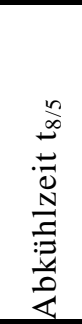 & 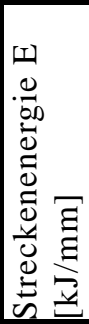 & 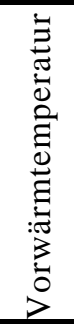 & 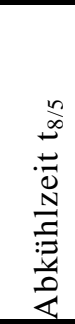 \\
\hline & & & & & \multicolumn{3}{|c|}{ Naht 1} & \multicolumn{3}{|c|}{ Naht 2} & \multicolumn{3}{|c|}{ Naht 3} & \multicolumn{3}{|c|}{ Naht 4} \\
\hline $\mid 2-001$ & 355 & 355 & 42 & \begin{tabular}{|l|}
4.1 \\
\end{tabular} & $\mid 0,97$ & 30 & -- & \begin{tabular}{|l|}
0,93 \\
\end{tabular} & 55 & 3,9 & 1,08 & 80 & $\overline{5,1}$ & 1,09 & 82 & $\overline{5,1}$ \\
\hline $2-0$ & 55 & 460 & 46 & \begin{tabular}{|l|}
4.1 \\
\end{tabular} & 0,88 & 30 & 3,7 & 1,04 & 52 & 4,3 & 1,09 & 68 & 5,0 & 0,98 & 85 & 5,1 \\
\hline $2-003$ & S355 & 690 & 42 & \begin{tabular}{|l|}
4.1 \\
\end{tabular} & 0,99 & 65 & 4,3 & 1,02 & 76 & 4,7 & 1,03 & 80 & 4,7 & 1,03 & 83 & 4,9 \\
\hline $2-005$ & 55 & S690 & 369 & 4.1 & 1,13 & 82 & 4,9 & 1,21 & 95 & 5,7 & 1,17 & 100 & 5,5 & 1,06 & 85 & 3,9 \\
\hline [2-006 & 460 & S690 & 346 & \begin{tabular}{|l|}
4.1 \\
\end{tabular} & 0,97 & 93 & 4,8 & 1,02 & 100 & 5,0 & 1,11 & 85 & 5,0 & 1,14 & 80 & 5,2 \\
\hline $2-008$ & 60 & 690 & 69 & 4.1 & 1,12 & 80 & 4,7 & 1,18 & 85 & 4,7 & 1,12 & 100 & 5,3 & 1,15 & 101 & 5,4 \\
\hline $2-019$ & 460 & S460 & $\overline{G 46}$ & \begin{tabular}{|l|}
4.5 \\
\end{tabular} & 1,40 & 20 & 5,3 & 1,38 & 52 & 5,5 & 1,46 & 80 & 6,3 & 1,46 & 90 & 6,4 \\
\hline $2-020$ & 460 & S460 & G69 & 4.4 & 1,08 & 75 & 4,7 & 1,10 & 82 & 4,4 & 1,07 & 93 & 4,9 & 1,06 & 102 & 5,1 \\
\hline $2-021$ & S460 & S460 & G46 & 4.4 & 1,10 & 30 & 3,6 & 1,14 & 45 & 4,2 & 1,20 & 60 & 5,0 & 1,27 & 70 & 5,6 \\
\hline $2-022$ & 460 & S460 & G46 & \begin{tabular}{|l|}
4.6 \\
\end{tabular} & 1,87 & 29 & 7,7 & 1,69 & 57 & 6,9 & 1,80 & 75 & 6,7 & 1,96 & 78 & 9,0 \\
\hline $2-0$ & 60 & S460 & G42 & \begin{tabular}{|l|}
4.4 \\
\end{tabular} & 1,24 & 27 & 5,1 & \begin{tabular}{|l|}
1,08 \\
\end{tabular} & 45 & 4,2 & 1,07 & 60 & 4,6 & 1,21 & 63 & 5,5 \\
\hline $2-0$ & 60 & S460 & 42 & \begin{tabular}{|l|}
4.6 \\
\end{tabular} & 1,12 & 35 & 3,8 & 1,24 & 50 & 4,8 & 1,30 & 80 & 5,0 & 1,29 & 60 & 5,7 \\
\hline $2-025$ & 60 & $\mathrm{~S} 460$ & G42 & 4.5 & 1,25 & 35 & 4,4 & 1,23 & 40 & 4,8 & 1,30 & 60 & 5,3 & 1,17 & 80 & 4,9 \\
\hline $2-026$ & 60 & S460 & G69 & 4.5 & 1,37 & 85 & 6,4 & 1,38 & 90 & 6,8 & 1,29 & 102 & 6,5 & 1,37 & 100 & 7,2 \\
\hline $2-030$ & S690 & S690 & G69 & \begin{tabular}{|l|}
4.5 \\
\end{tabular} & 1,50 & 80 & 6,6 & 1,48 & 85 & 8,2 & - & 102 & 8,7 & 1,68 & 96 & 8,1 \\
\hline 2-031 & S690 & S690 & G69 & 4.6 & 1,88 & 83 & 8,2 & 1,78 & 101 & 7,4 & 1,92 & 99 & - & 1,91 & 97 & $\begin{array}{l}19 * \\
*\end{array}$ \\
\hline $2-W 31$ & S690 & S690 & G69 & \begin{tabular}{|l|}
4.6 \\
\end{tabular} & & & & & & & & & & & & \\
\hline 2-032* & S690 & S690 & G69 & 4.4 & 1,11 & 90 & $2,9 *$ & 1,14 & 86 & $2,7 *$ & 1,11 & 96 & - & $5^{-}$ & 90 & $3,3^{*}$ \\
\hline $2-034 * *$ & S690 & S690 & G46 & \begin{tabular}{|l|}
4.4 \\
\end{tabular} & 1,18 & 97 & 9,4 & 1,18 & 92 & 11,8 & 1,17 & 87 & 10,5 & 1,13 & 88 & 11,5 \\
\hline 2-035** & S690 & S690 & G46 & 4.6 & 1,92 & 84 & 18,7 & 1,84 & 97 & 17,3 & 1,89 & 88 & 18,6 & 1,83 & 105 & 18,4 \\
\hline $2-036 * *$ & S690 & S690 & G46 & \begin{tabular}{|l|}
4.5 \\
\end{tabular} & 1,47 & 80 & 13,8 & 1,57 & 93 & 15,5 & 1,68 & 102 & & 1,75 & 100 & 20 \\
\hline
\end{tabular}


* nicht für statistische Auswertung verwendet

** Werte nicht plausibel, vermutlich Messfehler

\section{A.3 Überblick Schweißverbindungen nach [Zusatzversuche, 2008]}

\section{A.3.1 Chemische Kennwerte der verwendeten Grund- und Schweißzusatzwerk- stoffe}

Tabelle A.44: Chemische Zusammensetzung der Grundwerkstoffe gemäß Lieferzeugnis in [\%] nach [Zusatzversuche, 2008]

\begin{tabular}{|l|l|l|l|l|l|l|l|l|l|}
\hline Werkstoff & Blechdicke & $\mathrm{C}$ & $\mathrm{Si}$ & $\mathrm{Mn}$ & $\mathrm{P}$ & $\mathrm{S}$ & $\mathrm{Al}_{\text {ges }}$ & $\mathrm{N}$ & $\mathrm{Cu}$ \\
\hline S355J2 & 15 & 0,163 & 0,469 & 1,56 & 0,010 & 0,0007 & 0,043 & 0,0039 & 0,030 \\
\hline S690QL & 20 & 0,056 & 0,32 & 1,89 & 0,01 & 0,0007 & 0,032 & & 0,017 \\
\hline Werkstoff & Blechdicke & $\mathrm{Mo}$ & $\mathrm{Ni}$ & $\mathrm{Cr}$ & $\mathrm{Nb}$ & $\mathrm{Ti}$ & $\mathrm{N} 2$ & $\mathrm{CE}$ & $\mathrm{CET}$ \\
\hline S355J2 & 15 & 0,007 & 0,035 & 0,033 & & 0,009 & & 0,45 & 0,34 \\
\hline S690QL & 20 & 0,12 & & & 0,042 & 0,012 & 0,012 & 0,43 & 0,27 \\
\hline
\end{tabular}

Tabelle A.45: Chemische Zusammensetzung der Schweißzusatzwerkstoffe gemäß Lieferzeugnis in [\%] nach [Zusatzversuche, 2008]

\begin{tabular}{|l|l|l|l|l|l|l|l|l|l|l|l|l|l|l|}
\hline $\begin{array}{l}\text { Schweißzu- } \\
\text { satzwerkstoff }\end{array}$ & $\mathrm{C}$ & $\mathrm{Si}$ & $\mathrm{Mn}$ & $\mathrm{P}$ & $\mathrm{S}$ & $\mathrm{Al}$ & $\mathrm{Cu}$ & $\mathrm{Mo}$ & $\mathrm{Ni}$ & $\mathrm{Cr}$ & $\mathrm{V}$ & $\mathrm{CE}$ & $\mathrm{CET}$ \\
\hline $\begin{array}{l}\text { Union } \\
\text { NiMoCr }\end{array}$ & $\mathrm{G} 69$ & 0,10 & 0,45 & 1,26 & 0,004 & 0,014 & 0,007 & 0,10 & 0,49 & 1,33 & 0,27 & $<0,001$ & 0,56 & 0,33 \\
\hline $\begin{array}{l}\text { Union } \\
\text { X90 }\end{array}$ & $\mathrm{G} 89$ & 0,10 & 0,58 & 1,41 & 0,006 & 0,015 & $<0,001$ & 0,089 & 0,62 & 2,10 & 0,38 & 0,008 & 0,67 & 0,38 \\
\hline
\end{tabular}

\section{A.3.2 Versuchskörpertyp}

Tabelle A.46: Versuchskörpertyp 1 - Flankenkehlnahtverbindung, [Zusatzversuche, 2008]

\begin{tabular}{|c|c|c|c|c|c|c|c|c|c|}
\hline $\mathrm{VK}$ & $\mathrm{a}$ & $\mathrm{L}$ & $\mathrm{b} \_1$ & I__1 & $\mathrm{t} \_1$ & b_2 & $11 \_2$ & $\overline{\mathrm{t} \_2}$ & \\
\hline \begin{tabular}{|l|}
1.1 \\
\end{tabular} & 4 & 100 & 160 & 750 & 20 & 200 & 750 & 20 & {$[\mathrm{~mm}]$} \\
\hline 1.2 & 4 & 100 & 160 & 750 & 15 & 200 & 750 & 15 & {$[\mathrm{~mm}]$} \\
\hline 1.3 & 9 & 135 & 160 & 750 & 20 & 200 & 750 & 20 & {$[\mathrm{~mm}]$} \\
\hline
\end{tabular}




\section{A.3.3 Material und Schweißparameter der Flankenkehlnahtverbindungen}

Tabelle A.47: Durchgeführte Untersuchungen an Flankenkehlnahtverbindungen, [Zusatzversuche, 2008]

\begin{tabular}{|c|c|c|c|c|c|c|c|c|c|c|c|c|c|}
\hline$\sum_{\text {Z }}^{\sum_{1}^{\prime}}$ & 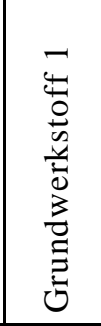 & 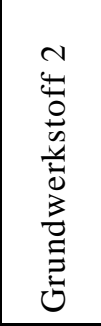 & 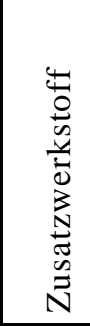 & 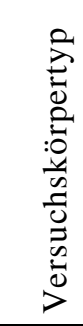 & 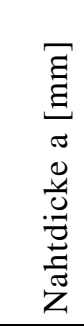 & 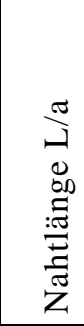 & 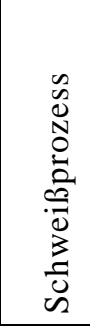 & 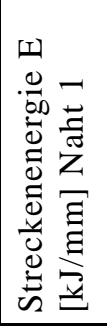 & 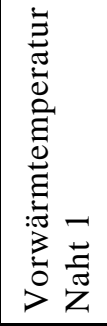 & 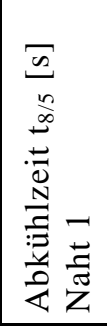 & 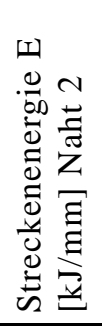 & 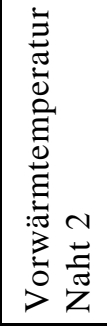 & 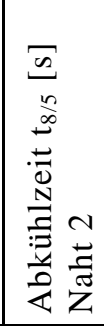 \\
\hline Z-001 & S690 & S690 & G69 & 1.1 & 4 & 25 & 135 & 1,60 & 90 & 7,5 & 1,61 & 91 & 7,6 \\
\hline Z-002 & S690 & S690 & G69 & 1.1 & 4 & 25 & 135 & 1,63 & 90 & 7,6 & 1,63 & 98 & 7,7 \\
\hline Z-003 & S690 & S690 & G69 & 1.1 & 4 & 25 & 135 & 1,94 & 90 & 10,0 & 1,98 & 94 & 10,1 \\
\hline Z-004 & S690 & S690 & G69 & 1.1 & 4 & 25 & 135 & 2,45 & 90 & 14,6 & 2,40 & 106 & 14,5 \\
\hline Z-005 & S690 & S690 & G69 & 1.1 & 4 & 25 & 135 & 1,55 & 92 & 6,7 & 1,55 & 93 & 8,0 \\
\hline Z-006 & S690 & S690 & G89 & 1.1 & 4 & 25 & 135 & 1,55 & 94 & 7,2 & 1,55 & 99 & 8,2 \\
\hline Z-007* & S690 & S690 & G89 & 1.2 & 9 & 15 & 135 & 1,59 & 92 & 8,6 & 1,56 & 78 & 8,6 \\
\hline Z-008* & S690 & S690 & G89 & 1.2 & 9 & 15 & 135 & 1,33 & 77 & 4,9 & 1,34 & 101 & 5,6 \\
\hline Z-009 & S355 & S355 & G89 & 1.3 & 4 & 25 & 135 & 1,32 & 102 & 4,9 & 1,35 & 104 & 5,6 \\
\hline
\end{tabular}




\section{A.4 Überblick Schweißverbindungen nach [FOSTA P812, 2011]}

\section{A.4.1 Chemische Kennwerte der verwendeten Grund- und Schweißzusatzwerk- stoffe}

Tabelle A.48: Chemische Zusammensetzung der verwendeten Grundwerkstoffe gemäß Lieferzeugnis in [\%] nach [FOSTA P812, 2011]

\begin{tabular}{|c|c|c|c|c|c|c|c|c|c|c|}
\hline Werkstoff & \begin{tabular}{|l} 
Dicke \\
{$[\mathrm{mm}]$}
\end{tabular} & $\mathrm{C}$ & $\mathrm{Si}$ & Mn & $\mathrm{P}$ & $S$ & $\mathrm{Al}_{\text {ges }}$ & $\mathrm{N}$ & \multicolumn{2}{|l|}{$\mathrm{Cu}$} \\
\hline S460M & 17,6 & 0,029 & 0,33 & 1,51 & 0,0074 & 0,0007 & 0,031 & & \multicolumn{2}{|l|}{0,017} \\
\hline S690QL1 & 15 & 0,170 & 0,282 & 1,28 & 0,013 & 0,0010 & - & 0,0045 & \multicolumn{2}{|l|}{0,026} \\
\hline S700MC & 15 & 0,056 & 0,320 & 1,620 & 0,013 & 0,0007 & 0,033 & 0,0041 & \multicolumn{2}{|l|}{0,014} \\
\hline S690Q & 20 & 0,161 & 0,284 & 1,29 & 0,014 & 0,0005 & & 0,0033 & \multicolumn{2}{|l|}{0,024} \\
\hline S700MC & 20 & 0,054 & 0,310 & 1,600 & 0,010 & 0,0006 & 0,031 & 0,0059 & \multicolumn{2}{|l|}{0,026} \\
\hline S690Q & 50 & 0,171 & 0,285 & 1,30 & 0,010 & 0,0005 & & 0,0030 & \multicolumn{2}{|l|}{0,017} \\
\hline S700MC & 50 & 0,054 & 0,310 & 1,600 & 0,010 & 0,0006 & 0,031 & 0,0059 & \multicolumn{2}{|l|}{0,017} \\
\hline Werkstoff & $\begin{array}{l}\text { Dicke } \\
{[\mathrm{mm}]}\end{array}$ & Mo & $\mathrm{Ni}$ & $\mathrm{Cr}$ & V & $\mathrm{Nb}$ & $\mathrm{Ti}$ & B & CE & CET \\
\hline S460M & 17,6 & 0,01 & & 0,2 & 0,003 & 0,041 & & & 0,330 & 0,191 \\
\hline S690QL1 & 15 & 0,112 & 0,048 & 0,046 & 0,001 & 0,024 & 0,003 & 0,0019 & 0,419 & 0,313 \\
\hline S700MC & 15 & 0,230 & 0,027 & 0,410 & 0,036 & 0,037 & 0,013 & 0,0012 & 0,464 & 0,263 \\
\hline S690Q & 20 & 0,113 & 0,051 & 0,068 & 0,001 & 0,025 & 0,002 & 0,0017 & 0,417 & 0,307 \\
\hline S700MC & 20 & 0,210 & 0,035 & 0,410 & 0,038 & 0,037 & 0,012 & 0,0014 & 0,456 & 0,258 \\
\hline S690QL & 50 & 0,261 & 0,126 & 0,319 & 0,001 & 0,030 & 0,003 & 0,0018 & 0,513 & 0,347 \\
\hline S700MC & 50 & 0,210 & 0,035 & 0,410 & 0,038 & 0,037 & 0,012 & 0,0014 & 0,456 & 0,258 \\
\hline
\end{tabular}

Tabelle A.49: Chemische Zusammensetzung der Schweißzusatzwerkstoffe gemäß Lieferzeugnis in [\%] nach [FOSTA P812, 2011]

\begin{tabular}{|l|l|l|l|l|l|l|l|l|l|l|}
\hline \multicolumn{2}{|l|}{ Schweißzusatzwerkstoff } & $\varnothing$ & $\mathrm{C}$ & $\mathrm{Si}$ & $\mathrm{Mn}$ & $\mathrm{Cr}$ & $\mathrm{Mo}$ & $\mathrm{Ni}$ & $\mathrm{P}$ & $\mathrm{S}$ \\
\hline Union K56 & G46 & 1,2 & 0,079 & 0,74 & 1,30 & 0,03 & 0,03 & 0,02 & 0,008 & 0,008 \\
\hline Union NiMoCr & G69A & 1,2 & 0,100 & 0,42 & 1,32 & 0,15 & 0,53 & 1,38 & 0,008 & 0,009 \\
\hline OK AristoRod 13.29 & G69B & 1,2 & 0,08 & 0,50 & 1,61 & 0,30 & 0,22 & 1,37 & 0,014 & 0,006 \\
\hline STEIN-Megafil 742 B & T69 & 1,2 & 0,090 & 0,377 & 1,450 & 0,450 & 0,343 & 2,178 & 0,016 & 0,011 \\
\hline Union X90 & G89 & 1,2 & 0,100 & 0,56 & 1,34 & 0,33 & 0,62 & 2,19 & 0,008 & 0,009 \\
\hline Schweißzusatzwerkstoff & $\varnothing$ & $\mathrm{Cu}$ & $\mathrm{V}$ & $\mathrm{Al}$ & $\mathrm{Ti}$ & $\mathrm{Zr}$ & $\mathrm{CE}$ & $\mathrm{CET}$ & \\
\hline Union K56 & G46 & 1,2 & 0,07 & $<0,001$ & 0,004 & 0,005 & $<0,001$ & 0,314 & 0,218 & \\
\hline Union NiMoCr & G69A & 1,2 & 0,12 & $<0,001$ & 0,004 & 0,023 & $<0,001$ & 0,556 & 0,333 \\
\hline OK AristoRod 13.29 & G69B & 1,2 & 0,02 & 0,07 & $<0,01$ & $<0,01$ & $<0,01$ & 0,559 & 0,313 \\
\hline STEIN-Megafil 742 B & T69 & 1,2 & - & - & - & - & - & 0,636 & 0,346 \\
\hline Union X90 & G89 & 1,2 & 0,15 & 0,001 & 0,005 & 0,022 & $<0,001$ & 0,670 & 0,375 & \\
\hline
\end{tabular}




\section{A.4.2 Versuchskörpertypen}

Tabelle A.50: Versuchskörpertyp 1 - Flankenkehlnahtverbindung, [FOSTA P812, 2011]

\begin{tabular}{|l|l|l|l|l|l|l|l|l|}
\hline & & & \\
\hline
\end{tabular}

Tabelle A.51: Versuchskörpertyp 2 - Flankenkehlnahtverbindung, [FOSTA P812, 2011]

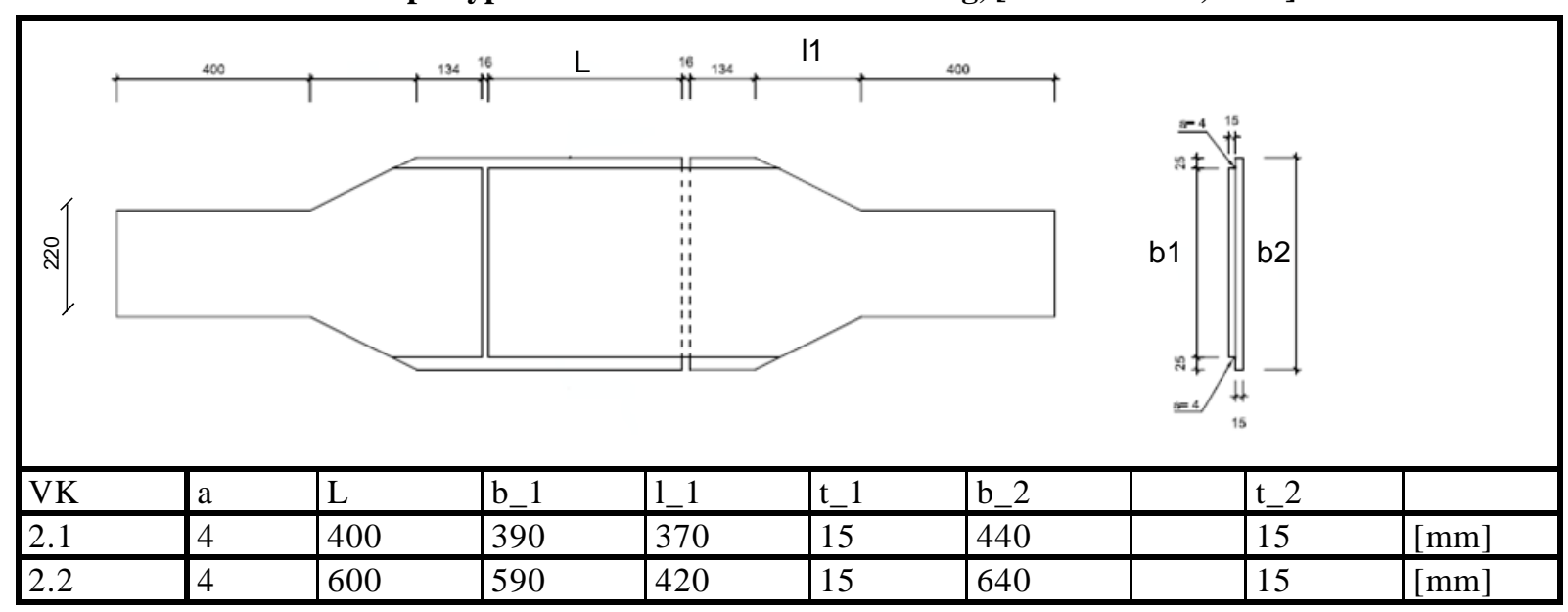

Tabelle A.52: Versuchskörpertyp 3 - Kreuzstoß, [FOSTA P812, 2011]

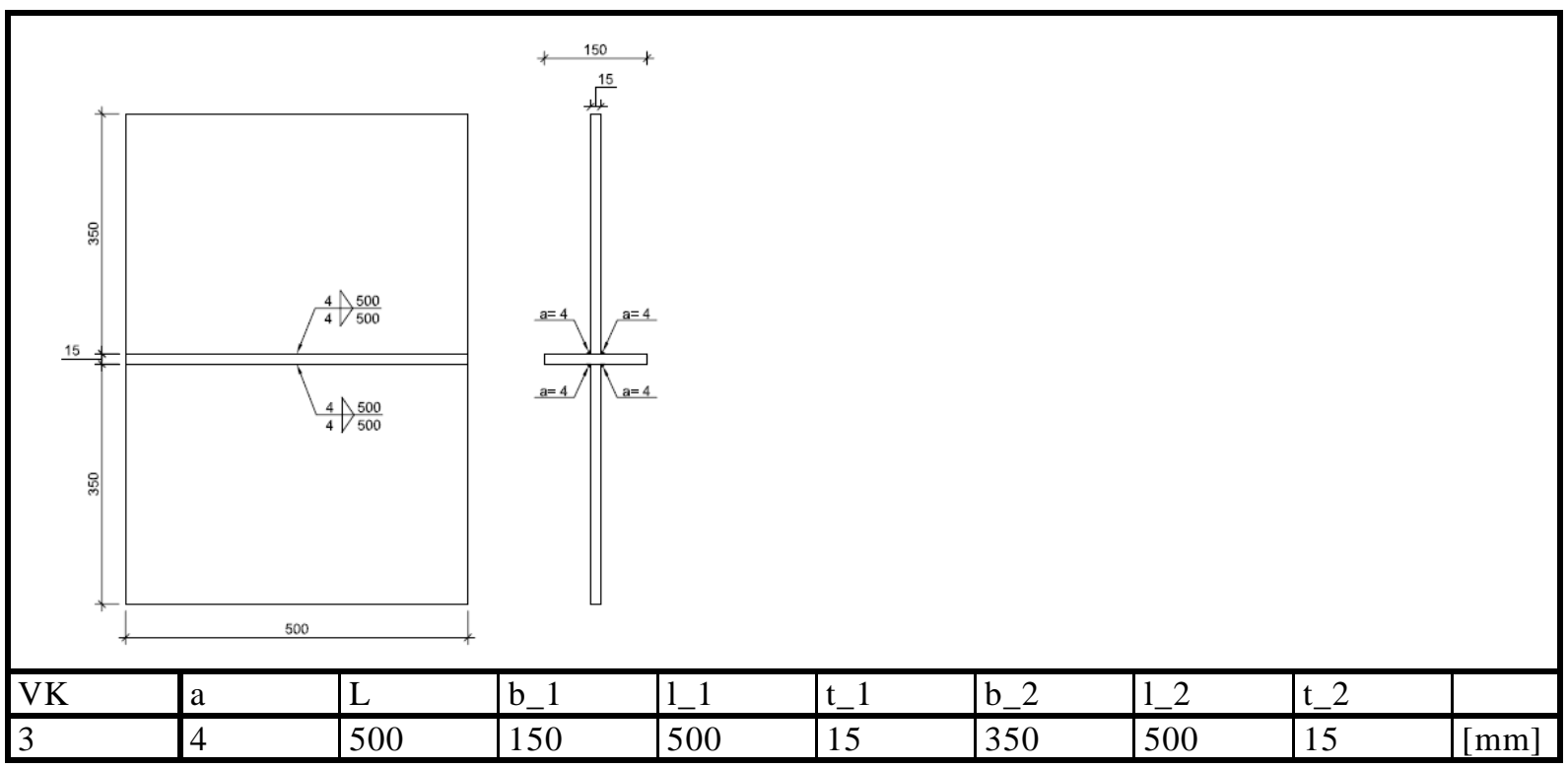




\section{A.4.3 Material und Schweißparameter der Flankenkehlnahtverbindungen}

Tabelle A.53: Durchgeführte Untersuchungen an Flankenkehlnahtverbindungen, [FOSTA P812, 2011]

\begin{tabular}{|c|c|c|c|c|c|c|c|c|c|c|c|c|c|}
\hline$\sum_{\text {乙 }}^{\sum_{1}^{\prime}}$ & 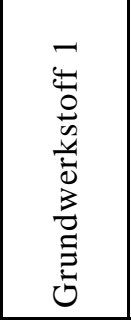 & 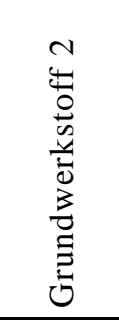 & 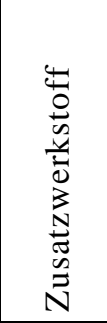 & 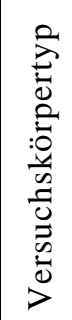 & 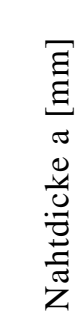 & 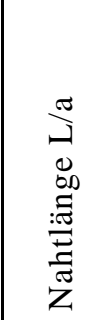 & 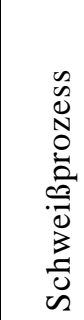 & 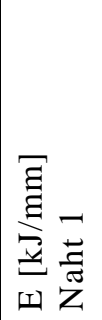 & 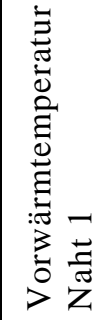 & 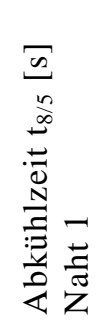 & 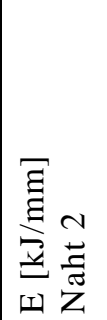 & 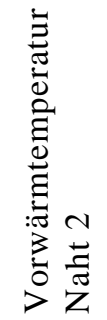 & 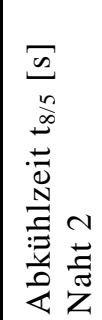 \\
\hline $1-01$ & S700A & S700A & G69A & 1.1 & 4 & 25 & 135 & 1,39 & 100 & 6,7 & 1,36 & 100 & - \\
\hline Z1-01 & S700A & S700A & G69A & 1.1 & 4 & 25 & 135 & 1,40 & 100 & 6,7 & 1,38 & 100 & 6,8 \\
\hline $1-02$ & S700A & S700A & G69A & 1.1 & 4 & 25 & 135 & 1,33 & 100 & 6,8 & 1,41 & 100 & 7,1 \\
\hline $1-03$ & S700A & S700A & G69B & 1.1 & 4 & 25 & 135 & 1,34 & 100 & - & 1,34 & 100 & 6,4 \\
\hline $1-04$ & S700A & S700A & G69B & 1.1 & 4 & 25 & 135 & 1,33 & 100 & 6,0 & 1,33 & 100 & 6,3 \\
\hline $1-05$ & S700A & S700A & T69 & 1.1 & 4 & 25 & 136 & 1,33 & 100 & 6,1 & 1,30 & 100 & 6,3 \\
\hline $1-06$ & S700A & S700A & T69 & 1.1 & 4 & 25 & 136 & 1,32 & 100 & 6,0 & 1,33 & 100 & 6,1 \\
\hline $1-07$ & S690B & S690B & G69A & 1.1 & 4 & 25 & 135 & 1,36 & 100 & - & 1,35 & 100 & - \\
\hline $1-08$ & S690B & S690B & G69A & 1.1 & 4 & 25 & 135 & 1,37 & 100 & 5,6 & 1,39 & 100 & - \\
\hline 1-09 & S690B & S690B & G69B & 1.1 & 4 & 25 & 135 & - & 100 & 5,3 & 1,31 & 100 & 5,0 \\
\hline $1-10$ & S690B & S690B & G69B & 1.1 & 4 & 25 & 135 & 1,32 & 100 & - & 1,30 & 100 & 5,6 \\
\hline $1-11$ & S690B & S690B & T69 & 1.1 & 4 & 25 & 136 & 1,32 & 100 & - & 1,34 & 100 & 5,7 \\
\hline $1-12$ & S690B & S690B & T69 & 1.1 & 4 & 25 & 136 & 1,35 & 100 & 5,7 & 1,34 & 100 & 5,7 \\
\hline $2-01$ & S700A & S700A & G69A & 1.1 & 4 & 25 & 135 & 1,36 & 175 & 9,3 & 1,37 & 175 & 10,0 \\
\hline Z2-01 & S700A & S700A & G69A & 1.1 & 4 & 25 & 135 & 1,38 & 175 & 10,1 & 1,38 & 175 & 8,6 \\
\hline $2-02$ & S700A & S700A & G69A & 1.1 & 4 & 25 & 135 & 0,00 & 175 & 9,3 & 1,35 & 175 & 9,3 \\
\hline $2-03$ & S700A & S700A & G69A & 1.1 & 4 & 25 & 135 & 1,37 & 235 & 16,2 & 1,36 & 235 & 15,0 \\
\hline Z2-03 & S700A & S700A & G69A & 1.1 & 4 & 25 & 135 & 0,00 & 235 & 15,4 & 1,36 & 235 & 13,3 \\
\hline $2-04$ & S700A & S700A & G69A & 1.1 & 4 & 25 & 135 & 1,36 & 235 & 13,4 & 1,37 & 235 & 11,6 \\
\hline $3-01$ & S700A & S700A & G69A & 1.3 & 4 & 50 & 135 & 1,27 & 100 & 6,0 & 1,30 & 100 & 6,0 \\
\hline 3-02 & S700A & S700A & G69A & 1.3 & 4 & 50 & 135 & 1,29 & 100 & 6,7 & 1,31 & 100 & 6,7 \\
\hline $3-03$ & S690B & S690B & G69B & 1.3 & 4 & 50 & 135 & 1,25 & 100 & 5,0 & 1,26 & 100 & 5,0 \\
\hline $3-04$ & S690B & S690B & G69B & 1.3 & 4 & 50 & 135 & 1,27 & 100 & 5,6 & 1,27 & 100 & 5,6 \\
\hline $3-05$ & S700A & S700A & G69A & 2.1 & 4 & 100 & 135 & 1,33 & 100 & - & 1,35 & 100 & - \\
\hline 3-06 & S700A & S700A & G69A & 2.1 & 4 & 100 & 135 & 1,32 & 100 & 6,8 & 1,33 & 100 & 6,8 \\
\hline $3-07$ & S690B & S690B & G69B & 2.1 & 4 & 100 & 135 & 1,27 & 100 & 5,6 & 1,28 & 100 & 5,6 \\
\hline $3-08$ & S690B & S690B & G69B & 2.1 & 4 & 100 & 135 & 1,28 & 100 & 4,9 & 1,26 & 100 & 4,9 \\
\hline 3-09 & S700A & S700A & G69A & 2.2 & 4 & 150 & 135 & 1,33 & 100 & 6,4 & 1,32 & 100 & 6,4 \\
\hline $3-10$ & S700A & S700A & G69A & 2.2 & 4 & 150 & 135 & 1,30 & 100 & 5,7 & 1,31 & 100 & 5,7 \\
\hline $3-11$ & S690B & S690B & G69B & 2.2 & 4 & 150 & 135 & 1,28 & 100 & - & 1,26 & 100 & - \\
\hline $3-12$ & S690B & S690B & G69B & 2.2 & 4 & 150 & 135 & 1,26 & 100 & 5,7 & 1,28 & 100 & 5,7 \\
\hline $4-01 *$ & S700A & S700A & G69A & 1.4 & 6 & 25 & 135 & 1,32 & 100 & 6,7 & 1,31 & 100 & 5,3 \\
\hline Z4-01* & S700A & S700A & G69A & 1.4 & 6 & 25 & 135 & 1,30 & 100 & 6,7 & 1,30 & 100 & 6,7 \\
\hline $4-02 *$ & S700A & S700A & G69A & 1.4 & 6 & 25 & 135 & 1,30 & 100 & - & 1,29 & 100 & 6,0 \\
\hline 4-03* & S690B & S690B & G69B & 1.4 & 6 & 25 & 135 & 1,27 & 100 & 5,7 & 1,26 & 100 & 5,7 \\
\hline 4-04* & S690B & S690B & G69B & 1.4 & 6 & 25 & 136 & 1,25 & 100 & 5,7 & 1,26 & 100 & 5,7 \\
\hline $4-05 *$ & S700A & S700A & G69A & 1.5 & 10 & 25 & 136 & 1,28 & 100 & 6,0 & 1,30 & 100 & 6,4 \\
\hline 4-06* & S700A & S700A & G69A & 1.5 & 10 & 25 & 135 & 1,29 & 100 & 6,4 & 1,29 & 100 & 5,6 \\
\hline Z4-06* & S700A & S700A & G69A & 1.5 & 10 & 25 & 135 & 1,24 & 100 & - & 1,28 & 100 & 6,4 \\
\hline $4-07 *$ & S690B & S690B & G69B & 1.5 & 10 & 25 & 135 & 1,23 & 100 & 6,3 & 1,22 & 100 & 6,0 \\
\hline $4-08^{*}$ & S690B & S690B & G69B & 1.5 & 10 & 25 & 135 & 1,22 & 100 & 6,0 & 1,21 & 100 & 4,9 \\
\hline
\end{tabular}


Forstsetzung Tabelle A.53: Durchgeführte Untersuchungen an Flankenkehlnahtverbindungen, [FOSTA P812, 2011]

\begin{tabular}{|c|c|c|c|c|c|c|c|c|c|c|c|c|c|}
\hline$\sum_{z}^{1}$ & 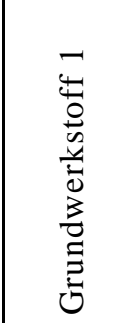 & 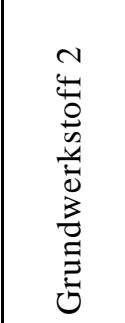 & 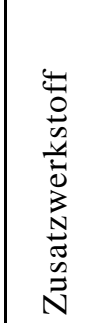 & 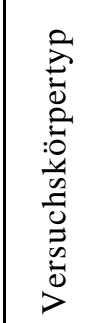 & 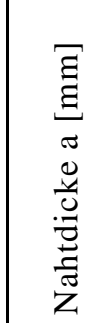 & 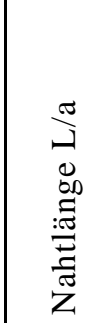 & 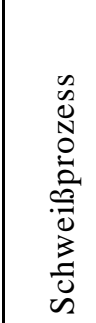 & 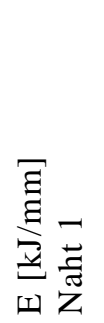 & 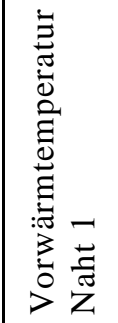 & 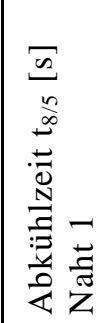 & 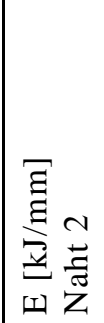 & 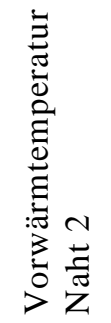 & 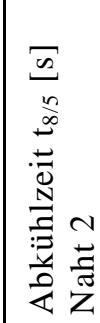 \\
\hline 5-01 & S700A & S700A & G46 & 1.1 & 4 & 25 & 135 & 1,31 & 100 & $\overline{6,0}$ & 1,32 & 100 & - \\
\hline Z5-01 & S700A & S700A & G46 & 1.1 & 4 & 25 & 135 & 1,32 & 100 & - & 1,33 & 100 & - \\
\hline \begin{tabular}{|l|}
$5-02$ \\
\end{tabular} & S700A & S700A & G46 & 1.1 & 4 & 25 & 135 & 1,31 & 100 & 5,7 & 1,31 & 100 & 6,3 \\
\hline \begin{tabular}{|l|}
$5-03$ \\
\end{tabular} & S700A & S700A & G89 & 1.1 & 4 & 25 & 135 & 1,29 & 100 & 4,9 & 1,29 & 100 & 5,0 \\
\hline Z5-03 & S700A & S700A & G89 & 1.1 & 4 & 25 & 135 & 1,27 & 100 & - & 1,26 & 100 & - \\
\hline \begin{tabular}{|l|}
$5-04$ \\
\end{tabular} & S700A & S700A & G89 & 1.1 & 4 & 25 & 135 & 1,28 & 100 & - & 1,28 & 100 & 4,9 \\
\hline $5-05$ & S690B & S690B & G46 & 1.1 & 4 & 25 & 135 & 1,28 & 100 & - & 1,31 & 100 & - \\
\hline 5-06 & S690B & S690B & G46 & 1.1 & 4 & 25 & 135 & 1,30 & 100 & - & 1,30 & 100 & 5,0 \\
\hline 5-07 & S690B & S690B & G89 & 1.1 & 4 & 25 & 135 & 1,27 & 100 & 3,9 & 1,27 & 100 & - \\
\hline \begin{tabular}{|l|}
$5-08$ \\
\end{tabular} & S690B & S690B & G89 & 1.1 & 4 & 25 & 135 & 1,29 & 100 & 5,0 & 1,23 & 100 & 4,6 \\
\hline \begin{tabular}{|l|}
$7-01$ \\
\end{tabular} & S460 & S460 & G46 & 1.2 & 4 & 25 & 135 & 1,24 & 100 & 5,6 & 1,28 & 100 & 6,0 \\
\hline Z7-01 & S460 & S460 & G46 & 1.2 & 4 & 25 & 135 & - & 20 & 3,9 & 1,27 & 20 & 4,2 \\
\hline \begin{tabular}{|l|}
$7-02$ \\
\end{tabular} & S460 & S460 & G46 & 1.2 & 4 & 25 & 135 & 1,29 & 100 & 5,3 & 1,29 & 100 & 5,7 \\
\hline \begin{tabular}{|l|}
$7-03$ \\
\end{tabular} & S460 & S460 & G46 & 1.2 & 4 & 25 & 135 & 1,27 & 50 & 5,0 & 1,29 & 50 & 4,8 \\
\hline 7-04 & S460 & S460 & G46 & 1.2 & 4 & 25 & 135 & 1,28 & 50 & 4,6 & 1,28 & 50 & 4,6 \\
\hline
\end{tabular}

\section{A.4.4 Material und Schweißparameter der Kreuzstöße}

Tabelle A.54: Durchgeführte Untersuchungen an Kreuzstößen, [FOSTA P812, 2011]

\begin{tabular}{|c|c|c|c|c|c|c|c|c|c|c|c|c|c|c|c|c|}
\hline$\sum_{Z}^{L}$ & 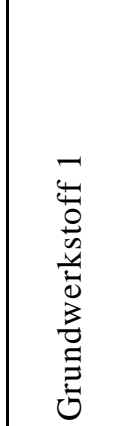 & 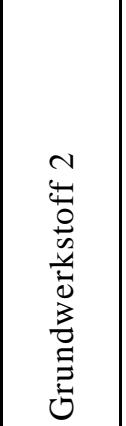 & 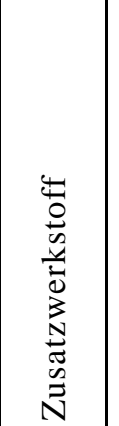 & 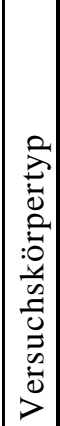 & 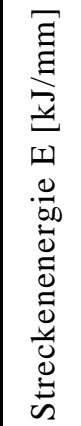 & 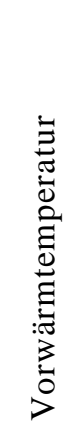 & 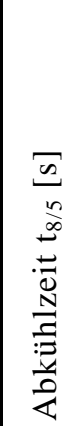 & 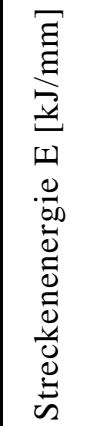 & 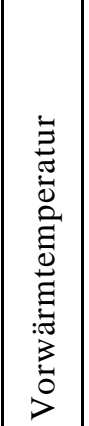 & 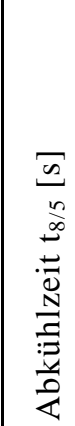 & 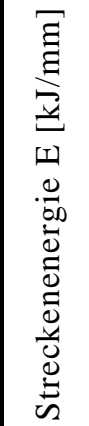 & 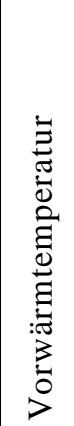 & 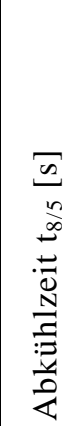 & 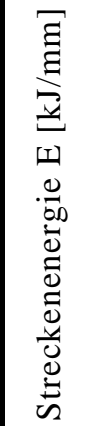 & 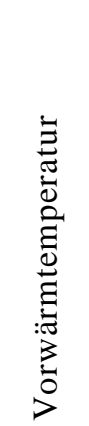 & 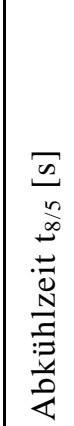 \\
\hline & & & & & \multicolumn{3}{|c|}{\begin{tabular}{|l} 
Naht 1 \\
\end{tabular}} & \multicolumn{3}{|c|}{ Naht 2} & \multicolumn{3}{|c|}{\begin{tabular}{|l|} 
Naht 3 \\
\end{tabular}} & \multicolumn{3}{|c|}{\begin{tabular}{|l|} 
Naht 4 \\
\end{tabular}} \\
\hline 6-01 & S700A & $700 \mathrm{~A}$ & G69A & 3 & 13,0 & 100 & 6,1 & 13,0 & \begin{tabular}{|l|}
100 \\
\end{tabular} & 6,0 & 13,0 & 100 & 5,3 & 13,1 & 100 & 6,0 \\
\hline 6-02 & S700A & S700A & G46 & 3 & 12,5 & 100 & & 12,8 & \begin{tabular}{|l|}
100 \\
\end{tabular} & 5,6 & 12,6 & 100 & 5,2 & 12,6 & 100 & 5,4 \\
\hline $6-03$ & S700A & S700A & G89 & 3 & 12,4 & 100 & 5,0 & 12,5 & 100 & 5,3 & 12,4 & 100 & 6,1 & 12,1 & 100 & 4,9 \\
\hline 6-04 & S690B & S690B & G69B & 3 & 12,9 & 100 & 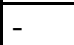 & 12,8 & \begin{tabular}{|l|}
100 \\
\end{tabular} & & 12,6 & 100 & 5,0 & 12,6 & 100 & 4,9 \\
\hline $6-05$ & S690B & S690B & G46 & 3 & 12,4 & 100 & 6,0 & 12,2 & 100 & 5,2 & 12,5 & 100 & 5,3 & 12,4 & 100 & 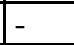 \\
\hline $6-06$ & S690B & S690B & G89 & 3 & 12,1 & 100 & 4,9 & 12,2 & \begin{tabular}{|l|}
100 \\
\end{tabular} & 5,0 & 12,2 & 100 & 4,9 & 12,2 & 100 & 4,6 \\
\hline Z6-01 & S700A & S700A & G69A & 3 & 13,1 & 100 & 6,0 & 13,0 & 100 & & 13,2 & 100 & & 13,2 & 100 & \\
\hline
\end{tabular}




\section{A.5 Statistische Auswertung zur Entwicklung eines Bemessungsvor- schlags}

\section{A.5.1 Allgemeines}

In Kapitel 10 werden alle Versuche, die in dieser Arbeit verwendet werden, vorgestellt. Die Versuche wurden getrennt nach Schweißzusatzwerkstoff statistisch ausgewertet, da sich für jeden Schweißzusatzwerkstoff ein anderer Beiwert $\beta_{\mathrm{w}}$ einstellt. Als Bemessungsgleichung dient der Vorschlag nach Gleichung (10.5), der in Gleichung (A.26) nochmal aufgeführt ist. Das heißt, die Versuchsergebnisse werden unter Berücksichtigung der Festigkeiten von Grund- und Schweißzusatzwerkstoffen statistisch ausgewertet.

$$
\text { Für Flankenkehlnähte } \tau_{\mathrm{II}}=\frac{0,25 \cdot \mathrm{f}_{\mathrm{u}, \mathrm{GW}}+0,75 \cdot \mathrm{f}_{\mathrm{u}, \mathrm{SZW}}}{\sqrt{3} \cdot \beta_{\mathrm{w}} \cdot \gamma_{\mathrm{M} 2}}
$$

Im Folgenden werden die Ergebnisse der statistischen Auswertung für die verschiedenen Schweißzusatzwerkstoffe aufgeführt. Neben der Angabe der statistischen Werte wird zusätzlich für die einzelnen Versuchsserien die Bemessungsgrenzspannung in Abhängigkeit der Mittelwerte von Grund- und Schweißzusatzwerkstoff unter Verwendung des ermittelten Beiwertes $\beta_{\mathrm{w}}$ angegeben.

\section{A.5.2 Schweißverbindungen mit dem Schweißzusatzwerkstoff der nominellen Streckgrenze $\mathrm{f}_{\mathrm{y}}=\mathbf{4 2 0} \mathrm{N} / \mathrm{mm}^{2}$}

Die Ergebnisse der statistischen Auswertung von Schweißverbindungen mit dem Schweißzusatzwerkstoffen G42 und E42 sind in Tabelle A.55 aufgeführt.

Tabelle A.55: Statistische Auswertung aller Flankenkehlnahtverbindungen G/E42

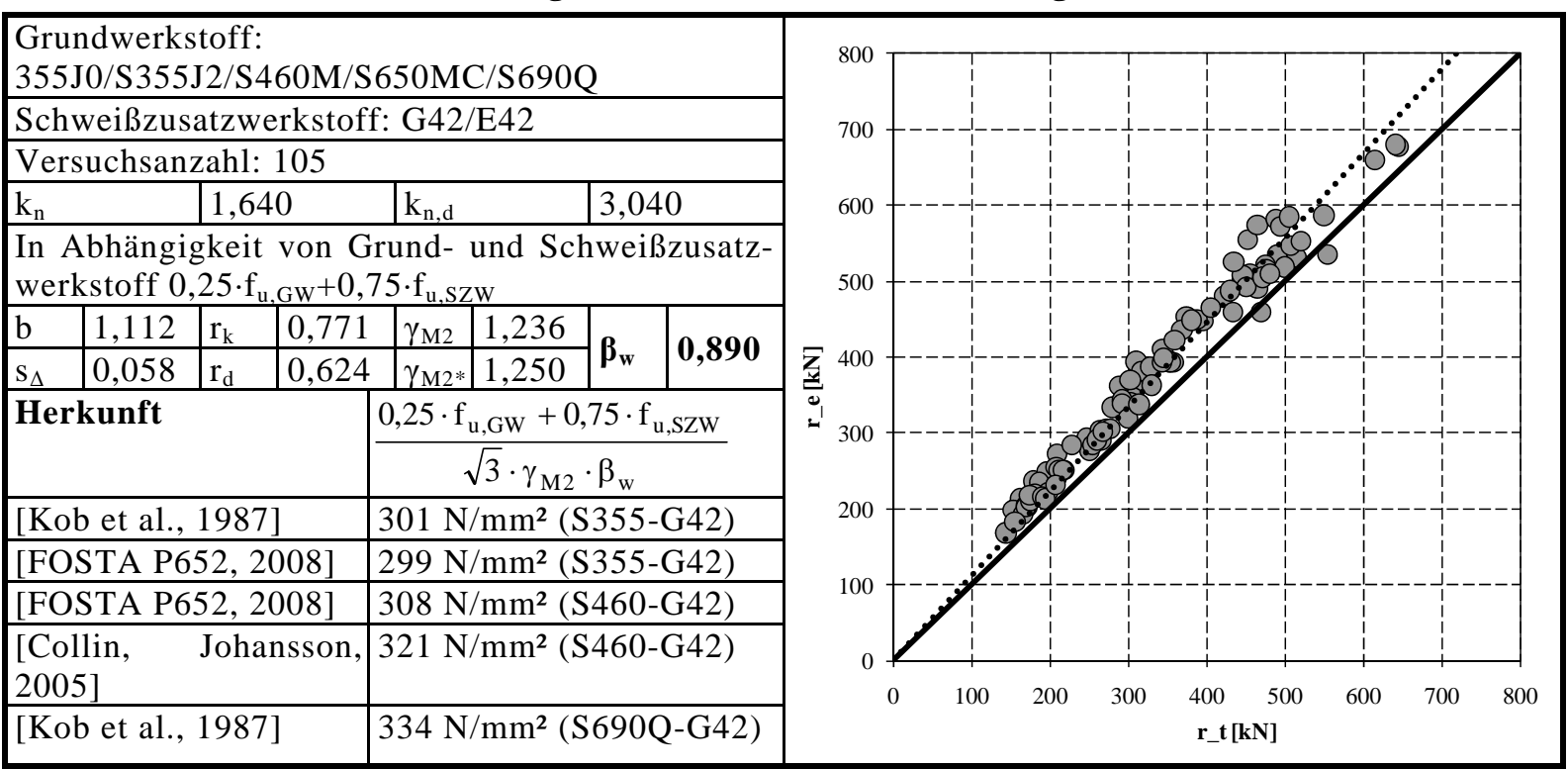




\section{A.5.3 Schweißverbindungen mit dem Schweißzusatzwerkstoff der nominellen Streckgrenze $f_{y}=460 \mathrm{~N} / \mathrm{mm}^{2}$}

Die Ergebnisse der statistischen Auswertung für Versuche mit den Schweißzusatzwerkstoffen G46, T46 und E46 sind in Tabelle A.56 aufgeführt.

Tabelle A.56: Statistische Auswertung aller Flankenkehlnahtverbindungen G/T/E46

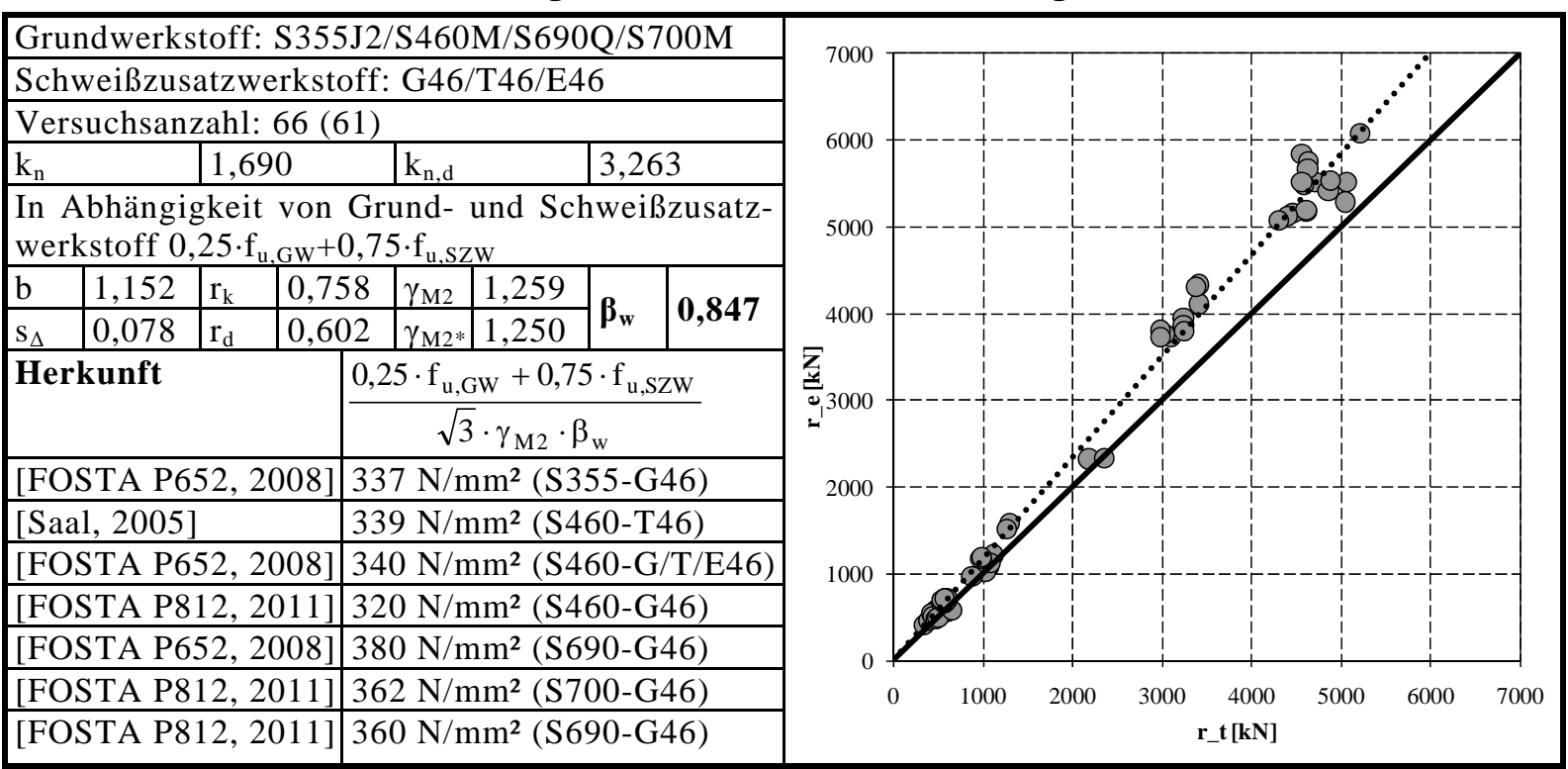

\section{A.5.4 Schweißverbindungen mit dem Schweißzusatzwerkstoff der nominellen Streckgrenze $\mathrm{f}_{\mathrm{y}}=550 \mathrm{~N} / \mathrm{mm}^{2}$}

Für Versuche mit dem Schweißzusatzwerkstoff E55 sind die Ergebnisse der statistischen Auswertung, in Tabelle A.57 vorgestellt.

Tabelle A.57: Statistische Auswertung aller Flankenkehlnahtverbindungen E55

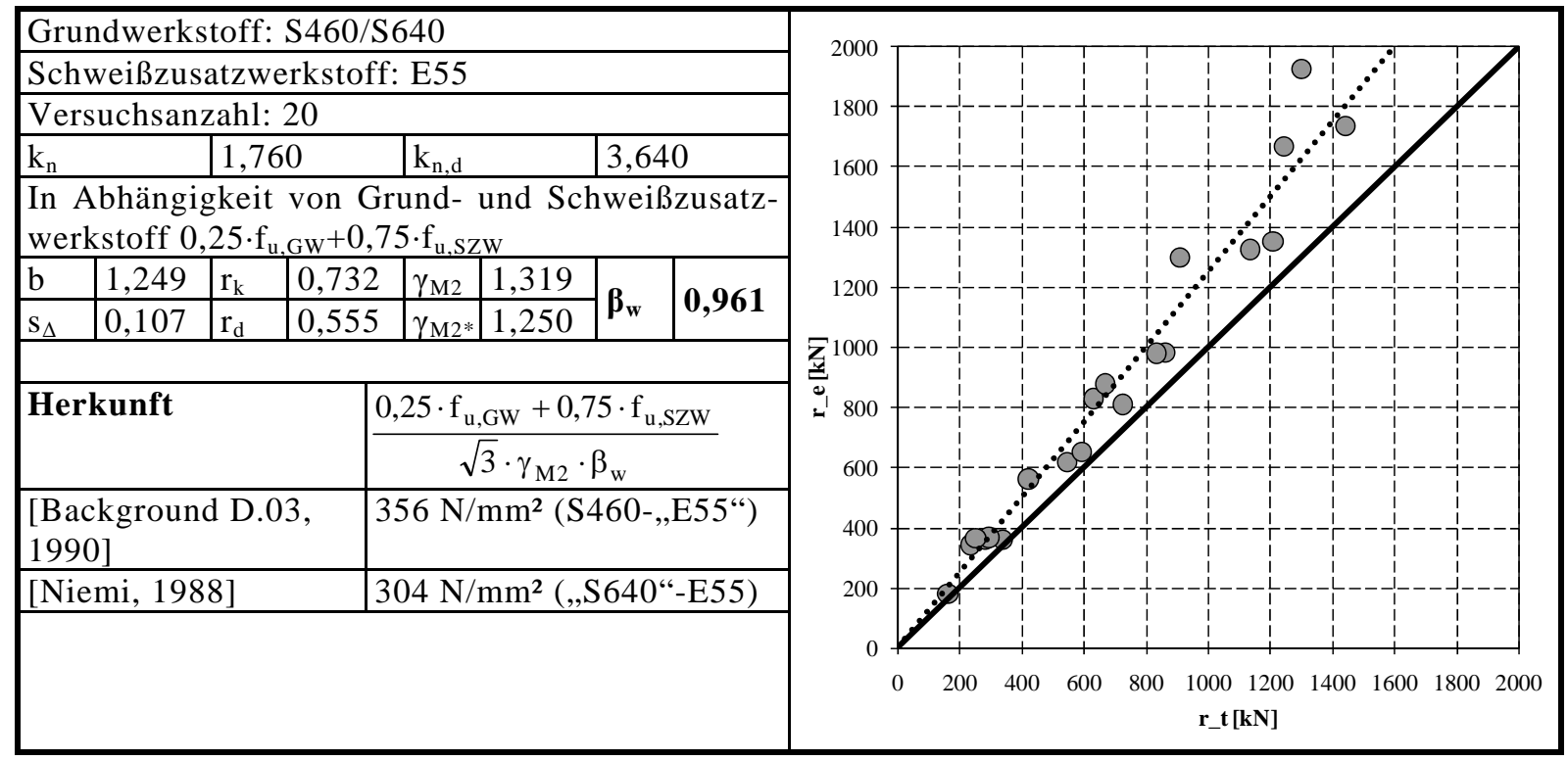




\section{A.5.5 Schweißverbindungen mit dem Schweißzusatzwerkstoff der nominellen Streckgrenze $f_{\mathbf{y}}=690 \mathrm{~N} / \mathrm{mm}^{2}$}

Die Ergebnisse der statistischen Auswertung für Schweißverbindungen mit den Schweißzusatzwerkstoffen G69 und T69 sind in Tabelle A.58 aufgeführt.

Tabelle A.58: Statistische Auswertung aller Flankenkehlnahtverbindungen G/T69

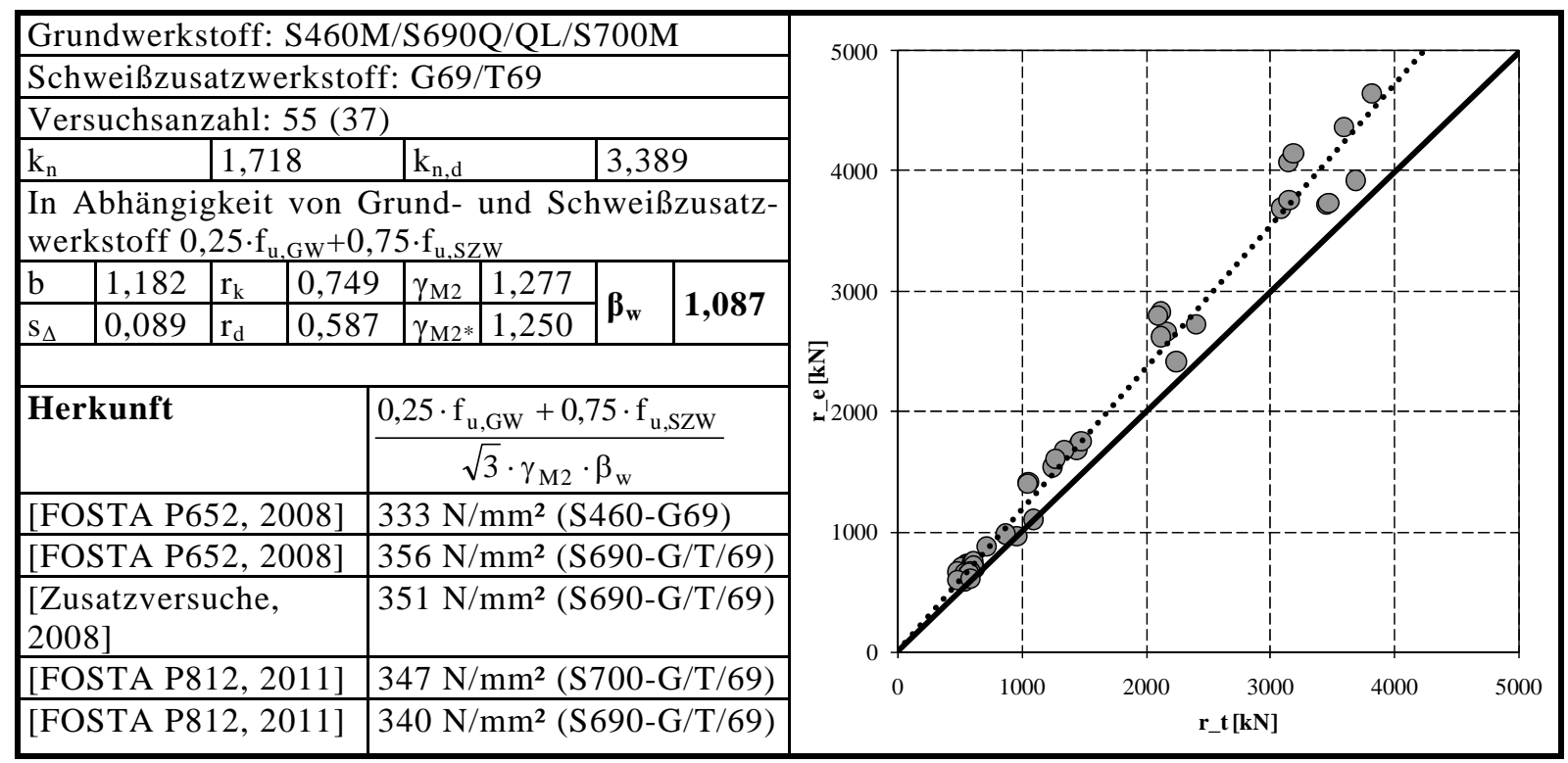

\section{A.5.6 Schweißverbindungen mit dem Schweißzusatzwerkstoff der nominellen Streckgrenze $\mathrm{f}_{\mathrm{y}}=\mathbf{8 9 0} \mathrm{N} / \mathrm{mm}^{2}$}

Die Ergebnisse der statistischen Auswertung für Schweißverbindungen mit dem Schweißzusatzwerkstoff G89 zeigt Tabelle A.59.

Tabelle A.59: Statistische Auswertung aller Flankenkehlnahtverbindungen G/T69

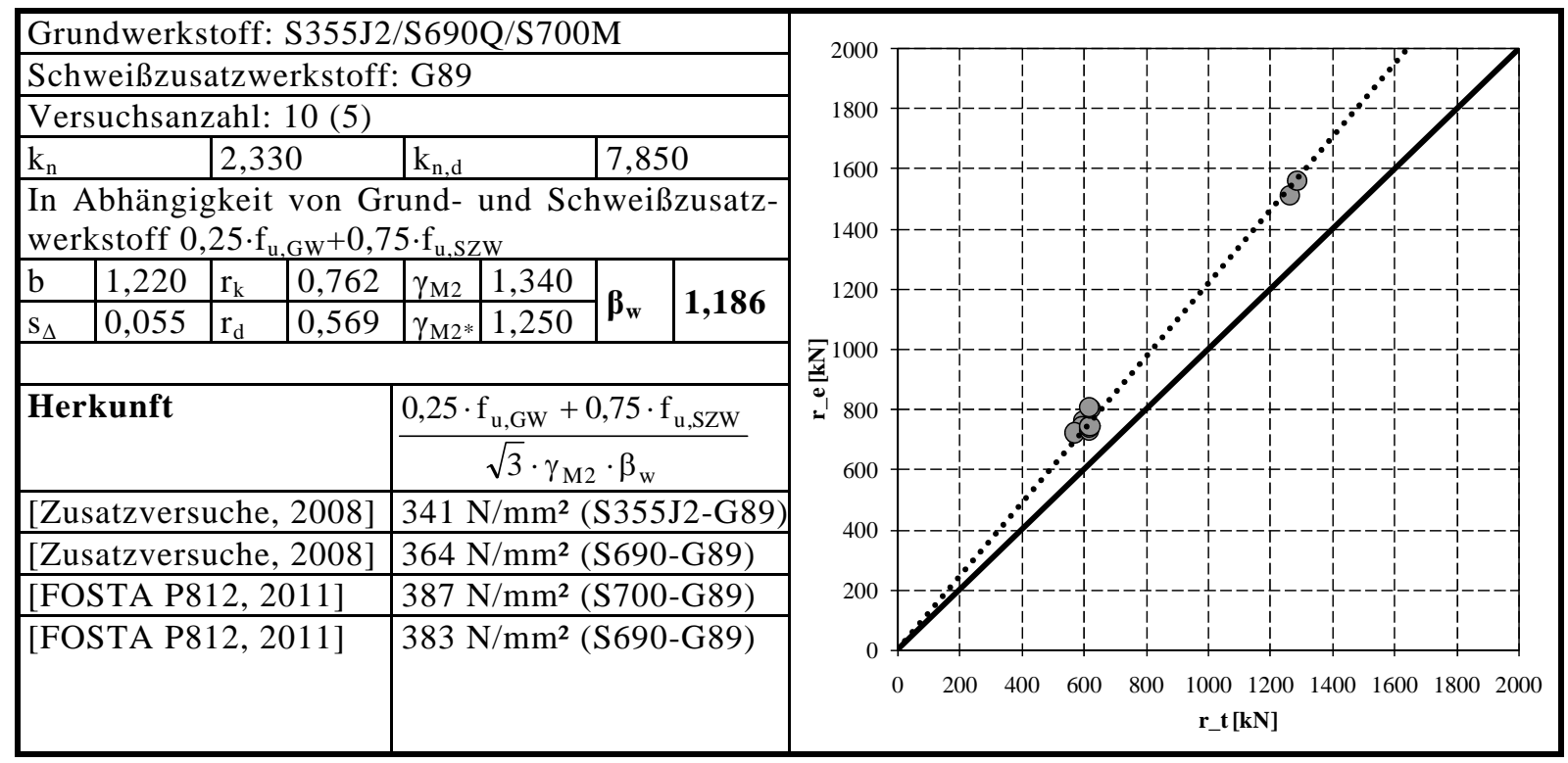

